Centro de Investigación del Cáncer

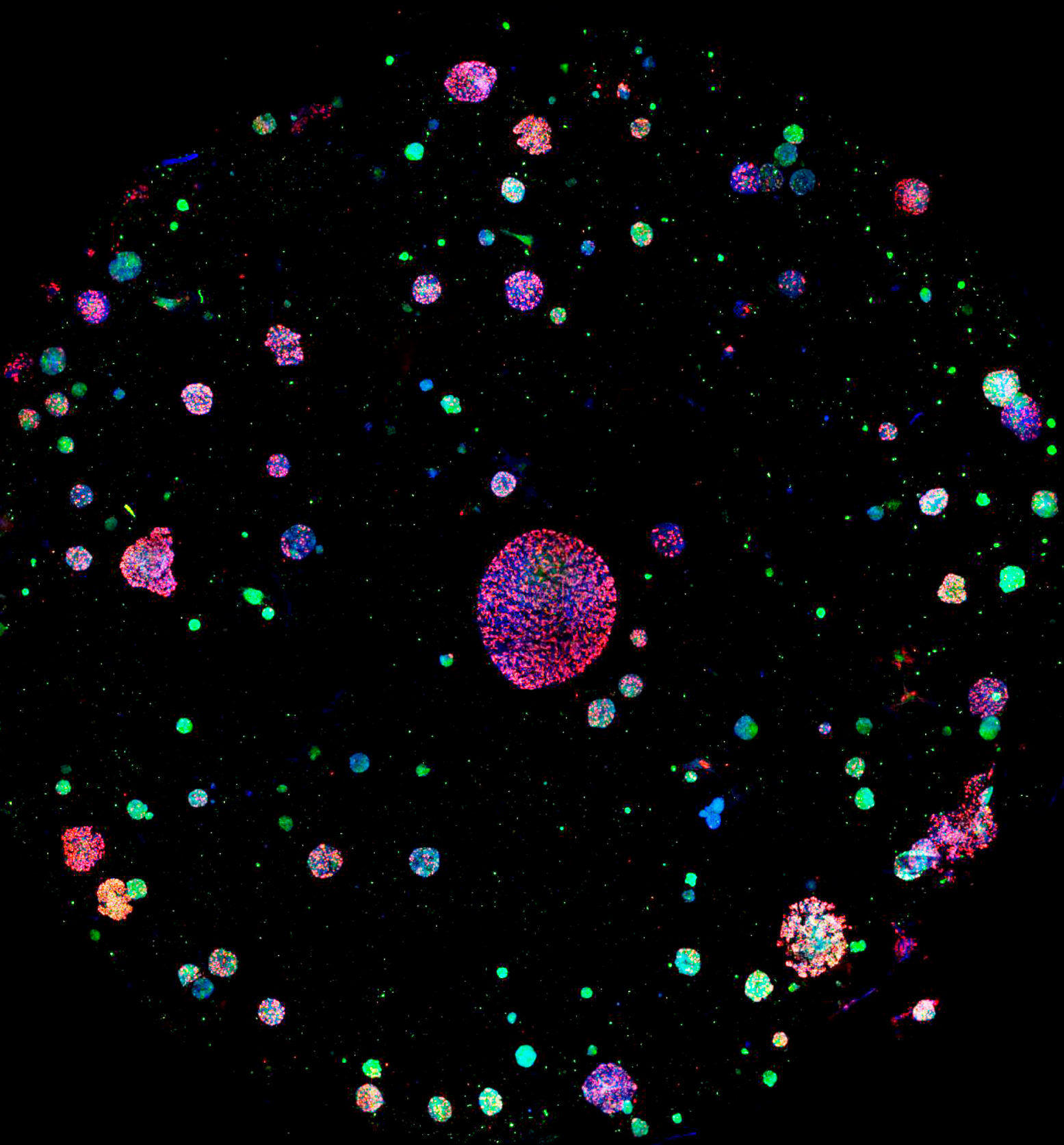

Role of HRas and NRas in murine lung development and neonatal survival

Ph.D. dissertation

Rocío Fuentes Mateos

2021 

Centro de Investigación del Cáncer / Instituto de Biología Molecular y Celular del Cáncer (CSIC-USAL)

Role OF HRAS AND NRAS IN MURINE LUNG DEVELOPMENT AND NEONATAL SURVIVAL

Thesis Dissertation

Rocío Fuentes Mateos

Salamanca, 2021 

D. Eugenio Santos, Catedrático del Departamento de Microbiología y Genética de la Universidad de Salamanca y, Director del Centro de Investigación del Cáncer de Salamanca; y D. Alberto Fernández Medarde, Profesor Contratado Doctor en el Departamento de Bioquímica y Biología Molecular de la Universidad de Salamanca,

\section{Certifican:}

Que la Tesis Doctoral titulada "Role of HRas and NRas in murine lung development and neonatal survival” presentada por Rocío Fuentes Mateos, graduada en Bioquímica y Biología molecular, ha sido realizada bajo su dirección en el Centro de Investigación del Cáncer de Salamanca y reúne, a su juicio, originalidad y contenidos suficientes para que sea presentada ante el tribunal correspondiente y para optar al grado de Doctor con Mención Internacional por la Universidad de Salamanca.

Y para que conste, expiden el presente certificado:

Salamanca, 02 de Noviembre de 2021

Eugenio Santos

Director
Alberto Fernández Medarde

Co-director 



\section{Abstract}

Ras GTPases are pivotal signal transduction molecules regulating cellular differentiation, proliferation, migration, apoptosis, and survival. They act as molecular switches by cycling between an inactive (GDP-bound) and active (GTP-bound) conformation in a process tightly modulated by GTPase activating proteins (GAPs, negative regulators) and Guanosine Exchange Factors (GEFs, positive regulators). Among the more than 150 known small GTPases, the canonical Ras subfamily includes HRas, NRas, as well as the two splicing isoforms, KRas4A and KRas4B. Despite their high similarity, the different Ras isoforms are not functionally redundant. Only the individual lack of KRas4B or the combined removal of HRas and NRas together with KRas haploinsufficiency result in embryonic lethality. Interestingly, we observed that HRas/NRas-devoid animals showed a significantly higher-than-expected mortality rate during the first hours after birth that was associated to severe respiratory distress. This dissertation presents an extensive analysis of knockout (KO) mouse models for HRas and/or NRas with an aim at uncovering specific or redundant functionalities of these two canonical Ras GTPases in lung embryonic development.

HRas/NRas-double mutant (DKO), and to a lesser extent HRas-knockout (HRas-KO) animals, exhibited delayed lung maturation as revealed by a reduced overall alveolar space, thicker separating alveoli septa, and increased retention of alveolar progenitors and of bi-potent progenitors in distal lung epithelium. Structural alterations were also present in the bronchiolar cell lineage, with all HRas, NRas and DKO mice showing evident flattening of Club and Ciliated cells. We also observed impaired lung proliferation in mice lacking HRas, with a retention of alveoli proliferation instead of differentiation at late stages of embryonic development; On the other hand, we observed an increase in cell death in alveolar regions of NRas-depleted mice, that was much further aggravated under concomitant HRas and NRas ablation. Additionally, increased levels of cellular ceramide and oxidative stress, together with mitochondrial electron transport chain alterations and infiltrated neutrophils were detected in lungs of HRas/NRas-devoid newborns. Furthermore, the few surviving DKO adult mice showed a reduced body size, facial dysmorphia and a patched lung phenotype with alveolar atelectasis areas next to emphysema lesions.

Treatment with dexamethasone, a glucocorticoid that boosts lung maturation, reverted the lung immaturity in our DKO animals, showing normal alveolar cells differentiation and lung inflation. However, it failed to abrogate lethality since the lifespan of the HRas/NRas-devoid pups was only extended for up to 6 days. We also evaluated the effect of antenatal antioxidant therapy on modulation of lung maturation by treatment with N-Acetylcysteine (NAC), which resulted in normal lung development and the survival of $50 \%$ of the DKO animals up to adulthood. 
Additionally, the redox imbalance in the lungs of the DKO pups was fully corrected with NAC, and only partially with dexamethasone, although mitochondrial respiration parameters were recovered after both treatments.

Using primary lung fibroblasts from newborn pups devoid of HRas and/or NRas we analysed whether the lung mesenchyme was responsible for the observed in vivo lung defects. Our results showed that primary lung fibroblasts lacking HRas had a dysregulation of the Hox5-Wnt2FGF7/10 pathway. Additionally, concomitant HRas and NRas ablation resulted in unbalanced redox status, and worsened mitochondrial respiration. These alterations were partially mitigated after in vitro treatment of the mesenchymal cells with dexamethasone, and more strongly bypassed after NAC addition. On the other hand, aberrant epithelial signalling downstream of mesenchymal FGF10 was observed in counterpart epithelium lacking HRas, with an increased expression and activation of the FGFR2b-KRas-SOX9 branching pathway.

Using lung organoids we investigated the epithelium-mesenchymal interactions with a wild-type epithelium in presence of HRas and/or NRas mutant mesenchyme. The organoids lacking HRas recapitulated the alterations observed in the lung fibroblasts cultures and newborn pups, with fewer alveolar organoids after 14-days of culture. This effect was corrected after NAC administration and, more strongly, after MAPK/Erk inhibition with UO126 inhibitor, obtaining increased alveolar differentiation levels. Additionally, activation of KRas and downstream Erk was higher in lungs from DKO pups, and these signalling defects were corrected by antenatal dexamethasone and more robustly with NAC.

Our data show that HRas, with partial overlapping contribution of NRas, exerts key roles in the last stages of mouse lung maturation controlling KRas activation. These effects are already visible in single HRas knockout embryos and become more visible when both HRas and NRas are absent. Moreover, surviving adult HRas/NRas-DKO mice exhibit a KRas-linked phenotype resembling that of known RASopathies. This study demonstrates the relevance of HRas in modulation of KRas-dependent signalling in tissue and cellular homeostasis. 


\section{Resumen}

Las GTPasas Ras controlan rutas de señalización implicadas en proliferación, migración, muerte y supervivencia celular, Actúan como interruptores moleculares, alternando entre una conformación inactiva (unido a GDP) y activa (unido a GTP), estando este proceso altamente regulado por proteínas activadoras de la actividad GTPasa intrínseca de Ras (GAPs, reguladores negativos), y factores de intercambio de nucleótidos de guanosina (GEFs, reguladores positivos). De entre las más de 150 GTPasas conocidas, la subfamilia de GTPasas Ras canonicas está constituida por HRas, NRas, y por las dos variantes Kras4A y Kras4B. A pesar de la gran homología, no son funcionalmente redundantes. Solo la pérdida individual de Kras4B, o la eliminación combinada de HRas y NRas junto con una haploinsuficiencia de Kras produce letalidad embrionaria. Sin embargo, hemos observado que la eliminación conjunta de HRas y NRas provocaba un aumento significativo de la letalidad perinatal, asociada con insuficiencia respiratoria. En esta Tesis Doctoral se ha llevado a cabo un análisis detallado de los modelos murinos knockouts (KO) para HRas y/o NRas con el fin de evaluar la especificidad o redundancia funcional de las dos GTPasas canónicas durante el desarrollo embrionario del pulmón.

Los animales doble mutantes para HRas y NRas (DKO), y en menor medida los mutantes sencillos para HRas (HRas-KO), mostraron un retraso en la maduración pulmonar, demostrado con una menor apertura de los alveolos, engrosamiento de los septos alveolares y un aumento del número de células progenitoras y bi-potentes alveolares en las zonas distales del pulmón. En relación al epitelio bronquial, se observaron notables alteraciones en la estructura de las células secretoras (Club) y Ciliadas, presentando una morfología cuadrada en lugar de columnar. Alteraciones en la proliferación fueron detectadas en animales carentes de HRas, presentando a estadios tardíos de desarrollo embrionario, una persistencia en la proliferación alveolar en lugar de diferenciación. Además, la ausencia de NRas se correlacionaba con un aumento de la muerte celular por apoptosis en zonas alveolares, fenotipo que se veía agravado con la eliminación concomitante de HRas y NRas. De manera adicional, se detectó un incremento en la deposición de ceramidas, desbalance del estrés oxidativo, junto con alteraciones en la respiración mitocondrial e infiltración de neutrófilos en pulmones de animales neonatos carentes de HRas y NRas. Además, el reducido número de animales DKO que sobrevivieron hasta la edad adulta presentaban una reducción notable del tamaño corporal, alteraciones de la morfología facialcraneal y un fenotipo pulmonar parcheado con zonas de atelectasia junto a enfisemas.

El tratamiento con dexametasona, un glucocorticoide que acelera la maduración alveolar, revierte la inmadurez pulmonar de los animales DKO, mostrando una diferenciación alveolar normal y una apertura correcta de los alveolos. Sin embargo, el tratamiento con dexametasona no es suficiente puesto que todos los DKO tratados con glucocorticoides morían entre día 5-6. Por otro 
lado, también evaluamos el efecto del tratamiento antenatal con antioxidantes, usando para ello $\mathrm{N}$-Acetilcisteína (NAC), produciendo un desarrollo normal del pulmón y la supervivencia del $50 \%$ de los animales DKO hasta la edad adulta. De manera adicional, las alteraciones en el balance redox presentes en los animales DKO recién nacidos se corrigieron tras el tratamiento con NAC, y de manera parcial con dexametasona.

Mediante el uso de fibroblastos primarios de pulmón de animales neonatos carentes de HRas y/o NRas, analizamos si los defectos observados en el modelo murino in vivo eran ocasionados por la falta de HRas y/o NRas en el mesénquima pulmonar. Nuestros resultados mostraron que los fibroblastos primarios de pulmón carentes de HRas presentaban un desbalance en la señalización Hox5-Wnt2-FGF7/10; que junto con la eliminación concomitante con NRas, provocaban alteraciones en el balance redox y en la respiración mitocondrial. Estas alteraciones se mitigaban parcialmente con el tratamiento in vitro con dexametasona, siendo prácticamente eliminadas con la adición de NAC. Por otro lado, se observaron alteraciones en rutas de señalización en el epitelio pulmonar downstream FGF7/10 en animales carentes de HRas, presentando un incremento en la expresión génica y activación de la ruta FGFR2b-KRas-SOX9.

Mediante el uso de un modelo de organoides de pulmón analizamos las interacciones entre el epitelio y el mesénquima pulmonar, empleando para ello células epiteliales controles y fibroblastos de pulmón primarios carentes de HRas y/o NRas. Los organoides generados a partir de células mesenquimales carentes de HRas recapitularon las alteraciones ya observadas en el modelo murino, generando un menor número de organoides alveolares tras 14 días de cultivo. Este defecto se corregía tras la administración de NAC, y de manera más potente, tras la inhibición de la vía MAPK/Erk con el inhibidor UO126, obteniendo una mayor tasa de diferenciación alveolar en los organoides. De manera adicional, los pulmones de animales DKO neonatos presentaban una mayor activación aberrante de KRas y Erk la cual era corregida tras el tratamiento antenatal con dexametasona y, de manera más evidente, con NAC.

Nuestros datos sugieren que HRas, con contribución parcial de NRas, posee un papel clave en las últimas etapas del desarrollo pulmonar murino controlando la activación de KRas. Estos efectos ya son aparentes en los embriones carentes de HRas, viéndose agravado el efecto cuando ambos HRas y NRas han sido eliminados. Además, los animales DKO que sobreviven hasta la edad adulta presentan un fenotipo ligado a la mayor activación de KRas que mimetiza aquellos observados en modelos murinos de RASopatías. Este estudio demuestra la relevancia de HRas en la modulación de la señalización dependiente de KRas en tejido y en la homeostasis celular. 


\section{LIST OF ABBREVIATIONS}

Ac-Tub Acetylated Tubulin

AEC1 Alveolar epithelial cell type 1

AEC2 Alveolar epithelial cell type 2

AKT Protein kinase B

BMP Bone morphogenic protein

BMP4 Bone morphogenic protein 4

BrdU Bromodeoxyuridine

BSA Bovine serum albumin

CFC Cardio-facial-cutaneous syndrome

CNrasGEF Cyclic Nucleotide dependent Ras GEF

CS Costello syndrome

DAPI 4',6-diamine-2-fenilindole

DCFH-DA 2'-7'dichlorofluorescin diacetate

DEX Dexamethasone

DHE Dihydroethidium

DKO Double knockout

DMEM Dulbecco's Modified

Eagle's Medium

EDTA Ethylenediaminetetraacetic acid

EGF Epidermal Growth Factor

FBS Fetal bovine serum

FGF Fibroblast growth factor

FGF10 Fibroblast growth factor 10

FGF7 Fibroblast growth factor 7

FGFR2b Fibroblast growth factor receptor $2 b$

GAPs GTPase activating proteins

GDP Guanosine 5'-diphosphate

GEFs Guanine nucleotide exchange factors
GSH Glutathione

GTP Guanosine 5'-triphosphate

h hours

IPTG Isopropil- $\beta$-D-1-

tiogalactopiranósido

KO knockout

min minutes

NAC N-Acetylcysteine

NE Neuroendocrine

NF1 Neurofibromin 1

$\mathrm{o} / \mathrm{n} \quad$ overnight

p.c. post coitum

PBS Phosphate saline buffer

PCR Polymerase chain reaction

PDGF Platelet Derived Growth Factor

PI3K Phosphoinositide 3-kinase

$\mathrm{PIP}_{2}$ Phosphatidylinositol 4,5-

bisphosphate

$\mathrm{PIP}_{3}$ Phosphatidylinositol-3,4,5-

triphosphate

PKC Protein kinase C

PLC Phospholipase C

RAF Rapidly accelerated fibrosarcoma

RalGDS Ras-like guanine nucleotide dissociator stimulator

RAS Rat sarcoma

RasGRF Ras-specific guanine nucleotide releasing factor

RasGRP Ras guanyl-releasing protein

RCA-I Ricinus communis agglutinin-I

ER Endoplasmic reticulum

Redox Reduction-oxidation 
REM Ras exchange motif

ROS Reactive oxygen species

RT Room temperature

Scgb1a1 Secretoglobulin 1a1

SDS Sodium dodecyl sulfate

sec seconds

SftpC Surfactant protein C

SH2 Src homology domain 2

SH3 Src homology domain 3

SHH Sonic hedgehog

SN Noonan syndrome
SOS Son of Sevenless

SOX2 Sex-determining region Y-box 2

SOX9 Sex-determining region Y-box 9

SP Surfactant proteins

Spry2 Sprouty 2

TBS-T Saline Tris buffer with Tween

TCR T-cell receptor

VEGF Vascular Endothelial Growth

Factor

WT Wildtype

$\beta$-Tub $\beta$-Tubulin 


\section{LIST OF FIGURES}

Figure 1: Comparison between human and mouse lung structure.........................

Figure 2: Adult lung cell composition...................................................

Figure 3: Signalling overview of the crosstalk between mesenchyme and epithelium during

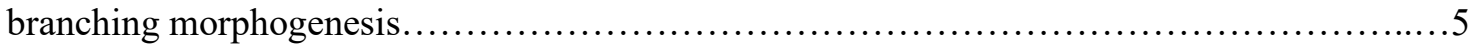

Figure 4: Origin of the lung cell lineages and their markers.............................6

Figure 5: RAS activation and deactivation cycle........................................ 12

Figure 6: Canonical RAS GTPases structure........................................ 14

Figure 7: Differential rAS processing and delivery to the plasma membrane.................15

Figure 8: RAS GEFs family members and main domains................................18

Figure 9: RAS GAPs family members and main domains................................

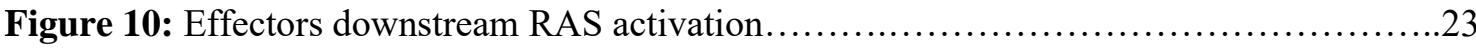

Figure 11: Representative images of PCR assays......................................

Figure 12: Antenatal dexamethasone (DEX) treatment...................................33

Figure 13: Antenatal N-Acetylcysteine (NAC) treatment.......................................

Figure 14: Agilent Seahorse MitoStress test modulators....................................44

Figure 15: Agilent Seahorse MitoStress lung tissue test profile.............................45

Figure 16: Lung fibroblast isolation................................................ 46

Figure 17: Agilent Seahorse MitoStress lung fibroblasts test profile.......................48

Figure 18: Lung organoid co-culture assay.......................................... 52

Figure 19: Analysis of the offspring from crosses between HRas-KO and/or NRas-KO

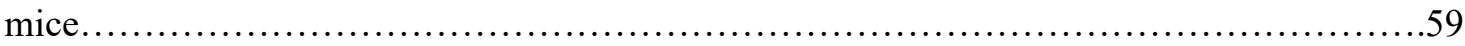

Figure 20: Histological analysis of the lungs of newborn pups (P0) and late embryos (E18.5) of

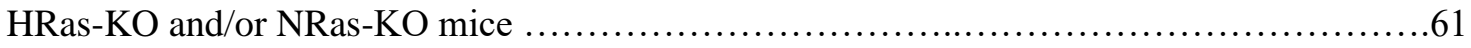

Figure 21: Immunostaining of alveolar differentiation markers in the lungs of HRas-KO and/or NRas-KO mice

Figure 22: Immunostaining of bronchiolar differentiation markers in the lungs of HRas-KO and/or NRas-KO mice.

Figure 23: Analysis of proliferative and apoptotic rates in the lungs of HRas-KO and/or NRasKO mice .66

Figure 24: Increased neutrophil infiltration in the lungs of HRas/NRas-DKO mice

Figure 25: Differential gene expression in the lungs of HRas/NRas-DKO newborn P0 mice 
Figure 26: Increased expression of enzymes implicated in ceramide synthesis in DKO lungs .70

Figure 27: Ceramide immunoassays in alveolar and bronchiolar regions of the lungs of HRas and/or NRas KO mice.

Figure 28: Adult HRas/NRas-DKO mice show partial lung atelectasis and facial dysmorphia .72

Figure 29: Craniomorphometric analyses of the skulls of adult CT and HRas/NRas-DKO mice. .73

Figure 30: Lungs of P0 HRas/NRas-DKO mice show an increment in reactive oxygen species production

Figure 31: Lungs of P0 HRas/NRas-DKO mice show lower basal respiration and ATP-linked respiration .75

Figure 32: Effect of dexamethasone and antioxidant antenatal therapy on survival of DKO mice .77

Figure 33: Histological analysis of the lungs of newborn pups (P0) and late embryos (E18.5) of HRas-KO and/or NRas-KO mice subjected to dexamethasone or $\mathrm{N}$-acetylcysteine treatment

Figure 34: PAS staining analysis of the lungs of newborn pups (P0) and late embryos (E18.5) of HRas-KO and/or NRas-KO mice subjected to dexamethasone or $\mathrm{N}$-acetylcysteine treatment . .80

Figure 35: Immunostaining of alveolar differentiation markers in the lungs of NT-, DEX- and NAC-treated HRas-KO and/or NRas-KO mice ....

Figure 36: Immunostaining of SOX9+ alveolar progenitors in the lungs of NT-, DEX- and NACtreated HRas-KO and/or NRas-KO mice

Figure 37: Immunostaining of bronchiolar differentiation markers in the lungs of DEX- or NACtreated HRas-KO and/or NRas-KO mice

Figure 38: Increased neutrophil infiltration in the lungs of HRas/NRas-DKO mice is completely rectified after DEX treatment and only partially corrected after NAC administration . .85

Figure 39: Differential gene expression in the lungs of Dexamethasone-treated, HRas/NRasDKO newborn P0 mice. . .86

Figure 40: Differential gene expression in the lungs of NAC-treated, HRas/NRas-DKO newborn P0 mice.

Figure 41: Ceramide immunoassays in alveolar and bronchiolar regions of DEX- and NACtreated lungs of HRas and/or NRas KO mice.

Figure 42: Analyses of oxidative stress in lungs of P0 HRas-KO and/or NRas-KO after DEX or NAC treatment.

Figure 43: Analyses of mitochondrial respiration parameters analyses of P0 HRas/NRas-DKO lungs after DEX or NAC antenatal administration .... 
Figure 44: Gene expression analysis of the three Ras isoforms in lung epithelium and mesenchyme of $\mathrm{P} 0$ mice

Figure 45: Analyses of the expression of genes known to participate in mesenchymal FGF production suggest a dysregulation of the Wnt-FGF signalling pathway in the DKO samples

Figure 46: Expression analyses of genes known to participate in the epithelial signalling downstream FGF reveal a dysregulation of this pathway in the DKO samples and a reduction in alveolar differentiation markers.

Figure 47: Lung mesenchymal cells of P0 HRas/NRas-DKO mice show augmented production of reactive oxygen species. .96

Figure 48: Altered gene expression of enzymes implicated in ROS detoxifying pathways in DKO lung mesenchymal cells.

Figure 49: Lung mesenchymal cells devoid of HRas show lower basal respiration, spare respiratory capacity, and ATP-linked respiration.

Figure 50: Lung mesenchymal cells of P0 HRas/NRas-DKO mice show an increment in production of reactive oxygen species production.

Figure 51: Dexamethasone rescues the lower basal respiration, spare respiratory capacity and ATP-linked respiration observed in HRas-KOs and DKO lung fibroblasts. 100

Figure 52: NAC and DEX treatment of lung mesenchymal cells decreases expression of FGF and related genes as well as SOD3 in DKO cells... 101

Figure 53: Lung organoids obtained from untreated and DEX- and NAC- treated samples 103

Figure 54: Effects of DEX and NAC treatment on lung organoid differentiation 104

Figure 55: Effect of KRas, MEK1/2, PI3K and p38 inhibitors on lung organoid formation...106

Figure 56: Exposure to KRas, MEK1/2, PI3K and p38 inhibitors modulates lung organoid differentiation

Figure 57: Effect of KRas, MEK1/2, PI3K and p38 inhibitors on double-negative, doublepositive, alveolar or bronchiolar organoid proportions .108

Figure 58: Altered KRas expression and ERK activation in HRas-KO and/or NRAs-KO lung fibroblasts

Figure 59: Increased KRas expression in HRas-KO and/or NRas-KO lung fibroblasts after Fendiline and UO126 treatment. .110

Figure 60: UO126 efficiently decreases ERK activation in primary lung fibroblasts 111

Figure 61: AKT activation is not altered in primary lung fibroblasts devoid of HRas and/or NRas .112

Figure 62: Inhibition of p38 with SB202190 leads to a strong compensatory effect in primary lung fibroblasts

Figure 63: NAC and UO126 decrease FGF7 concentration in the culture media of lung organoids 
Figure 64: Ras activation and pERK levels in primary lung fibroblasts treated with DEX, NAC

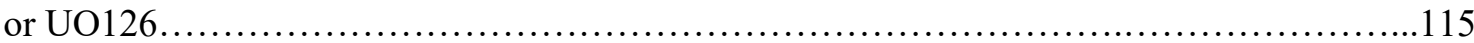

Figure 65: Increased KRas epithelial activation in DKO lungs is reversed after NAC antenatal

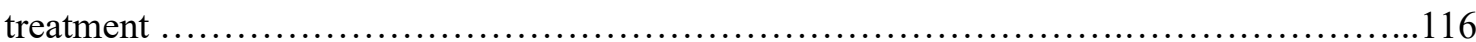

Figure 66: Increased epithelial ERK activation in DKO lungs is reversed after NAC antenatal treatment 


\section{Contents}

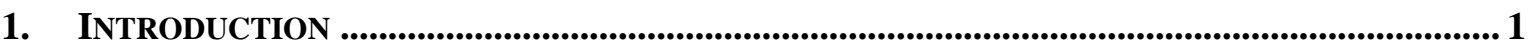

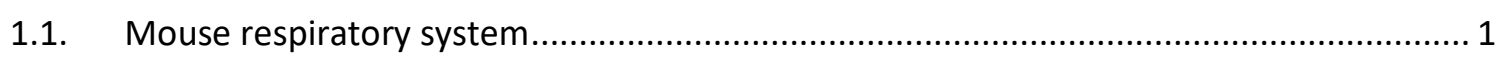

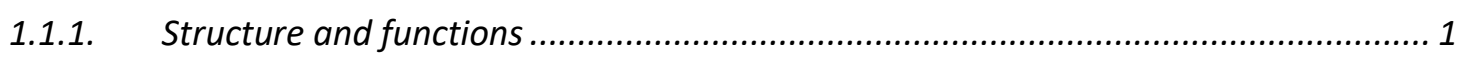

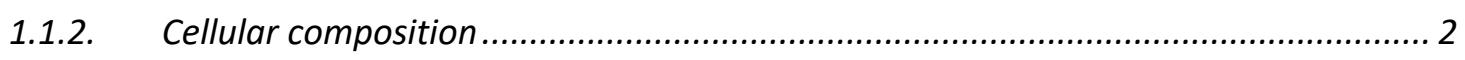

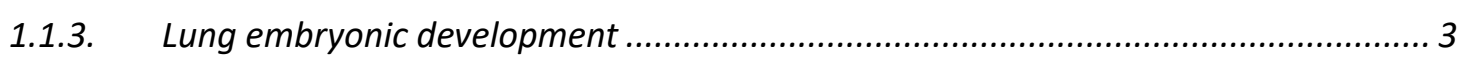

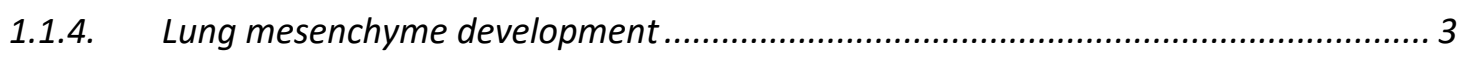

1.1.5. Epithelium-Mesenchyme interactions during lung development............................... 4

1.1.6. Lung epithelial cells differentiation ................................................................... 6

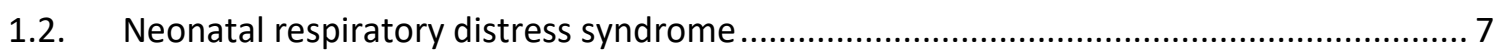

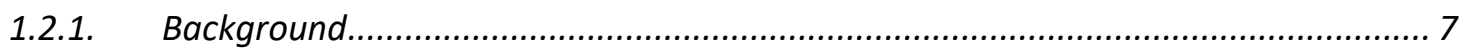

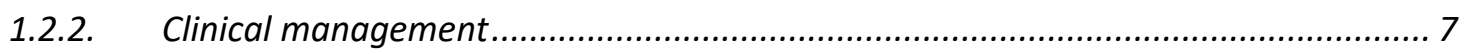

1.2.3. Genes and proteins implicated in NRDS susceptibility .......................................... 8

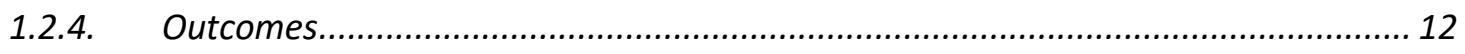

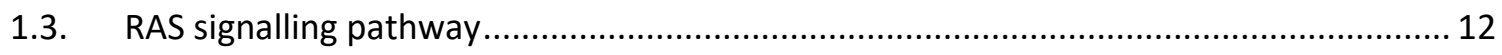

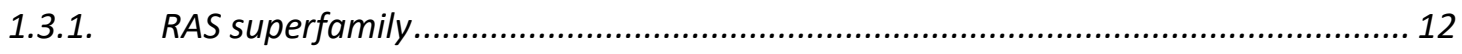

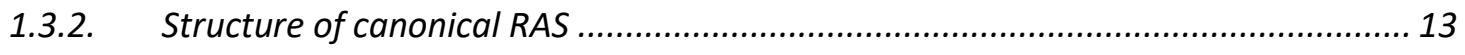

1.3.3. Post-translational modifications of canonical RAS................................................. 14

1.3.4. Differences in plasma membrane and endomembrane signalling among canonical

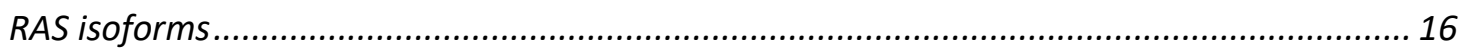

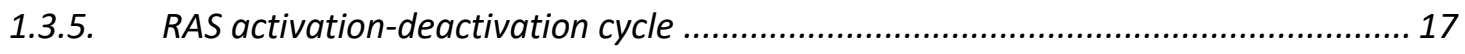

1.3.6. RAS activators (GEFs) and inhibitors (GAPS) proteins......................................... 18

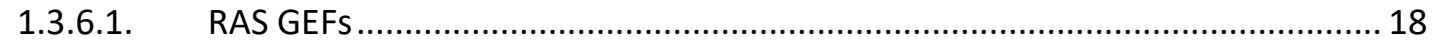

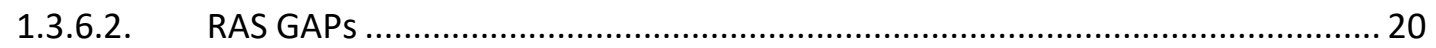

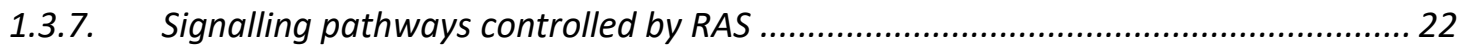

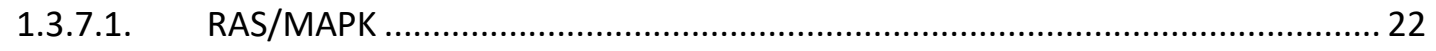

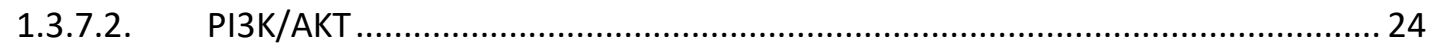

1.3.8. Functional specificity and redundancy of canonical Ras isoforms in KO mouse models

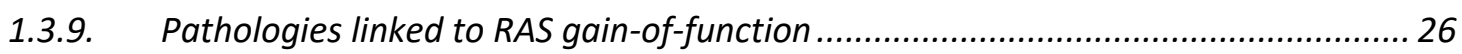

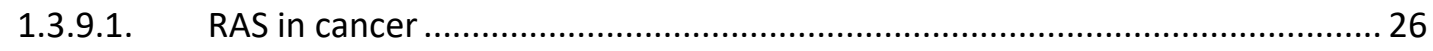

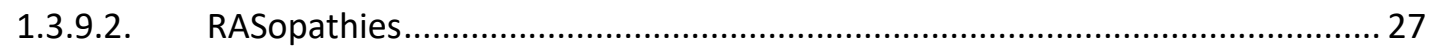

2. AIMS OF THE THESIS / OBJETIVOS........................................................................................... 29

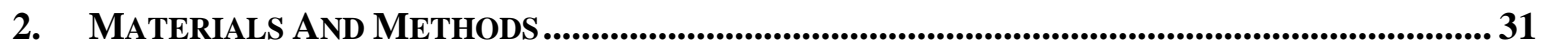

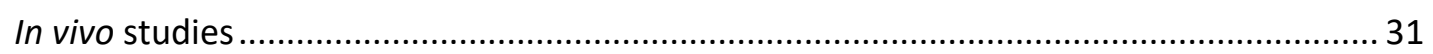

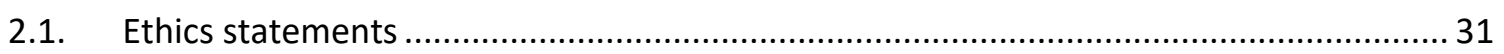


2.2. Generation of HRas and/or NRas null mice

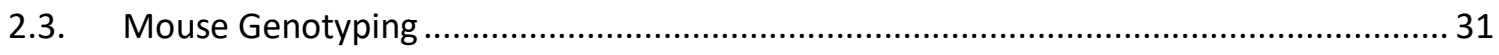

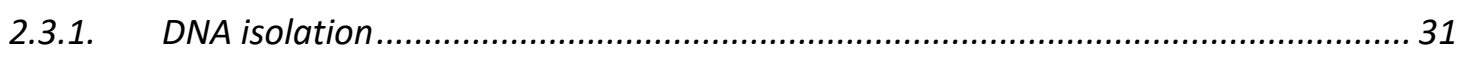

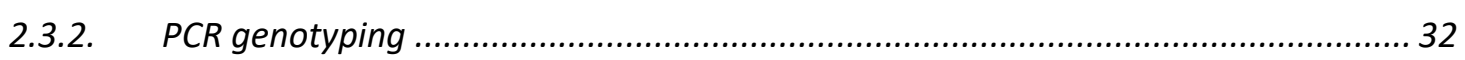

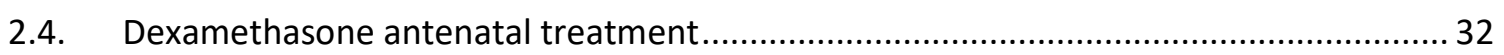

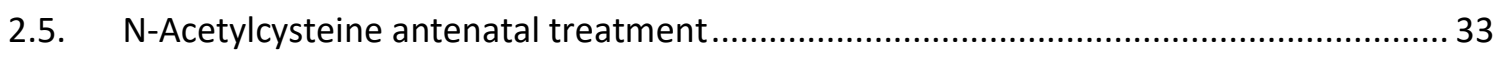

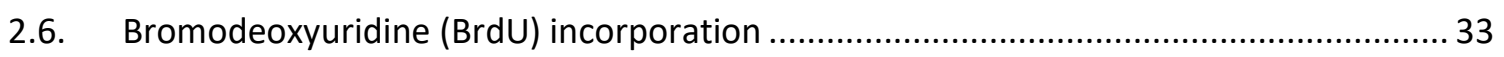

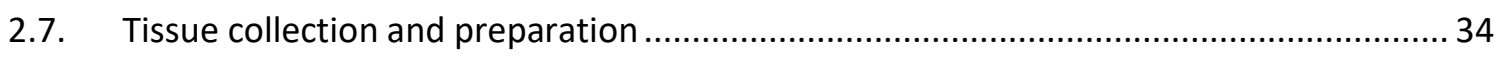

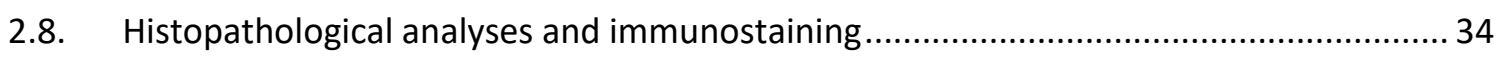

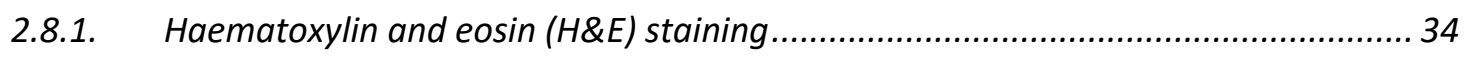

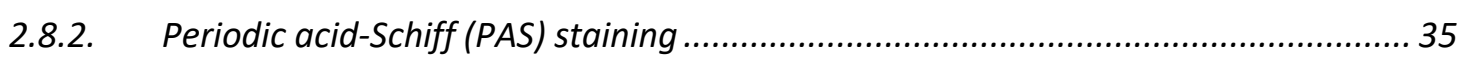

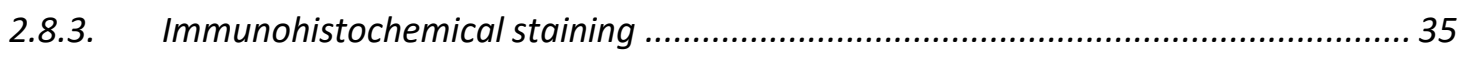

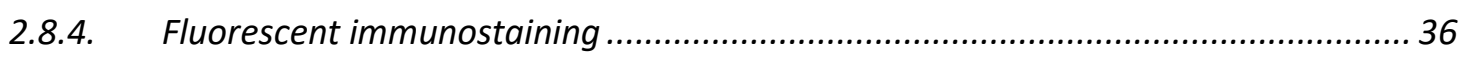

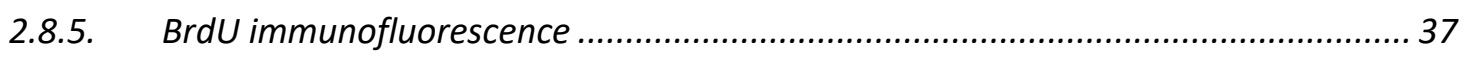

2.9. RNA isolation, RT-qPCR and microarray hibridations/lung transcriptome analysis......... 38

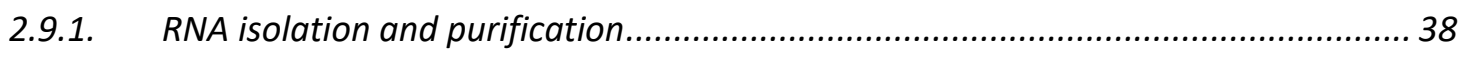

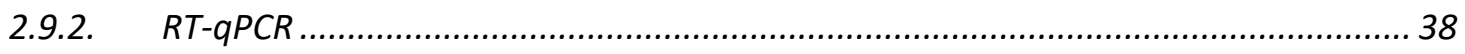

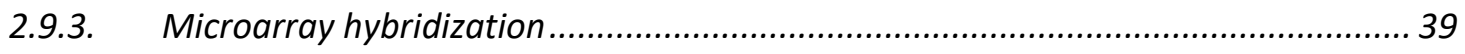

2.10. Western blot analysis in whole lung homogenates ..................................................... 40

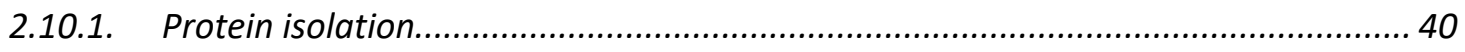

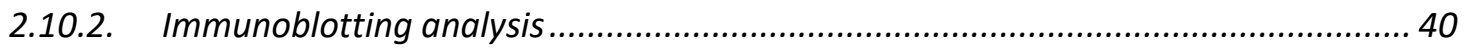

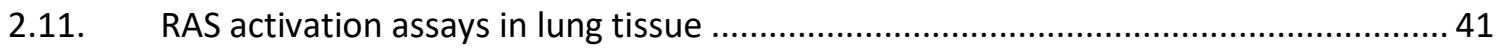

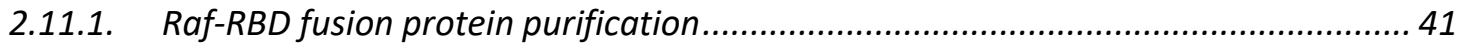

2.11.2. Binding of GST-Raf-RBD to Glutathione-Sepharose beads .................................... 42

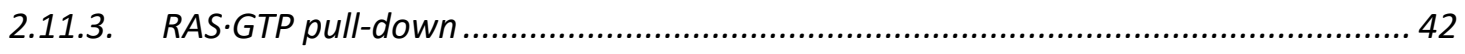

2.12. Reactive oxygen species analysis in lung tissue through flow cytometry.................... 43

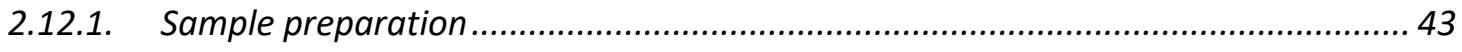

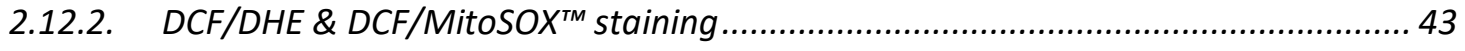

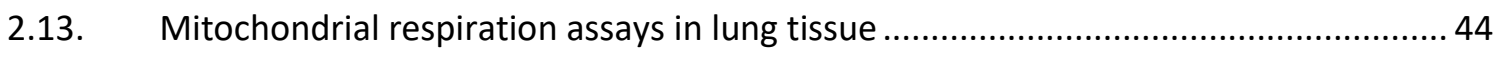

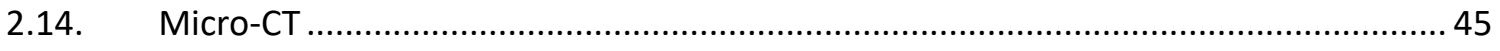

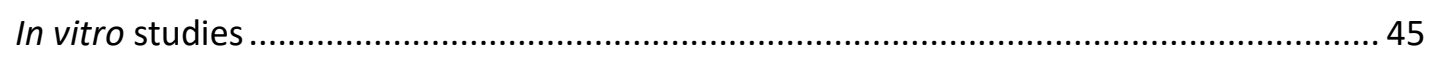

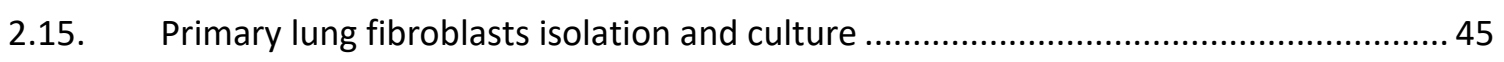

2.16. Reactive oxygen species assays in primary lung fibroblasts through flow cytometry 47

2.17. Mitochondrial respiration assays in primary lung fibroblasts ..................................... 47

2.18. RNA isolation and RT-qPCR analysis in primary lung fibroblasts................................ 48

2.19. Immunoblotting assays in primary lung fibroblasts ................................................... 50

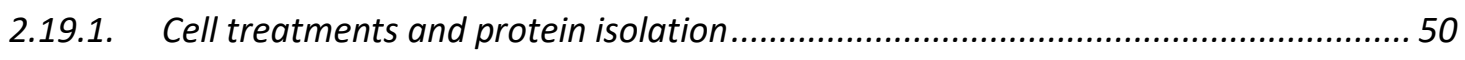


2.19.2. Immunoblotting analysis.

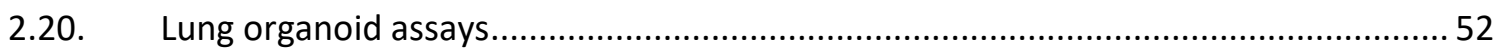

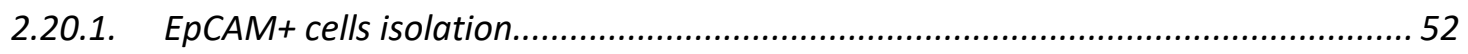

2.20.2. Proliferation-inactivation of primary lung fibroblasts.............................................5 54

2.20.3. Organoid co-culture seeding and treatments ................................................ 54

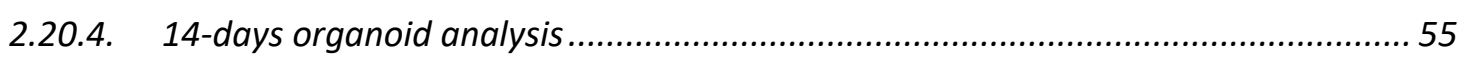

2.20.5. Organoid fixation and immunofluorescence staining ......................................... 55

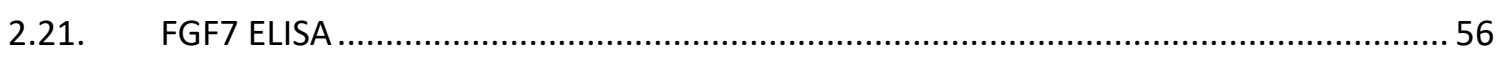

2.22. Ras activation assay in primary lung fibroblasts ............................................ 56

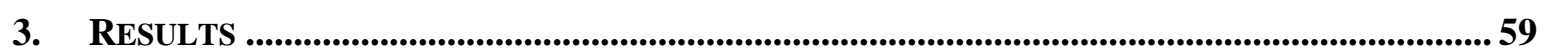

3.1. Phenotypes resulting from genetic deletion of HRas and/or NRas in mice..................59

3.1.1. HRas and NRas simultaneous ablation causes neonatal death ............................59

3.1.2. HRas/NRas double-null mice suffer neonatal death caused by respiratory failure.. 60

3.1.3. Alveolar cell lineage differentiation is altered in both HRas-KO and HRas/NRas-DKO animals.....

3.1.4. Individual or combined ablation of HRas and NRas causes structural alterations of bronchiolar cells................................................................................................ 62

3.1.5. Ablation of HRas leads to increased alveolar cell proliferation............................... 65

3.1.6. Increased apoptotic rates in PO lungs of NRas-ablated mice ................................. 65

3.1.7. Increased neutrophil infiltration in HRas/NRas-double null animals ....................... 67

3.1.8. HRas and NRas deficiency in PO mice causes strong transcriptional alterations in the lungs of $P O$ newborn mice ......................................................................................... 68

3.1.9. HRas and NRas deficiency alters lung sphingolipid metabolism and increases ceramide levels.................................................................................................. 69

3.1.10. Metabolic alterations linked to HRas and NRas ablation..................................... 70

3.1.11. Surviving HRas/NRas-DKO mice display facial dysmorphia and a patched lung

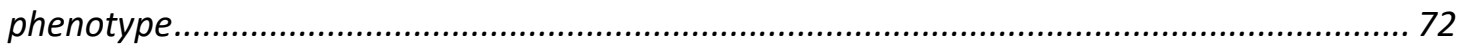

3.2. Altered lung redox homeostasis and mitochondrial respiration in mice lacking HRas and

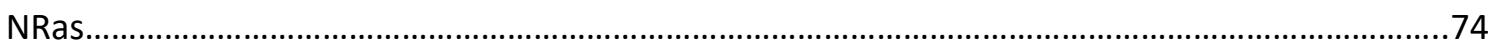

3.2.1. HRas/NRas double-KO mouse lungs exhibit an increase in reactive oxygen species production 74

3.2.2. The lungs of HRas/NRas-DKO mice show altered mitochondrial respiratory parameters and a decrease in ATP production

3.3. Characterization of the effect of antenatal glucocorticoid or $\mathrm{N}$-acetylcysteine administration on lung development and postnatal survival of HRas/NRas-double null mice ... 76

3.3.1. Antenatal glucocorticoid administration extends the lifespan of DKO pups up to six

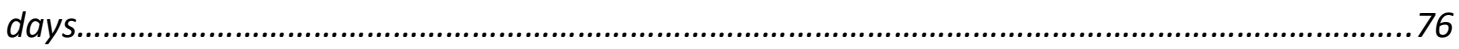

3.3.2. Treatment with $\mathrm{N}$-Acetylcysteine ameliorates the neonatal lethality of HRas/NRasDKO mice .76

3.3.3. Recovery of normal lung differentiation after DEX and NAC antenatal treatments 78 
3.3.4. Bronchiolar cell lineages show no alterations after DEX or NAC treatments.....

3.3.5. Neutrophil infiltration.

3.3.6. Transcriptomic changes induced by antenatal dexamethasone treatment in the lungs of newborn DKO mice. 84

3.3.7. Transcriptomic changes induced after NAC treatment in PO lungs of DKO mice .....86

3.3.8. Ceramide levels are depleted after antenatal administration of either DEX or NAC88

3.3.9. Effect of antenatal administration of DEX or NAC on ROS generation .................. 89

3.3.10. Seahorse tests of lungs from mice treated with either NAC or DEX...................... 90

3.4. Effect of HRas/NRas ablation on epithelium-mesenchyme interaction ........................ 91

3.4.1. HRas and KRas expression is increased in NRas deficient lung epithelium whereas KRas expression is increased in lung mesenchyme lacking both HRas and NRas .................. 91

3.4.2. Lack of HRas in lung mesenchyme causes higher levels of FGF7 and FGF10 .......... 93

3.4.3. FGF7 and FGF10 lung epithelial downstream signalling is altered in the lung epithelium after HRas/NRas ablation.......................................................................... 94

3.4.4. Lung mesenchyme lacking HRas and NRas GTPases show increased ROS and dysregulation of ROS detoxifying enzymes ............................................................. 96

3.4.5. Concomitant ablation of HRas and NRas in lung mesenchymal cells worsens mitochondrial respiration...

3.4.6. NAC treatment reduces mesenchymal oxidative stress and reduces mitochondrial respiration whereas DEX treatment partially lower ROS production and enhances mitochondrial respiration. 99

3.4.7. Mesenchymal gene expression changes linked to DEX and NAC treatment ......... 100

3.5. HRas isoform function is crucial for KRas-driven lung branching termination, with NRas exhibiting also a partial overlapping function.... 102

3.5.1. Loss of HRas and NRas is associated to augmented yields of small lung organoids .102

3.5.2. HRas and NRas activity is necessary for proper lung organoid differentiation and NAC partially rescues the aberrant phenotype. 103

3.5.3. KRas signalling is crucial for lung organoid formation onset. 105

3.5.4. Inhibition of Ras-MEK-ERK signalling rescues alveolar differentiation in HRas/NRasDKO lung organoids..... 105

3.5.5. Effect of dexamethasone and $N$-Acetylcysteine on Ras downstream signalling pathways... 108

3.5.6. Effect of different inhibitors of Ras signalling on KRas expression and the activation of downstream signalling pathways in KO and CT lung mesenchymal cells ........................ 109

3.5.7. Lack of HRas enhances FGF7 production in HRas-KO and DKO lung organoids..... 113

3.5.8. Ras activation is not significantly affected in the lung mesenchyme after DEX or NAC addition..

3.5.9. Combined absence of HRas and NRas leads to increased epithelial KRas activation and $p E R K$ levels. 116

4. DISCUSSION 119 
4.1. Phenotypes resulting from genetic deletion of HRas and/or NRas in mice

4.2. Altered lung redox homeostasis and mitochondrial respiration in mice lacking HRas and NRas.

4.3. Characterization of the effect of antenatal glucocorticoid administration on lung development and postnatal survival of HRas/NRas-double null mice

4.4. Characterization of the effect of antenatal $\mathrm{N}$-Acetylcysteine administration on lung development and postnatal survival of HRas/NRas-double null mice.....

4.5. Effect of HRas/NRas ablation on epithelium-mesenchyme interaction 125

4.6. HRas function is crucial for KRas-driven lung branching termination, with NRas exhibiting also a partial overlapping function.

4.7. Adult HRas/NRas-deficient mouse exhibit partial atelectasis and a KRas-linked phenotype resembling RASopathies.

4.8. General discussion................................................................................ 130

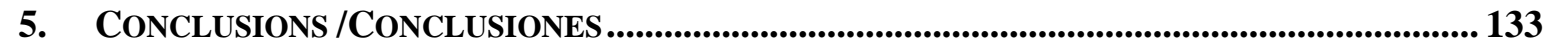

6. BIBLIOGRAPHY ........................................................................................................... 135

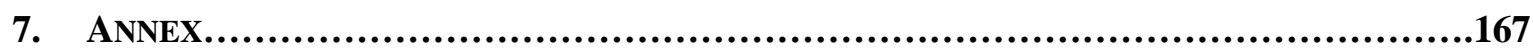





\section{INTRODUCTION}





\section{INTRODUCTION}

1.1. Mouse respiratory system

\subsubsection{Structure and functions}

The respiratory system of mammals includes three different structures: the trachea and main bronchi, the bronchioles and the alveoli. The upper respiratory system (trachea, bronchi and bronchioles) consists of tubular and well-ramified structures in charge of conducting the air to and from the distal parts of the lung. Alveoli are located at the end of the bronchioles, and are small sacs surrounded by a dense capillary network responsible of the gas exchange between the air and the blood (Herriges \& Morrisey, 2014; Hogan et al., 2014; Rock et al., 2010; Rock \& Hogan, 2011).

In this regard, a proper gas exchange function depends on a proper specification and organization of both epithelial and endothelial cells. Each lung epithelial cell type accomplishes one specific function: innate immunity, mucus secretion and clearance, maintenance of the surface tension with the production of surfactant, fluids, electrolytic transport and gas exchange. All these epithelial cells are organized in a complex ramified system closely related with vascular and lymphatic ducts (Cardoso, 2008; Herriges \& Morrisey, 2014).

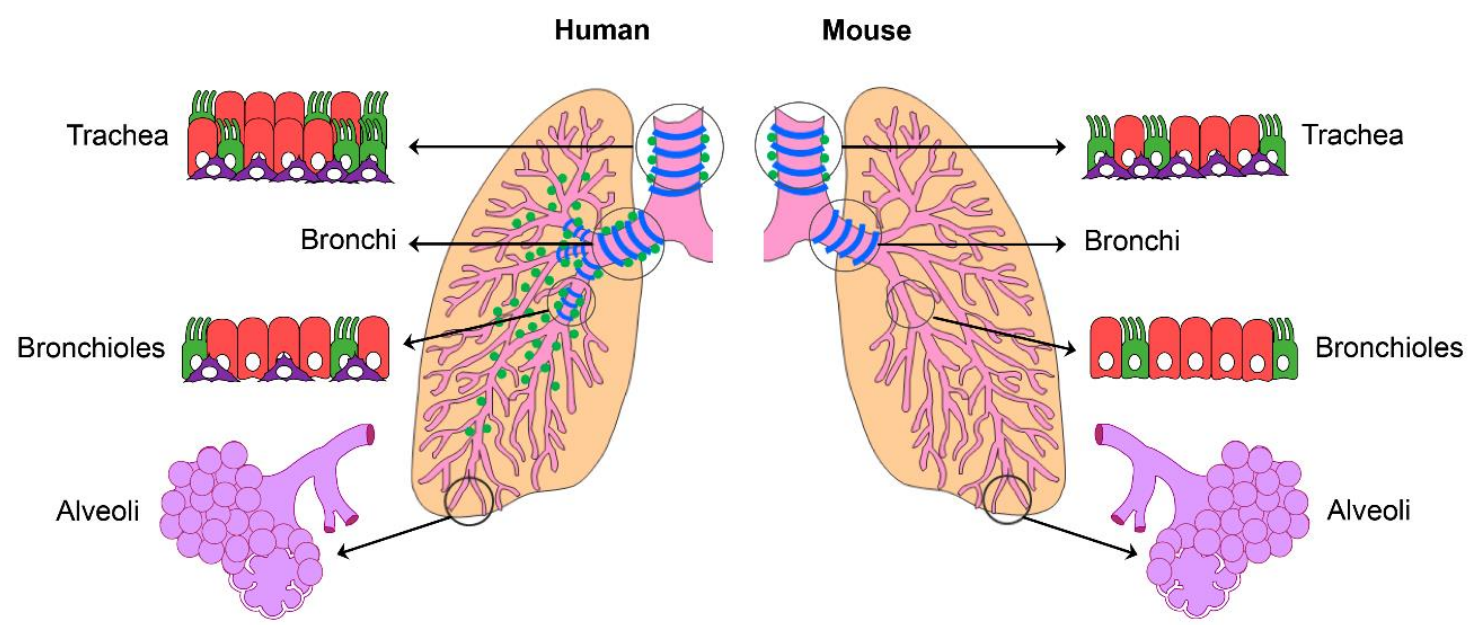

Figure 1: Comparison between human and mouse lung structure. Schemes of the human (left), and mouse (right) lung showing cartilage rings (blue), submucosal glands (green dots), Basal (purple), secretory (red), and Ciliated cells (green).

The lung of vertebrates is a highly conserved organ among the species, but notable differences exist when comparing between the human and mouse lung. The general anatomic organization of the lung is similar between the two species. Both human and mouse lungs are divided in 5 lobes, but they are differently distributed: 3 lobes in the right lung and 2 in the left lung in humans, but 4 lobes in the right lung (cranial, medial, caudal and accessory) and 1 in the left lung in mice. The trachea is composed by a pseudostratified epithelium, being thicker in human when compared to mouse. Moreover, in mice, the cartilage rings are only present in the 


\section{Introduction}

trachea, but in the human lung, these cartilage rings extend for several bronchial generations into the lung. Human lung Basal cells are found from the trachea to the bronchioles, in contrast with mouse where the can only be found in the tracheal regions. Mucin-producing submucosal glands are located only in the proximal trachea in the mouse lung, however, in the human lung, we can find submucosal glands deep into the human lung (Fig. 1) (Cardoso, 2008; Meyerholz et al., 2018; Rock et al., 2010).

\subsubsection{Cellular composition}

The lung is a crucial organ comprised by numerous cell types that mediate respiration and gas exchange, immune response to microbial and environmental insults and tissue protection and repair. These highly coordinated functions are mediated by diverse cell types, which includes epithelial and endothelial cells, fibroblasts and immune cells (Herriges \& Morrisey, 2014; Rock \& Hogan, 2011).

Single cell RNA sequencing analysis of mouse lungs have identified 4 major cell types (mesenchymal, epithelial, endothelial and immune cells) and 20 cell sub-types. Moreover, the cellular composition of the lung vary along its proximo-distal axis (Deprez et al., 2020; Guo et al., 2019; Montoro et al., 2018; Zepp et al., 2017).

A pseudostratified columnar epithelium composed by Basal, Ciliated, Club, neuroendocrine (NE) cells and a low percentage of Ionocytes, which control the fluid regulation, constitute the mouse trachea and main bronchi (Fig. 2). The bronchioles are formed by simple columnar epithelium, containing Ciliated, secretory Club and goblet cells and NE cells. In this region, it should be noticed that Club cells predominate over the Ciliated cells in number (approximately $60 \%-40 \%$ respectively), being both implicated in the mucociliary clearance, the process by which inhaled particles and microorganism are cleared from the lungs (Fig. 2). Next to the bronchioles are the alveoli, and the connecting region between them is called bronchioalveolar duct junction (BADJ). In the BADJ we can find a few cells that have been proposed to be the bronchioalveolar stem cells (BASCs), that co-express both markers of Club and alveolar epithelial type 2 cells (AEC2). A complex network of alveoli comprises the most distal region of the lung, lined by squamous alveolar epithelial type 1 cells (AEC1) and cuboidal AEC2 (Fig. 2). AEC1 are the responsible of the gas exchange and regulation of fluid homeostasis. This is due to its close apposition to the capillary network, the high expression of ion channels and pores (including Aquororin 5, AQP5), and its high membrane to cytoplasm ratio, representing the $95 \%$ of the internal surface of each alveolus. On the other hand, the AEC2 produce the surfactant, a mix of extracellular proteins and lipids that maintain the alveolar tension, contributes to host defence and keeps alveolar homeostasis. Of note, both AEC1 and AEC2 arise from an alveolar bi-potent progenitor cell (Fig. 4), but it has been described that AEC1 emerge 
from differentiated AEC2 cells. This is due to the fact that AEC2 cells act like alveolar Basal cells giving rise to both AEC1 and AEC2 in normal lung development and during the alveolar regeneration after an epithelial injury (Barkauskas et al., 2013; Brandt \& Mandiga, 2021; Desai et al., 2014; Hogan et al., 2014; Rawlins \& Hogan, 2006; Rock \& Hogan, 2011).

\section{Trachea and main bronchi}
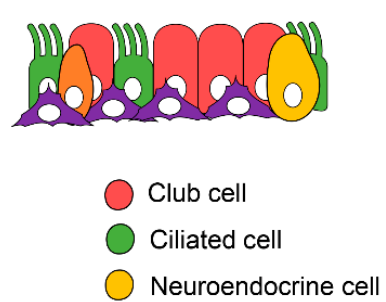
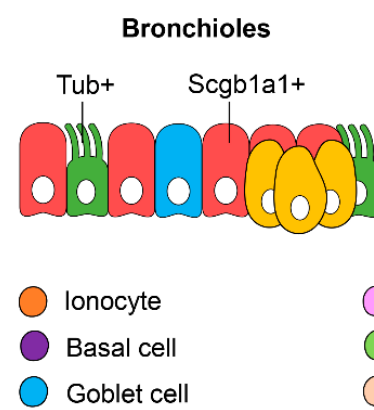
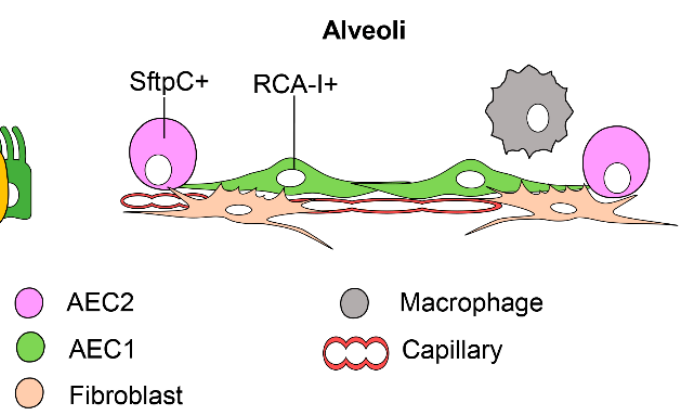

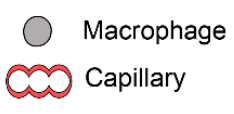

Figure 2: Adult lung cell composition. Cellular composition of the lung in proximo-distal axis order: trachea and main bronchi (left), bronchioles (middle) and alveoli (right). Specific cell markers for Ciliated ( $\beta$-Tubulin), Club (Secretoglobulin 1a1, Scgb1a1), alveolar epithelial cell type 1 (AEC1, Ricinus communis agglutinin-I RCA-I) and alveolar epithelial cell type 2 (AEC2, Surfactant protein C, SftpC) are shown.

\subsubsection{Lung embryonic development}

The transition from fetal to postnatal life is critical to perinatal survival, it is necessary that all the cell types, including those from the conducting airways to distal alveoli, perform their function properly. Therefore, a correct lung development is crucial for proper lung function.

The trachea and lungs both arise from the anterior foregut endoderm, starting at embryonic day 9.0 (E9.0) with the expression of the transcription factor $\mathrm{Nkx} 2.1$ in endodermal cells on the ventral side. An evagination of these epithelial cells results in the origination of the trachea and two lung buds between E9.5-E12.5. This is called the "embryonic stage" of lung development. The branching programme regulates the generation of the tree-like network of the airways during the embryonic and pseudoglandular stages (E12.5-E16.5), giving rise to thousands of terminal branches. In the last two stages of lung development, the canalicular (E16.5-E17.5) and saccular (E18.5-postnatal day 5 (P5)) stages, the terminal branches will develop into clusters of epithelial sacs that will later form the alveoli. The lung development programme ends with the alveolarization stage (P0-P14), in which the full maturation of the alveolus occurs (Cardoso, 2008; Schittny, 2017; Warburton et al., 2000).

\subsubsection{Lung mesenchyme development}

During all the stages of endodermal development, the lung mesoderm (or mesenchyme) develops closely and interacts with the lung endoderm to promote and drive the branching, 


\section{Introduction}

differentiation and generation of the different lineages of the lung. Alterations in this critical interaction can lead to severe anatomical and functional defects in the airway and alveoli lead to high neonatal mortality in humans (Ahlfeld \& Conway, 2012; Chao et al., 2015; Herriges \& Morrisey, 2014; Hines \& Sun, 2014; McCulley et al., 2015; Warburton et al., 2000; Yuan et al., 2018; Zepp et al., 2017).

Lung mesoderm is composed by several specialized cell types, including pericytes, airway smooth muscle, myofibroblasts, alveolar fibroblasts and lipofibroblasts. Mesoderm proliferation and differentiation must coordinate with the endoderm through complex signalling networks that implies some key players including Sonic Hedgehog (SHH), Bone Morphogenic Proteins (BMP), Wnts, Vascular Endothelial Growth Factor (VEGF), Platelet-Derived Growth Factor (PDGF), Fibroblast Growth Factor (FGF), Transforming Growth Factor-beta (TGF $\beta$ ) and Retinoid Acid (RA). The distal mesoderm multipotent progenitors will give rise to the different lineages depending on the inductive signals they receive. Cells committed to become airway smooth muscle move to the proximal part of the endodermal tubes and envelop them; meanwhile, those committed to be vascular smooth muscle incorporate into vessels (Cardoso, 2008; Herriges \& Morrisey, 2014; Hines \& Sun, 2014; Morrisey \& Hogan, 2010; Schittny, 2017).

\subsubsection{Epithelium-Mesenchyme interactions during lung development}

The three well-established main pathways that are implicated in lung specification, branching and patterning include the Wnt, BMP, FGF mediated pathways; and its expression is produced in the lung mesenchyme (Herriges \& Morrisey, 2014; Hines \& Sun, 2014; J. Yang \& Chen, 2014).

Two family members of the Wnt/ $\beta$-catenin signalling pathway, Wnt2 and Wtn2a, are expressed in the mesenchyme surrounding the anterior foregut region and drive the expression of NK2 homeobox 1 (NKX2.1) in the respiratory endoderm progenitors (Fig. 4). Moreover, the action of BMP-mediated signalling is necessary, together with Wnt signalling, in order to coordinate the process. In this regard, BMP4, expressed in the mesoderm surrounding the anterior foregut region where the NKX2.1+ progenitors are located, and, through blocking the transcription factor sex-determining region Y-box 2 (SOX2), allows the early specification and development of lung (Fig. 4) (Aros et al., 2021; Goss et al., 2009; Herriges \& Morrisey, 2014).

The branching morphogenesis is the process by which the early buds of the main bronchi will extend into the surrounding mesenchyme, generating the airway tree-like structure. This essential process is orchestrated by signals between the developing endoderm and the surrounding mesoderm. At this point, the main driver is the FGF signalling pathway. In 
particular, FGF10 from the mesoderm and FGFR2b located in the developing endoderm, is crucial for a correct formation of the branch points (Fig. 3). FGFR2b downstream signalling is mediated by KRas and $\beta$-Catenin, signals that maintain the epithelial proliferation through sexdetermining region Y-box 9 (SOX9) transcription factor activation (Fig. 3). In addition, other signalling pathways, including those mediated by BMP4 and SHH, which regulate FGF10 expression in the distal lung mesenchyme, indicating that a complex interplay of signalling molecules participate in the branch formation and outgrowth (Fig. 3) (Chang et al., 2013; McCulley et al., 2015; Nyeng et al., 2008; Ostrin et al., 2018; Ustiyan et al., 2016; Volckaert \& De Langhe, 2015; Weaver et al., 2000).

FGF10 also establishes the appropriate direction of branch growth through activation of Ras/Sprouty2 (Spry2) pathway. KRas drives proliferation of the epithelial cells, leading to the loss of new branch points in the expansive proximal zones. Moreover, other pathways such as the planar cell polarity (PCP) pathway may have a subtle role in the regulation of tube shape during branching, as well as integrin $\beta 1$-mediated cell-matrix interactions. These distal signalling networks play a key role in determining the ultimate size and morphology of the lung (Chang et al., 2013; Shaw et al., 2008; Tang et al., 2011; Ustiyan et al., 2016; Yates et al., 2010).

During the canalicular stage, the terminal buds, with distally located SOX9+ progenitors, become narrower and give rise to small saccules. Then, during the saccular stage, these small saccules develop to mature alveoli, the functional units for gas exchange, with the differentiation of the specialized cell types of the alveoli, AEC1 and AEC2. Unlike SOX2 function in conducting airway differentiation, up to date, no specific transcription factors have been yet identified to be specific for alveolar cells. In all this developmental process, the blood vessels develop in parallel and become tightly apposed with the AEC1, allowing an efficient gas exchange (Barkauskas et al., 2013; Cardoso, 2008; Morrisey \& Hogan, 2010; J. Yang \& Chen, 2014).

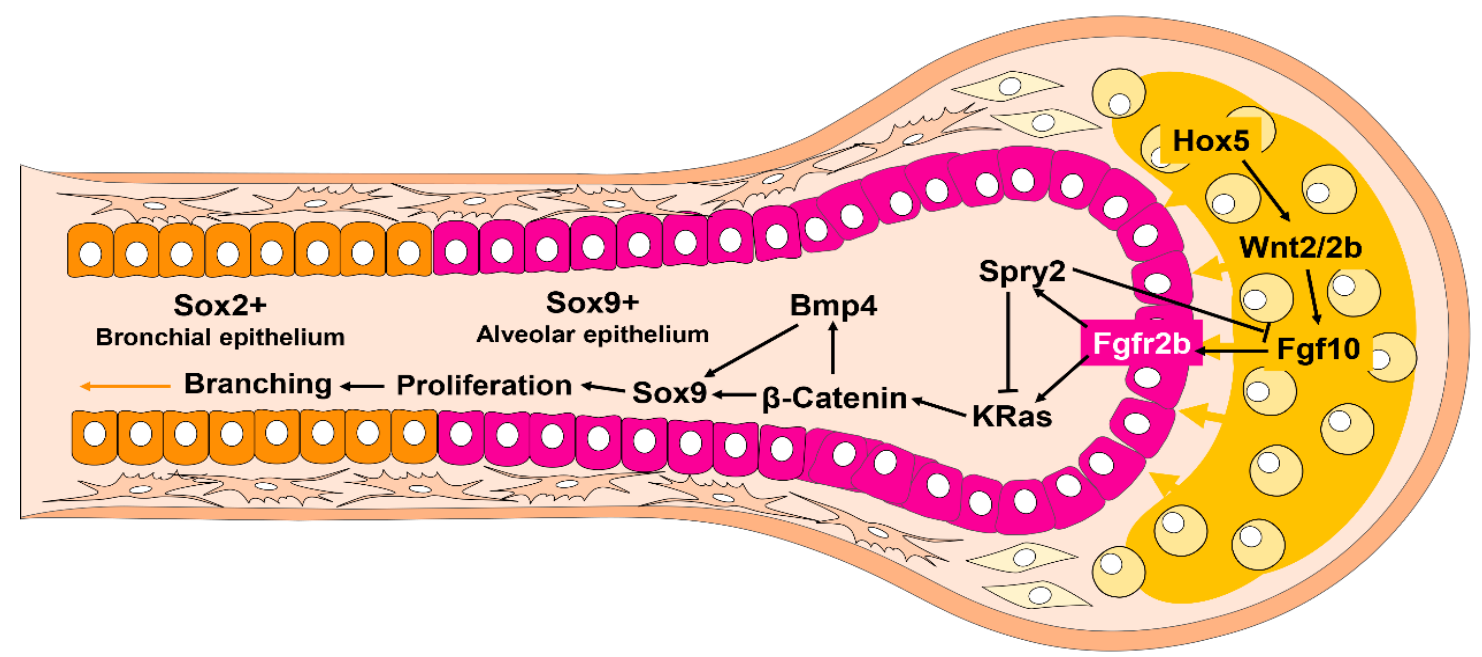

Figure 3: Signalling overview of the crosstalk between mesenchyme and epithelium during branching morphogenesis. Distal-tip branched structures (SOX9+ epithelium) in the developing lung are surrounded by 


\section{Introduction}

mesenchymal cells expressing FGF10 (yellow). This mesenchyme secretes growth factors that control the changes in the developing lung epithelium.

\subsubsection{Lung epithelial cells differentiation}

During the branching programme, the lung endoderm starts to develop the different cell lineages along its proximal-distal axis through the expression of several transcription factors. The proximal endoderm progenitor lineage cells express SOX2, whereas the distal endoderm progenitor lineage is expressing concomitantly SOX9 and the transcriptional regulator DNAbinding protein inhibitor (ID2). These two populations have distinct fate: on one hand, the proximal progenitors give rise to all the bronchiolar cell lineage, including NE, secretory, Ciliated and mucosal cells; on the other hand, the distal progenitors give rise to the two specialized alveolar cell types, AECI and AEC2 (Fig. 4) (Frank et al., 2019; Warburton et al., 2000; J. Yang \& Chen, 2014).

SOX2 and SOX9/ID2 expression in the proximal-distal axis is crucial since loss of SOX2 or SOX9/ID2 expression lead to a complete loss of mature secretory and Ciliated cells or distal cell lineages, respectively. In addition, ID2/NKX2.1 expressing cells maintain its multipotency up to E13.5, being able to generate both alveolar and bronchiolar epithelial cells up to this period of time (Fig. 4) (El Agha \& Bellusci, 2014; Herriges \& Morrisey, 2014; J. Yang \& Chen, 2014).

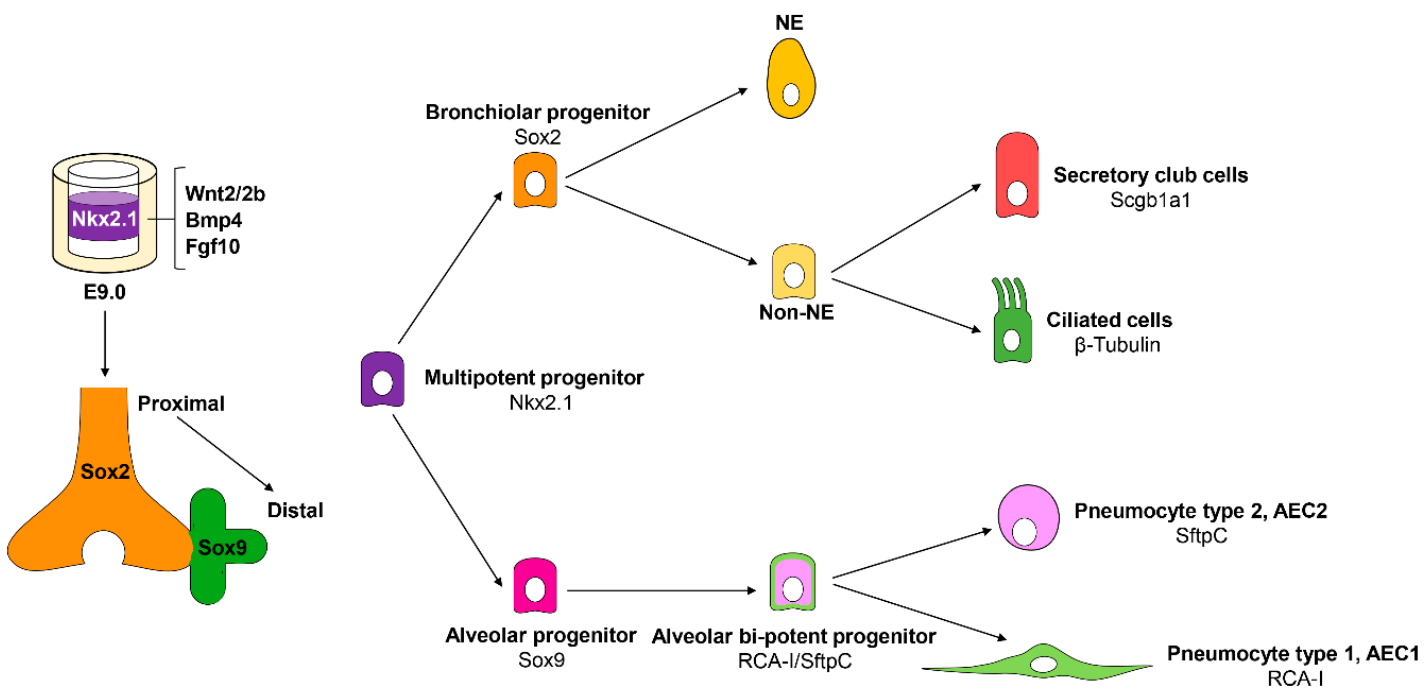

Figure 4: Origin of lung cell lineages and their markers. Proximo-distal cell lineages specification during lung development and key molecules in early lung specification (Mesenchymal Wnt2/2b, BMP4, FGF10 and epithelial $\mathrm{Nkx2}$ ). The proximal areas will give rise to bronchiolar cell lineages, meanwhile the distal areas will originate specialised alveolar cells. All differentiated cells originate from a multipotent progenitor expressing Nkx2.1 transcription factor, present in murine lungs until E13 day of embryonic development. RCA-I: Ricinus communis agglutinin-I, SftpC: Surfactant protein C, Scgb1a1: Secretoglobulin 1a1. 


\subsection{Neonatal respiratory distress syndrome}

\subsubsection{Background}

Premature birth, intrauterine infection, growth restriction, or genetic mutations can disrupt the normal developmental cues, resulting in defective lungs in the neonates. Thus, while a normal lung can undergo repair, some prenatal deficiencies cannot be rescued by postnatal regeneration, resulting in lifelong respiratory problems and further clinical burdens (Ahlfeld \& Conway, 2012; Hallman \& Haataja, 2003; Jo, 2014; Y. Li et al., 2019; Najafian \& Hossein Khosravi, 2020; Ye et al., 2020).

The neonatal respiratory distress syndrome (NRDS) affects about $1 \%$ of newborns and is the leading cause of death in preterm infants, being most common in males. The most common clinical signs are nasal flaring, cyanosis, chest wall retractions, breathing efforts and expiratory grunting. It can be caused by a variety of factors: developmental insufficiency of surfactant production and structural immaturity of the lungs, neonatal infection, genetic issues within the surfactant synthesis and maternal diabetes; leading to a severe hypoxemia in the infant (Jo, 2014; Li et al., 2019; Najafian \& Hossein Khosravi, 2020; Wambach et al., 2010).

\subsubsection{Clinical management}

Currently, the antenatal steroid therapy, which is applied to pregnant women with a high risk of preterm delivery, has been shown to decrease the neonatal mortality of NRDS by accelerating fetal lung maturation and surfactant production. Both dexamethasone and betamethasone are the most widely used as therapeutic option preventing neonatal morbidity, although there is no consensus in the scientific community about the proper administration regimen protocol (optimal doses, timing, frequency and via of administration), being different between countries (Brownfoot et al., 2013; Floros et al., 1985; Göggell et al., 2004; Roberts et al., 2017).

In the initial management of the syndrome, mechanical ventilation is used, together with oxygen therapy. However, they are associated with higher incidence of bronchopulmonary dysplasia (BDP). For this reason, a combination of early respiratory support and surfactant therapy may improve the outcomes. Lung surfactant is a mixture of phospholipids and proteins secreted by AEC2 that reduces the surface tension in terminal air spaces. Lack of proper surfactant production is another feature of NRDS; thus, surfactant therapy can augment respiratory function and pulmonary compliance (Keszler \& Sant'Anna, 2015; Najafian \& Hossein Khosravi, 2020; Nkadi et al., 2009; Rey-Santano et al., 2020; Sweet et al., 2013).

An active metabolite of bromhexine, the ambroxol hydrochloride, is used in clinical treatment of NRDS. It has a mucolytic activity, increasing the natural mucus clearance 


\section{Introduction}

mechanism of the lungs; and additionally, it is a secretolytic agent, enhancing the production of serous mucus, which contributes making the phlegm less viscous. This will allow Ciliated cells to expel the mucus by transporting it out of the lungs through the cilia movement. Moreover, a wide range of advantages has been reported, from reducing the production of hydrogen peroxide, stimulating surfactant secretion, reducing lung damage and attenuating the inflammatory response (Najafian \& Hossein Khosravi, 2020; Shi et al., 2020; Xiang \& Wang, 2019).

In order to alleviate and reduce pulmonary edema, inflammation, vascular resistance and hypoxia, inhaled nitric oxide (iNO) is given to the infants, making respiratory difficulties easier for them. Moreover, it has been shown that iNO improves pulmonary angiogenesis and protects against pulmonary infections with no side effects in growth or neurodevelopmental status. For these reasons, the use of iNOin NRDS , alone or in combination with surfactant therapy, notably improves oxygenation and reduces the mortality rate of preterm infants, (Dzierba et al., 2014; Najafian \& Hossein Khosravi, 2020; Schreiber et al., 2003).

\subsubsection{Genes and proteins implicated in NRDS susceptibility}

Many studies suggested that deficiency in the production and secretion of extracellular surfactant is the major pathogenic factor for developing NRDS, and those defective levels of surfactant are associated with the prematurity. Nevertheless, some infants born at term develop NRDS, while others born extremely premature do not develop the syndrome; thus, there is an individual variation in surfactant production based on the genetic background, and prematurity by itself does not determine the risk of developing the disorder (Copland \& Post, 2012; Hallman et al., 2001; Hallman \& Haataja, 2003; Jo, 2014).

Surfactant is composed by a mixture of lipids (dipalmitoylphosphatidylcholine (DPPC), other phospholipids (PC), neutral lipids and cholesterol) and proteins (plasma proteins and surfactant proteins SP-A, SP-B, SP-C and SP-D). SP-A and SP-D are collectins involved in host defense, they act by coating bacteria and viruses and promoting their macrophage-mediated phagocytosis, meanwhile SP-B and SP-C are hydrophobic membrane proteins required for proper lung function (Haczku, 2008; Hawgood, 2004; McCormack \& Whitsett, 2002).

The critical function of each surfactant protein has been extensively studied through directed mutations in mouse animal models. Deletion or inactivation of SP-A do not cause respiratory failure but induce a higher predisposition to lung infections. However, some single nucleotide polymorphisms (SNPs) have been reported in newborns as a risk factor for developing RDS, meanwhile others have been identified as protective factors for the syndrome, although the 
precise mechanism is still not known (Bersani et al., 2012; Rämet et al., 2000; Tsitoura et al., 2016; Wang et al., 2020).

Altered or lack of synthesized SP-B causes death by respiratory in mice at birth, with congenital alveolar proteinosis, excess of SP-A and SP-D but no mature SP-C. In humans, loss or deficiency of SP-B caused by genetic mutations, as well as SNPs or length variations (insertions/deletions polymorphic changes) have been identified as major developers of NRDS, (Table 1) (Clark \& Clark, 2005; Jo, 2014; Marttila et al., 2003; X. Wang et al., 2020).

SP-C is expressed as a precursor, proSP-C, that matures to SP-C. Lack of SP-C expression or mutation does not result in neonatal lung disease resembling RDS in animal models, however, with aging, they develop a severe pulmonary disorder with interstitial pneumonitis, progressive emphysema, monocytes infiltrates, and AEC2 hyperplasia as main symptoms. Genetic mutations have been identified in humans as key developers of lung diseases, and some SNPs in SFTPC have been associated with RDS in preterm infants (Table 1) (Hallman \& Haataja, 2007; Jo, 2014; Mulugeta \& Beers, 2006; Wambach et al., 2010).

SP-D, as SP-A, is a key component of the innate pulmonary immune system. Some specific polymorphisms of SFTPD gene have been detected to alter the structure, function and concentration of SP-D. There have been reported haplotypes associated with both RDS development and protection (Hallman \& Haataja, 2003; McCormack \& Whitsett, 2002; Nkadi et al., 2009).

The ABC (ATP-binding cassette) transporters are highly conserved transmembrane proteins, which transport various substrates using the energy extracted from ATP hydrolysis. Several members of the $\mathrm{ABC}$ superfamily are involved in phospholipids and sterols transport; $\mathrm{ABCA} 1$, ABCA3 and cystic fibrosis transmembrane conductance regulator (CFTR) are expressed in the lung, playing an important function in pulmonary lipid metabolism. Mice lacking ABCA1 develop respiratory distress, alveolar proteinosis and surfactant composition alterations. In the lung, ABCA3 is exclusively expressed in AEC2, having an essential role in lipid transport, lamellar body biogenesis and normal surfactant homeostasis. Loss-of-functions mutations in the $A B C A 3$ gene have been identified not only as a cause of surfactant deficiency in newborns, but also with milder phenotypes of severe respiratory distress and interstitial lung disease (Table 1) (Clark \& Clark, 2005; Hallman \& Haataja, 2007; J. Y. Hu et al., 2020; McGillick et al., 2021).

\begin{tabular}{ccccc}
\hline \multirow{2}{*}{ Gene } & \multicolumn{2}{c}{ Mutation } & & \multicolumn{2}{c}{ Predicted result } \\
\cline { 2 - 5 } & Genetic change & Type & mRNA/protein & Clinical outcome \\
\hline $\begin{array}{c}1549>\text { GAA } \\
\text { homozygote (in exon } \\
\text { 4) }\end{array}$ & Frameshift & $\begin{array}{c}\text { Complete absence of } \\
\text { mRNA and SP-B }\end{array}$ & $\begin{array}{c}\text { Fatal respiratory } \\
\text { failure in early } \\
\text { newborn period }\end{array}$ \\
& $\begin{array}{c}\text { 1549>GAA/457delC } \\
\text { (in exon4/exon 2) }\end{array}$ & Frameshift & $\begin{array}{c}\text { Complete absence of } \\
\text { SP-B }\end{array}$ & $\begin{array}{c}\text { Fatal respiratory } \\
\text { failure in early } \\
\text { newborn period }\end{array}$ \\
\end{tabular}




\begin{tabular}{|c|c|c|c|c|}
\hline & $\begin{array}{c}1549 \mathrm{C}>\mathrm{GAA} / 4380 \mathrm{C}> \\
\mathrm{T}(236 \mathrm{R}>\mathrm{C})(\text { in exon } \\
\text { 4/exon } 7)\end{array}$ & Missense & $\begin{array}{c}\text { Near normal content } \\
\text { of mRNA, a low but } \\
\text { detectable level of } \\
\text { SP-B }\end{array}$ & $\begin{array}{l}\text { Rapidly severe } \\
\text { respiratory distress } \\
\text { and persistent oxygen } \\
\text { requirement (lethal) }\end{array}$ \\
\hline & $\begin{array}{c}2479 \mathrm{G}>\mathrm{T}(\mathrm{c} .479 \mathrm{G}>\mathrm{T}) \\
\text { homozygote (in exon } \\
5)\end{array}$ & $\begin{array}{c}\text { Splice (a } \\
\text { frameshift } \\
\text { and a } \\
\text { termination } \\
\text { codon in } \\
\text { exon } 7 \text { ) }\end{array}$ & $\begin{array}{l}\text { Reduced amount of } \\
\text { mature SP-B }\end{array}$ & $\begin{array}{l}\text { Case 1: need lung } \\
\text { transplantation } \\
\text { Case 2: persistent } \\
\text { oxygen requirement }\end{array}$ \\
\hline & $\begin{array}{c}\text { c.673-1248del2959 } \\
\text { homozygote (in exon } \\
7-8 \text { ) }\end{array}$ & Deletion & - & $\begin{array}{l}\text { Fatal respiratory } \\
\text { failure in early } \\
\text { newborn period }\end{array}$ \\
\hline & $\begin{array}{c}2417 \mathrm{G}>\mathrm{A}(\mathrm{G} 135 \mathrm{~S}) \\
\text { heterozygote (in exon } \\
5)\end{array}$ & Missense & $\begin{array}{c}\text { Transient absence of } \\
\text { SP-B }\end{array}$ & $\begin{array}{l}\text { Respiratory distress in } \\
\text { early newborn period } \\
\text { with continuous } \\
\text { oxygen requirement }\end{array}$ \\
\hline \multirow{4}{*}{ SFTPC } & $\begin{array}{c}+128 \mathrm{~T}>\mathrm{A} \\
\text { heterozygote (in exon } \\
5 \text { ) }\end{array}$ & - & $\begin{array}{l}\text { Misfolding and } \\
\text { trapping proSP-C }\end{array}$ & $\begin{array}{l}\text { Familial pulmonary } \\
\text { fibrosis }\end{array}$ \\
\hline & $\begin{array}{c}\text { p.I73T }(218 \mathrm{~T}>\mathrm{C}) \\
\text { heterozygote (in exon } \\
3 \text { ) }\end{array}$ & - & $\begin{array}{l}\text { Misfolding and } \\
\text { trapping of proSP-C }\end{array}$ & $\begin{array}{l}\text { Familial interstitial } \\
\text { lung disease, } \\
\text { asymptomatic parent } \\
\text { of the patient }\end{array}$ \\
\hline & p.L188Q & - & $\begin{array}{l}\text { Partially trafficked to } \\
\text { lamellar bodies }\end{array}$ & $\begin{array}{c}\text { Interstitial lung } \\
\text { disease }\end{array}$ \\
\hline & p.P30L & - & $\begin{array}{c}\text { Arrested poSP-C in } \\
\text { the endoplasmic } \\
\text { reticulum }\end{array}$ & $\begin{array}{l}\text { Interstitial lung } \\
\text { disease }\end{array}$ \\
\hline \multirow{3}{*}{$A B C A 3$} & $\begin{array}{c}\text { E292V (c.875AZT) } \\
\text { heterozygote (in exon } \\
9 \text { ) }\end{array}$ & $\begin{array}{l}\text { Missense } \\
\text { (termination } \\
\text { codon in } \\
\text { exon 33) }\end{array}$ & $\begin{array}{l}\text { Defects in } \\
\text { phosphocholine } \\
\text { transport }\end{array}$ & $\begin{array}{c}\text { Less severe } \\
\text { (interstitial lung } \\
\text { disease) }\end{array}$ \\
\hline & $\begin{array}{c}\text { L1580P } \\
(4739 \mathrm{~T}>\mathrm{C}) / 4552 \text { insT } \\
(\text { in exon 31/exon 30) }\end{array}$ & $\begin{array}{l}\text { Missense/fra } \\
\text { meshift }\end{array}$ & Trafficking defects & $\begin{array}{l}\text { Rapidly fatal } \\
\text { respiratory failure }\end{array}$ \\
\hline & $\begin{array}{c}\mathrm{G} 1221 \mathrm{~S}(3661 \mathrm{G}>\mathrm{A}) / \mathrm{L} \\
982 \mathrm{P}(2945 \mathrm{~T}>\mathrm{C} 9(\mathrm{in} \\
\text { exon } 24 / \text { exon } 21)\end{array}$ & $\begin{array}{l}\text { Missense/mi } \\
\text { ssense }\end{array}$ & Trafficking defects & $\begin{array}{l}\text { Rapidly fatal } \\
\text { respiratory failure }\end{array}$ \\
\hline
\end{tabular}

Table 1: Representative human mutations of SFTPB, SFTPC, and ABCA3. SP-B, surfactant protein B; SP-C, surfactant protein C; proSP-C, surfactant protein C precursor. Adapted from (Jo, 2014).

In addition to mutation in surfactant protein genes, NRDS can also be caused by alterations in several proteins implicated in the pulmonary surfactant synthesis and maturation. In this regard, some transcription factors that regulate the gene expression of surfactant proteins have been identified either in animal models or human as drivers of NRDS. This is due to their role in lung formation, surfactant production and homeostasis in late gestation. These transcription factors include: thyroid transcription factor (TTF-I, also known as NK2 homeobox 1 [NKX2.1], or thyroid-specific-enhancer-binding protein $[\mathrm{T} / \mathrm{EBP}]$ ), CCAAT enhancer binding protein- $\alpha$ (C/EBP $\alpha$ ), and forkhead box A2 (FOXA2) (Jo, 2014; Martis et al., 2006; Sparkman et al., 2006; Wan et al., 2005; Whitsett et al., 2004). 
TTF-I is expressed during early lung development, being also an early marker of lung differentiation and a regulator of the expression of SP-A, SP-B, SP-C and ABCA3; therefore, mutations affecting NKX2-1 results in NRDS and respiratory failure, as well as in interstitial lung disease in older children. Additionally, it has been proved that ceramide, an sphingolipid and second messenger mediating inflammation and apoptosis, interfere with SP-B metabolism through the reduction of TTF-I binding activity to SP-B promoter (Cardoso, 2008; Salerno et al., 2014; Sparkman et al., 2006).

The basic leucine zipper transcription factor $\mathrm{C} / \mathrm{EBP} \alpha$ plays an important role in surfactant lipids and proteins synthesis and metabolism. Expectedly, deletion of Cebpa gene results in murine lung epithelial cell proliferation and differentiation inhibition (Bassères et al., 2006; Martis et al., 2006).

FOXA2 modulates lung gene expression by interacting with other transcription factors. An animal study suggests that its action is crucial for surfactant homeostasis, and, moreover, deletion of Foxa 2 has been shown to inhibit lung branching morphogenesis and epithelial cell differentiation in mouse (Wan et al., 2004, 2005).

Several enzymes implicated in the synthesis of the surfactant lipid component have been studied and described to be associated with RDS development. Phosphatidylcholine (PC) constitute the most abundant phospholipid in the surfactant (80\% of the total phospholipids), and a key enzyme in the pathway leading to its production is the lysophospholipid acyltransferase (LPCAT1). LPCAT1 is the major regulator of surfactant production, and its activity is regulated by glucocorticoids. An hypomorphic animal model study with a reduced expression of Lpcat1 showed perinatal respiratory failure with NRDS hallmarks, and, moreover, it has been identified some LPCATI SNPs that may be protective against RDS possibly by generating a more stable and efficient mRNA translation (Bridges et al., 2010; Shen et al., 2020).

Preterm infants have an immaturity of the antioxidant systems, thus, leading to a lower ability to maintain overall homeostasis and control the overproduction of cell-damaging free radicals. The newborns infants with NRDS have an increment in lipid peroxidation, DNA and protein oxidation damage. This oxidative damage also contributes to the pathogenesis of neonatal syndrome. Additionally, several studies have proved that oxidative stress can modulate embryonic development since some key signalling pathways, such as Wnt and NF- $\kappa \beta$, both important in normal lung development, are modulated depending on oxygen radicals and its action as second messengers. However, it should be noted that certain levels of ROS are necessary in normal developments, since they regulate transcription factors, and completely blocking oxidative stress has a negative impact on growth. On the other hand, the use of invasive mechanical ventilation and oxygenation therapy (hyperoxia) could also contribute to the 


\section{Introduction}

severityof NRDS (Abdel Hamid et al., 2019; Alvira, 2014; Dennery, 2007; Elkabany et al., 2020; Londhe et al., 2008; Marseglia et al., 2019; Sah et al., 2020; Zafarullah et al., 2003).

\subsubsection{Outcomes}

Neonatal respiratory distress syndrome is a major cause of premature death. Moreover, part of the survivors may develop bronchopulmonary dysplasia and suffer chronic pulmonary diseases. NRDS is a multifactorial and multigenetic disease, and its prognosis is related to the treatment and management. Inherited mutations resulting in defective surfactant metabolism are usually fatal, and the association of genetic polymorphisms with the development of RDS needs to be confirmed with large scale association studies (Agrons et al., 2005; Ahlfeld \& Conway, 2012; Najafian \& Hossein Khosravi, 2020).

\subsection{RAS signalling pathway}

\subsubsection{RAS superfamily}

Signalling through RAS GTPases constitute one of the key systems controlling signal transduction, regulating the majority of cellular functions. Small GTPases act as molecular switches, continuously cycling between two states: the active state (GTP-bound) and the inactive form (GDP-bound) (Fig. 5) (Boguski \& McCormick, 1993; Santos \& Nebreda, 1989; Vetter \& Wittinghofer, 2001).

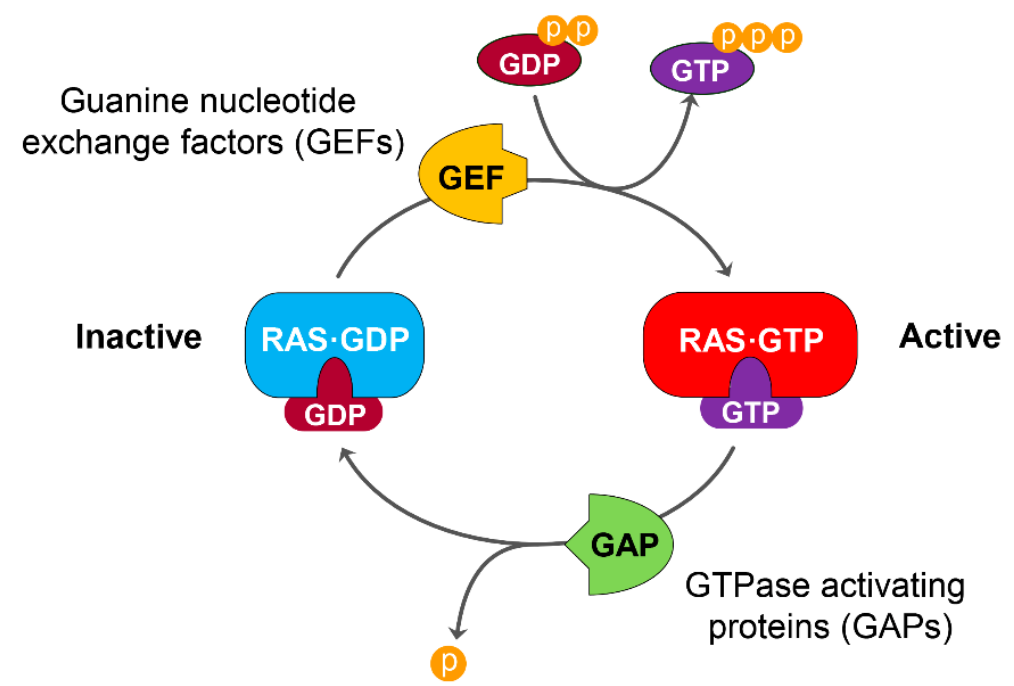

Figure 5: RAS activation and deactivation cycle. Ras activation (RAS.GTP) and deactivation (RAS. GDP) cycle is modulated by the Guanine nucleotide exchange factors (GEFs) and GTPase activating proteins (GAPs). 
In humans, RAS GTPases superfamily is composed of more than 150 members, with evolutionary conserved orthologues in other species such D. melanogaster, C. elegans, $S$. cerevisiae, S. pombe, Dictyostelium and plants. RAS superfamily is subdivided in five main branches of the corresponding families RAB, RAS, ARF, RHO and RAN, maintaining all of them similarities in their structure and mechanism of action (Fernández-Medarde et al., 2021; Wennerberg et al., 2005).

\subsubsection{Structure of canonical RAS}

Canonical HRAS, NRAS and KRAS GTPases are best known due to their mitogenic properties. They contribute to cell differentiation and organ development since they serve as signalling nodes, activated in response to diverse extracellular stimuli. Activating mutations, on position 12, 13 or 61 of these proteins in vitro transform cells, and such mutations are commonly found in a wide variety of human tumours, thus, HRAS, NRAS and KRAS are oncoproteins. These mutations found in cancer strongly diminish their catalytic activity, leading to long-term activation of the downstream signalling pathways. Blockage of RAS GTPase activity has been found not only during tumour development, but also has been related to several developmental syndromes called RASopathies (Aoki et al., 2016; Fernández-Medarde \& Santos, 2011a; Newlaczyl et al., 2017; Simanshu et al., 2017).

The three loci encode four different isoforms, HRAS, NRAS, KRAS4A and KRAS4B, resulting the last two from an alternative splicing of KRAS locus (Fig. 6). These four isoforms share a high percentage of structural homology (around 80\%), with a nearly identical N-terminal domain. The differences between them are mainly located in their C-terminal domain, the socalled hypervariable region (HVR, residues 166-188/189). This HVR, comprised by 24 aminoacids, exhibits different combinations of post-translational modifications together with different aminoacid residues that will confer each isoform distinct dynamics, trafficking, membrane attachment and localization (Fig. 6). However, not all the differences among the isoforms are located in the $\mathrm{C}$-terminal domain, since significant variations are also found in the allosteric lobe (residues 87-166). Other highly conserved structures include domains responsible for binding and hydrolysis of guanine nucleotides, and functional interactions with activators and downstream effectors (Fig. 6) (Barbacid, 1987; Esther Castellano \& Santos, 2011; Henis et al., 2009; Parker \& Mattos, 2015; Prior \& Hancock, 2012). 


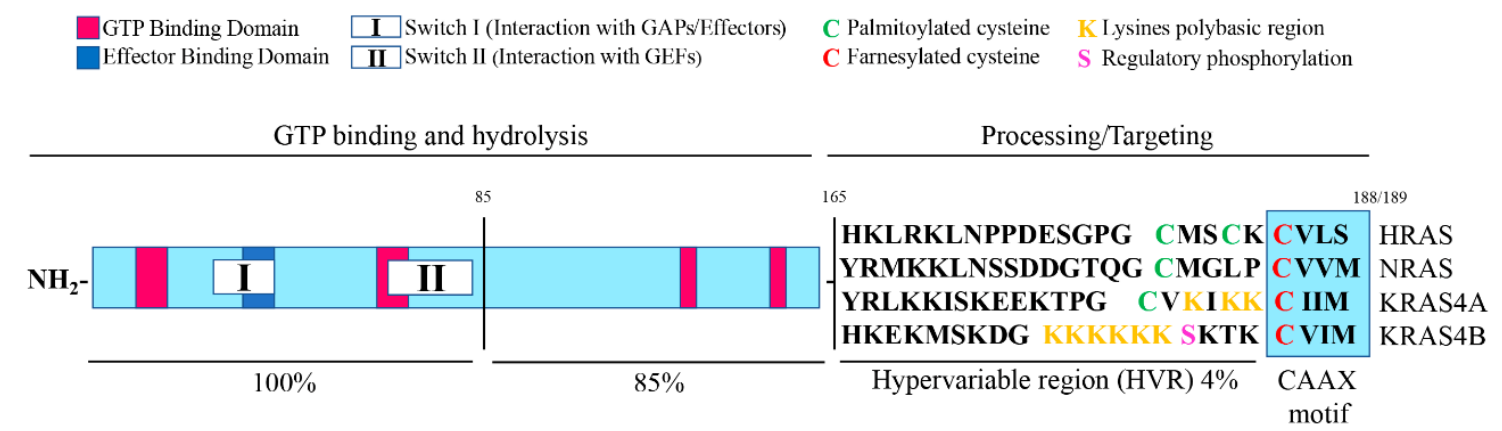

Figure 6: Canonical RAS GTPases structure. HRas, NRas, KRas4A and KRas4B have a high degree of homology, sharing the domains implicated in the regulation of signalling. However, their carboxy-terminus region is very different showing specific modification sites responsible for controlling their activity. Image modified from (Esther Castellano \& Santos, 2011).

\subsubsection{Post-translational modifications of canonical RAS}

The three canonical Ras isoforms, HRAS, NRAS and KRAS, exhibit a high degree of structural similarity since they share the domains for GTP/GDP binding and those to interact with regulators and effectors. However, despite sharing more than $80 \%$ of homology, they are not functionally redundant, and thus, the specificity of these proteins may be due to the HVR localized in the C-terminal domain of the proteins. Depending on the biochemical properties of the aminoacids localized in the HVR, each isoform will undergo different (1) post-translational modifications, (2) cellular and subcellular localizations and (3) protein turnover. Moreover, although they have a ubiquitous expression, there are differences depending on the tissue and the moment of organism development among isoforms (Ahearn et al., 2012; Arozarena et al., 2011; Prior \& Hancock, 2012).

After their synthesis, RAS proteins undergo a sequence of lipidic modifications in the CAAX motif, which is present in the three isoforms in the $\mathrm{C}$-terminal domain. Those modifications follow a well-defined sequence (Fig. 7): first, (1) the cysteine is farnesylated by a farnesyltransferase that will facilitate the association with the endoplasmic reticulum (ER), (2) where AAX motif is cleaved by Ras Converter Enzyme-1 (RCE1), and finally, (3) the farnesylated cysteine is carboxymethylated by an Isoprene-Cysteine methyltransferase (ICMT) (Ahearn et al., 2018; Ahearn et al., 2012; Quinlan \& Settleman, 2009).

HRAS, NRAS and KRAS4A need additional post-translational modifications in the HVR sequence for a better association of the protein with the membrane. KRAS4B does not need further modifications since it presents a polybasic region of lysines which stabilizes its union to the membrane through electrostatic interactions (Fig. 6). Although KRAS4B requires no more modifications after CAAX processing, the strength of the interaction with the plasma membrane is regulated negatively by phosphorylation of Ser181, and to a lesser extent at Ser171 and Thr183, by Protein Kinase C (PKC) (which partially neutralizes the positive charge), shifting 
the distribution and localization of KRAS4B in favour of endomembranes structures. On the other hand, HRAS and NRAS lack of such polybasic lysins region, therefore, in order to have a correct binding to the membrane they must suffer, respectively, a double palmitoylation and a mono-palmitoylation in the Golgi apparatus apparatus. KRAS4A uses a mixed mechanism for membrane binding, because it contains two small polybasic regions together with a single palmitoylation (Ahearn et al., 2018; Cho, 2020; Quinlan \& Settleman, 2009).

Farnesylation is an irreversible modification, but palmitoylation is reversible, which allow a dynamic transition of palmitoylated proteins between the plasma membrane and endomembranes, where they can also signal. Thereby, both the polybasic region of KRAS and palmitoyl groups in HRAS and NRAS allow a dynamic association between the different cellular compartments (Arozarena et al., 2011; Quinlan \& Settleman, 2009).

On top of these cycles regulating the spatial organization of RAS GTPases, phosphodiesterase delta (PDE $\delta)$ and $\mathrm{Ca} 2+$-binding protein calmodulin $(\mathrm{CaM})$ interact with farnesylated KRAS4B through a hydrophobic binding pocket for farnesyl. The interaction between PDE $\delta$ and farnesylated KRAS4B, independent of GDP/GTP loading, solubilizes KRas4B and facilitates its cytoplasmatic diffusion and delivery to the plasma membrane. On the other hand, $\mathrm{Ca} 2+/ \mathrm{CaM}$ plays opposite roles: negatively charged $\mathrm{Ca} 2+/ \mathrm{CaM}$ binds to the polybasic KRAS4B region, thus reversing its ionic charge and repulsing KRAS4B from the plasma membrane, since farnesylation alone is not sufficient to maintain the RAS GTPases anchored to the plasmatic membrane (Chandra et al., 2011; Cho, 2020; Schmick et al., 2014; Sperlich et al., 2016).

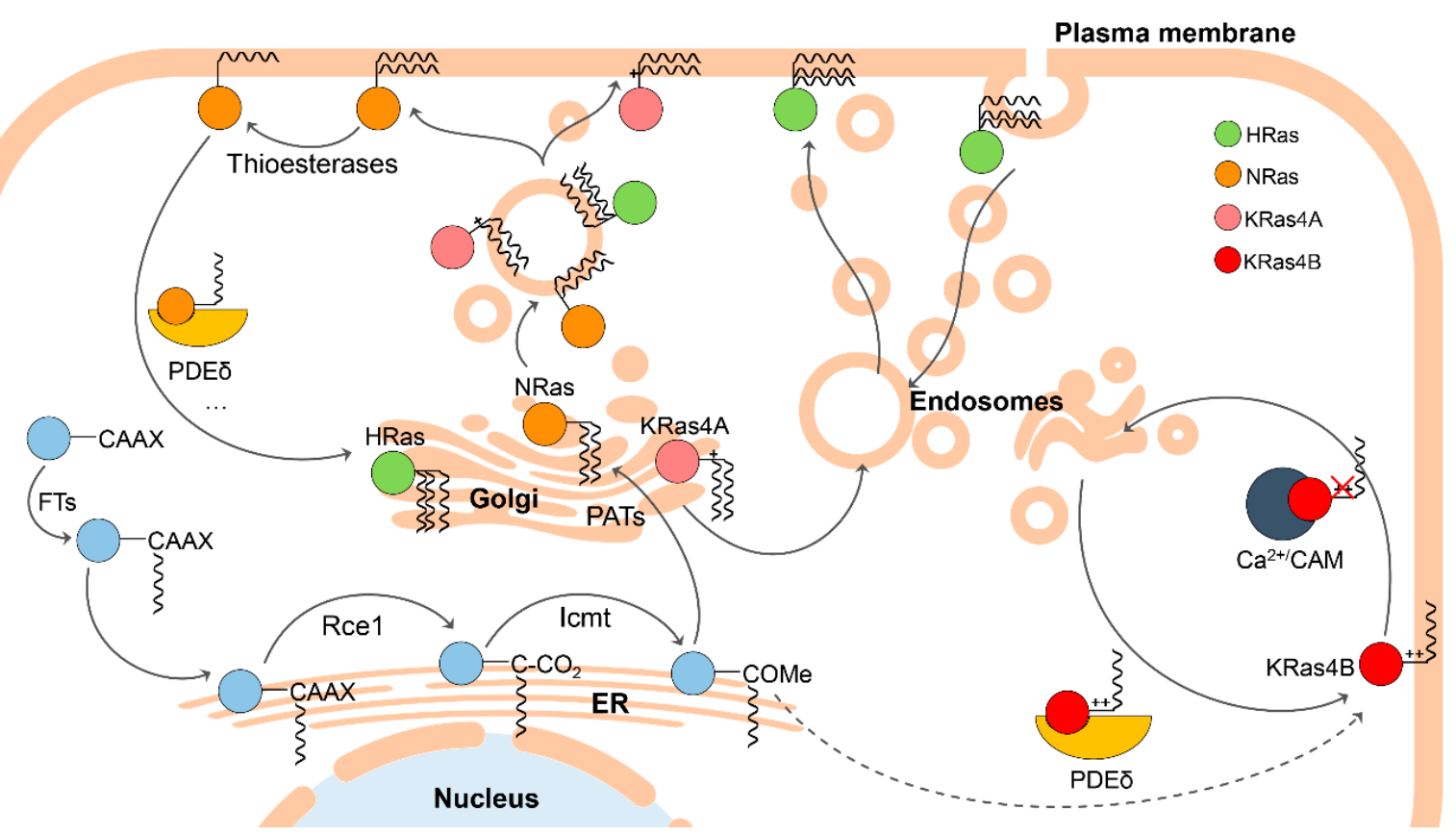

Figure 7: Differential RAS processing and delivery to the plasma membrane. Scheme representing some of the described Ras targeting to and from the plasma membrane. HRas (green), NRas (orange), KRas4A (light red), KRas4B (deep red) isoforms are represented as coloured circles. Both HRas and NRas isoforms undergo the 
acylation/deacylation cycle, but only NRas is represented. Similarly, HRas and NRas switch between the plasma membrane and Golgi apparatus can be facilitated by phosphodiesterase $\delta$ action, although this is only one of the mechanisms of non-vesicular transport described. FTs: Farnesyl transferases, Rce1: Ras-converting enzyme 1, Imct: Isoprenylcysteine carboxyl methyltransferase, PATs: palmitoyl-transferases, PDE $\delta$ : Phosphodiesterase $\delta$, CAM: Calmodulin, ER: Endoplasmic reticulum.

\subsubsection{Differences in plasma membrane and endomembrane signalling among canonical RAS} isoforms

RAS GTPases interact with a wide range of activators and effectors. However, different RAS-mediated signalling outputs are obtained depending on the plasma membrane and microdomains localization, as well as endomembrane association. In this regard, RAS GTPases have been found in Golgi apparatus, ER, mitochondria and endosomes, being able to activate its downstream signalling pathways from these endomembranes.

Specifically, RAS activation in the Golgi apparatus has been observed in both fibroblasts and more strongly in T lymphocytes downstream T-cell receptor (TCR) signalling. In this context, RAS activation in the Golgi apparatus is mainly mediated by Ras Guanyl nucleotideReleasing Protein (RasGRP1) guanine nucleotide exchange factor (GEF). Additionally, activation of HRAS, by Ras Guanine nucleotide-Releasing Factor (RasGRF) GEF, in the ER has also been documented. Interestingly, it seems that the signalling pathways downstream RAS activation in Golgi apparatus or ER are slightly different, since ERK and AKT are efficiently activated from the Golgi apparatus but not c-Jun N-terminal kinase (JNK), and vice versa in ER. Altogether, these findings further prove the existence of spatially restricted pools of RAS activation capable of interacting with different downstream effectors (Arozarena et al., 2004; Chiu et al., 2002; Fehrenbacher et al., 2009; Hernandez-Valladares \& Prior, 2015; Matallanas et al., 2006; Mor et al., 2007).

Association of RAS isoforms with the mitochondria has also been documented, with KRAS exhibiting a key role in BCL-driven programmed cell death. Furthermore, activated receptor tyrosine kinases (RTKs), adaptors proteins including Src homology 2 domain-containingtransforming protein $\mathrm{C}$ (SHC), Growth factor receptor-bound protein 2 homolog (GRB2), and Son of Sevenless (SOS) are localised at the endosome, facilitating GDP/GTP exchange and increasing RAS activity in these endomembranous compartments. Moreover, RAS effectors such as Phosphatidylinositol 3-Kinase (PI3K) and Cell Division Control protein 42 (CDC42) have been also identified to interact with active endosomal RAS. Nonetheless, opposing published data demonstrate that endogenous HRAS mainly signals from receptors activated at the plasma membrane, and not from internal endomembranous organelles. In fact, it should be noted that these findings have been obtained from model systems expressing high levels of receptors and signalling components, and future research clarifying the endogenous RAS 
activity is still required (Bivona et al., 2006; Cheng et al., 2011; Fehrenbacher et al., 2009; Hernandez-Valladares \& Prior, 2015; Miaczynska \& Bar-Sagi, 2010; Moretó et al., 2008; Pinilla-Macua et al., 2016; Platta \& Stenmark, 2011; Tsutsumi et al., 2009; Wolfman et al., 2006)

In line with the GEF-endomembrane activation specificity, it has been postulated that there is a different GEF modulation among the canonical RAS. For instance, RasGRF1 is able to activate HRAS but not NRAS or KRAS, and in contrast RasGRF2 has been reported to preferably stimulate GDP/GTP exchange in NRAS and KRAS and less efficiently in HRAS. Additionally, RasGRP2 can activate both NRAS and KRAS but no HRAS, meanwhile RasGRP1 and RasGRP3 exhibits stronger HRAS activation and lower GDP/GTP exchange activity for NRAS and KRAS. Lastly, SOS GEFs are known to induce activation of all canonical Ras isoforms. (Jaumot et al., 2002; Matallanas et al., 2003; Rojas \& Santos, 2006).

Additionally, different downstream activation of RAF proto-oncogene serine/threonineprotein kinase (RAF1) and PI3K by HRAS and KRAS has been documented, with KRAS being the most efficient isoform in recruiting RAF1 to the plasma membrane, whereas HRAS seems to preferable activate PI3K (Yan et al., 1998).

In summary, RAS isoforms can signal from different organelles, can be activated after the action of different GEF activators and have different efficacies in inducing distinct downstream effectors.

\subsubsection{RAS activation-deactivation cycle}

RAS activation-deactivation cycle is given by two properties of GTPases proteins or GTP hydrolases: (1) their high affinity binding guanosine nucleotides, and (2) their ability to hydrolyse the GTP to GDP. Both processes are accomplished by conserving structural elements, the G-domain, constituted by five preserve polypeptide loops (G-motif 1-G-motif 5, G1-G5), which allow the binding of the nucleotides by electrostatic interactions through conserved aminoacids in G1, G4 and G5 loops (Bos et al., 2007; Hennig et al., 2015; Vetter \& Wittinghofer, 2001).

GTP binding to RAS results in a conformational change which orientate two switches: switch 1, located in G2; and switch 2, located in G3 (Fig. 6). This induces the reaction between the catalytic $\mathrm{Mg}^{2+}$ and a water molecule, facilitating GTP hydrolysis. At the same time, switches 1 and 2 exhibit the effectors interaction surface of RAS.GTP in this active conformation (Hennig et al., 2015; G. Li \& Zhang, 2004). 
As previously described, RAS acts as a GTPase, having the ability to hydrolyse the bound GTP to GDP, and therefore, changing from its active to the inactive state, respectively. However, RAS intrinsic GTPase activity and exchange of nucleotides are very slow and incompatible with the observed RAS kinetics. This is due to the existence of two essential protein families: (1) the guanine nucleotide exchange factors (GEFs) that promote GTP binding to RAS and its activation, and (2) the GTPase activating proteins (GAPs) which speed-up the internal GTPase activity of RAS, inducing GTP hydrolysis to GDP and RAS inactivation (Ahearn et al., 2012; Bos et al., 2007; Hennig et al., 2015).

\subsubsection{RAS activators (GEFs) and inhibitors (GAPs) proteins}

Guanine nucleotide exchange factors (GEFs) and GTPase activating proteins (GAPs) regulate the activity of RAS binding to guanine nucleotide to control cellular functions. GAPs and GEFs interact with switch 1 and 2 of RAS proteins in order to control its activity. These switches undergo conformational changes depending on RAS binding to GDP or GTP, with RAS.GDP being able to bind to its GEF activators and RAS.GTP interacting with the GAPs that will switch-off RAS signalling (Bos et al., 2007; Hennig et al., 2015).

\subsubsection{RAS GEFS}

In mammals there are five families of RAS GEFs: SOS, RasGRP, RasGRF, CNrasGEF and PLC $\varepsilon$; all of them highly homologous in their catalytically competent unit, the RAS Exchange Motif (REM) domain, in combination with the catalitic CDC25 homology domain (CDC5HD/RasGEF) (Fig. 8) (Bowtell et al., 1992; Ebinu et al., 1998; I et al., 2000; Rojas et al., 2011; Jose M. Rojas \& Santos, 2006).

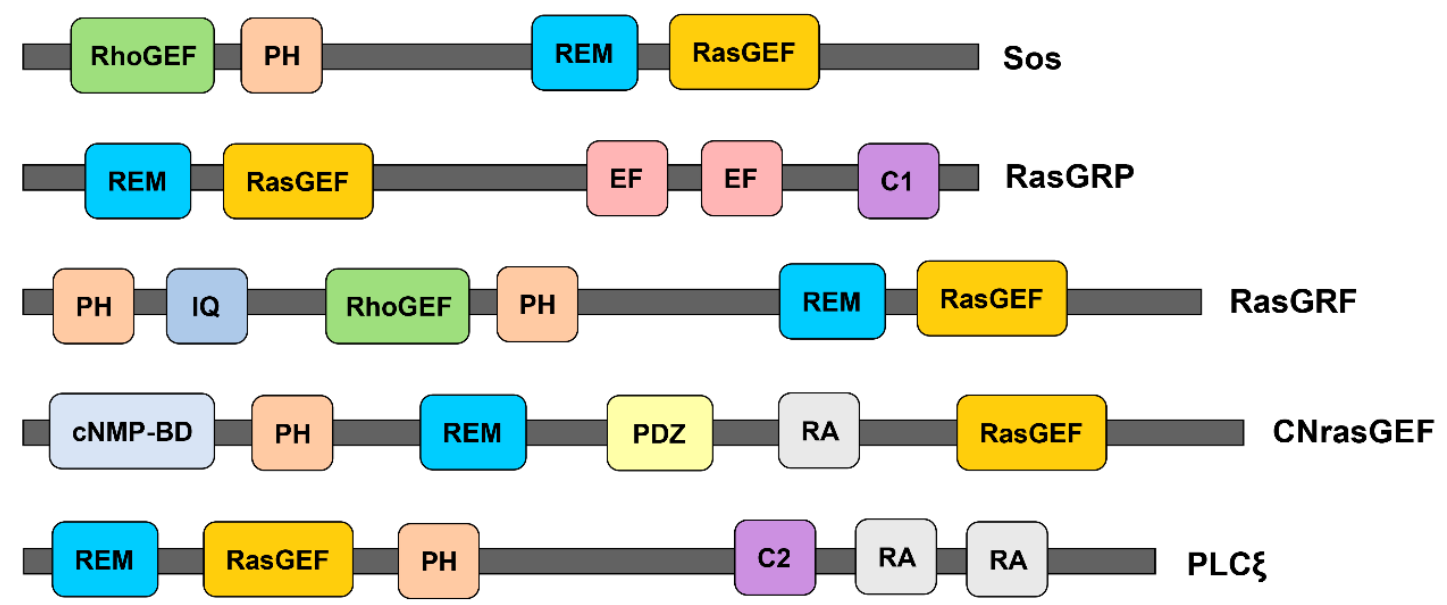

Figure 8: RAS GEFs family members and main domains. GEFs with nucleotide exchange activity towards Ras. Cdc25 catalytic domain is represented as RasGEF (yellow), and Ras exchange motif as REM (blue). PH: Pleckstrin homology, $\mathrm{EF}: \mathrm{Ca}^{2+}$ binding EF hand, IQ: calmodulin binding motif, cNMP-BD: cAMP and cGMP binding domain, 
PDZ: targeting to the cell membrane through interactions with phosphatidylinositol, RA: Ras/Rap1-associating domain, C1: Diacylglycerol-binding C1 domain, C2: protein kinase C conserved region 2.

\section{SOS (Son of Sevenless)}

SOS proteins (Son of Sevenless) (Fig. 8) were initially discovered as regulators of eye development studies in Drosophila melanogaster, being essential in the development of a cluster of photoreceptors cells. Moreover, the RAS activators found in yeasts, Cdc25, Scd25 and Ste6, share a high homology with SOS from $D$. melanogaster and a common domain, the catalytic CDC25 domain, and further studies identified other SOS homologues in C. elegans, $M$. musculus, and humans, although in mammals there are two homologous SOS proteins: SOS1 and SOS2 (Rogge et al., 1991; Simon et al., 1991).

The expression of mammalian SOS (SOS1 and SOS2) is ubiquitous, having an extensive expression during both embryonic development and adult stage, including almost all cell lineages, tissues and organs. Despite the high homology between SOS1 and SOS2, there are key biochemical and functional differences between them. The C-terminal domain is the most variable region among SOS1 and SOS2, presenting also differences in this region on consensus phosphorylation regions, and ubiquitination zones present in SOS2 but not on SOS1. All these features may explain the different affinity of SOS with the adaptor protein GRB2 (SOS2 shows more affinity than SOS1), the differences in regulation and protein stability (SOS1 is more stable than SOS2 since SOS1 lacks ubiquitination zones) (Baltanás et al., 2020b; Rojas et al., 2011).

In addition, knockout (KO) mouse animal models for Sos1 and/or Sos2 have revealed that, while Sos2-KO animals develop normally with no apparent phenotype, Sos1-KO mice died during embryonic development as a cause of placental malformation. However, the lack of Sos1 in adult the stage does not affect organism survival. In spite of this, the combined elimination in adult animals of Sos 1 and Sos2 (Sos1/2-DKO) leads to mice dead within 15 days, revealing a functional redundancy of Sos proteins in adult organism homeostasis and survival (Baltanás et al., 2013, 2020; Liceras-Boillos et al., 2018; Qian et al., 2000; Wang et al., 1997).

\section{RasGRP (Ras Guanyl nucleotide-Releasing Protein)}

Ras guanyl nucleotide-releasing proteins (RasGRPs) belong to a family of 4 are guanyl nucleotide exchange factors described in vertebrates (RasGRP1-4). They share many structural domains but with subtle differences between each one. Moreover, whereas SOS is ubiquitously expressed, RasGRP expression is restricted, with predominance in blood cells; and they activate $\mathrm{H}-, \mathrm{N}-$, and KRAS, as well as RAP. As in the case of SOS proteins, RasGRP exhibits the REMCDC25, but also presents two calcium binding domains and one diacylglycerol (DAG) binding domain, being all of them essential for its activation (Fig. 8) (Ebinu et al., 1998; Ksionda et al., 2013; Stone, 2011). 


\section{RasGRF (Ras Guanine nucleotide-Releasing Factor)}

Ras Guanine nucleotide-Releasing Factors (RasGRF) family encode RasGRF1 and RasGRF2 members, being predominantly expressed in the nervous system, albeit they can be found in pancreas, lung, muscle and kidney (Fig. 8). In particular, RasGRF1 is involved in $\beta$ cell homeostasis, in neuronal differentiation, learning and memory process, meanwhile RasGRF2 has a key role in the alcohol-induced reinforcement process, photoreception, nuclear migration in retinal cone photoreceptors, lymphocyte proliferation and T-cells signalling (Fernández-Medarde \& Santos, 2011b; Font De Mora et al., 2003; Guerrero et al., 1996; Jimeno $\&$ Santos, 2017; Manyes et al., 2018; Stacey et al., 2012).

\section{CNrasGEF (Cyclic Nucleotide Ras Guanine Exchange Factor)}

Cyclic Nucleotide Ras Guanine Exchange Factors (CNrasGEF) are RAS and RAP1 activator GEFs, predominantly expressed in the brain and localised at the plasma membrane (Fig. 8). CNrasGEF RAS activation is mediated in response to cAMP or cGMP, meanwhile RAP1 activation is constitutive and independent of cAMP (Amsen et al., 2006; Pak et al., 2002; Pham et al., 2000).

\section{PLCE (Phospholipase C Epsilon)}

Phospholipase C Epsilon (PLCE) activation generates 1,4,5-triphosphate (IP3) and diacylglycerol (DAG) that stimulates the release of stored $\mathrm{Ca}^{2+}$ reserves and Protein Kinase $\mathrm{C}$ (PKC) respectively. It has been shown that HRAS and RAS-related proteins have the ability to bind and activate PLC $\varepsilon$ through the tandem RAS associated domains (RA) present in the Cterminal region of PLC $\varepsilon$. Additionally, PLC $\varepsilon$ structure contains a CDC25 domain located in the $\mathrm{N}$-terminal region, with evidences pointing at the possible role of PLC $\varepsilon$ as a RAS and RAP GEF (Fig. 8) (Dusaban \& Brown, 2015; Jin et al., 2001; Lopez et al., 2001; Tyutyunnykova et al., 2017).

\subsubsection{RAS GAPS}

Although RAS are GTPases with an intrinsic ability to hydrolase bound GTP to GDP, the intrinsic reaction rate exhibits a half-life of about $30 \mathrm{~min}$, being this rate incompatible with the rapidly activation and deactivation cycles observed in RAS cellular kinetics. For an efficient hydrolysis, GAPs function is needed to accelerate the reaction, and this is achieved through interaction with RAS. GTP through its RasGAP or catalytic domain. The four main RAS GAPs are p120GAP, NF1, GAP1 family members and SynGAP (Fig. 9). 


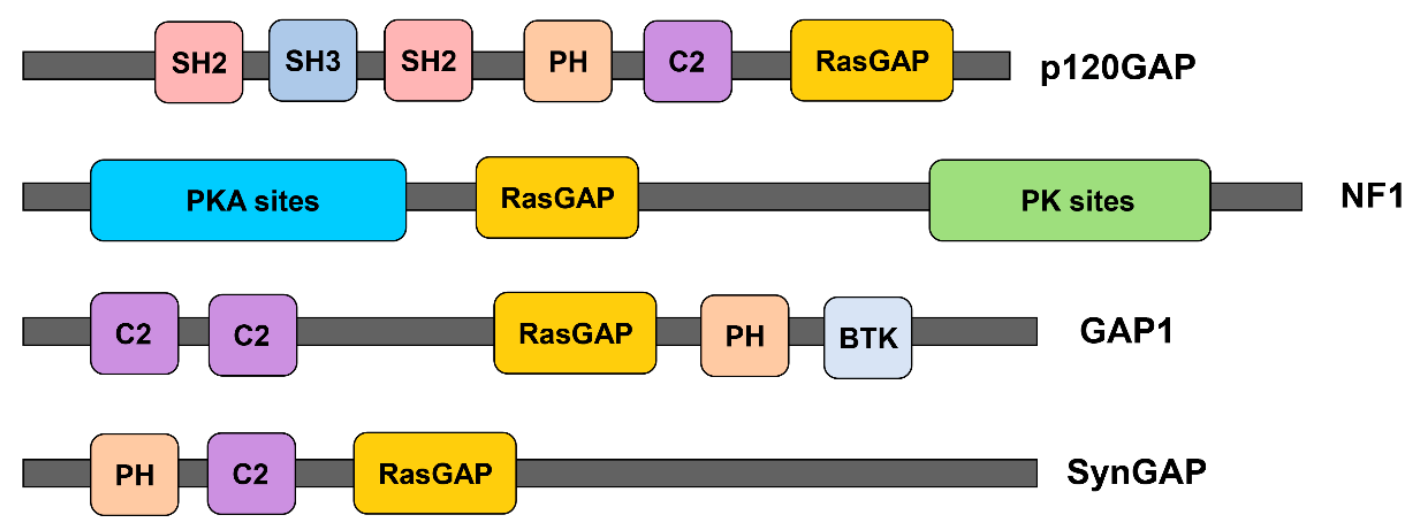

Figure 9: RAS GAPs family members and main domains. Domain architecture of Ras GAP with proven catalytic activity towards Ras. RasGAP: catalytic domain of GTPase activating proteins, C2: protein kinase C conserved region 2, PH: pleckstrin homology, SH2: Src homology 2 domain, SH3: Src homology 3 domain, PKA sites: protein kinase A phosphorylation sites, PK sites: protein kinase phosphorylation sites, BTK: Bruton's tyrosine kinase motif.

\section{p120GAP (Ras GTPase-activating protein 1 / RASA1)}

p120GAP or RASA1 was the first negative regulator of RAS identified, although some studies indicate a dual role, acting as a RAS negative regulator/positive effector (Fig. 9). Of the two p120GAP isoforms, type I is the most widely expressed in cells meanwhile type II splicing isoform is more expressed in placental trophoblasts. Additionally, RASA1 KO mice dye during embryonic development due to severe vascular abnormalities, and in concordance, RASA1 mutations have been identified in several arteriovenous malformations, pointing to a possible tissue-specific function in the vascular cell-growth control (Drugan et al., 2000; Hennig et al., 2015; Rajalingam et al., 2007; Rojas \& Santos, 2006).

\section{NF1 (Neurofibromin 1)}

Neurofibromin (NF1) is the protein product of the tumour suppressor NF1 (Fig. 9), being lost in the inherited disorder neurofibromatosis type 1, a common autosomal dominant disorder where RasGAP domain is found to be mutated. NF1 null mice died embryonically between E12.5-E13.5 due to anomalous growth of hematopoietic cells and markedly cardiac defects, and NF1+- animals showed deficits in spatial memory and learning and increased astrocyte number, indicating that NF1 is necessary to control RAS activation in myeloid cells and brain (Hennig et al., 2015; Rojas \& Santos, 2006; Yzaguirre et al., 2015; Zhang et al., 1998).

\section{GAP1 (Ras GTPase-activating protein 1)}

GAP1 family members encompasses GAP1 ${ }^{\mathrm{m}}, \mathrm{GAP} 1^{\mathrm{IB} 4 \mathrm{BP}}, \mathrm{CAPRI}$ and RASAL, being able to exert its activity on both RAS and RAP GTPases (Fig. 9). CAPRI and RASAL are GAPs stimulated by $\mathrm{Ca} 2+$ levels, thereby connecting $\mathrm{Ca} 2+$ oscillations and spikes with RAS regulation. On the other hand, GAP $1^{\mathrm{m}}, \mathrm{GAP} 1^{1 \mathrm{~B} 4 \mathrm{BP}}$ lack the $\mathrm{Ca} 2+$ levels-responding residues, 


\section{Introduction}

being GAP1 $1^{\mathrm{IB} 4 \mathrm{BP}}$ constitutively bound to plasma membrane and $\mathrm{GAP} 1^{\mathrm{m}}$ is localized in the perinuclear region, being recruited to the plasma membrane after PI3K activation (Hennig et al., 2015; Kupzig et al., 2006; Rojas \& Santos, 2006).

\section{SynGAP (Synaptic Ras-GTPase activating protein)}

SynGAP (Synaptic Ras-GTPase activating protein) is a negative regulator of RAS and RAP selectively expressed in brain, and specially in excitatory synapses, where regulates synaptic plasticity, neuronal homeostasis and MAPK signalling in neurons (Fig. 9). SynGAP mutations have been found in human patients with autism spectrum disorder, intellectual disability and epilepsy. SynGAP null mice die postnatally within a week, so SynGAP haploinsufficiency models have been extensively studied to analyse its implication in physiology and cognition (Jeyabalan \& Clement, 2016; Kim et al., 2003; Komiyama et al., 2002; Nakajima et al., 2019).

\subsection{7. $\quad$ Signalling pathways controlled by RAS}

Active RAS GTPases transient activate downstream signalling pathways, transducing extracellular signals to its effectors and regulating a huge variety of cellular processes associated with survival, proliferation, migration, apoptosis and differentiation. The activation of a certain downstream effector would depend on both the received stimulus and cell type, being the most studied the RAS/MAPK, PI3K/AKT, RAS/RAL guanine nucleotide dissociation stimulator (RALGDS) and RAS/Mitogen-activated protein kinase p38 (p38), among others. Although numerous RAS downstream effectors have been described (Fig. 10), we are focusing only on the pathways related to our study (Anton \& Wennogle, 1998; Campbell et al., 1998; Joneson \& Bar-Sagi, 1997; Rajalingam et al., 2007).

\subsubsection{RAS/MAPK}

The family of MAPKs are Ser-Thr kinases that modulate signal transduction and gene expression in response to external signals and environmental changes. The MAPKs family is composed by four different groups: ERK1/2, JNK1/2/3, p38 $(\alpha / \beta / \gamma / \delta)$, ERK5, and the called atypical MAPKs ERK3/4, ERK7 and Nemo-like kinase (NLK) (Cargnello \& Roux, 2011; Kassouf \& Sumara, 2020; Turjanski et al., 2007).

MAPKs signalling pathways are conserved among organisms, from plants fungi, nematodes insects and mammals. Their activation is achieved through a consecutive and sequential activation cascade in which a specific MAPK kinase kinase (MAPKKK) phosphorylates and activates a MAPK kinase (MAPKK) that will in turn phosphorylate and activate their downstream MAPKs (Turjanski et al., 2007). 


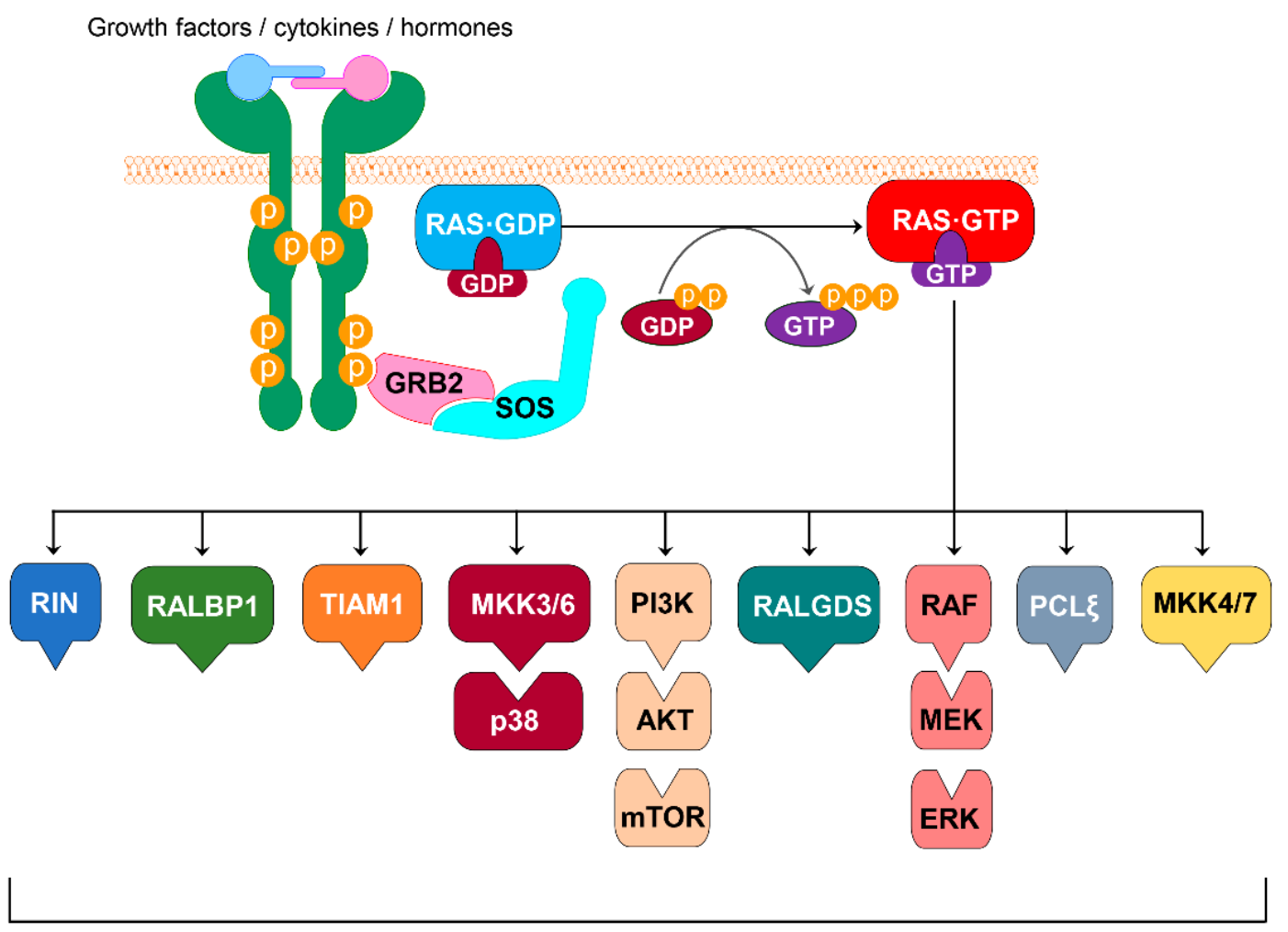

Proliferation, survival, apoptosis, differentiation, angiogenesis, migration, endocytosis

Figure 10: Effectors downstream RAS activation. Scheme representing Ras activation and some of its effectors. Only Ras/p38, Ras/PI3K and Ras/ERK pathways are described in the text.

\section{RAS/ERK}

The first MAPKKK component of Ras/ERK pathway is the threonine-serine kinase RAF (CRAF, A-RAF, B-RAF). These RAF kinases only bind to the effector domain of RAS when the GTPase is in its active state (GTP bound). For this purpose, they contain a RAS-BindingDomain (RBD), that is common to all RAS effectors. The specific RAF activation mechanism involves a tight regulation though the interaction with other proteins, and by phosphorylation/dephosphorylation events were kinases such as Proto-oncogene tyrosineprotein kinase SRC (SRC), PKC, Serine/threonine-protein kinase PAK (PAK) or Serine/threonine-protein phosphatase PP2A (PP2A) phosphatases are involved. Active RAF phosphorylates and activates Dual specificity mitogen-activated protein kinase kinase $1 / 2$ (MEK1/2) MAPKKs, serine-threonine kinases that will ultimately phosphorylate and activate the ERK1/2 MAPKs (Fig. 10) (Roberts \& Der, 2007; Terrell \& Morrison, 2019; Turjanski et al., 2007).

ERK1/2, also named p44/p42 MAPK, were the first MAPKs described in mammals. Cytoplasmic ERK is inactive under basal conditions, however, active ERK distributes throughout different subcellular components, including translocation to the nucleus where regulates gene expression, but also interacts with cytoplasmic scaffold proteins (such as Kinase 


\section{Introduction}

suppressor of Ras 1 (KSR1), $\beta$-arrestin and Interleukin-17 receptor D or Sef homolog (Sef)), leading to a set of cytoplasmic responses. Its signalling involves multiple cellular processes such proliferation, survival, metabolism, differentiation and cell death through the phosphorylation of more than 175 effector proteins, including transcription factors, phosphatases, kinases, apoptosis regulators, etc (Casar et al., 2008; DeFea et al., 2000; Z. Lu \& Xu, 2006; Mebratu \& Tesfaigzi, 2009; Meloche \& Pouysségur, 2007; Sugimoto et al., 1998; Tohgo et al., 2002; Torii et al., 2004; Wortzel \& Seger, 2011).

\section{RAS/p38}

p38 family includes four isoforms: p38 $\alpha$ (MAPK14), p38 $\beta$ (MAPK11), p38 $\gamma$ (MAPK12), and p38 (MAPK13), and mediate stress signals, inflammatory responses and cell death. They exhibit a dual-nature since they were originally described as tumour-suppressor kinases, but extensive experimental data support also its role as a tumour promoter. Of the four isoforms, $\mathrm{p} 38 \alpha / \beta$ exhibit a more ubiquitous expression, meanwhile $\mathrm{p} 38 \gamma / \delta$ have a more restricted expression pattern and may have more specific functions. RAS can stimulate p38 activation through MKK3 and MKK6 activation (Fig. 10), leading to stress/ROS-driven senescence. Activation of p38 takes place through a dual phosphorylation on Thr and Tyr residues on the Thr-Gly-Tyr motif located in the activation loop, enabling a more open conformation for substrate recognition. They play key roles in immune response, survival, senescence, differentiation and proliferation, being also able to activate ERK1/2. The mechanisms through which p38 play opposing effects is likely to be due to differences in strong and sustained or low p38 activation, but more experimental data is needed to clarify these dual-effects (Cuadrado \& Nebreda, 2010; Houliston et al., 2001; Martínez-Limón et al., 2020; Wang et al., 2002; Zarubin \& Han, 2005).

\subsubsection{PI3K/AKT}

PI3K is composed by a regulatory subunit (p85) and a catalytic subunit (p110) and are recruited to the plasma membrane after the activation of RTKs or GPCRs in response to insulin, growth factors and cytokines. They have the ability of binding directly to the activated receptor or through the SH2 domain of p85 subunits and scaffold proteins, producing a conformational change in p85 subunit that is further transmitted to p110. Additionally, PI3K encompasses an RBD domain that interact with active RAS.GTP inducing downstream activation of PI3K effectors (Fig. 10). HRAS is the isoform that more efficiently activates PI3K in comparison to KRAS, that is a weaker activator of the pathway (Castellano \& Downward, 2011; Hoxhaj \& Manning, 2020; Rodriguez-Viciana et al., 1994; Yan et al., 1998). 
Activated PI3Ks catalyse the formation of the plasma membrane phospholipids phosphorylating phosphoinositide 4,5-biphosphate $\left(\mathrm{PIP}_{2}\right)$ to phosphoinositide 3,4,5triphosphate $\left(\mathrm{PIP}_{3}\right)$, a key second messenger for several effector proteins such as 3phosphoinositide-dependent protein kinase 1 (PDK1) and Protein kinase B (PKB or AKT). PIP3 recruits $\mathrm{AKT}$ and PKD1 to the plasma membrane through the binding to their pleckstrin homology domains (PH), inducing PDK1 activation and subsequent AKT phosphorylation by PDK1 in Thr308. This phosphorylation is sufficient to activate AKT, but fully activated AKT needs in addition its phosphorylation in Ser473 which can be mediated by Rapamycininsensitive companion of mTOR (mTORC2), DNA-protein kinase (DNA-PK), or by AKT autophosphorylation. Activated AKT mediates the phosphorylation of large and diverse downstream substrates, influencing a variety of cellular functions such as proliferation, growth, metabolism and survival. On the other hand, Phosphatidylinositol 3,4,5-trisphosphate 3phosphatase and dual-specificity protein phosphatase (PTEN) and Phosphatidylinositol 3,4,5trisphosphate 5-phosphatase (SHIP) phosphatases negatively regulates PI3K-mediated signalling through the dephosphorylation of $\mathrm{PIP}_{3}$ and regeneration of $\mathrm{PIP}_{2}$ in the plasma (Castellano \& Downward, 2011; Fruman et al., 2017; Hoxhaj \& Manning, 2020; Sarbassov et al., 2005; Toker \& Newton, 2000).

\subsubsection{Functional specificity and redundancy of canonical Ras isoforms in KO mouse models}

Expression of the canonical RAS isoforms is conserved between species and almost ubiquitous, although notable differences have been pointed out such as different expression levels depending on the tissue and organism developmental stage. To better understand the in vivo role of the RAS GTPases and to analyse whether these highly homologous GTPases exert redundant functions or not, several mouse knockouts $(\mathrm{KO})$ models have been generated and studied.

Of the three isoforms, only the removal of KRas was found to be embryonically lethal, with the KRas-ablated mouse embryos dying between embryonic day 12 and 14 (E12-E14), showing markedly liver defects and anaemia. However, subsequent studies showed the dispensability of KRas4A during embryonic development in presence of a functional KRas4B, pointing to a specific need of the KRas4B isoform for normal embryonic development (Johnson et al., 1997; Koera et al., 1997; Plowman et al., 2003).

On the other hand, individual lack of HRas or NRas was demonstrated to be irrelevant for proper embryonic development and adult mice survival. However, a combination of complete removal of NRas with single allele elimination in KRas (NRas-; ${ }^{--} \mathrm{KRas}^{+-}$) led to early embryonic lethality, with almost all mice dying between E10-E12, thus showing that NRas and KRas exert, to some extent, overlapping functions during embryonic development, and that NRasKO 


\section{Introduction}

embryos need a wild-type KRas. Interestingly, HRas--;KRas"- embryos died earlier than KRas ${ }^{--}$ mutant embryos, suggesting overlapping functions of HRas and KRas between E9.5-E11.5 of embryonic development (Esteban et al., 2001; Johnson et al., 1997; Nakamura et al., 2008; Umanoff et al., 1995).

HRas $^{--}$;NRas ${ }^{--}$were viable, with the adult mice showing no obvious external phenotype. Despite this, the number of adult HRas ${ }^{--}$;NRas ${ }^{--}$double-knockout (DKO) mice was significantly lower than expected, even if the ratio of DKO embryos at E17.5 was normal, suggesting some overlapping functions of HRas and NRas between E18.5 and postnatal growth that could not be substituted by KRas (Esteban et al., 2001; Weyandt et al., 2016).

In another study aiming to analyse the specificity of these isoforms, KRas embryonic functional specificity was bypassed using a HRas knock-in (KI) mice expressing HRas under KRas promoter. With this approach, KRas function during embryonic development was successfully replaced by HRas, pointing to a spatiotemporal specificity of the KRas promoter as a reason for the specificity of this isoform in embryonic development. In spite of this, adult HRasKI mice showed markedly cardiovascular defects, which demonstrated a protein-specific role for KRas in the cardiovascular system (Nakamura et al., 2008; Potenza et al., 2005).

In summary, despite the canonical Ras isoforms exert some tissue or cell-dependent expression with KRas4B function being the most critical for organism survival, there are strong evidence supporting functional redundancy during some stages of embryonic development.

\subsubsection{Pathologies linked to RAS gain-of-function}

Somatic activating mutations affecting $R A S$ and associated signalling pathway components such RAF and MEK have been found frequently mutated in 10-30\% of human cancers; but also germline mutations on $R A S$ and related pathway genes such as NF1, Protein-tyrosine phosphatase 2C (SHP2) and SOS1 have been strongly implicated in a broad group of developmental disorders named RASopathies (RAS/mitogen-activated protein kinase (MAPK) syndromes) (Fernández-Medarde \& Santos, 2011a; Muñoz-Maldonado et al., 2019; Prior et al., 2020; Riller \& Rieux-Laucat, 2021).

\subsubsection{RAS in cancer}

$R A S$ gain-of-function mutations include those producing an enhanced GTP binding activity and impairment of GTPase activity or GAP binding, mostly located at codons 12, 13 and 61. Of the three codons, mutations located at codons 12 and 13 are thought to interfere with GAPs binding, meanwhile codon 61 mutations affect GTP intrinsic hydrolysis mechanism. Of the three canonical RAS GTPases, KRAS is the most frequently mutated in human tumours, 
harbouring the $85 \%$ of total RAS mutations, followed by NRAS (11\%) and HRAS (4\%) (MuñozMaldonado et al., 2019; Prior et al., 2020).

The mutation rates at each codon differ between the RAS GTPases, with notable differences regarding among the three isoforms. In this regard, G12 mutations are found in an $81 \%$ of KRAS cancers, followed by G13 (14\%) and Q61 (2\%); meanwhile the most mutated codon in NRAS and HRAS human cancers is Q61 (62 and 38\%, respectively), followed by G12 (23 and 26\%, respectively) and G13 (11 and 23\%, respectively) (Dunnett-Kane et al., 2020; MuñozMaldonado et al., 2019; Prior et al., 2020).

KRAS mutations are frequently found in solid tumours such as pancreatic ductal adenocarcinoma, colorectal adenocarcinoma, non-small-cell lung cancer and small intestine adenocarcinoma and are highly associated with a higher malignancy when compared with HRAS and NRAS. On the other hand, NRAS has been found frequently mutated in skin melanoma, haematological malignancies and thyroid carcinoma. Finally, HRAS mutations have been reported in bladder cancer, thyroid carcinoma and head and neck squamous cell carcinoma. All this data demonstrates some tissue-specific RAS differences with significant preference of some mutations with a specific tumour type (Castellano \& Santos, 2011; Dunnett-Kane et al., 2020; Fernández-Medarde \& Santos, 2011a; Muñoz-Maldonado et al., 2019; Prior et al., 2020).

\subsubsection{RASopathies}

Somatic RAS mutations and related regulators cause a group of developmental disorders socalled RASopathies. These illnesses are a heterogeneous group exhibiting overlapping phenotypic features such as craniofacial dysmorphism, neocutaneous abnormalities, cardiovascular defects, risk of tumorigenesis, developmental delay, learning disabilities and short stature. They include neurofibromatosis 1 (NF1), Noonan syndrome (NS), Noonan syndrome with multiple lentigines (NSML), Costello syndrome (CS), Legius syndrome (LS), LEOPARD, cardiofaciocutaneous syndrome (CFC), capillary malformation-arteriovenous malformation syndrome (CM-AVM) and autosomal dominant intellectual disability type 5 (Castellano \& Santos, 2011; Fernández-Medarde \& Santos, 2011a; Riller \& Rieux-Laucat, 2021; Tajan et al., 2018).

Besides the known cardio-facio-cutaneous phenotypes present in these patients, a strong incidence of neonatal respiratory distress syndrome has been described in several clinical cases. Germline mutations in HRAS GTPases, being the most common G12S substitution causing CS, have been reported to cause respiratory distress at birth in the $45 \%$ of the neonates ( 5 cases of a total of 12 reported clinical cases), retardation of normal alveolar development or congenital alveolar dysplasia, lung fibrosis, bronchopulmonary dysplasia, etc. Altogether, 65\% of CS newborn (19 of 30 reported clinical cases) exhibit respiratory distress syndrome at birth. Furthermore, $33 \%$ of CFC newborns (4 out of 12 reported clinical cases) exhibit respiratory 


\section{Introduction}

distress, with a clinical case reporting a mutation in $K R A S$ with P34R substitution. Additionally, NS clinical cases have reported to exhibit respiratory distress syndrome at birth, with RAF1 P261R mutation, SOS1 substitution F623I in the patients, and $42 \%$ of neonates showing respiratory distress syndrome. Interestingly, these observations regarding respiratory distress syndrome were not attributable to cardiovascular defects (Baltanás et al., 2020a; Fabretto et al., 2010; Gomez-Ospina et al., 2016; Morcaldi et al., 2015; Myers et al., 2014; Ratola et al., 2015).

Additionally, there are several mouse models recapitulating different RASopathies syndromes. Of interest for our work, mouse models harbouring P34R KRas mutation, described in CFC patients, exhibit a markedly perinatal lethality from respiratory failure due to defective lung maturation associated with increased aberrant pERK activity in lung epithelium. In contrast, KRas T58I mouse (NS mutation) do not exhibit neonatal death and display a lung maturation phenotype intermediate between the wild-type and P34R mice (Wong et al., 2020).

Thus, it is of interest how some mutations in RAS GTPases and related pathway components trigger lung abnormalities and immaturity but not all of them result in such phenotypic defects 
AIMs / OBJETIVOS 



\section{AIMS OF THE THESIS}

The overall aim of this Thesis was to investigate the involvement of HRas and NRas in late embryonic mouse lung development. The specific proposed objectives were:

1. To analyse the differential phenotypes that arose from the genetic deletion in mice of HRas and NRas, alone and in combination.

2. To study the effect of HRas and/or NRas ablation in lung oxidative stress and mitochondrial respiration.

3. To evaluate the effect of antenatal glucocorticoids and antioxidant treatment on the lung development and postnatal survival.

4. To investigate the specific implication of HRas and/or NRas in lung mesenchyme signalling and oxidative stress.

5. To analyse the contribution of HRas and/or NRas to lung development in a 3D-lung organoid model. 


\section{OBJETIVOS}

El objetivo principal de la Tesis fue investigar el papel de HRas y NRas en las últimas etapas del desarrollo pulmonar murino. Específicamente los objetivos eran:

1. Analizar las diferencias fenotípicas que se originan tras la eliminación de HRas y NRas, de manera individual o conjunta, en ratones.

2. Estudiar el efecto de la eliminación de HRas y/o NRas en el control del estrés oxidativo y respiración mitocondrial pulmonar.

3. Evaluar el efecto del tratamiento perinatal con glucocorticoides o antioxidantes en el desarrollo pulmonar y supervivencia neonatal.

4. Investigar el papel de HRas y NRas en la señalización y estrés oxidativo del mesénquima pulmonar.

5. Analizar la contribución de HRas y/o NRas en el desarrollo pulmonar en un modelo de organoides de pulmón. 
MATERIALS AND METHODS 



\section{MATERIAls AND Methods}

\section{In vivo studies}

\subsection{Ethics statements}

Experiments and animal procedures were conducted following the guidelines laid down by the European Communities Council Directive of 20 March 2015 (ECC/566/2015), Spanish (RD53/2015), and approved by the Junta de Castilla y León Government and the bioethics committee from the University of Salamanca (ref 409). Additionally, the experimentation conducted in The Netherlands was carried out in accordance with ethical permit AVD1050020209205 approved by the Centrale Commissie Dierproeven (Den Haag, The Netherlands).

\subsection{Generation of HRas and/or NRas null mice}

HRas (Esteban et al., 2001) and NRas (Umanoff et al., 1995) simple and DKO mice were maintained in pure C57Bl/6 background and kept on a 12 hour light/dark cycle. HRas ${ }^{+-}$;NRas ${ }^{--}$ and HRas ${ }^{--} \mathrm{NRas}^{+-}$animals were crossed to generate descendants with equal proportion of the four genotypes under study: HRas ${ }^{+-}$;NRas ${ }^{+-}$(hereinafter control (CT)), HRas ${ }^{--} \mathrm{NRas}^{+-}$(HRasKO), HRas ${ }^{+-}$;NRas"- (NRas-KO), HRas ${ }^{--}$;NRas"- (HRas/NRas-DKO (DKO)).

\subsection{Mouse Genotyping}

Mice were genotyped by Polymerase Chain Reaction (PCR) analysis of genomic DNA isolated from mouse tails using specific primers for the wildtype (WT) or null-mutant alleles of $H R A S$ or $N R A S$, as appropriate.

\subsubsection{DNA isolation}

Genomic DNAs were obtained from embryos, newborn and adult mice tails. Tissue was homogenized in $500 \mu \mathrm{l}$ of lysis buffer (Tris $100 \mathrm{mM} \mathrm{pH} \mathrm{8,} \mathrm{EDTA} 5 \mathrm{mM} \mathrm{pH} \mathrm{8,} \mathrm{NaCl} \mathrm{200mM,}$ SDS $0.2 \%$, Proteinase $\mathrm{K} 0.26 \mathrm{mg} / \mathrm{ml}$, MiliQ water) overnight (o/n) at $56^{\circ} \mathrm{C}$ with gentle agitation. Following the enzymatic digestion, the samples were centrifuged 10 minutes (min) at 13000 rpm and the supernatants were collected $(500 \mu 1)$. DNA was precipitated with $900 \mu$ of $100 \%$ ethanol $(\mathrm{EtOH})$ and centrifuged $10 \mathrm{~min}$ at $13000 \mathrm{rpm}$, the supernatants were discarded, and the DNA was washed with $500 \mu 1$ of cold $70 \%$ EtOH. Next, the samples were centrifuged for $5 \mathrm{~min}$ at $13000 \mathrm{rpm}$, the supernatants were discarded, and the precipitated DNA was dried during 10 
min at room temperature (RT). DNA was resuspended in 200-500 $\mu 1$ of MiliQ water, depending on DNA amount.

\subsubsection{PCR genotyping}

Presence of WT and/or mutant alleles was detected using the primers indicated in Table 2. HRAS WT allele forward (F) and reverse (R) set of primers generated a specific 434bp fragment, meanwhile $H R A S$ null-mutant allele $\mathrm{F}$ and $\mathrm{R}$ primers generated a specific 336bp fragment. On the other hand, for NRAS WT allele $\mathrm{F}$ and R primers generated a specific $185 \mathrm{bp}$ fragment, and NRAS null-mutant allele set of $\mathrm{F}$ and $\mathrm{R}$ primers generated a specific $315 \mathrm{bp}$ fragment (Fig. 11).

\begin{tabular}{cc}
\hline Gene & Sequence \\
HRas WT & F- AGCTCCCTGGCCCCTTGTGG \\
\cline { 2 - 2 } & R-ACCTGCCAATGAGAAGCACACTTAGCC \\
\hline \multirow{2}{*}{ HRas mutant } & F- AGCTCCCTGGCCCCTTGTGG \\
\cline { 2 - 2 } NRas WT & R- CTACCGGTGGATGTGGAATGTGTGCGA \\
\cline { 2 - 2 } & F- CCAGGATTCTTACCGAAAGCAAGTGGTG \\
\hline \multirow{2}{*}{ NRas mutant } & F- CATGGCAAATACACAGAGGAACCCTTCG \\
\cline { 2 - 2 } & R- CATATGCGGTGTTACCAAATACAGCAAGTGGTG \\
\hline
\end{tabular}

Table 2: Primers used for mouse genotyping.

The composition of the reaction mixture for the PCR assays was: $5.5 \mu 1$ of DreamTaq ${ }^{\mathrm{TM}}$ Green Master Mix 2X ( $\mathrm{MgCl}_{2} 4 \mathrm{mM}, 0.4 \mathrm{mM}$ from each $\left.\mathrm{dNTP}\right), 0.3 \mu \mathrm{l}$ from each primer, $0.7 \mu \mathrm{l}$ of DNA ( 200ng) and 3.2 $\mu$ l of MiliQ water. The PCR conditions were as follows: $94^{\circ} \mathrm{C}$ for 4 $\min , 30$ cycles of $94^{\circ} \mathrm{C}$ for $1 \mathrm{~min}, 64.5^{\circ} \mathrm{C}$ for $1 \mathrm{~min}$ and $72^{\circ} \mathrm{C}$ for $1 \mathrm{~min}$ and a final incubation of $72^{\circ} \mathrm{C}$ for $10 \mathrm{~min}$.
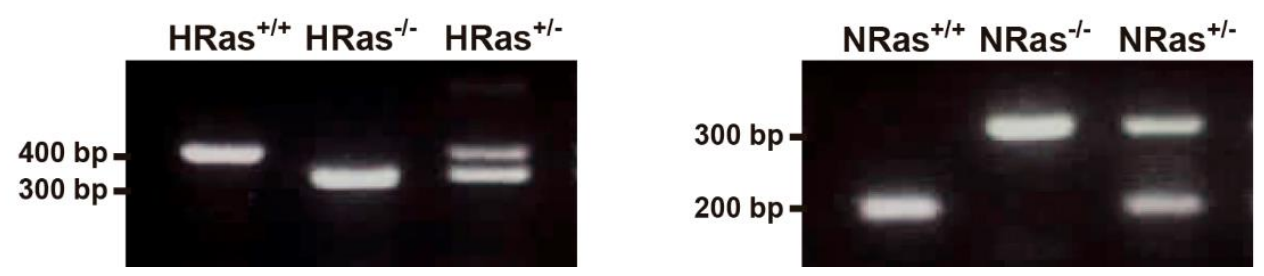

Figure 11: Representative images of PCR assays. Sizes for amplified bands: HRas WT (+/+) 434bp and HRas mutant (-/-) 336bp; NRas WT (+/+) 185bp and NRas mutant (-/-) 315bp.

\subsection{Dexamethasone antenatal treatment}

The beginning of gestation (E0.5) was timed through the detection of vaginal plugs, with further confirming the pregnancy by weighting the females from day 10 post coitum (pc). Either $0.4 \mathrm{mg} / \mathrm{kg}$ of dexamethasone (DEX, Sigma, D2915) or saline control $(\mathrm{NaCl} 0.9 \%)$ were injected 
subcutaneously (sb) to pregnant females on days E17.5 and E18.5 of embryonic development (Fig. 12). Survival of the newborn pups was monitored daily (weight, phenotype, activity). Embryos collected at embryonic day E18.5 received only one dose of DEX at E17.5 (Fig. 12).
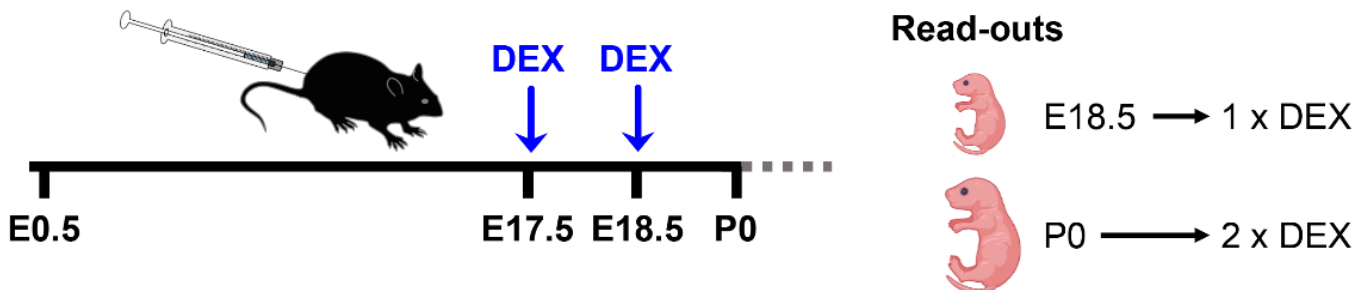

Figure 12: Antenatal dexamethasone (DEX) treatment. Schedule of subcutaneous injections with $0.4 \mathrm{mg} / \mathrm{kg}$ DEX of pregnant mice on embryonic days (E) 17.5 and 18.5. Pregnancy was verified with the observation of vaginal plug (E0.5). The read-outs include the analysis of E18.5 embryos, that received only one dose of DEX, and newborn pups (P0), that received two doses of DEX.

\subsection{N-Acetylcysteine antenatal treatment}

Pregnancy was timed at E0.5 after the observation of vaginal plugs. N-Acetylcysteine (NAC, Sigma, A7250) was administered in tap water (0.5\%, pH 7.4) using the breeding bottle $a d$ libitum, throughout the pregnancy, starting on E0.5 embryonic day (Fig. 13). NAC solution was changed weekly and pregnant females, together with the resulting litters, were monitored daily (weight, phenotype, activity, survival).

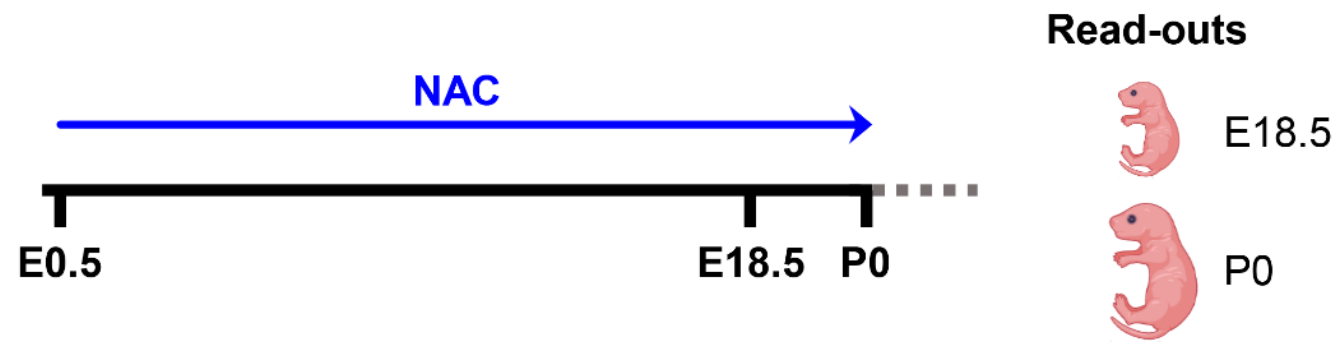

Figure 13: Antenatal N-Acetylcysteine (NAC) treatment. Schedule of NAC administration $(0.5 \%$ in tap water, ad libitum) in pregnant mice throughout pregnancy. Pregnancy was verified with the observation of vaginal plug (E0.5). The read-outs include the analysis of E18.5 embryos and P0 pups.

\subsection{Bromodeoxyuridine (BrdU) incorporation}

Nuclear incorporation of BrdU was used to measure the lung cellular proliferation in E18.5 embryos. To achieve this, BrdU (0.1mg/g body weight, Sigma, B5002) was injected intraperitoneally into pregnant female mice at E18.5 day of gestation. Mice were then euthanized $2 \mathrm{~h}$ after BrdU injection. 


\section{Materials and Methods}

\subsection{Tissue collection and preparation}

Adult animals were euthanized with an isoflurane (IsoFlo®, Esteve) overdose, followed by cervical dislocation after loss of pedal reflex. Newborns and E18.5 embryos were weighted and euthanized by decapitation.

Adult lungs, as well as whole body from newborns and embryos, untreated and subjected either to DEX or NAC treatments, were harvested and washed in 1X PBS prior to fixation with 4\% paraformaldehyde (PFA, PanReac BioChem, 252931.1214) at $4^{\circ} \mathrm{C}$ for three days before dehydration and paraffin embedding. For Haematoxylin-Eosin (H\&E) and immunostainings $3 \mu \mathrm{m}$ sections were used, meanwhile $5 \mu \mathrm{m}$-thick sections were used to perform Periodic acidSchiff (PAS) staining.

To perform other analyses, newborn lung lobes were separated and snap-frozen in dry ice for quantitative real-time PCR (RT-qPCR), RAS.GTP pull-down assays, western blot (WB) and ELISA analyses. For Seahorse assays, lung lobes were maintained in full-supplemented DMEM $\mathrm{o} / \mathrm{n}$ at $4^{\circ} \mathrm{C}$. To analyse the levels of ROS by flow cytometry and/or to isolate primary lung fibroblasts, lungs were washed in 1X Hank's balance salt solution, pH 7.2 (HBSS, Gibco, 14175095) and used immediately after harvesting.

\subsection{Histopathological analyses and immunostaining}

\subsubsection{Haematoxylin and eosin $(H \& E)$ staining}

$\mathrm{H} \& \mathrm{E}$ staining was performed to evaluate anatomo-pathological of lung histology (overall lung structure, ramification, alveoli condensed areas and emphysema lesions), analyses of the morphology and structure of epithelial cells (bronchiolar and alveolar), and quantification of alveoli spaces, as well as for the initial whole-body anatomo-pathological evaluation of all organs and tissues from newborns and E18.5 embryos. For lung studies, all the analyses were performed using a minimum of 10 images taken from equivalent sections. For alveolar area quantitation, images were manually transformed into binary images using ImageJ $®$ software (NIH), where the alveolar spaces were recognized as positive elements (black). The area of each alveolus $\left(\mu \mathrm{m}^{2}\right)$ was calculated using the ImageJ software.

Paraffin-embedded $3 \mu \mathrm{m}$-thick sections were incubated with Histoclear II (National Diagnostics, HS-202) $3 \times 5$ min, and transferred sequentially into EtOH as follows: $100 \%, 90 \%$, $80 \%, 50 \%$ ( $5 \mathrm{~min}$ each), followed by a wash in deionized water for $4 \mathrm{~min}$. Sections were then stained with Harris haematoxylin (PanReac) during $5 \mathrm{~min}$ at RT, followed by a wash in tap water during $20 \mathrm{~min}$ to eliminate the excess of staining. Sections were subsequently stained with an alcoholic solution of $0.5 \%$ eosin (PanReac) for 30 seconds (s), followed by a wash in distilled water. Finally, sections were dehydrated sequentially by immersion in $70 \%, 80 \%, 90 \%$ and 
100\% EtOH solutions for 1-2 min each, washed with Histoclear II 3x5 min, mounted and photodocumented under a light microscope (Olympus BX51 with Olympus DP70 camera).

\subsubsection{Periodic acid-Schiff (PAS) staining}

$5 \mu$ m-thick paraffin embedded sections were incubated with alcian blue ( $\mathrm{pH} 2.5)$ for 20 min, washed with distilled water, incubated in $0.5 \%$ periodic acid for $3 \mathrm{~min}$ and washed again with distilled water followed by the incubation with Schiff reactive for $20 \mathrm{~min}$, washed with distilled water and stained with Harris haematoxylin (PanReac) for 1-2 min. Sections were then washed in tap water to eliminate the excess of haematoxylin and dehydrated sequentially in $70 \%, 80 \%$, 90\% and 100\% EtOH solutions (1-2 min each). Finally, samples were washed with Histoclear II 3x5 min, mounted and photo-documented under a light microscope (Olympus BX51 with Olympus DP70 camera).

PAS staining served to assess the epithelial Club cell abundance and morphology, as well as for analysing alveolar glycogen deposition in newborns and E18.5 embryos. Quantification of PAS+ regions was performed using a minimum of 10 digital images taken from equivalent sections of the different genotypes and conditions. Images were transformed into binary images in which only PAS+ stainied elements appeared as black pixels. Then, the PAS+ area was delimited, using the original image as reference, and average PAS staining was measured as the black/white pixel ratio.

\subsubsection{Immunohistochemical staining}

Avidin-biotin-peroxidase procedure was carried out to detect neutrophils, using the neutrophil-elastase (NE) as a cellular marker; lymphocytes, using the CD3 cell marker, and pERK, in lungs of untreated, DEX or NAC treated newborn animals. Neutrophil infiltration grade was expressed as the number of NE+ cells relative to the total cell numbers, evaluating at least 10 fields per animal.

Paraffin-embedded sections were rehydrated using xylene for $10 \mathrm{~min}$, and sequentially transferred to $100 \%$ (twice), 90\%, 80\%, 70\%, 50\% EtOH, finalizing in deionized water (5 min in each step). Antigen retrieval was performed to facilitate antibody binding to antigen using citrate buffer $0.01 \mathrm{M} \mathrm{pH} 6$ and heating in a microwave oven at $250 \mathrm{~W} 3 \times 3 \mathrm{~min}$, followed by a 20 min cooling down at RT in the same buffer. Then, sections were washed 3x10 min in $1 \mathrm{X}$ PBS, and washed in PBS-Triton (0.1\%, Sigma, X100) for $10 \mathrm{~min}$. Endogenous peroxidase activity was blocked with $0.03 \%$ hydrogen peroxide for $15 \mathrm{~min}$, washed $3 \times 10$ min with $1 \mathrm{X}$ PBS and blocked with $1 \mathrm{X}$ PBS containing $0.1 \%$ Triton, 5\% Bovine serum albumin (BSA) (Sigma 
Aldrich, 9048-46-8) and 2\% goat serum (Sigma, G9023) for $1 \mathrm{~h}$ at RT. Primary antibodies (Table 3) were diluted in $1 \mathrm{X}$ PBS containing $0.1 \%$ Triton, $2 \%$ BSA and $2 \%$ goat serum, and incubated with the sections $\mathrm{o} / \mathrm{n}$ at $4^{\circ} \mathrm{C}$. The following day, samples were washed $3 \times 10 \mathrm{~min} 1 \mathrm{X}$ PBS, and incubated in 1:250 biotinylated goat anti rabbit IgG (Vector) for NE and goat anti mouse IgG (Vector) for pERK, diluted in the antibody solution (1X PBS containing $0.1 \%$ Triton, $2 \%$ BSA and $2 \%$ goat serum), during $1 \mathrm{~h}$ at RT. Sections were then washed $3 \times 10$ min $1 \mathrm{X}$ PBS and incubated with 1:250 Vectastain Elite ABC reagent (Vector) in PBS for $1 \mathrm{~h}$ and $30 \mathrm{~min}$ at RT. After 3x10 min washes with 1 X PBS, the reaction product was visualized by incubating the sections in $0.05 \%$ 3, $3^{\prime}$-diaminobenzidine and $0.0033 \%$ hydrogen peroxide in PBS until the desired staining intensity was reached. Finally, sections were counterstained with diluted Harris hematoxylin during $1 \mathrm{~min}$, washed in distilled water and dehydrated using 70\%, 80\%, 90\% and $100 \%$ EtOH solutions, 1-2 min each one, washed with Histoclear II 3x5 min, mounted and photo-documented under a light microscope (Olympus BX51 with Olympus DP70 camera).

\begin{tabular}{ccccc}
\hline Antibody & Supplier & Type & Dilution & Reference \\
\hline$\alpha$-Neutrophil elastase & Abcam & Rabbit polyclonal & $1: 400$ & ab68672 \\
\hline$\alpha$-CD3 & Abcam & Rabbit polyclonal & $1: 500$ & ab5690 \\
\hline$\alpha$-pERK1/2 & Cell Signaling & Rabbit polyclonal & $1: 200$ & 9101 \\
\hline
\end{tabular}

Table 3: Primary antibodies used for immunohistochemistry (IHC).

\subsubsection{Fluorescent immunostaining}

Bronchiolar and alveolar epithelial cell composition was evaluated using specific cell markers as follows: Secretoglobulin 1a1 (Scgb1a1, Club cells), $\beta$-Tubulin (Tub, Ciliated cells), Surfactant protein C (SftpC, AT2), Ricinus communis agglutinin-I fluorescein (RCA-I, AT1), and SRY-Box Transcription Factor 9 (SOX9, alveolar progenitor). Quantitation of Scgb1a1+, Tub+, SftpC+, and SOX9+ cells was expressed as the number of positive cells per total cell number. For RCA-I quantification, the area was delimited in each digital image, and both fluorescence intensity and total number of cell nuclei were measured in the chosen area using the Image J software. A minimum of 10 fields per animal were analysed.

Cellular death was assessed using Cleaved Caspase-3 (CC3) detection. Quantification of overall death in the lungs was expressed as the number of CC $3+$ cells per field, analysing a total of 10 fields per animal.

On the other hand, presence of ceramide was detected in both bronchiolar and alveolar areas and was quantified as in the case of RCA-I staining, delimiting the areas of interest, and analysing the fluorescence intensity per total cell number in the selected area. A minimum of 10 digital images per animal were studied. 
Paraffin-embedded sections were deparaffinised using xylene for $10 \mathrm{~min}$, and sequentially transferred to $100 \%$ (twice), 90\%, 80\%, 70\%, 50\% EtOH, finalizing in deionized water (5 min in each step). Antigen retrieval was performed to facilitate antibody binding to antigen using citrate buffer $0.01 \mathrm{M} \mathrm{pH} 6$ and heating in a microwave oven at 250W $3 \times 3 \mathrm{~min}$, followed by a 20 min cool down to RT in the same buffer. Then, sections were washed 3x10 min in 1 X PBS, and $10 \mathrm{~min}$ in PBS-Triton $(0.1 \%)$, and blocked during $1 \mathrm{~h}$ at RT in $1 \mathrm{X}$ PBS containing $0.1 \%$ Triton, $5 \% \mathrm{BSA}$ and $2 \%$ goat serum. Slides were then incubated at $4{ }^{\circ} \mathrm{C} \mathrm{o} / \mathrm{n}$ with the primary antibodies (Table 4) diluted in 1X PBS containing 0.1\% Triton, 2\% BSA and 2\% goat serum. After $3 \times 10$ min $1 \mathrm{X}$ PBS washes and $10 \mathrm{~min}$ in PBS-Triton $(0.1 \%)$, sections were incubated with the secondary antibodies, goat anti-mouse Alexa 488 or Cy3, goat anti-rabbit Alexa 488 or Cy3, diluted 1:500, and counterstained with nuclear DAPI (Sigma), in the aforementioned antibody solution during $1 \mathrm{~h}$ at RT. Additionally, RCA-I labelled with fluorescein was incubated with the secondary antibodies. Finally, sections were washed 3x10 min with 1X PBS and mounted with ProLong Diamond antifading reagent (Life Technologies, P36970) and examined using a confocal microscope (Leica TCS SP5) or a Leica DM6000B (with Hamamatsu ocar-er C474280 digital camera).

\begin{tabular}{ccccc}
\hline Antibody & Supplier & Type & Dilution & Reference \\
\hline$\alpha$ - $\beta$-Tubulin & Sigma & Mouse monoclonal & $1: 1000$ & T5293 \\
\hline$\alpha$-Bromodeoxyuridine & Accurate Chemical & Rat monoclonal & $1: 2000$ & OBT0030CX \\
\hline$\alpha$-Ceramide & Enzo & Mouse monoclonal & $1: 100$ & ALX-804-196 \\
\hline$\alpha$-Cleaved Caspase-3 & Cell Signaling & Rabbit polyclonal & $1: 400$ & 9661 \\
\hline$\alpha-$ Secretoglobulin1a1 & Abcam & Rabbit polyclonal & $1: 1000$ & ab40876 \\
\hline$\alpha-S O X 9$ & Cell Signaling & Rabbit polyclonal & $1: 500$ & 87630 \\
\hline$\alpha$-Surfactant protein C & MERK Millipore & Rabbit polyclonal & $1: 500$ & AB3786 \\
\hline Ricinus communis & Atom & Fluorescein & $1: 1000$ & FL-1081 \\
agglutinin-I & Santa Cruz & Mouse monoclonal & $1: 200$ & sc-23950 \\
\hline Ac-Tub & & & & \\
\hline
\end{tabular}

Table 4: Primary antibodies and fluorescein used for immunofluorescence (IF).

\subsubsection{BrdU immunofluorescence}

Cell proliferation in the lungs was assessed by means of BrdU incorporation in at least 10 digital images per animal. Quantification of BrdU+ cells was expressed as the number of BrdUpositive cells per total cell number in each field.

Sections were deparaffinised and rehydrated using xylene (2x10 min), and 100\% (twice), $90 \%, 80 \%, 70 \%, 50 \% \mathrm{EtOH}$, and deionized water (5 min in each step). Antigen retrieval was 


\section{Materials and Methods}

performed using citrate buffer $0.01 \mathrm{M} \mathrm{pH} 6$ and heating in a microwave oven at $250 \mathrm{~W} 3 \times 3 \mathrm{~min}$, followed by a $20 \mathrm{~min}$ cool down to RT in the same buffer. Sections were then treated with $2 \mathrm{~N}$ $\mathrm{HCl}$ for $45 \mathrm{~min}$ at $37^{\circ} \mathrm{C}$, neutralized with borate buffer $0.1 \mathrm{M} \mathrm{pH} 8.53 \times 10 \mathrm{~min}$, washed in $1 \mathrm{X}$ PBS, 10min in PBS-Triton (0.1\%), and blocked during $1 \mathrm{~h}$ at RT in $1 \mathrm{X}$ PBS containing $0.1 \%$ Triton, 5\% BSA and 2\% goat serum. Primary BdrU antibody (Table 4) was incubated o/n at $4^{\circ} \mathrm{C}$ in $1 \mathrm{X}$ PBS containing $0.1 \%$ Triton, $2 \%$ BSA and $2 \%$ goat serum. Sections were washed with PBS and incubated with the secondary antibody goat anti-rat Alexa 488 diluted 1:500, (Jackson ImmunoResearch) and counterstained with nuclear marker DAPI for $1 \mathrm{~h}$ at RT. Preparations were then washed with 1X PBS and mounted with ProLong Diamond antifading reagent and examined under a confocal microscope (Leica TCS SP5) or using a Leica DM6000B (with Hamamatsu ocar-er C4742-80 digital camera).

2.9. RNA isolation, RT-qPCR and microarray hybridization/lung transcriptome analysis

\subsubsection{RNA isolation and purification}

Lungs were dissected from untreated, DEX and NAC-treated newborn mice, rinsed in 1X PBS to eliminate blood excess and homogenized in 500 $\mu 1$ of NZYol (nzytech, MB18501) using a GentleMACS dissociator (Miltenyi Biotec). Total RNA was subsequently isolated following NZYol manufacturer's instructions and further purified using RNAse Mini Kit columns (QIAGEN, 74104). RNA quantity and quality was checked by RNA capillary electrophoresis columns (Agilent Technologies, RNA 6000 Nanochips) and stored at $-80^{\circ} \mathrm{C}$.

\subsection{2. $R T-q P C R$}

Total RNA obtained from individual mouse lungs was subsequent used for RT-qPCR, using for this purpose the Luna ${ }^{\circledR}$ Universal One-Step RT-qPCR Kit (New England BioLabs, E3005) on a QuantStudio ${ }^{\mathrm{TM}} 3$ - 96-Well and/or QuantStudio ${ }^{\mathrm{TM}} 5$ - 384-Well (ThermoFisher). The expression levels of genes of interest (Table 5) were measured, $\beta 2$-microglobulin (B2M) was used as housekeeping gene to have an endogenous control to normalize results, and fold-change was calculated by the $2-\Delta \Delta \mathrm{Ct}$ method (Rao et al., 2013).

The one-step RT-qPCR conditions were as follows: $55^{\circ} \mathrm{C}$ for $10 \mathrm{~min}$ (reverse transcription), followed by $95^{\circ} \mathrm{C}$ for $1 \mathrm{~min}$ and 45 cycles of $95^{\circ} \mathrm{C}$ for $10 \mathrm{~s}, 60-65^{\circ} \mathrm{C}$ for $30 \mathrm{~s}$ (depending on the primers used), and a melt curve step of $95^{\circ} \mathrm{C}$ for $15 \mathrm{~s}, 60^{\circ} \mathrm{C}$ for $1 \mathrm{~min}$ and $95^{\circ} \mathrm{C}$ for $15 \mathrm{~s}$.

\begin{tabular}{ccc}
\hline Gene & id & \multicolumn{1}{c}{ Sequence } \\
\hline HRas & C0H5X4 & F- AGAAGAGTATAGTGCCATGC \\
\cline { 2 - 3 } & & R-AAGGACTTGGTGTTGTTGAT \\
\hline
\end{tabular}




\begin{tabular}{|c|c|c|}
\hline \multirow{2}{*}{ NRas } & \multirow{2}{*}{ Q9D091 } & F- CCATATTTGCTCCCTGGCTA \\
\hline & & P- GAGGTATGGACTCTGGCTTC \\
\hline \multirow{2}{*}{ KRas } & \multirow{2}{*}{ P32883 } & F- TTCCGCTGACCTAGGGAAT \\
\hline & & R- GCAGTACGGTTCATGACAAAAAT \\
\hline \multirow{2}{*}{ Wnt7a } & \multirow{2}{*}{ P24383 } & F- TACACAATAACGAGGCGGGT \\
\hline & & R- TGTGGTCCAGCACGTCTTAG \\
\hline \multirow{2}{*}{ Wnt7b } & \multirow{2}{*}{ P28047 } & F- GGATGCCCGTGAGATCAAAA \\
\hline & & R- CACACCGTGACACTTACATTCCA \\
\hline \multirow{2}{*}{ Fgfr2 } & \multirow{2}{*}{ P21803 } & F- TCCCCCTGCGGAGACA \\
\hline & & R- TGCCCAGCGTCAGCTTAT \\
\hline \multirow{2}{*}{ Spry2 } & \multirow{2}{*}{ Q9QXV8 } & F- AGAGGATTCAAGGGAGAGGG \\
\hline & & R- CATCAGGTCTTGGCAGTGTG \\
\hline \multirow{2}{*}{ Bmp4 } & \multirow{2}{*}{$\mathrm{P} 21275$} & F- TGGACTGTTATTATGCCTTGTTT \\
\hline & & R- CTCCTAGCAGGACTTGGCAT \\
\hline \multirow{2}{*}{ Nkx2.1 } & \multirow{2}{*}{ P50220 } & F- TCCAGCCTATCCCATCTGAACT \\
\hline & & R- CAAGCGCATCTCACGTCTCA \\
\hline
\end{tabular}

Table 5: Primers used in lung tissue RT-qPCR assays

\subsubsection{Microarray hybridization}

Chip microarray hybridizations and data generated with Affymetrix GeneChip Mouse Gene 2.0 ST Array (26,515 genes) were used in this study. All microarray hybridization data were deposited and are available at the NCBI Gene Expression Omnibus (GEO) database (GEO GSE130415 Accession viewer, GEO GSE186161 Accession viewer). The RNAs were preamplified prior to microarray hybridization using the Gene Chip Expression 3'-Amplification Two-Cycle cDNA Synthesis kit (Affymetrix, Santa Clara, CA, USA; \#900432), the Gene Chip Sample Cleanup Module (Affymetrix \#900371) and the MEGAscript T7 High Yield Transcription Kit (Ambion, Austin, TX, USA; \#1334), according to Affymetrix instruction manual \#701025 rev. 5. The pre-amplified RNAs were then submitted to the Gene Chip microarray hybridization protocol (Affymetrix Expression Analysis Technical Manual, as previously described (Castellano et al., 2007). Using Bioconductor (Gentleman et al., 2004) and R (R Development Core Team \& Development Core Team, 2011) as computational tools, the robust microarray analysis (RMA) algorithm (Irizarry et al., 2003) was applied for background correction and normalization of fluorescent hybridization signals. The significance analysis of microarrays (SAM) algorithm (Tusher et al., 2001) was used to identify probe sets displaying significant differential expression when comparing the KO samples to their respective controls. This method uses permutations to provide robust statistical inference of the most significant genes and provides $\mathrm{P}$ values adjusted to multiple testing using false discovery rate (FDR) (Benjamini et al., 2001). The GeneCodis (Gene Annotation Co-occurrence Discovery) software 


\section{Materials and Methods}

package (http://genecodis.cnb.csic.es/) was used for functional annotation analysis of differentially expressed gene sets in order to identify specific gene subsets sharing co-occurrent functional annotations linking them, with high statistical significance, to particular Gene Ontology (GO) Biological Process or Molecular Function categories and KEGG Signalling Pathways (Tabas-Madrid et al., 2012).

2.10. Western blot analysis in whole lung homogenates

\subsubsection{Protein isolation}

Lung lobes were dissected, rinsed in 1X PBS and subsequently homogenized using the GentleMACS dissociator (Miltenyi Biotec) in 500 $\mu 1$ of cold lysis buffer (Cell Signaling, 9803) supplemented with $1 \mathrm{mM} \mathrm{NaF}$ (as phosphatases inhibitor), $1 \mathrm{mM} \mathrm{Na}_{3} \mathrm{VO}_{4}, 1 \mathrm{mM}$ (Phenylmethylsulfonyl fluoride) PMSF (as proteases inhibitor) and EDTA-free protease inhibitor cocktail Complete ${ }^{\circledR}(1$ tablet/50 ml, Roche, 11873580001$)$, followed by a $10 \mathrm{~min}$ centrifugation at $13000 \mathrm{rpm}$ at $4^{\circ} \mathrm{C}$. The supernatant was transferred into $1.5 \mathrm{ml}$ Eppendorf tubes and protein concentration was determined using Bradford assay (BioRad, 5000006) following the manufacturer's instructions.

\subsubsection{Immunoblotting analysis}

Protein lysates were denaturalized in 6x Laemmli loading buffer (200mM Tris-HCl pH 6.8, $40 \%$ glycerol, 4\% Sodium dodecyl sulfate (SDS), 4\% $\quad \beta$-mecaptoethanol and $0.04 \%$ bromophenol blue) and heated during $5 \mathrm{~min}$ at $100^{\circ} \mathrm{C}$.

According to their molecular weight, samples were separated using poly-acrylamide gels (30\% Acrylamide Solution, BioRad, 1610158) (Tables 6 and 7) in 1X SDS-PAGE electrophoresis buffer (25mM Tris- $\mathrm{HCl} \mathrm{pH} 8.3,200 \mathrm{mM}$ glycine, $0.05 \%$ SDS). Gels were equilibrated in $20 \% \mathrm{EtOH}$ during $10 \mathrm{~min}$ at $\mathrm{RT}$, and proteins were further transferred into Polyvinylidene Fluoride (PVDF) membranes (iBlot®2 PVDF Regular Stacks, Invitrogen, IB24001) using the iBlot 2 Dry Blotting System (Invitrogen).

Then, membranes were incubated during $1 \mathrm{~h}$ at RT in blocking solution, consisting in Trisbuffered saline-Tween (TBS-T, 20mM Tris pH 7.5, $137 \mathrm{mM} \mathrm{NaCl}, 0.05 \%$ Tween-20) with 5\% of non-fat dry milk (NFDM), followed by the incubation with the primary antibodies (Table 8), washed $3 \times 5 \mathrm{~min}$ in TBS-T and incubated $1 \mathrm{~h}$ at RT with the corresponding secondary antibody goat anti-mouse DyLight 800 (Invitrogen, 35521) or goat anti-rabbit Alexa fluor 680 (Invitrogen, A21076) diluted 1:10000 in TBS-T in 5\% NFDM and washed again 3x10 min. 
Results were observed by scanning the membranes in a LI-COR Odyssey infrared imaging system (LI-COR Biosciences) and quantified using Image $\mathbf{J}$ software.

\begin{tabular}{ccc}
\cline { 2 - 2 } & \multicolumn{2}{c}{ Percentage } \\
\cline { 2 - 3 } & $\mathbf{1 0 \%}$ & $\mathbf{1 2 \%}$ \\
\hline LOWER Tris & $2.5 \mathrm{ml}$ & $2.5 \mathrm{ml}$ \\
\hline $\mathbf{H}_{2} \mathrm{O}$ MiliQ & $4 \mathrm{ml}$ & $3.3 \mathrm{ml}$ \\
\hline 30\% Acrylamide & $3.3 \mathrm{ml}$ & $4 \mathrm{ml}$ \\
\hline Tetramethylethylenediamine (TEMED) & $5 \mu \mathrm{l}$ & $5 \mu \mathrm{l}$ \\
\hline $\mathbf{1 0 \%}$ Ammonium persulfate (APS) & $100 \mu \mathrm{l}$ & $100 \mu \mathrm{l}$ \\
\hline
\end{tabular}

Table 6: Acrylamide separating gel reagents for one gel. LOWER Tris: Tris- $\mathrm{HCl} 1.5 \mathrm{M} \mathrm{pH} 8,10 \%$ SDS.

\begin{tabular}{ccccc}
\hline UPPER Tris & $\mathbf{H}_{2} \mathrm{O}$ MiliQ & 30\% Acrylamide & TEMED & 10\% APS \\
\hline $1.26 \mathrm{ml}$ & $2.91 \mathrm{ml}$ & $0.83 \mathrm{ml}$ & $5 \mu \mathrm{l}$ & $50 \mu \mathrm{l}$
\end{tabular}

Table 7: Acrylamide stacking gel reagents for one gel. UPPER Tris: Tris-HCl 1.M pH 6.5, 10\% SDS.

\begin{tabular}{|c|c|c|c|c|c|c|}
\hline Antibody & Supplier & Reference & Type & Dilution & $\begin{array}{c}\text { Antibody } \\
\text { solution }\end{array}$ & Incubation \\
\hline $\begin{array}{c}\alpha-\beta- \\
\text { Tubulin }\end{array}$ & Sigma & T5293 & $\begin{array}{c}\text { Mouse } \\
\text { monoclonal }\end{array}$ & $1: 10000$ & $5 \% \mathrm{BSA}$ & $1 \mathrm{~h}, \mathrm{RT}$ \\
\hline$\alpha$-ERK1/2 & $\begin{array}{c}\text { Cell } \\
\text { Signaling }\end{array}$ & 4696 & $\begin{array}{l}\text { Mouse } \\
\text { monoclonal }\end{array}$ & 1:2000 & $5 \%$ NFDM & $1 \mathrm{~h}, \mathrm{RT}$ \\
\hline $\begin{array}{c}\alpha- \\
\mathrm{pERK} 1 / 2\end{array}$ & $\begin{array}{c}\text { Cell } \\
\text { Signaling }\end{array}$ & 9101 & $\begin{array}{c}\text { Rabbit } \\
\text { polyclonal }\end{array}$ & $1: 2000$ & $5 \%$ NFDM & $\mathrm{o} / \mathrm{n}, 4^{\circ} \mathrm{C}$ \\
\hline$\alpha$-KRas & Sigma & $\begin{array}{c}\text { WH003845 } \\
\text { M1 }\end{array}$ & $\begin{array}{c}\text { Mouse } \\
\text { monoclonal }\end{array}$ & $1: 500$ & $5 \%$ NFDM & $48 \mathrm{~h}, 4^{\circ} \mathrm{C}$ \\
\hline
\end{tabular}

Table 8: Primary antibodies used in tissue immunoblotting assays.

\subsection{RAS activation assays in lung tissue}

Following Ras GTPases activation, active Ras (RAS.GTP) specifically binds to Raf serine/threonine kinase through its Ras binding domain (RBD). This specific interaction allows us to isolate the RAS.GTP from our samples and, thus, to analyse its activation status.

\subsubsection{Raf-RBD fusion protein purification}

Escherichia coli (E. coli) bacteria, DH10B strain, were transfected with a construct containing the Raf1 RBD domain inserted in pGEX-2T plasmid (GE Healthcare, 28-9546-53), containing ampicillin resistance gene. Cells were grown at $37^{\circ} \mathrm{C}$ in Lysogeny broth/Luria- 


\section{Materials and Methods}

Bertani (LB) containing $100 \mu \mathrm{g} / \mathrm{ml}$ ampicillin to a $600 \mathrm{~nm}$ optical density (OD) between $0.5-0.7$ and stimulated with $1 \mu \mathrm{g} / \mathrm{mL}$ Isopropyl- $\beta$-D-1-thiogalactopyranoside (IPTG) during $4 \mathrm{~h}$ at $37^{\circ} \mathrm{C}$ with agitation. Cultures were then centrifuged during $20 \mathrm{~min}$ at $8000 \mathrm{rpm}$ at $4^{\circ} \mathrm{C}$, and the cell pellets were resuspended in lysis buffer ( $2 \mathrm{mM}$ EDTA, $0.1 \% \beta$-mercaptoethanol, $0.2 \mathrm{mM}$ PMSF in PBS-Tween) and sonicated (Vibra-Cell, SONICS) with $20 \mathrm{~s}$ pulses (5 times), on ice. Disrupted cells were centrifuged at $15000 \mathrm{rpm}$ during $30 \mathrm{~min}$ at $4^{\circ} \mathrm{C}$ and the supernatant was collected in $1 \mathrm{ml}$ aliquots and quickly frozen at $-80^{\circ} \mathrm{C}$ until use.

\subsubsection{Binding of GST-Raf-RBD to Glutathione-Sepharose beads}

To selectively precipitate the GST-Raf-RBD, bacterial cell lysates containing the GST-RafRBD fusion protein were incubated with Glutathione-Sepharose beads (GE Healthcare, 175132-01) during $90 \mathrm{~min}$ at $4^{\circ} \mathrm{C}$ with gentle rotation. Beads were then washed 3 times with $1 \mathrm{X}$ MLB buffer (5X MLB, 5mM Na$V_{3} \mathrm{VO}_{4}, 1 \mathrm{mM}$ PMSF, EDTA-free protease inhibitor cocktail Complete $\AA$ ( 1 tablet/50ml), bacterial cell lysates containing the fusion protein GST-Raf-RBD (500 $\mu 1 / 50 \mathrm{ml})$; $5 \mathrm{X}$ MLB: 125mM HEPES, 750mM NaCl, 5\% Igepal CA-360, 50mM MgCl 2 , 5mM EDTA, $10 \%$ glycerol) and centrifuged every time between washes at 10000xg during 1 min. In the last wash, the supernatant was removed and the pellet, containing the beads with the GST-Raf-RBD attached, was resuspended in $1 \mathrm{X}$ MLB ( $1 \mathrm{ml} \mathrm{MLB} / 1 \mathrm{ml}$ bacterial cell lysate), and frozen at $-80^{\circ} \mathrm{C}$ until use.

\subsubsection{RAS.GTP pull-down}

Lung left lobes from newborn mice were used in these studies. For each animal, the left lobe was homogenized in 500 $\mu 1$ of $1 \mathrm{X}$ MLB using the GentleMACS dissociator, followed by a 10 min centrifugation at $13000 \mathrm{rpm}$ and $4^{\circ} \mathrm{C}$. Supernatants were collected into $1.5 \mathrm{ml}$ eppendorf tubes and the protein concentration was measured using the Bradford assay.

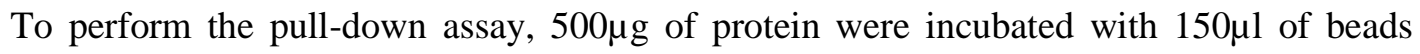
attached to GST-Raf-RBD during $30 \mathrm{~min}$ at $4^{\circ} \mathrm{C}$ with gentle rotation. The samples were then washed 2 times with $1 \mathrm{X}$ MLB with centrifugation between washes at $13000 \mathrm{rpm}$ and $4^{\circ} \mathrm{C}$. After the last centrifugation, supernatants were discarded, and the pellets were resuspended in $60 \mu 1$ of $4 \mathrm{X} \mathrm{LB}$ and quickly frozen at $-80^{\circ} \mathrm{C}$ until use.

For total protein analysis, $100 \mu \mathrm{l}$ of lung lysates were mixed with $20 \mu \mathrm{l}$ of $6 \mathrm{X} \mathrm{LB}$ and frozen at $-80^{\circ} \mathrm{C}$ until use. Finally, $20 \mu \mathrm{l}$ of each pull-down assay sample and $25 \mu \mathrm{g}$ of total protein lysates were loaded into $12 \%$ acrylamide gels, and levels of KRAS.GTP and total-KRAS were analysed 
by western blot assays with the incubation of the membranes with a specific primary antibody against KRAS (Table 8) during $48 \mathrm{~h}$ at $4^{\circ} \mathrm{C}$.

2.12. Reactive oxygen species analysis in lung tissue through flow cytometry

Total hydrogen peroxide $\left(\mathrm{H}_{2} \mathrm{O}_{2}\right)$, total and mitochondrial superoxide $\left(\mathrm{O}_{2}^{-}\right)$ROS were assessed in total lung homogenates from newborn animals, of the indicated genotypes, using the 2',7'-dichlorofluorescin diacetate (DCFH-DA; hereinafter DCF, Molecular Probes, D-399), dihydroethidium (DHE, Molecular Probes, D11347), and MitoSOX ${ }^{\mathrm{TM}}$ (Molecular Probes, M36008), respectively.

DCFH-DA easily diffuse through the cellular membranes, being subsequently hydrolysed by intracellular esterases to diclorofluoroscin (DCFH), a non-fluorescent compound that undergo oxidation in the presence of ROS, producing its highly fluorescent oxidized form, DCF. On the other hand, DHE exhibits a blue fluorescence in the cytosol, however, oxidation of DHE by superoxide results in hydroxylation at 2-position, generating 2-hydroxyethidium, a compound able to intercalate into the DNA and stain the nucleus with red fluorescence. Finally, MitoSOX $^{\mathrm{TM}}$ is a fluorogenic dye specifically directed to the mitochondria of live cells. Oxidation of MitoSOX ${ }^{\mathrm{TM}}$ by mitochondrial superoxide produces red fluorescence.

\subsubsection{Sample preparation}

Lungs were dissected, quickly minced using scissors, digested in $500 \mu 1$ of $1 \mathrm{mg} / \mathrm{ml}$ Collagenase/Dispase ${ }^{\circledR}$ (Roche, 10269638001) and diluted in HBSS during $30 \mathrm{~min}$ at $37^{\circ} \mathrm{C}$ in gentle agitation. Digested lungs were then passed through a $100 \mu \mathrm{m}$ cell strainer (Falcon, 352360) and each sample was diluted to 3ml with HBSS and distributed in 6 eppendorf cups, each one containing 500 $\mu$ ( 3 replicates/animal for DCF/DHE staining, and 3 replicates/animal for DCF/MitoSOX staining).

\subsection{2. $D C F / D H E \& D C F / M i t o S O X^{\mathrm{TM}}$ staining}

For DCF/DHE staining, samples were incubated with $5 \mu \mathrm{M}$ of each probe in DMEM (Gibco, A14430.01) supplemented with $0.1 \%$ of fetal bovine serum (FBS) and $10 \mathrm{mM}$ of glucose (Glu) during $30 \mathrm{~min}$ at $37^{\circ} \mathrm{C}$ in the dark with gentle agitation. On the other hand, for DCF/MitoSOX ${ }^{\mathrm{TM}}$, samples were incubated first with $5 \mu \mathrm{M}$ of MitoSOX ${ }^{\mathrm{TM}}$, in the same media, during $30 \mathrm{~min}$ at $37^{\circ} \mathrm{C}$ in the dark with gentle agitation, then $5 \mu \mathrm{M}$ of DCF was added to each sample, followed 


\section{Materials and Methods}

by $30 \mathrm{~min}$ incubation at $37^{\circ} \mathrm{C}$ in the dark with gentle agitation (due to the needed of incubating MitoSOX ${ }^{\mathrm{TM}}$ during $60 \mathrm{~min}$, but only $30 \mathrm{~min}$ for DCF probe).

The reactions were quickly stopped by adding $1 \mathrm{ml}$ of cold HBSS, followed by a centrifugation of the samples at $13000 \mathrm{rpm}$ at $4^{\circ} \mathrm{C}$ during $5 \mathrm{~min}$. Supernatants were discarded and cell pellets were resuspended in $200 \mu \mathrm{l}$ of DMEM supplemented with $0.1 \%$ FBS. Samples were analysed using a BD Accuri ${ }^{\mathrm{TM}} \mathrm{C} 6$ cytometer.

\subsection{Mitochondrial respiration assays in lung tissue}

Using Seahorse-based technology and the MitoStress Test (Agilent, 103015-100) we evaluated the mitochondrial respiration status, as well as function, by means of measuring the oxygen consumption rate (OCR) of the cells in real time.

The assay consisted of adding four different modulators (divided in three sequential injections) that specifically block one electron transport chain (ETC) complex, modulating, thus, mitochondrial respiration and revealing its function. The sequence of inhibitor injections was as follow: (1) Oligomycin (OL), that inhibits ATP synthase or complex V, revealing the oxygen consumption linked to ATP production; (2) Carbonyl cyanide-4 (trifluoromethoxy) phenylhydrazone (FCCP), an uncoupling compound that collapses the mitochondrial proton gradient and disrupts its membrane potential, showing the ability of the cell to respond under stress or under high energy demand situations; (3) Rotenone and Antimycin A (ROT/AA) block complexes I and III, respectively, completely shutting down mitochondrial respiration and exhibiting the non-mitochondrial respiration (Fig. 14).

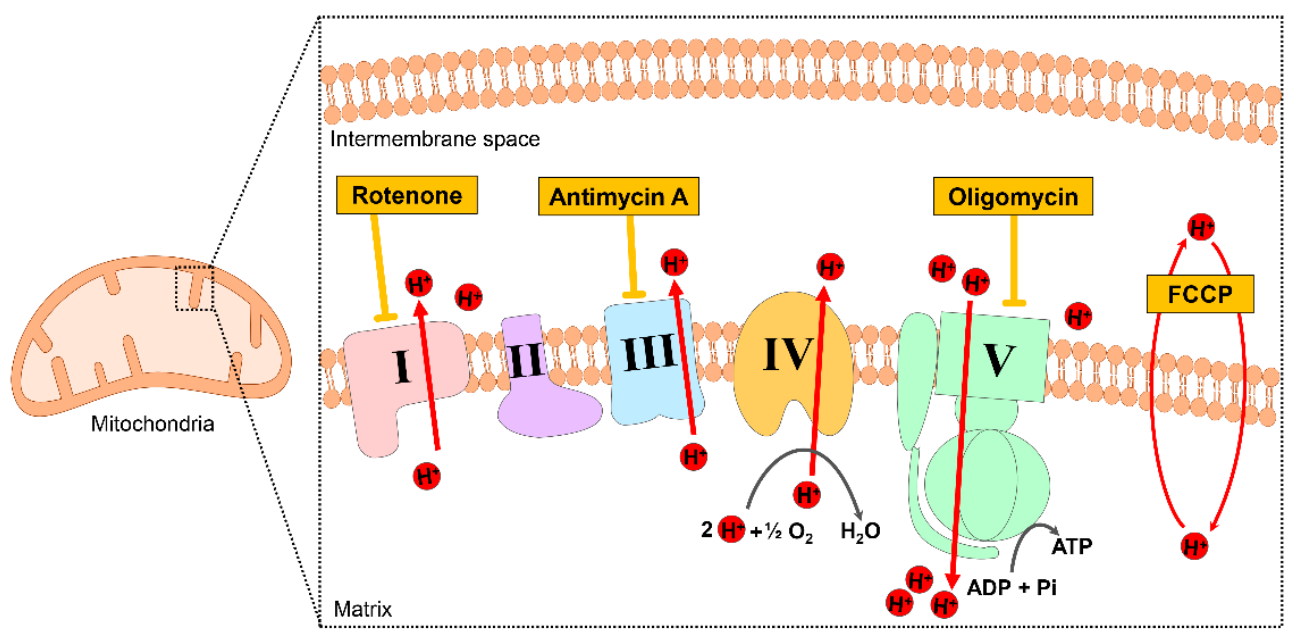

Figure 14: Agilent Seahorse MitoStress test modulators. Scheme representing the electron transport chain targets for each inhibitor.

Lung lobes were dissected from newborn animals, rinsed in 1X PBS and maintained o/n at $4^{\circ} \mathrm{C}$ in a $2 \mathrm{ml}$ Eppendorf filled with full DMEM supplemented with $10 \% \mathrm{FBS}$ and $1 \%$ PenicillinStreptomycin (PS). Lung lobes were subsequently sliced in 1mg pieces and inserted onto the 
Islet capture microplate (Agilent, 101122-100) following manufacturer's instructions, followed by an incubation at $37^{\circ} \mathrm{C}$ without $\mathrm{CO}_{2}$ during $45 \mathrm{~min}$ in Seahorse XF DMEM medium, $\mathrm{pH} 7.4$ (Agilent, 103575-100) supplemented with 10mM Glucose, 1mM Pyruvate, and 2mM LGlutamine. Cartridge injection ports were filled with OL, FCCP and ROT/AA so the final concentration in each well was 15,16 and $3 / 12 \mu \mathrm{M}$ respectively.

Microplates were assessed in a Seahorse XFe24 Extracellular Flux Analyzer system (Agilent, Santa Clara, CA), following MitoStress Test protocol with some alterations: 3 measures of basal OCR, 6 measures of OCR after OL injection, 3 measures after FCCP injection and 5 measures after ROT/AA injection (Fig. 15). Data was analysed using Wave Desktop 2.6 software.

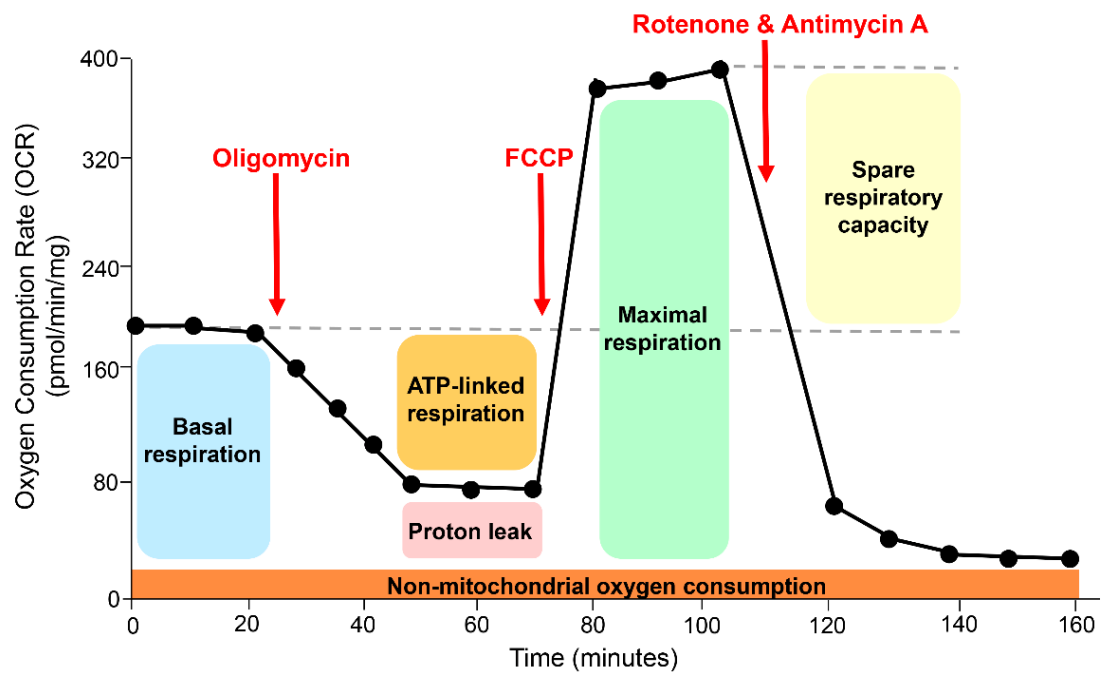

Figure 15: Agilent Seahorse MitoStress lung tissue test profile. Scheme illustrating the key parameters of mitochondrial function as obtained in a typical lung tissue Seahorse analysis. Oxygen consumption rate (OCR) is represented as $\mathrm{pmol} / \mathrm{min} / \mathrm{mg}$.

\subsection{Micro-CT}

6-months old male mice were anaesthetised and they were imaged using the SuperArgus Micro-CT (SEDECAL, Madrid (Spain)). Images were taken with 720 plane projections, with $100 \mathrm{~ms}$ exposure time per projection, $\mathrm{X}$-ray energies of $45 \mathrm{kVp}$ and $400 \mu \mathrm{A}$. Images were reconstructed and converted to 3D volumes (Micro-CT Sedecal ACQ).

\section{In vitro studies}

2.15. Primary lung fibroblasts isolation and culture

Primary lung fibroblasts were isolated from newborn animals to analyse the effect of the lack of HRas and NRas, alone and/or in combination, in the mesenchymal cells of the lungs. 
To achieve this, $\mathrm{HRas}^{+-} ; \mathrm{NRas}^{--}$and $\mathrm{HRas}^{--}$; $\mathrm{NRas}^{+-}$animals were crossed to generate descendants with equal proportion of the 4 genotypes under study: CT, HRas-KO, NRas-KO and DKO. The genotype of each animal was determined by PCR analysis of DNA extracted from tail tissue. Newborn mice were briefly washed in $70 \% \mathrm{EtOH}$ and euthanized by decapitation followed by a small incision from the neck to the chest without reaching the peritoneal cavity. The lungs were removed using forceps and placed into a Petri dish containing drops of HBSS. Non-lung tissue (heart, oesophagus, etc.) was removed, and lung lobes were rinsed in HBSS to clean out blood.

Then, lung lobes were placed and minced using scissors into a $1.5 \mathrm{ml}$ eppendorf containing $500 \mu 11 \mathrm{mg} / \mathrm{ml}$ Collagenase/Dispase diluted in HBSS (Fig. 16), followed by a digestion during $30 \mathrm{~min}$ at $37^{\circ} \mathrm{C}$ with gentle agitation. After the first digestion with Collagenase/Dispase, samples were centrifuged $5 \mathrm{~min}$ at $1000 \mathrm{xg}$, the supernatants were discarded, and the pellets were washed by adding $1 \mathrm{ml}$ of HBSS and centrifuged for $5 \mathrm{~min}$ at $1000 \mathrm{xg}$.

After decanting the supernatants, pellets were subsequently processed for a second enzymatic digestion with 500 $\mu 1$ of $0.25 \%$ Trypsin-EDTA (Gibco, 25200-056) during 20 min at $37^{\circ} \mathrm{C}$ with gentle agitation (Fig. 16). Following the second digestion, samples were centrifuged for $5 \mathrm{~min}$ at $1000 \mathrm{xg}$, the supernatants were discarded, and the pellets were resuspended and incubated with $100 \mu \mathrm{l}$ of Red Blood Cell lysis buffer (RBC or ACK buffer, $0.155 \mathrm{M} \mathrm{NH}_{4} \mathrm{Cl}$, $10 \mathrm{mM} \mathrm{KHCO}_{3}, 10 \mathrm{mM}$ EDTA, pH 7.4) for $1 \mathrm{~min}$ at RT to eliminate the erythrocytes. The reaction was quickly neutralized by adding $1.2 \mathrm{ml}$ of $1 \mathrm{X}$ PBS, followed by a centrifugation at $1000 x g$ for $5 \mathrm{~min}$.

Finally, supernatants were discarded, and cell pellets were resuspended in $500 \mu \mathrm{l}$ of fibroblast culture media (DMEM supplemented with $10 \%$ FBS, $1 \%$ PS) and culture in a 6-well plates with a final volume of $2.5 \mathrm{ml} /$ well. Cells were maintained at $37^{\circ} \mathrm{C}$ and $5 \% \mathrm{CO}_{2}$, and the medium was changed every 2 days. Cells were split to a 100mm Petri dish when confluent, and frozen (1 confluent 100mm Petri dish/vial) in DMEM containing 10\% dimethyl sulfoxide (DMSO) and $20 \%$ FBS.

Lung fibroblasts were used for experiments with a maximum of 5 splits, in order to maintain them as primary cells.

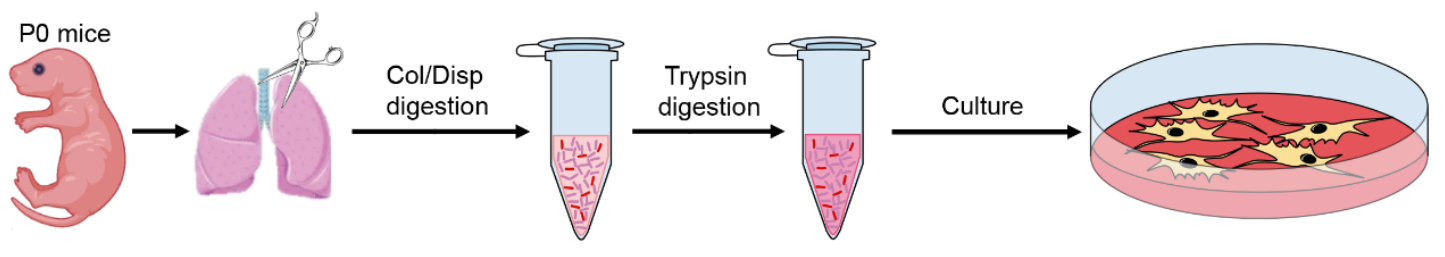

Figure 16: Lung fibroblast isolation Scheme illustrating the steps to isolate primary lung fibroblasts from newborn mice. 
2.16. Reactive oxygen species assays in primary lung fibroblasts through flow cytometry

As previously described in section 3.12., oxidative stress status of the cells was analysed using DCF/DHE and DCF/MitoSOX ${ }^{\mathrm{TM}}$ probes.

Primary lung fibroblasts were seeded and grown in 6-well plates until 80\%-confluency and subsequently treated (in steady state) with either DMEM supplemented with 10\% FBS and 1\% PS; or DMEM with $60 \mathrm{nM}$ DEX or $10 \mathrm{mM} \mathrm{NAC}$, during $24 \mathrm{~h}$ at $37^{\circ} \mathrm{C}$ and $5 \% \mathrm{CO}_{2}$; Antimycin A was used as positive ROS production control. Then, media was aspirated, and cells were washed twice with DMEM without phenol red and incubated with $5 \mu \mathrm{M} \mathrm{DCF} / 5 \mu \mathrm{M}$ DHE or $5 \mu \mathrm{M}$ DCF/ $5 \mu \mathrm{M}$ MitoSOX ${ }^{\mathrm{TM}}$, as detailed in section 3.12.2. Cells were then trypsinized, collected in $1.5 \mathrm{ml}$ eppendorf tubes and centrifuged during $5 \mathrm{~min}$ at $1200 \mathrm{rpm}$ at $4^{\circ} \mathrm{C}$. Finally, after discarding the supernatant, cell pellets were resuspended in $300 \mu 1$ of cold DMEM without phenol red supplemented with $0.1 \%$ FBS and analysed using a BD Accuri ${ }^{\mathrm{TM}} \mathrm{C} 6$ cytometer.

2.17. Mitochondrial respiration assays in primary lung fibroblasts

Analyses of cellular bioenergetics in primary lung fibroblasts were performed using the Seahorse XFe24 Extracellular flux Analyzer as stated in section 3.13. Primary lung fibroblasts were seeded in 100mm Petri dishes and treated with either DMEM supplemented with 10\% FBS and $1 \%$ PS, or DMEM with 60nM DEX or 10mM NAC, during $24 \mathrm{~h}$. XFe24 microplates (Agilent, 100777-004) were coated with $2 \mu \mathrm{g} / \mathrm{ml}$ fibronectin at $4^{\circ} \mathrm{C}$ during $12 \mathrm{~h}$.

Cells were then detached and re-plated at a density of $50 \times 10^{4}$ cells/well into fibronectincoated XFe24 microplates in the same media conditions $24 \mathrm{~h}$ prior to the assay. Then, medium was discarded, and the cells were washed twice with DMEM without phenol red, and 500 $\mu$ of Seahorse XF DMEM medium, pH 7.4, supplemented with 10mM Glucose, 1mM Pyruvate, $2 \mathrm{mM}$ L-Glutamine were added to each well.

Cells were incubated for $1 \mathrm{~h}$ prior to the assay in a non- $\mathrm{CO}_{2}$ incubator at $37^{\circ} \mathrm{C}$. Cartridge injection ports were filled with OL, FCCP and ROT/AA so the final concentration in each well was 5.5, 1 and $1 / 1 \mu \mathrm{M}$ respectively. Finally, MitoStress test protocol (see section 3.13.) was carried out with some modifications: 3 measures of basal OCR, 4 measures of OCR after OL injection, 3 measures after FCCP injection and 4 measures after ROT/AA injection (Fig. 17). Data was analysed using Wave Desktop 2.6 software. 


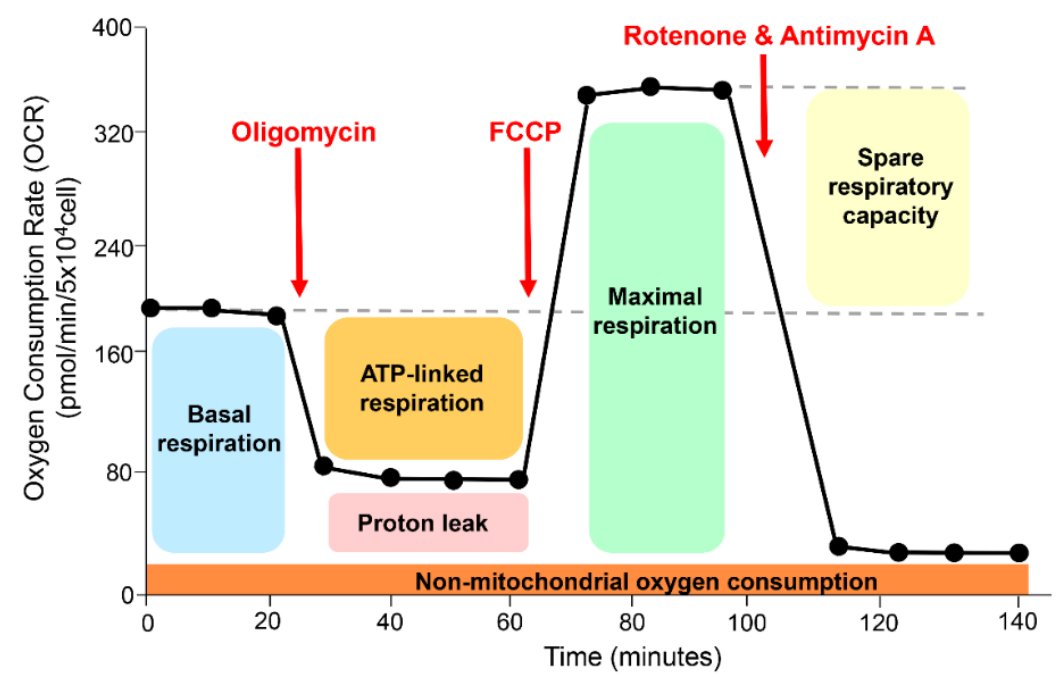

Figure 17: Agilent Seahorse MitoStress lung fibroblasts test profile. Scheme illustrating the key paramt mitochondrial function as obtained in a typical primary lung fibroblasts Seahorse analysis. Oxygen consumption rate (1 represented as $\mathrm{pmol} / \mathrm{min} / 5 \times 10^{4}$ cell.

\subsection{RNA isolation and RT-qPCR analysis in primary lung fibroblasts}

Primary lung fibroblasts from the four genotypes under study were treated during $48 \mathrm{~h}$ with 60nM DEX or 10mM NAC in DMEM supplemented with 10\% FBS and 1\% PS. Cells were then washed with $1 \mathrm{X}$ PBS, 500 $\mu 1$ of NZYol were added, and cells were detached using a cellscraper. Total RNA was subsequently isolated following NZYol manufacturer's instructions and further purified using RNAse Mini Kit columns. RNA quantity and quality was checked by RNA capillary electrophoresis columns (Agilent Technologies, RNA 6000 Nanochips) and stored at $-80^{\circ} \mathrm{C}$. RT-qPCR assays were performed as described in section 3.9.2, and the expression levels of different genes were measured (Table 9).

\begin{tabular}{|c|c|c|}
\hline Gene & Accession number & Sequence \\
\hline \multirow{2}{*}{ HRas } & \multirow{2}{*}{$\mathrm{C} 0 \mathrm{H} 5 \mathrm{X} 4$} & F- AGAAGAGTATAGTGCCATGC \\
\hline & & R- AAGGACTTGGTGTTGTTGAT \\
\hline \multirow{2}{*}{ NRas } & \multirow{2}{*}{ Q9D091 } & F- CCATATTTGCTCCCTGGCTA \\
\hline & & R- GAGGTATGGACTCTGGCTTC \\
\hline \multirow{2}{*}{ KRas } & \multirow{2}{*}{ P32883 } & F- TTCCGCTGACCTAGGGAAT \\
\hline & & R- GCAGTACGGTTCATGACAAAAAT \\
\hline \multirow{2}{*}{ Wnt2 } & \multirow{2}{*}{ P21552 } & F- TCTTGAAACAAGAATGCAAGTGTCA \\
\hline & & R- GAGATAGTCGCCTGTTTTCCTGAA \\
\hline \multirow{2}{*}{ Wnt2b } & \multirow{2}{*}{$\mathrm{O} 70283$} & F- CTGCTGCTGCTACTCCTGACT \\
\hline & & R- GGGGATGTTGTCACAGATCA \\
\hline \multirow{2}{*}{ Fgf7 } & \multirow{2}{*}{ Q544I6 } & F- CTGCTCCACGCTAACTTCCA \\
\hline & & R- GAGTTTACGCACCAGCACAC \\
\hline Fgf9 & P54130 & F- TTCATGCGGTGGGTTCTTATT \\
\hline
\end{tabular}




\begin{tabular}{|c|c|c|}
\hline & & R- TCCTCATCCAAGCTTCCATCA \\
\hline \multirow{2}{*}{ Fgf10 } & \multirow{2}{*}{ O35565 } & F- GTCAGCGGGACCAAGAATGA \\
\hline & & R- GTCGTTGTTAAACTCTTTTGAGCC \\
\hline \multirow{2}{*}{ Axin 2} & \multirow{2}{*}{ O88566 } & F- CAGTGAGCTGGTTGTCACCT \\
\hline & & R- TCCTCAAAAACTGCTCCGCA \\
\hline \multirow{2}{*}{ Nkd1 } & \multirow{2}{*}{ Q99MH6 } & F- TAGACCTGGCGGGGATAGAG \\
\hline & & R- GTCAAGGAGGTGGAAGGAGC \\
\hline \multirow{2}{*}{ Lef1 } & \multirow{2}{*}{ P27782 } & F- AAATGGGTCCCTTTCTCCAC \\
\hline & & R- CTCGTCGCTGTAGGTGATGA \\
\hline \multirow{2}{*}{ Hoxa5 } & \multirow{2}{*}{ P09021 } & F- CAGGGTCTGGTAGCGAGTGT \\
\hline & & R- CTCAGCCCCAGATCTACCC \\
\hline \multirow{2}{*}{ Hoxb5 } & \multirow{2}{*}{ P09079 } & F- CTGGTAGCGAGTATAGGCGG \\
\hline & & R- AGGGGCAGACTCCACAGATA \\
\hline \multirow{2}{*}{ Hoxc5 } & \multirow{2}{*}{ P32043 } & F- TTCTCGAGTTCCAGGGTCTG \\
\hline & & R- ATTTACCCGTGGATGACCAA \\
\hline \multirow{2}{*}{ Sod1 } & \multirow{2}{*}{ NM_011434 } & F- GTGATTGGGATTGCGCAGTA \\
\hline & & R- TGGTTTGAGGGTAGCAGATGAGT \\
\hline \multirow{2}{*}{ Sod2 } & \multirow{2}{*}{ NM_013671 } & F- TTAACGCGCAGATCATGCA \\
\hline & & R- GGTGGCGTTGAGATTGTTCA \\
\hline \multirow{2}{*}{ Sod3 } & \multirow{2}{*}{ NM_011435 } & F- CATGCAATCTGCAGGGTACAA \\
\hline & & R- AGAACCAAGCCGGTGATCTG \\
\hline \multirow{2}{*}{ Cat } & \multirow{2}{*}{ NM_009804 } & F- TGAGAAGCCTAAGAACGCAATTC \\
\hline & & R- CCCTTCGCAGCCATGTG \\
\hline \multirow{2}{*}{ Nrf1 } & \multirow{2}{*}{ NM_001164226.1 } & F- TCGGGCATTTATCCCAGAGATGCT \\
\hline & & R- TACGAGATGAGCTATACTGTGTGT \\
\hline \multirow{2}{*}{ Nrf2 } & \multirow{2}{*}{ NM_008065.2 } & F- GCAATGTGAGAGCAGGTTCA \\
\hline & & R- GTGGCTACACCAGGCTGTTT \\
\hline \multirow{2}{*}{ GPX1 } & \multirow{2}{*}{ NM_001329527 } & F- GAAGAACTTGGGCCATTTGG \\
\hline & & R- TCTCGCCTGGCTCCTGTTT \\
\hline CDYo & 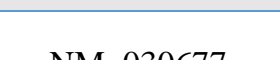 & F- ACCGATCCCAAGCTCATCAT \\
\hline UI A & NIV_OSOOT & R- CAAAGTTCCAGGACACGTCTGA \\
\hline CDY2 & NM 000161 & F- ACAATTGTCCCAGTGTGTGCAT \\
\hline UFAJ & N1V1_008101 & R- TGGACCATCCCTGGGTTTC \\
\hline $\mathrm{CST}=$ & D10610 & F- CGTCCACCTGCTGGAACTTC \\
\hline Usiaz & P10048 & R- GCCTTCAGCAGAGGGAAAGG \\
\hline GSTm2 & P15626 & F- GCTCTTACCACGTGCAGCTT \\
\hline US 1 IIIL & 115020 & R- GGCTGGGAAGAGGAAATGGA \\
\hline $\mathrm{CSTm}_{3}$ & P10630 & F- CACCCGCATACAGCTCATGAT \\
\hline (4) & (1905 & R- TTCTCAGGGATGGCCTTCAA \\
\hline TRX & P10639 & F- CCGCGGGAGACAAGCTT \\
\hline
\end{tabular}




\begin{tabular}{ccc}
\hline & & R- GGAATGGAAGAAGGGCTTGATC \\
\hline \multirow{2}{*}{ GSR } & Q3TWI5 & F- GCTATGCAACATTCGCAGATG \\
\cline { 2 - 2 } PRX1 & R- AGCGGTAAACTTTTTCCCATTG \\
\hline \multirow{2}{*}{ NQ01 } & F- GATCCCAAGCGCACCATT \\
\hline \multirow{2}{*}{ Q6- TAATAAAAAGGCCCCTGAAAGAGAT } \\
\hline
\end{tabular}

Table 9: Primers used in lung fibroblasts RT-qPCR assays.

2.19. Immunoblotting assays in primary lung fibroblasts

\subsubsection{Cell treatments and protein isolation}

Steady state cells seeded in 6-well plates at $80 \%$ confluency were treated during $24 \mathrm{~h}$ at $37^{\circ} \mathrm{C}$ and 5\% of $\mathrm{CO}_{2}$ with the compounds and concentrations described in Table 10. Then, cells were washed with 1X PBS and homogenized in $100 \mu$ l of cold lysis buffer (Cell Signaling) supplemented with $1 \mathrm{mM} \mathrm{NaF}, 1 \mathrm{mM} \mathrm{Na} \mathrm{VO}_{4}, 1 \mathrm{mM}$ PMSF and EDTA-free protease inhibitor cocktail Complete ${ }^{\circledR}(1 \mathrm{tablet} / 50 \mathrm{ml})$ followed by a $10 \mathrm{~min}$ centrifugation at $13000 \mathrm{rpm}$ at $4^{\circ} \mathrm{C}$. Supernatants were collected into $1.5 \mathrm{ml}$ Eppendorf tubes and protein concentration was determined using Bradford assay following the manufacturer's instructions. Samples were then stored at $-80^{\circ} \mathrm{C}$ until use.

\begin{tabular}{cccc}
\hline Treatment & Final concentration & Supplier & Reference \\
\hline DMEM+10\%FBS+1\%P/S & - & - & - \\
\hline Dexamethasone (DEX) & $60 \mathrm{nM}$ & Sigma & D2915 \\
\hline N-Acetylcysteine (NAC) & $10 \mathrm{mM}$ & Sigma & A7250 \\
\hline Treatment & Final concentration & Supplier & Reference \\
\hline DMSO & $0.1 \%$ & Merck Millipore & 1029521000 \\
\hline Fendiline & $10 \mu \mathrm{M}$ & Cayman Chemical & 17295 \\
\hline UO126 & $10 \mu \mathrm{M}$ & Promega & V1121 \\
\hline Ly294002 & $10 \mu \mathrm{M}$ & Calbiochem & 440204 \\
\hline SB202190 & $10 \mu \mathrm{M}$ & Calbiochem & 559388 \\
\hline
\end{tabular}

Table 10: Treatments used on primary lung fibroblasts cultures. Upper part: Dexamethasone (DEX) and NAcetylcysteine (NAC, pH adjusted to 7.4) were diluted in DMEM supplemented with 10\% FBS and 1\% P/S (vehicle). Lower part: Fendiline (KRas inhibitor), UO126 (pan-MEK inhibitor), Ly294002 (pan-PI3K inhibitor) and SB202190 (pan-p38 inhibitor), DMSO (vehicle) was added as a control. 


\subsubsection{Immunoblotting analysis}

Protein lysates were processed as stated in section 3.10.2, and $25 \mu \mathrm{g}$ of total protein from each sample were loaded onto $10 \%$ or $12 \%$ acrylamide gels. Proteins were then transferred into PVDF membranes using the iBLOT2 system as described previously and blocked in 5\% NFDM diluted in TBS-T during $1 \mathrm{~h}$ at RT. Primary antibodies were incubated with the conditions described in Table 11, washed and incubated at RT with the corresponding secondary antibodies as shown in 3.10.2 section. Finally, membranes were scanned in a LI-COR Odyssey infrared imaging system (LI-COR Biosciences) and quantified using Image $\mathbf{J}$ software.

\begin{tabular}{|c|c|c|c|c|c|c|}
\hline Antibody & Supplier & Reference & Type & Dilution & $\begin{array}{c}\text { Antibody } \\
\text { solution }\end{array}$ & Incubation \\
\hline $\begin{array}{c}\alpha-\beta- \\
\text { Tubulin }\end{array}$ & Sigma & T5293 & $\begin{array}{c}\text { Mouse } \\
\text { monoclonal }\end{array}$ & $1: 10000$ & $5 \% \mathrm{BSA}$ & $1 \mathrm{~h}, \mathrm{RT}$ \\
\hline$\alpha$-ERK1/2 & $\begin{array}{c}\text { Cell } \\
\text { Signaling }\end{array}$ & 4696 & $\begin{array}{l}\text { Mouse } \\
\text { monoclonal }\end{array}$ & $1: 2000$ & $5 \%$ NFDM & $1 \mathrm{~h}, \mathrm{RT}$ \\
\hline $\begin{array}{c}\alpha- \\
\mathrm{pERK} 1 / 2\end{array}$ & $\begin{array}{c}\text { Cell } \\
\text { Signaling }\end{array}$ & 9101 & $\begin{array}{c}\text { Rabbit } \\
\text { polyclonal }\end{array}$ & 1:2000 & $5 \%$ NFDM & $\mathrm{o} / \mathrm{n}, 4^{\circ} \mathrm{C}$ \\
\hline$\alpha$-panRas & $\begin{array}{c}\text { Merck } \\
\text { Millipore }\end{array}$ & $005-516$ & $\begin{array}{l}\text { Mouse } \\
\text { monoclonal }\end{array}$ & $1: 1000$ & $5 \%$ BSA & $\mathrm{o} / \mathrm{n}, 4^{\circ} \mathrm{C}$ \\
\hline $\begin{array}{c}\alpha- \\
\mathrm{pMEK} 1 / 2\end{array}$ & $\begin{array}{c}\text { Cell } \\
\text { Signaling }\end{array}$ & 9121 & $\begin{array}{c}\text { Rabbit } \\
\text { polyclonal }\end{array}$ & $1: 500$ & $5 \% \mathrm{BSA}$ & $\mathrm{o} / \mathrm{n}, 4^{\circ} \mathrm{C}$ \\
\hline$\alpha-\mathrm{MEK} 1 / 2$ & $\begin{array}{l}\text { Santa } \\
\text { Cruz }\end{array}$ & sc- 436 & $\begin{array}{c}\text { Rabbit } \\
\text { polyclonal }\end{array}$ & $1: 500$ & $5 \%$ BSA & $\mathrm{o} / \mathrm{n}, 4^{\circ} \mathrm{C}$ \\
\hline$\alpha-\mathrm{pAKT}$ & $\begin{array}{c}\text { Cell } \\
\text { Signaling }\end{array}$ & 4060 & $\begin{array}{c}\text { Rabbit } \\
\text { polyclonal }\end{array}$ & $1: 1000$ & $5 \% \mathrm{BSA}$ & $\mathrm{o} / \mathrm{n}, 4^{\circ} \mathrm{C}$ \\
\hline$\alpha-\mathrm{AKT}$ & $\begin{array}{c}\text { Cell } \\
\text { Signaling }\end{array}$ & 2920 & $\begin{array}{c}\text { Mouse } \\
\text { monoclonal }\end{array}$ & $1: 1000$ & $5 \% \mathrm{BSA}$ & $1 \mathrm{~h}, \mathrm{RT}$ \\
\hline$\alpha-p-p 38$ & Abcam & ab32557 & $\begin{array}{l}\text { Rabbit } \\
\text { monoclonal }\end{array}$ & $1: 500$ & $5 \%$ NFDM & $\mathrm{o} / \mathrm{n}, 4^{\circ} \mathrm{C}$ \\
\hline$\alpha-p 38$ & Abcam & ab31828 & $\begin{array}{c}\text { Mouse } \\
\text { monoclonal }\end{array}$ & $1: 500$ & $5 \%$ BSA & $1 \mathrm{~h}, \mathrm{RT}$ \\
\hline$\alpha-\mathrm{PCNA}$ & $\begin{array}{c}\text { Cell } \\
\text { Signaling }\end{array}$ & 13110 & $\begin{array}{l}\text { Rabbit } \\
\text { polyclonal }\end{array}$ & $1: 1000$ & $5 \%$ BSA & $\mathrm{o} / \mathrm{n}, 4^{\circ} \mathrm{C}$ \\
\hline
\end{tabular}

Table 11: Primary antibodies used in lung fibroblasts immunoblotting assays.

When necessary, antibodies were detached from the membranes by treating them 2x10 min with stripping buffer (1.5\% Glycine, $0.1 \%$ SDS, 1\% Tween20 (Sigma, 93773) in MiliQ water), followed by 3x10 min washes with 1X TBS-T and blocking with 5\% NFDM during $1 \mathrm{~h}$ at RT. Then, membranes were again incubated with primary and secondary antibodies. 


\subsection{Lung organoid assays}

Lung organoids were used as an in vitro approach to study the interactions between epithelium and mesenchyme necessary for a proper lung development and maturation. Lung mesenchymal cells provide the fundamental factors that support epithelial survival, proliferation and differentiation. Meanwhile, epithelial cells provide the fibroblasts with feedback signalling to control the different stages of lung development and differentiation.

The method is based on a co-culture composed by primary lung fibroblasts, isolated as described in section 3.14, constituting the mesenchyme, and alveolar epithelial progenitors (expressing Epithelial cell adhesion molecule, hereinafter denominated as EpCAM+ cells) as the epithelial component. Specifically, in these experiments, CT, HRas-KO, NRas-KO and DKO primary lung fibroblasts and WT EpCAM+ cells were used (Fig. 18).

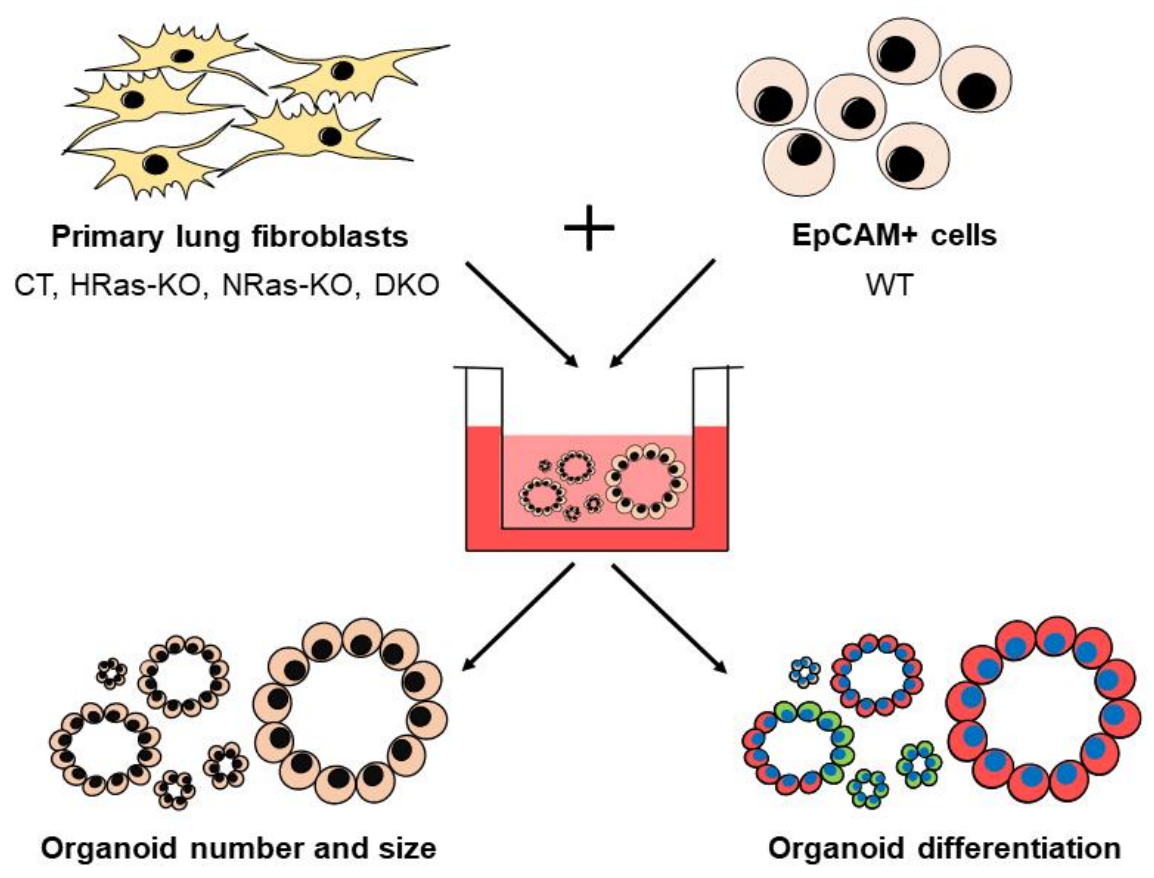

Figure 18: Lung organoid co-culture assay. Scheme illustrating the organoid co-culture assay protocol and readouts. The read-outs of the 14-days organoids include measures or organoid size, number, and differentiation. Organoid differentiation is represented as follows: bronchiolar (red), alveolar (green), double-positive (red/green) and double-negative (no colour).

\subsubsection{EpCAM+ cells isolation}

Lung epithelial cells were isolated from adult 8-weeks old C57B1/6 mice. Mice were anesthetized and sacrificed, and lungs were washed through the heart with $20-30 \mathrm{ml}$ of cold $1 \mathrm{X}$ PBS to eliminate blood, and subsequently intra-tracheal instilled with $1.5 \mathrm{ml}$ dispase (VWR, 11553550) and covered with ice for 5-10 min. Then, lungs were dissected and collected into a $15 \mathrm{ml}$ tube containing $1 \mathrm{ml}$ dispase and digested during $45 \mathrm{~min}$ at $\mathrm{RT}$. 
Digested lungs were transferred to a $100 \mathrm{~mm}$ Petri dish and lobes were separated, discarding any non-lung tissue. Then, lung lobes were homogenized with forceps in $10 \mathrm{ml}$ of "+ medium" (Table 12), disaggregating any remaining tissue by pipetting up and down with a $10 \mathrm{ml}$ stripette and transferred to a $50 \mathrm{ml}$ tube thought a $100 \mu \mathrm{m}$ cell strainer, adding $30 \mathrm{ml}$ more of "+ medium". The cell suspension was centrifuged at $300 \mathrm{xg}$ for $5 \mathrm{~min}$ at $15^{\circ} \mathrm{C}$, resuspended in $10 \mathrm{ml}$ of "+ medium", transferred into a $15 \mathrm{ml}$ tube and centrifuged again at $300 \mathrm{xg}$ for $5 \mathrm{~min}$ at $15^{\circ} \mathrm{C}$.

Supernatant was discarded and pellet was resuspended in $80 \mu$ l of MACS buffer with 5\% BSA (1500ml MACS rising solution (Miltenyi Biotec, 130-091-222) with 75ml MACS BSA Stock Solution (Miltenyi Biotec, 130-091-376)), and negative selection to eliminate hematopoietic and endothelial cells was performed by adding $10 \mu 1$ of CD45 microbeads (Miltenyi Biotec, 130-052-301) and 10 $\mu 1$ of CD31 microbeads (Miltenyi Biotec, 130-097-418) respectively. Samples were then incubated during $20 \mathrm{~min}$ at $4^{\circ} \mathrm{C}$ without mixing. $10 \mathrm{ml}$ of MACS buffer was added and samples were centrifuged at 300xg during $5 \mathrm{~min}$ at $15^{\circ} \mathrm{C}$. Supernatant was discarded, pellet was resuspended in $8 \mathrm{ml}$ of MACS buffer and processed by magnetic separation using the QuadroMACS ${ }^{\mathrm{TM}}$ separator (Miltenyi Biotec). Magnetic column separators (Miltenyi Biotec, 130-042-401) and 30 $\mu \mathrm{m}$ pre-separation filters (Miltenyi Biotec, 130-041-407) were prepared and washed following manufacturer's instructions. Cell suspensions were loaded onto the columns, keeping the cells in the flow-through in a $15 \mathrm{ml}$ tube and discarding the column with the hematopoietic and endothelial cells attached.

\begin{tabular}{|c|c|c|c|c|}
\hline $\begin{array}{l}\text { Minus (-) } \\
\text { medium }\end{array}$ & $\begin{array}{c}\text { Plus (+) medium } \\
\text { (50ml/mouse) }\end{array}$ & $\begin{array}{l}10 \% \text { FBS } \\
\text { medium }\end{array}$ & $\begin{array}{c}\text { Organoid base } \\
\text { medium }\end{array}$ & Organoid medium \\
\hline DMEM & $\begin{array}{l}\text { 50ml minus } \\
\text { medium }\end{array}$ & $\begin{array}{l}\text { DMEM:Ham's } \\
\text { F-12 1:1 }\end{array}$ & $\begin{array}{c}\text { DMEM:Ham's } \\
\text { F-12 1:1 }\end{array}$ & $\begin{array}{l}\text { Organoid base } \\
\text { medium }\end{array}$ \\
\hline \multirow[t]{4}{*}{$1 \% \mathrm{P} / \mathrm{S}$} & 2mg DNase & $10 \% \mathrm{FBS}$ & $5 \% \mathrm{FBS}$ & $0.025 \%$ EGF \\
\hline & & $1 \% \mathrm{P} / \mathrm{S}$ & $1 \% \mathrm{P} / \mathrm{S}$ & $\begin{array}{c}1 \% \text { Insulin- } \\
\text { transferrin-selenium }\end{array}$ \\
\hline & & $\begin{array}{c}1 \% \mathrm{~L}- \\
\text { Glutamine }\end{array}$ & $\begin{array}{c}1 \% \mathrm{~L}- \\
\text { Glutamine }\end{array}$ & $\begin{array}{l}1.75 \% \text { Bovine } \\
\text { pituitary extract }\end{array}$ \\
\hline & & $\begin{array}{c}1 \% \\
\text { Amphotericin B }\end{array}$ & $\begin{array}{c}1 \% \\
\text { Amphotericin B }\end{array}$ & \\
\hline
\end{tabular}

Table 12: EpCAM+ cells and organoids culture mediums. Penicillin/Streptomycin (P/S), Fetal bovine serum (FBS), Epithelial grown factor (EFG), DMEM medium (Gibco, 11965092), Ham's F12 medium (Lonza, BE12-615F).

Samples were centrifuged at $300 \mathrm{xg}$ for $5 \mathrm{~min}$ at $15^{\circ} \mathrm{C}$ and pellets were processed for the positive selection of EpCAM+ cells by resuspending and incubating $\left(4^{\circ} \mathrm{C}, 20 \mathrm{~min}\right)$ each sample

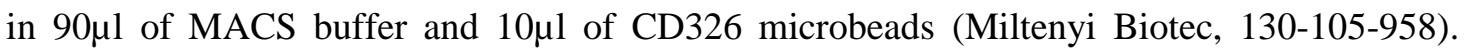
Samples were then diluted with $10 \mathrm{ml}$ of MACS buffer, centrifuged at $300 \mathrm{xg}$ for $5 \mathrm{~min}$ at $15^{\circ} \mathrm{C}$, resuspended in $8 \mathrm{ml}$ of MACS buffer and separated using the QuadroMACS ${ }^{\mathrm{TM}}$ separator as described before. The flow-through was discarded and the EpCAM+ cells were detached from 


\section{Materials and Methods}

the column by adding $5 \mathrm{ml}$ of MACS buffer and using the plunger, collecting the cells into $15 \mathrm{ml}$ tubes. Samples were centrifuged at $300 \mathrm{xg}$ for $5 \mathrm{~min}$ at $15^{\circ} \mathrm{C}$ and EpCAM+ cells were resuspended in $1000 \mu \mathrm{l}$ of $10 \%$ FBS medium (Table 12) and counted to determine the number of cells $/ \mathrm{ml}$. Cells were maintained in ice until use.

\subsubsection{Proliferation-inactivation of primary lung fibroblasts}

Primary lung fibroblasts from the four genotypes under study were seeded into 100mm Petri dishes or $25 \mathrm{~cm}^{2}$ ventilated flasks and growth until $80-90 \%$ confluency. The day of EpCAM+ cells isolation, lung fibroblasts proliferation was inactivated by adding $10 \mu \mathrm{g} / \mathrm{ml}$ of Mitomycin $\mathrm{C}$ (Sigma, M4287) diluted in 10\% FBS medium during $2 \mathrm{~h}$ in a $37^{\circ} \mathrm{C}$ and $5 \% \mathrm{CO}_{2}$ incubator. Cells were washed twice with $1 \mathrm{X}$ PBS and incubated for at least $1 \mathrm{~h}$ at $37^{\circ} \mathrm{C}$ and $5 \% \mathrm{CO}_{2}$ in DMEM supplemented with $10 \%$ FBS.

Lung fibroblasts were then trypsinized and counted manually to determine the number of cells $/ \mathrm{ml}$ and maintained in ice until use.

\subsubsection{Organoid co-culture seeding and treatments}

Each $3 \mathrm{D}$ co-culture was established mixing $10^{4}$ primary lung fibroblasts with $10^{4}$ EpCAM+ cells in Matrigel ${ }^{\circledR}$ (Corning, 356230). Then, $40 \%$ cells (in DMEM with 10\% FBS) and $60 \%$ Matrigel ${ }^{\circledR}$ (Corning, 353095) (in a total volume of $100 \mu$ l per insert) were placed in a 24 -well cell culture plate. Samples were incubated into $37^{\circ} \mathrm{C}$ and $5 \% \mathrm{CO}_{2}$ incubators during 30 min to allow the Matrigel ${ }^{\circledR}$ solidify. Then, $410 \mu$ l of organoid medium (Table 12) with $0.0032 \mathrm{mg} / \mathrm{ml}$ of Y27632 ROCK inhibitor (ROCKi, Axon, 1683) were added to each sample beneath the insert during $24 \mathrm{~h}$. After $24 \mathrm{~h}$ ROCKi was eliminated, and media was replaced every 2-3 days with $410 \mu \mathrm{l}$ of organoid medium.

Organoids were subjected to the treatment with different compounds (Table 13), all of them diluted in the organoid culture medium, starting on the second day of culture and throughout the remaining 13 days. The culture medium from each treatment and genotype was collected throughout the 14 days of culture and frozen at $-80^{\circ} \mathrm{C}$ for further analysis.

\begin{tabular}{cccc}
\hline Treatment & Final concentration & Supplier & Reference \\
\hline Organoid medium & - & - & - \\
\hline Dexamethasone (DEX) & $60 \mathrm{nM}$ & Sigma & D2915 \\
\hline N-Acetylcysteine (NAC) & $1 \mathrm{mM}$ & Sigma & A7250
\end{tabular}




\begin{tabular}{cccc} 
Treatment & Final concentration & Supplier & Reference \\
\hline DMSO & $0.1 \%$ & Merck Millipore & 1029521000 \\
Fendiline & $10 \mu \mathrm{M}$ & Cayman Chemical & 17295 \\
\hline UO126 & $10 \mu \mathrm{M}$ & Promega & V1121 \\
\hline Ly294002 & $10 \mu \mathrm{M}$ & Calbiochem & 440204 \\
\hline SB202190 & $10 \mu \mathrm{M}$ & Calbiochem & 559388 \\
\hline
\end{tabular}

Table 13: Treatments used on organoid cultures. Upper part: Dexamethasone (DEX) and N-Acetylcysteine (NAC, $\mathrm{pH}$ adjusted to 7.4) were diluted in organoid medium (vehicle). Lower part: Fendiline (KRas inhibitor), UO126 (panMEK inhibitor), Ly294002 (pan-PI3K inhibitor) and SB202190 (pan-p38 inhibitor) were added to the organoid culture medium diluted with DMSO (vehicle), which was added as a control to non-treated samples.

\subsubsection{4-days organoid analysis}

Lung organoids start to differentiate toward alveolar or bronchiolar phenotypes on day 7 of culture, and mature organoids are obtained on day 14. As a first approach, using bright field microscopy, we can distinguish two different phenotypes depending on the organoid shape: alveolar (smaller and darker), bronchiolar (bigger, clearer and with visible cilia movement).

On culture day 14, 3 different areas per well were analysed by taking images, using a Nikon Eclipse Ti-S microscope, of different focus planes of each area, and a single image of one field with all organoids focused was created using ImageJ software and "Stack focuser" plugin (https://imagej.nih.gov/ij/plugins/stack-focuser.html). Organoid size and number were determineed using the stacked images using ImageJ software.

\subsubsection{Organoid fixation and immunofluorescence staining}

Culture media from 14-days organoids was collected and frozen at $-80^{\circ} \mathrm{C}$ and wells were washed by adding $1 \mathrm{ml}$ of $1 \mathrm{X}$ PBS beneath the inserts. PBS was removed and $500 \mu 1$ of ice-cold methanol-acetone (1:1) was added beneath the insert, and additional $150 \mu 1$ of ice-cold methanolacetone (1:1) was added on the top of the insert. The 24 -well plate was incubated at $-20^{\circ} \mathrm{C}$ for $15 \mathrm{~min}$, then, methanol-acetone from both the well and top of the insert was collected carefully without disturbing the gel and $1 \mathrm{ml}$ of $1 \mathrm{X}$ PBS with $0.02 \%$ sodium azide was added beneath the inserts. The fixed organoids were maintained at $4^{\circ} \mathrm{C}$ until use.

Immunofluorescence was performed in order to identify whether the morphology of the 14days organoids was predominantly alveolar (Surfactant protein $\mathrm{C}$ alveolar marker, SftpC+), bronchiolar (Acetylated tubulin bronchiolar marker, AcTub+), double-positive (SftpC+/AcTub+) or double-negative (SftpC-/AcTub-). All the following solutions were added 


\section{Materials and Methods}

into the top of the insert: Organoids were permeabilized with 1X PBS-0.1\% Triton during 10 min, and then, samples were blocked using 5\% BSA, $2 \%$ goat serum, $0.1 \%$ Triton in $1 \mathrm{X}$ PBS $\mathrm{o} / \mathrm{n}$ at $4^{\circ} \mathrm{C}$. Blocking solution was removed and primary antibodies (Table 4) diluted in 1X PBS with $2 \%$ BSA, $2 \%$ goat serum and $0.1 \%$ Triton were incubated during $48 \mathrm{~h}$ at $4{ }^{\circ} \mathrm{C}$ with gentle rocking. Primary antibodies were removed, and samples were washed 3x30 min with 1X PBS and gentle rocking, followed by 10 min with $1 \mathrm{X}$ PBS+Triton and subsequently incubated with the corresponding fluorescent secondary antibodies goat anti-mouse $\mathrm{Cy} 3$ and goat anti-rabbit Alexa 488, diluted 1:500 in antibody solution during $2 \mathrm{~h}$ at RT with gentle rocking. Then, organoids were washed $3 \times 30$ min with $1 \mathrm{X}$ PBS, and $1 \mathrm{X}$ PBS was left o/n at $4^{\circ} \mathrm{C}$. Finally, the membrane of each insert was cut out using a scalpel and mounted into glass slides using Fluoroshield mounting medium with DAPI (Abcam, ab104139). Digital images were acquired using a Leica DM4000b microscope, a minimum of 10 images per genotype and condition were analysed using Photoshop CS6 software and organoids were counted and divided in alveolar bronchiolar, double-positive or double-negative (Fig. 18).

\subsection{FGF7 levels measured by ELISA assay}

Fibroblast growth factor-7 (FGF7) levels were determined in culture mediums collected from the different organoid culture's conditions using the mouse FGF-7 ELISA Kit (Invitrogen, EM32RB), following manufacturer's guidelines, and determination of optical density of each well was performed in a colorimetric microplate reader (TECAN infinite M200 PRO).

\subsection{Ras activation assay in primary lung fibroblasts}

Production and binding of GST-Raf-RBD fusion protein to Glutathione-Sepharose beads were performed as described in sections 3.11.1 and 3.11.2, respectively.

Primary lung fibroblasts seeded in $100 \mathrm{~mm}$ Petri dishes at $80 \%$ confluency were treated in

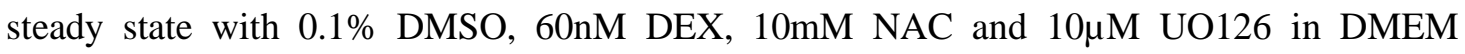
supplemented with 10\% FBS during $24 \mathrm{~h}$. In addition, as a control of the experiment, one Petri dish was starved in DMEM without FBS for $10 \mathrm{~h}$.

Following the completion of the treatments, reactions were stopped in ice, mediums were discarded, and cells were washed with cold 1 X PBS. Then, cells were homogenized in 500 $\mu 1$ of cold 1X MLB using a cell scraper, followed by a 10 min centrifugation at $13000 \mathrm{rpm}$ and $4^{\circ} \mathrm{C}$.

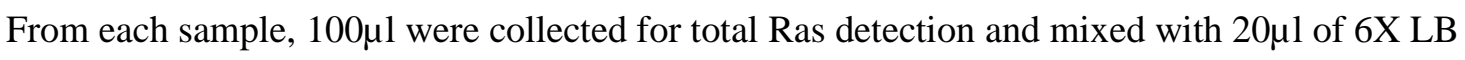
and frozen at $-80^{\circ} \mathrm{C}$ until use. Meanwhile, the remaining $400 \mu \mathrm{l}$ were further processed for RAS. GTP pull-down assays, by incubating them with $100 \mu$ of GST-Raf-RBD attached to the glutathione-Sepharose beads during $30 \mathrm{~min}$ at $4^{\circ} \mathrm{C}$ with gentle rotation. The samples were then 
washed 2 times with 1X MLB, with centrifugation steps between washes at $13000 \mathrm{rpm}$ and $4^{\circ} \mathrm{C}$. Supernatants were discarded and pellets were resuspended in $40 \mu 1$ of $4 \mathrm{X}$ LB, and quickly frozen at $-80^{\circ} \mathrm{C}$ until use.

Finally, $20 \mu 1$ of total Ras samples and $20 \mu 1$ of RAS.GTP pull-down assay samples were loaded onto $12 \%$ acrylamide gels, and levels of RAS. GTP and total-RAS were analysed through Western blot assays, as indicated in section 3.18.2., with the incubation of the membranes with a specific primary antibody against pan-RAS (Table 11) o/n at $4^{\circ} \mathrm{C}$. 



\section{RESULTS}





\section{RESUltS}

3.1. Phenotypes resulting from genetic deletion of HRas and/or NRas in mice.

\subsubsection{HRas and NRas simultaneous ablation causes neonatal death}

To determine the effect of HRas and NRas loss, alone and in combination, we crossed $\mathrm{HRas}^{+/}$ ;NRas ${ }^{-/}$and $\mathrm{HRas}^{-/} ; \mathrm{NRas}^{+/-} \mathrm{C} 57 \mathrm{Bl} / 6$ mice, as indicated in section 3.2 of Material and Methods, and analysed the phenotypic traits of the litters resulting from these crossings: $\mathrm{HRas}^{+/} ; \mathrm{NRas}^{+-}$

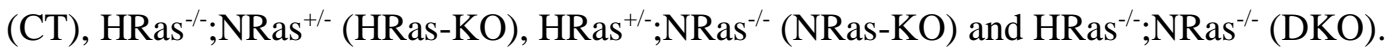

Pregnant females and resulting litters were monitored daily and newborn (P0) pups were counted and genotyped at $\mathrm{P} 0$ and on weaning day (P21). The numbers of born CT, HRas-KO, NRas-KO and DKO animals followed expected mendelian rates (around 25\% for each genotype) (Fig. 19A). Additionally, DKO mice showed an important reduction in body weight at birth compared with CT and HRas-KO littermates, and similar tendency was observed in NRas-KO animals compared with CT littermates (Fig. 19C, D).

A

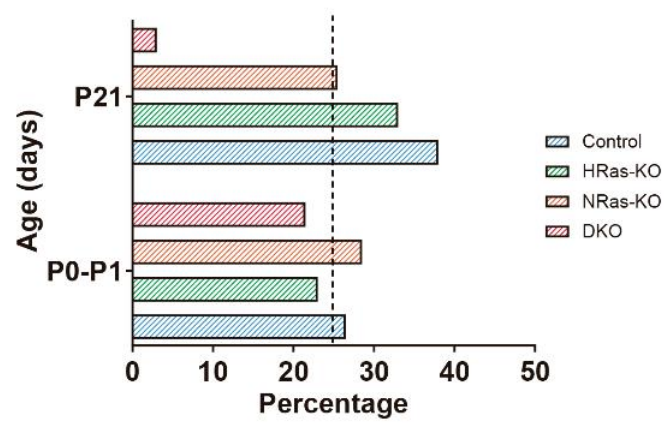

C

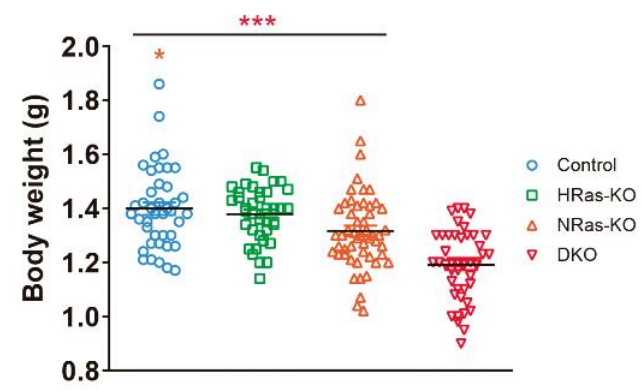

B

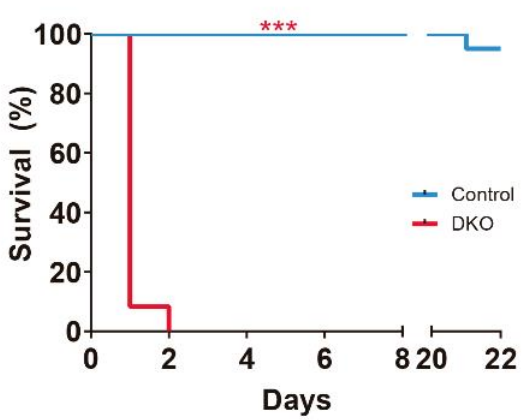

D

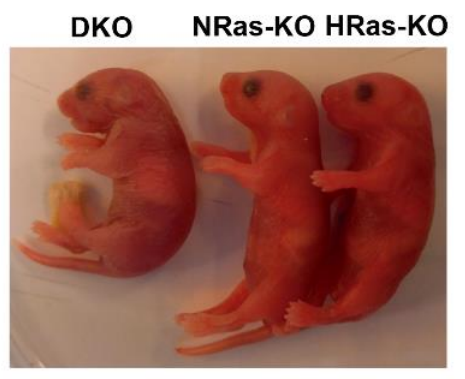

Figure 19: Analysis of the offspring from crosses between HRas-KO and/or NRas-KO mice. A. Bar graphs depicting percentage of individuals of the indicated genotypes (Control, HRas-KO, NRas-KO, DKO) counted at time of birth (P0) or at weaning time (P21) in the litters resulting from parental crosses between $\mathrm{HRas}^{+-} ; \mathrm{NRas}^{--}$and vice versa. B. Kaplan-Meier plot comparing the survival rates of Control and DKO littermates. $n=20$ for Controls and $n=12$ for DKO individuals. $* * * p>0.001$ for comparison between Control and DKO mice. C. Body weight distribution of living, newborn P0 mice of the indicated genotypes at time of birth. Data represented as the mean \pm s.e.m. for each genotype. Control, $\mathrm{n}=59$; HRas-KO, $\mathrm{n}=39$; NRas-KO, $\mathrm{n}=52$; DKO, $\mathrm{n}=45 . * \mathrm{p}<0.05$, *** $\mathrm{p}<0.001$. D. Representative picture of a cyanotic DKO pup (left) next to healthy HRas-KO and NRas-KO littermates, immediately after birth (P0). 
However, although all genotypes were born at normal rates, the number of surviving DKO mice was decreased at P21 (Fig. 19A), meaning that DKO animals were dying at some point between P0 and P21 days. Given this observation, we carefully examined the newborn litters and found that the DKO pups died between $0-48 \mathrm{~h}$ after birth (Fig. 19B).

\subsubsection{HRas/NRas double-null mice suffer neonatal death caused by respiratory failure}

Interestingly, most DKO neonates became cyanotic and showed severe respiratory distress as observed by their noticeable breathing efforts, with some mice showing these features dying within minutes. None of these phenotypes were present on the individual CT, HRas-KO and NRas-KO genotypes, suggesting that the lack of both GTPases led to impaired respiratory activity and postnatal death.

Full anatomo-pathological analyses of P0 CT, HRas-KO, NRas-KO and DKO animals were performed on histological samples, but no alterations were found in heart, brain, digestive system, kidney, or liver. In contrast, the lungs of newborn DKO mice showed extensive atelectasis with occasional haemorrhages, probably caused by the breathing efforts (Fig. 20A). Additionally, H\&E assays also revealed that only DKO animals, but not the rest of genotypes, presented a significant reduction of the alveolar saccular space, with notably thicker alveoli separating septa (Fig. 20A).

Interestingly, alveolar condensation was already noticeable at earlier embryonic stages (E18.5) not only in DKO animals, but also in HRas-KO mice (Fig. 20A). Nevertheless, the lungs of newborn HRas-KO were completely indistinguishable from those of CT mice, indicating that the defect observed at the E18.5 embryonic stage had already disappeared at birth in the HRasKO but not in the DKO mice.

\subsubsection{Alveolar cell lineage differentiation is altered in both HRas-KO and HRas/NRas-DKO animals}

There is a known connection between neonatal respiratory distress syndrome (NRDS) and impaired lung maturation (Hallman \& Haataja, 2003; Najafian \& Hossein Khosravi, 2020). Hence, we decided to study the alveolar differentiation in the lungs of CT, HRas-KO, NRas-KO and DKO animals at P0 and E18.5 stages, since crucial and quick changes take place during the alveolarization stage: the alveolar precursors cells stop proliferating and start to differentiate into alveolar epithelial cells type I (AECI) and alveolar epithelial cells type II (AECII) (Desai et al., 2014; Frank et al., 2019). 
A
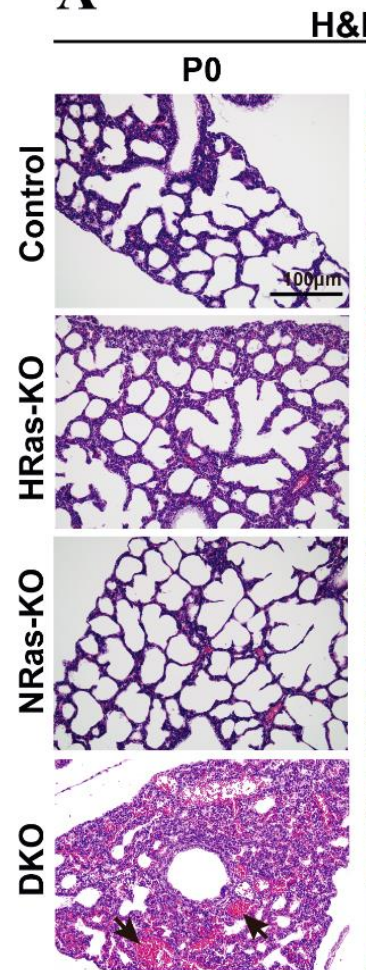

H\&E
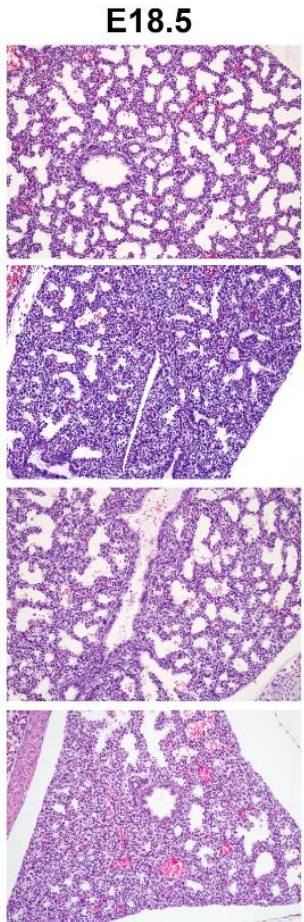

B

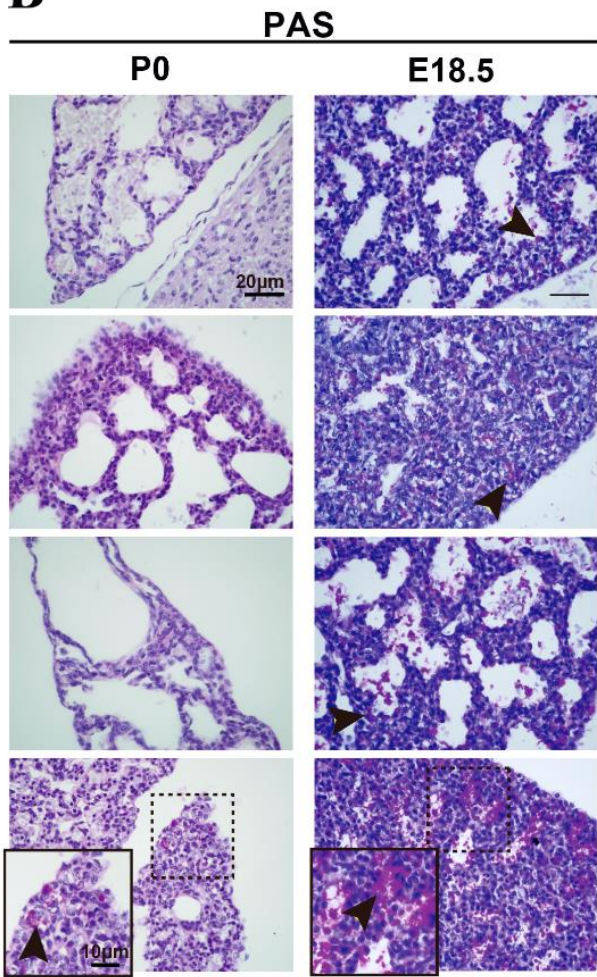

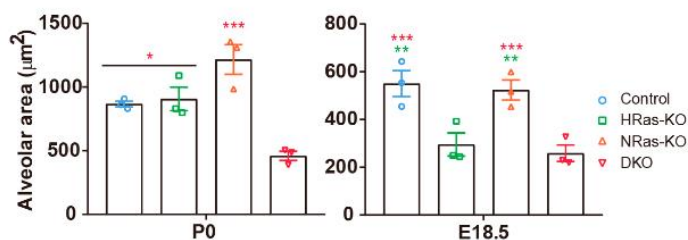

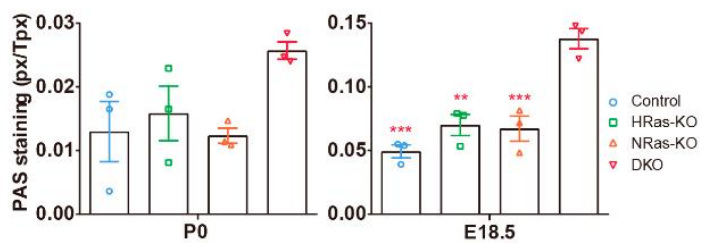

Figure 20: Histological analysis of the lungs of newborn pups (P0) and late embryos (E18.5) of HRas-KO and/or NRas-KO mice. A. Representative images of Haematoxylin-Eosin (H\&E)-stained sections of lungs from P0 newborn pups or from E18.5 embryos of the indicated genotypes. Arrows indicate haemorrhagic regions. Scale bars: $100 \mu \mathrm{m}$. The bar graphs at bottom of the panels quantify the average area $(\mu \mathrm{m} 2)$ of the individual alveolar sacs in the lungs of, respectively, P0 and E18.5 individuals of the indicated genotypes. Data is expressed as the mean \pm s.e.m. $\mathrm{n}=3$ individuals for each genotype. $* \mathrm{p}<0.05, * * \mathrm{p}<0.01$, *** $\mathrm{p}<0.001$. B. Representative images of PAS-stained lung sections from P0 newborn mice or from E18.5 embryos of the indicated genotypes. Scale bars: $20 \mu \mathrm{m}$ and $10 \mu \mathrm{m}$ in magnified, boxed areas. Black arrowheads point to cytoplasmic and extracellular accumulations of PAS-positive label in alveolar areas of the indicated genotypes. The bar graphs in this panel quantify the relative levels of PAS-staining (ratio of PAS+ pixels relative to total number of pixels) in the lungs of P0 and E18.5 individuals of the indicated genotypes. Data is expressed as the mean \pm s.e.m. $n=3$ individuals for all genotypes in E18.5 lungs; $n=4$ for DKO and $\mathrm{n}=3$ for the rest of genotypes of P0 lungs. $* \mathrm{p}<0.05, * * \mathrm{p}<0.01, * * * \mathrm{p}<0.001$.

Glycogen granules, present in the cytoplasm of AECII, are the building blocks for surfactant phospholipids, and the presence of glycogen deposits at later stages of lung development is a hallmark of immature AECII (Ridsdale \& Post, 2004; Young et al., 1991). Through the analysis of PAS staining of lung sections, we detected a strong enrichment in PAS+ glycogen intracellular content in the alveolar regions of P0 and E18.5 animals as compared to the other genotypes (Fig. 20B).

We further evaluated the differentiation of the pneumocytes, into gas-exchanging AECI and surfactant-producing AECII cuboidal cells, with specific cell markers. Using the fluoresceinlabelled Ricinus communis agglutinin-I (RCA-I) as a marker of AECI, and Surfactant protein C (SftpC) as marker a for AECII cells, we can distinguish three different cells: AECI (RCA-I+), 
AECII (SftpC+) and alveolar bi-potent progenitors (RCA-I+/SftpC+). These alveolar bi-potent progenitors are present in the mouse embryonic lungs until E18.3, giving rise to the mature AECI and AECII cells (Desai et al., 2014).

We firstly analysed the AECI and AECII populations in P0 lungs, observing no differences in RCA-I+ staining among the four genotypes under study. However, a significant increment in SftpC+ cell number was noticed in HRas-KO and DKO newborn lungs (Fig. 21A, D, G), that was consistent with the notion of pulmonary immaturity (Ringvall \& Kjelln, 2010). Moreover, these SftpC+ pneumocytes were placed in circular accumulations instead of being distributed throughout the luminal surface of the alveolar sacs (Fig. 21A, B-arrowheads).

Secondly, we studied the presence or absence of alveolar bi-potent progenitors using simultaneous immunoassays against RCA-I and SftpC. An abnormally higher percentage of RCA-I+/SftpC+ alveolar bi-potent cells were detected in both HRas-KO and DKO lung sections at E18.5 whereas, however, at P0 only the DKO lungs maintained a higher number of these cells in contrast with the other genotypes (Fig. 21B, E, H).

Additionally, the retention of undifferentiated alveolar progenitors in our HRas-KO and DKO strains was further monitored with immunoassays against Sex-determining region Y-box 9 (SOX9), a well-established marker of alveolar distal-tip progenitors. We detected a strong nuclear SOX9 staining in lung distal-tip structures of HRas-KO and DKO mice at E18.5 stage as compared to CT and NRas-KO lungs. This increment in SOX9+ cells in both mice lacking HRas and HRas/NRas-double null animals was observed not only in the peripheral parts of the lungs where usually distal-tip structures are located, but also in the inner parenchyma (Fig. 21C, F, I).

Altogether, we have demonstrated a delayed differentiation of the alveolar cell lineages in DKO mice, and a partial delay in HRas single-KO, through (1) the increment in PAS+ glycogen deposits in the alveoli, (2) the higher number of SftpC+ AECII cells, (3) the retained presence of RCA-I+/SftpC+ alveolar bi-potent progenitors and (4) the higher amount of SOX9+ alveolar distal-tip progenitors.

\subsubsection{Individual or combined ablation of HRas and NRas causes structural alterations of bronchiolar cells}

The bronchiolar cell lineage was studied by histochemical PAS staining and immunostaining with Secretoglobulin 1a1 (Scgb1a1), as specific Club cell marker, and $\beta$-Tubulin ( $\beta$-Tub) as Ciliated cell marker. These analyses revealed significant alterations in the structure of the Club cells, located in the luminal layer of the bronchioles. These alterations were present in DKO and single KO PO littermates and included overall reduction of glycosaminoglycan (PAS+) 
labelling, corresponding to the apical vesicle characteristic of the Club cells (Fig. 22A), as well as noticeable morphological flattening from its typical columnar to a square shape, as confirmed by means of immunostainings with Scgb1a1, possibly linked to the observed shortening of their apical vesicular area (Fig. 22A, B, C). On the other hand, a significant shortening of the Ciliated cells' structure was only observed in the DKO when compared to the rest of the genotypes as assessed through $\beta$-Tub immunostainings (Fig. 22B, D). No alterations were detected when the Club-Ciliated cell composition was studied in the P0 lungs of all four genotypes (Fig. 22E).

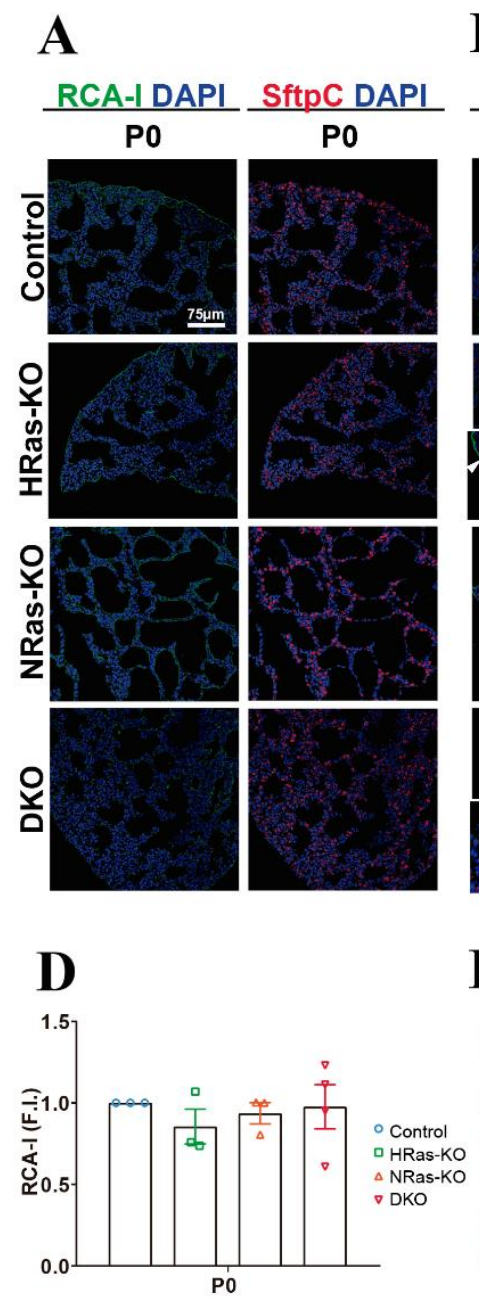

B
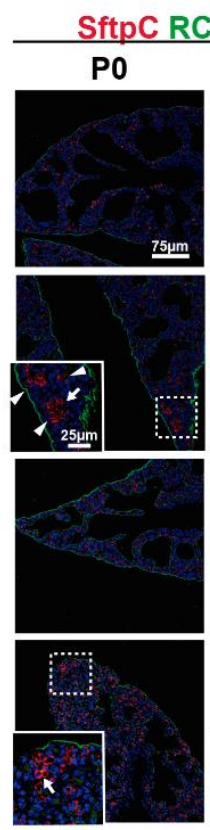

$\mathbf{E}$
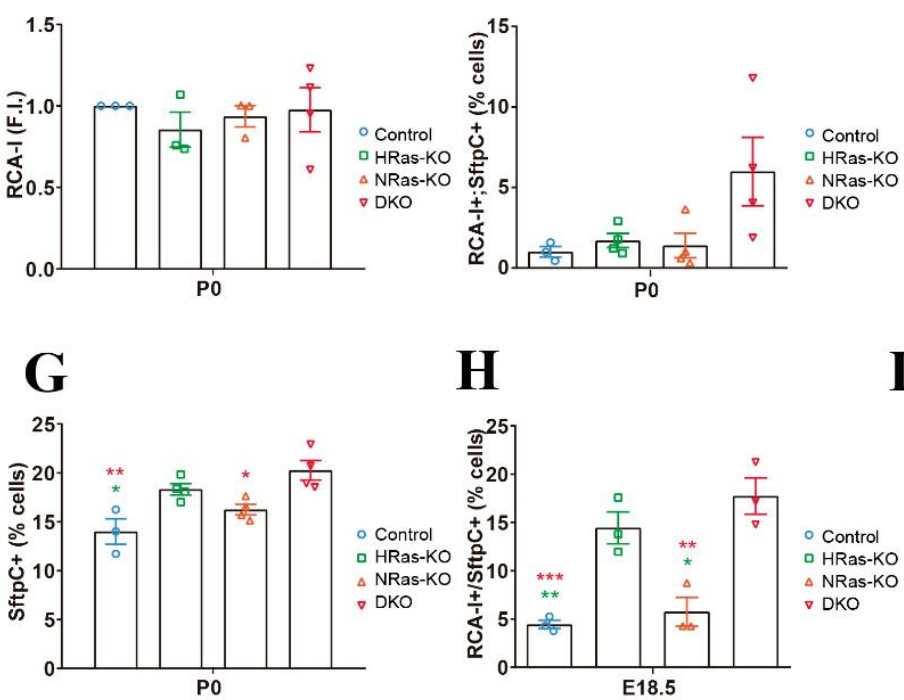

Sox9 DAPI

\section{E18.5 Periphery E18.5 Parenchyma}
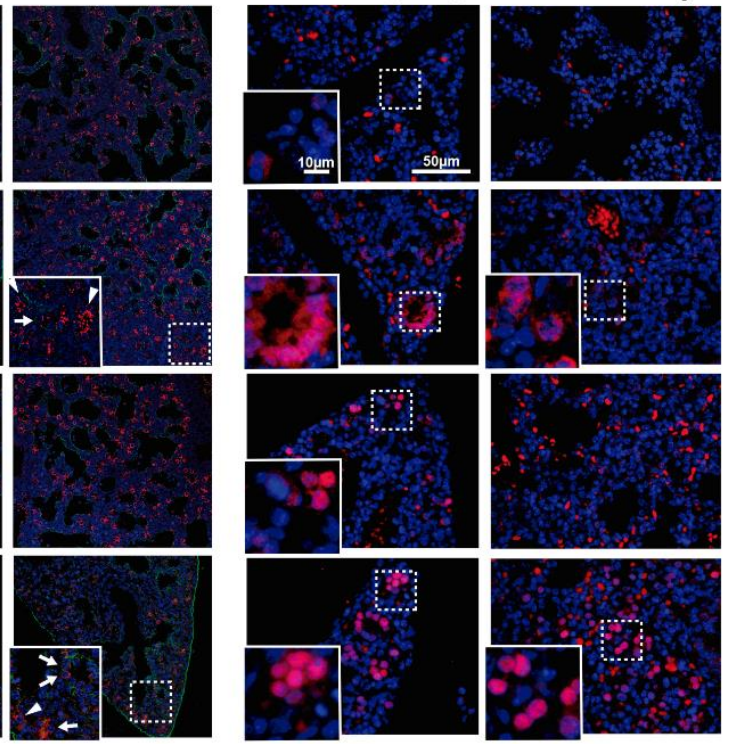

$\mathbf{F}$

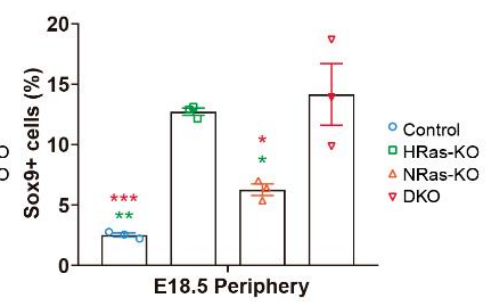

I

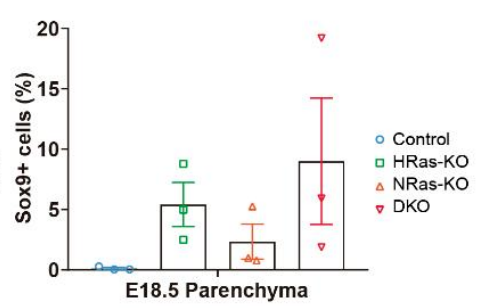

Figure 21: Immunostaining of alveolar differentiation markers in the lungs of HRas-KO and/or NRas-KO mice. A. Representative images of immunostaining for Ricinus communis agglutinin-I (RCA-I, AT1 lineage, green) and Surfactant protein-C (SftpC, AT2 lineage, red) in paraffin sections of the lungs of newborn P0 mice of the indicated genotypes. Regions of SftpC + cell accumulations are marked by tailed arrows. Scale bar: $75 \mu \mathrm{m} . \mathbf{B}$. Representative images of immunostaining for Surfactant protein-C (SftpC, AT2 lineage, red) and Ricinus communis agglutinin-I (RCA-I, AT1 lineage, green) in paraffin sections of the lungs of newborn P0 and E18.5 mice of the indicated genotypes. Distal-tip like alveolar structures are marked by arrow heads. Co-immunolabeled, alveolar bi-potent progenitor cells are marked by tailed arrows. Scale bar: $75 \mu \mathrm{m}$ and $25 \mu \mathrm{m}$ in the magnified boxed 
areas. C. Representative images of immunostainings for SOX9 (distal-tip progenitors, red) in peripheral or inner parenchymal areas of E18.5 lungs from the indicated genotypes. Scale bar: $50 \mu \mathrm{m}$ and $10 \mu \mathrm{m}$ in the magnified boxed areas. D, G. The bar graphs quantify, respectively, the average fluorescence intensity (F.I.) of the RCA-I immunoassay signals (relative to Control animals), and the percentage of SftpC+ pneumocytes (relative to total nuclei) in the lungs of P0 individuals of the indicated genotypes. Data expressed as the mean \pm s.e.m. Ten separate microscopic fields were quantified for each individual analysed in each genotype, $n=3$ for all genotypes in RCAI quantification, and $\mathrm{n}=3$ for Control and $\mathrm{n}=4$ for the rest of genotypes in SftpC quantification. $* \mathrm{p}<0.05, * * \mathrm{p}<0.01$. E, H. The bar graphs quantify the percentage of alveolar bi-potent cells (RCA-I+/SftpC+) relative to total number of SftpC+ cells in the lungs of P0 or E18.5 individuals of the indicated genotypes. Data expressed as the mean \pm s.e.m. Ten separate microscopic fields were quantified for each individual analysed. $n=3$ for all genotypes of E18.5 lungs, $\mathrm{n}=3$ for Controls and $\mathrm{n}=4$ for the rest of genotypes in $\mathrm{P} 0$ lungs. $* \mathrm{p}<0.05, * * \mathrm{p}<0.01, * * * \mathrm{p}<0.001$. F, I. The bar graphs quantify the percentage of SOX9+ cells relative to total number of cells. Data expressed as the mean \pm s.e.m. Ten separate microscopic fields were quantified for each of the 3 individuals analysed in each genotype. $\mathrm{n}=3$ individuals for all genotypes. $* \mathrm{p}<0.05, * * \mathrm{p}<0.01, * * * \mathrm{p}<0.001$.

A
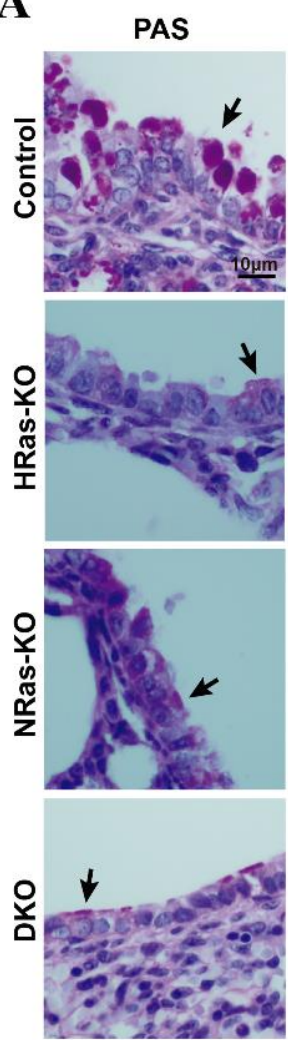

B

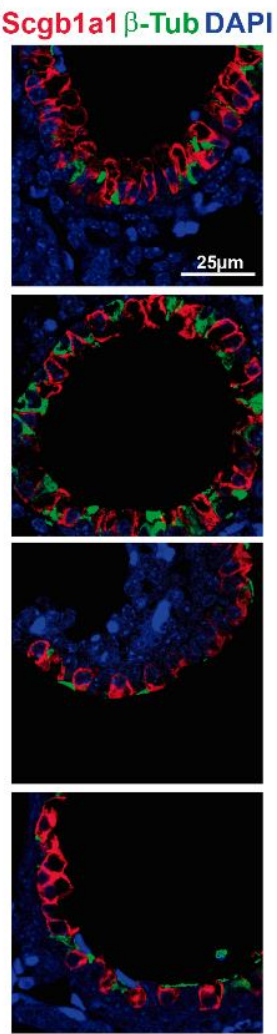

C

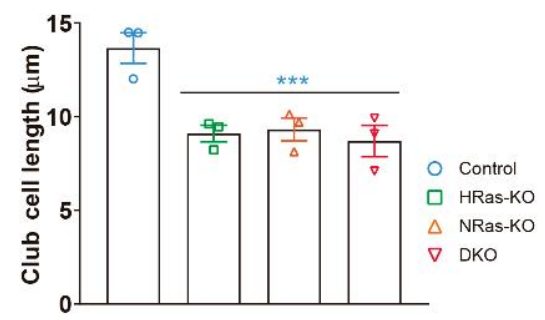

D

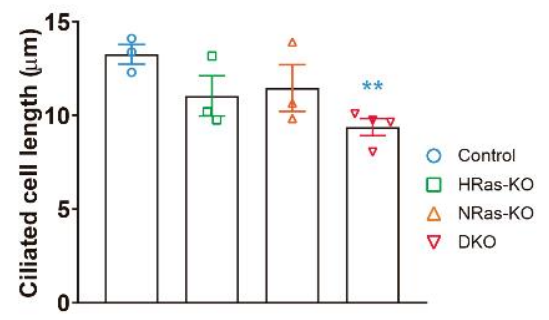

$\mathbf{E}$

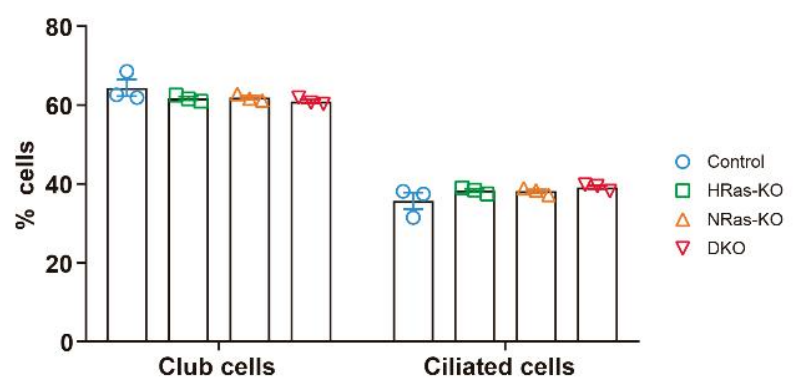

Figure 22: Immunostaining of bronchiolar differentiation markers in the lungs of HRas-KO and/or NRasKO mice. A. Representative images of PAS-stained lung sections from P0 mice of the indicated genotypes. Tailed arrows point to PAS+ accumulations located in the apical cytoplasmic region of bronchiolar Clara cells. Scale bar: $10 \mu \mathrm{m}$. B. Representative images of immunostaining for Secretoglobulin (Scgb1a1, Club cells, red) and $\beta$-Tubulin ( $\beta$-Tub, Ciliated cells, green), counterstained with DAPI (blue), in paraffin sections of bronchiolar regions of lungs from P0 mice of the indicated genotypes. Scale bar: $25 \mu \mathrm{m}$. C, D. Cell length measurements (from basal to apical membrane) of Club (panel C) and Ciliated (panel D) cells stained as in panel B. Data expressed as the mean \pm s.e.m. Ten separate microscopy fields were quantified for each individual analysed in each genotype. $n=4$ individuals for DKO and $\mathrm{n}=3$ for rest of genotypes. $* * \mathrm{p}<0.01$, *** $\mathrm{p}<0.001$. E. Bar graph quantification of Club 
and Ciliated cell percentage relative to total number of bronchiolar cells. Data expressed as the mean \pm s.e.m. Ten separate fields were quantified. $n=3$ individuals for all genotypes.

\subsubsection{Ablation of HRas leads to increased alveolar cell proliferation}

Lung proliferation is a well-balance process that take place during embryonic development, giving rise to new branching tubes that ultimately will form the distal sacs or alveoli. For this reason, during the last stages of embryonic lung development, proliferation rate diminishes, and, in contrast, cell differentiation takes place in the distal parts of the lung (Cardoso, 2008; Desai et al., 2014; Morrisey \& Hogan, 2010).

To analyse the state of cell proliferation in the lungs of E18.5 embryos of the four genotypes under study, we studied Bromodeoxyuridine (BrdU) incorporation by injection into pregnant females and immunostaining using a specific antibody against BrdU (see methods). We observed that the number of BrdU+ or proliferating cells was significantly higher in E18.5 lungs from HRas-KO and DKO mice than in CT and NRas-KO littermates (Fig. 23A, E).

Additionally, in an attempt to identify what cell types were proliferating, we performed multiple-immunostainings against BrdU, SftpC, and Scgb1a1, markers for proliferative, AECII and Club cells respectively. The lung bronchiolar epithelium, composed by Club cells, did not show many BrdU+ cells (Fig. 23B); however, the vast majority of these proliferative cells corresponded to SftpC+ alveolar cells (Fig. 23C).

These results are in concordance with the previous observations regarding delayed alveolar differentiation and SftpC+ cell cumuli in the alveolar zone, affecting HRas-KO (only at E18.5 stage) and DKO, with a possible key role of HRas in controlling these processes (Castellano et al., 2007, 2009).

\subsubsection{Increased apoptotic rates in PO lungs of NRas-ablated mice}

To compare cell death levels, apoptotic rate was evaluated in newborn lungs through Cleaved-caspase 3 (CC3) immunoassays, resulting in overall low rates in absolute terms, but the lungs of DKO showed statistically significantly higher levels of apoptosis, with a not statistically significant increment in NRas single-KO animals (Fig. 23D, F). These observations are in concordance with previous studies were NRas lack was linked to apoptotic pathways activation (Castellano et al., 2007, 2009). However, these apoptotic rates appear to be low, and it is difficult to assume that they may have any physiological relevance. 
A
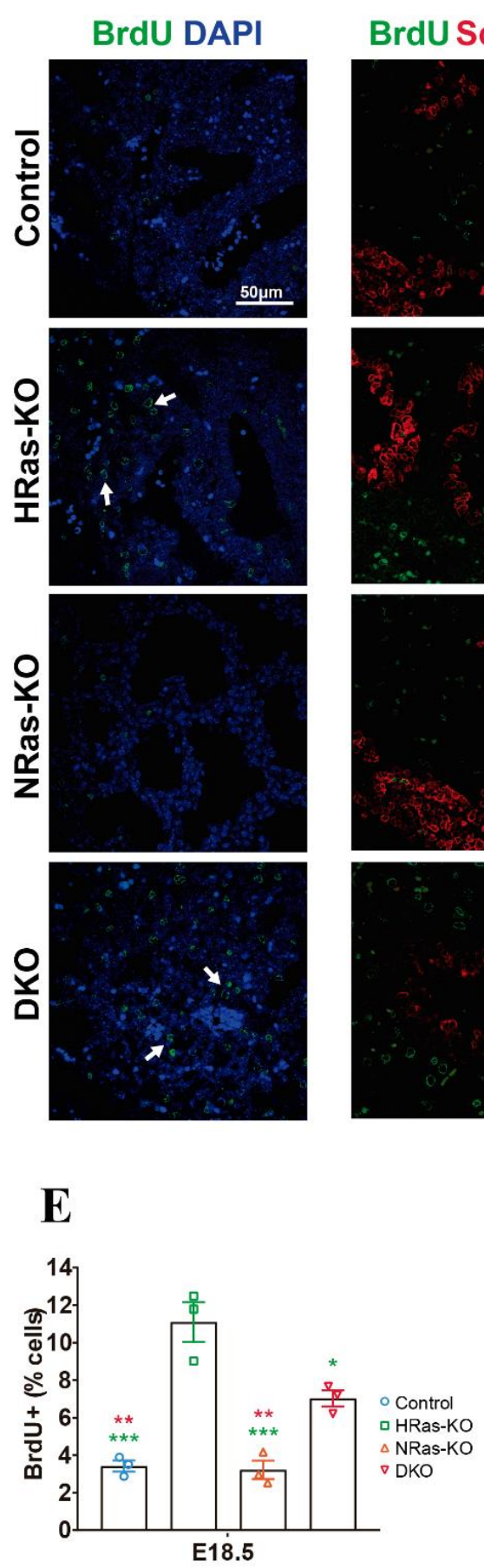

B
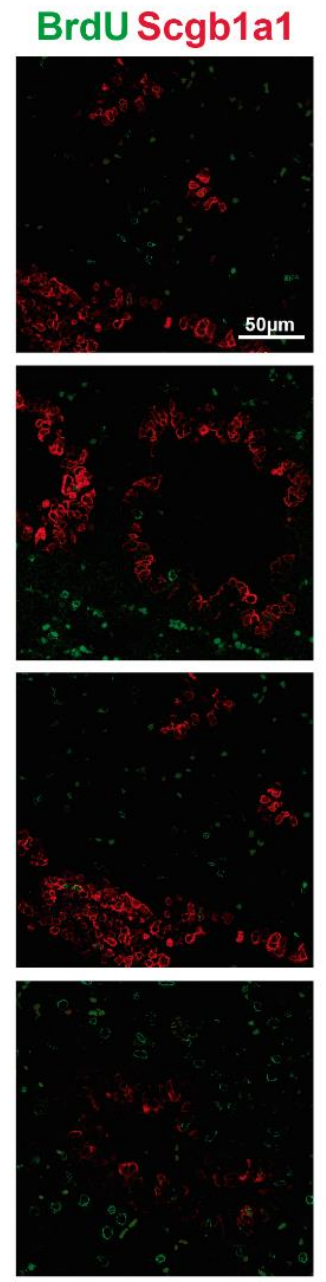

C
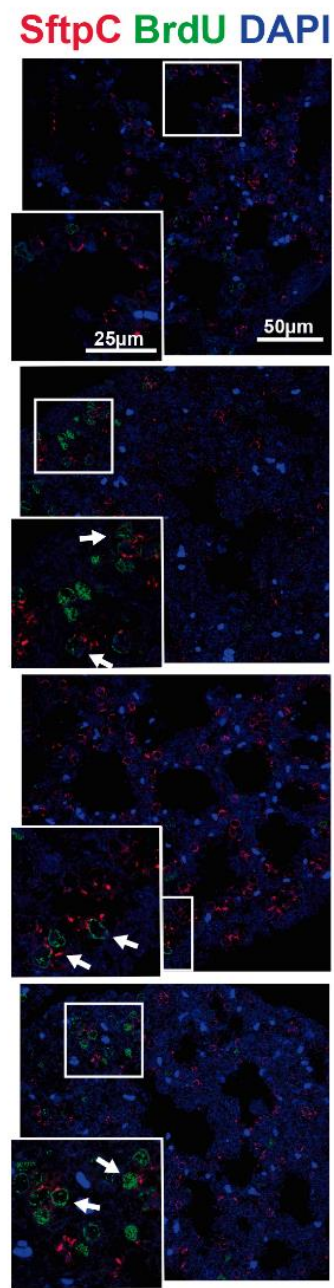

D
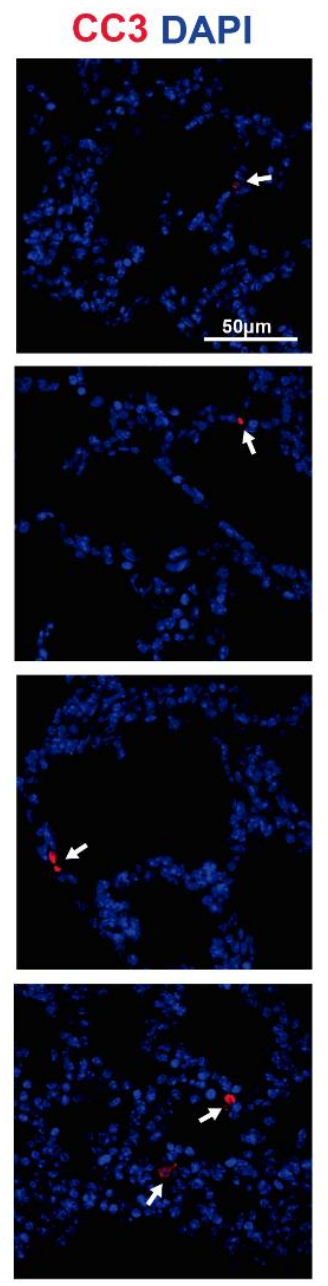

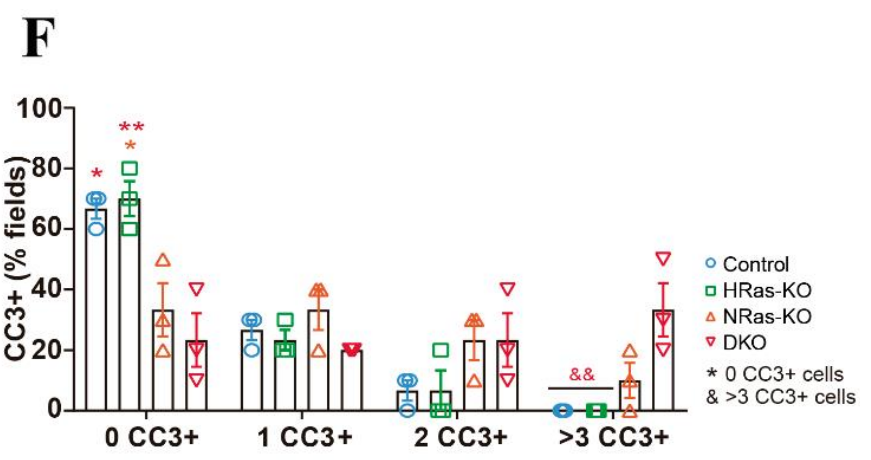

Figure 23: Analysis of proliferative and apoptotic rates in the lungs of HRas-KO and/or NRas-KO mice. A. Representative immunostaining images for Bromodeoxyuridine (BrdU, green) counterstained with DAPI (blue) in paraffin sections of E18.5 lungs of the indicated genotypes. Tailed arrows point to BrdU+ cells. Scale bar: $50 \mu \mathrm{m}$. B. Representative images of immunostainings for Secretoglobulin (Scgb1a1, red) and Bromodeoxyuridine (BrdU, green) in E18.5 paraffin sections. Scale bar: $50 \mu \mathrm{m}$. C. Representative images of immunostaining for Bromodeoxyuridine (BrdU, green) and SftpC (red), counterstained with DAPI (blue), in paraffin sections of E18.5 lungs of the indicated genotypes. Tailed arrows point to cells presenting double staining with BrdU and SftpC. Scale bar: $50 \mu \mathrm{m}$ and $25 \mu \mathrm{m}$ for magnified box areas. D. Representative images of immunostaining for Cleaved caspase-3 (CC3, red) and counterstaining with DAPI (blue) in paraffin sections of P0 lungs of the indicated genotypes. Scale bar: $50 \mu \mathrm{m}$. E. Bar graphs quantifying percentage of BrdU+ cells relative to total number of cells. Data expressed as mean \pm s.e.m. Ten separate microscopy fields were quantified for each individual analysed. $n=3$ individuals for all genotypes. $* \mathrm{p}<0.05, * * \mathrm{p}<0.01, * * * \mathrm{p}<0.001$. F. For quantitation of apoptotic $(\mathrm{CC} 3+)$ cells, the bar plots represent the percentage $(\%)$ of microscopy fields containing the specified number of apoptotic cells $(0$, 1,2 , or $>3$ per individual field) as indicated. Ten separate microscopy fields were quantified for each individual analysed. $n=3$ animals for all genotypes. For analysis of statistical significance, the $*$ and \& characters in the bar plot correspond, respectively, to comparisons between frequencies of samples of each genotype containing 0 
apoptotic cells per microscopy field, and frequencies of samples of each genotype containing $>3-4$ apoptotic cells per microscopic field. $* \mathrm{p}<0.05, * * \mathrm{p}<0.01, \& \& \mathrm{p}<0.01$.

\subsubsection{Increased neutrophil infiltration in HRas/NRas-double null animals}

A key hallmark of NRDS is the presence of infiltrating neutrophils, exhibiting a dual role: neutrophils participate in lung renewal after tissue damage through the release of metalloproteinases (MMP-9) and the activation of the Wnt//-Catenin pathway (Blázquez-Prieto et al., 2018; Zemans et al., 2011). However, on the other hand, activated neutrophils rapidly migrate to inflamed lung tissue, releasing its cytotoxic granular content and further boosting lung injury and contributing to the progression of the damage through the generation of oxidative stress (Yang et al., 2020).

To evaluate the levels of neutrophils infiltration in lung sections of newborn pups we performed immunoassays using the neutrophil elastase (NE) as a marker. Interestingly, only the

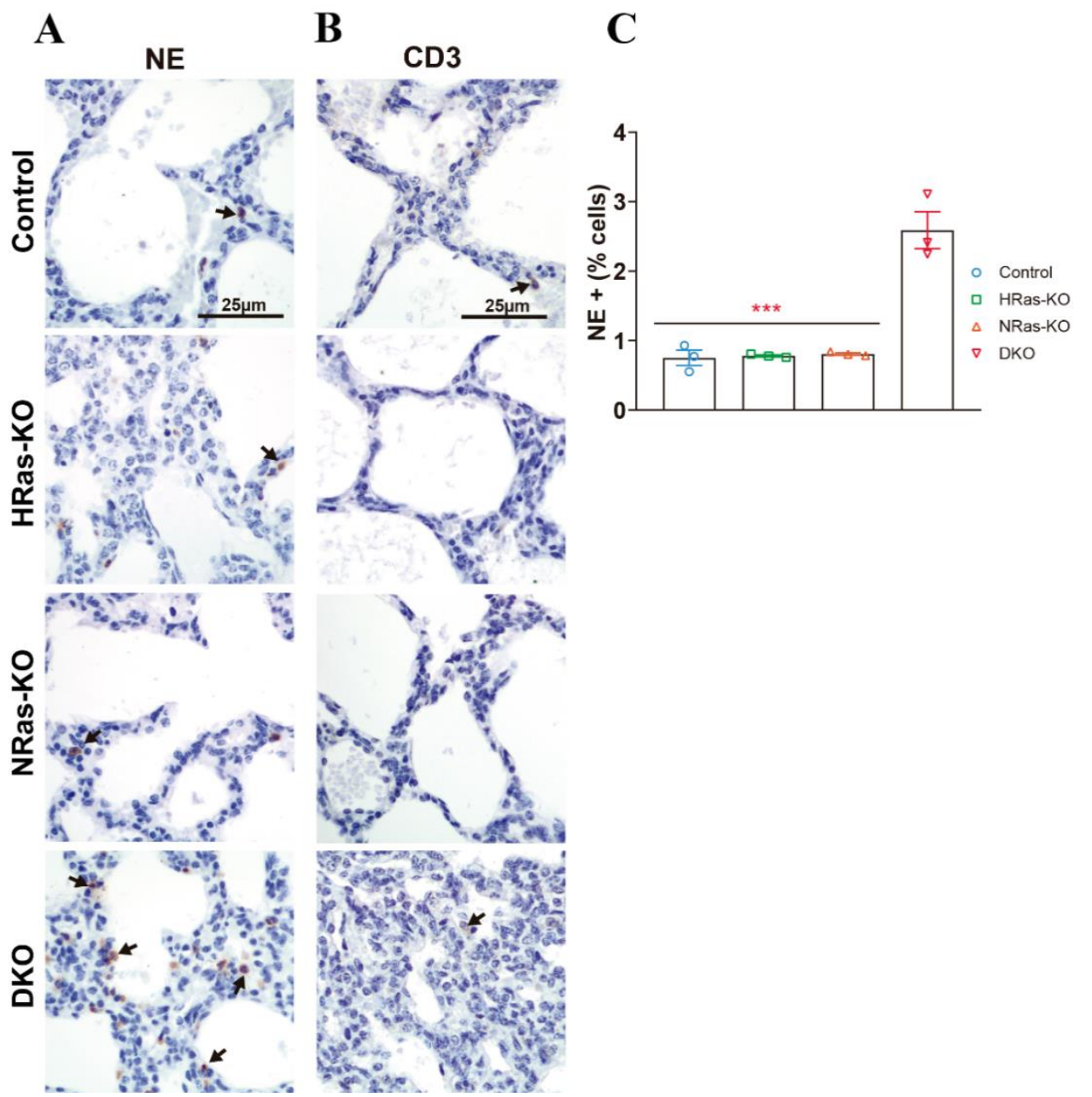

Figure 24: Increased neutrophil infiltration in the lungs of HRas/NRas-DKO mice. A. Representative images of immunostaining for neutrophil elastase (NE), counterstained with haematoxylin (blue) in lung paraffin sections of untreated neonates. Arrows point to NE+ cells. Scale bar: $25 \mu \mathrm{m}$. B. Representative images of immunostaining for lymphocytes (CD3), counterstained with haematoxylin (blue) in lung paraffin sections of untreated neonates. Arrows point to CD3+ cells. Scale bar: $25 \mu \mathrm{m}$. C. The bar graph quantitates percentage of NE+ cells relative to total cells. Mean \pm s.e.m. for each genotype. $n=3$ individuals for all genotypes. $* * * p<0.001$. 
DKO P0 pups presented significantly higher levels of infiltrating neutrophils in alveolar regions as compared to the other genotypes (Fig. 24A, C). Nevertheless, no differential cell counts for lymphocytes were detected since very low number of CD3+ lymphocytes were observed in all the genotypes (Fig. 24B).

3.1.8. HRas and NRas deficiency in PO mice causes strong transcriptional alterations in the lungs of P0 newborn mice

To determine the impact of HRas and NRas deficiency, alone and in combination, on global lung RNA expression, we performed a transcriptomic analysis using commercial microarrays to compare the transcriptional profiles of lung tissues from P0 neonate littermates of the four genotypes under study. Multiclass comparisons of microarray expression data profiles, generated with high stringency (False Discovery Rate, $(\mathrm{FDR})=0.1)$ produced a dendrogram that clearly discriminated all independent DKO samples from a separate group encompassing the rest of genotypes, indicating the existence of a distinct pattern of transcriptional alterations specifically linked to the disappearance of HRas and NRas in the lungs (Fig. 25A) (Data submitted to Gene Expression Omnibus, accession number GSE130415).

Most of the differentially expressed probesets in DKO lungs (around 75\%) were overexpressed, whereas only $25 \%$ were repressed, suggesting that transcriptional repression is the predominant consequence of HRas and NRas-driven signals in mouse lungs at this developmental stage (Fig. 25A).

The most significantly affected probesets included enrichment in component of various biological processes, molecular functions and signalling pathways that may be mechanistically linked with the phenotypic alterations observed in newborn DKO lungs. More specifically, the upregulated genes annotated in DKO samples showed a highly statistical enrichment in distinct Gene Ontology (GO) functional categories, including “Transport and Metabolic Processes” or "Hydrolase activity" (Fig. 25B). On one hand, 34 overexpressed genes in DKO lungs were related with different subcategories of transport, as "Protein transport", "Vesicle-mediated transport", "Intracellular transport", "Ion transport", "Proton transport" or "Transmembrane transport". On the other hand, a separate group of 21 upregulated genes was specifically concerned with various "Protein metabolic processes" like proteolysis, ubiquitination and dephosphorylation, as well as "Carbohydrate and Glycogen Metabolism" (Fig. 25B). Additionally, consistent with the GO annotations, the list of upregulated genes in DKO lungs was significantly enriched with components of different Kyoto Encyclopaedia of Genes and Genomes (KEGG) signalling pathways, including "Oxidative phosphorylation", "N-glycan biosynthesis" and "Sphingolipid metabolism", processes functionally significant for lung homeostasis (Fig. 25B). 
A

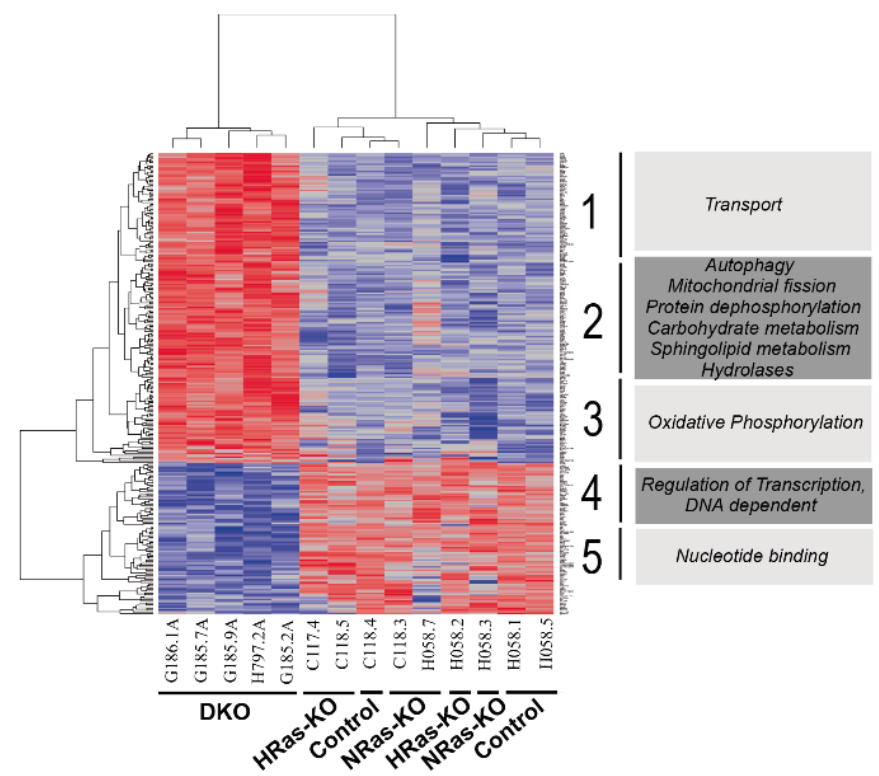

B

GO Biological Process \& Molecular Function

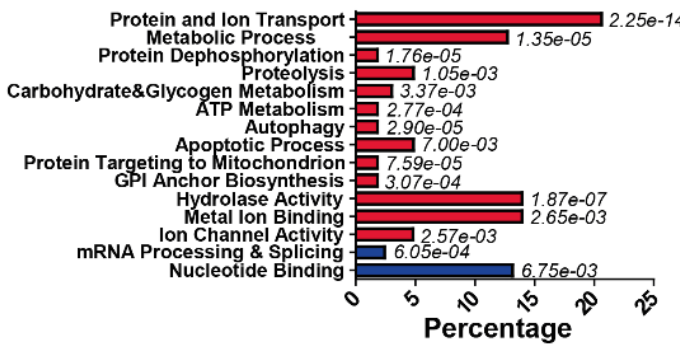

Signaling Pathways (KEGG)

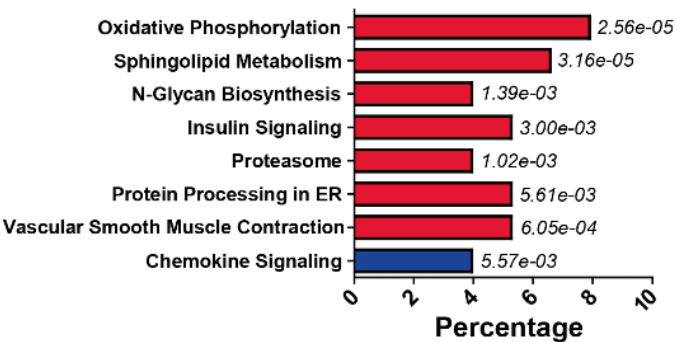

Figure 25: Differential gene expression in the lungs of HRas/NRas-DKO newborn P0 mice. A. A set of 14 independent chip microarray hybridizations were performed using RNA extracted from the lungs of at least 3 independent, newborn P0 mice belonging to each of the 4 indicated genotypes and analysed jointly as described in Materials \& Methods. The heatmap depicts the results of hierarchical clustering and multiclass comparisons of 265 gene probesets that showed differential expression $(\mathrm{FDR}=0.1)$ in the lungs of DKO mice as compared to the rest of genotypes. Labels on the right side of the dendrogram identify specific functional categories that are enriched at high statistical significance within the indicated, individual horizontal clusters (blocks 1-5). B. Each individual bar in the horizontal bar plots represents the percentage of the total number of differentially expressed, overexpressed (red bars) or repressed (blue bars), gene probesets corresponding to specific groups of genes of the dendrogram that were identified by GeneCodis as significantly enriched (hypergeometric p-values in italics) for the indicated GO and KEGG functional categories.

\subsubsection{HRas and NRas deficiency alters lung sphingolipid metabolism and increases ceramide}

levels

The significant upregulation of genes related to "Sphingolipid metabolism" or "GPI anchor biosynthesis" were relevant for the defective DKO lung phenotypes in view of the critical roles that ceramides and surfactants play in multiple physiological and pathological lung processes (Barnes, 2004; Göggell et al., 2004; Petrache \& Berdyshev, 2016; Pettus et al., 2002; Sparkman et al., 2006; van Mastrigt et al., 2018). Of note, our transcriptomic analyses uncover significant overexpression in DKO lungs of an important number of loci involved in sphingolipid metabolic pathways controlling the levels of ceramide, such as Sptlc1 (Serine palmitoyltransferase, long 
chain base subunit 1), Cers5 (Ceramide synthase 5), Degs1 (Delta (4)-desaturase sphingolipid 1), Sgppl (Sphingosine-1-phosphate phosphatase 1) or Acer3 (Alkaline ceramidase 3) (Fig. 27A).

Consistent with the transcriptomic alterations, our western blot analyses of whole P0 lung homogenates also showed a higher expression of some of the named enzymes (Fig. 26). Additionally, through immunohistochemical studies of the lungs of neonate mice we detected significantly elevated levels of ceramide in alveolar and bronchiolar regions of DKO animals as compared to all three other genotypes (Fig. 27B, C), thus showing that the transcriptomic changes observed in this pathway are translated into physiological alterations in vivo.
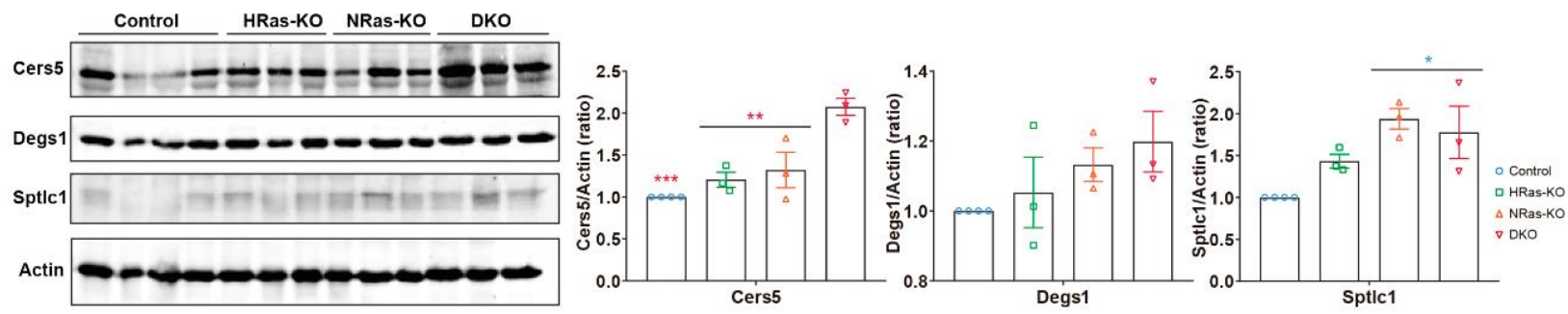

Figure 26: Increased expression of enzymes implicated in ceramide synthesis in DKO lungs. A. Representative western blot assays of total lung lysates protein extracts showing the expression of Ceramide synthase 5 (Cers5), Delta 4-desaturase sphingolipid 1 (Degs1), and Serine palmitoyltransferase, long chain base subunit 1 (Sptlc1). Actin was used as loading control. Expression levels quantitated as fold change relative to expression in CT samples. Data presented as mean \pm s.e.m. $\mathrm{n}=4$ for Controls and $\mathrm{n}=3$ for the rest of genotypes. ${ }^{*} \mathrm{p}<0.05, * * \mathrm{p}<0.01, * * * \mathrm{p}<0.001$.

\subsubsection{Metabolic alterations linked to HRas and NRas ablation}

A mechanistic link was readily apparent between some transcriptional alterations and the phenotypic defects observed in our HRas/NRas double-null animals. The increased apoptotic levels detected in P0 lungs (Fig. 23D, F) were accompanied by overexpression of various loci coding for regulatory components of "Apoptotic and Autophagy processes". Furthermore, the significant increase in alveolar glycogen deposits detected through PAS+ elements (Fig. 20B) or the morphological flattening of secretory Club cells (Fig. 22A) correlated with transcriptional upregulation of various loci involved in regulation of "Carbohydrate and glycogen metabolism" as well as "Intracellular vesicle-mediated transport". Interestingly, the severe respiratory distress exhibited by P0 neonates was paralleled by transcriptional upregulation of loci involved in "Oxidative phosphorylation", "ATP metabolism" or "Protein targeting to mitochondria", suggesting that redox signalling may be affected in the lungs of mice lacking HRas and NRas GTPases. 
A

De novo synthesis pathway

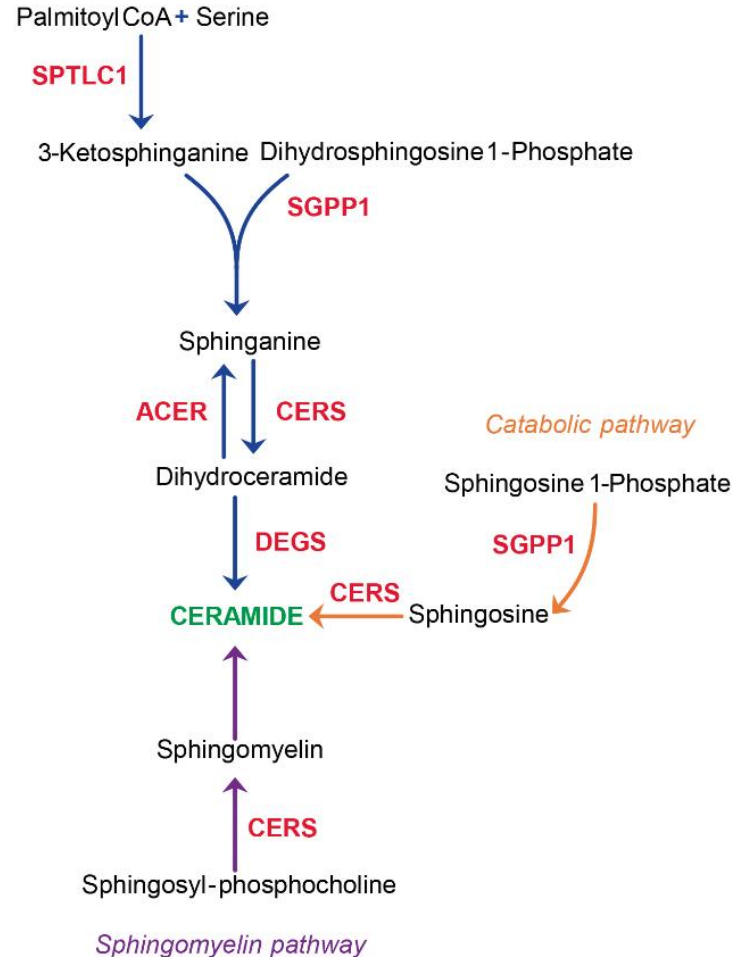

Sphingomyelin pathway

$\mathbf{C}$

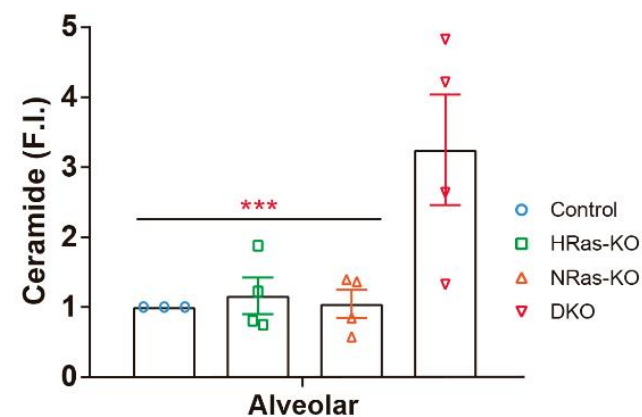

B
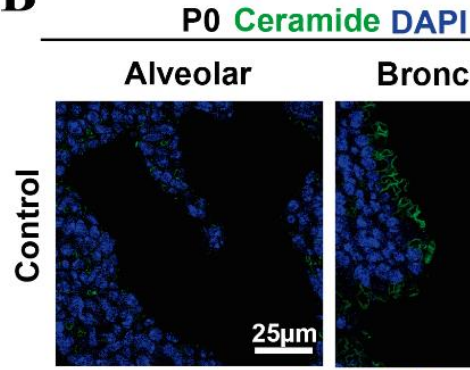

\section{Bronchiolar}
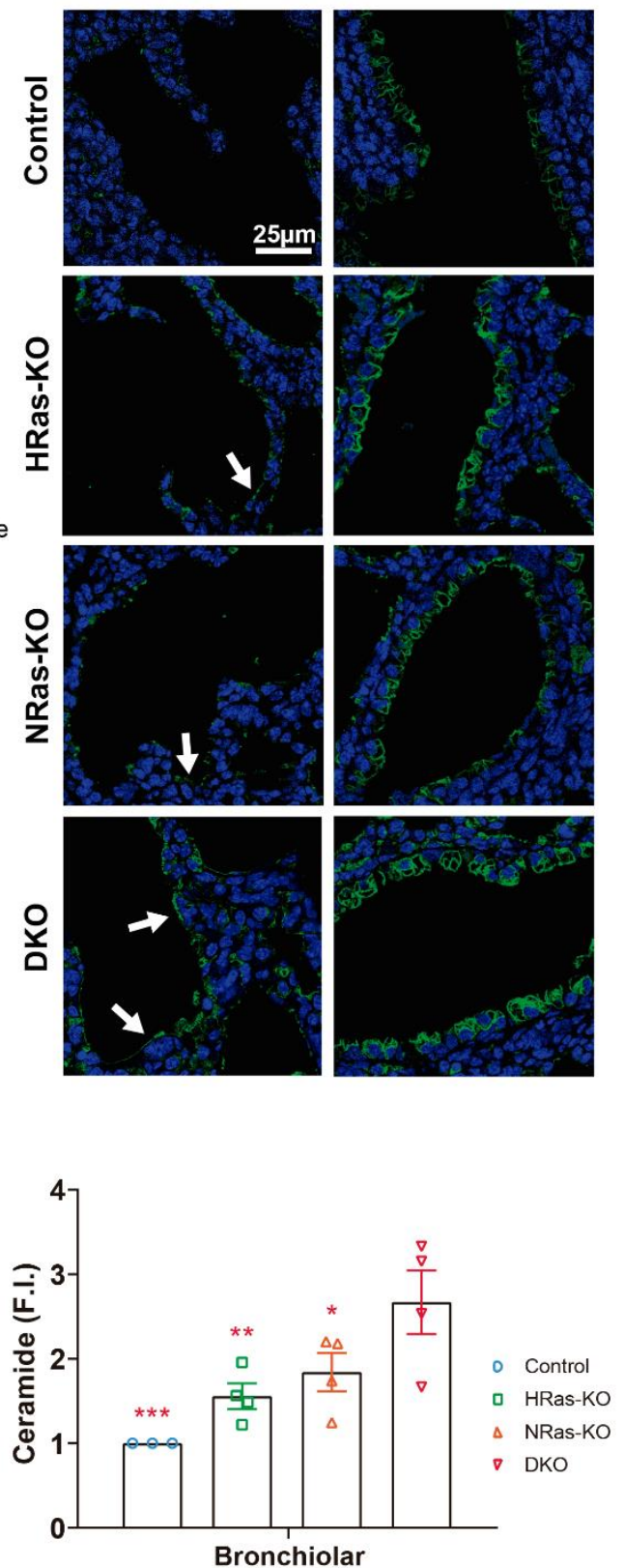

Figure 27: Ceramide immunoassays in alveolar and bronchiolar regions of the lungs of HRas and/or NRas KO mice. A. The enzymatic components of sphingolipid metabolism pathways that showed differential expression in our transcriptomic analyses of P0 lungs extracts are marked in red (genes overexpressed in DKO lungs). Ceramide is indicated in green. Enzymatic reactions driving either de novo synthesis (Blue arrows), catabolic pathway (orange arrows) and sphingomyelin pathway (purple arrows) lead to ceramide accumulation in the DKO lungs. SGPP1- Sphinganine phosphate phosphatase 1, SPTLC1- Serine Palmitoyltransferase Long Chain Base Subunit 1, CERS- Ceramide Synthase, ACER- Alkaline Ceramidase, DEGS- Delta 4-Desaturase, Sphingolipid 1. B. Representative images of immunostaining for Ceramide (green) and DAPI counterstaining (blue) in lung paraffin sections of newborn P0 mice of the indicated genotypes. Alveolar and bronchiolar areas are shown, respectively, in each column of this panel. Arrows point to zones with significantly increased ceramide levels observed in alveolar regions of the indicated genotypes. Scale bar: $25 \mu \mathrm{m}$. C, D. The bar plots represent the average values of ceramide fluorescence intensity (F.I.) in alveoli (C) and bronchiole (D) relative to Control Data expressed as the mean \pm s.e.m. Ten separate microscopy fields were quantified for each individual analysed. $n=3$ individuals for Controls and $\mathrm{n}=4$ individuals for the rest of genotypes. $* \mathrm{p}<0.05, * * \mathrm{p}<0.01, * * * \mathrm{p}<0.01$. 
3.1.11. Surviving HRas/NRas-DKO mice display facial dysmorphia and a patched lung phenotype

The reduced number of DKO mice surviving up to adulthood were analysed to determine if there were some phenotypic differences with the control littermates. In concordance with the previous observations in $\mathrm{P} 0$ mice, adult DKO mice were smaller in size as compared with the CT littermates (Fig. 28A). Additionally, in contrast to the CT mice, 6 months old DKO animals showed a marked defect in spinal curvature (Fig. 28A). Along with these spinal defects, we also detected significant facial dysmorphia in all adult DKO animals (Fig. 28A \& Fig. 29), and ocular defects (anophthalmia, microphthalmia and/or blindness).

A
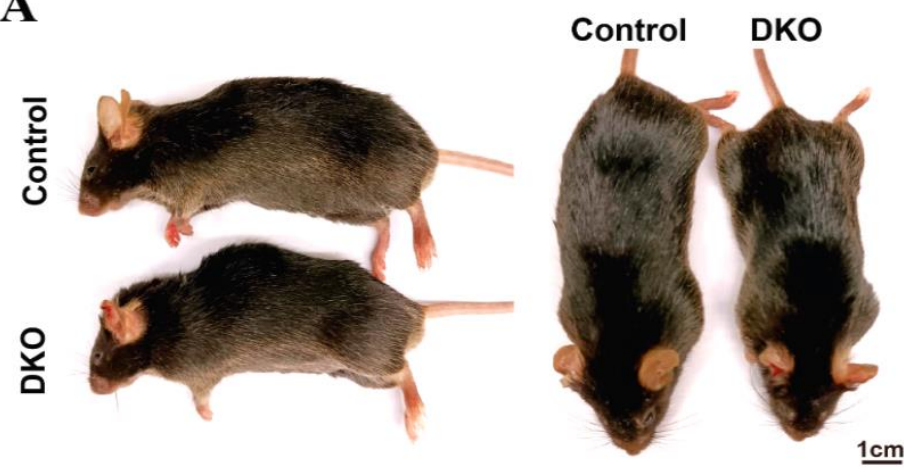

B

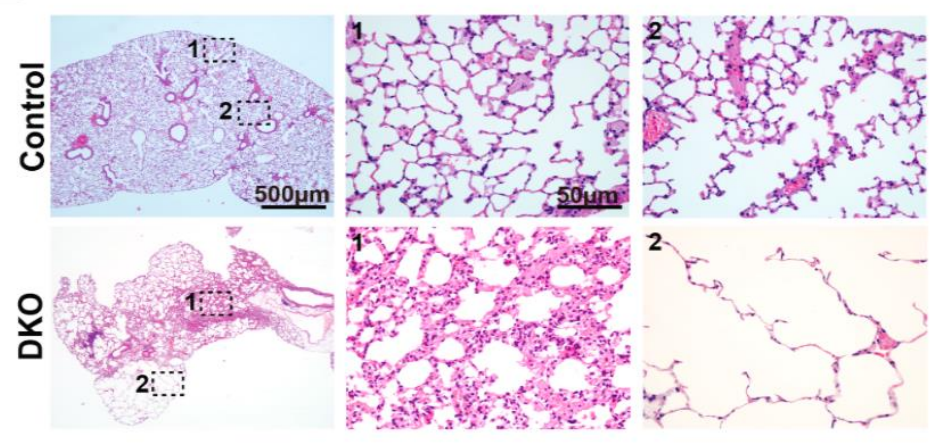

C
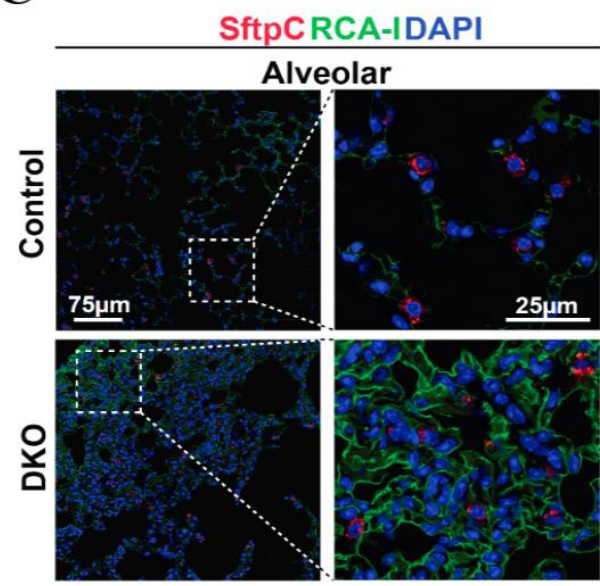

D
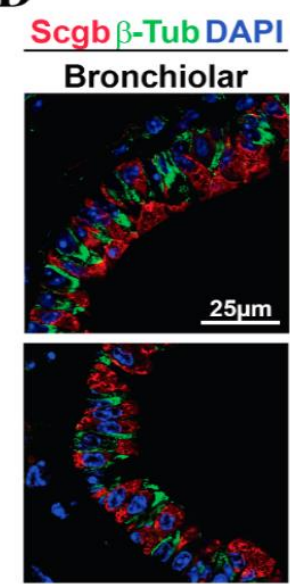

Figure 28: Adult HRas/NRas-DKO mice show partial lung atelectasis and facial dysmorphia. A. Typical body and facial morphology of CT and DKO 6 months old littermates male mice. B. Representative images of 
H\&E staining of lung sections from one year-old mice of the indicated genotypes (Control and DKO). Scale bar: $500 \mu \mathrm{m}$ for the pictures in the first column and $50 \mu \mathrm{m}$ for the magnifications in the second and third columns. $\mathrm{n}=4$ individuals for DKO and $\mathrm{n}=3$ for Control. C. Representative images of immunostaining for SftpC (red) and RCAI (green), counterstained with DAPI, in alveolar areas of lung paraffin sections from adult mice of the indicated genotype. Scale bars: $75 \mu \mathrm{m}$, and $25 \mu \mathrm{m}$ on the higher magnification of boxed areas. $\mathrm{n}=4$ individuals for DKO and $\mathrm{n}=3$ for Control. D. Representative images of immunostaining for Scgb1a1 (red) and $\beta$-Tubulin (green) counterstained with DAPI, in bronchiolar areas of lung paraffin sections from adult mice of the indicated genotypes. Scale bar: $25 \mu \mathrm{m}$. $\mathrm{n}=4$ individuals for $\mathrm{DKO}$ and $\mathrm{n}=3$ for Control.
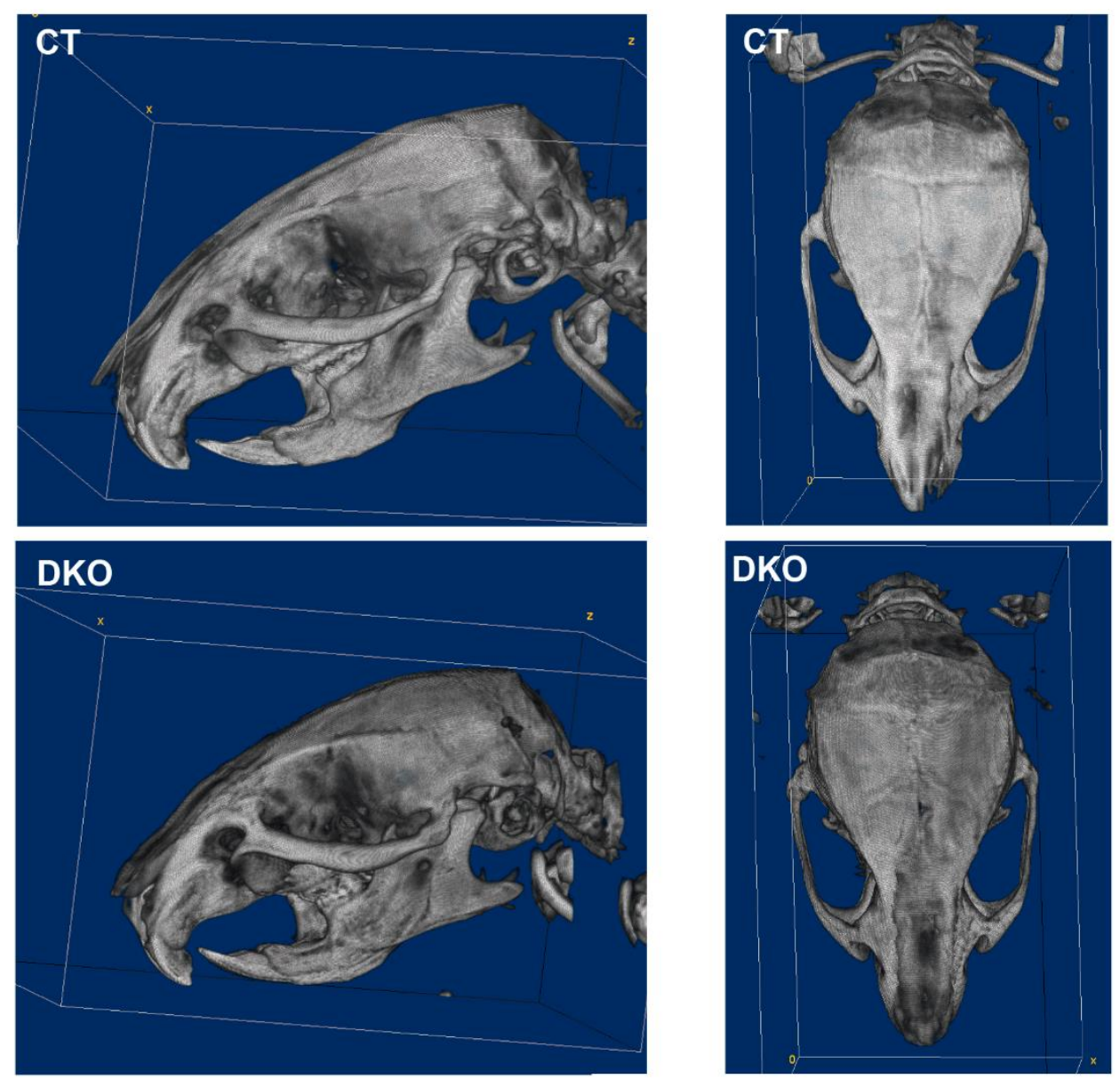

Figure 29: Craniomorphometric analyses of the skulls of adult CT and HRas/NRas-DKO mice. A. 3D reconstructions of Micro-CT scans showing the lateral (left) and dorsal (right) views of CT and DKO 6 months old littermates male mice skulls.

Interestingly, the lungs of the surviving DKO mice displayed patches of atelectasis (condensed alveolar areas) and alveolar emphysemas, probably developed as a compensatory effect of the atelectasis (Fig. 28B). Consistently with the observations in H\&E sections, lack of normal inflated saccular areas was found next to normal lung regions (Fig. 28C). However, contrary to the bronchiolar phenotypes observed in the P0 DKO mice, no alterations were detected in the structure of Club or Ciliated cells (Fig. 28D). 
3.2. Altered lung redox homeostasis and mitochondrial respiration in mice lacking HRas and

NRas

3.2.1. HRas/NRas double-KO mouse lungs exhibit an increase in reactive oxygen species production

To gain some insights about the transcriptional alterations observed related to redox homeostasis and mitochondrial function (including an enrichment in enzymes implicated in the reactive oxygen species (ROS) detoxifying pathways, alterations in proteins of the different electron transport chain (ECT) complexes, ATP metabolism and protein targeting to the mitochondria), we sought to study the levels of ROS present in whole lung tissue homogenates of newborn mice (Fig. 30A).

Interestingly, whole lungs of DKO animals showed a significant increment in intracellular hydrogen peroxide $\left(\mathrm{H}_{2} \mathrm{O}_{2}\right)$ and total oxygen superoxide $\left(\mathrm{O}_{2}^{-}\right)$in comparison with $\mathrm{CT}$, HRas-KO and NRas-KO littermates, whereas no significant changes were observed between the CT or simple-KO lungs (Fig. 30B). Additionally, a slight, albeit significant, increment in mitochondrial $\mathrm{O}_{2}^{-}$was detected in the NRas-KO and DKO lungs compared with CT and HRasKO (Fig. 30B).

A

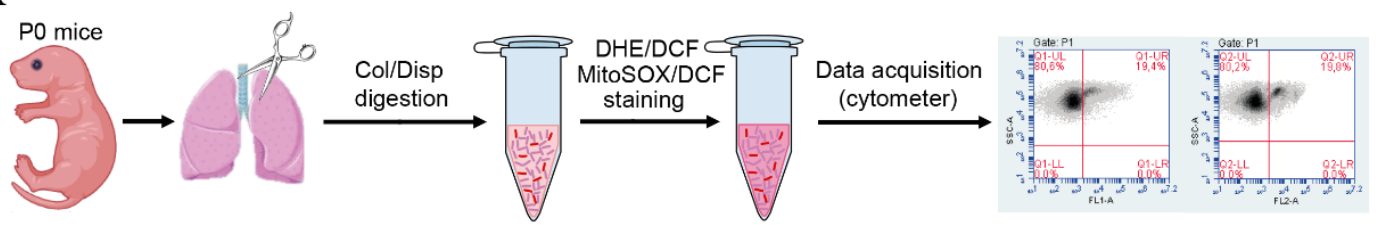

B
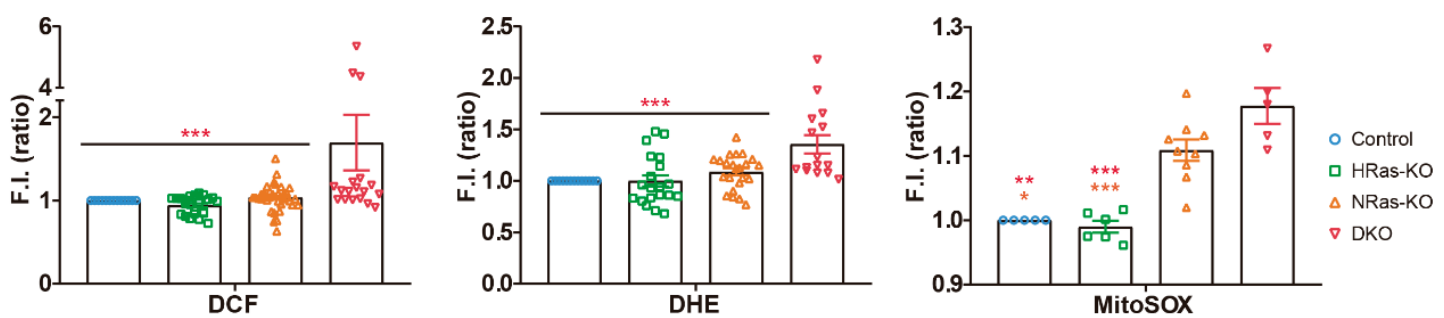

Figure 30: Lungs of PO HRas/NRas-DKO mice show an increment in reactive oxygen species production. A. Scheme representing the steps for reactive oxygen species (ROS) labelling in whole lung lysates. B. The bar plots represent the in vivo quantitation, relative to Control, of redox parameters carried out by means of FACS fluorescence measurements, performed (30.000 events in each case) on fresh whole lung lysates using specific fluorophores for intracellular hydrogen peroxide (DCF, $5 \mu \mathrm{M}$ ), intracellular total superoxide (DHE, $5 \mu \mathrm{M})$ and mitochondrial superoxide (MitoSOX ${ }^{\mathrm{TM}}, 5 \mu \mathrm{M}$ ) as described in Materials and Methods. Data expressed as the mean \pm s.e.m. DCF: CT $n=13$, HRas-KO $n=25$, NRas-KO n=35, DKO $n=18$; DHE: CT $n=13$, HRas-KO $n=20$, NRasKO $n=23$, DKO $n=15$; MitoSOX ${ }^{\mathrm{TM}} \mathrm{CT} \mathrm{n}=5$, HRas-KO $\mathrm{n}=6$, NRas-KO $\mathrm{n}=9$, DKO $\mathrm{n}=5$. * $\mathrm{p}<0.05$, ** $<<0.01$, $* * * \mathrm{p}<0.001$. 


\subsubsection{The lungs of HRas/NRas-DKO mice show altered mitochondrial respiratory parameters} and a decrease in ATP production

To ascertain whether the higher ROS production in the lungs of HRas/NRas-double null mice was also reflected in related mitochondrial functional alterations, we analysed the respiratory profile of CT, HRas-KO, NRas-KO and DKO newborn lung tissue using Seahorse-based technology (Fig. 31A). OCR measurements detected reduced rate of basal respiration (a parameter indicating the energetic demand of the cells under baseline conditions) in DKO lungs, albeit they were not significative ( $\mathrm{p}=0.056$ vs $\mathrm{CT}, \mathrm{p}=0.06$ vs NRas-KO) (Fig. 31B, C). Furthermore, spare respiratory capacity (measurement showing how closely the cells are respiring in relation to its theoretical maximum) OCR levels of DKO lungs were significantly higher when compared with $\mathrm{CT}$ and HRas-KO lungs, meaning that the electron transport chain of DKO lung tissue is working at higher levels (Fig. 31B). However, the ATP production of the DKO lungs was significantly reduced as compared with CT and NRas-KO littermates (Fig. 31B).

A

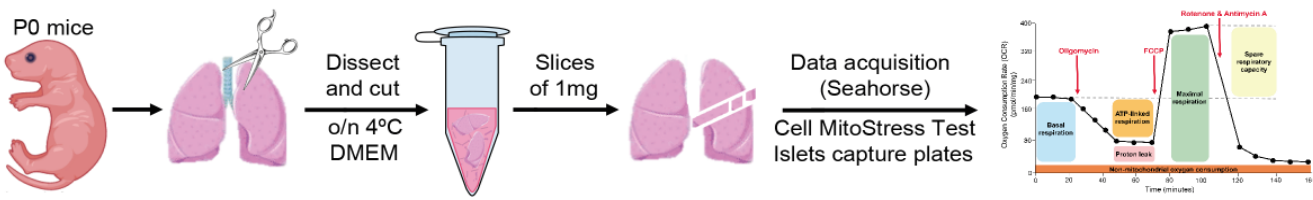

B

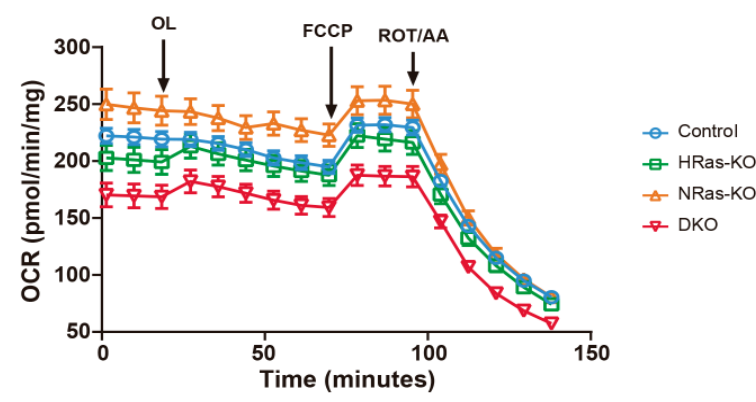

C
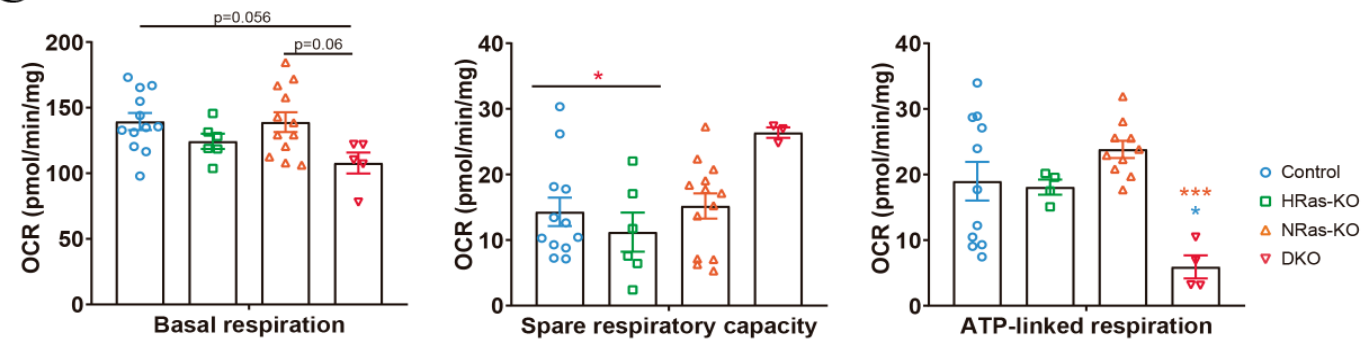

Figure 31: Lungs of P0 HRas/NRas-DKO mice show lower basal respiration and ATP-linked respiration. A. Scheme representing the steps for Seahorse XF MitoStress Test, performed on fresh lung slices (1mg/well) as described in Materials and Methods. B. MitoStress profile from P0 mice of the indicated genotypes. OCR (oxygen consumption rate) was measured under basal conditions followed by the sequential addition of $15 \mu \mathrm{M}$ Oligomycin (OL), $16 \mu \mathrm{M}$ FCCP, and $3 \mu \mathrm{M}$ Rotenone and $12 \mu \mathrm{M}$ Antimycin A (ROT/AA) following manufacturer's instruction. C. Quantitation of parameters for basal respiration, spare respiratory capacity and ATP-linked respiration. Data presented are the mean \pm s.e.m. using at least five technical replicates per experiment per genotype. Basal respiration: $\mathrm{CT} n=12$, HRas-KO n=6, NRas-KO n=12, DKO n=5; Spare respiratory capacity: $\mathrm{CT} n=12$, HRas-KO 
$\mathrm{n}=6$, NRas-KO n=14, DKO n=4; ATP-linked respiration: CT n=11, HRas-KO n=4, NRas-KO n=10, DKO n=4. * $\mathrm{p}<0.05, * * * \mathrm{p}<0.001$.

3.3. Characterization of the effect of antenatal glucocorticoid or $\mathrm{N}$-acetylcysteine administration on lung development and postnatal survival of HRas/NRas-double null mice

\subsubsection{Antenatal glucocorticoid administration extends the lifespan of DKO pups up to six days}

To test the possibility of reversing the alveolar maturation delay observed in the lungs of E18.5 and newborn DKO mice by means of antenatal administration of glucocorticoids (Brownfoot et al., 2013; Roberts et al., 2017), we gave two subcutaneous injections of dexamethasone (DEX) to pregnant female mice on consecutive days E17.5 and E18.5 of embryonic gestation (Full detailed protocol in 3.4 section, materials and methods). E18.5 embryos who received one dose of DEX at E17.5, and newborn pups (P0) received two doses of DEX, were subsequently examined.

Interestingly, antenatal DEX treatment extended the lifespan of DKO mice, whereas most untreated DKOs died within the two first postnatal days, DEX-treated pups exhibited a delay of 4-5 days in the time of death (Fig. 32A, B). However, as with untreated DKO animals, DEXtreated DKO pups still showed a significantly lower body weight and size in comparison with CT, HRas-KO and NRas-KO untreated or treated with DEX (Fig. 32C, D).

\subsubsection{Treatment with $N$-Acetylcysteine ameliorates the neonatal lethality of HRas/NRas-DKO mice}

Since newborn DKO pups exhibited higher levels of ROS, and knowing the existing oral antioxidant/mucolytic therapy to treat premature infants (Shi et al., 2020; Xiang \& Wang, 2019), we sought to analyse the effect of the oral administration of N-Acetylcysteine (NAC) on the neonatal survival and lung maturation of the DKO pups. NAC is a thiol compound, which provides sulfhydryl groups, and acts as a mucolytic agent by hydrolysing the disulfide bonds within mucin, breaking down its oligomers and making the mucin less viscous. On the other hand, NAC acts as a general antioxidant scavenger by itself and through the participation in the synthesis of the antioxidant glutathione, a known the precursor of reduced glutathione. In this way, NAC can interfere with several signalling pathways controlling redox, inflammatory response, apoptosis, as well as enhancing overall lung function due to its role as antioxidant and mucolytic (Pei et al., 2018; Zafarullah et al., 2003).

To perform this treatment, we administered NAC in the breeding bottle ad libitum throughout the pregnancy (Full detailed protocol is described in materials and methods, section 3.5), and 
evaluated the resulting litters. Approximately around 53\% of the NAC-treated DKO born animals survived up to adulthood (Fig. 32A, B) but, however, they still exhibited a reduced body size and weight at birth (Fig. 32C, D), albeit some of the NAC-treated DKO P0 pups were indistinguishable from the rest of genotypes under study.

A

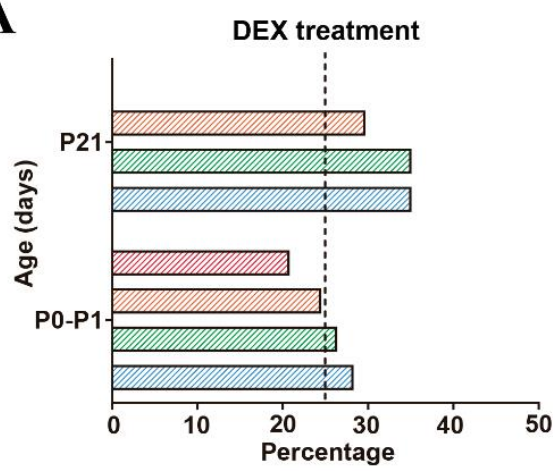

B

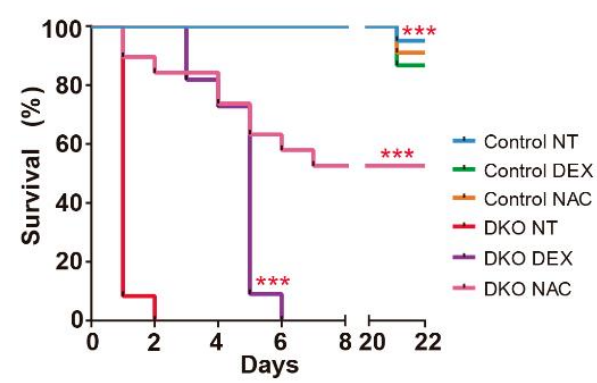

D

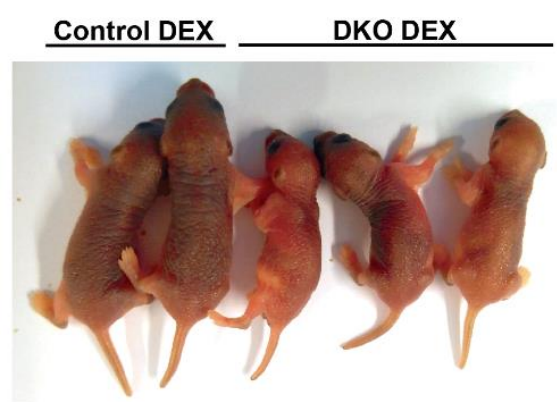

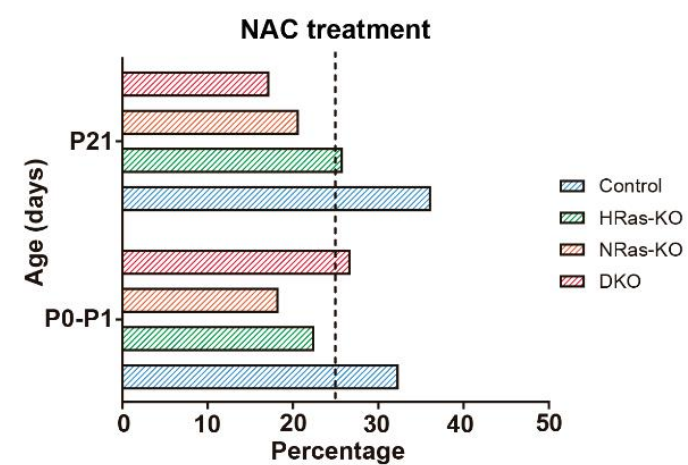

C

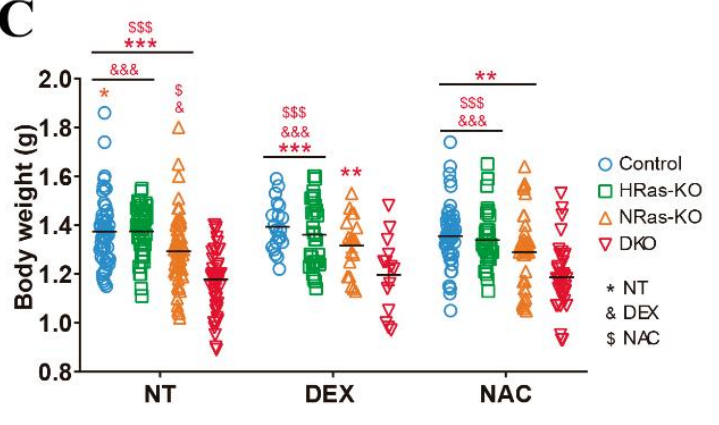

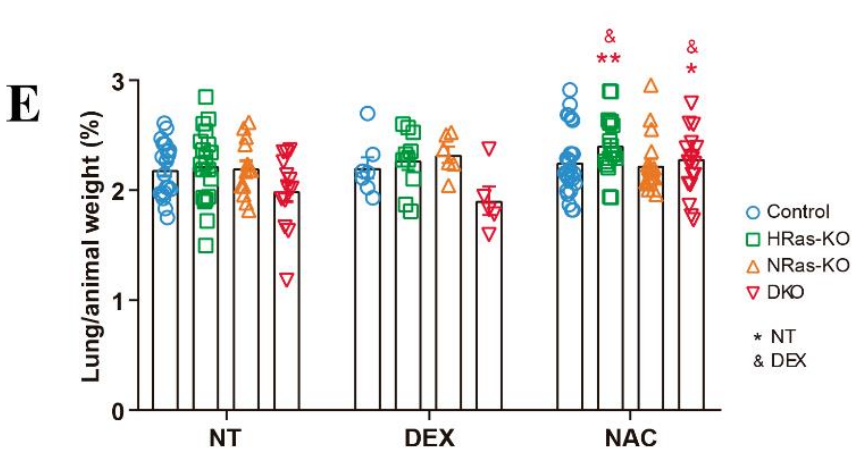

Figure 32: Effect of dexamethasone and antioxidant antenatal therapy on survival of DKO mice. A. Bar graphs depicting percentage and absolute numbers (in italics) of individuals of the indicated genotypes (Control, HRas-KO, NRas-KO, DKO) counted at time of birth (P0) or at weaning time (P21) in the litters resulting from parental crosses between $\mathrm{HRas}^{+-}$; $\mathrm{NRas}^{--}$and vice versa, from dexamethasone-treated (DEX) and $\mathrm{N}$-acetylcysteinetreated (NAC, right). B. Kaplan-Meier plot comparing the survival rates of untreated (NT), DEX and NAC-treated Control and DKO littermates. NT: CT $n=20$, DKO n=12; DEX: CT $n=14$, DKO n=11; NAC: CT $n=23$, DKO $\mathrm{n}=19$. $* * * \mathrm{p}<0.001$ for comparison between $v s$ DKO NT mice. C. Body weight distribution of living, newborn P0 
mice of the indicated genotypes and treatments at time of birth. Data represented as the mean \pm s.e.m. for each genotype. NT: CT $n=59$, HRas-KO n=39, NRas-KO n=52, DKO n=45; DEX: CT $n=21$, HRas-KO $n=31$, NRasKO $n=19$, DKO $n=15$; NAC: CT $n=50$, HRas-KO $n=38$, NRas-KO $n=43$, DKO $n=39$. *vs NT, \&vs DEX, \$vs NAC, $* \mathrm{p}<0.05, * * / \& \& \$ \$ \mathrm{p}<0.01, * * * / \& \& \& \$ \$ \$ \mathrm{p}<0.001$. D. Representative pictures of DEX-treated (left) and NACtreated (right) of Control and DKO P4 littermates. E. Graph bar quantitation of individual lung weight relative to animal body weight of newborn mice of the different genotypes and treatments. Data represented as the mean \pm s.e.m. for each genotype. NT: CT $n=21$, HRas-KO n=21, NRas-KO n=14, DKO n=14; DEX: CT n=7, HRas-KO $\mathrm{n}=10$, NRas-KO n=6, DKO n=5; NAC: CT $\mathrm{n}=27$, HRas-KO $\mathrm{n}=20$, NRas-KO n=18, DKO $\mathrm{n}=18$ *vs NT, \&vs DEX, $* \mathrm{p}<0.05, * * / \& \& \mathrm{p}<0.01$

\subsubsection{Recovery of normal lung differentiation after DEX and NAC antenatal treatments}

The lung weight/body weight ratios in the DEX treated P0 pups were similar to those of the untreated animals; but both HRas-KO and DKO lungs treated with NAC displayed significant higher lung/body weight ratio as compared with the NT and DEX-treated DKO lungs (Fig. 32E).

Histological analyses of HRas-KO and DKO E18.5 embryos as well as DKO P0 pups treated antenatally with either DEX or NAC showed normal alveoli architecture and a reduced thickness of alveoli separating septa (being more evident in the P0 DEX-treated animals in comparison with the P0 NAC-treated) when compared with untreated mice, producing a complete reversion to normal values (Fig. 33A, B). Additionally, no differences related to lung structure were observed among CT, HRas-KO, NRas-KO or DKO animals treated with either DEX or NAC, being completely indistinguishable (Fig. 33A, B). Notably, comparison between E18.5 untreated HRas-KO and DKO lungs and DEX and NAC-treated samples showed that, both, a single antenatal DEX injection (at E17.5) and antenatal NAC administration were enough to correct the altered saccular architecture and reduced alveolar area (Fig. 33A).

To get some insights of the alveolar differentiation status we performed PAS staining on DEX and NAC-treated E18.5 lungs. As expected, treatment with glucocorticoids significantly decreased the PAS+ glycogen deposits of alveolar areas in DKO mice treated with DEX as compared with the untreated DKO (Fig. 34). On the other hand, treatment with NAC resulted in overall slight increase in PAS+ glycogen structures, being more evident in NRas-KO and DKO NAC-treated samples when compared with the untreated CT (Fig. 34). However, even though the reduction was not as strong as the observed with DEX treatment, DKO NAC-treated lungs showed statistically fewer alveolar glycogen deposits than the untreated DKO ones (Fig. 34).

Additionally, to validate the assumption of normal alveolar development after treating the animals with DEX or NAC, alveolar bi-potent cells were analysed by means of double positive RCA-I+/SftpC+ cells and SOX9+ cells in lungs of E18.5 embryos. Consistently with the previous observations of $\mathrm{H} \& \mathrm{E}$-stained sections, $\mathrm{RCA}-\mathrm{I}+/ \mathrm{SftpC}+$ bi-potent cells were practically absent (Fig. 35), followed by a significant reduction of SOX9+ distal-tip progenitors after both 
treatments (Fig. 36), further confirming that, both, a single dose of DEX or NAC administration were able to rescue the defective differentiation of pneumocytes in the DKO.

A
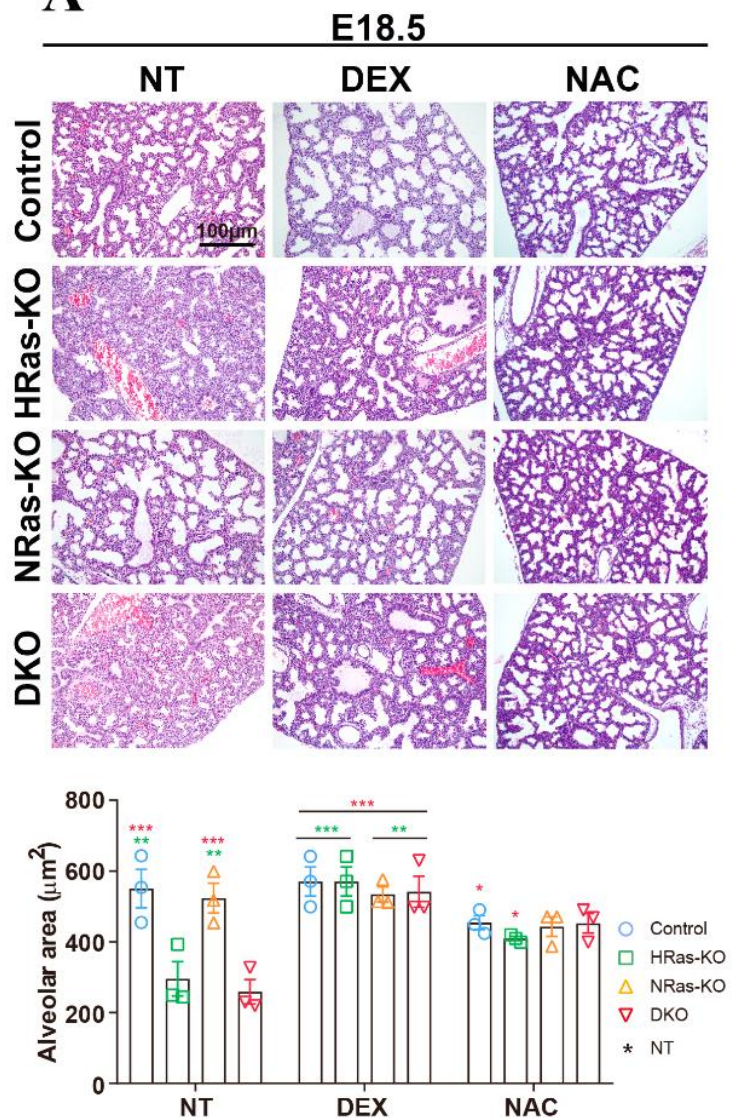

B
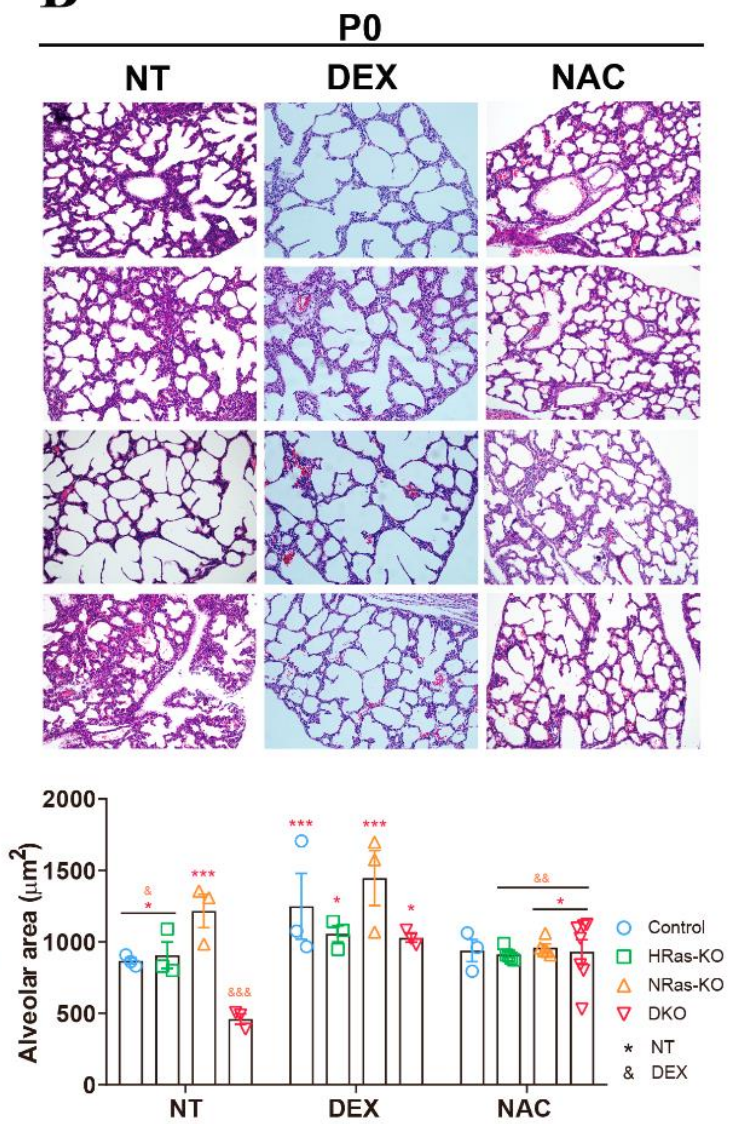

Figure 33: Histological analysis of the lungs of newborn pups (P0) and late embryos (E18.5) of HRas-KO and/or NRas-KO mice subjected to dexamethasone or $\mathbf{N}$-acetylcysteine treatment. A, B Representative images of Haematoxylin-Eosin (H\&E)-stained sections of lungs of E18.5 embryos (A) and P0 (B) newborn pups of the indicated genotypes and treatments. Scale bars: $100 \mu \mathrm{m}$. The bar graphs at bottom of the panels quantify the average area $(\mu \mathrm{m} 2)$ of the individual alveolar sacs in the lungs of, respectively, E18.5 (A) and P0 (B) individuals of the indicated genotypes and treatments. Data is expressed as the mean \pm s.e.m. $n=3$ individuals for E18.5 NT, DEX, NAC and P0 NT, DEX. NAC: CT $\mathrm{n}=3$, HRas-KO, NRas-KO $\mathrm{n}=5$, DKO $\mathrm{n}=7, * v s$ NT, \& vs DEX, */\& $<<0.05$, $* * / \& \& \mathrm{p}<0.01, * * * / \& \& \& \mathrm{p}<0.001$.

Even though the lungs of the DEX-treated DKO pups were apparently normal, the lethality was not abrogated since these DEX-treated DKO pups kept dying 5-6 days after birth-time (Fig. 32A, B). On the other hand, the NAC-treated animals displayed significantly higher survival rates (Fig. 32A, B) together with normal lung maturation, suggesting that there might be other factors implicated in neonatal death of DKO animals that are mitigated with NAC but not with glucocorticoids. 
E18.5 PAS
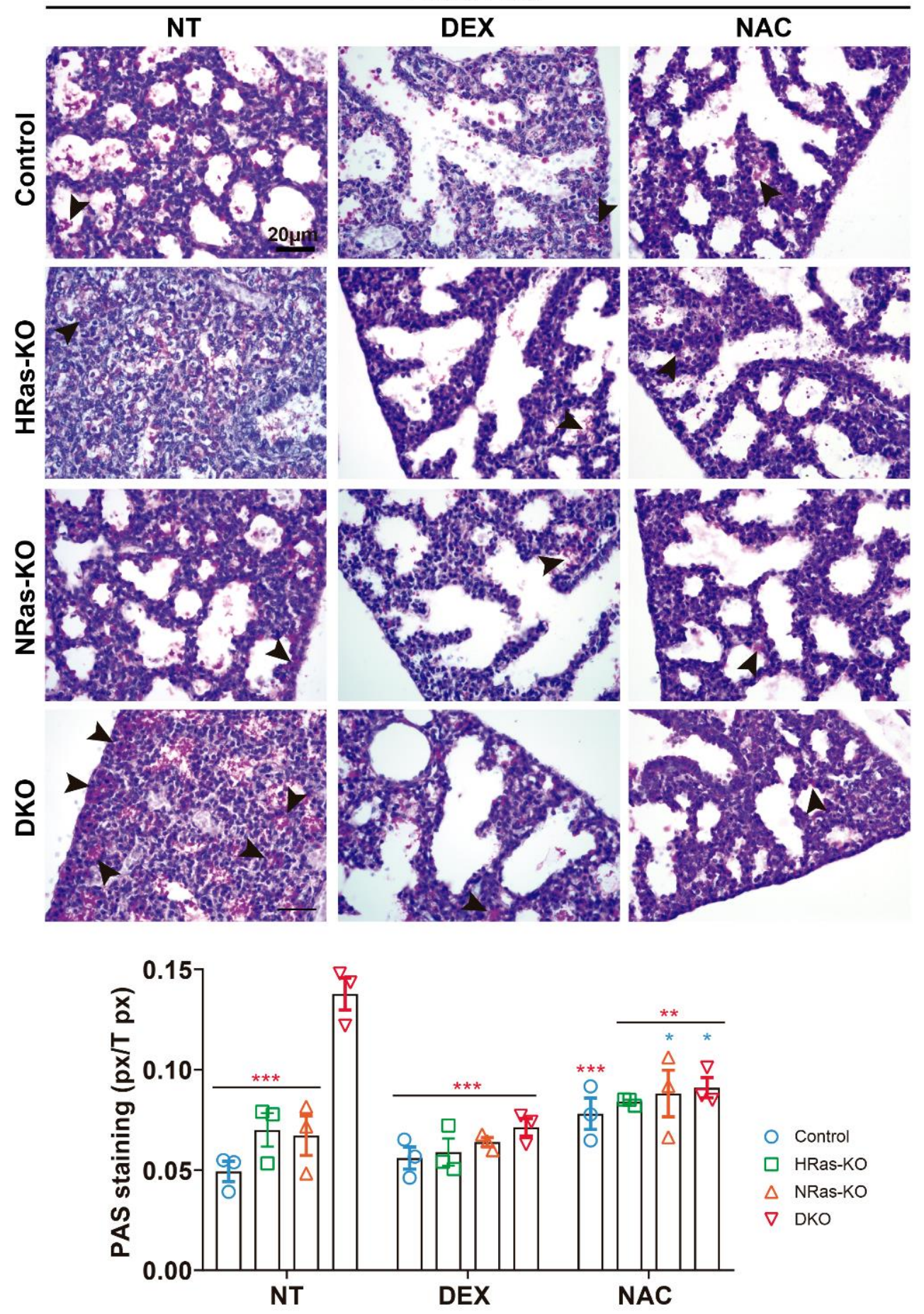

Figure 34: PAS staining analysis of the lungs of newborn pups (P0) and late embryos (E18.5) of HRas-KO and/or NRas-KO mice subjected to dexamethasone or $\mathbf{N}$-acetylcysteine treatment. Representative images of PAS-stained lung sections from E18.5 embryos of the indicated genotypes and treatments. Scale bars: $20 \mu \mathrm{m}$ and $10 \mu \mathrm{m}$ in magnified, boxed areas. Black arrowheads point to cytoplasmic and extracellular accumulations of PAS-positive label in alveolar areas of the indicated genotypes. The bar graphs in this panel quantify the relative levels of PAS-staining (ratio of PAS+ pixels relative to total number of pixels) in the lungs of E18.5 individuals of the indicated genotypes and treatments. Data is expressed as the mean \pm s.e.m. $\mathrm{n}=3$ individuals for all genotypes and conditions. ${ }^{*} v s \mathrm{NT}, * \mathrm{p}<0.05, * * \mathrm{p}<0.01$, $* * \mathrm{p}<0.01, * * * \mathrm{p}<0.001$. 

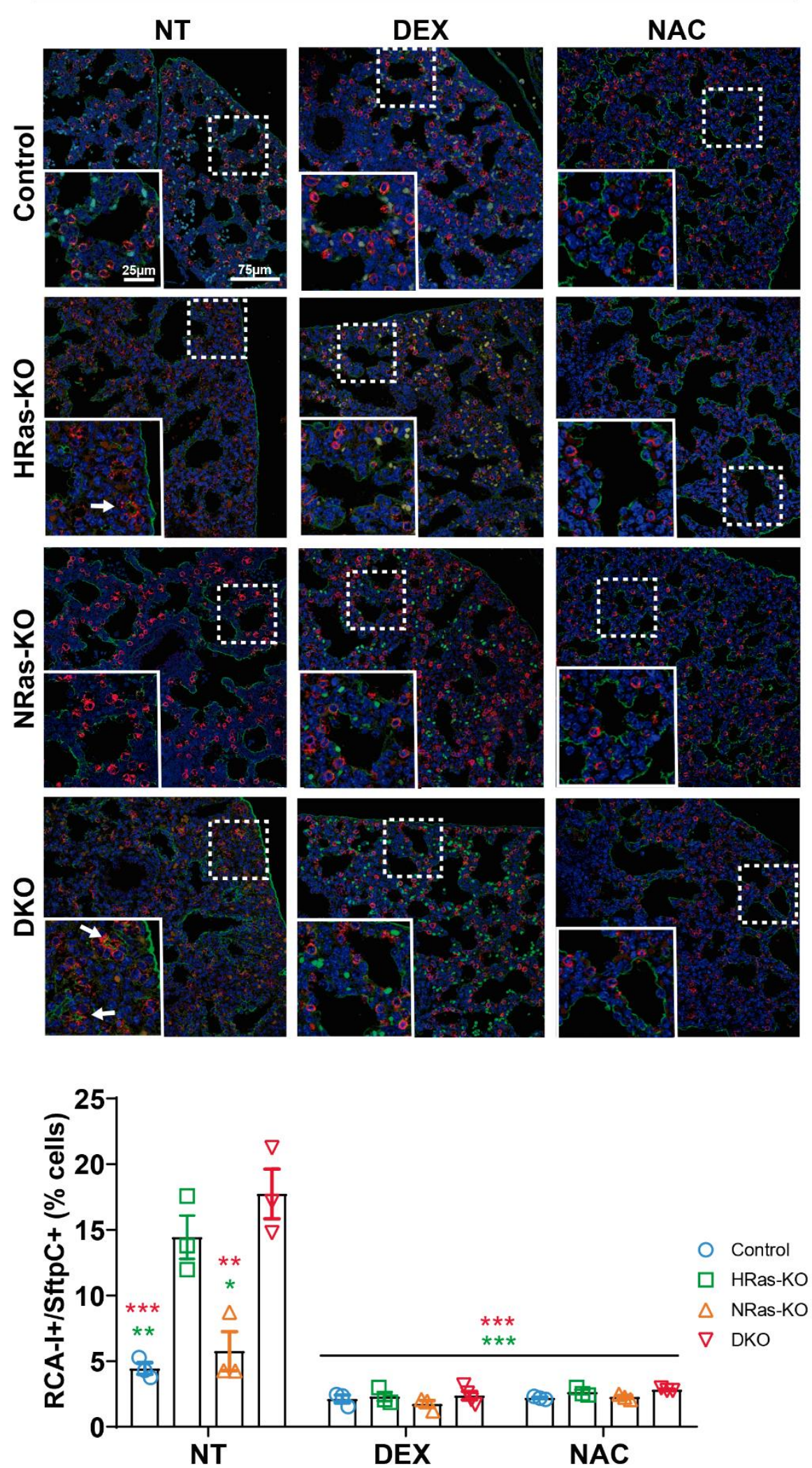

Figure 35: Immunostaining of alveolar differentiation markers in the lungs of NT-, DEX-and NAC-treated HRas-KO and/or NRas-KO mice. A. Representative images of immunostaining for Surfactant protein-C (SftpC, AT2 lineage, red) and Ricinus communis agglutinin-I (RCA-I, AT1 lineage, green) in paraffin sections of the lungs of E18.5 mice of the indicated genotypes. Co-immunolabeled, alveolar bi-potent progenitor cells are marked by tailed arrows. Scale bar: $75 \mu \mathrm{m}$ and $25 \mu \mathrm{m}$ in the magnified boxed areas. The bottom bar graph quantifies the percentage of alveolar bi-potent cells $(\mathrm{RCA}-\mathrm{I}+/ \mathrm{SftpC}+$ ) relative to total number of $\mathrm{SftpC}+$ cells in the lungs of E18.5 individuals of the indicated genotypes and treatment. Data expressed as the mean \pm s.e.m. Ten separate 
microscopic fields were quantified for each individual analysed in each genotype. $n=4$ for DKO-DEX, $n=3$ for the rest of genotypes and conditions. *vs $\mathrm{NT} * \mathrm{p}<0.05, * * \mathrm{p}<0.01, * * * \mathrm{p}<0.001$.

\section{E18.5 Sox9 DAPI}
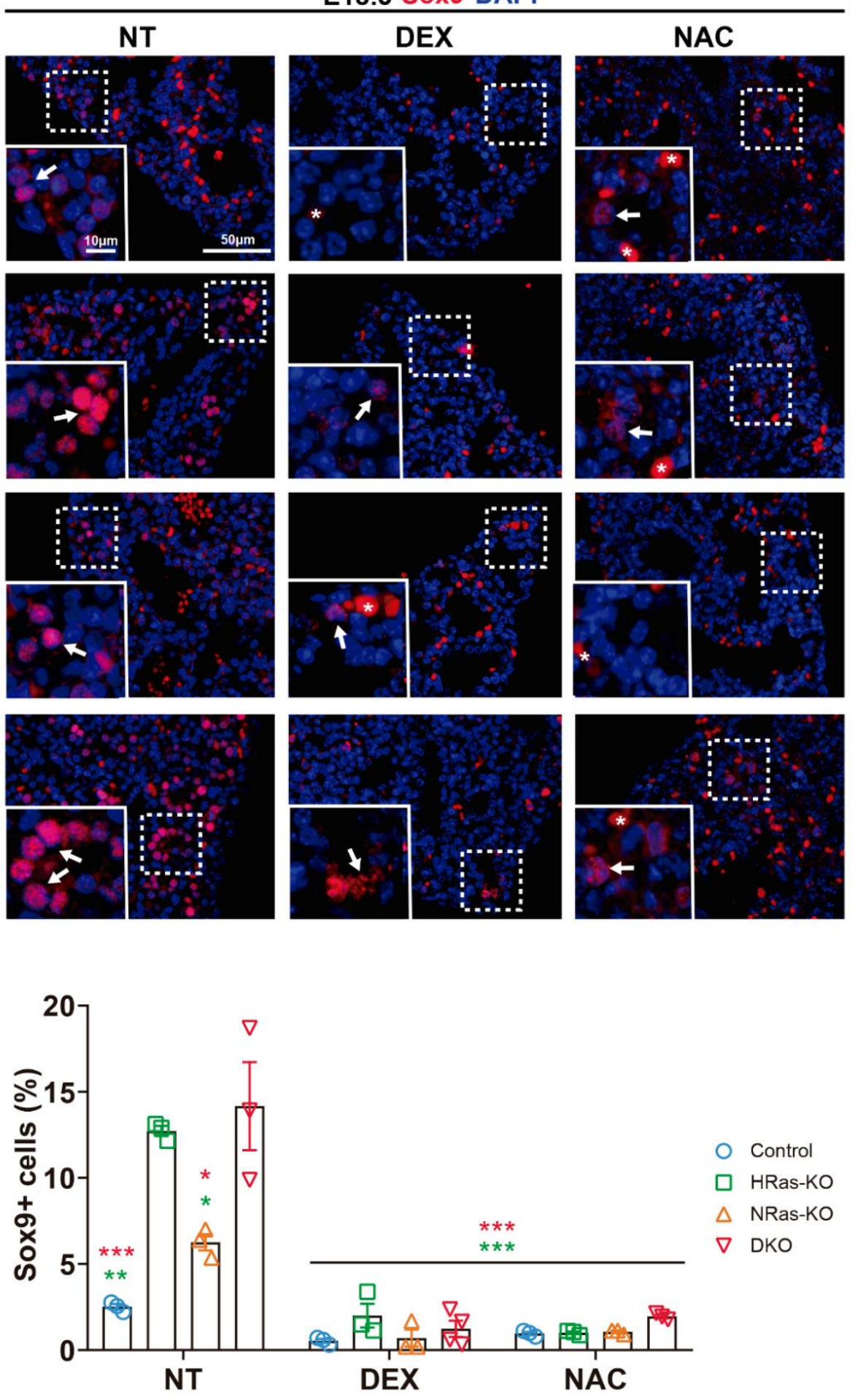

Figure 36: Immunostaining of SOX9+ alveolar progenitors in the lungs of NT-, DEX- and NAC-treated HRas-KO and/or NRas-KO mice. Representative images of immunostainings for SOX9 (distal-tip progenitors, red) in peripheral or inner parenchymal areas of E18.5 lungs from the indicated genotypes. Arrows point to SOX9+ cells, asterisk point to unspecific staining of erythrocytes. Scale bar: $50 \mu \mathrm{m}$ and $10 \mu \mathrm{m}$ in the magnified boxed areas. The bottom bar graph quantifies the percentage of SOX9+ cells relative to total number of cells. Data expressed as the mean \pm s.e.m. Ten separate microscopic fields were quantified in each animal. $\mathrm{n}=4$ for DKODEX, $\mathrm{n}=3$ for the rest of genotypes and conditions. *vs NT * $\mathrm{p}<0.05, * * \mathrm{p}<0.01, * * * \mathrm{p}<0.001$. 


\subsubsection{Bronchiolar cell lineages show no alterations after DEX or NAC treatments}

Previous results showed a significant alteration in Club cells (HRas and NRas single and double-KO) and Ciliated cells (only in DKO lungs) morphology (Fig. 22A-D). Given these observations, we studied the structure of these cells in P0 mice that received either antenatal DEX or NAC, showing no differences between the different genotypes and being indistinguishable when compared to the untreated controls (Fig. 37A, E). Additionally, no differences in Club cell (Scgb1a1+) - Ciliated cell $(\beta$-Tub+) proportions were observed after DEX or NAC treatments (Fig. 37B-D). These findings indicate that both DEX and NAC treated animals exhibit a normal bronchiolar cells columnar morphology and that cell number is not modified.

A
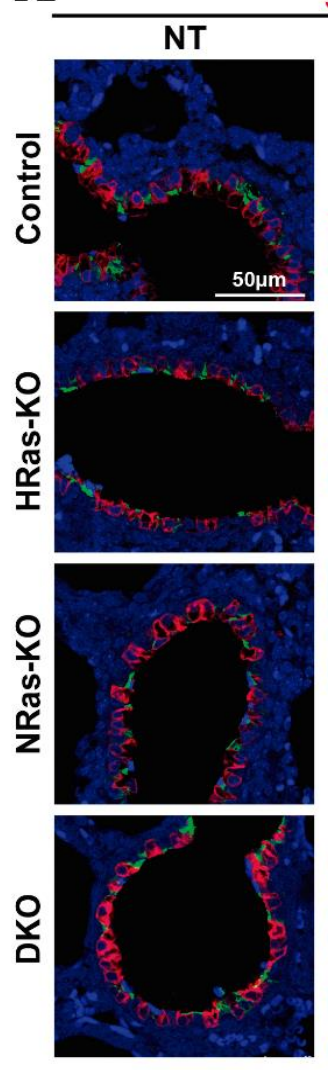
Scgb1a1 $\beta$-Tub DAPI
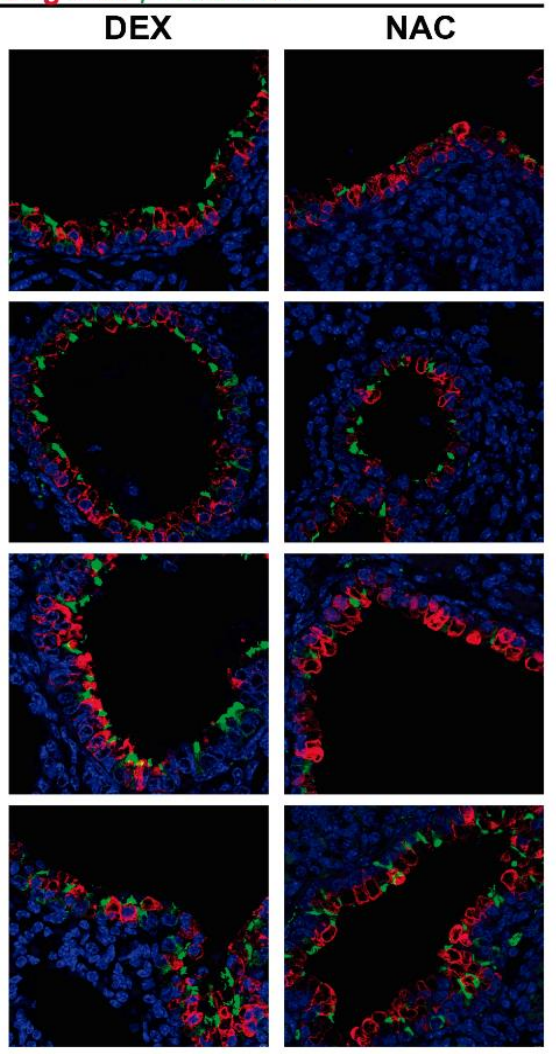

B
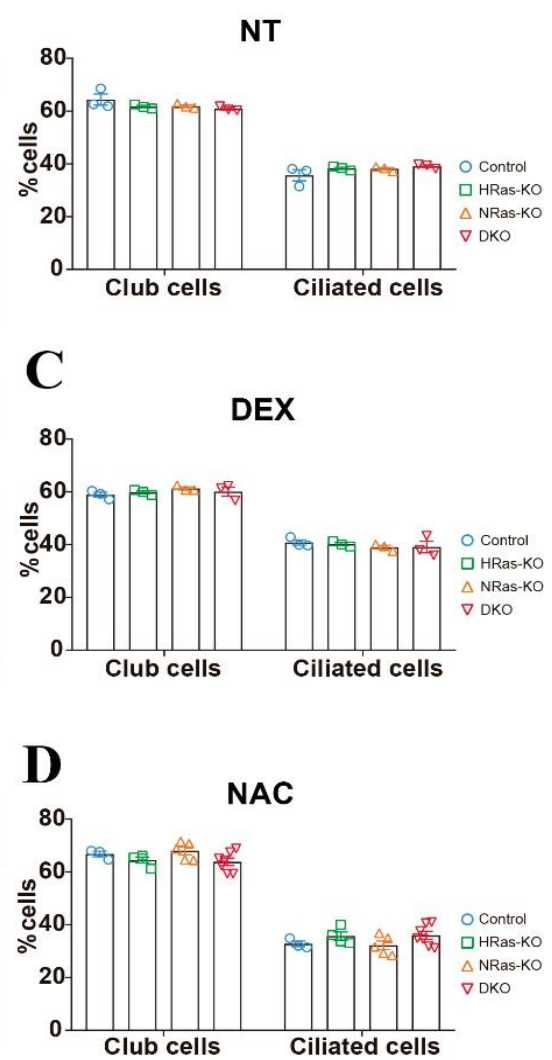

$\mathbf{E}$
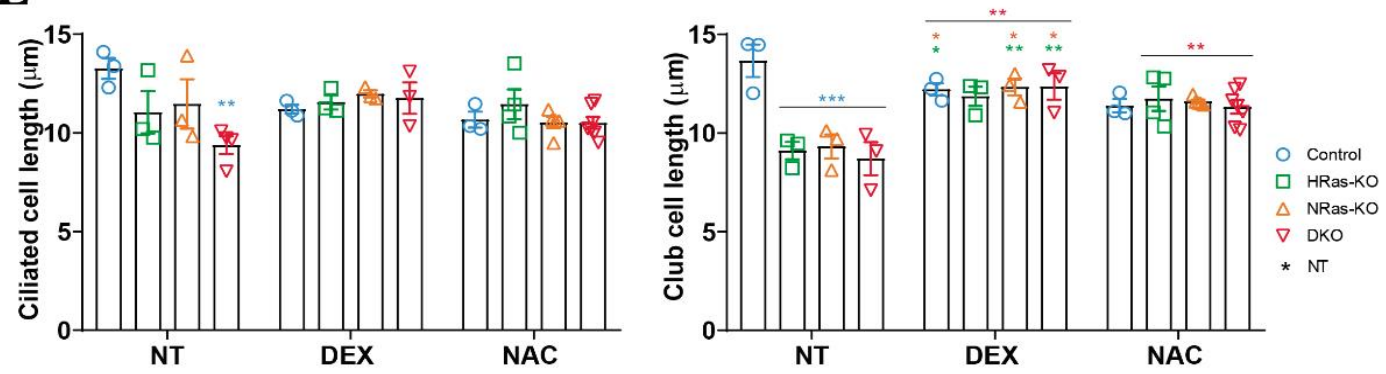

Figure 37: Immunostaining of bronchiolar differentiation markers in the lungs of DEX- or NAC-treated HRas-KO and/or NRas-KO mice. A. Representative images of immunostaining for Secretoglobulin (Scgb1a1, Club cells, red) and $\beta$-Tubulin ( $\beta$-Tub, Ciliated cells, green), counterstained with DAPI (blue), in paraffin sections of bronchiolar regions of lungs from NT/DEX/NAC-treated P0 mice of the indicated genotypes. Scale bar: $50 \mu \mathrm{m}$. B. C, D. Bar graphs quantifications of Club and Ciliated cell percentage relative to total number of bronchiolar 
cells from NT (B), DEX (C) and NAC-treated animals (D). Data expressed as the mean \pm s.e.m. Ten separate microscopy fields were quantified for each individual analysed in each genotype. $n=3$ individuals for NT and DEX animals, NAC: CT n=3, HRas-KO n=4, NRas-KO n= 5, DKO n=7. E. Cell length measurements (from basal to apical membrane) of Club (left) and Ciliated (right) cells stained as in panel A from NT/DEX/NAC-treated animals. Data expressed as the mean \pm s.e.m. Ten separate microscopy fields were quantified for each individual analysed in each genotype. Data expressed as the mean \pm s.e.m. Ten separate fields were quantified. $n=3$ individuals for NT and DEX animals, NAC: CT n=3, HRas-KO n=4, NRas-KO $n=5$, DKO n=7. *vs NT, *p<0.05, $* * \mathrm{p}<0.01, * * * \mathrm{p}<0.001$.

\subsubsection{Neutrophil infiltration}

Given that we previously detected an increase in neutrophil infiltration in the lungs of DKO pups (Fig. 24A, C), we sought to analyse whether DEX or NAC antenatal treatment had some effect on the lung neutrophil extravasation. As expected, dexamethasone, a glucocorticoid that inhibits immune response (Ricci et al., 2021; Ronchetti et al., 2018), produced a complete reversion of neutrophil infiltration in the DKO lungs (Fig. 38). Consistently, treatment with NAC also reduced the number of neutrophils in alveolar regions of DKO pups, albeit the effect was lower than with DEX treatment (Fig. 38).

\subsubsection{Transcriptomic changes induced by antenatal dexamethasone treatment in the lungs of newborn DKO mice}

The transcriptional profiles of untreated P0 DKO lungs and DEX-treated P0 DKO lungs were compared, and unsupervised hierarchical clustering of the normalized expression data profiles generated under high stringency produced a dendrogram where samples corresponding to the DEX-treated DKO mice were clearly discriminated from the untreated DKO lung samples (Fig. 39A). As expected, glucocorticoid treatment (dexamethasone) resulted in a downregulation of more than $90 \%$ of differentially expressed genes, whereas less than $10 \%$ were upregulated as a result of this treatment.

Functional annotation analyses of the DEX-treated samples differentially expressed genes identified a series of GO functional categories and KEGG signalling pathways (Fig. 39B) that, mainly, mirrored in opposite direction (downregulation) the transcriptional behaviour of similar functional categories that were previously found upregulated in the untreated DKO lungs. Interestingly, "Protein Transport", "Metabolic/phosphorylation or apoptotic cellular processes" were significantly downregulated. It was also striking the significant downregulation of various components of signalling pathways and processes, that were upregulated in the untreated DKO samples, and are known to be significant for lung functionality, such as "Oxidative phosphorylation", "N-glycan metabolism" and "Sphingolipid metabolism" (Fig. 39B). (Data submitted to Gene Expression Omnibus, accession number GSE130415). 
Interestingly, no significant changes were detected when untreated CT / HRas-KO / NRasKO were compared against DEX-treated CT / HRas-KO / NRas-KO, as well as between DEXtreated DKO versus DEX-treated CT / HRas-KO / NRas-KO samples.
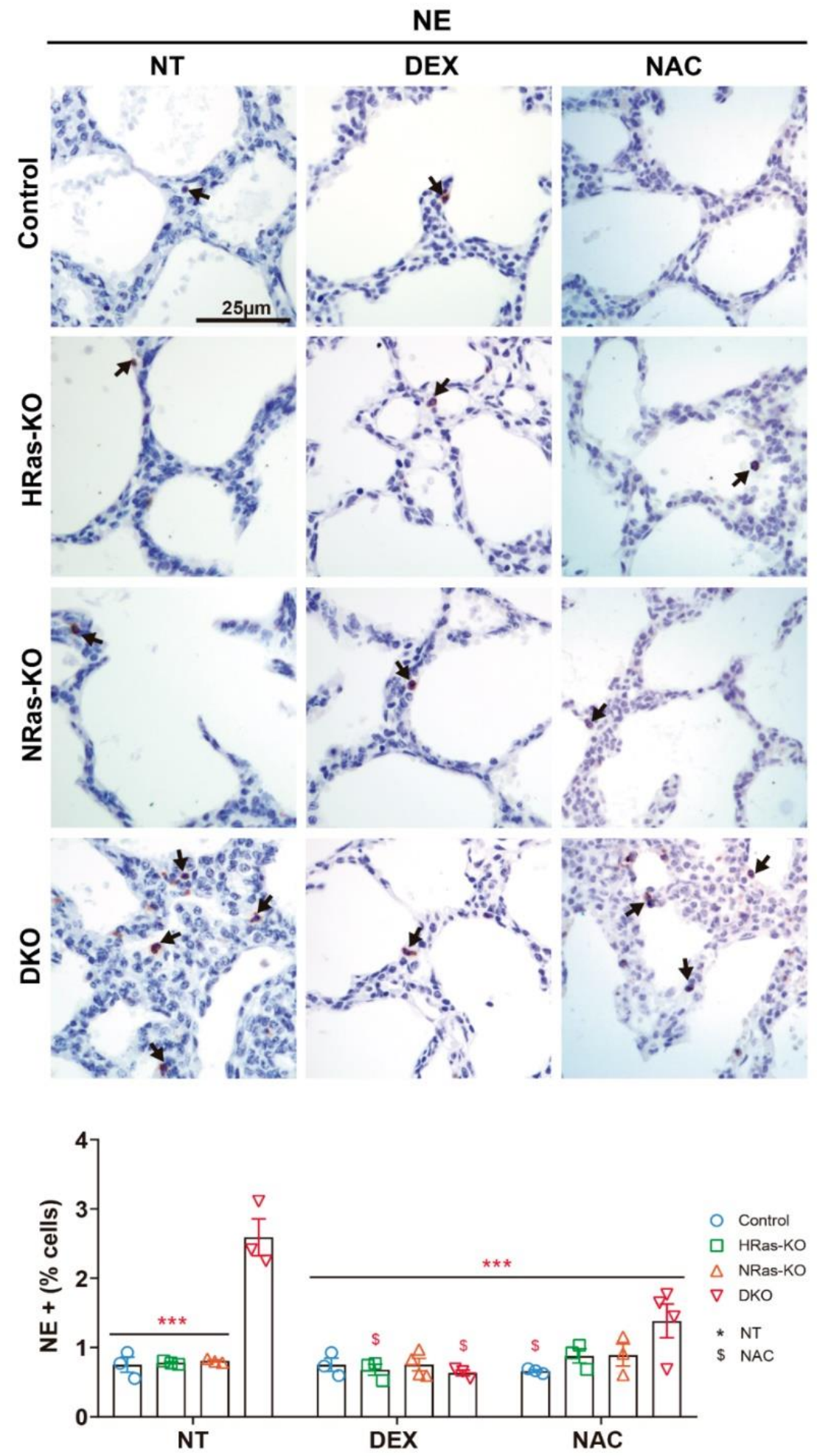

Figure 38: Increased neutrophil infiltration in the lungs of HRas/NRas-DKO mice is completely rectified after DEX treatment and only partially corrected after NAC administration. A. Representative images of immunostaining for neutrophil elastase (NE), counterstained with haematoxylin (blue) in lung paraffin sections of NT, DEX or NAC-treated neonates. Arrows point to NE+ cells. Scale bar: $25 \mu \mathrm{m}$. The bar graph quantitates percentage of NE+ cells relative to total cells. Data expressed as the mean \pm s.e.m. for each genotype. $n=4$ individuals for NRas-KO-DEX and DKO-NAC and $\mathrm{n}=3$ for the rest of genotypes and conditions. ${ }^{*} v s$ NT, ${ }^{\$} v s$ $\mathrm{NAC},{ }_{\mathrm{p}}<0.05, * * * \mathrm{p}<0.001$. 
A

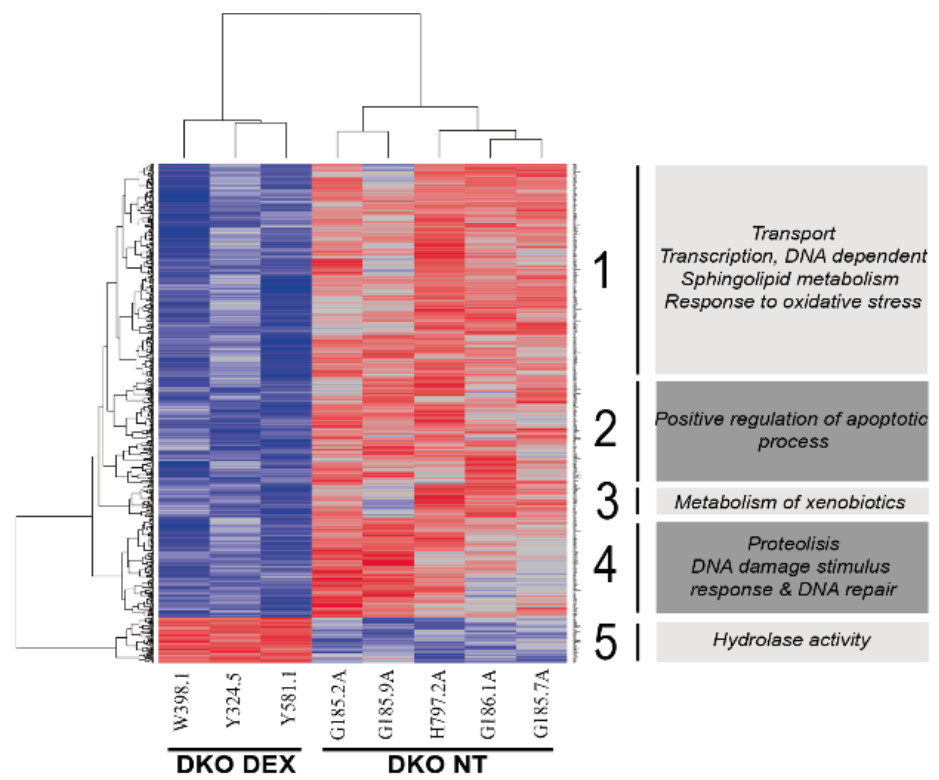

B

GO Biological Process \& Molecular Function DEX

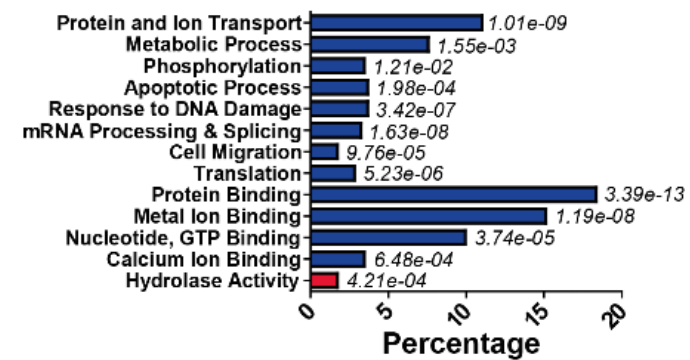

Signaling Pathways (KEGG) DEX

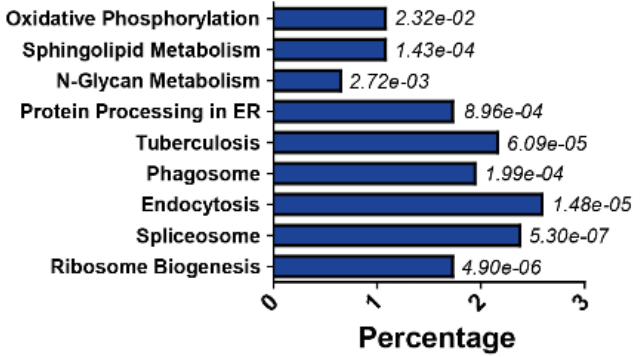

Figure 39: Differential gene expression in the lungs of Dexamethasone-treated, HRas/NRas-DKO newborn P0 mice. A. RNAs extracted from the lungs of 3 independent, newborn P0 DKO mice that had been previously treated in utero with dexamethasone as described (Materials \& Methods) were submitted to microarray hybridizations and their transcriptional profiles were compared to those of 5 independent, untreated DKO P0 neonates. The heatmap depicts the results of hierarchical clustering and multiclass comparison of 509 gene probesets that showed differential expression $(F D R=0.15)$ between the untreated and the dexamethasone-treated lung DKO samples. Labels on the right side of the dendrograms identify specific functional categories that are enriched at high statistical significance within the indicated individual horizontal clusters (blocks 1-5) of the heatmap. B. The horizontal bar plots depict color-coded functional annotations (hypergeometric p-values indicated in italics) corresponding to specific groups of genes that are overexpressed (red) or repressed (blue) in dexamethasone-treated DKO lung samples as compared to untreated DKO counterparts and were identified by GeneCodis as significantly enriched for the indicated functional categories. Values in the X-axis represent the percentage of the total number of differentially expressed gene probesets corresponding to each individual functional category identified in the graphs.

\subsubsection{Transcriptomic changes induced after NAC treatment in PO lungs of DKO mice}

In order to get some clues explaining the better survival of the NAC-treated DKO mice, we compared the transcriptional profiles of lung tissues from P0 untreated DKO mice with the NAC-treated DKO samples. The resulting dendrogram clearly discriminated NAC-treated and untreated samples. Of the differentially expressed genes resulted from the comparison between NAC-treated and untreated DKO samples, $50.3 \%$ were overexpressed whereas $49.7 \%$ were 
repressed, suggesting that there was no predominance in transcriptional overexpression or repression after NAC treatment (Fig. 40A).

A

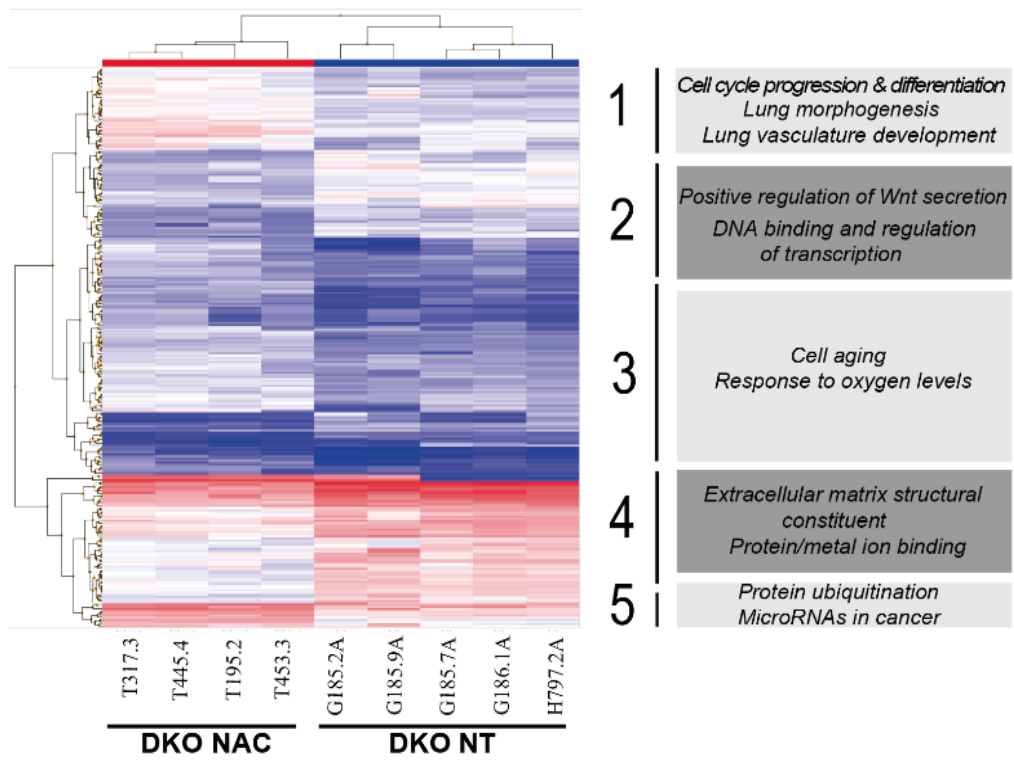

B
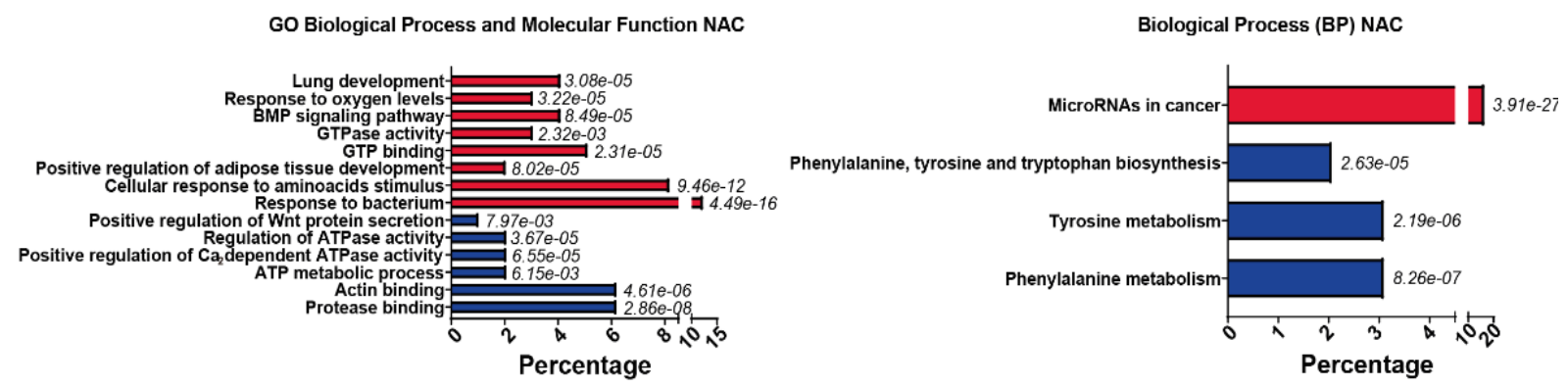

Figure 40: Differential gene expression in the lungs of NAC-treated, HRas/NRas-DKO newborn P0 mice. A. RNAs extracted from the lungs of 4 independent, newborn P0 DKO mice that had been previously treated in utero with N-Acetylcysteine as described (Materials \& Methods) were submitted to microarray hybridizations and their transcriptional profiles were compared to those of 5 independent, untreated DKO P0 neonates. The heatmap depicts the results of hierarchical clustering and multiclass comparison of 261 gene probesets that showed differential expression $(\mathrm{p}<0.05)$ between the untreated and the NAC-treated lung DKO samples. Labels on the right side of the dendrograms identify specific functional categories that are enriched at high statistical significance within the indicated individual horizontal clusters (blocks 1-5) of the heatmap. B. The horizontal bar plots depict color-coded functional annotations (hypergeometric p-values indicated in italics) corresponding to specific groups of genes that are overexpressed (red) or repressed (blue) in NAC-treated DKO lung samples as compared to untreated DKO counterparts and were identified by GeneCodis as significantly enriched for the indicated functional categories. Values in the $\mathrm{X}$-axis represent the percentage of the total number of differentially expressed gene probesets corresponding to each individual functional category identified in the graphs.

Functional annotation of the detected probe sets identified significant enrichment in various biological processes, molecular functions and signalling pathways that may explain the observed better survival of DKO pups after NAC administration. The upregulated group of genes in DKO NAC-treated lungs included statistically significant enrichment in distinct GO 
functional categories such as "Lung development", "Response to oxygen levels", "BMP signalling pathway", processes functionally related and significant for the developmental processes of lungs, as well as "GTPase activity", "GTP binding" and "Positive regulation of adipose tissue development" (Fig. 40A, B). In contrast, the downregulated GO categories included "Positive regulation of Wnt protein secretion", which constitutes one of the major pathways regulating epithelial-mesenchymal communication during lung development, "Regulation of ATPase activity", "ATP metabolic process" and "Actin \& protease binding". Interestingly, the list of upregulated genes in DKO NAC-treated lungs was significantly enriched with 16 probes related with "MicroRNAs in cancer" KEGG signalling pathway, being them linked with cell proliferation inhibition (Fig. 40A, B). (Data submitted to Gene Expression Omnibus, accession number GSE186161).

When the rest of the NAC-treated genotypes were compared with the correspondent untreated counterpart no biologically significant enrichments were obtained, as well as between NAC-treated DKO versus NAC-treated CT / HRas-KO / NRas-KO samples.

\subsubsection{Ceramide levels are depleted after antenatal administration of either DEX or NAC}

Microarray analyses of DEX-treated DKO lungs showed a significant downregulation of several components of sphingolipid metabolic pathways that were previously found upregulated in untreated DKO lungs (Fig. 39B). Specifically, glucocorticoid treatment resulted in a downregulation of loci such Alkaline ceramidase 2 (Acer2), Alkaline ceramidase 3 (Acer3), Delta (4)-desaturase, sphingolipid 1 (Degs1), Neuraminidase 3 (Neu3), and Sphingosine kinase 1 (Sphkl). To further study whether this downregulation was translated to a lower ceramide accumulation, immunoassays against ceramide were performed in lung sections of P0 DEXtreated mice, resulting in a significant reduction of the ceramide accumulations observed in the alveolar regions of non-treated DKO lungs, as well as a significant reduction in bronchiolar ceramide levels (Fig. 41A, B).

Additionally, NAC-treated P0 lung slices also showed alveolar and bronchiolar ceramide levels similar to the controls in all the genotypes (Fig. 41A, B). These results indicate that both dexamethasone and $\mathrm{N}$-acetylcysteine antenatal treatments were able to alleviate the ceramide accumulation that DKO lungs exhibited. 
A
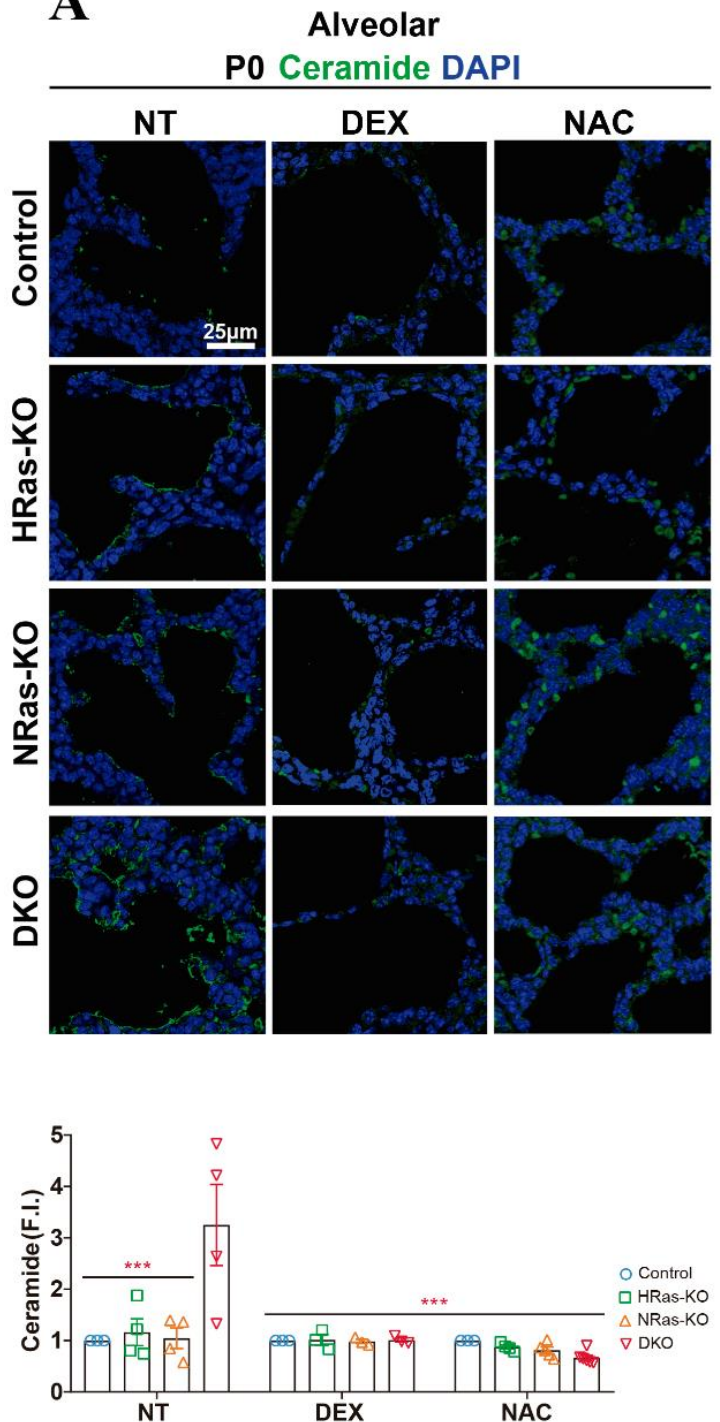

B

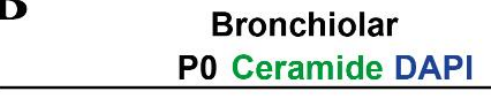

PO Ceramide DAPI
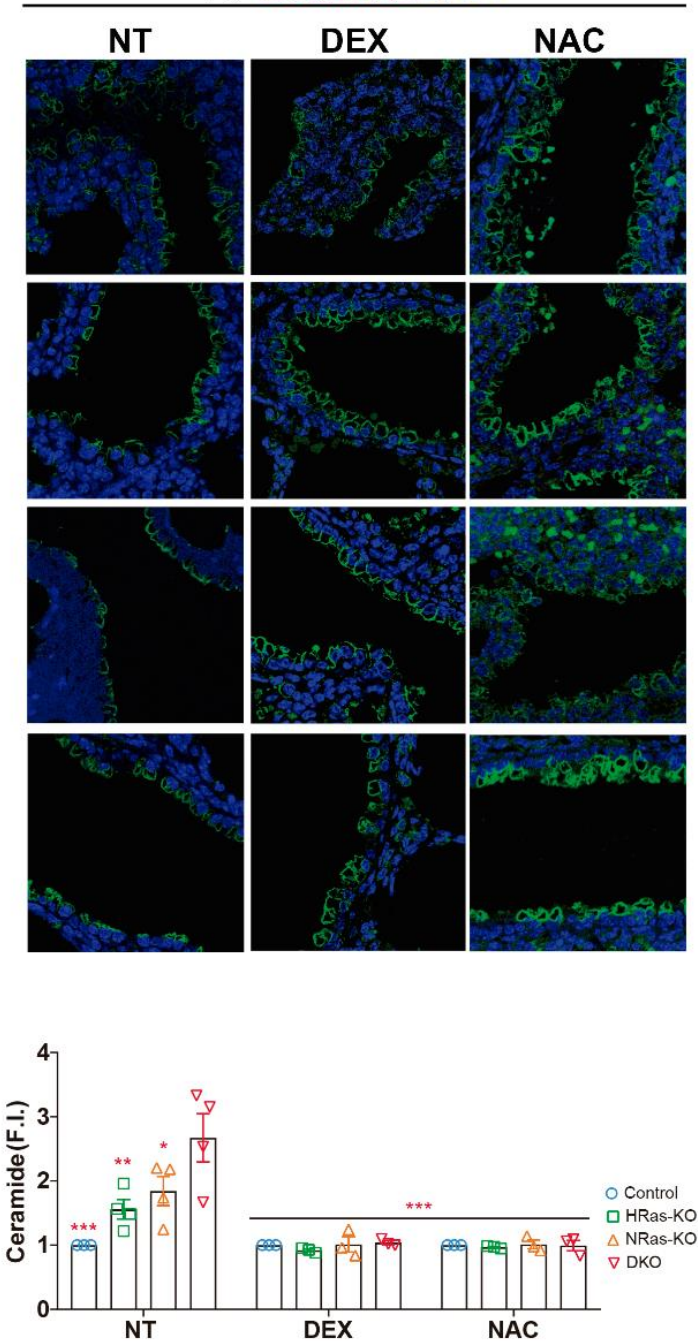

Figure 41: Ceramide immunoassays in alveolar and bronchiolar regions of DEX- and NAC-treated lungs of HRas and/or NRas KO mice. A. Representative alveolar images of immunostaining for Ceramide (green) and DAPI counterstaining (blue) in lung paraffin sections of newborn NT/DEX/NAC-treated P0 mice of the indicated genotypes. Scale bar: $25 \mu \mathrm{m}$. The bottom bar plots represent the average values of ceramide fluorescence intensity (F.I.) in alveoli, relative to Control. Data expressed as the mean \pm s.e.m. Ten separate microscopy fields were quantified for each individual analysed. NT: CT $n=3$ and $n=4$ individuals for the rest of genotypes; DEX: $n=3$ for all the genotypes; NAC: CT $n=3$, HRas-KO, NRas-KO $n=5$, DKO $n=7$. ${ }^{*} \mathrm{p}<0.05$, **p<0.01, ***p<0.01. B. Representative bronchiolar images of immunostaining for Ceramide (green) and DAPI counterstaining (blue) in lung paraffin sections of newborn NT/DEX/NAC-treated P0 mice of the indicated genotypes. The bar plots represent the average values of ceramide fluorescence intensity (F.I.) in bronchiole relative to Control. Data expressed as the mean \pm s.e.m. Ten separate microscopy fields were quantified for each individual analysed. NT: $\mathrm{CT} n=3$ and $\mathrm{n}=4$ individuals for the rest of genotypes; DEX/NAC: $\mathrm{n}=3$ for all the genotypes. ${ }^{*} v s \mathrm{NT},{ }^{*} \mathrm{p}<0.05$, $* * \mathrm{p}<0.01, * * * \mathrm{p}<0.01$.

\subsubsection{Effect of antenatal administration of DEX or NAC on ROS generation}

Since we had previously observed that only DKO lungs presented a higher production of $\mathrm{H}_{2} \mathrm{O}_{2}$ and total $\mathrm{O}_{2}^{-}$, and both NRas-KO and DKO lungs showed a significant increment in mitochondrial $\mathrm{O}_{2}^{-}$, we sought to analyse the effect of DEX and NAC antenatal treatment on ROS production. For this purpose, lungs from P0 mice treated either with DEX or NAC were studied. 
Interestingly, NAC administration throughout the pregnancy resulted in a significant decrease of both $\mathrm{H}_{2} \mathrm{O}_{2}$ and total $\mathrm{O}_{2}{ }^{-}$in DKO lungs, but conversely, mitochondrial $\mathrm{O}_{2}{ }^{-}$was not affected after NAC treatment (Fig. 42). Moreover, HRas-KO, NAC-treated lungs showed a significant increment in MitoSOX ${ }^{\mathrm{TM}}$ (mitochondrial $\mathrm{O}_{2}{ }^{-}$) labelling when compared with treated and untreated controls, and HRas-KO-untreated ones (Fig. 42). On the other hand, DEX antenatal administration produced a significant diminution of total and mitochondrial $\mathrm{O}_{2}^{-}$levels in the DKO lungs, but it was not efficient in reducing the $\mathrm{H}_{2} \mathrm{O}_{2}$ levels (Fig. 42). Additionally, NRas-KO DEX treated lungs showed an increment in $\mathrm{H}_{2} \mathrm{O}_{2}$ when compared to the controls, and the augmented mitochondrial $\mathrm{O}_{2}^{-}$was not lowered after DEX treatment (Fig. 42).
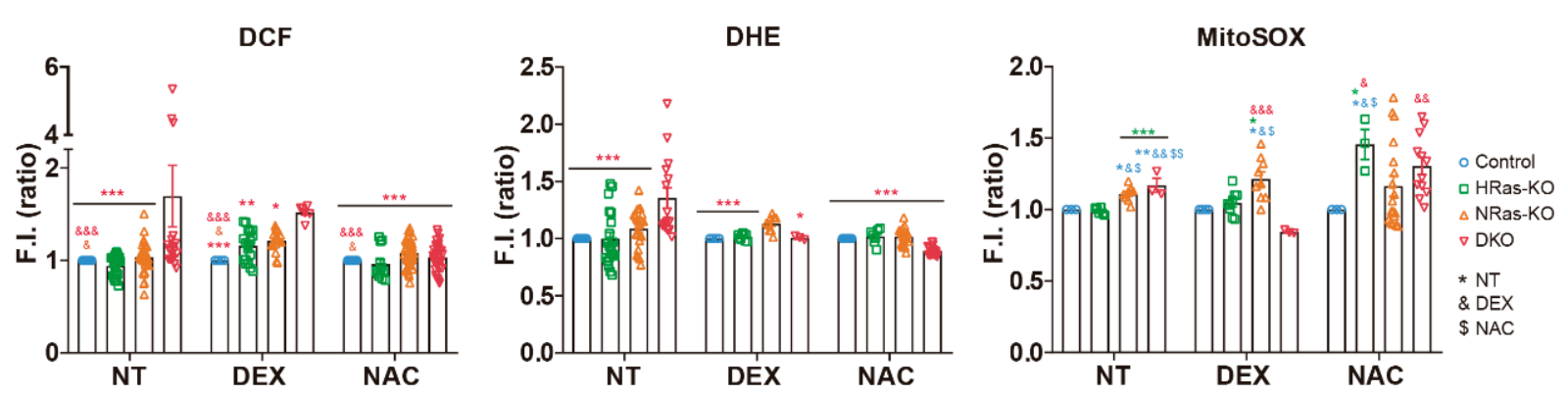

Figure 42: Analyses of oxidative stress in lungs of P0 HRas-KO and/or NRas-KO after DEX or NAC treatment. The bar plots represent the in vivo quantitation, relative to Control, of redox parameters carried out by means of FACS fluorescence measurements performed (30.000 events in each case) on fresh whole lung lysates using specific fluorophores for intracellular hydrogen peroxide (DCF, $5 \mu \mathrm{M}$ ), intracellular total superoxide (DHE, $5 \mu \mathrm{M})$ and mitochondrial superoxide (MitoSOX ${ }^{\mathrm{TM}}, 5 \mu \mathrm{M}$ ) as described in Materials and Methods. Data expressed as the mean \pm s.e.m. DCF NT: CT n=13, HRas-KO n=25, NRas-KO n=35, DKO n=18; DEX: CT n=4, HRas-KO n=18, NRas-KO $n=20$, DKO n=6; NAC: CT n=9, HRas-KO n=12, NRas-KO n=40, DKO n=33; DHE NT: CT n=13, HRas-KO n=20, NRas-KO n=23, DKO n=15; DEX: CT $n=4$, HRas-KO $n=7$, NRas-KO n=9, DKO n=3; NAC: CT $n=7$, HRas-KO $n=9$, NRas-KO n=20, DKO n=21; MitoSOX ${ }^{\text {TM }}$ NT: CT n=5, HRas-KO n=6, NRas-KO n=9, DKO n=5; DEX: CT $\mathrm{n}=4$, HRas-KO n=9, NRas-KO n=9, DKO n=3; NAC: CT n=3, HRas-KO n=3, NRas-KO n=18, DKO n=12. *vs NT, $\&_{v s}$ DEX, ${ }^{\$} v s$ NAC, $* / \& / \$ \mathrm{p}<0.05, * * / \& \& / \$ \mathrm{p}<0,01, * * * / \& \& \& / \$ \$ \mathrm{p}<0.001$.

\subsubsection{Seahorse tests of lungs from mice treated with either NAC or DEX}

To determine the impact of DEX and NAC antenatal treatment on mitochondrial respiration, we analysed the respiratory profile of DEX or NAC-treated lungs from newborn CT, HRas-KO, NRas-KO and DKO pups using Seahorse MitoStress kit assay. No differences between genotypes and conditions were detected when OCR measurements of basal respiration were compared (Fig. 43A, B). Interestingly, spare respiratory capacity OCR levels were significantly lower in DEX and NAC-treated DKO lungs when compared with the untreated DKO samples (Fig. 43B). Correspondingly, CT, HRas-KO and NRas-KO genotypes, treated either with DEX or NAC, showed a decrease in the spare respiratory capacity, albeit the differences were not statistically significative, indicating that the ETC from DEX or NAC-treated lungs was working at lower levels (Fig. 43B). 
In contrast with the previous observations, mitochondrial respiration linked to ATP production was significantly higher in DKO lungs treated with either DEX or NAC as compared with the untreated DKO samples (Fig. 43B).
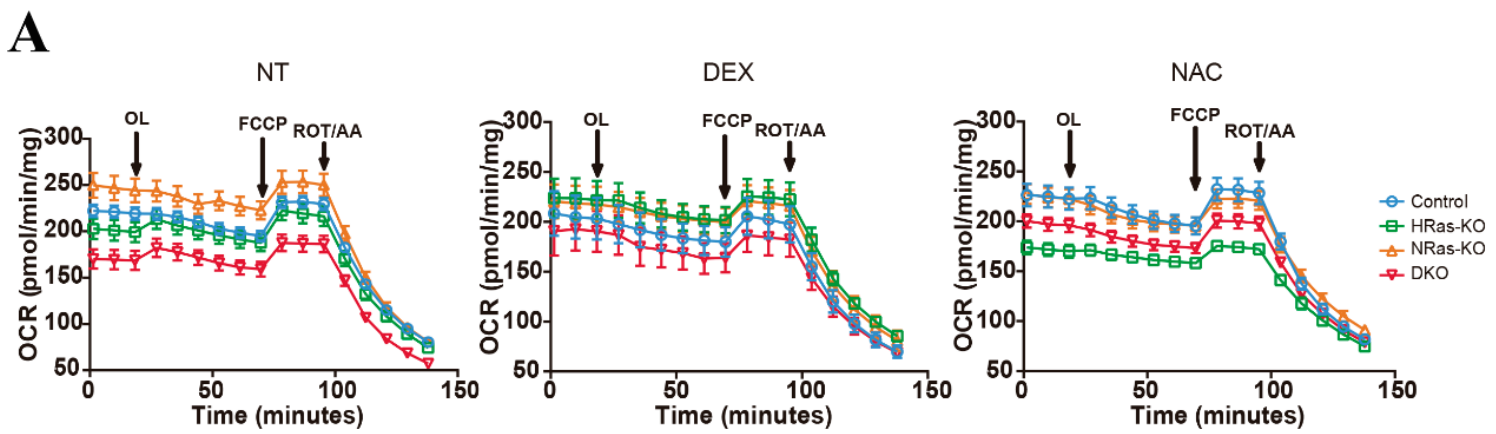

B
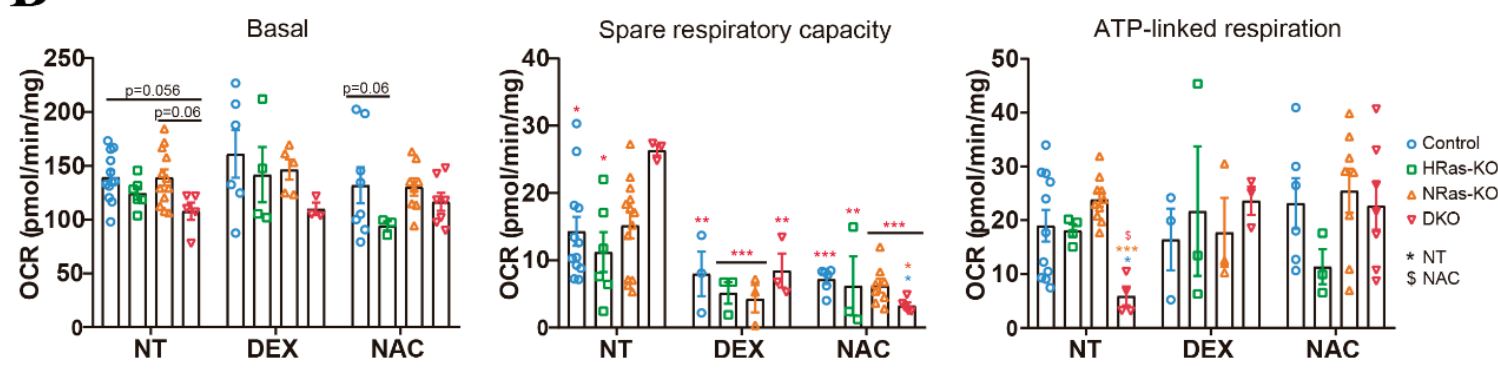

Figure 43: Analyses of mitochondrial respiration parameters analyses of P0 HRas/NRas-DKO lungs after DEX or NAC antenatal administration A. MitoStress profile from P0 mice of the indicated genotypes and treatments. OCR (oxygen consumption rate) was measured under basal conditions followed by the sequential addition of $15 \mu \mathrm{M}$ Oligomycin (OL), $16 \mu \mathrm{M}$ FCCP, and $3 \mu \mathrm{M}$ Rotenone and $12 \mu \mathrm{M}$ Antimycin A (ROT/AA) following manufacturer's instruction. B. Quantitation of parameters for basal respiration, spare respiratory capacity and ATP-linked respiration. Data presented are the mean \pm s.e.m. using at least five technical replicates per experiment per genotype and condition. Basal respiration NT: CT $n=12$, HRas-KO n=6, NRas-KO n=12, DKO n=5; DEX: CT n=6, HRas-KO n=4, NRas-KO n=5, DKO n=3; NAC: CT n=8, HRas-KO n=3, NRas-KO n=8, DKO n=7; Spare respiratory capacity NT: CT $n=12$, HRas-KO n=6, NRas-KO n=14, DKO n=4; DEX: CT n=6, HRas-KO n=3, NRas-KO $n=3$, DKO $n=3$; NAC: CT $n=6$, HRas-KO n=3, NRas-KO $n=8$, DKO $n=5$; ATP-linked respiration NT: CT $n=11$, HRas-KO $n=4$, NRas-KO n=10, DKO n=4; DEX: CT n=3, HRas-KO n=3, NRas-KO n=3, DKO n=3; NAC: CT $\mathrm{n}=6$, HRas-KO $\mathrm{n}=3$, NRas-KO $\mathrm{n}=8$, DKO $\mathrm{n}=7 ; *_{v s} \mathrm{NT},{ }^{\$}{ }_{v s} \mathrm{NAC}, * / \mathrm{p}<0.05, * * \mathrm{p}<0,01, * * * \mathrm{p}<0.001$.

3.4. Effect of HRas/NRas ablation on epithelium-mesenchyme interaction

\subsubsection{HRas and KRas expression is increased in NRas deficient lung epithelium whereas KRas}

expression is increased in lung mesenchyme lacking both HRas and NRas

It has been described that, despite the high structural homology shared among the three canonical Ras GTPases, they are not functionally redundant (Esther Castellano \& Santos, 2011; C. W. Johnson et al., 2017). However, in certain contexts, Ras isoforms exhibit partial overlapping functions, as demonstrated by the viability of HRas-KO or NRas-KO but not DKO mice, or that the strong dependence on KRas signalling for embryonic development, can be partially rescued with a HRas transgene inserted under KRas promoter (Esteban et al., 2001; Johnson et al., 1997; Nakamura et al., 2008; Potenza et al., 2005). In this regard, to validate 
whether there was a compensatory effect of the other isoforms after the genomic ablation of HRas and/or NRas we performed RT-qPCR against HRas, NRas and KRas in both lung mesenchymal (Fig. 44B) cells and whole lung homogenates (Fig. 44A).

Interestingly, NRas ablation in whole lung samples led to a significant increase in HRas expression but, however, there was no similar compensatory effect on NRas expression in the HRas-KO lungs (Fig. 44A). In addition, when only the mesenchyme was analysed, no compensatory expression was observed in single HRas-KO or NRas-KO cells, pointing to a compensatory overexpression of HRas only in the epithelial cells of the NRas KO lungs (Fig. 44A). Additionally, HRas-KO, NRas-KO and DKO lungs showed slightly higher KRas expression (albeit not statistically significant), and an increased KRas gene expression was also detected in DKO lung fibroblasts, with a slight increment (but not statistically significant) in HRas-KO lung mesenchymal cells (Fig. 44A, B).

A
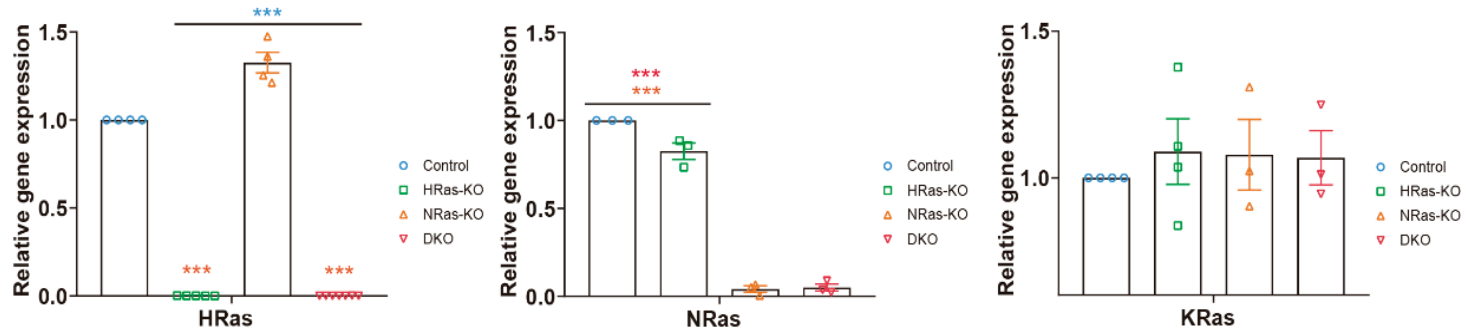

\section{B}
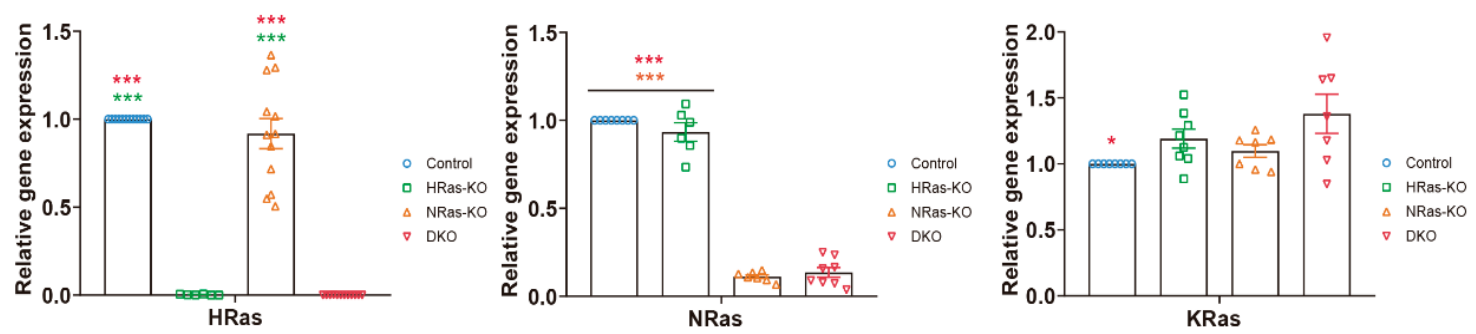

Figure 44: Gene expression analysis of the three Ras isoforms in lung epithelium and mesenchyme of P0 mice. A. mRNA expression levels of the indicated canonical Ras GTPases (HRas, NRas, KRas) were determined by quantitative RT-PCR analysis of RNA samples extracted from whole lung homogenates of the four relevant genotypes. The expression levels of $\beta$-2-microglobulin were used as internal controls for normalization in all cases. Sequence of the oligonucleotide primers used in RT-qPCR assays are shown in Materials and Methods. *p<0.05, $* * \mathrm{p}<0.01, * * * \mathrm{p}<0.001$. B. mRNA expression levels of the indicated canonical Ras GTPases (HRas, NRas, KRas) were determined by quantitative RT-PCR analysis of RNA samples extracted from primary lung fibroblasts isolated from newborn lungs of the four relevant genotypes. The expression levels of $\beta$-2-microglobulin were used as internal controls for normalization in all cases. Sequence of the oligonucleotide primers used in RT-qPCR assays are shown in Materials and Methods. ${ }^{*} \mathrm{p}<0.05, * * \mathrm{p}<0.01,{ }^{* * *} \mathrm{p}<0.001$. 


\subsubsection{Lack of HRas in lung mesenchyme causes higher levels of FGF7 and FGF10}

To investigate whether the defects observed in vivo in our mouse models were due to the lack of HRas and NRas in lung mesenchyme we isolated primary lung fibroblasts from newborn CT, HRas-KO, NRas-KO and DKO pups, as described in Materials and Methods. Knowing that Wnt signalling is crucial not only for lung epithelial development, but also has a key role in mediating FGF signalling in the adjacent mesenchyme (Aros et al., 2021; Herriges \& Morrisey, 2014; Volckaert et al., 2013).

For this reason, we analysed different components of the Wnt-FGF mesenchymal pathway, starting with the three different FGFs molecules (FGF7, FGF9, FGF10), known to be important to trigger the KRas-SOX9 signalling in the epithelium (FGF7 and FGF10), and in mesenchyme proliferation (FGF9) (Aros et al., 2021; Chang et al., 2013; Ostrin et al., 2018; PM et al., 2006; Ustiyan et al., 2016). RT-qPCR analyses on RNA extracted from these primary lung fibroblasts showed a significant increment in Fgf7 expression in those lung fibroblasts lacking HRas, alone (HRas-KO) and in combination with NRas (DKO) (Fig. 45). Additionally, only in DKO lung fibroblasts an increase in Fgflo gene expression was detected, but no differences were detected when $F g f 9$ expression levels were studied (Fig. 45).

To further analyse how HRas ablation affected the lung mesenchyme signalling, we also investigated whether the genes controlling the Fgfs expression were dysregulated. In this regard, $W n t 2$ and $W n t 2 b$ genes, key morphogens regulating lung mesenchyme signalling to the epithelium during lung development (Cardoso, 2008; Volckaert \& De Langhe, 2015), were analysed through RT-qPCR assays. In line with the overexpression of Fgf7 and Fgf10, higher levels of Wnt 2 mRNA were detected in lung fibroblasts lacking both HRas and NRas GTPases, however no changes were observed in $W n t 2 b$ gene expression (Fig. 45).

In addition, mesenchymal Wnt $2 / 2 \mathrm{~b}$ signalling is controlled by the upstream regulators Hox 5 genes (Hrycaj et al., 2015). For this, we analysed the expression levels of Hoxa5, Hoxb5 and Hoxc5 genes in our primary lung fibroblasts, observing no expression changes in Hoxa5, but a significant decrease in Hoxb5 expression in our DKO lung fibroblasts, followed by a significant upregulation in Hoxc5 genes when compared with the rest of genotypes (Fig. 45).

Interestingly, opposed to the observed higher expression of $W n t 2 b$, we observed a significant lower expression of Axin2 (Wnt/B-Catenin target) in both HRas-KO fibroblasts and DKO samples; but only significant lower expression of Lefl (Wnt/ $\beta$-Catenin target) was detected in DKO mesenchymal cells (Fig. 45). 

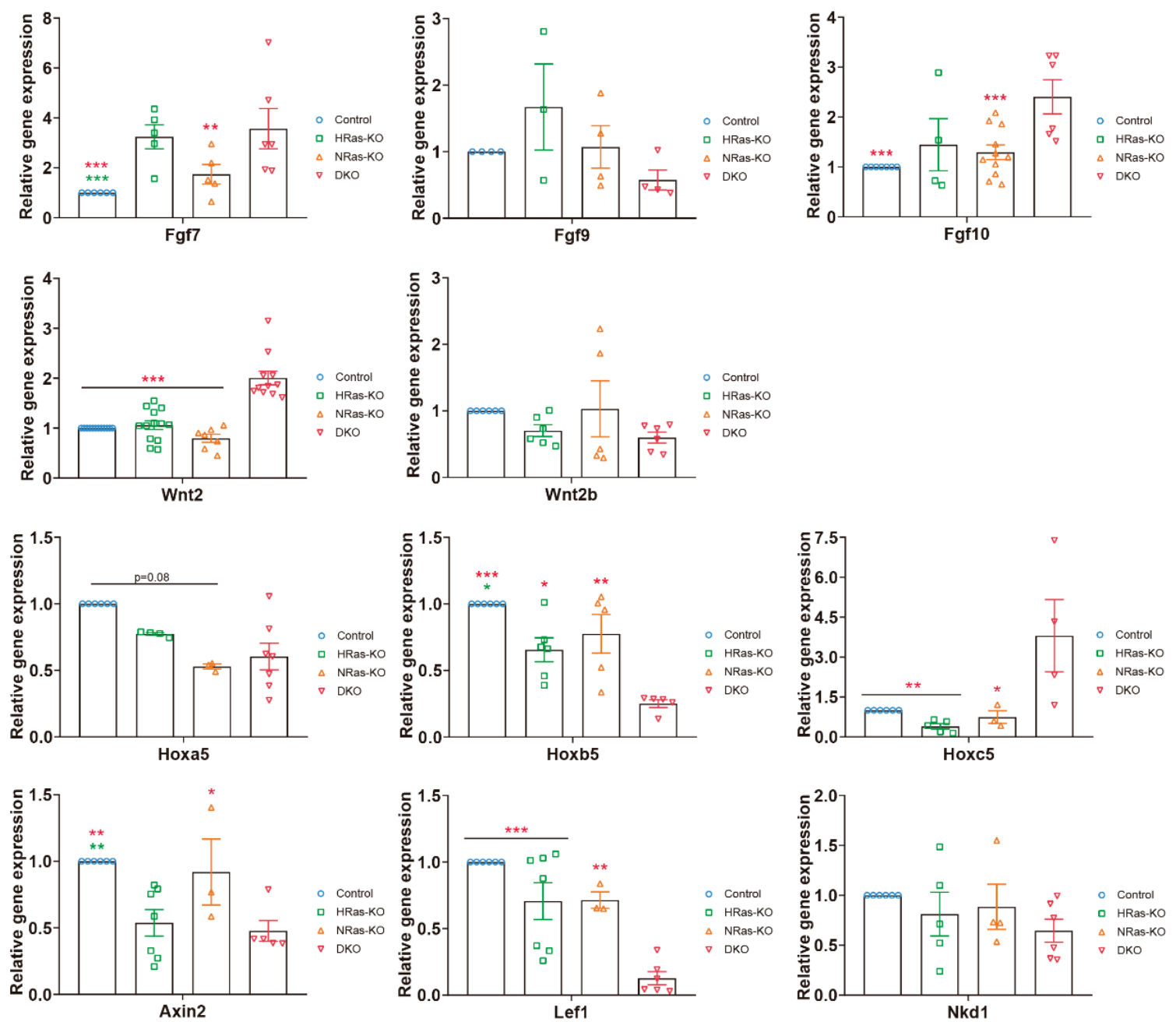

Figure 45: Analyses of the expression of genes known to participate in mesenchymal FGF production suggest a dysregulation of the Wnt-FGF signalling pathway in the DKO samples. mRNA expression levels of the indicated genes of Hox-Wnt-FGF signalling in the mesenchyme (Fgf7, Fgf9, Fgf10, Wnt2, Wnt2b, Hoxa5, Hoxb5, Hoxc5, Axin2, Lef1, Nkd1) were determined by quantitative RT-qPCR analysis of RNA samples extracted from primary lung fibroblasts isolated from P0 lungs of the four relevant genotypes. The expression levels of $\beta$-2microglobulin were used as internal controls for normalization in all cases. Sequence of the oligonucleotide primers used in RT-qPCR assays are shown in Materials and Methods. *p $<0.05, * * \mathrm{p}<0.01, * * * \mathrm{p}<0.001$.

3.4.3. FGF7 and FGF10 lung epithelial downstream signalling is altered in the lung epithelium after HRas/NRas ablation

FGF7 and FGF10 signalling in the lung epithelium is mediated through FGFR2b, which induces KRas activation, that ultimately would maintain the epithelial proliferation through SOX9 action. This loop is negatively controlled by Spry2, which acts on KRas activity, and BMP4 signalling, which limits FGFs-mediated bud outgrowth in the adjacent mesenchyme. Additionally, Wnt7a/Wnt7b are specific epithelial Wnt ligands that controls epithelial branching and mesenchymal proliferation (Chang et al., 2013; Ostrin et al., 2018; Volckaert \& De Langhe, 2015). 
In concordance with the previously observed Fgf7 and FgflO alterations in lung mesenchymal cells lacking HRas, whole lung extracts from HRas-KO and DKO mice exhibited a higher expression of the $F g f r 2 b$ receptor (Fig. 46). When epithelial Wnt ligands were analysed, no significant changes were detected in $W n t 7 b$ expression, albeit a slight increment was noticed in DKO lungs (Fig. 46). Additionally, a strong increase in Wnt7a expression was observed only in DKO lung samples (Fig. 46).

Interestingly, a reduction in Spry2 gene expression, the negative regulator or KRas, was present in HRas/NRas-null lungs, together with a small decrease (not statistically significant) in HRas-KO samples (Fig. 46). On the other hand, BMP4 was upregulated in DKO lungs when compared with the control samples (Fig. 46).

We then checked Nkx2/TTF-I epithelial expression, a known transcription factor specific of progenitor cells which acts orchestrating lung development and epithelial differentiation (Little et al., 2021; Warburton et al., 2000). In concordance with the observed increase in the number of SOX9+ cells, DKO lung samples showed a significant rise in $N k x 2$ gene expression as compared with the rest of genotypes (Fig. 46).
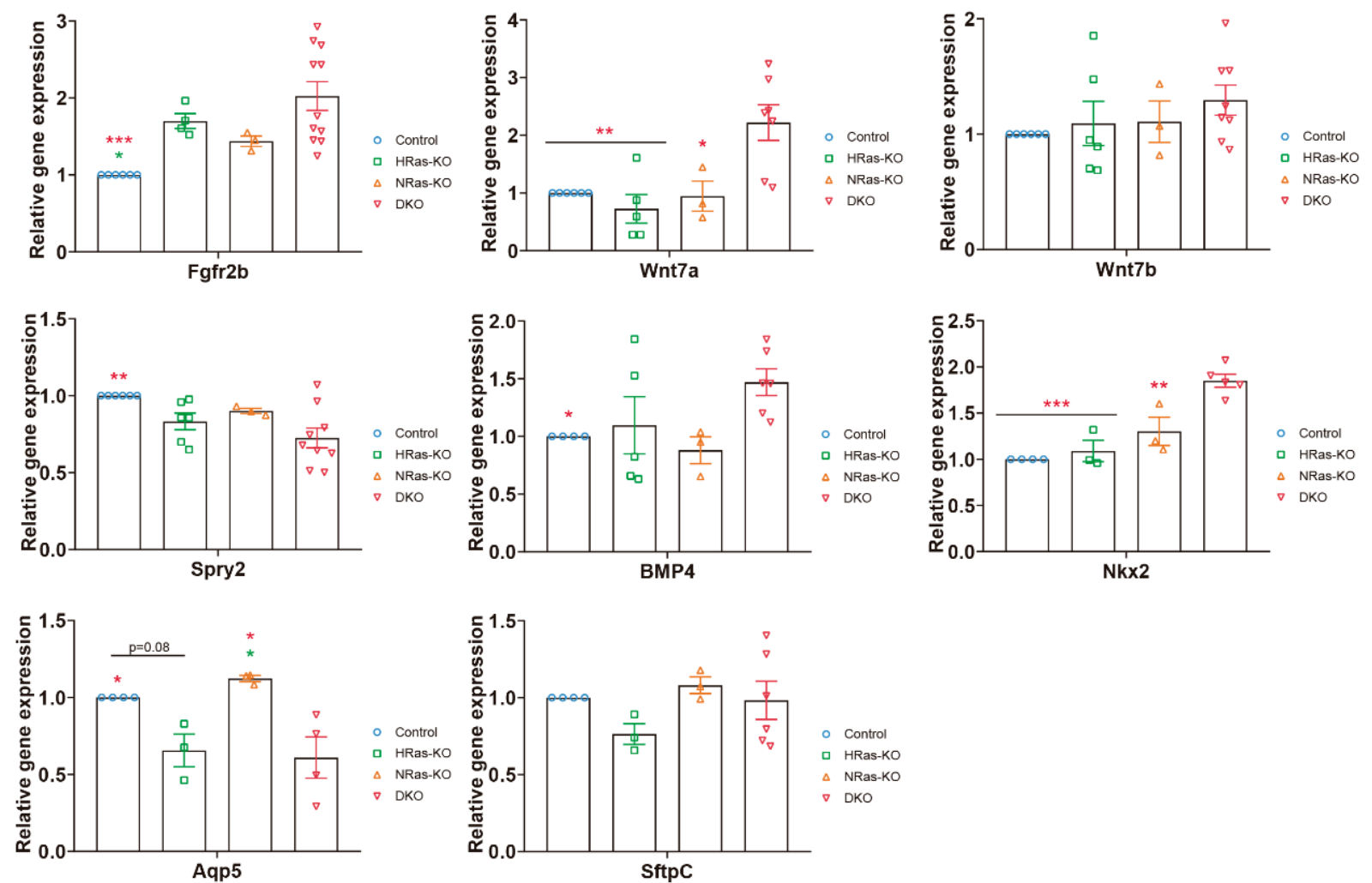

Figure 46: Expression analyses of genes known to participate in the epithelial signalling downstream FGF reveal a dysregulation of this pathway in the DKO samples and a reduction in alveolar differentiation markers. mRNA expression levels of the indicated genes of FGF-mediated signalling in the epithelium (Fgfr2b, Wnt7, Wnt7b, Spry2, $B M P 4, N k x 2, A q p 5$, SftpC) were determined by quantitative RT-PCR analysis of RNA samples extracted from whole P0 lung homogenates from the four relevant genotypes. The expression levels of $\beta$-2-microglobulin were used as internal controls for normalization in all cases. Sequence of the oligonucleotide primers used in RT-qPCR assays are shown in Materials and Methods. ${ }^{*} \mathrm{p}<0.05,{ }^{* *} \mathrm{p}<0.01, * * * \mathrm{p}<0.001$. 
To confirm delayed alveolar differentiation, we analysed Aqp5 gene expression, a specific marker of mature AT1 not expressed in bi-potent alveolar progenitors. A significant reduction in Aqp5 expression was detected in HRas/NRas-double mutant lungs when compared with CT and NRas-KO; and, in HRas-KO samples when compared with NRas-KO lungs (albeit HRas changes were not statistically significant versus $\mathrm{CT}$ samples $(\mathrm{p}=0.08)$ ) (Fig. 46). Nevertheless, no significant changes were noticed in SftpC gene expression, a marker of alveolar cells expressed from the bi-potent progenitors to mature AT2 cells (Fig. 46).

\subsubsection{Lung mesenchyme lacking HRas and NRas GTPases show increased ROS and dysregulation of ROS detoxifying enzymes}

Based on previously obtained results regarding ROS misbalancing in DKO whole lung samples we sought to investigate whether the HRas/NRas-DKO lung mesenchyme was also exhibiting the same phenotype. To achieve this, primary lung fibroblasts from the four genotypes under study were subjected to DCF, DHE and MitoSOX ${ }^{\mathrm{TM}}$ assays to detect $\mathrm{H}_{2} \mathrm{O}_{2}$, total $\mathrm{O}_{2}{ }^{-}$and mitochondrial $\mathrm{O}_{2}^{-}$respectively. In concordance with the above-mentioned results, lung fibroblasts lacking both HRas and NRas GTPases exhibited significant higher levels of $\mathrm{H}_{2} \mathrm{O}_{2}$, and of total and mitochondrial $\mathrm{O}_{2}^{-}$(Fig. 47). Interestingly, NRas-KO lung fibroblasts showed a slight increment in the three ROS analysed (not statistically significant), indicating a possible key role of NRas in controlling redox signalling (Fig. 47).
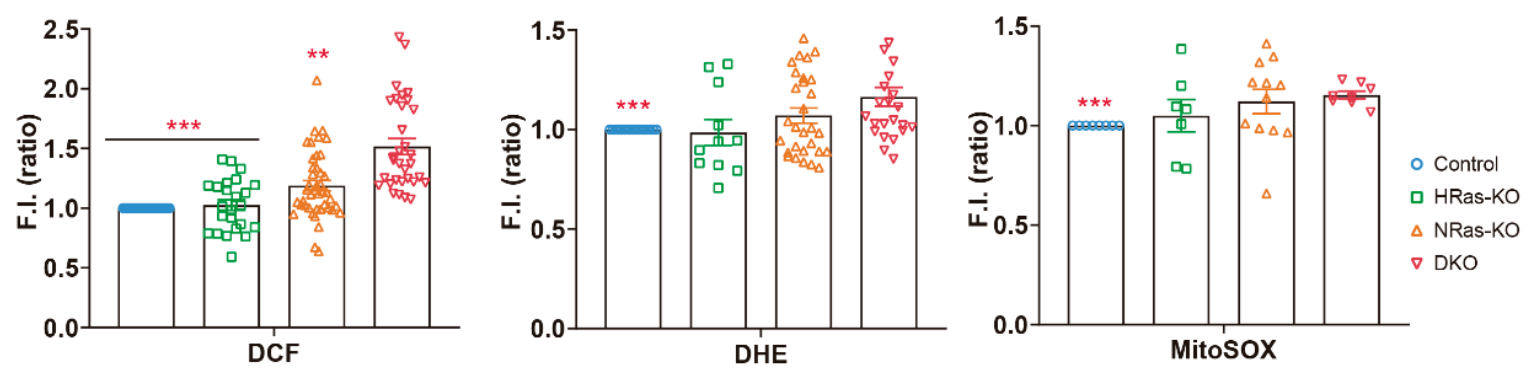

Figure 47: Lung mesenchymal cells of P0 HRas/NRas-DKO mice show augmented production of reactive oxygen species. The bar plots represent the in vivo quantitation, relative to Control, of redox parameters carried out by means of FACS fluorescence measurements performed (10.000 events in each case) on primary lung fibroblasts using specific fluorophores for intracellular hydrogen peroxide (DCF, $5 \mu \mathrm{M}$ ), intracellular total superoxide (DHE, $5 \mu \mathrm{M})$ and mitochondrial superoxide (MitoSOX ${ }^{\mathrm{TM}}, 5 \mu \mathrm{M}$ ) as described in Materials and Methods. Data expressed as the mean \pm s.e.m. DCF: CT $n=27$, HRas-KO n=24, NRas-KO n=43, DKO n=32; DHE: CT n=17, HRas-KO $n=11$, NRas-KO n=29, DKO n=24; MitoSOX ${ }^{\mathrm{TM}} \mathrm{CT} n=8$, HRas-KO n=7, NRas-KO n=12, DKO $n=8 . * * p<0.01, * * * p<0.001$.

The mechanisms regulating redox homeostasis include activation of several enzymes implicated in different ROS detoxifying pathways. Thus, we analysed the expression levels of different components of ROS scavenger pathways in lung fibroblasts by RT-qPCR assays. Among the three different superoxide dismutase (SOD) isoforms (SOD1 or cytoplasmic, SOD2 or mitochondrial, SOD3/EC-SOD or extracellular), no differences were observed in SOD1 and 
SOD2 expression in lung fibroblasts, however a strong SOD3 overexpression was detected in DKO lung fibroblasts samples (Fig. 48). Additionally, a slight decrease in the expression of glutathione peroxidases (GPX1, GPX2, GPX3) was observed in both HRas-KO and DKO mesenchymal cells (Fig. 48). Within the different glutathione S-transferases (GST), a statistical increment in GSTa2 was noticed in the DKO samples, together with a noticeable (but not statistically significant) increase in both HRas-KO and NRas-KO lung fibroblasts (Fig. 48).
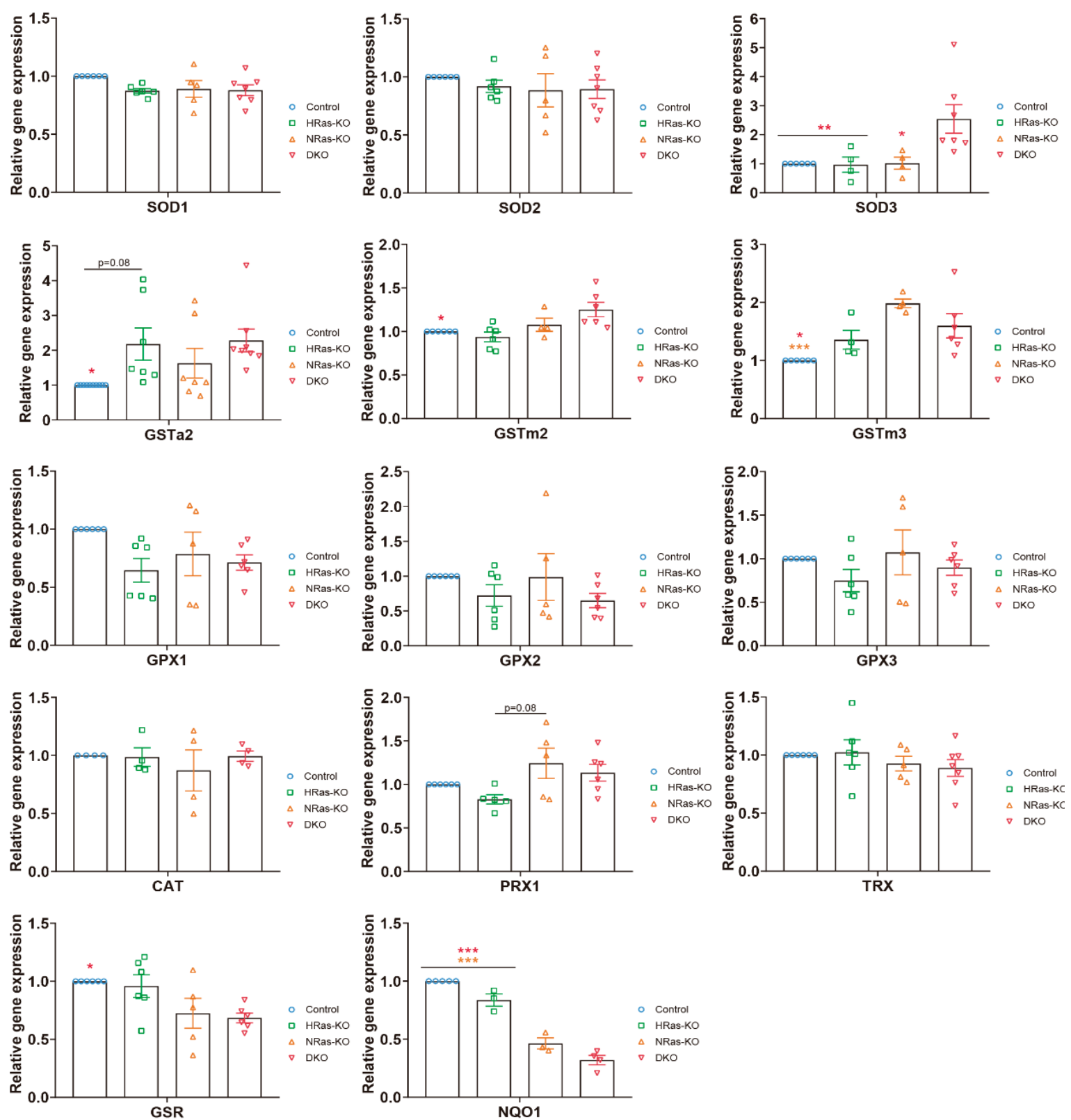

Figure 48: Altered gene expression of enzymes implicated in ROS detoxifying pathways in DKO lung mesenchymal cells. mRNA expression levels of the indicated genes of redox and antioxidant detoxifying enzyme isoforms (SOD1, SOD2, SOD3, GSTa2, GSTm2, GSTm2, GPX1, GPX2, GPX3, CAT, PRX1, TRX, GSR, NQO1) were determined by quantitative RT-PCR analysis of RNA samples extracted from whole P0 lung homogenates from the four relevant genotypes. The expression levels of $\beta$-2-microglobulin were used as internal controls for normalization in all cases. Sequence of the oligonucleotide primers used in RT-qPCR assays are shown in Materials and Methods. ${ }^{*} \mathrm{p}<0.05, * * \mathrm{p}<0.01, * * * \mathrm{p}<0.001$.

We detected also an increment in GSTm2 expression in HRas/NRas-double null samples, and of GSTm3 in both NRas-KO and DKO mesenchymal cells (Fig. 48). No differences were observed when the catalase (CAT), peroxiredoxin 1 (PRXI) and thioredoxins (TRXI) enzymes 
were analysed (Fig. 48). Nevertheless, a significant downregulation in glutathione S-reductase $(G S R)$ was noticed in DKO samples, as well as in NRas-KO samples (not statistically significant) (Fig. 48). Regarding NAD(P)H Quinone Dehydrogenase 1 (NQO1), key enzyme controlling ubiquinone and vitamin $\mathrm{E}$ quinone metabolism, and thus, protecting against oxidative stress, appeared downregulated in both NRas-KO and DKO lung mesenchymal cells when compared to the control samples (Fig. 48).

3.4.5. Concomitant ablation of HRas and NRas in lung mesenchymal cells worsens mitochondrial respiration

To determine whether the increased oxidative stress observed in lung mesenchymal cells reflected in mitochondrial alterations, we analysed the mitochondrial respiration using Seahorse-based technology and MitoStress tests (Fig. 49A).

OCR measurements detected significantly reduced rate of basal respiration in those lung

$\mathbf{A}$

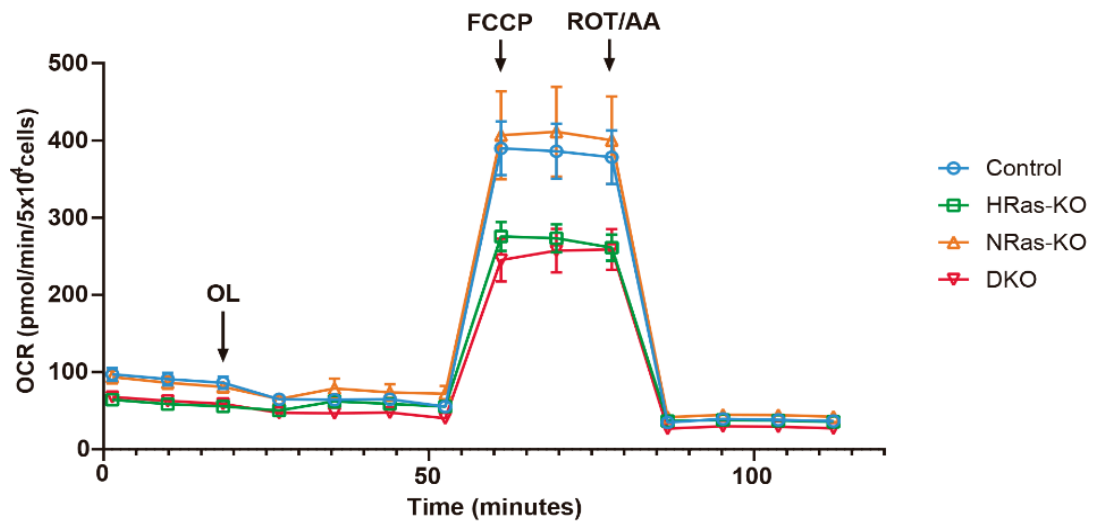

B
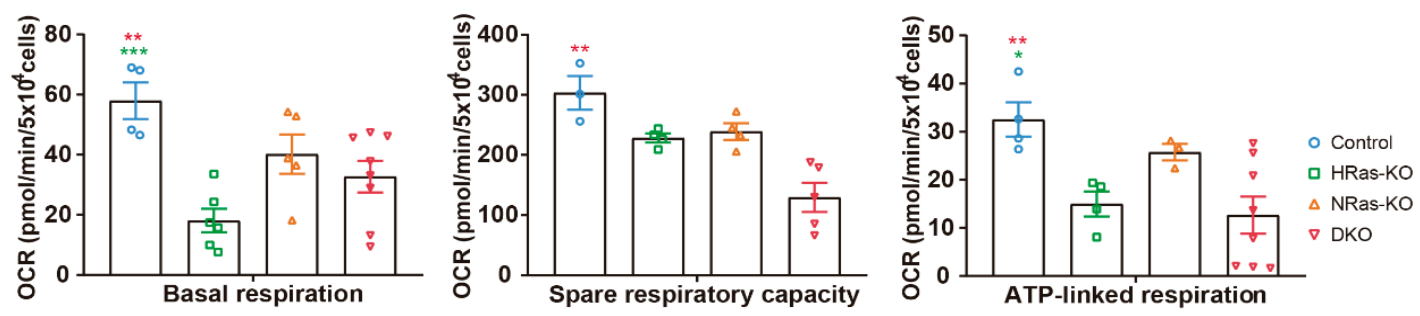

Figure 49: Lung mesenchymal cells devoid of HRas show lower basal respiration, spare respiratory capacity, and ATP-linked respiration. A. MitoStress profile from primary lung fibroblasts of the indicated genotypes. OCR (oxygen consumption rate) was measured under basal conditions followed by the sequential addition of 5.5 $\mu$ M Oligomycin (OL), $1 \mu \mathrm{M}$ FCCP, and $1 \mu \mathrm{M}$ Rotenone and $1 \mu \mathrm{M}$ Antimycin A (ROT/AA) following manufacturer's instruction. B. Quantitation of parameters for basal respiration, spare respiratory capacity and ATP-linked respiration. Data presented as the mean \pm s.e.m. using at least five technical replicates per experiment per genotype. Basal respiration: $\mathrm{CT} n=4$, HRas-KO n=6, NRas-KO n=5, DKO n=7; Spare respiratory capacity: CT n=3, HRas-KO n=4, NRas-KO n=4, DKO $\mathrm{n}=5$; ATP-linked respiration: $\mathrm{CT} \mathrm{n}=4$, HRas-KO n=4, NRas-KO n=3, DKO n=8. * $\mathrm{p}<0.05, * * * \mathrm{p}<0.001$.

fibroblasts lacking HRas (HRas-KO and DKO samples) (Fig. 49A, B). Additionally, significant 
diminished spare respiratory capacity and ATP-linked respiration was observed only in DKO lung fibroblasts (Fig. 49B). Interestingly, despite the previously observed possible link between NRas loss and oxidative stress, no significant alterations in mitochondrial respiration parameters were detected, meaning that mitochondrial malfunction might not be the cause of increased ROS production, pointing to a potential effect driven by the altered ROS detoxifying metabolism (Fig. 49B).

3.4.6. NAC treatment reduces mesenchymal oxidative stress and reduces mitochondrial respiration whereas DEX treatment partially lower ROS production and enhances mitochondrial respiration

To explore the effects of NAC and DEX specifically on lung mesenchymal cells, we treated primary lung fibroblasts during $48 \mathrm{~h}$ with either 10mM NAC or 60nM DEX. Quantification of DCF showed a strong reduction in $\mathrm{H}_{2} \mathrm{O}_{2}$ in NAC-treated cells, but DEX produced no changes in $\mathrm{H}_{2} \mathrm{O}_{2}$ production and even producing a slight increment in DEX-treated HRas-KO and NRasKO cells as compared with the untreated ones (Fig. 50). On the other hand, both DEX and NACtreated cells showed a decrease in total $\mathrm{O}_{2}^{-}$production, as measured with DHE. Interestingly, no changes were observed in mitochondrial $\mathrm{O}_{2}{ }^{-}$production after DEX treatment, and NAC only reduced the mitochondrial $\mathrm{O}_{2}{ }^{-}$in DKO samples, but not in NAC-treated NRas-KO mesenchymal cells (Fig. 50).

When the mitochondrial respiration values were analysed, no changes were detected between DEX-treated CT, HRas-KO, NRas-KO and DKO lung fibroblasts (Fig. 51A). Furthermore, an increment was detected in Spare respiratory capacity of DKO cells treated with DEX when
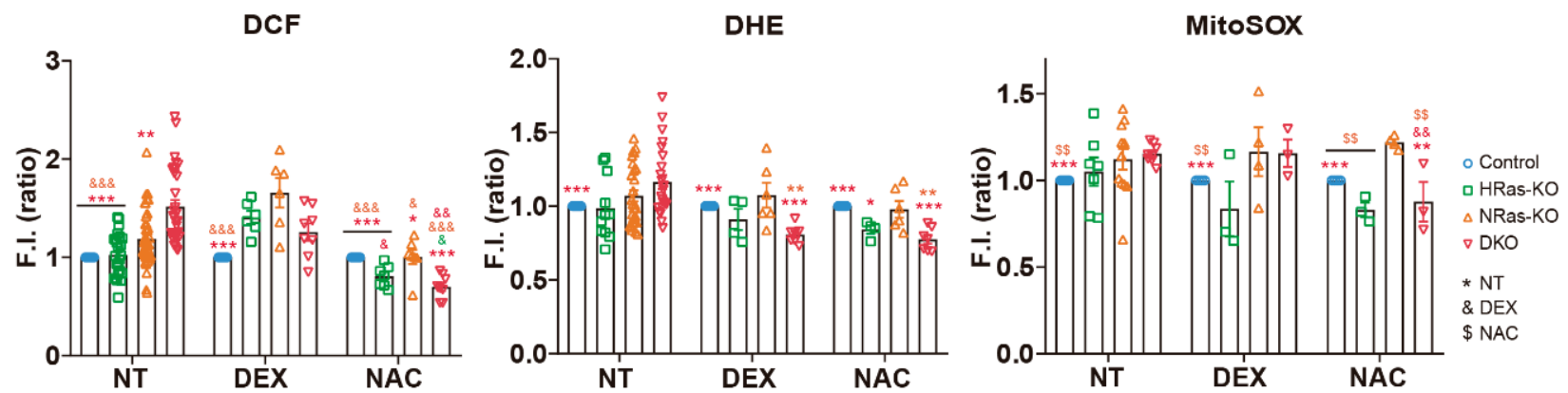

Figure 50: Lung mesenchymal cells of P0 HRas/NRas-DKO mice show an increment in production of reactive oxygen species production. The bar plots represent the in vivo quantitation, relative to Control, of redox parameters carried out by means of FACS fluorescence measurements performed (10.000 events in each case) on primary lung fibroblasts, either untreated (NT), DEX or NAC-treated, using specific fluorophores for intracellular hydrogen peroxide (DCF, $5 \mu \mathrm{M}$ ), intracellular total superoxide (DHE, $5 \mu \mathrm{M}$ ) and mitochondrial superoxide (MitoSOX ${ }^{\mathrm{TM}}, 5 \mu \mathrm{M}$ ) as described in Materials and Methods. Data is expressed as the mean \pm s.e.m. DCF NT: CT $n=27$, HRas-KO $n=24$, NRas-KO n=43, DKO n=32; DEX: CT n=11, HRas-KO n=6, NRas-KO n=6, DKO n=9; NAC: CT n=11, HRas-KO $\mathrm{n}=7$, NRas-KO n=7, DKO n=9; DHE NT: CT $n=17$, HRas-KO $n=11$, NRas-KO n=29, DKO n=24; DEX/NAC: CT $\mathrm{n}=8$, HRas-KO n=4, NRas-KO n=6, DKO n=7; MitoSOX ${ }^{\mathrm{TM}}$ NT: CT n=8, HRas-KO n=7, NRas-KO n=6, DKO n=8; DEX/NAC: CT $\mathrm{n}=4$, HRas-KO $\mathrm{n}=3$, NRas-KO $\mathrm{n}=4$, DKO $\mathrm{n}=3 *{ }_{v s} \mathrm{NT},{ }^{\&}{ }_{v s}$ DEX; ${ }^{*}{ }_{v s} \mathrm{NAC}, * /{ }_{\mathrm{p}}<0.05$, $* * / \& \& / \$ \$ \mathrm{p}<0,01, * * * / \& \& \& \mathrm{p}<0.001$. 
compared with the untreated ones, as well as an increase (albeit not statistically significant) in ATP production linked-respiration (Fig. 51B). In contrast, NAC treatment of the cells caused an overall subtle attenuation of mitochondrial respiration parameters, affecting all genotypes; being more evident in the spare respiratory capacity OCR values of mesenchymal cells lacking HRas (HRas-KO and DKO) (Fig. 51A, B).

A
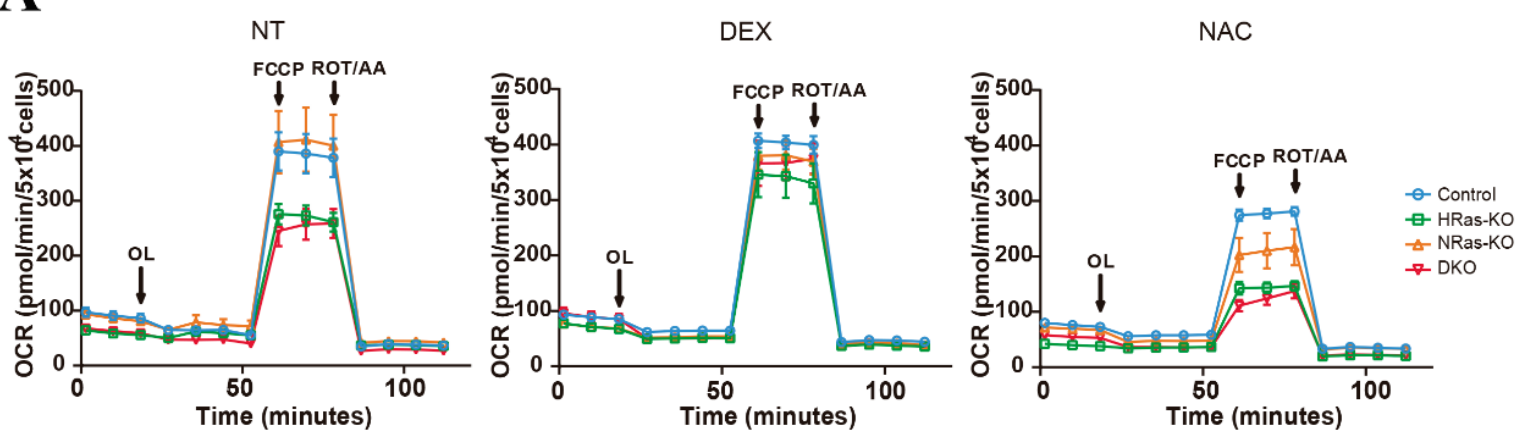

\section{B}
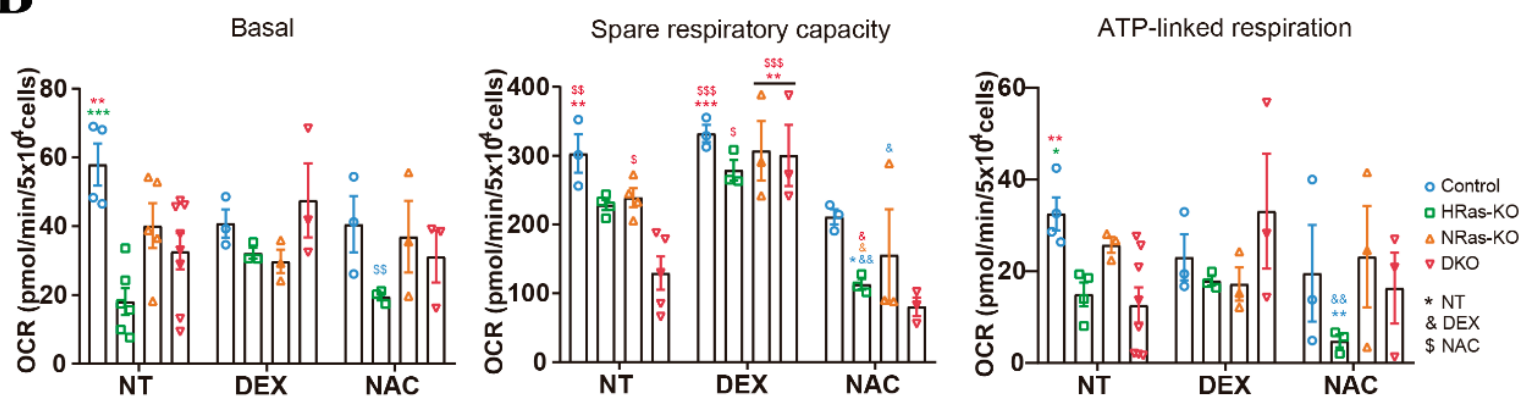

Figure 51: Dexamethasone rescues the lower basal respiration, spare respiratory capacity and ATP-linked respiration observed in HRas-KOs and DKO lung fibroblasts. A. MitoStress profile from primary lung fibroblasts of the indicated genotypes and treatments. OCR (oxygen consumption rate) was measured under basal conditions followed by the sequential addition of $5.5 \mu \mathrm{M}$ Oligomycin $(\mathrm{OL}), 1 \mu \mathrm{M}$ FCCP, and $1 \mu \mathrm{M}$ Rotenone and $1 \mu \mathrm{M}$ Antimycin A (ROT/AA) following manufacturer's instruction. B. Quantitation of parameters for basal respiration, spare respiratory capacity and ATP-linked respiration. Data presented as the mean \pm s.e.m. using at least five technical replicates per experiment per genotype. Basal respiration NT: CT $n=4$, HRas-KO n=6, NRas-KO n=5, DKO n=7; DEX/NAC $n=3$ for all genotypes; Spare respiratory capacity NT: CT $n=3$, HRas-KO n=4, NRas-KO n=4, DKO n=5; DEX/NAC $n=3$ for all genotypes; ATP-linked respiration NT: CT $n=4$, HRas-KO n=4, NRas-KO n=3, DKO n=8; DEX/NAC $\mathrm{n}=3$ for all genotypes. ${ }^{*} v s \mathrm{NT},{ }^{\&} v s \mathrm{DEX} ;{ }^{\$}{ }_{v s} \mathrm{NAC},{ }^{*} \mathrm{p}<0.05, * * / \& \& / \$ \mathrm{p}<0,01, * * * \$ \$ \$ \mathrm{p}<0.001$.

\subsubsection{Mesenchymal gene expression changes linked to DEX and NAC treatment}

To test whether DEX or NAC administration was affecting mesenchymal signalling, we performed RT-qPCR assays of some of the key genes analysed before in the untreated cells.

A strong reduction in $\mathrm{Fgf7}$ gene expression was noticed in lung mesenchymal cells lacking HRas after both DEX and NAC treatments, but, interestingly, only NAC induced a decrease in Fgf10 expression levels in DKO samples (Fig. 52). The increased Wnt2 levels observed in the untreated DKO cells were reverted to normal values after the addition of both DEX or NAC during $48 \mathrm{~h}$, and no significant changes were noticed in $W n t 2 b$ expression levels between the different conditions (Fig. 52). 
Among Hox 5 genes, none of the treatments produced differences in Hoxa 5 gene expression. However, a strong increase in Hoxb5 was detected in HRas-KO and DKO cells, but Hoxc5 appeared downregulated in DKO mesenchymal cells after DEX or NAC treatment, with HRasKO and NRas-KO samples showing also almost a null expression of this factor (Fig. 52).

Fgf7

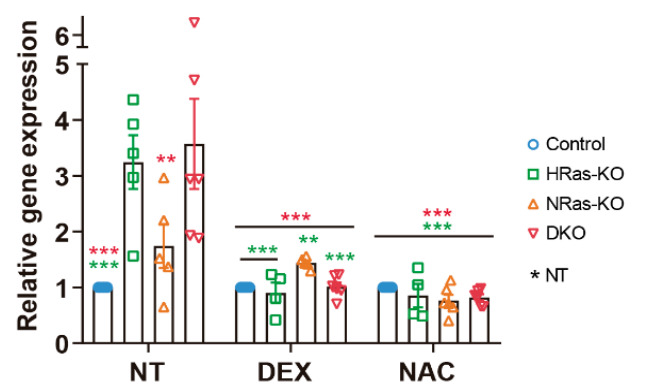

Wnt2

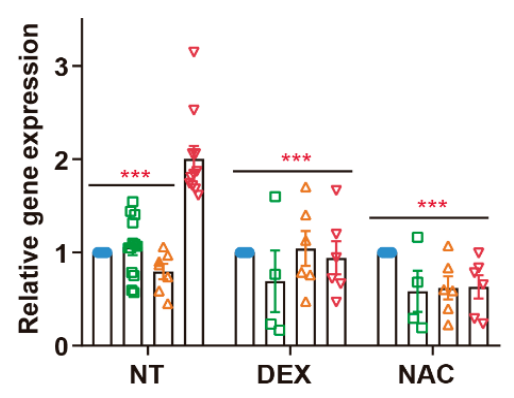

Hoxa5

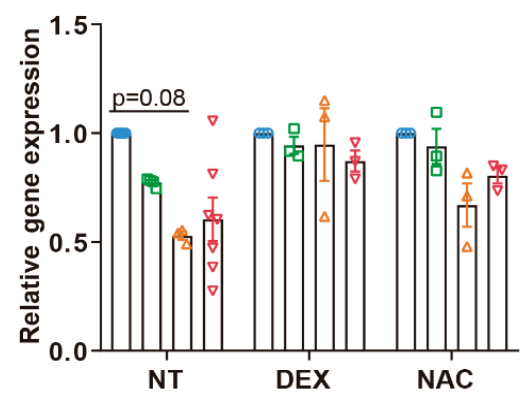

Hoxc5

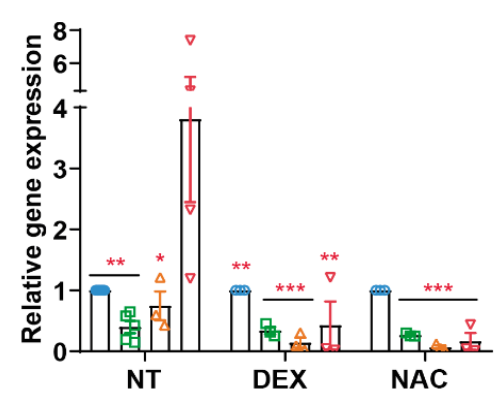

Fgf10

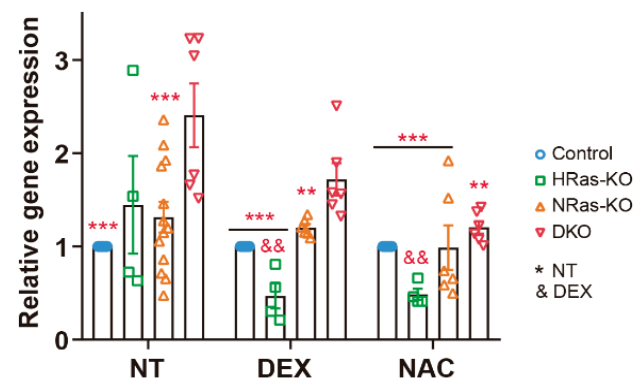

Wnt2b
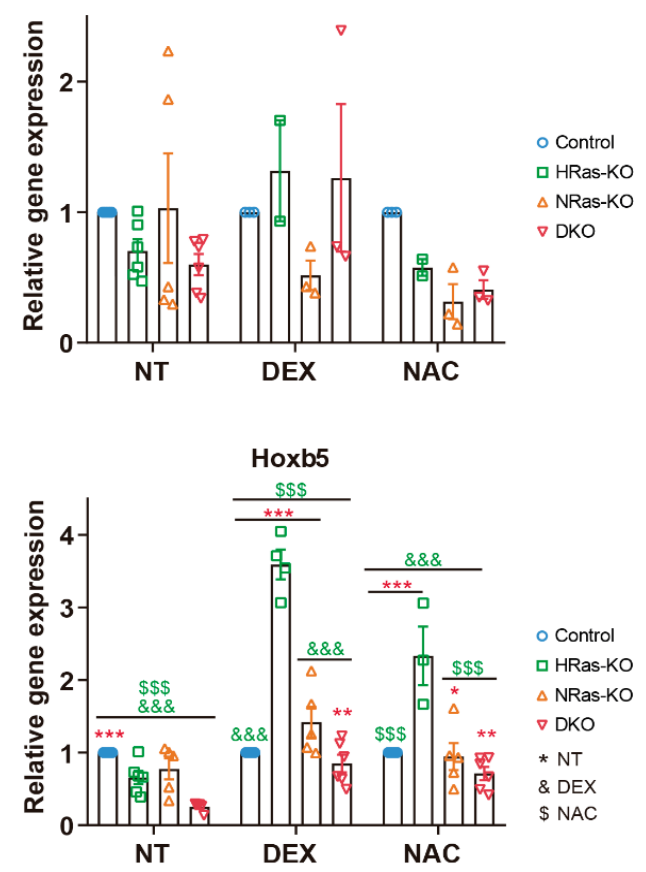

SOD3

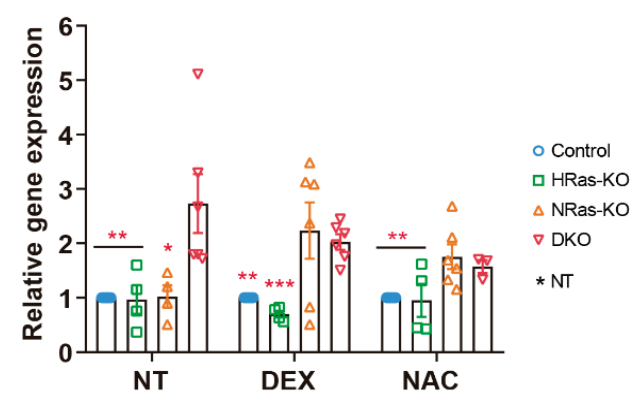

Figure 52: NAC and DEX treatment of lung mesenchymal cells decreases expression of FGF and related genes as well as SOD3 in DKO cells. mRNA expression levels of the indicated genes of Hox-Wnt-FGF signalling in the mesenchyme (Fgf7, Fgf10, Wnt2, Wnt2b, Hoxa5, Hoxb5, Hoxc5) as well as SOD3 expression were determined by quantitative RT-PCR analysis of RNA samples extracted from primary lung fibroblasts isolated from P0 lungs of the four relevant genotypes subjected either to DEX, NAC or no treatment. The expression levels of $\beta$ 2-microglobulin were used as internal controls for normalization in all cases. Sequence of the oligonucleotide primers used in RT-qPCR assays are shown in Materials and Methods. Mean \pm s.e.m, ${ }^{*} v s$ NT, ${ }^{\&} v s$ DEX; ${ }^{\$} v s$ NAC, $* \mathrm{p}<0.05, * * / \& \& / \$ \mathrm{p}<0,01, * * * / \& \& \& \mathrm{p}<0.001$ 
Finally, we sought to analyse SOD3 expression levels due to its key role not only in controlling lung oxidative stress, but also in modulating signalling pathways such as AKT and Ras activation (Laukkanen, 2016; Laurila et al., 2009; Sah et al., 2020). In this regard, a decrease in SOD3 expression levels was detected in NAC-treated DKO mesenchyme (not statistically significant), whereas DEX treatment induced no observable changes in HRas/NRas-null samples and weakly increased SOD3 levels in NRas-KO treated samples (albeit not statistically significant) (Fig. 52).

These results indicate a potential role of HRas isoform in the control of late lung embryonic development signalling, being HRas loss partially modulated with DEX and NAC treatments.

3.5. HRas isoform function is crucial for KRas-driven lung branching termination, with NRas exhibiting also a partial overlapping function

\subsubsection{Loss of HRas and NRas is associated to augmented yields of small lung organoids}

We evaluated the effect of HRas and/or NRas ablation (alone and in combination) in the mesenchyme, in presence of a WT epithelium, in lung organoids formation. HRas null mesenchyme resulted in higher organoid yield numbers, which increased when we also removed NRas, producing statistically significant higher organoid numbers compared with CT, HRasKO and NRas-KO samples (Fig. 53A, B). Interestingly, both HRas-KO and DKO organoids were slightly smaller as compared to CT and NRas-KO genotypes (Fig. 53A, C).

Treatment with Dexamethasone resulted in overall fewer organoids in all the genotypes, but the elevated organoid number in HRas-KO DEX-treated (not statistically significant) and DKO DEX-treated was still present, and no differences in size were observed after the treatment with DEX (Fig. 53A-C).

We also tested the effect of NAC, which resulted in a general increment in organoid numbers in all the genotypes, with no differences among them. In addition, all the NAC-treated culture wells showed an increment in the number of small organoids (Fig. 53A-C). 
A
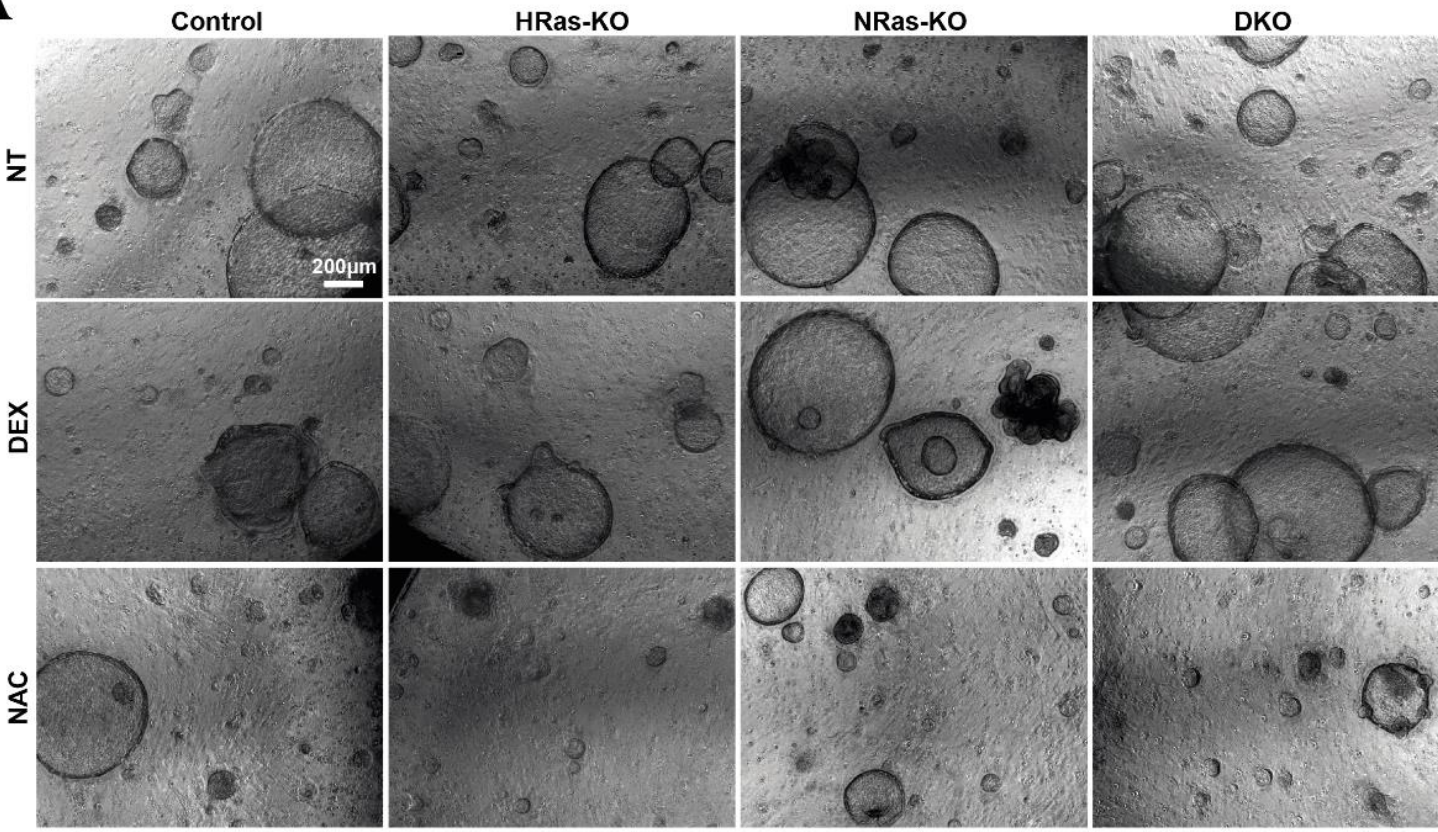

B

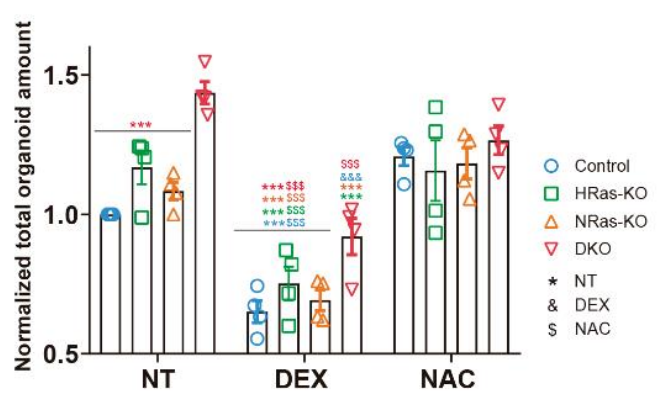

C

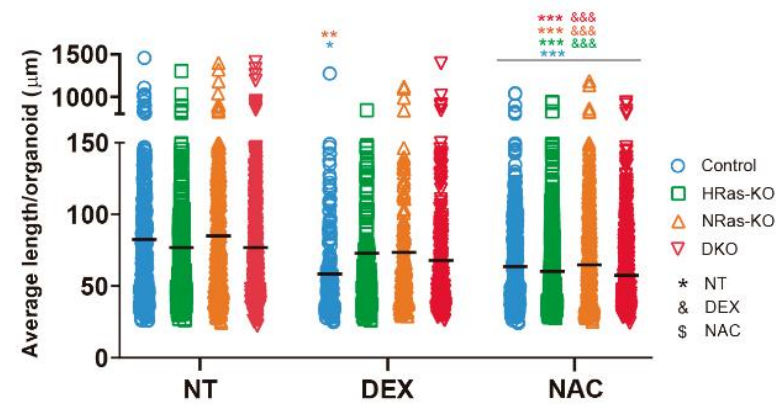

Figure 53: Lung organoids obtained from untreated and DEX- and NAC- treated samples. A. Representative stack images of 14-days organoids obtained from the different combinations of genotypes and treatments. Untreated (NT), dexamethasone (DEX), N-Acetylcysteine (NAC), scale bar $200 \mu \mathrm{m} . \mathrm{n}=4$ for all genotypes and conditions. B. Total organoid number relativized against the untreated control. Mean \pm s.e.m. $\mathrm{n}=4$ for all genotypes and conditions. $*_{v s} \mathrm{NT},{ }^{\&}{ }_{v s} \mathrm{DEX} ;{ }^{\$}{ }_{v s} \mathrm{NAC}, * * * / \& \& \& / \$ \$ \$ \mathrm{p}<0.001$. C. Graph representing the average organoid size. Median values are represented, $\mathrm{n}=4$ for all genotypes and conditions, $<200$ organoids per sample were measured. *vs $\mathrm{NT}$, ${ }^{\star} v s$ DEX; $* \mathrm{p}<0.5, * * \mathrm{p}<0.01, * * * / \& \& \& \mathrm{p}<0.001$.

\subsubsection{HRas and NRas activity is necessary for proper lung organoid differentiation and NAC}

\section{partially rescues the aberrant phenotype}

To evaluate the organoid differentiation, we performed immunoassays against SftpC and Acetylated-Tubulin (AcTub) in order to determine the percentage of organoids that had an alveolar, bronchiolar, double-positive or double-negative phenotype. As expected, based on our in vivo observations in mice, the resulting DKO organoids were mainly poorly differentiated double-negative and bronchiolar organoids, with a few numbers of the alveolar type (Fig. 54AC). Interestingly, HRas-KO samples exhibited a high percentage of double-positive organoids, and there were no differences between CT and NRas-KO (Fig. 54A-C). 
A Sftpc / AcTub / DAPI

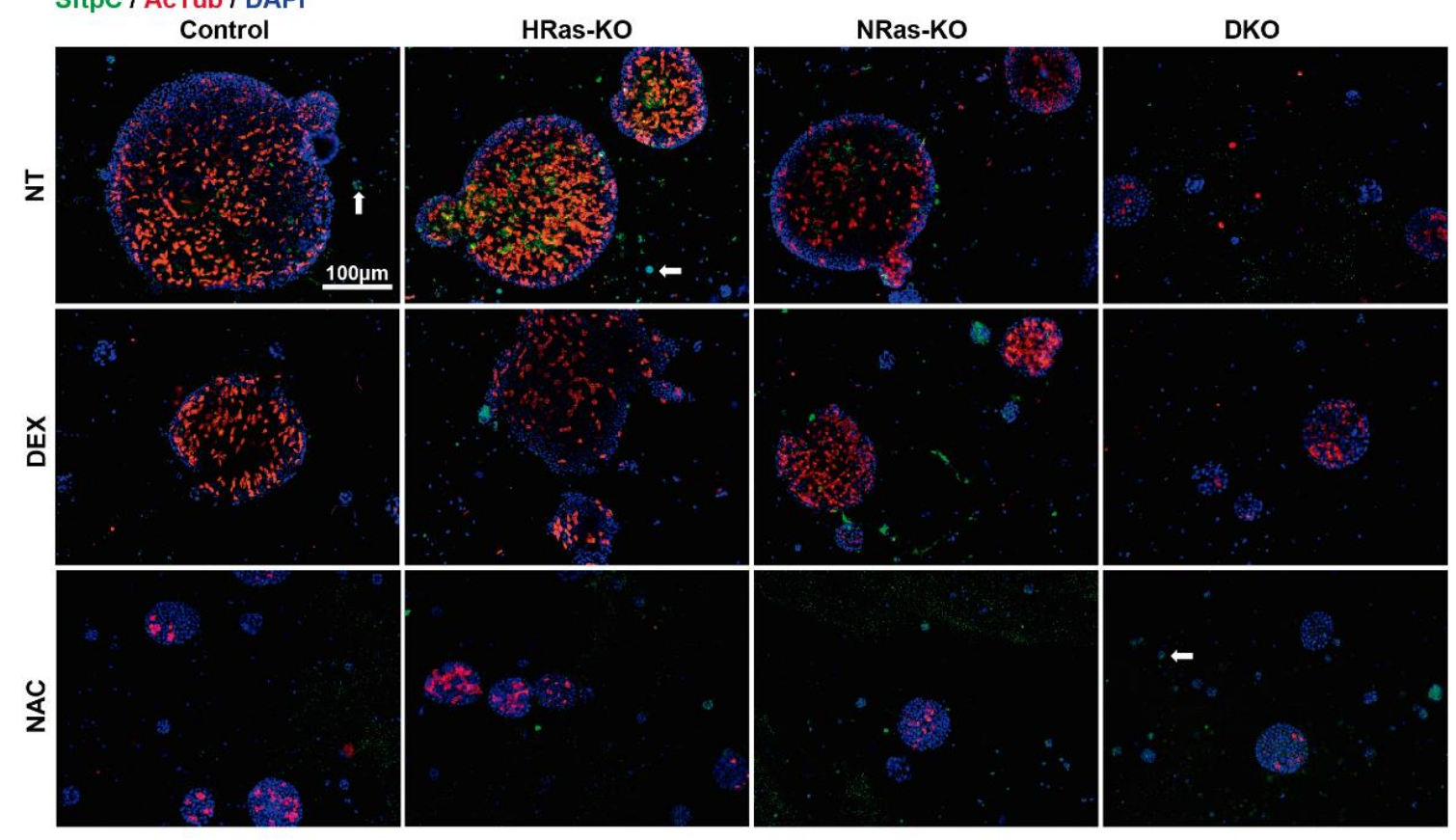

B

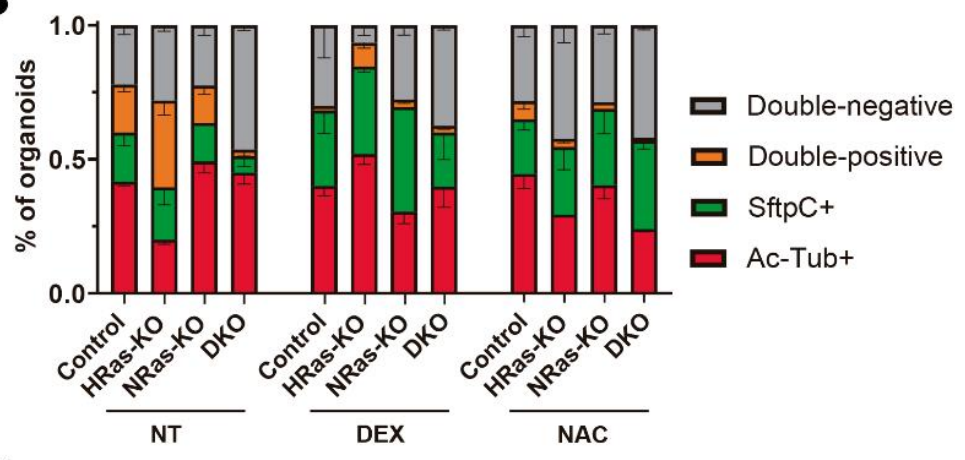

C
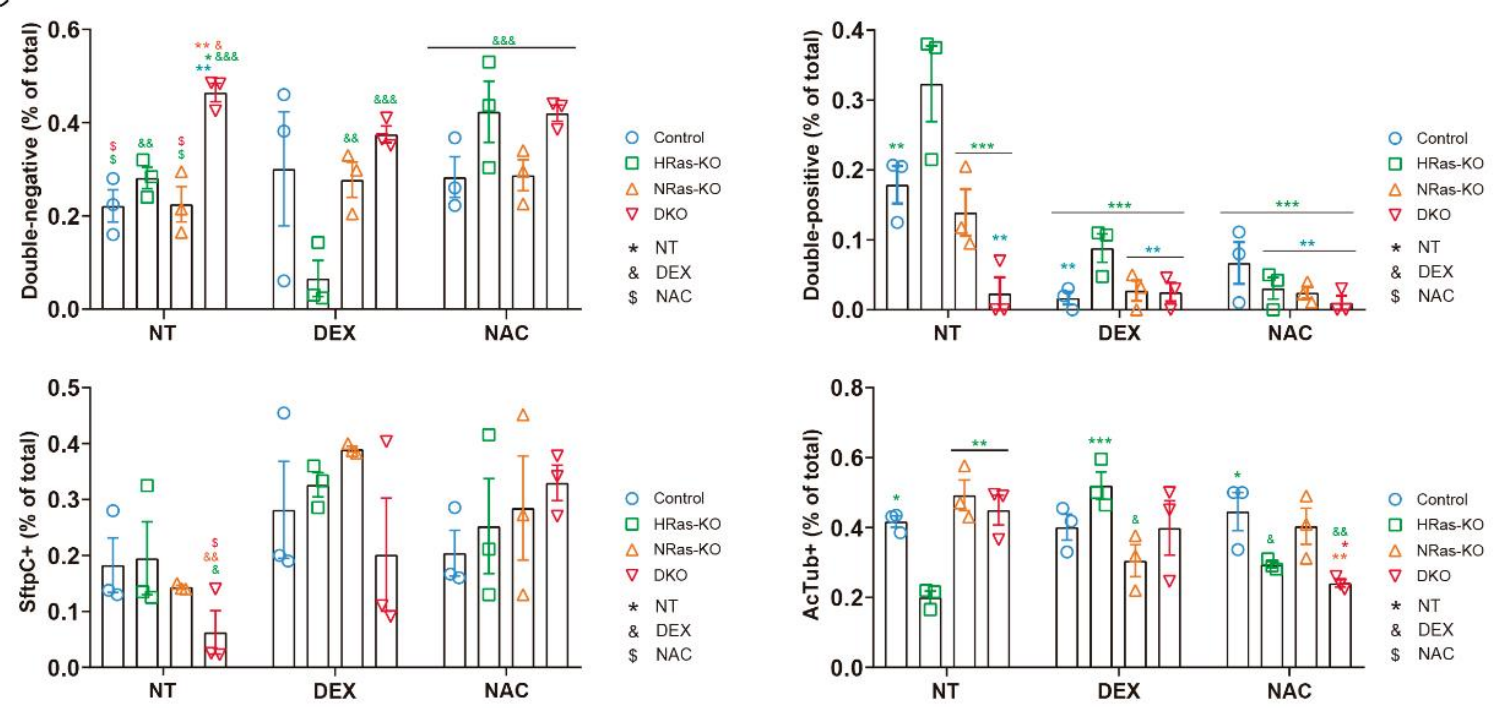

Figure 54: Effects of DEX and NAC treatment on lung organoid differentiation. A. Representative immunofluorescence images of 14-days organoids obtained from the different combinations of genotypes and treatments. Untreated (NT), dexamethasone (DEX), N-Acetylcysteine (NAC). Markers: Blue DAPI (nuclei), green SftpC (alveolar), red Ac-Tub (bronchiolar). White arrows point to small alveolar organoids. Scale bar 100 $\mu \mathrm{m}$. $\mathrm{n}=3$ for all the genotypes and conditions. B. Quantification of organoid proportion expressing SftpC (green), Ac-Tub (red), both markers (double-positive, orange) and neither (double-negative, grey). $n=3$ for all the genotypes and 
conditions. C. Individual quantification of organoid proportion. Data is represented as mean \pm s.e.m. $n=3$ for all the genotypes and conditions. ${ }^{*} v s \mathrm{NT},{ }^{\&}{ }_{v s} \mathrm{DEX},{ }^{\$} v s \mathrm{NAC} ; * / \& / \$ \mathrm{p}<0.5, * * / \& \& \mathrm{p}<0.01, * * * / \& \& \& \mathrm{p}<0.001$.

\subsubsection{KRas signalling is crucial for lung organoid formation onset}

To get further insights on the mechanism underling the aberrant communication between epithelium and mesenchyme in our DKO, and to a lesser extent HRas-KO samples, we tested different inhibitors of Ras downstream signalling pathways, such as MAPK-ERK (UO126), PI3K-AKT (Ly294002), and p38 (SB202190), and a selective KRas inhibitor (Fendiline).

KRas inhibition resulted in almost complete abrogation of organoid formation, being the few resulting organoids much smaller in size in all the genotypes compared with the DMSO-treated samples (Fig. 55A-C). However, slightly fewer organoids were obtained with UO126 pan-MEK inhibitor in comparison with the DMSO treated (not statistically significant), but those organoids were smaller in size without differences between the different genotypes (Fig. 55AC).

In contrast, inhibition of PI3K pathway with Ly294002 gave rise to an increment in organoid formation in all the genotypes (not statistically significant), showing no important changes regarding to organoid size compared with the DMSO treated (Fig. 55A-C). Finally, the effect of treating the organoids with SB202190 p38 inhibitor was a strong increment in organoid number, accompanied with no changes in organoid sizes as compared with the vehicle treated ones (Fig. 55A-C).

\subsubsection{Inhibition of Ras-MEK-ERK signalling rescues alveolar differentiation in HRas/NRas-} DKO lung organoids

Additionally, we evaluated the effect of these treatments on lung organoid cell differentiation. The DMSO-treated organoids followed the same pattern than that observed in the previous experiment, with the DKO exhibiting a significant reduction in alveolar organoids (Fig. 56A, B \& Fig.57). Interestingly, treatment with Fendiline resulted in a decrease in bronchiolar organoids in all the genotypes except the NRas-KO samples (Fig. 56A, B \& Fig.57). On the other hand, total inhibition of Ras-MEK-ERK pathway with UO126 derived in an important increment of alveolar organoids when compared with the DMSO-treated (Fig. 56A, B \& Fig. 57). Ly294002-treated organoids did not show important changes regarding organoid differentiation except for a decrease in alveolar and an increment in bronchiolar organoids in the control samples (Fig. 56A, B \& Fig. 75). Lastly, samples subjected to p38 inhibition showed a significant increment of double-negative organoids, representing more than $90 \%$ of total (Fig. 56A, B \& Fig. 57). 


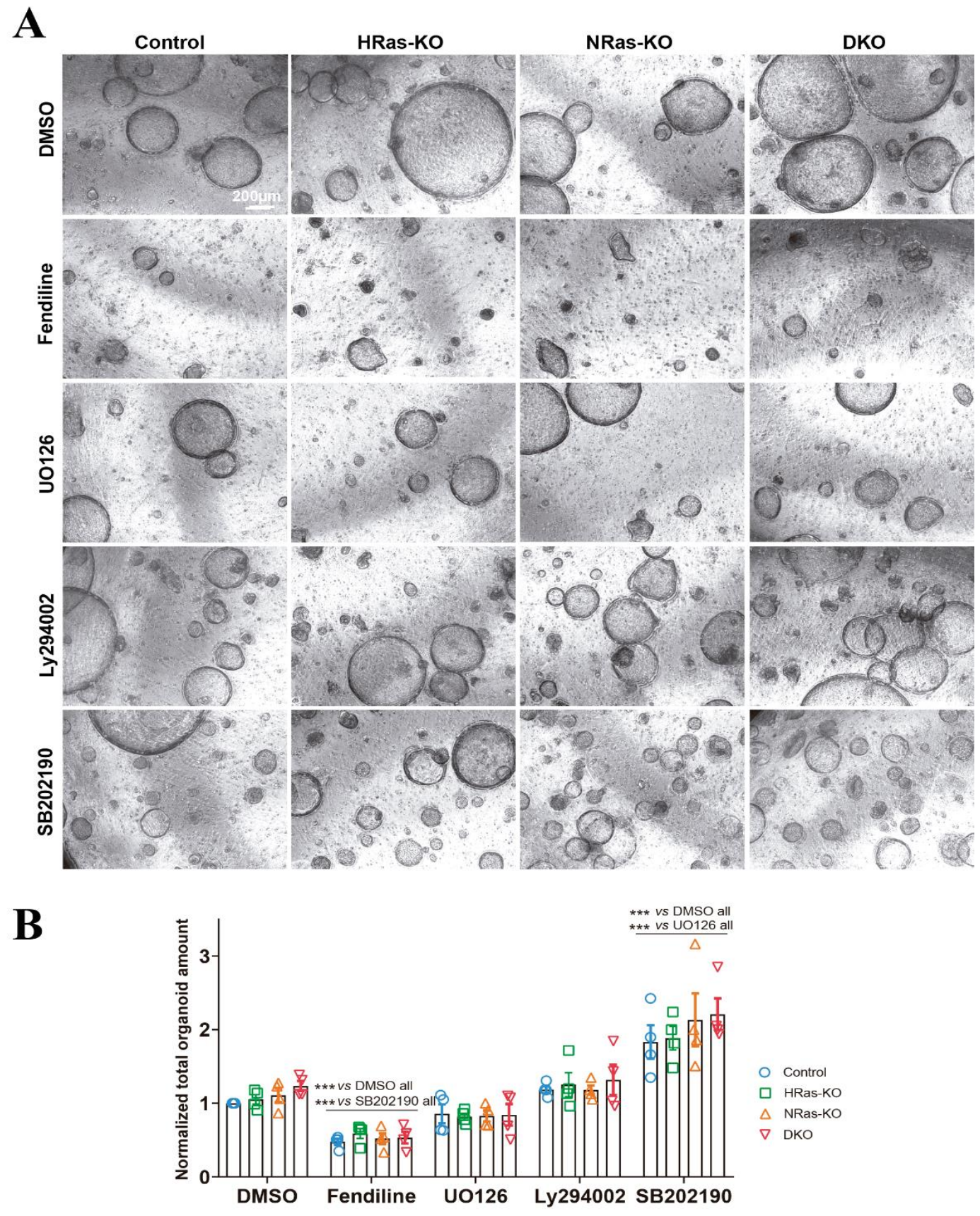

C

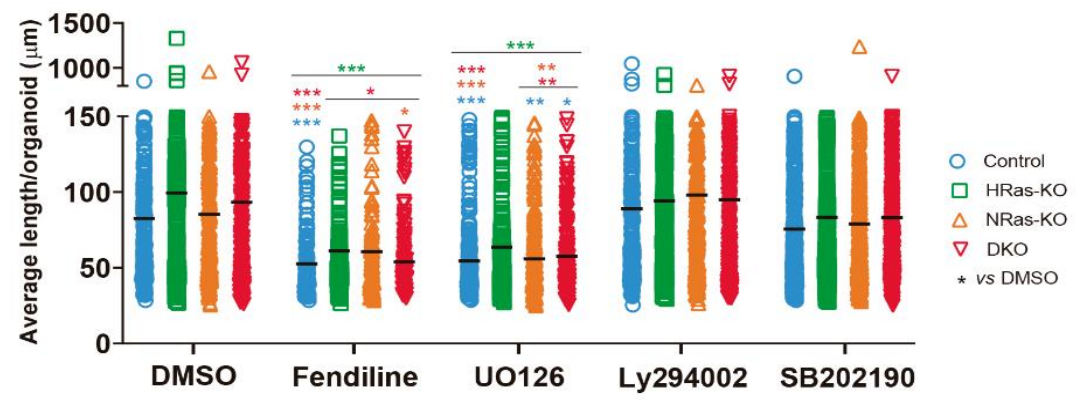

Figure 55: Effect of KRas, MEK1/2, PI3K and p38 inhibitors on lung organoid formation. A. Representative stack images of 14-days organoids obtained from the different combinations of genotypes and treatments. Vehicle (DMSO), Fendiline (KRas inhibitor), UO126 (MEK1/2 inhibitor), Ly294002 (PI3K inhibitor) and SB202190 (p38 inhibitor). Scale bar $200 \mu \mathrm{m} . \mathrm{n}=4$ for all the genotypes and conditions. B. Total organoid number relativized against the DMSOtreated control. Mean \pm s.e.m. $\mathrm{n}=4$ for all the genotypes and conditions. *vs DMSO, $* * * \mathrm{p}<0.001$. C. Graph representing the average organoid size. Median values are represented, $\mathrm{n}=4$ for all the genotypes and conditions. *vs DMSO, * $\mathrm{p}<0.5$, $* * \mathrm{p}<0.01, * * * \mathrm{p}<0.001$. 
A

SftpC / AcTub / DAPI

Control

HRas-KO

NRas-KO

DKO
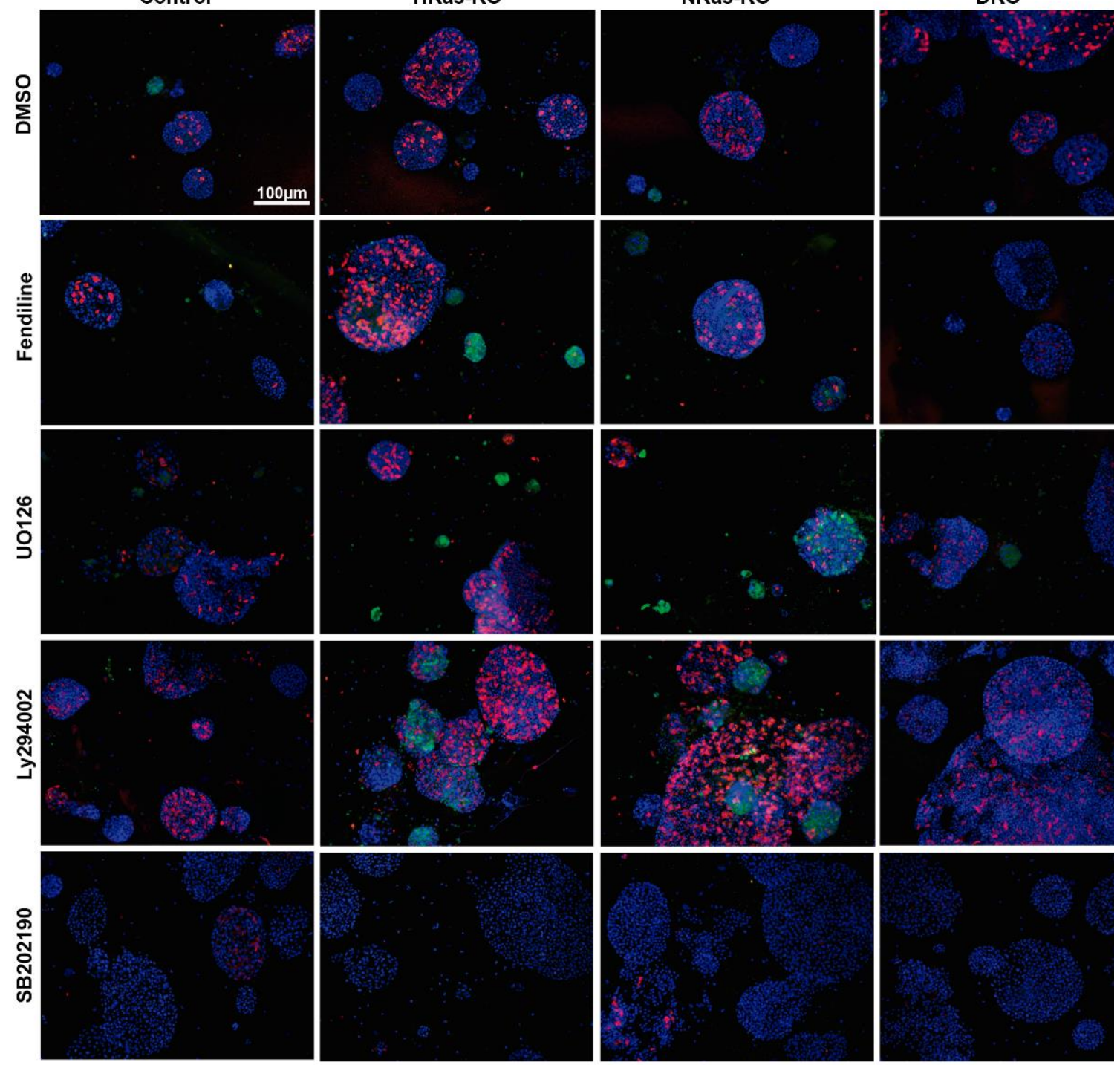

B

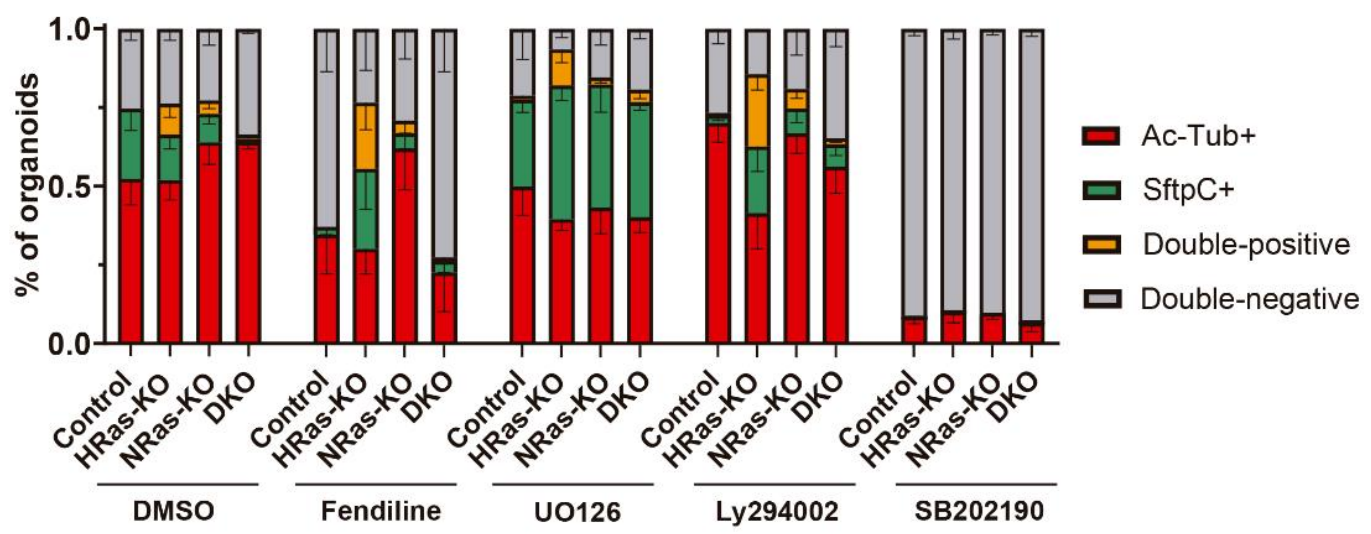

Fig 56: Exposure to KRas, MEK1/2, PI3K and p38 inhibitors modulates lung organoid differentiation. A. Representative immunofluorescence images of 14-days organoids obtained from the different combinations of genotypes and treatments. Vehicle (DMSO), Fendiline (KRas inhibitor), UO126 (MEK1/2 inhibitor), Ly294002 (PI3K inhibitor) and SB202190 (p38 inhibitor). Markers: Blue DAPI (nuclei), green SftpC (alveolar), red Ac-Tub (bronchiolar). Scale bar $100 \mu \mathrm{m} . \mathrm{n}=4$ for all the genotypes and conditions. B. Quantification of organoid proportion 
expressing SftpC (green), Ac-Tub (red), both markers (double-positive, orange) and neither (double-negative, grey). Data is represented as mean \pm s.e.m. $n=4$ for all the genotypes and conditions.
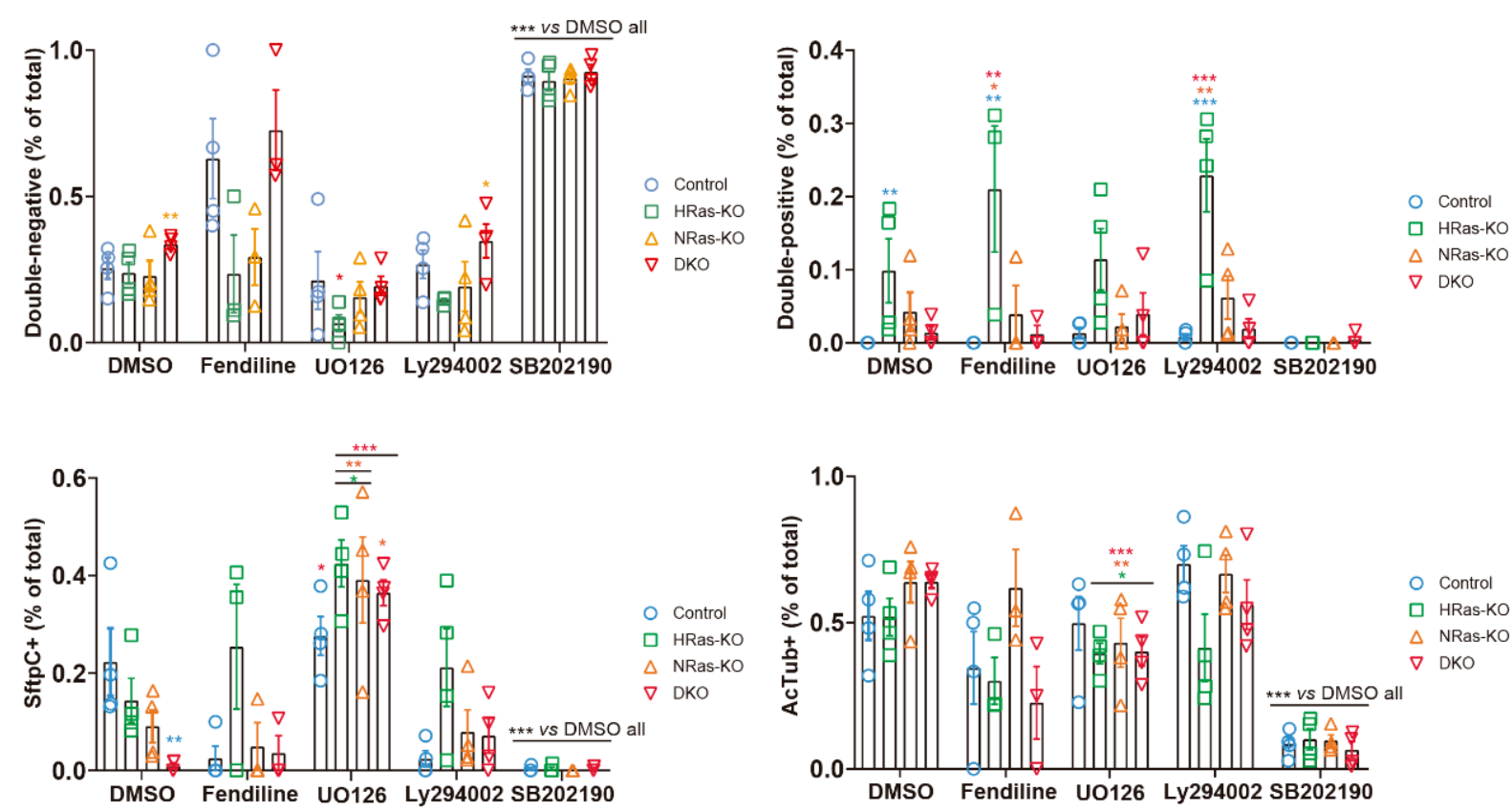

Figure 57: Effect of KRas, MEK1/2, PI3K and p38 inhibitors on double-negative, double-positive, alveolar or bronchiolar organoid proportions. Individual quantification of organoid proportions. Data is represented as mean \pm s.e.m. $\mathrm{n}=4$ for all the genotypes and conditions. $* v s$ DMSO, $* \mathrm{p}<0.5, * * \mathrm{p}<0.01, * * * \mathrm{p}<0.001$.

\subsubsection{Effect of dexamethasone and N-Acetylcysteine on Ras downstream signalling pathways}

To test whether DEX or NAC were triggering changes in lung fibroblasts that could explain some of the changes observed in the organoid model, we treated lung fibroblasts in steady state growth with either 60nM DEX or 10mM NAC during $24 \mathrm{~h}$. Interestingly, and as previously detected through RT-qPCR assays, a significantly higher KRas expression was noticed in both HRas-KO and DKO samples, and to a lesser extent in NRas-KO cells (but not statistically significant) as compared with the untreated CT (Fig. 58A). Moreover, neither DEX nor NAC treatment had an effect on KRas expression levels when compared with the untreated counterparts, except for a slight increment in KRas levels in DEX/NAC-treated CT (but not statistically significant) (Fig. 58A).

Additionally, despite that the HRas-KO, DKO and, to a lesser extent NRas-KO lung mesenchymal cells exhibited higher KRas levels, downstream ERK1/2 activation was lower in all mutant lung fibroblasts as compared with CT, although this reduction was not statistically significant for HRas-KO cells (Fig. 58B). Of interest, DEX or NAC treatment to the cells did not change the ERK activation levels as compared with the untreated HRas-KO, NRas-KO or DKO counterparts, however led to a slight reduction in the pERK/ERK ratio of DEX/NACtreated CT (not statistically significant) (Fig. 58B). 
In contrast with the Ras-ERK signalling pathway, no considerable differences were observed when AKT activation was analysed, with only a slight increment in untreated NRas-KO lung mesenchymal cells as compared with the untreated CT (Fig. 58C). Treatment with DEX resulted in no changes in pAKT levels, since the tendency was comparable with the observed in the NT samples. Finally, NAC-treated cells showed a slight (not statistically significant) reduction in AKT activation levels in all the genotypes (Fig- 58C).
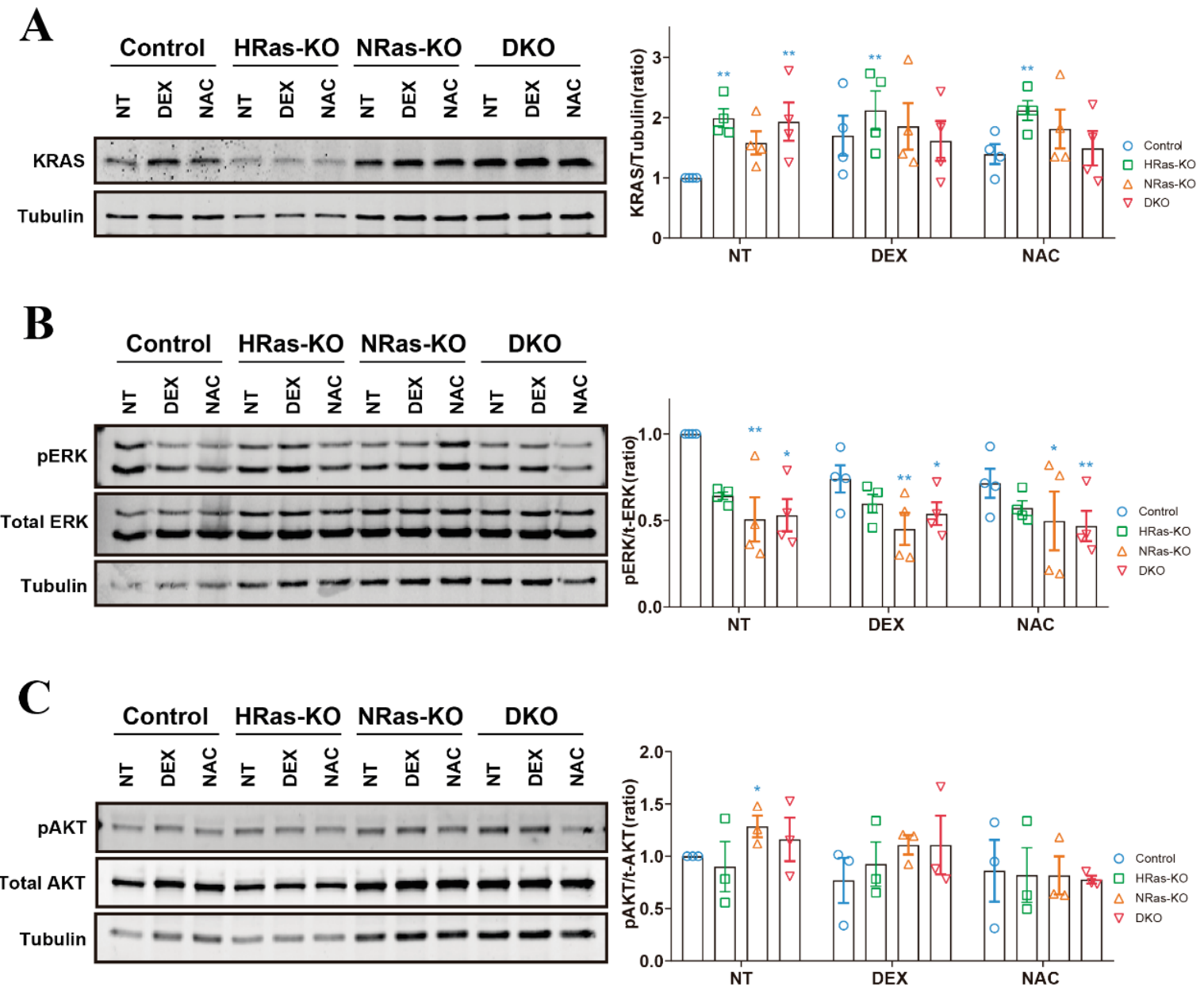

Figure 58: Altered KRas expression and ERK activation in HRas-KO and/or NRAs-KO lung fibroblasts. A-C Representative western blot assays of total primary lung fibroblasts protein extracts, from different genotypes and conditions, showing the expression of KRas (panel A), pERK and total ERK levels (pERK/total ERK, panel B), and pAKT and total AKT levels (pAKT/total AKT, panel C). Tubulin was used as loading control. Levels quantitated as fold change relative to those measured in CT samples. Data presented as mean \pm s.e.m. $n=4$ for all the different genotypes in panel A and B; $\mathrm{n}=3$ for all the different genotypes and conditions in panel C. ${ }^{*} v s \mathrm{NT},{ }^{*} \mathrm{p}<0.05, * * \mathrm{p}<0.01, * * * \mathrm{p}<0.001$.

\subsubsection{Effect of different inhibitors of Ras signalling on KRas expression and the activation of downstream signalling pathways in KO and CT lung mesenchymal cells}

In order to check whether some inhibitor of Ras signalling influenced mesenchymal cells, we treated the primary lung fibroblasts in steady state growth for $24 \mathrm{~h}$ with $10 \mu \mathrm{M}$ of Fendiline, UO126 and Ly294002, and SB202190 (KRas, MEK, PI3K and p38 inhibitors, respectively). 
As observed in the previous section, DKO lung mesenchymal cells treated with the vehicle (DMSO) exhibited a significant increase in KRas expression as compared with the CT cells. Similarly, but to a lesser extent, HRas-KO cells also showed more KRas levels (not statistically significant) (Fig. 59). KRas inhibition with Fendiline resulted in higher KRas levels in all HRasKO, NRas-KO and DKO mesenchymal cells, but not in the Fendiline-treated CT (Fig. 59). Similarly, inhibition of MEK1/2 kinase activity with UO126 derived in overall increment in KRas protein levels in all the genotypes, being stronger in HRas-KO and DKO cells (Fig. 59). Interestingly, the same tendency than that observed with the pan-MEK inhibitor was obtained after the inhibition of PI3K with the pan-inhibitor Ly294002 (Fig. 59). However, only CT and NRas-KO mesenchymal cells experienced augmented protein levels of KRas after the inhibition of p38 with the pan-inhibitor SB202190, as compared with the DMSO-treated counterparts (Fig. 59).
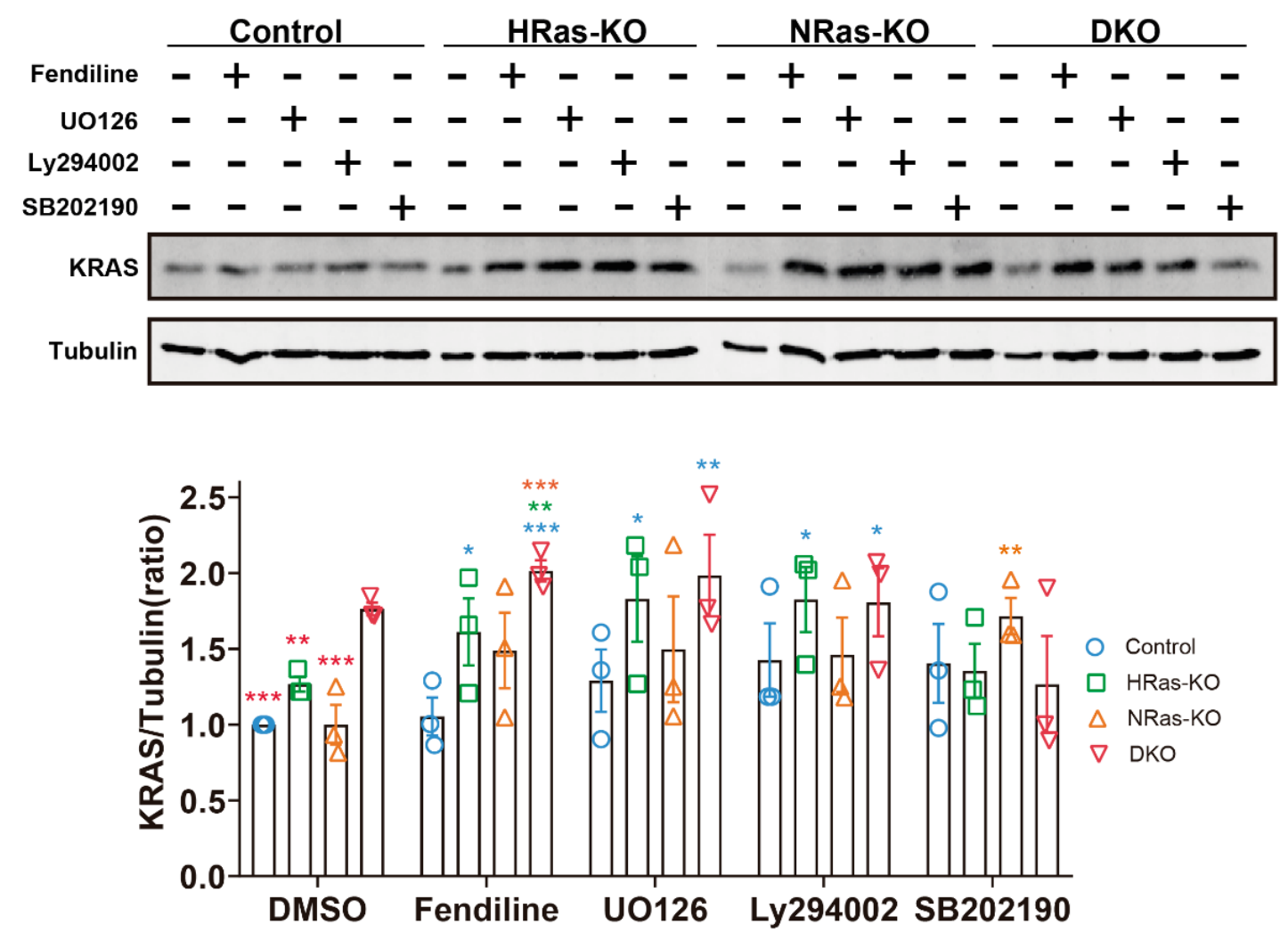

Figure 59: Increased KRas expression in HRas-KO and/or NRas-KO lung fibroblasts after Fendiline and UO126 treatment. Representative western blot assays of total primary lung fibroblasts protein extracts, from different genotypes and conditions, showing the expression of KRas. Tubulin was used as loading control. Expression levels quantitated as fold change relative to expression in CT samples. Data presented as mean \pm s.e.m. $\mathrm{n}=3$ for all the different genotypes and conditions. ${ }^{*} v s$ DMSO, ${ }^{*} \mathrm{p}<0.05, * * \mathrm{p}<0.01, * * * \mathrm{p}<0.001$.

No differences were noticed in MEK1/2 activation levels between the different genotypes and treatments, but a strong compensation of pMEK and MEK levels was observed in those cells treated with the MEK kinase activity inhibitor UO126 (Fig. 60). A slight decrease in ERK1/2 activation was detected after KRas inhibition with Fendiline, but strong and significant reduction in pERK levels were obtained with the pan-MEK inhibitor UO126 in the four 
genotypes as compared with the DMSO-treated cells (Fig. 60). Neither PI3K nor p38 inhibition resulted in changes in ERK activation, except for a slight decrease in pERK levels in CT treated with p38 inhibitor SB202190 when compared with the DMSO-treated CT mesenchymal cells (Fig. 60).
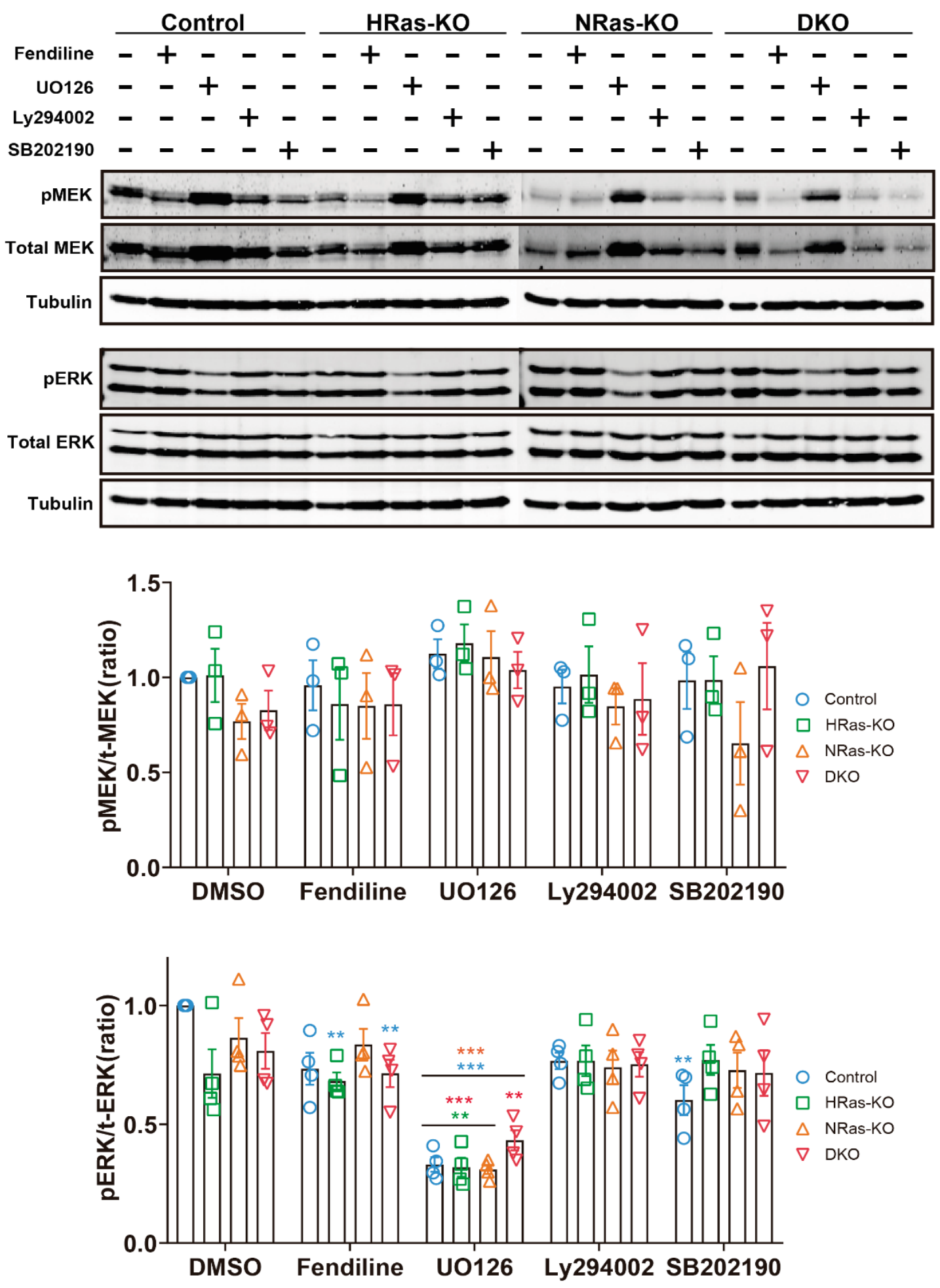

Figure 60: UO126 efficiently decreases ERK activation in primary lung fibroblasts. Representative western blot assays of total primary lung fibroblasts protein extracts, showing the cellular levels of pMEK and total MEK (upper part of the panel), and pERK and total ERK. Tubulin was used as loading control. Levels quantitated as fold change relative to those measured in CT samples. Data presented as mean \pm s.e.m. $n=3$ for all the different genotypes for pMEK/total MEK; $\mathrm{n}=4$ for all the genotypes for pERK/total ERK. $* v s$ DMSO, ${ }^{*} \mathrm{p}<0.01,{ }^{* * *} \mathrm{p}<0.001$. 
Next, pAKT levels were analysed in all the genotypes and conditions in our primary lung fibroblasts. No changes were observed in pAKT levels after KRas or MEK inhibition, but a slight decrease was detected in cells treated with either both Ly294002 (Pi3K inhibitor) or SB202190 (P38 inhibitor) (Fig. 61). The inhibitory effect of Ly294002 on AKT activation in treated cells was attenuated because the concentration used was the lowest described, nevertheless the effect of PI3K inhibition was apparent in our lung organoid cultures, suggesting that the inhibitor may be affecting the lung epithelial cells with more efficacy compared with the observed in lung mesenchyme.
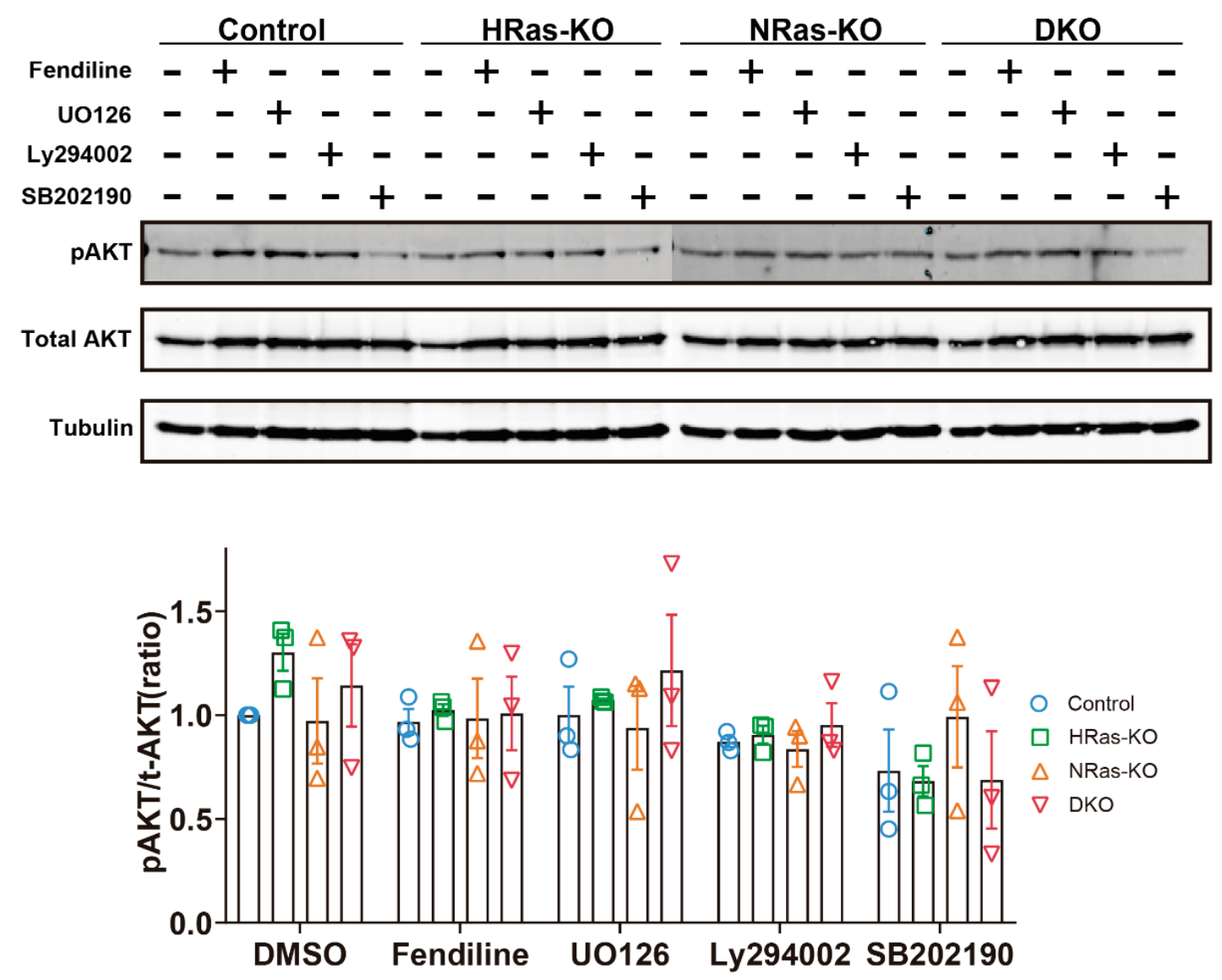

Figure 61: AKT activation is not altered in primary lung fibroblasts devoid of HRas and/or NRas. Representative western blot assays of total primary lung fibroblasts protein extracts, showing the levels of pAKT and total AKT. Tubulin was used as loading control. Levels quantitated as fold change relative to those measured in CT samples. Data presented as mean \pm s.e.m. $n=3$ for all the different genotypes and conditions.

Lastly, we sought to analyse p-p38 levels after the different treatments in our four different genotypes. No differences were detected between the different genotypes in the DMSO-treated cells, and neither in those treated with KRas, MEK or PI3K inhibitor (Fig. 62). Interestingly, p38 inhibition resulted in a strong compensatory effect caused by significant higher total p38 protein levels in those cells treated with the inhibitor (Fig. 62). However, despite the lung fibroblasts exhibited this compensation, the effect of p38-inhibition on lung organoids was apparent, so it may have a more intense effect on lung epithelial cells rather than on lung mesenchyme. 

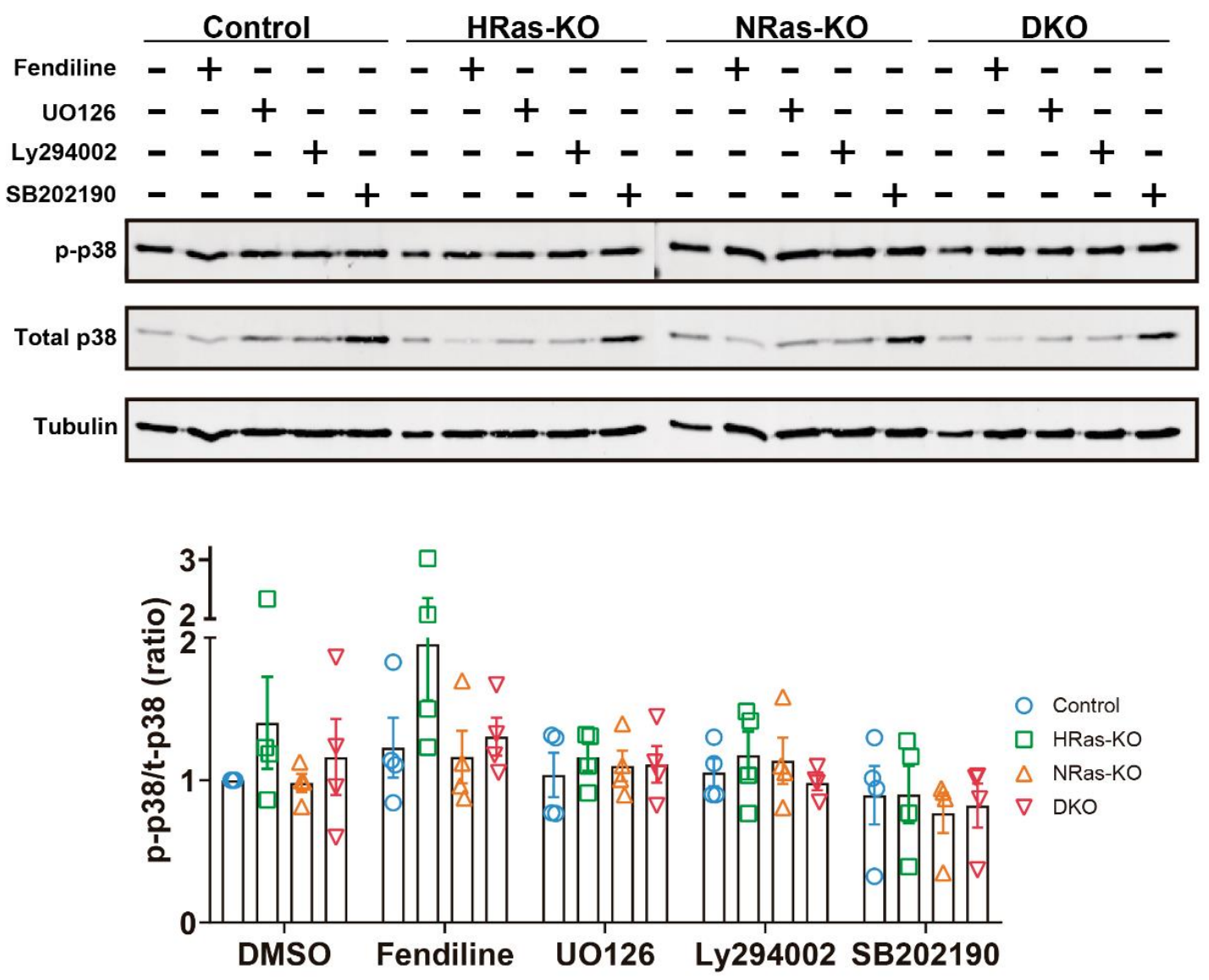

Figure 62: Inhibition of p38 with SB202190 leads to a strong compensatory effect in primary lung fibroblasts. Representative western blot assays of total primary lung fibroblasts protein extracts, from different genotypes and conditions, showing the cellular levels of p-p38 and total p38. Tubulin was used as loading control. Levels quantitated as fold change relative to those measured in CT samples. Data presented as mean \pm s.e.m. $n=4$ for all the different genotypes and conditions.

\subsubsection{Lack of HRas enhances FGF7 production in HRas-KO and DKO lung organoids}

In order to study whether the increased gene expression levels of Fgf7 observed in lung mesenchymal cells lacking HRas-KO isoforms were transduced in an increment in FGF7 factor, we collected and analysed the FGF7 levels in the culture mediums of the organoid cultures using an ELISA assay.

Interestingly, we detected increased FGF7 levels, albeit not statistically significant, in NT organoid-derived mediums originated using HRas-KO and DKO lung mesenchymal cells (Fig. 63A). This tendency was corrected in those NAC, and UO126-treated organoids (Fig. 63A, B). Additionally, FGF7 concentration in the organoid-derived culture media from the SB202190treated samples was lower, probably due to the fact that SB202190-treated organoids were mostly composed by Basal cells that do not need a FGF7 distal-lung signalling (Fig. 63B). 
A

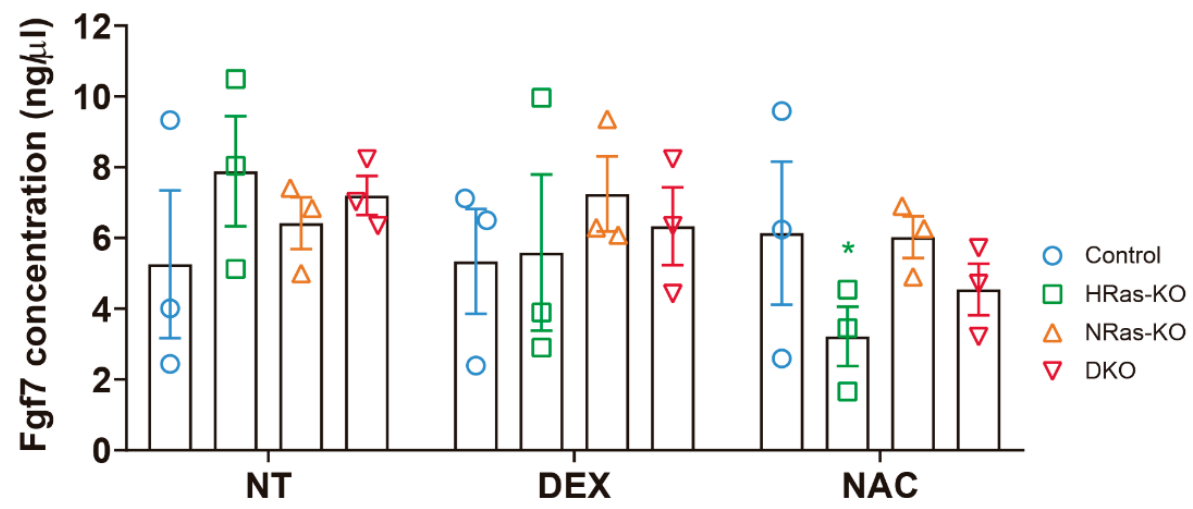

B

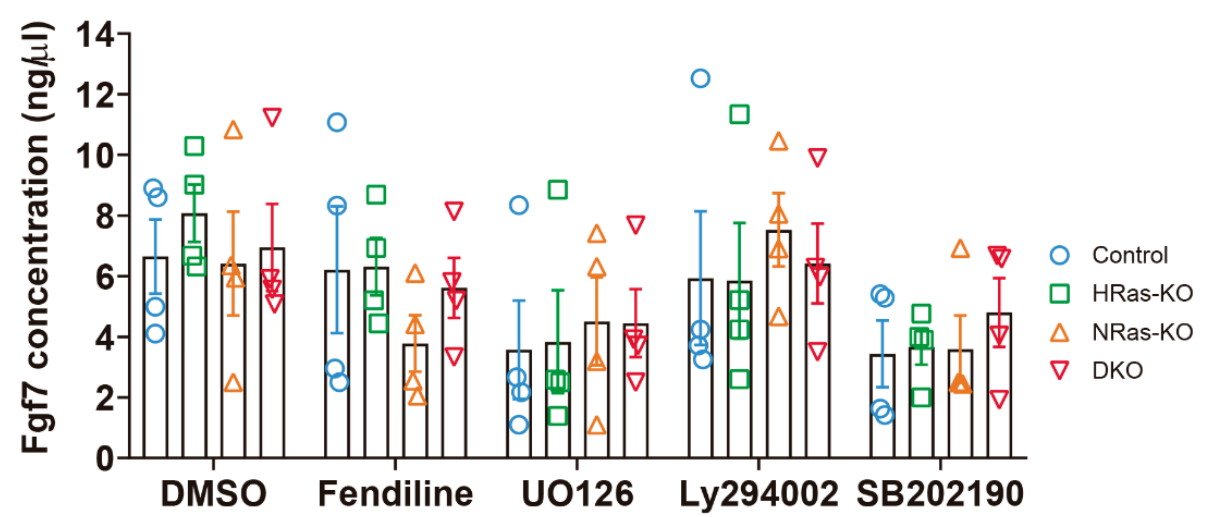

Figure 63: NAC and UO126 decrease FGF7 concentration in the culture media of lung organoids. A. ELISA analysis of mouse FGF7 secretion in lung organoids culture medium from the different genotypes subjected to NT, DEX or NAC treatments. Data presented as mean \pm s.e.m. $n=3$ for all the different genotypes and conditions. B. ELISA analysis of mouse FGF7 secretion in lung organoids culture medium from the different genotypes subjected to DMSO, Fendiline, UO126, Ly294002 or SB202190 treatments. Data presented as mean \pm s.e.m. $n=4$ for all the different genotypes and conditions.

3.5.8. Ras activation is not significantly affected in the lung mesenchyme after DEX or NAC addition

In the assay using organoids both NAC and UO126 MEK inhibitor showed an important effect on promoting alveolar differentiation in our DKO samples, nevertheless, dexamethasone had a partial impact on the process. For this reason, we analysed the influence of the three treatments on Ras and downstream ERK activation in primary lung fibroblasts.

The cells were treated during $24 \mathrm{~h}$ in steady state, to mimic how they were seeded for organoid assays, and starved cells were included in every experiment as a control. For these assays, each treatment was compared with untreated cells in steady state. In all the cases, treatment of the cells with the MEK inhibitor resulted in an increment in RAS. GTP as compared with the steady state levels, however, the ERK activation was significantly lower and almost identical to cells depleted of serum for $10 \mathrm{~h}$. 
CT cells treated with either DEX or NAC during $24 \mathrm{~h}$ resulted in a slight (not statistically significant) increment in Ras activation but showed no changes in ERK activation. Similarly, HRas-KO lung mesenchymal cells exhibited no significant changes in both Ras or ERK activation. No significant alterations were found in RAS·GTP levels in NRas-KO cells after DEX or NAC treatment, but a slight decrease in ERK activation (not statistically significant) was detected. Lastly, DEX produced a minor decline in Ras activation in the DKO lung fibroblasts, however NAC-treatment resulted in significantly fewer RAS.GTP/Total RAS levels. Additionally, as observed previously in NRas-KO cells, DKO samples treated with DEX, or NAC displayed a slight reduction in ERK activation (Fig. 64).
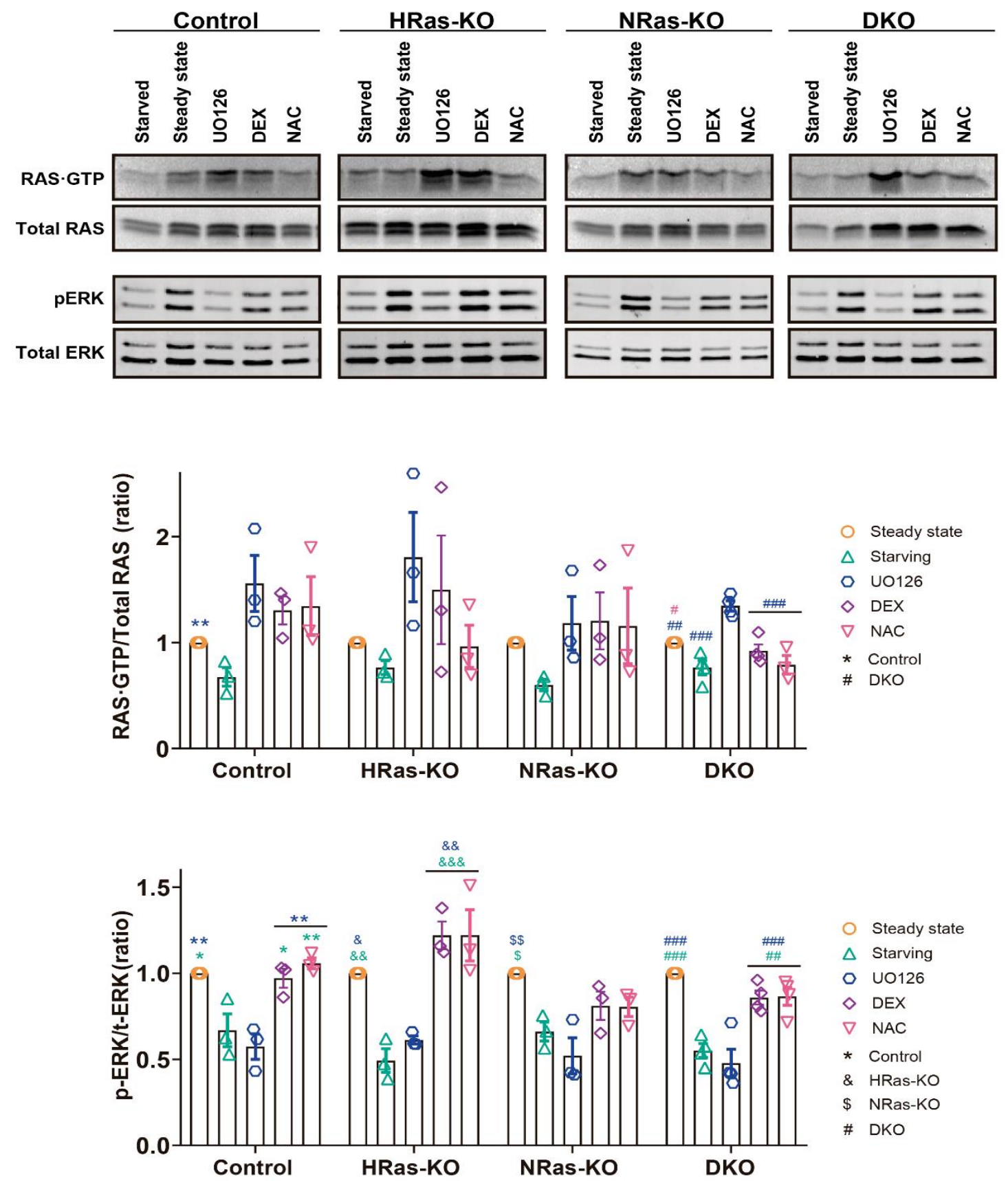

Figure 64: Ras activation and pERK levels in primary lung fibroblasts treated with DEX, NAC or UO126. Representative western blot assays of RAS. GTP pull-down assays and total protein extract from primary lung fibroblasts from different genotypes and conditions. . Graphs show the analyses of the Pan-RAS.GTP/total RAS (upper) and pERK/total ERK (bottom) ratios in the different samples and conditions. Activation levels quantitated as fold change 
relative to the expression in samples grown in steady state conditions. Data presented as mean \pm s.e.m. $n=3$ for all the different genotypes and conditions.

\subsubsection{Combined absence of HRas and NRas leads to increased epithelial KRas activation and pERK levels}

In order to analyze if the role observed for KRas in the lung organoids was mirrored in changes in KRas activation in vivo, we carried out RAS.GTP pull-down assays in whole lung homogenates from untreated (NT), DEX-treated and NAC-treated newborn pups to detect the levels of KRas activation. Interestingly, the lungs lacking both HRas and NRas isoforms displayed significantly elevated levels of KRAS.GTP as compared with the NT-CT littermates (Fig. 65). It is worth mentioning that HRas-KO lungs showed two well distinguishable phenotypes, one being more similar to the DKO samples and the other one with fewer KRAS·GTP (Fig. 65).

DEX-treated lungs exhibited a tendency similar to untreated samples, albeit the KRAS.GTP levels in DKO lungs were slightly decreased (not statistically significant versus the NT-DKO lungs) (Fig. 65). Finally, levels of KRAS·GTP were significantly decreased in NAC-treated DKO lungs as compared with the NT-DKO samples (Fig. 65).

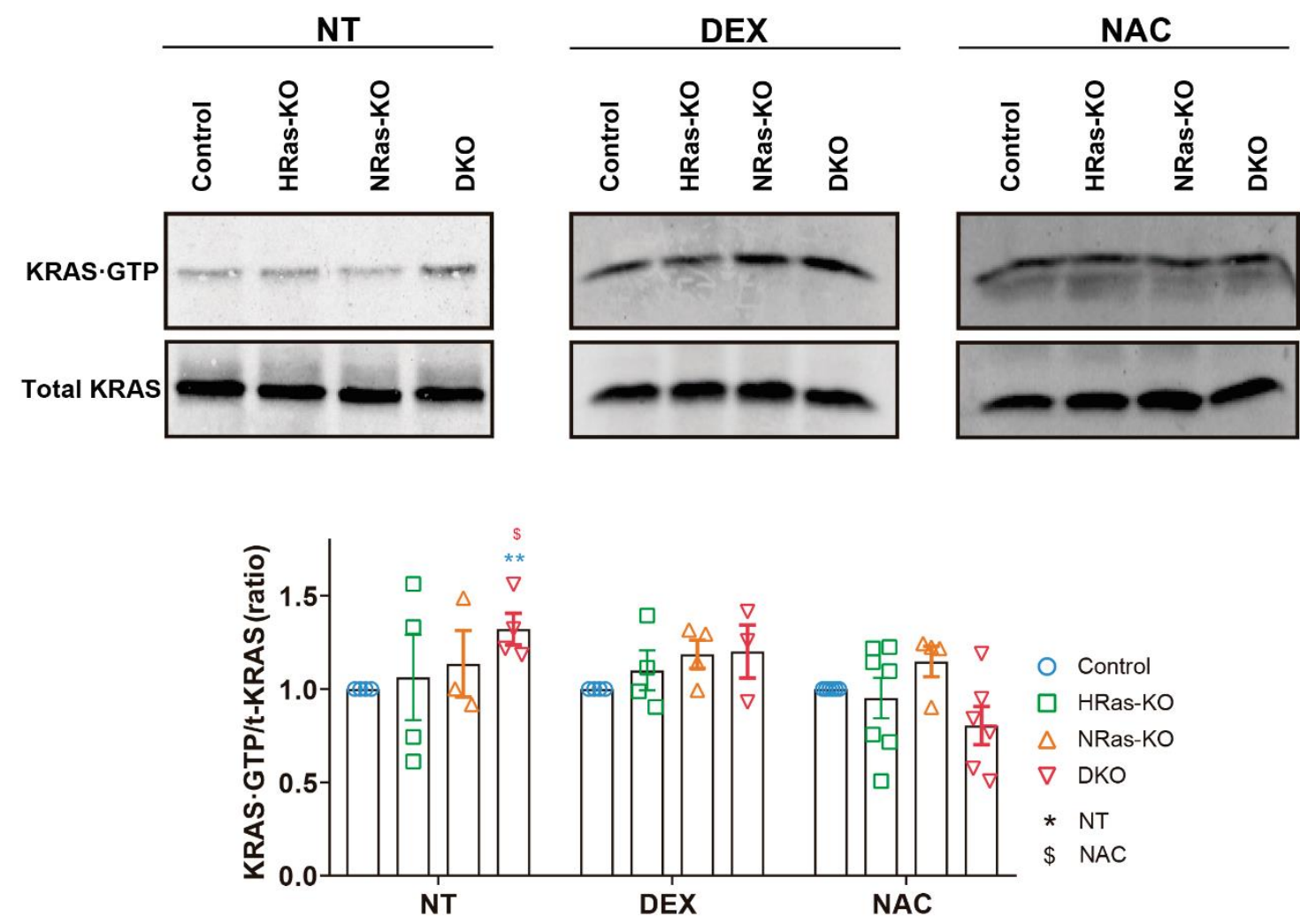

Figure 65: Increased KRas epithelial activation in DKO lungs is reversed after NAC antenatal treatment. Representative western blot assays of KRAS. GTP pull-down assays and total protein extract from whole lung tissue samples from different genotypes and conditions. Graph shows the analysis of the KRAS·GTP/total KRAS ratios for the different treatments and genotypes. Activation levels were quantitated as fold change relative to those measured in CT-NT/CT-DEX/CT-NAC samples. Data presented as mean \pm s.e.m. NT: CT/HRas-KO/DKO n=4, NRas-KO n=3; 
DEX: CT/HRas-KO/NRas-KO n=4, DKO n=3; NAC: CT n=6, HRas-KO/DKO n=7, NRas-KO n=4. *vs NT, ${ }^{\$} v s$ NAC, ${ }_{\mathrm{p}}<0.05, * * \mathrm{p}<0.01$.

A
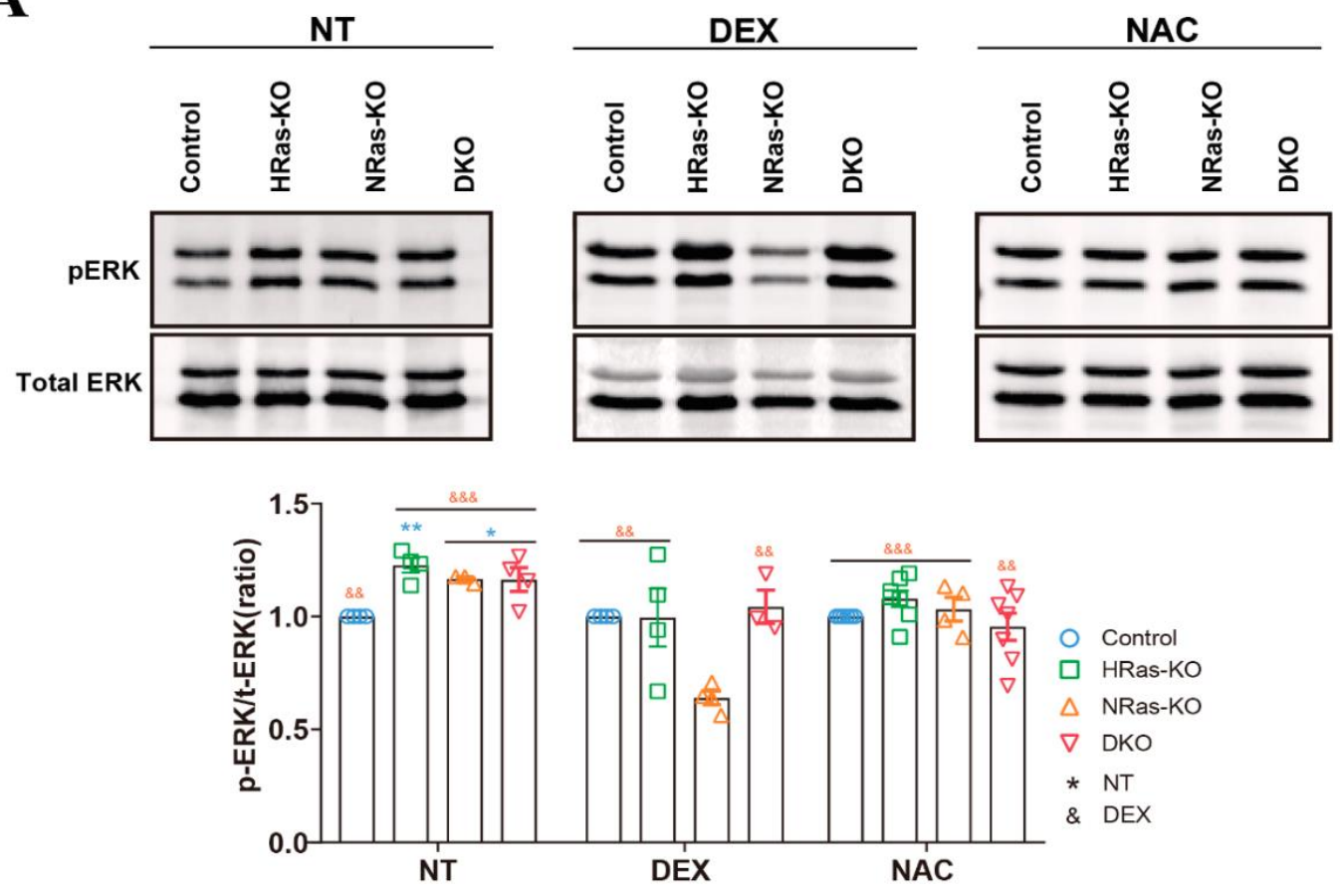

B

NT

DEX

NAC

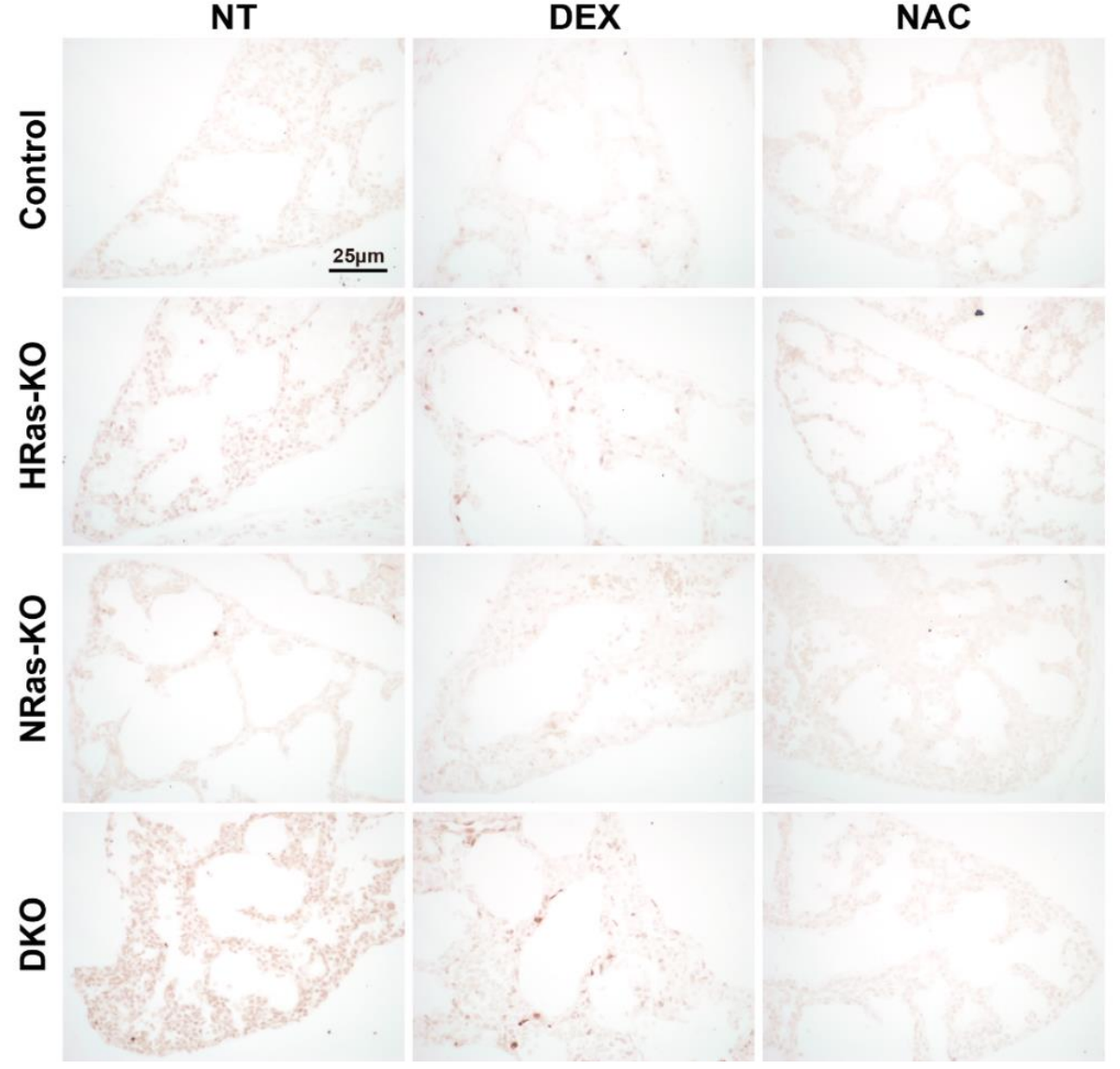

Figure 66: Increased epithelial ERK activation in DKO lungs is reversed after NAC antenatal treatment. A. Representative western blot assays of pERK/total ERK from whole lung tissue total protein extracts from the different genotypes and conditions. Activation levels were quantitated as fold change relative to those measured in CT-NT/CTDEX/CT-NAC samples. Data presented as mean \pm s.e.m. NT: CT/HRas-KO/DKO n=4, NRas-KO n=3; DEX: CT/HRasKO/NRas-KO n=4, DKO n=3; NAC: CT $\mathrm{n}=6$, HRas-KO/DKO $\mathrm{n}=7$, NRas-KO $\mathrm{n}=4$. ${ }^{*} v s \mathrm{NT}$, ${ }^{\&} v s$ DEX, ${ }^{*} \mathrm{p}<0.05$, 
${ }^{* * * \& \&} \mathrm{p}<0.01, \& \& \& \mathrm{p}<0.001$. B. Representative immunohistochemical assays for $\mathrm{pERK}$ in lung sections from P0 mice from the different genotypes and conditions. Scale bar $25 \mu \mathrm{m}$.

Additionally, to further analyse the activation of Ras-MAPK pathway in our P0 lungs, we performed western blot assays against pERK1/2 and total-ERK1/2. In concordance with the previously observed results regarding higher KRas activation in DKO lungs, increased levels of ERK activation were found in HRas-KO, NRas-KO and DKO samples (Fig. 66A). Interestingly, both DEX and NAC decreased ERK activation, with a strong inhibition in DEX-treated NRas-KO lungs (Fig. 66A). To further confirm these observations, we evaluated pERK levels by means of immunohistochemical assays in lung sections from newborn mice, untreated, DEX or NAC-treated. Consistently with the results obtained from the Western blot assays, DKO lung slices showed a more intense pERK staining as compared with the rest of genotypes (Fig. 66B). In addition, HRas-KO untreated lungs exhibited staining levels with an intensity situated between the DKO and CT/NRas-KO samples (Fig. 66B). Of note, both DEX and NAC-treated lungs displayed fewer pERK levels, however, some regions of the DEX-treated DKO lungs exhibited higher pERK staining levels that the rest of phenotypes (Fig. 66B). 


\section{DISCUSSION}





\section{DISCUSSION}

\subsection{Phenotypes resulting from genetic deletion of HRas and/or NRas in mice}

Our present results confirmed previous observations of our lab on the viability of HRas/NRas-DKO animals kept on a mixed genetic background (Esteban et al., 2001; Weyandt et al., 2016), by demonstrating that HRas/NRas-double null animals kept on pure C57B1/6 genetic background die during the first postnatal 24-48 hours, while showing clear signs of neonatal respiratory distress, cyanosis and respiratory failure. Thus, despite the recognized dominance and critical requirement of KRas-dependent signalling for embryonic development and adult viability (L. Johnson et al., 1997; Koera et al., 1997; Plowman et al., 2003), as well as for lung tumorigenesis (Adderley et al., 2019; Castellano \& Santos, 2011; Drosten et al., 2018; Gazdar et al., 2016; Lohinai et al., 2017; Simanshu et al., 2017), here we showed that the HRas and NRas family members also exert critical functions in control of the last stages of murine embryonic lung development and organism survival.

DKO neonates and E18.5 embryos showed unaltered overall lung morphology, but exhibited significant defects of alveolar lung maturation, as observed through the markedly reduced alveolar saccular space and thicker separating septa, abnormal accumulation of glycogen in alveolar regions and delayed differentiation of AEC1 and AEC2. Consistent with these observations, higher alveolar distal-tip progenitors (Chang et al., 2013; Perl et al., 2005) and bipotent alveolar progenitors (Desai et al., 2014) were present in the lungs of DKO as compared to Control and NRas-KO littermates. Additionally, E18.5 HRas-KO embryos were phenotypically equal to the DKO littermates; however, they achieved a normal alveolar development at birth. These data suggest that HRas exerts a key signalling role during pneumocyte differentiation, that are partially redundant with NRas. However, when both HRas and NRas are absent, KRas action cannot substitute these functions. Interestingly, mutations in components of the Ras/MAPK/ERK pathway have been described to induce an aberrant lung embryonic development. Mice lacking EGF receptor (EGFR-KO) exhibited a lung maturation delay with lack of alveolarization and impaired branching (Miettinen et al., 1997); lack of maturation and retarded alveolar epithelium differentiation is also stated in ERK3-KO (Klinger et al., 2009; Pew et al., 2016) and STK40 serine-threonine kinase (Yu et al., 2013); and major lung abnormalities like lung agenesis and lack of lung progenitor cells in MEK1/2 and ERK1/2 mutants in murine lung epithelium (Boucherat et al., 2014b); indicating the pivotal role of ERK/MAPK pathway for fetal lung growth, survival and morphogenesis.

In addition to the abnormal alveolar phenotypes, structural alterations of the bronchiolar cells were also linked to HRas or NRas deficiency, that resulted in a significant flattening affecting 


\section{Discussion}

both the Club and Ciliated cells. This defect, together with the altered PAS-staining pattern, suggests a secretory deficiency at the bronchiolar level which may also contribute to the respiratory stress of newborn DKO mice since a correct mucin production is essential for normal lung function (Roy et al., 2011), and further demonstrates the requirement of Ras/Raf/ERK pathway activation to stimulate mucin production (Thai et al., 2008). However, in addition to the structural abnormalities, no further alterations in Club-Ciliated cell number, nor in distribution were observed after HRas and/or NRas ablation.

The delayed alveolar differentiation is accompanied with increased rates of proliferation, detected in the HRas-KO and HRas/NRas-DKO animals. Interestingly, and in concordance with prior transcriptomic analyses of Ras-KO mouse embryonic fibroblasts (MEFs) of our group (Castellano et al., 2007, 2009), both HRas ablation and concomitant HRas/NRas-deficiency result in significant increment in lung cellular proliferation, although this phenotypic effect is clearly stronger in the HRas-KO. This effect may be caused by a compensation between HRas and NRas, with HRas/NRas-DKO exhibiting an intermediate phenotype. On the other hand, the increase of apoptotic rates in NRas-KO and DKO mice are linked to NRas deficiency (Castellano et al., 2007, 2009) and may have also contribution to the respiratory distress in these animals. Thus, our findings, concurrently with the previous observations, point to a preferential link of HRas with control of cellular proliferation and of NRas with control of cell death, with the HRas/NRas-DKO animals showing a halfway phenotype.

The elevated levels of infiltrating neutrophils detected in the lungs of DKO animals may also contribute to perinatal lethality since neutrophil migration into the lungs is a well-stablished hallmark in acute respiratory distress syndromes (Castillo et al., 2015; Potey et al., 2019; Sun et al., 2019). They play a central role in inflammation, reactive oxygen species production and subsequent tissue damage, which may also contribute to the increased apoptotic rates in our DKO lungs. In particular, activation of KRas, the only remaining Ras isoform in our DKO mice, is linked to increased neutrophil activation and extravasation in KRas-gain-of-function lung tumours (Ji et al., 2006; Phan et al., 2013).

Transcriptional analyses yielded additional potential mechanistic clues regarding the defective lung phenotypes of DKO mice. In particular, genes coding for enzymes implicated in sphingolipid metabolism, Cers, Sptlc1, Sggp1, Degs1, and Acer, are overexpressed in lungs from DKO P0 neonates in comparison to the rest of genotypes. This overexpression may be especially relevant since all the named enzymes are regulating the production of ceramide from different pathways, ultimately leading to ceramide production and accumulation. We have confirmed by means of immunoassays the ceramide accumulation in both alveolar and bronchiolar areas of DKO lungs. Thus, given the recognised relation of ceramide as key second messenger promoting and enhancing stress signals, tissue injury and apoptosis (Barnes, 2004; Göggell et al., 2004; Petrache et al., 2005; Petrache \& Berdyshev, 2016; Pettus et al., 2002; 
Sparkman et al., 2006; van Mastrigt et al., 2018), as well as with decreased surfactant production and various lung pathologies, including respiratory distress syndromes (Boggaram, 2009; Göggell et al., 2004; Petrache \& Berdyshev, 2016; Sparkman et al., 2006; van Mastrigt et al., 2018), we postulate that ceramide accumulation is a major factor for the respiratory stress contributing to neonatal death of the DKO mice. Additionally, in the context of the Ras genotypes studied, it is worth mentioning that sphingomyelin and ceramide (SM/ceramide) balance regulates phosphatidylserine (PtdSer) localization at the plasma membrane, and thus, regulates KRas function and plasma membrane localization. In this context, an increment or external supplementation of ceramide restores PtdSer and KRas to the plasmatic membrane, whereas an inhibition of ceramide production with Fendiline induces PtdSer depletion and KRas mislocalization (K. Cho et al., 2015; van der Hoeven et al., 2013; P. Wang et al., 2021), being also a potential approach to inhibit oncogenic KRAS (Gorfe \& Cho, 2021; Henkels et al., 2021).

\subsection{Altered lung redox homeostasis and mitochondrial respiration in mice lacking HRas}

\section{and NRas}

Reactive oxygen species (ROS) are key second messengers, necessary for maintaining organism homeostasis. However, an excessive generation of ROS that surpasses the endogenous detoxifying pathways, and its high reactivity may lead to important tissue injuries (Patel et al., 2018). Our transcriptomic analyses of P0 DKO lungs also revealed an overexpression of several distinct genes coding for components required for correct oxidative phosphorylation, suggesting a possible dysfunction in redox processes and homeostasis. Our results showed clear signs of oxidative stress in the lungs of our DKO mice as demonstrated by the increased levels of hydrogen peroxide, total cellular superoxide, and mitochondrial superoxide. In this regard, increased levels of oxidative stress have been detected in lung diseases and respiratory distress syndromes as a result of inflammation and cytokines release (Abdel Hamid et al., 2019; Castillo et al., 2015; Kellner et al., 2017; Marseglia et al., 2019), and the observed redox imbalance of our DKO lungs may be caused by the ceramide accumulation, as well as by the increased levels of infiltrating neutrophils in alveolar areas. Interestingly, NRas-KO lungs also show an increase in mitochondrial superoxide, but not in the other reactive oxygen species, indicating a possible implication of NRas in control of mitochondrial oxidative stress.

Alterations of mitochondrial respiration are linked to redox imbalance, and vice versa (Murphy, 2009; Zhao et al., 2019), and our results indicated a similar connection between the aforementioned ROS accumulation and mitochondria alterations. Particularly, DKO lung tissue showed worsened mitochondrial respiration, displaying reduced basal respiration and ATPlinked respiration even though the mitochondria of DKO P0 mice were respiring close to its maximum as indicated with the increment in spare respiratory capacity. These analyses suggest 


\section{Discussion}

that only when both HRas and NRas are absent, the lung mitochondria are working up to its maximum levels but are still incapable to achieve the basal and ATP-linked production respiration rates seen in the littermates. To further validate the observation of reduced ATP production, colorimetric multiplate assays to detect ATP levels in lung homogenates are needed.

\subsection{Characterization of the effect of antenatal glucocorticoid administration on lung development and postnatal survival of HRas/NRas-double null mice}

Treatment with glucocorticoids alleviates lung pathologies by decreasing lung inflammation (Janahi et al., 2018; Prescott \& Rice, 2020), but also induces and boosts alveolar differentiation through the binding to the glucocorticoid receptor (GR) in the mesenchyme adjacent to the developing alveoli (Gerber, 2015; Habermehl et al., 2011; Laresgoiti et al., 2016). Our data indicated a clear reversion of the defects observed in alveolar cell lineages differentiation after antenatal dexamethasone treatment and extended the lifespan of HRas/NRas-devoid newborn mice for 5-6 more days. Our transcriptional analyses showed also that many transcriptional alterations observed in the untreated DKO lungs are reversed, including those related to sphingosine and ceramide metabolism, are reversed, as demonstrated by the reduction of the ceramide alveolar accumulations.

In line with the above observations, the structural alterations of bronchiolar cells linked to HRas and/or NRas ablation were also corrected after treatment with dexamethasone, and, in addition, no alterations in Club/Ciliated cell counts were observed after glucocorticoid administration. This observation is in line with previous reports describing that dexamethasone administration to pregnant rabbits stimulates the secretory potential of Club cells by elevating the level of uteroglobin secretory protein (Fernández-Renau et al., 1984; Lombardero \& Nieto, 1981; Plopper \& Fanucchi, 2014).

In concordance with the inhibitory effect of dexamethasone on inflammatory response and neutrophil extravasation (Janahi et al., 2018; Ricci et al., 2021; Ronchetti et al., 2018), the neutrophil infiltrates present in mice devoid of HRas and NRas were completely absent in those animals treated antenatally with dexamethasone. Furthermore, given this dramatic reduction in neutrophil infiltrates, we would expect a strong reduction in ROS production in the lungs of P0 DKO animals. However, our results demonstrated that dexamethasone antenatal administration could alleviate both total and mitochondrial superoxide generation but failed in reducing the hydrogen peroxide levels, thus exerting only a partial rescuing effect. Interestingly, the NRasKO lungs treated with dexamethasone were not showing reduction of the mitochondrial oxidative stress and exhibited more hydrogen peroxide accumulation. Hence, more detailed analyses are needed to investigate the connection between NRas and redox homeostasis control. 
Additional analyses of dexamethasone treated lungs showed an overall decrease in mitochondrial spare respiratory capacity affecting all genotypes, meaning that glucocorticoids reduce mitochondria function. This observation is in concordance with previously published data where high non-basal levels of glucocorticoids exposure decrease mitochondrial function in neurons , adipocytes and hepatocytes (Du et al., 2009; Luan et al., 2019; Suwanjang et al., 2019; Welberg, 2009). Additionally, dexamethasone did not correct the reduced basal respiration in the HRas/NRas-devoid lungs, but, however, ATP-linked respiration in DKO lung tissue was augmented, although this data needs to be further examined through direct measurement assays of ATP levels.

Our observations of dexamethasone-treated animals indicated a complete reversion of alveolar abnormal differentiation, a finding that, together with the decreased ceramide accumulations and reduction in neutrophil infiltrates, contributes to alleviate the perinatal lethality of our DKO newborn mice. However, the dexamethasone-treated DKO animals died 5-6 days later, an effect possibly occasioned by the partial effect of this glucocorticoid on reducing oxidative stress since all postnatal day 5 DKO animals treated antenatally with dexamethasone showed drastically augmented levels of apoptotic cells in the bronchiolar cells (Data included in the Annex, Fig. 7C \& Fig. 8D).

\subsection{Characterization of the effect of antenatal N-Acetylcysteine administration on lung development and postnatal survival of HRas/NRas-double null mice}

$\mathrm{N}$-Acetylcysteine (NAC) is a widely used mucolytic and general antioxidant agent facilitating mucus clearance out of the lungs and acting as a direct and indirect antioxidant scavenger (Zafarullah et al., 2003). Its direct role as an antioxidant is mediated through the binding to free - $\mathrm{SH}$ thiol groups, whereas its action as an indirect antioxidant is achieved by acting as reduced glutathione (GSH) precursor (Pei et al., 2018). Taken together, NAC improves lung function, and removal of ROS by antioxidants has been an attractive strategy in the treatment of different respiratory diseases with a clear inflammatory and oxidative stress phenotype, including respiratory distress syndromes, and to reduce the prematurity-related morbidity (Assimakopoulos et al., 2021; Buhimschi et al., 2020; X. Lu et al., 2019; Suter et al., 1994; Y. Zhang et al., 2017). Our results with antenatal NAC administration indicated a clear beneficial effect on perinatal to adulthood survival of the HRas/NRas-double null animals, with the survival of $53 \%$ of the NAC-treated DKO mice.

One key action of NAC is the induction of normal lung development, as demonstrated by a complete opening and maturation of the alveoli and the significative reduction of alveolar precursors in our HRas-KO and DKO E18.5 embryos. Additionally, this observation was further 


\section{Discussion}

supported by our microarray data assays were, comparisons between untreated DKO P0 lung and NAC-treated DKO P0 lungs uncovered an enrichment of pathways related to lung development and morphogenesis.

Our observations indicate that antenatal NAC administration had also a beneficial effect on normal bronchiolar Club cell columnar shape and apical vesicle formation. This effect could be explained by the known effect of NAC on enhancing secretory protein CC16 expression (Liao et al., 2010; Nie et al., 2005), the main secretory product of Club cells that plays an important protective role against oxidative stress in the respiratory tract.

Interestingly, our results showed an effect of NAC on the reduction of ceramide accumulation, in concordance with the notion of the inhibitory effect of GSH on neutral sphingomyelinase (nSMase) activity and reduction of ceramide formation upon NAC treatment in hypoxic cells (Yoshimura et al., 1999) and skeletal muscle (Nikolova-Karakashian \& Reid, 2011), as well as in blood plasma and heart in a model of obesity (Hodun et al., 2021).

Our analyses of the NAC-treated newborn DKO lungs suggested an effect of this antioxidant on the process of neutrophil extravasation. This role of NAC on neutrophils has been already described, acting as an inhibitor of chemotaxis inhibition (Atayoğlu et al., 2017; Kharazmi et al., 1988; Sadowska et al., 2006). The effect obtained with NAC treatment is less profound than that observed in the DEX-treated animals, meaning that, even though NAC is preventing the accumulation and negative action of the neutrophils on lung tissue, it is not as effective as glucocorticoid treatment.

The reduction of ceramide accumulation and neutrophil infiltrates in the lungs of NACtreated HRas/NRas-DKO mice was accompanied by a significant reduction of the redox imbalance, affecting hydrogen peroxide and total superoxide ROS levels. Interestingly, mitochondrial superoxide levels are not decreased after NAC antioxidant treatment. This is in concordance with previous observations of our laboratory where it has been proven that, in contrast to recognized mitochondria-targeted antioxidants such as MitoTempo (Rósula GarcíaNavas et al., 2021) or MitoQ (Tauskela, 2007), NAC is not effective as a mitochondrial ROS scavenger (unpublished data). In line with the impact of NAC on mitochondrial ROS, an overall reduction of mitochondrial respiration was observed in lung tissue of NAC-treated neonates, that was similar to that obtained with dexamethasone treatment, suggesting that both compounds act inhibiting mitochondria respiration, although further studies on mitochondrial membrane potential, morphology and functions are needed to clarify these observations.

Thus, our results clearly indicate a strong therapeutic role of NAC antenatal administration resulting in improvement of lung maturation and function improvement, as well as in survival of our DKO newborn mice, that was achieved through the reduction of redox imbalance and 
decrease in ceramide and neutrophils levels, further contributing to the observed decrease in oxidative stress levels.

\subsection{Effect of HRas/NRas ablation on epithelium-mesenchyme interaction}

After extensively describing the phenotypes of our HRas-KO and/or NRas-KO neonates and the effect of antenatal dexamethasone and NAC administration on lung maturation and survival, we next asked whether the observed phenotypes were caused by a cell-autonomous defect of the lack of HRas and/or NRas in lung epithelium or by the lack of HRas and/or NRas in the lung mesenchyme. Our results indicated that, in lung epithelium, NRas deletion is compensated with an overexpression of HRas, whereas KRas is slightly more expressed after HRas and/or NRas ablation. Interestingly, no compensatory effect of NRas expression was observed in HRasdevoid epithelium. On the other hand, only HRas/NRas-DKO lung mesenchymal cells showed a compensatory increment in KRas gene expression levels. These observations indicate that HRas and KRas are exhibiting a compensatory effect in lung epithelium, whereas only KRas is showing that effect on mesenchyme.

Detailed analyses of lung epithelium-mesenchyme interactions revealed a key role of HRas in control of the Hox5-Wnt-FGF7/10 mesenchymal pathway and the FGFR2b-Spry2-Wnt7Bpm4 epithelial pathway (El Agha \& Bellusci, 2014; Hrycaj et al., 2015; Klinkhammer et al., 2019; Shiraishi et al., 2019; Volckaert \& De Langhe, 2015). Particularly, lack of HRas led to a significant upregulation of the pathway in both, lung mesenchymal and epithelial cells, with the HRas/NRas-DKO exhibiting a more pronounced effect. Interestingly, mesenchymal FGF7/10 binds to its FGFR2b in the adjacent epithelium, triggering the activation of epithelial KRas and Spry2, a negative regulator of KRas activation in this context (Shaw et al., 2008; Tang et al., 2011; Warburton et al., 2000). Additionally, epithelial Wnt7 generates positive feedback by both inducing more FGF7/10 mesenchymal secretion (Aros et al., 2021; Volckaert \& De Langhe, 2015) and epithelial BMP4 (Whitsett et al., 2019) that will signal through $\beta$-Catenin, together with KRas, to induce proliferation and maintenance of SOX9+ distal tip alveolar progenitors (Chang et al., 2013; Ostrin et al., 2018; Ustiyan et al., 2016), which we previously identified to be retained at later stages of lung development in our DKO lungs and HRas-KO E18.5 embryos. Furthermore, SOX9 induces epithelial branching and inhibits alveolar differentiation (Chang et al., 2013). Additionally, the Nkx2 transcription factor present in basal and immature cells (Boggaram, 2009; Harris-Johnson et al., 2009) also appeared upregulated in HRas/NRas-devoid lung epithelium. Further supporting the notion of alveolar maturation delay, a decrease in the mature pneumocyte type I (mature AEC1) cell marker Aquoporin 5 was noticed in both HRasKO and DKO lungs. These observations further support previously described results and 


\section{Discussion}

reinforce the notion of a key functional role of HRas in branching termination and alveolar differentiation.

Overwhelming evidence suggests a link between redox imbalance and lung maturation delay since ROS can act as second messenger by regulating key transcription factors that alter gene expression in the embryo (Dennery, 2007) and are involved in neonatal respiratory distress syndrome pathogenesis (Abdel Hamid et al., 2019; McGillick et al., 2021; Xiang \& Wang, 2019). Among the transcription factors modulated by ROS, it is worth mentioning Wnt morphogens, since they are activated by hydrogen peroxide (Hwang et al., 2011). Together with the previously described ROS accumulation in lung epithelium, we also detected an increase of oxidative stress in lung primary mesenchymal cells lacking both HRas/NRas. Interestingly, these HRas/NRas-devoid cells also exhibited a dysregulation of the ROS detoxifying enzymes such as SOD3 or extracellular superoxide dismutase (EC-SOD), the predominantly expressed lung isoform catalysing oxygen superoxide (Fattman et al., 2000, 2003). Besides its role in oxidative stress clearance, SOD3 also exert functions controlling Ras/MAPK pathway activation in the context of tissue repair after injury (Laurila et al., 2009). Three members of glutathione S-transferases, (GSTa2, GSTm2 and GSTm3) appear upregulated after concomitant HRas/NRas deletion in lung mesenchyme. These enzymes are in charge of catalysing the conjugation of glutathione with toxic oxidant compounds, and associated with acute and chronic inflammatory lung diseases (Fletcher et al., 2015). In contrast, a reduction in Glutathione Sreductase (GSR) and $\mathrm{NAD}(\mathrm{P}) \mathrm{H}$ Quinone Dehydrogenase 1 (NQO1) was apparent in HRas/NRas-deficient lung mesenchymal cells. They play key roles in restoring the reduced glutathione (GSH) (Robbins et al., 2021) and in superoxide scavenging (Ross \& Siegel, 2017) respectively. Interestingly, and in concordance with our results, there is a clear link between GSH deficiency and impaired lung development (Robbins et al., 2021).

Our data also indicated that primary lung mesenchymal cells lacking HRas, alone and in combination with NRas, displayed worsened mitochondrial respiration parameters, with significantly reduced basal respiration, and ATP production-linked respiration. Thus, our data suggest that HRas and NRas deficiency significantly alters redox homeostasis and may be implicated in the dysregulation of developmental pathways and lung maturation delay. HRas clearly emerged as the more functionally prevalent of these two Ras family members regarding the generation of these phenotypic defects. Nonetheless, the significant defects observed in single HRas lungs were always further worsened by concomitant NRas ablation.

Evaluation of the effect of dexamethasone and NAC on lung mesenchymal cells showed a complete decrease of oxidative stress upon NAC treatment, and only a partial response upon dexamethasone administration. However, contrary to the results obtained in lung tissue assays, dexamethasone treatment of lung primary fibroblasts rescued the mitochondrial respiration, whereas NAC did not. In fact, NAC administration to primary fibroblasts resulted in a decrease 
of mitochondrial respiration in all the genotypes, further supporting similar previous phenotypes observed in our laboratory (unpublished data).

On the other hand, the observed dysregulation of late embryonic development signalling mostly attributed to HRas loss, is also partially modulated with dexamethasone and NAC treatment in lung mesenchyme. In this regard, a strong decrease on FGF7 gene expression levels was achieved after both treatments, but only NAC fully reverted the increased FGF10 gene expression levels up to control levels in both HRas and HRas/NRas-double deficient cells. This was accompanied by a downregulation of the expression levels of Wnt2, as well as of Hoxc5 and SOD3. These results, along with the reduction of oxidative stress levels, could explain the partial effect of in vivo antenatal dexamethasone administration, and the better survival rates with NAC antenatal treatment, and reveal key roles of NAC in regulating lung development, probably due to the reduction of ROS levels in both epithelial and mesenchymal cells.

\subsection{HRas function is crucial for KRas-driven lung branching termination, with NRas exhibiting also a partial overlapping function}

Characterization of the dysregulated epithelium-mesenchyme signalling pathway towards maintenance of the FGF10-KRas-SOX9 branching loop led us to further investigate whether HRas and HRas/NRas-double null lung mesenchymal cells, in presence of a wildtype epithelium, could recapitulate the in vivo observations in the mouse model through an in vitro organoid approach that mimics the lung epithelium-mesenchyme interactions during development and differentiation (Y. Hu et al., 2020). Our data showed higher organoid formation yields in DKO samples and, to a lesser extent, in HRas-KO samples, but the lack of NRas was indistinguishable from CT. Interestingly, the absence of HRas and NRas in the mesenchyme caused around 50\% generation of smaller, double-negative undifferentiated organoids, as well as similar percentages of bronchiolar. These observations support previous findings where we identified higher proliferation rates in both HRas-KO and DKO lungs accompanied with alveolar differentiation delay in E18.5 embryos and P0 (only DKO). When dexamethasone was added to the organoid cultures, we obtained an overall reduction in organoid number affecting the four different genotypes under study. However, despite obtaining fewer organoid yield numbers, the tendency of HRas-KO and DKO ones was comparable to the untreated ones, with a slight increment in organoid formation in HRas-KO samples and a more notable increment in DKO organoids. Furthermore, dexamethasone incremented alveolar organoid yield number in those samples lacking HRas or NRas, but this effect was less evident in the CT and DKO ones. These results showing reduced organoid formation and increased alveolar differentiation upon dexamethasone administration is in line with the notion of the inhibitory effect of glucocorticoids restraining lung proliferation during development (Daniel 


\section{Discussion}

Bird et al., 2015); and with a premature and accelerated distal lung maturation and distorted branching when glucocorticoids, such as dexamethasone, are administered in earlier embryonic stages of lung development where the bronchiolar proximal epithelium is not fully developed (Oshika et al., 1998). Thus, our observations would also explain the partial rescue noticed in the in vivo DKO mice treated with glucocorticoids.

Our previous findings indicated that $\mathrm{N}$-acetylcysteine treatment produced a reduction of ROS levels in both lung epithelium and mesenchymal cells, ameliorating the perinatal lethality observed in HRas/NRas-devoid pups and enhancing normal alveoli differentiation. Consistent with these observations, we demonstrated that in vitro treatment of the organoids with NAC caused an overall increment in organoid numbers across all CT and KO genotypes, but also increased SftpC-expressing alveolar organoids. Interestingly, the reduced levels of mesenchymal FGF7 and FGF10 upon NAC treatment could explain this increment in alveolar organoids.

To get further mechanistic insights in our HRas/NRas-DKO model, we examined several key signalling pathways acting downstream of Ras activation, which are known to be involved in lung development. In particular: (1) KRas signalling during lung development regulates lung branching together with Wnt and SOX9 signalling (Ostrin et al., 2018; Ustiyan et al., 2016); (2) additionally, ERK/MAPK signalling has been proved to be crucial in lung mesenchyme and epithelium for correct lung organogenesis (Boucherat et al., 2014a, 2017); (3) importantly, $\mathrm{PI} 3 \mathrm{~K} / \mathrm{AKT}$ signalling pathway is involved in the control of survival signals and branching morphogenesis (Carter et al., 2014; J. Wang et al., 2005); as well as the (4) mitogen activated protein kinase p38 (Liu et al., 2008). Our results indicated a strong dependence on KRas signalling for a correct organoid formation since its inhibition with Fendiline (van der Hoeven et al., 2013) abrogated almost completely the correct organoid generation in all genotypes. Interestingly, MEK1/2 inhibition with the UO126 inhibitor resulted not only in a decrease of overall lung organoid yield numbers, but also in a strong differentiation of the organoids towards alveolar phenotype in all genotypes under study. These contradictory effects between Fendiline (KRas inhibition) and UO126 (MEK inhibition) may be explained with the inhibitory effect of Fendiline on voltage-gated L-type calcium channel (Tripathi et al., 1993). In this regard, although the concentration used for KRas inhibition $(10 \mu \mathrm{M})$ is slightly lower that used for voltage-gated L-type calcium channel inhibition $(17 \mu \mathrm{M})$, we observed a completely distorted branching in lung organoids treated with Fendiline, a process that is also regulated through the voltage-gated calcium channels (Brennan et al., 2013). On the other hand, PI3K inhibition resulted in a slight increment in bronchiolar organoids without affecting alveoli organoid proportion, being these observations consistent with previous studies where PI3K inhibition is linked to an enhancement of branching morphogenesis (Carter et al., 2014). Last, p38 inhibition results in a complete abrogation of differentiation, resulting in a strong increase in organoid 
yield numbers in all genotypes, being all these organoids similar to a pseudostratified epithelium conformed by basal-like organoids, thus, suggesting that p38 has a key role in the maintenance of these cells in lung organoids.

Interestingly, our results suggest that HRas and HRas/NRas organoids produce higher levels of FGF7, as detected in the organoid culture medium. Interestingly, a reduction on FGF7 concentration in the culture media derived from NAC- and UO126-treated lung organoids was noticeable in HRas and HRas/NRas samples (NAC), whereas UO126 affected to all the genotypes. On the other hand, no strong changes in Ras/ERK/PI3K/p38 signalling pathways were observed in mesenchymal cells upon treatment with the compounds during $24 \mathrm{~h}$, except for UO126 and NAC, that produced a strong decrease ERK activation (in all UO126-treated cells), and only a decrease in RAS.GTP in DKO NAC-treated cells. These results possibly suggest that the observed effect on the lung organoids could be due to the activity of these compounds on epithelial cells rather than on mesenchymal cells, since no strong effect was observed in NAC-treated cells, although further detailed assays are needed to clarify these observations.

On the other hand, consistent with the notion of a predominant role of KRas in maintaining branching morphogenesis loop and, thus, preventing alveolar differentiation (Chang et al., 2013; Ostrin et al., 2018), our results indicated more KRas activation in HRas/NRas-devoid lungs together with higher pERK levels. HRas-KO lungs exhibited a highly variable phenotype, with some of them showing similar KRas levels that DKO lungs, whereas others exhibited reduced KRas epithelial activation. However, HRas-KO lungs also exhibited incremented levels of pERK. Dexamethasone, and more efficiently NAC, decreased both KRas and pERK activation in lungs of HRas-KO and HRas/NRas-double null pups.

Taking all these results in context, our data uncover a crucial role of HRas, with partial overlapping from NRas, in controlling KRas activation in the context of distal lung alveolar differentiation. Consistently, inhibiting KRas or MEK activation results in increased alveoli differentiation rates.

\subsection{Adult HRas/NRas-deficient mouse exhibit partial atelectasis and a KRas-linked phenotype resembling RASopathies}

The few surviving adult DKO animals that could be studied exhibited smaller body size as compared with CT littermates, plus marked craniofacial dysmorphias, splenomegaly (not show in this dissertation), and a partial patched lung phenotype with atelectatic areas within normal lung tissue. It is worth mentioning that all these features, together with the observed neonatal KRas-gain-of-function, and perinatal death, are consistent with previously published data 


\section{Discussion}

describing a murine model of KRas-gain-of-function mutations inducing RASopathy-like phenotypes (Wong et al., 2020). Overall, our data in HRas/NRas-DKO mice point to a KRasdependent phenotype (Resembling RASopathies, without other mutations in the pathway) resulting from the absence of physiological inhibitory effects of HRas (With overlapping function of NRas), over KRas activation in normal lung cells and tissues.

\subsection{General discussion}

In this dissertation, we have analysed the functional specificity of HRas, NRas and KRas in lung developmental processes through the use of in vivo studies of specific knockout mouse models as well as in vitro analyses of lung organoids. For this purpose, we have made an extensive use of the HRas-KO, NRas-KO and HRas/NRas-DKO mouse strains previously generated in our group.

Initial in vivo studies on newborn mice revealed that both HRas and NRas are necessary for neonatal survival since HRas/NRas-DKO mice exhibited a severe neonatal respiratory, distresslike phenotype accompanied by high mortality rates. Our data indicate that both HRas and NRas are necessary for normal, late lung development. Of the two isoforms, HRas shows a stronger mechanistic link with alveolar maturation, although the defective alveolar phenotype of HRasKO mice is further aggravated when both HRas and NRas are absent. Regarding the delayed pneumocyte differentiation observed in HRas-KO and DKO mice, HRas appears to be the main functional regulator responsible for the abnormally increased proliferation rates measured in that cell lineage, whereas NRas appears to exert a more dominant functional role regarding control of cell death.

We also studied the functional implication of HRas and NRas in control of redox homeostasis control in lung epithelium and mesenchyme. Thus, ablation of both HRas and NRas in DKO lungs resulted in significant accumulation of reactive oxygen species and signs of mitochondrial transport chain alterations, with single NRas-KO lungs exhibiting a much milder phenotype. On the other hand, no significant differences were found when comparing ROS accumulation levels in the lungs of $\mathrm{CT}$ and HRas-KO mice.

Interestingly, a normal alveolar differentiation was achieved in HRas/NRas-DKO lungs by means of the antenatal administration of dexamethasone (DEX) or N-Acetylcysteine (NAC). However, only NAC mitigated perinatal lethality, presumably due to its potential as an antioxidant and its effect on ROS reduction in the lung epithelium and mesenchyme.

In vitro analysis of lung organoids mimicking lung development recapitulated the previous in vivo observations. In particular, these studies showed a crucial dependence on HRas for normal organoid yield number and alveolar differentiation. Additionally, only NAC and UO126 
(MEK inhibitor) rescued alveolar differentiation in HRas/NRas-ablated lung organoids, probably due to a reduction in KRas and ERK activation.

These results support the notion that a well-balanced coordination between the three canonical Ras isoforms is crucial for murine development. In particular, we have documented here that HRas/NRas-ablation causes significant defects of lung developmental processes and also causes a RASopathy-like phenotype linked to KRas activity. Regarding lung development, although only the concomitant absence of both HRas and NRas results in neonatal lethality due to respiratory failure, it is fairly apparent that HRas has a functional prevalence over NRas regarding the control of late lung maturation process. An interesting speculation is that the relevant deleterious effect caused by HRas ablation on mouse lung development may be mechanistically linked, at least in part, to the absence of its functional contribution(s) counteracting activation of KRas in the lung epithelium under physiological conditions. 



\section{Conclusions / CONCLUSIONES}





\section{CONCLUSIONS}

1. Simultaneous ablation of HRas and NRas in mice causes perinatal lethality with markedly respiratory distress caused by a delayed lung maturation, characterized by strong retention of undifferentiated alveolar progenitor cells. Single deletion of HRas, but not of NRas, shows also a partial time delay in alveolar differentiation as compared to HRas/NRas-DKO lungs. These observations underscore the critical requirement of active HRas/NRas signalling during the late stages of lung development.

2. Absence of HRas, but not of NRas, is linked to increased signalling through the mesenchymal FGF7/10 - epithelial SOX9 axis, as well as strongly enhanced cell proliferation, thus leading to maintained branching-proliferative signalling that inhibits alveolar differentiation. This deleterious effect is further aggravated when both HRas and NRas are ablated. These data uncover a direct mechanistic link between HRas and control of proliferation and branching termination.

3. Single NRas ablation causes an overall increase of cell apoptosis and oxidative stress in whole lungs and in primary lung fibroblasts, whereas single HRas ablation appears to be dispensable in this regard. However, simultaneous HRas/NRas disruption results in a stronger redox imbalance, consistent with alterations in mitochondrial respiration, and higher apoptotic rates. These data point to a direct mechanistic link between NRas and control of apoptosis and mitochondrial redox balance.

4. Antenatal administration of $\mathrm{N}$-Acetylcysteine and dexamethasone rescues normal lung alveolar development, but only N-Acetylcysteine efficiently decreases perinatal HRas/NRas-DKO mortality, redox imbalance and FGF7/10 expression. These observations highlight the therapeutic relevance of antioxidant treatments to reduce lethality due to respiratory distress by decreasing oxidative stress and modulating the branching signalling axis in the lung.

5. MEK inhibition rescues the in vitro alveolar differentiation defects of HRas/NRas-DKO lung organoids, and N-Acetylcysteine treatment only does this partially, revealing a corrective role of Ras downstream signalling in lung epithelium rather than in lung mesenchyme. Interestingly, the higher KRas-ERK activation of HRas/NRas-DKO epithelium, and to a 
lesser extent of HRas-KO lungs, is mitigate by N-Acetylcysteine and only partially by dexamethasone antenatal treatment. These results indicate that inhibition of aberrant KRasERK activation in lung epithelium rescues alveolar differentiation and suggest that KRas downstream signalling is critical needed for physiological modulation of the process of branching.

Collectively, these findings provide some mechanistic insights about the individual functional role(s) of HRas, with partial overlapping contribution from NRas, in the control of late murine lung developmental processes that are mainly dependent on the regulatory functions of activated KRas. 


\section{Conclusiones}

1. Le eliminación simultánea de HRas y NRas en ratones provoca muerte perinatal por fallo respiratorio, causado por un retraso en la maduración pulmonar caracterizado por una retención de progenitores alveolares indiferenciados. La eliminación de HRas, pero no de NRas, presenta un retraso parcial en la diferenciación alveolar comparado con los pulmones de los animales HRas/NRas-DKO.

2. La ausencia de HRas, pero no de NRas, repercute en una mayor señalización de la vía FGF7/10 (mesénquima) - SOX9 (epitelio), junto con un incremento significativo de la proliferación, dando lugar a una señal de branching mantenida que inhibe la diferenciación alveolar. Este efecto se ve agravado cuando ambos HRas y NRas han sido delecionados. Estos datos revelan un link mecanístico directo de HRas en el control de la proliferación y la terminación del proceso de branching.

3. La eliminación de NRas causa un incremento de la muerte celular por apoptosis, así como del estrés oxidativo en pulmones y fibroblastos de pulmón; mientras que la eliminación de HRas no muestra ningún efecto en estos procesos. Sin embargo, la eliminación conjunta de HRas y NRas provoca un desbalance redox, alteraciones en la respiración mitocondrial y elevados niveles de muerte celular por apoptosis. Estos datos sugieren la existencia de un link de NRas en el control de la apoptosis y balance redox mitocondrial.

4. La administración antenatal de N-Acetilcisteína y dexametasona rescata la maduración alveolar defectuosa, pero solo el tratamiento con N-Acetilcisteína disminuye de manera eficiente la letalidad perinatal de los animales carentes de HRas y NRas, así como reduce el desbalance redox y la expresión de FGF7/10. Estas observaciones destacan la relevancia terapéutica del tratamiento con antioxidantes para reducir la letalidad por fallo respiratorio a través de la disminución del estrés oxidativo y la modulación del branching.

5. La inhibición de MEK rescata in vitro los defectos en la diferenciación alveolar derivados de la falta de HRas y NRas, mientras que N-Acetilcisteina provoca una recuperación parcial, a través del efecto sobre la vía de señalización de Ras en el epitelio pulmonar. De manera concordante, los mayores niveles de activación de KRas-ERK en el epitelio de los animales HRas/NRas-DKO, y en menor medida de HRas-KO, son mitigados tras el tratamiento antenatal con $\mathrm{N}$-Acetilcisteína y de manera parcial con la administración de dexametasona. Estos datos sugieren que la inhibición de la activación aberrante de Kras-ERK en el epitelio 


\section{Conclusions / Conclusiones}

pulmonar recuperan la diferenciación alveolar, y sugieren que la señalización de KRas es necesaria para una modulación fisiológica del proceso de branching.

De manera conjunta, nuestros resultados proporcionan conocimientos clave sobre el(los) papel(es) individual(es) de HRas, con una contribución parcial de NRas, en el control de los procesos que tienen lugar en el desarrollo pulmonar murino tardío, los cuales dependen principalmente de las funciones reguladores sobre la activación de KRas. 


\section{BIBLIOGRAPHY}

1. Abdel Hamid, E. R., Ali, W. H., Azmy, A., Ahmed, H. H., Sherif, L. S., \& Saleh, M. T. (2019). Oxidative stress and anti-oxidant markers in premature infants with respiratory distress syndrome. Open Access Macedonian Journal of Medical Sciences. https://doi.org/10.3889/oamjms.2019.534

2. Adderley, H., Blackhall, F. H., \& Lindsay, C. R. (2019). KRAS-mutant non-small cell lung cancer: Converging small molecules and immune checkpoint inhibition. In EBioMedicine (Vol. 41, pp. 711-716). Elsevier. https://doi.org/10.1016/j.ebiom.2019.02.049

3. Agrons, G. A., Courtney, S. E., Stocker, J. T., \& Markowitz, R. I. (2005). Lung disease in premature neonates: Radiologic-pathologic correlation. Radiographics, 25(4), 1047-1073. https://doi.org/10.1148/rg.254055019

4. Ahearn, I. M., Haigis, K., Bar-Sagi, D., \& Philips, M. R. (2012). Regulating the regulator: Post-translational modification of RAS. In Nature Reviews Molecular Cell Biology (Vol. 13, Issue 1, pp. 39-51). https://doi.org/10.1038/nrm3255

5. Ahearn, I., Zhou, M., \& Philips, M. R. (2018). Posttranslational Modifications of RAS Proteins. In Cold Spring Harbor perspectives in medicine (Vol. 8, Issue 11). Cold Spring Harbor Laboratory Press. https://doi.org/10.1101/cshperspect.a031484

6. Ahlfeld, S. K., \& Conway, S. J. (2012). Aberrant signaling pathways of the lung mesenchyme and their contributions to the pathogenesis of bronchopulmonary dysplasia. In Birth Defects Research Part A - Clinical and Molecular Teratology. https://doi.org/10.1002/bdra.22869

7. Alvira, C. M. (2014). Nuclear factor-kappa-B signaling in lung development and disease: One pathway, numerous functions. Birth Defects Research Part A - Clinical and Molecular Teratology, 100(3), 202-216. https://doi.org/10.1002/bdra.23233

8. Amsen, E. M., Pham, N., Pak, Y., \& Rotin, D. (2006). The guanine nucleotide exchange factor CNrasGEF regulates melanogenesis and cell survival in melanoma cells. Journal of Biological Chemistry, 281(1), 121-128. https://doi.org/10.1074/jbc.M507595200

9. Anton, Y., \& Wennogle, L. P. (1998). The RAF family: An expanding network of posttranslational controls and protein-protein interactions. Cell Research, 8(2), 81-98. https://doi.org/10.1038/cr.1998.9

10. Aoki, Y., Niihori, T., Inoue, S. I., \& Matsubara, Y. (2016). Recent advances in RASopathies. In Journal of Human Genetics (Vol. 61, Issue 1, pp. 33-39). J Hum Genet. https://doi.org/10.1038/jhg.2015.114

11. Aros, C. J., Pantoja, C. J., \& Gomperts, B. N. (2021). Wnt signaling in lung development, regeneration, and disease progression. In Communications Biology (Vol. 4, Issue 1). 
Bibliography

https://doi.org/10.1038/s42003-021-02118-w

12. Arozarena, I., Calvo, F., \& Crespo, P. (2011). Ras, an actor on many Stages: Posttranslational modifications, Localization, and Site-Specified Events. Genes and Cancer, 2(3), 182-194. https://doi.org/10.1177/1947601911409213

13. Arozarena, I., Matallanas, D., Berciano, M. T., Sanz-Moreno, V., Calvo, F., Muñoz, M. T., Egea, G., Lafarga, M., \& Crespo, P. (2004). Activation of H-Ras in the endoplasmic reticulum by the RasGRF family guanine nucleotide exchange factors. Molecular and Cellular Biology, 24(4), 1516-1530. https://doi.org/10.1128/MCB.24.4.1516-1530.2004

14. Assimakopoulos, S. F., Aretha, D., Komninos, D., Dimitropoulou, D., Lagadinou, M., Leonidou, L., Oikonomou, I., Mouzaki, A., \& Marangos, M. (2021). N-acetyl-cysteine reduces the risk for mechanical ventilation and mortality in patients with COVID-19 pneumonia: a two-center retrospective cohort study. Infectious Diseases, 53(11), 847-854. https://doi.org/10.1080/23744235.2021.1945675

15. Atayoğlu, K., Gürleyik, G., Demirel, G., \& Özkara, S. (2017). Deneysel akut pankreatitte nötrofil fonksiyonları üzerine N-asetilsistein'in etkisi. Ulusal Travma ve Acil Cerrahi Dergisi, 23(2), 100-106. https://doi.org/10.5505/tjtes.2016.59844

16. Baltanás, F. C., Pérez-Andrés, M., Ginel-Picardo, A., Diaz, D., Jimeno, D., Liceras-Boillos, P., Kortum, R. L., Samelson, L. E., Orfao, A., \& Santos, E. (2013). Functional Redundancy of Sos1 and Sos2 for Lymphopoiesis and Organismal Homeostasis and Survival. Molecular and Cellular Biology, 33(22), 4562-4578. https://doi.org/10.1128/mcb.01026-13

17. Baltanás, F. C., Zarich, N., Rojas-Cabañeros, J. M., \& Santos, E. (2020a). SOS GEFs in health and disease. In Biochimica et Biophysica Acta - Reviews on Cancer. https://doi.org/10.1016/j.bbcan.2020.188445

18. Baltanás, F. C., Zarich, N., Rojas-Cabañeros, J. M., \& Santos, E. (2020b). SOS GEFs in health and disease. In Biochimica et Biophysica Acta - Reviews on Cancer (Vol. 1874, Issue 2, p. 188445). Elsevier. https://doi.org/10.1016/j.bbcan.2020.188445

19. Barbacid, M. (1987). ras GENES1. Annual Review of Biochemistry, 56, 779-827. www.annualreviews.org

20. Barkauskas, C. E., Cronce, M. J., Rackley, C. R., Bowie, E. J., Keene, D. R., Stripp, B. R., Randell, S. H., Noble, P. W., \& Hogan, B. L. M. (2013). Type 2 alveolar cells are stem cells in adult lung. The Journal of Clinical Investigation, 123(7), 3025. https://doi.org/10.1172/JCI68782

21. Barnes, P. J. (2004). Ceramide lances the lungs. In Nature Medicine (Vol. 10, Issue 2, pp. 130131). Nat Med. https://doi.org/10.1038/nm0204-130 
22. Bassères, D. S., Levantini, E., Ji, H., Monti, S., Elf, S., Dayaram, T., Fenyus, M., Kocher, O., Golub, T., Wong, K., Halmos, B., \& Tenen, D. G. (2006). Respiratory Failure Due to Differentiation Arrest and Expansion of Alveolar Cells following Lung-Specific Loss of the Transcription Factor C/EBP $\alpha$ in Mice. Molecular and Cellular Biology, 26(3), 1109-1123. https://doi.org/10.1128/MCB.26.3.1109-1123.2006

23. Benjamini, Y., Drai, D., Elmer, G., Kafkafi, N., \& Golani, I. (2001). Controlling the false discovery rate in behavior genetics research. Behavioural Brain Research. https://doi.org/10.1016/S0166-4328(01)00297-2

24. Bersani, I., Speer, C. P., \& Kunzmann, S. (2012). Surfactant proteins A and D in pulmonary diseases of preterm infants. In Expert Review of Anti-Infective Therapy (Vol. 10, Issue 5, pp. 573-584). Expert Rev Anti Infect Ther. https://doi.org/10.1586/eri.12.34

25. Bivona, T. G., Quatela, S. E., Bodemann, B. O., Ahearn, I. M., Soskis, M. J., Mor, A., Miura, J., Wiener, H. H., Wright, L., Saba, S. G., Yim, D., Fein, A., Pérez de Castro, I., Li, C., Thompson, C. B., Cox, A. D., \& Philips, M. R. (2006). PKC regulates a farnesyl-electrostatic switch on K-Ras that promotes its association with Bcl-XL on mitochondria and induces apoptosis. Molecular Cell, 21(4), 481-493. https://doi.org/10.1016/j.molcel.2006.01.012

26. Blázquez-Prieto, J., López-Alonso, I., Amado-Rodríguez, L., Huidobro, C., González-López, A., Kuebler, W. M., \& Albaiceta, guillermo M. (2018). Impaired lung repair during neutropenia can be reverted by matrix metalloproteinase-9. Thorax, 73(4), 321-330. https://doi.org/10.1136/thoraxjnl-2017-210105

27. Boggaram, V. (2009). Thyroid transcription factor-I (TTF-I/Nkx2.I/TITFI) gene regulation in the lung. In Clinical Science (Vol. 116, Issue 1, pp. 27-35). Portland Press. https://doi.org/10.1042/CS20080068

28. Boguski, M. S., \& McCormick, F. (1993). Proteins regulating Ras and its relatives. In Nature (Vol. 366, Issue 6456, pp. 643-654). Nature Publishing Group. https://doi.org/10.1038/366643a0

29. Bos, J. L., Rehmann, H., \& Wittinghofer, A. (2007). GEFs and GAPs: Critical Elements in the Control of Small G Proteins. In Cell (Vol. 129, pp. 865-877). https://doi.org/10.1016/j.cell.2007.05.018

30. Boucherat, O., Landry-Truchon, K., Aoidi, R., Houde, N., Nadeau, V., Charron, J., \& Jeannotte, L. (2017). Lung development requires an active ERK/MAPK pathway in the lung mesenchyme. Developmental Dynamics. https://doi.org/10.1002/dvdy.24464

31. Boucherat, O., Nadeau, V., Bérubé-Simard, F.-A., Charron, J., \& Jeannotte, L. (2014a). Crucial requirement of ERK/MAPK signaling in respiratory tract development. Development, 141(16), 3197-3211. https://doi.org/10.1242/DEV.110254 
Bibliography

32. Boucherat, O., Nadeau, V., Bérubé-Simard, F. A., Charron, J., \& Jeannotte, L. (2014b). Crucial requirement of ERK/MAPK signaling in respiratory tract development. Development (Cambridge). https://doi.org/10.1242/dev.110254

33. Bowtell, D., Fu, P., Simon, M., \& Senior, P. (1992). Identification of murine homologues of the Drosophila son of sevenless gene: potential activators of ras. Proceedings of the National Academy of Sciences, 89(14), 6511-6515. https://doi.org/10.1073/PNAS.89.14.6511

34. Brandt, J. P., \& Mandiga, P. (2021). Histology, Alveolar Cells. In StatPearls. StatPearls Publishing. https://www.ncbi.nlm.nih.gov/books/NBK557542/

35. Brennan, S. C., Finney, B. A., Lazarou, M., Rosser, A. E., Scherf, C., Adriaensen, D., Kemp, P. J., \& Riccardi, D. (2013). Fetal calcium regulates branching morphogenesis in the developing human and mouse lung: Involvement of voltage-gated calcium channels. PLoS ONE, 8(11), 80294. https://doi.org/10.1371/journal.pone.0080294

36. Bridges, J. P., Ikegami, M., Brilli, L. L., Chen, X., Mason, R. J., \& Shannon, J. M. (2010). LPCAT1 regulates surfactant phospholipid synthesis and is required for transitioning to air breathing in mice. Journal of Clinical Investigation, 120(5), 1736-1748. https://doi.org/10.1172/JCI38061

37. Brownfoot, F. C., Gagliardi, D. I., Bain, E., Middleton, P., \& Crowther, C. A. (2013). Different corticosteroids and regimens for accelerating fetal lung maturation for women at risk of preterm birth. In Cochrane Database of Systematic Reviews. https://doi.org/10.1002/14651858.CD006764.pub3

38. Buhimschi, C. S., Bahtiyar, M. O., Zhao, G., Abdelghany, O., Schneider, L., Razeq, S. A., Dulay, A. T., Lipkind, H. S., Mieth, S., Rogers, L., Bhandari, V., \& Buhimschi, I. A. (2020). Antenatal $\mathrm{N}$-acetylcysteine to improve outcomes of premature infants with intra-amniotic infection and inflammation (Triple I): randomized clinical trial. Pediatric Research 2020 89:1, 89(1), 175-184. https://doi.org/10.1038/s41390-020-01106-w

39. Campbell, S. L., Khosravi-Far, R., Rossman, K. L., Clark, G. J., \& Der, C. J. (1998). Increasing complexity of Ras signaling. In Oncogene (Vol. 17, Issue 11 REV. ISS. 1, pp. 1395-1413). Oncogene. https://doi.org/10.1038/sj.onc.1202174

40. Cardoso. (2008). Specification and patterning of the respiratory system. StemBook. https://doi.org/10.3824/stembook.1.10.1

41. Cargnello, M., \& Roux, P. P. (2011). Activation and Function of the MAPKs and Their Substrates, the MAPK-Activated Protein Kinases. Microbiology and Molecular Biology Reviews, 75(1), 50-83. https://doi.org/10.1128/mmbr.00031-10

42. Carter, E., Miron-Buchacra, G., Goldoni, S., Danahay, H., Westwick, J., Watson, M. L., Tosh, D., \& Ward, S. G. (2014). Phosphoinositide 3-kinase alpha-dependent regulation of branching 
morphogenesis in murine embryonic lung: Evidence for a role in determining morphogenic properties of FGF7. PLoS ONE. https://doi.org/10.1371/journal.pone.0113555

43. Casar, B., Pinto, A., \& Crespo, P. (2008). Essential Role of ERK Dimers in the Activation of Cytoplasmic but Not Nuclear Substrates by ERK-Scaffold Complexes. Molecular Cell, 31(5), 708-721. https://doi.org/10.1016/j.molcel.2008.07.024

44. Castellano, E., De Las Rivas, J., Guerrero, C., \& Santos, E. (2007). Transcriptional networks of knockout cell lines identify functional specificities of H-Ras and N-Ras: Significant involvement of N-Ras in biotic and defense responses. Oncogene. https://doi.org/10.1038/sj.onc.1209845

45. Castellano, E., Guerrero, C., A Núñez, De Las Rivas, J., \& E Santos. (2009). Serum-dependent transcriptional networks identify distinct functional roles for H-Ras and N-Ras during initial stages of the cell cycle. Genome Biology, 10(11). https://doi.org/10.1186/GB-2009-10-11R123

46. Castellano, Esther, \& Downward, J. (2011). Ras interaction with PI3K: More than just another effector pathway. In Genes and Cancer (Vol. 2, Issue 3, pp. 261-274). Genes Cancer. https://doi.org/10.1177/1947601911408079

47. Castellano, Esther, \& Santos, E. (2011). Functional specificity of Ras isoforms: So similar but so different. Genes and Cancer, 2, 216-231. https://doi.org/10.1177/1947601911408081

48. Castillo, R. ., Carrasco Loza, R., \& Romero-Dapueto, C. (2015). Pathophysiological Approaches of Acute Respiratory Distress syndrome: Novel Bases for Study of Lung Injury. The Open Respiratory Medicine Journal, 9(Suppl 2: M2), 83-91. https://doi.org/10.2174/1874306401509010083

49. Chandra, A., Grecco, H. E., Pisupati, V., Perera, D., Cassidy, L., Skoulidis, F., Ismail, S. A., Hedberg, C., Hanzal-Bayer, M., Venkitaraman, A. R., Wittinghofer, A., \& Bastiaens, P. I. H. (2011). The GDI-like solubilizing factor PDE $\delta$ sustains the spatial organization and signalling of Ras family proteins. Nature Cell Biology 2012 14:2, 14(2), 148-158. https://doi.org/10.1038/ncb2394

50. Chang, D. R., Alanis, D. M., Miller, R. K., Ji, H., Akiyama, H., McCrea, P. D., \& Chen, J. (2013). Lung epithelial branching program antagonizes alveolar differentiation. Proceedings of the National Academy of Sciences of the United States of America, 110(45), 18042-18051. https://doi.org/10.1073/pnas.1311760110

51. Chao, C. M., El Agha, E., Tiozzo, C., Minoo, P., \& Bellusci, S. (2015). A breath of fresh air on the mesenchyme: Impact of impaired mesenchymal development on the pathogenesis of bronchopulmonary dysplasia. In Frontiers in Medicine. https://doi.org/10.3389/fmed.2015.00027 
Bibliography

52. Cheng, C.-M., Li, H., Gasman, S., Huang, J., Schiff, R., \& Chang, E. C. (2011). Compartmentalized Ras Proteins Transform NIH 3T3 Cells with Different Efficiencies. Molecular and Cellular Biology, 31(5), 983-997. https://doi.org/10.1128/mcb.00137-10

53. Chiu, V. K., Bivona, T., Hach, A., Sajous, J. B., Silletti, J., Wiener, H., Johnson, R. L. 2nd, Cox, A. D., \& Philips, M. R. (2002). Ras signalling on the endoplasmic reticulum and the Golgi. Nature Cell Biology, 4(5), 343-350. https://doi.org/10.1038/ncb783

54. Cho, K.-J. (2020). K-Ras Plasma Membrane Interactions: A Tractable Therapeutic Target. American Journal of Biomedical Science \& Research, 9(5), 414-417. https://doi.org/10.34297/ajbsr.2020.09.001440

55. Cho, K., van der Hoeven, D., Zhou, Y., Maekawa, M., Ma, X., Chen, W., Fairn, G. D., \& Hancock, J. F. (2015). Inhibition of acid sphingomyelinase depletes cellular phosphatidylserine and mislocalizes K-Ras from the plasma membrane. Molecular and Cellular Biology, 36(2), MCB.00719-15. https://doi.org/10.1128/mcb.00719-15

56. Clark, H., \& Clark, L. S. (2005). The genetics of neonatal respiratory disease. In Seminars in Fetal and Neonatal Medicine. https://doi.org/10.1016/j.siny.2005.02.004

57. Copland, I. B., \& Post, M. (2012). Understanding the Mechanisms of Infant Respiratory Distress and Chronic Lung Disease. Https://Doi.Org/10.1165/Ajrcmb.26.3.F231, 26(3), 261265. https://doi.org/10.1165/AJRCMB.26.3.F231

58. Cuadrado, A., \& Nebreda, A. R. (2010). Mechanisms and functions of p 38 MAPK signalling. In Biochemical Journal (Vol. 429, Issue 3, pp. 403-417). Biochem J. https://doi.org/10.1042/BJ20100323

59. Daniel Bird, A., McDougall, A. R. A., Seow, B., Hooper, S. B., \& Cole, T. J. (2015). Minireview: Glucocorticoid regulation of lung development: Lessons learned from conditional GR knockout mice. Molecular Endocrinology, 29(2), 158-171. https://doi.org/10.1210/me.2014-1362

60. DeFea, K. A., Zalevsky, J., Thoma, M. S., Dery, O., Mullins, R. D., \& Bunnett, N. W. (2000). $\beta$-Arrestin-dependent endocytosis of proteinase-activated receptor 2 is required for intracellular targeting of activated ERK1/2. Journal of Cell Biology, 148(6), 1267-1281. https://doi.org/10.1083/jcb.148.6.1267

61. Dennery, P. A. (2007). Effects of oxidative stress on embryonic development. In Birth Defects Research Part C - Embryo Today: Reviews. https://doi.org/10.1002/bdrc.20098

62. Deprez, M., Zaragosi, L. E., Truchi, M., Becavin, C., García, S. R., Arguel, M. J., Plaisant, M., Magnone, V., Lebrigand, K., Abelanet, S., Brau, F., Paquet, A., Pe'er, D., Marquette, C. H., Leroy, S., \& Barbry, P. (2020). A single-cell atlas of the human healthy airways. American Journal of Respiratory and Critical Care Medicine. https://doi.org/10.1164/rccm.201911- 
$21990 \mathrm{C}$

63. Desai, T. J., Brownfield, D. G., \& Krasnow, M. A. (2014). Alveolar progenitor and stem cells in lung development, renewal and cancer. Nature. https://doi.org/10.1038/nature12930

64. Drosten, M., Guerra, C., \& Barbacid, M. (2018). Genetically engineered mouse models of KRas-driven lung and pancreatic tumors: Validation of therapeutic targets. Cold Spring Harbor Perspectives in Medicine, 8(5). https://doi.org/10.1101/cshperspect.a031542

65. Drugan, J. K., Rogers-Graham, K., Gilmer, T., Campbell, S., \& Clark, G. J. (2000). The Ras/p120 GTPase-activating Protein (GAP) Interaction Is Regulated by the p120 GAP Pleckstrin Homology Domain *. Journal of Biological Chemistry, 275(45), 35021-35027. https://doi.org/10.1074/jbc.M004386200

66. Du, J., McEwen, B., \& Manji, H. K. (2009). Glucocorticoid receptors modulate mitochondrial function: A novel mechanism for neuroprotection. Communicative and Integrative Biology, 2(4), 350-352. https://doi.org/10.4161/cib.2.4.8554

67. Dunnett-Kane, V., Burkitt-Wright, E., Blackhall, F. H., Malliri, A., Evans, D. G., \& Lindsay, C. R. (2020). Germline and sporadic cancers driven by the RAS pathway: parallels and contrasts. Annals of Oncology: Official Journal of the European Society for Medical Oncology, 31(7), 873-883. https://doi.org/10.1016/j.annonc.2020.03.291

68. Dusaban, S. S., \& Brown, J. H. (2015). PLC $\varepsilon$ mediated sustained signaling pathways. In Advances in Biological Regulation (Vol. 57, pp. 17-23). Adv Biol Regul. https://doi.org/10.1016/j.jbior.2014.09.014

69. Dzierba, A. L., Abel, E. E., Buckley, M. S., \& Lat, I. (2014). A review of inhaled nitric oxide and aerosolized epoprostenol in acute lung injury or acute respiratory distress syndrome. In Pharmacotherapy. https://doi.org/10.1002/phar.1365

70. Ebinu, J. O., Bottorff, D. A., Chan, E. Y. W., Stang, S. L., Dunn, R. J., \& Stone, J. C. (1998). RasGRP, a Ras guanyl nucleotide- releasing protein with calcium- and diacylglycerol-binding motifs. Science, 280(5366), 1082-1086. https://doi.org/10.1126/science.280.5366.1082

71. El Agha, E., \& Bellusci, S. (2014). Walking along the Fibroblast Growth Factor 10 Route: A Key Pathway to Understand the Control and Regulation of Epithelial and Mesenchymal CellLineage Formation during Lung Development and Repair after Injury. Scientifica, 2014, 120. https://doi.org/10.1155/2014/538379

72. Elkabany, Z. A., El-Farrash, R. A., Shinkar, D. M., Ismail, E. A., Nada, A. S., Farag, A. S., Elsayed, M. A., Salama, D. H., Macken, E. L., \& Gaballah, S. A. (2020). Oxidative stress markers in neonatal respiratory distress syndrome: advanced oxidation protein products and 8-hydroxy-2-deoxyguanosine in relation to disease severity. Pediatric Research, 87(1), 7480. https://doi.org/10.1038/s41390-019-0464-y 
Bibliography

73. Esteban, L. M., Vicario-Abejon, C., Fernandez-Salguero, P., Fernandez-Medarde, A., Swaminathan, N., Yienger, K., Lopez, E., Malumbres, M., McKay, R., Ward, J. M., Pellicer, A., \& Santos, E. (2001). Targeted Genomic Disruption of H-ras and N-ras, Individually or in Combination, Reveals the Dispensability of Both Loci for Mouse Growth and Development. Molecular and Cellular Biology, 21, 1444-1452. https://doi.org/10.1128/mcb.21.5.14441452.2001

74. Fabretto, A., Kutsche, K., Harmsen, M. B., Demarini, S., Gasparini, P., Fertz, M. C., \& Zenker, M. (2010). Two cases of Noonan syndrome with severe respiratory and gastroenteral involvement and the SOS1 mutation F623I. European Journal of Medical Genetics, 53(5), 322-324. https://doi.org/10.1016/J.EJMG.2010.07.011

75. Fattman, C. L., Enghild, J. J., Crapo, J. D., Schaefer, L. M., Valnickova, Z., \& Oury, T. D. (2000). Purification and characterization of extracellular superoxide dismutase in mouse lung. Biochemical and Biophysical Research Communications, 275(2), 542-548. https://doi.org/10.1006/bbrc.2000.3327

76. Fattman, C. L., Schaefer, L. M., \& Oury, T. D. (2003). Extracellular superoxide dismutase in biology and medicine. Free Radical Biology and Medicine, 35(3), 236-256. https://doi.org/10.1016/S0891-5849(03)00275-2

77. Fehrenbacher, N., Bar-Sagi, D., \& Philips, M. (2009). Ras/MAPK signaling from endomembranes. Molecular $\quad$ Oncology, 3(4), 297-307. https://doi.org/10.1016/j.molonc.2009.06.004

78. Fernández-Medarde, A., De, J., Rivas, L., \& Santos, E. (2021). 40 Years of RAS-A Historic Overview. https://doi.org/10.3390/genes12050681

79. Fernández-Medarde, A., \& Santos, E. (2011a). Ras in cancer and developmental diseases. In Genes and Cancer (Vol. 2, pp. 344-358). https://doi.org/10.1177/1947601911411084

80. Fernández-Medarde, A., \& Santos, E. (2011b). The RasGrf family of mammalian guanine nucleotide exchange factors. Biochimica et Biophysica Acta (BBA) - Reviews on Cancer, 1815(2), 170-188. https://doi.org/10.1016/J.BBCAN.2010.11.001

81. Fernández-Renau, D., Lombardero, M., \& Nieto, A. (1984). Glucocorticoid-dependent uteroglobin synthesis and uteroglobulin mRNA levels in rabbit lung explants cultured in vitro. European Journal of Biochemistry, 144(3), 523-527. https://doi.org/10.1111/j.14321033.1984.tb08497.x

82. Fletcher, M. E., Boshier, P. R., Wakabayashi, K., Keun, H. C., Smolenski, R. T., Kirkham, P. A., Adcock, I. M., Barton, P. J., Takata, M., \& Marczin, N. (2015). Influence of glutathioneS-transferase (GST) inhibition on lung epithelial cell injury: Role of oxidative stress and metabolism. American Journal of Physiology - Lung Cellular and Molecular Physiology, 
308(12), 1274-1285. https://doi.org/10.1152/ajplung.00220.2014

83. Floros, J., Post, M., \& Smith, B. T. (1985). Glucocorticoids affect the synthesis of pulmonary fibroblast-pneumonocyte factor at a pretranslational level. Journal of Biological Chemistry, 260, 2265-2267. https://doi.org/10.1016/0045-6039(85)90361-6

84. Font De Mora, J., Esteban, L. M., Burks, D. J., Núñez, A., Garcés, C., García-Barrado, M. J., Iglesias-Osma, M. C., Moratinos, J., Ward, J. M., \& Santos, E. (2003). Ras-GRF1 signaling is required for normal $\beta$-cell development and glucose homeostasis. EMBO Journal, 22(12), 3039-3049. https://doi.org/10.1093/emboj/cdg280

85. Frank, D. B., Penkala, I. J., Zepp, J. A., Sivakumar, A., Linares-Saldana, R., Zacharias, W. J., Stolz, K. G., Pankin, J., Lu, M. Q., Wang, Q., Babu, A., Li, L., Zhou, S., Morley, M. P., Jain, R., \& Morrisey, E. E. (2019). Early lineage specification defines alveolar epithelial ontogeny in the murine lung. Proceedings of the National Academy of Sciences of the United States of America. https://doi.org/10.1073/pnas.1813952116

86. Fruman, D. A., Chiu, H., Hopkins, B. D., Bagrodia, S., Cantley, L. C., \& Abraham, R. T. (2017). The PI3K Pathway in Human Disease. In Cell (Vol. 170, Issue 4, pp. 605-635). NIH Public Access. https://doi.org/10.1016/j.cell.2017.07.029

87. García-Navas, R., Liceras-Boillos, P., Anta, B., Lillo, C., Gómez, C., Jimeno, D., Calzada, N., Jimenez, M. S., Baltanás, F. C., \& Santos, E. (2016). Sos1 disruption increases mitochondrial oxidative stress in primary MEFs. European Journal of Cancer. https://doi.org/10.1016/s0959-8049(16)61591-5

88. García-Navas, Rósula, Liceras-Boillos, P., Gómez, C., Baltanás, F. C., Calzada, N., NuevoTapioles, C., Cuezva, J. M., \& Santos, E. (2021). Critical requirement of SOS1 RAS-GEF function for mitochondrial dynamics, metabolism, and redox homeostasis. Oncogene, 40(27), 4538-4551. https://doi.org/10.1038/s41388-021-01886-3

89. Gazdar, A. F., Hirsch, F. R., \& Minna, J. D. (2016). From mice to men and back: An assessment of preclinical model systems for the study of lung cancers. In Journal of Thoracic Oncology (Vol. 11, Issue 3, pp. 287-299). J Thorac Oncol. https://doi.org/10.1016/j.jtho.2015.10.009

90. Gentleman, R. C., Carey, V. J., Bates, D. M., Bolstad, B., Dettling, M., Dudoit, S., Ellis, B., Gautier, L., Ge, Y., Gentry, J., Hornik, K., Hothorn, T., Huber, W., Iacus, S., Irizarry, R., Leisch, F., Li, C., Maechler, M., Rossini, A. J., ... Zhang, J. (2004). Bioconductor: open software development for computational biology and bioinformatics. Genome Biology. https://doi.org/10.1186/gb-2004-5-10-r80

91. Gerber, A. N. (2015). Glucocorticoids and the lung. Advances in Experimental Medicine and Biology, 872, 279-298. https://doi.org/10.1007/978-1-4939-2895-8_12 
Bibliography

92. Göggell, R., Winoto-Morbach, S., Vielhaber, G., Imai, Y., Lindner, K., Brade, L., Brade, H., Ehlers, S., Slutsky, A. S., Schütze, S., Gulbins, E., \& Uhlig, S. (2004). PAF-mediated pulmonary edema: A new role for acid sphingomyelinase and ceramide. Nature Medicine, 10(2), 155-160. https://doi.org/10.1038/nm977

93. Gomez-Ospina, N., Kuo, C., Ananth, A. L., Myers, A., Brennan, M.-L., Stevenson, D. A., Bernstein, J. A., \& Hudgins, L. (2016). Respiratory system involvement in Costello syndrome. American Journal of Medical Genetics. Part A, 170(7), 1849-1857. https://doi.org/10.1002/ajmg.a.37655

94. Gorfe, A. A., \& Cho, K. J. (2021). Approaches to inhibiting oncogenic K-Ras. In Small GTPases (Vol. 12, Issue 2, pp. 96-105). Taylor \& Francis. https://doi.org/10.1080/21541248.2019.1655883

95. Goss, A. M., Tian, Y., Tsukiyama, T., Cohen, E. D., Zhou, D., Lu, M. M., Yamaguchi, T. P., $\&$ Morrisey, E. E. (2009). Wnt2/2b and $\beta$-Catenin Signaling Are Necessary and Sufficient to Specify Lung Progenitors in the Foregut. Developmental Cell. https://doi.org/10.1016/j.devcel.2009.06.005

96. Guerrero, C., Rojas, J. M., Chedid, M., Esteban, L. M., Zimonjic, D. B., Popescu, N. C., Font de Mora, J., \& Santos, E. (1996). Expression of alternative forms of Ras exchange factors GRF and SOS1 in different human tissues and cell lines. Oncogene, 12(5), 1097-1107.

97. Guo, M., Du, Y., Gokey, J. J., Ray, S., Bell, S. M., Adam, M., Sudha, P., Perl, A. K., Deshmukh, H., Potter, S. S., Whitsett, J. A., \& Xu, Y. (2019). Single cell RNA analysis identifies cellular heterogeneity and adaptive responses of the lung at birth. Nature Communications. https://doi.org/10.1038/s41467-018-07770-1

98. Habermehl, D., Parkitna, J. R., Kaden, S., Brügger, B., Wieland, F., Gröne, H. J., \& Schütz, G. (2011). Glucocorticoid activity during lung maturation is essential in mesenchymal and less in alveolar epithelial cells. Molecular Endocrinology, 25(8), 1280-1288. https://doi.org/10.1210/me.2009-0380

99. Haczku, A. (2008). Protective role of the lung collectins surfactant protein A and surfactant protein D in airway inflammation. In Journal of Allergy and Clinical Immunology. https://doi.org/10.1016/j.jaci.2008.10.014

100. Hallman, M., Glumoff, V., \& Rämet, M. (2001). Surfactant in respiratory distress syndrome and lung injury. Comparative Biochemistry and Physiology - A Molecular and Integrative Physiology. https://doi.org/10.1016/S1095-6433(01)00324-5

101. Hallman, M., \& Haataja, R. (2003). Genetic influences and neonatal lung disease. In Seminars in Neonatology. https://doi.org/10.1016/S1084-2756(02)00196-3

102. Hallman, M., \& Haataja, R. (2007). Genetic basis of respiratory distress syndrome. In 
Frontiers in Bioscience. https://doi.org/10.2741/2263

103. Harris-Johnson, K. S., Domyan, E. T., Vezina, C. M., \& Sun, X. (2009). $\beta$-Catenin promotes respiratory progenitor identity in mouse foregut. Proceedings of the National Academy of Sciences of the United States of America. https://doi.org/10.1073/pnas.0902274106

104. Hawgood, S. (2004). Surfactant protein B: Structure and function. Biology of the Neonate. https://doi.org/10.1159/000078169

105. Henis, Y. I., Hancock, J. F., \& Prior, I. A. (2009). Ras acylation, compartmentalization and signaling nanoclusters (Review). Molecular Membrane Biology, 26(1-2), 80-92. https://doi.org/10.1080/09687680802649582

106. Henkels, K. M., Rehl, K. M., \& Cho, K. (2021). Blocking K-Ras Interaction With the Plasma Membrane Is a Tractable Therapeutic Approach to Inhibit Oncogenic K-Ras Activity. Frontiers in Molecular Biosciences, 8, 511. https://doi.org/10.3389/fmolb.2021.673096

107. Hennig, A., Markwart, R., Esparza-Franco, M. A., Ladds, G., \& Rubio, I. (2015). Ras activation revisited: Role of GEF and GAP systems. In Biological Chemistry (Vol. 396, pp. 831-848). https://doi.org/10.1515/hsz-2014-0257

108. Hernandez-Valladares, M., \& Prior, I. A. (2015). Comparative proteomic analysis of compartmentalised Ras signalling. Scientific Reports, 5(1), 17307. https://doi.org/10.1038/srep17307

109. Herriges, M., \& Morrisey, E. E. (2014). Lung development: Orchestrating the generation and regeneration of a complex organ. Development (Cambridge), 141(3), 502-513. https://doi.org/10.1242/dev.098186

110. Hines, E. A., \& Sun, X. (2014). Tissue crosstalk in lung development. In Journal of Cellular Biochemistry. https://doi.org/10.1002/jcb.24811

111. Hodun, K., Sztolsztener, K., \& Chabowski, A. (2021). Antioxidants Supplementation Reduces Ceramide Synthesis Improving the Cardiac Insulin Transduction Pathway in a Rodent Model of Obesity. Nutrients, 13(10), 3413. https://doi.org/10.3390/nu13103413

112. Hogan, B. L. M., Barkauskas, C. E., Chapman, H. A., Epstein, J. A., Jain, R., Hsia, C. C. W., Niklason, L., Calle, E., Le, A., Randell, S. H., Rock, J., Snitow, M., Krummel, M., Stripp, B. R., Vu, T., White, E. S., Whitsett, J. A., \& Morrisey, E. E. (2014). Repair and regeneration of the respiratory system: Complexity, plasticity, and mechanisms of lung stem cell function. In Cell Stem Cell. https://doi.org/10.1016/j.stem.2014.07.012

113. Houliston, R. A., Pearson, J. D., \& Wheeler-Jones, C. P. D. (2001). Agonist-specific cross talk between ERKs and p38mapk regulates PGI2 synthesis in endothelium. American Journal of Physiology - Cell Physiology, 281(4 50-4). https://doi.org/10.1152/ajpcell.2001.281.4.c1266

114. Hoxhaj, G., \& Manning, B. D. (2020). The PI3K-AKT network at the interface of oncogenic 
Bibliography

signalling and cancer metabolism. In Nature Reviews Cancer (Vol. 20, Issue 2, pp. 74-88). Nature Publishing Group. https://doi.org/10.1038/s41568-019-0216-7

115. Hrycaj, S. M., Dye, B. R., Baker, N. C., Burke, A. C., Spence, J. R., \& Wellik Correspondence, D. M. (2015). Hox5 Genes Regulate the Wnt2/2b-Bmp4-Signaling Axis during Lung Development. CellReports, 12, 903-912. https://doi.org/10.1016/j.celrep.2015.07.020

116. Hu, J. Y., Yang, P., Wegner, D. J., Heins, H. B., Luke, C. J., Li, F., White, F. V., Silverman, G. A., Cole, F. S., \& Wambach, J. A. (2020). Functional Characterization of 4 ATP-Binding Cassette Transporter A3 Gene (ABCA3) Variants. Human Mutation, 41(7), 1298. https://doi.org/10.1002/HUMU.24014

117. Hu, Y., Ng-Blichfeldt, J.-P., Ota, C., Ciminieri, C., Ren, W., Hiemstra, P. S., Stolk, J., Gosens, R., \& Königshoff, M. (2020). Wnt/ $\beta$-catenin signaling is critical for regenerative potential of distal lung epithelial progenitor cells in homeostasis and emphysema. Stem Cells (Dayton, Ohio), 38(11), 1467-1478. https://doi.org/10.1002/stem.3241

118. Hwang, J., Wen, J., \& Kelly, G. (2011). Activation of Wnt signaling by reactive oxygen species. Developmental Biology, 356(1), 134. https://doi.org/10.1016/j.ydbio.2011.05.112

119. I, A., DS, A., D, M., V, S., N, A., SP, T., H, T., T, I., JC, Z., JS, G., \& P, C. (2000). The Rho family GTPase Cdc42 regulates the activation of Ras/MAP kinase by the exchange factor RasGRF. The Journal of Biological Chemistry, 275(34), 26441-26448. https://doi.org/10.1074/JBC.M002992200

120. Irizarry, R. A., Hobbs, B., Collin, F., Beazer-Barclay, Y. D., Antonellis, K. J., Scherf, U., \& Speed, T. P. (2003). Exploration, normalization, and summaries of high density oligonucleotide array probe level data. Biostatistics (Oxford, England). https://doi.org/10.1093/biostatistics/4.2.249

121. Janahi, I. A., Rehman, A., \& Baloch, N. U.-A. (2018). Corticosteroids and Their Use in $\begin{array}{llll}\text { Respiratory In Disorders. IntechOpen. } & \text { Corticosteroids. }\end{array}$ https://doi.org/10.5772/intechopen.72147

122. Jaumot, M., Yan, J., Clyde-Smith, J., Sluimer, J., \& Hancock, J. F. (2002). The linker domain of the Ha-Ras hypervariable region regulates interactions with exchange factors, Raf-1 and phosphoinositide 3-kinase. The Journal of Biological Chemistry, 277(1), 272-278. https://doi.org/10.1074/jbc.M108423200

123. Jeyabalan, N., \& Clement, J. P. (2016). SYNGAP1: Mind the Gap. Frontiers in Cellular Neuroscience, 10, 32. https://doi.org/10.3389/fncel.2016.00032

124. Ji, H., Houghton, A. M., Mariani, T. J., Perera, S., Kim, C. B., Padera, R., Tonon, G., McNamara, K., Marconcini, L. A., Hezel, A., El-Bardeesy, N., Bronson, R. T., Sugarbaker, D., Maser, R. S., Shapiro, S. D., \& Wong, K. K. (2006). K-ras activation generates an 
inflammatory response in lung tumors. Oncogene, 25(14), 2105-2112. https://doi.org/10.1038/sj.onc.1209237

125. Jimeno, D., \& Santos, E. (2017). A new functional role uncovered for RASGRF2 in control of nuclear migration in cone photoreceptors during postnatal retinal development. In Small GTPases. https://doi.org/10.1080/21541248.2016.1189989

126. Jin, T. G., Satoh, T., Liao, Y., Song, C., Gao, X., Kariya, K. I., Hu, C. D., \& Kataoka, T. (2001). Role of the CDC25 Homology Domain of Phospholipase C $\varepsilon$ in Amplification of Rap1dependent Signaling. Journal of Biological Chemistry, 276(32), 30301-30307. https://doi.org/10.1074/jbc.M103530200

127. Jo, H. S. (2014). Genetic risk factors associated with respiratory distress syndrome. In Korean Journal of Pediatrics. https://doi.org/10.3345/kjp.2014.57.4.157

128. Johnson, C. W., Reid, D., Parker, J. A., Salter, S., Knihtila, R., Kuzmic, P., \& Mattos, C. (2017). The small GTPases K-Ras, N-Ras, and H-Ras have distinct biochemical properties determined by allosteric effects. Journal of Biological Chemistry, 292, 12981-12993. https://doi.org/10.1074/jbc.M117.778886

129. Johnson, L., Greenbaum, D., Cichowski, K., Mercer, K., Murphy, E., Schmitt, E., Bronson, R. T., Umanoff, H., Edelmann, W., Kucherlapati, R., \& Jacks, T. (1997). K-ras is an essential gene in the mouse with partial functional overlap with $\mathrm{N}$-ras.

130. Joneson, T., \& Bar-Sagi, D. (1997). Ras effectors and their role in mitogenesis and oncogenesis. In Journal of Molecular Medicine (Vol. 75, Issue 8, pp. 587-593). J Mol Med (Berl). https://doi.org/10.1007/s001090050143

131. Kassouf, T., \& Sumara, G. (2020). Impact of conventional and atypical mapks on the development of metabolic diseases. Biomolecules, 10(9), 1-34. https://doi.org/10.3390/biom10091256

132. Kellner, M., Noonepalle, S., Lu, Q., Srivastava, A., Zemskov, E., \& Black, S. M. (2017). ROS signaling in the pathogenesis of Acute Lung Injury (ALI) and Acute Respiratory Distress Syndrome (ARDS). In Advances in Experimental Medicine and Biology (Vol. 967, pp. 105137). Adv Exp Med Biol. https://doi.org/10.1007/978-3-319-63245-2_8

133. Keszler, M., \& Sant'Anna, G. (2015). Mechanical Ventilation and Bronchopulmonary Dysplasia. In Clinics in Perinatology. https://doi.org/10.1016/j.clp.2015.08.006

134. Kharazmi, A., Nielsen, H., \& Schiøtz, P. O. (1988). N-acetylcysteine inhibits human neutrophil and monocyte chemotaxis and oxidative metabolism. International Journal of Immunopharmacology, 10(1), 39-46. https://doi.org/10.1016/0192-0561(88)90148-8

135. Kim, J. H., Lee, H.-K., Takamiya, K., \& Huganir, R. L. (2003). The role of synaptic GTPaseactivating protein in neuronal development and synaptic plasticity. The Journal of 
Bibliography

Neuroscience: The Official Journal of the Society for Neuroscience, 23(4), 1119-1124. https://doi.org/10.1523/JNEUROSCI.23-04-01119.2003

136. Klinger, S., Turgeon, B., Lévesque, K., Wood, G. A., Aagaard-Tillery, K. M., \& Meloche, S. (2009). Loss of ERK3 function in mice leads to intrauterine growth restriction, pulmonary immaturity, and neonatal lethality. Proceedings of the National Academy of Sciences of the United States of America, 106(39), 16710-16715. https://doi.org/10.1073/pnas.0900919106

137. Klinkhammer, K., Bellusci, S., Hopkins, S., Riches, D., Günther, A., Yuan, T., Chao, C., Yao, C., Volckaert, T., DeLanghe, S., Wasnick, R., Redente, E., Yuan, J., Zhang, J., Stripp, B., Thannickal, V., \& Majka, S. (2019). FGF10-FGFR2B Signaling Generates Basal Cells and Drives Alveolar Epithelial Regeneration by Bronchial Epithelial Stem Cells after Lung Injury. Stem Cell Reports, 12(5). https://doi.org/10.1016/j.stemcr.2019.04.003

138. Koera, K., Nakamura, K., Nakao, K., Miyoshi, J., Toyoshima, K., Hatta, T., Otani, H., Aiba, A., \& Katsuki, M. (1997). K-Ras is essential for the development of the mouse embryo. Oncogene, 15(10), 1151-1159. https://doi.org/10.1038/sj.onc.1201284

139. Komiyama, N. H., Watabe, A. M., Carlisle, H. J., Porter, K., Charlesworth, P., Monti, J., Strathdee, D. J. C., OltextquoterightCarroll, C. M., Martin, S. J., Morris, R. G. M., OltextquoterightDell, T. J., \& Grant, S. G. N. (2002). SynGAP Regulates ERK/MAPK Signaling, Synaptic Plasticity, and Learning in the Complex with Postsynaptic Density 95 and NMDA Receptor. Journal of Neuroscience, 22(22), 9721-9732. https://doi.org/10.1523/JNEUROSCI.22-22-09721.2002

140. Ksionda, O., Limnander, A., \& Roose, J. P. (2013). RasGRP Ras guanine nucleotide exchange factors in cancer. In Frontiers in Biology (Vol. 8, Issue 5, pp. 508-532). NIH Public Access. https://doi.org/10.1007/s11515-013-1276-9

141. Kupzig, S., Deaconescu, D., Bouyoucef, D., Walker, S. A., Liu, Q., Polte, C. L., Daumke, O., Ishizaki, T., Lockyer, P. J., Wittinghofer, A., \& Cullen, P. J. (2006). GAP1 family members constitute bifunctional Ras and Rap GTPase-activating proteins. The Journal of Biological Chemistry, 281(15), 9891-9900. https://doi.org/10.1074/jbc.M512802200

142. Laresgoiti, U., Niko, M. Z., Rao, C., Brady, J. L., Richardson, R. V., Batchen, E. J., Chapman, K. E., \& Rawlins, E. L. (2016). Lung epithelial tip progenitors integrate glucocorticoid- and STAT3-mediated signals to control progeny fate. Development (Cambridge), 143(20), 36863699. https://doi.org/10.1242/dev.134023

143. Laukkanen, M. O. (2016). Extracellular Superoxide Dismutase: Growth Promoter or Tumor Suppressor? https://doi.org/10.1155/2016/3612589

144. Laurila, J. P., Castellone, M. D., Curcio, A., Laatikainen, L. E., Haaparanta-Solin, M., Gronroos, T. J., Marjamaki, P., Martikainen, S., Santoro, M., \& Laukkanen, M. O. (2009). 
Extracellular Superoxide Dismutase Is a Growth Regulatory Mediator of Tissue Injury Recovery. Molecular Therapy: The Journal of the American Society of Gene Therapy, 17(3), 448. https://doi.org/10.1038/MT.2008.282

145. Li, G., \& Zhang, X. C. (2004). GTP hydrolysis mechanism of Ras-like GTPases. In Journal of Molecular Biology (Vol. 340, Issue 5, pp. 921-932). Academic Press. https://doi.org/10.1016/j.jmb.2004.06.007

146. Li, Y., Wang, W., \& Zhang, D. (2019). Maternal diabetes mellitus and risk of neonatal respiratory distress syndrome: a meta-analysis. In Acta Diabetologica. https://doi.org/10.1007/s00592-019-01327-4

147. Liao, J., Chi, C., Li, H., \& Tang, X. (2010). Effects of N-acetylcysteine on Clara cells in rats with cigarette smoke exposure. Chinese Medical Journal, 123(4), 412-417.

148. Liceras-Boillos, P., García-Navas, R., Ginel-Picardo, A., Anta, B., Pérez-Andrés, M., Lillo, C., Gómez, C., Jimeno, D., Fernández-Medarde, A., Baltanás, F. C., \& Santos, E. (2016). Sos1 disruption impairs cellular proliferation and viability through an increase in mitochondrial oxidative stress in primary MEFs. Oncogene. https://doi.org/10.1038/onc.2016.169

149. Liceras-Boillos, P., Jimeno, D., García-Navas, R., Francisco Lorenzo-Martín, L., MenachoMarquez, M., Segrelles, C., Gómez, C., Calzada, N., Fuentes-Mateos, R., Paramio, J. M., Bustelo, X. R., Baltanás, F. C., \& Santos, E. (2018). Differential role of the RasGEFs Sos1 and Sos2 in mouse skin homeostasis and carcinogenesis. Molecular and Cellular Biology, 38(16). https://doi.org/10.1128/MCB.00049-18

150. Little, D. R., Lynch, A. M., Yan, Y., Akiyama, H., Kimura, S., \& Chen, J. (2021). Differential chromatin binding of the lung lineage transcription factor NKX2-1 resolves opposing murine alveolar cell fates in vivo. Nature Communications, 12(1). https://doi.org/10.1038/s41467021-22817-6

151. Liu, Y., Martinez, L., Ebine, K., \& Abe, M. K. (2008). Role for mitogen-activated protein kinase $\mathrm{p} 38 \alpha$ in lung epithelial branching morphogenesis. Developmental Biology. https://doi.org/10.1016/j.ydbio.2007.12.003

152. Lohinai, Z., Klikovits, T., Moldvay, J., Ostoros, G., Raso, E., Timar, J., Fabian, K., Kovalszky, I., Kenessey, I., Aigner, C., Renyi-Vamos, F., Klepetko, W., Dome, B., \& Hegedus, B. (2017). KRAS-mutation incidence and prognostic value are metastatic site-specific in lung adenocarcinoma: Poor prognosis in patients with KRAS mutation and bone metastasis. Scientific Reports, 7(1), 1-8. https://doi.org/10.1038/srep39721

153. Lombardero, M., \& Nieto, A. (1981). Glucocorticoid and developmental regulation of uteroglobin synthesis in rabbit lung. Biochemical Journal, 200(3), 487-494. https://doi.org/10.1042/bj2000487 
154. Londhe, V. A., Nguyen, H. T., Jeng, J. M., Li, X., Li, C., Tiozzo, C., Zhu, N., \& Minoo, P. (2008). NF-kB induces lung maturation during mouse lung morphogenesis. Developmental Dynamics, 237(2), 328-338. https://doi.org/10.1002/dvdy.21413

155. Lopez, I., Mak, E. C., Ding, J., Hamm, H. E., \& Lomasney, J. W. (2001). A Novel Bifunctional Phospholipase C That Is Regulated by $\mathrm{G} \alpha 12$ and Stimulates the Ras/Mitogen-activated Protein Kinase Pathway. Journal of Biological Chemistry, 276(4), 2758-2765. https://doi.org/10.1074/jbc.M008119200

156. Lu, X., Ma, Y., He, J., Li, Y., Zhu, H., \& Yu, X. (2019). N-acetylcysteine for adults with acute respiratory distress syndrome: A meta-analysis of randomized controlled trials. Hong Kong Journal of Emergency Medicine, 26(5), 288-298. https://doi.org/10.1177/1024907918794559

157. Lu, Z., \& Xu, S. (2006). ERK1/2 MAP kinases in cell survival and apoptosis. In IUBMB Life (Vol. 58, Issue 11, pp. 621-631). IUBMB Life. https://doi.org/10.1080/15216540600957438

158. Luan, G., Li, G., Ma, X., Jin, Y., Hu, N., Li, J., Wang, Z., \& Wang, H. (2019). DexamethasoneInduced Mitochondrial Dysfunction and Insulin Resistance-Study in 3T3-L1 Adipocytes and Mitochondria Isolated from Mouse Liver. Molecules, 24(10). https://doi.org/10.3390/MOLECULES24101982

159. Manyes, L., Holst, S., Lozano, M., Santos, E., \& Fernandez-Medarde, A. (2018). Spatial learning and long-term memory impairments in RasGrf1 KO, Pttg1 KO, and double KO mice. Brain and Behavior. https://doi.org/10.1002/brb3.1089

160. Marseglia, L., D’Angelo, G., Granese, R., Falsaperla, R., Reiter, R. J., Corsello, G., \& Gitto, E. (2019). Role of oxidative stress in neonatal respiratory distress syndrome. In Free Radical Biology and Medicine (Vol. 142, pp. 132-137). Free Radic Biol Med. https://doi.org/10.1016/j.freeradbiomed.2019.04.029

161. Martínez-Limón, A., Joaquin, M., Caballero, M., Posas, F., \& de Nadal, E. (2020). The p38 pathway: From biology to cancer therapy. In International Journal of Molecular Sciences (Vol. 21, Issue 6, p. 1913). Multidisciplinary Digital Publishing Institute. https://doi.org/10.3390/ijms21061913

162. Martis, P. C., Whitsett, J. A., Xu, Y., Perl, A. K. T., Wan, H., \& Ikegami, M. (2006). C/EBP $\alpha$ is required for lung maturation at birth. Development, 133(6), 1155-1164. https://doi.org/10.1242/dev.02273

163. Marttila, R., Haataja, R., Rämet, M., Löfgren, J., \& Hallman, M. (2003). Surfactant protein B polymorphism and respiratory distress syndrome in premature twins. Human Genetics. https://doi.org/10.1007/s00439-002-0835-y

164. Matallanas, D., Arozarena, I., Berciano, M. T., Aaronson, D. S., Pellicer, A., Lafarga, M., \& Crespo, P. (2003). Differences on the Inhibitory Specificities of H-Ras, K-Ras, and N-Ras 
(N17) Dominant Negative Mutants Are Related to Their Membrane Microlocalization*. Journal of Biological Chemistry, 278(7), 4572-4581. https://doi.org/https://doi.org/10.1074/jbc.M209807200

165. Matallanas, D., Sanz-Moreno, V., Arozarena, I., Calvo, F., Agudo-Ibáñez, L., Santos, E., Berciano, M. T., \& Crespo, P. (2006). Distinct utilization of effectors and biological outcomes resulting from site-specific Ras activation: Ras functions in lipid rafts and Golgi complex are dispensable for proliferation and transformation. Molecular and Cellular Biology, 26(1), 100116. https://doi.org/10.1128/MCB.26.1.100-116.2006

166. McCormack, F. X., \& Whitsett, J. A. (2002). The pulmonary collectins, SP-A and SP-D, orchestrate innate immunity in the lung. In Journal of Clinical Investigation. https://doi.org/10.1172/JCI0215293

167. McCulley, D., Wienhold, M., \& Sun, X. (2015). The pulmonary mesenchyme directs lung development. In Current Opinion in Genetics and Development. https://doi.org/10.1016/j.gde.2015.01.011

168. McGillick, E. V., Orgeig, S., Allison, B. J., Brain, K. L., Niu, Y., Itani, N., Skeffington, K. L., Kane, A. D., Herrera, E. A., Morrison, J. L., \& Giussani, D. A. (2021). Molecular regulation of lung maturation in near-term fetal sheep by maternal daily vitamin $\mathrm{C}$ treatment in late gestation. Pediatric Research. https://doi.org/10.1038/s41390-021-01489-4

169. Mebratu, Y., \& Tesfaigzi, Y. (2009). How ERK1/2 activation controls cell proliferation and cell death is subcellular localization the answer? In Cell Cycle (Vol. 8, Issue 8, pp. 11681175). Cell Cycle. https://doi.org/10.4161/cc.8.8.8147

170. Meloche, S., \& Pouysségur, J. (2007). The ERK1/2 mitogen-activated protein kinase pathway as a master regulator of the G1- to S-phase transition. In Oncogene (Vol. 26, Issue 22, pp. 3227-3239). Nature Publishing Group. https://doi.org/10.1038/sj.onc.1210414

171. Meyerholz, D. K., Suarez, C. J., Dintzis, S. M., \& Frevert, C. W. (2018). Respiratory System. Comparative Anatomy and Histology, 147-162. https://doi.org/10.1016/B978-0-12-8029008.00009-9

172. Miaczynska, M., \& Bar-Sagi, D. (2010). Signaling endosomes: Seeing is believing. In Current Opinion in Cell Biology (Vol. 22, Issue 4, pp. 535-540). Elsevier Current Trends. https://doi.org/10.1016/j.ceb.2010.05.007

173. Miettinen, P. J., Warburton, D., Bu, D., Zhao, J. S., Berger, J. E., Minoo, P., Koivisto, T., Allen, L., Dobbs, L., Werb, Z., \& Derynck, R. (1997). Impaired lung branching morphogenesis in the absence of functional EGF receptor. Developmental Biology, 186(2), 224-236. https://doi.org/10.1006/dbio.1997.8593

174. Montoro, D. T., Haber, A. L., Biton, M., Vinarsky, V., Lin, B., Birket, S. E., Yuan, F., Chen, 
Bibliography

S., Leung, H. M., Villoria, J., Rogel, N., Burgin, G., Tsankov, A. M., Waghray, A., Slyper, M., Waldman, J., Nguyen, L., Dionne, D., Rozenblatt-Rosen, O., ... Rajagopal, J. (2018). A revised airway epithelial hierarchy includes CFTR-expressing ionocytes. Nature. https://doi.org/10.1038/s41586-018-0393-7

175. Mor, A., Campi, G., Du, G., Zheng, Y., Foster, D. A., Dustin, M. L., \& Philips, M. R. (2007). The lymphocyte function-associated antigen-1 receptor costimulates plasma membrane Ras via phospholipase D2. Nature Cell Biology, 9(6), 713-719. https://doi.org/10.1038/ncb1592

176. Morcaldi, G., Bellini, T., Rossi, C., Maghnie, M., Boccardo, F., Bonioli, E., \& Bellini, C. (2015). LYMPHODYSPLASIA AND KRAS MUTATION: A CASE REPORT AND LITERATURE REVIEW. Lymphology, 48(3), 121-127.

177. Moretó, J., Lladó, A., Vidal-Quadras, M., Calvo, M., Pol, A., Enrich, C., \& Tebar, F. (2008). Calmodulin modulates H-Ras mediated Raf-1 activation. Cellular Signalling, 20(6), 10921103. https://doi.org/10.1016/j.cellsig.2008.01.022

178. Morrisey, E. E., \& Hogan, B. L. M. (2010). Preparing for the First Breath: Genetic and Cellular Mechanisms in Lung Development. In Developmental Cell (Vol. 18, pp. 8-23). https://doi.org/10.1016/j.devcel.2009.12.010

179. Mulugeta, S., \& Beers, M. F. (2006). Surfactant protein C: Its unique properties and emerging immunomodulatory role in the lung. In Microbes and Infection. https://doi.org/10.1016/j.micinf.2006.04.009

180. Muñoz-Maldonado, C., Zimmer, Y., \& Medová, M. (2019). A Comparative Analysis of Individual RAS Mutations in Cancer Biology. Frontiers in Oncology, 9, 1088. https://doi.org/10.3389/fonc.2019.01088

181. Murphy, M. P. (2009). How mitochondria produce reactive oxygen species. Biochemical Journal, 417(Pt 1), 1. https://doi.org/10.1042/BJ20081386

182. Myers, A., Bernstein, J. A., Brennan, M.-L., Curry, C., Esplin, E. D., Fisher, J., Homeyer, M., Manning, M. A., Muller, E. A., Niemi, A.-K., Seaver, L. H., Hintz, S. R., \& Hudgins, L. (2014). Perinatal features of the RASopathies: Noonan syndrome, Cardiofaciocutaneous syndrome and Costello syndrome. American Journal of Medical Genetics Part A, 164(11), 2814-2821. https://doi.org/10.1002/AJMG.A.36737

183. Najafian, B., \& Hossein Khosravi, M. (2020). Neonatal Respiratory Distress Syndrome: Things to Consider and Ways to Manage. In Update on Critical Issues on Infant and Neonatal Care. https://doi.org/10.5772/intechopen.90885

184. Nakajima, R., Takao, K., Hattori, S., Shoji, H., Komiyama, N. H., Grant, S. G. N., \& Miyakawa, T. (2019). Comprehensive behavioral analysis of heterozygous Syngap1 knockout mice. Neuropsychopharmacology Reports, 39(3), 223-237. 
https://doi.org/10.1002/npr2.12073

185. Nakamura, K., Ichise, H., Nakao, K., Hatta, T., Otani, H., Sakagami, H., Kondo, H., \& Katsuki, M. (2008). Partial functional overlap of the three ras genes in mouse embryonic development. Oncogene, 27, 2961-2968. https://doi.org/10.1038/sj.onc.1210956

186. Newlaczyl, A. U., Coulson, J. M., \& Prior, I. A. (2017). Quantification of spatiotemporal patterns of Ras isoform expression during development. Scientific Reports, 7, 41297. https://doi.org/10.1038/srep41297

187. Nie, X., Li, Q., Cai, G., Dai, Y., \& Zhang, J. (2005). The effect of N-acetylcysteine on Clara cells and Clara cell $16 \mathrm{kDa}$ protein in a murine model of allergen-induced airway inflammation. Respirology, 10(2), 157-163. https://doi.org/10.1111/j.14401843.2005.00698.x

188. Nikolova-Karakashian, M. N., \& Reid, M. B. (2011). Sphingolipid Metabolism, Oxidant Signaling, and Contractile Function of Skeletal Muscle. Antioxidants \& Redox Signaling, 15(9), 2501. https://doi.org/10.1089/ARS.2011.3940

189. Nkadi, P. O., Merritt, T. A., \& Pillers, D. A. M. (2009). An overview of pulmonary surfactant in the neonate: Genetics, metabolism, and the role of surfactant in health and disease. In Molecular Genetics and Metabolism. https://doi.org/10.1016/j.ymgme.2009.01.015

190. Nyeng, P., Norgaard, G. A., Kobberup, S., \& Jensen, J. (2008). FGF10 maintains distal lung bud epithelium and excessive signaling leads to progenitor state arrest, distalization, and goblet cell metaplasia. BMC Developmental Biology 2008 8:1, 8(1), 1-15. https://doi.org/10.1186/1471-213X-8-2

191. Oshika, E., Liu, S., Ung, L. P., Singh, G., Shinozuka, H., Michalopoulos, G. K., \& Katyal, S. L. (1998). Glucocorticoid-induced effects on pattern formation and epithelial cell differentiation in early embryonic rat lungs. Pediatric Research, 43(3), 305-314. https://doi.org/10.1203/00006450-199803000-00001

192. Ostrin, E. J., Little, D. R., Gerner-Mauro, K. N., Sumner, E. A., Ríos-Corzo, R., Ambrosio, E., Holt, S. E., Forcioli-Conti, N., Akiyama, H., Hanash, S. M., Kimura, S., Huang, S. X. L., \& Chen, J. (2018). B-Catenin Maintains Lung Epithelial Progenitors After Lung Specification. Development (Cambridge), 145(5), 1-13. https://doi.org/10.1242/dev.160788

193. Pak, Y., Pham, N., \& Rotin, D. (2002). Direct Binding of the $\beta 1$ Adrenergic Receptor to the Cyclic AMP-Dependent Guanine Nucleotide Exchange Factor CNrasGEF Leads to Ras Activation. Molecular and Cellular Biology, 22(22), 7942-7952. https://doi.org/10.1128/mcb.22.22.7942-7952.2002

194. Parker, J. A., \& Mattos, C. (2015). The Ras-Membrane Interface: Isoform-Specific Differences in the Catalytic Domain. Molecular Cancer Research, 13(4), 595-603. 
Bibliography

https://doi.org/10.1158/1541-7786.MCR-14-0535

195. Patel, R., Rinker, L., Peng, J., \& Chilian, W. M. (2018). Reactive Oxygen Species: The Good and the Bad. In Reactive Oxygen Species (ROS) in Living Cells. IntechOpen. https://doi.org/10.5772/intechopen.71547

196. Pei, Y., Liu, H., Yang, Y., Yang, Y., Jiao, Y., Tay, F. R., \& Chen, J. (2018). Biological activities and potential oral applications of $\mathrm{N}$-acetylcysteine: Progress and prospects. In Oxidative Medicine and Cellular Longevity. https://doi.org/10.1155/2018/2835787

197. Perl, A. K. T., Kist, R., Shan, Z., Scherer, G., \& Whitsett, J. A. (2005). Normal lung development and function after Sox9 inactivation in the respiratory epithelium. Genesis, 41(1), 23-32. https://doi.org/10.1002/gene.20093

198. Petrache, I., \& Berdyshev, E. V. (2016). Ceramide Signaling and Metabolism in Pathophysiological States of the Lung. In Annual Review of Physiology (Vol. 78, pp. 463480). Annu Rev Physiol. https://doi.org/10.1146/annurev-physiol-021115-105221

199. Petrache, I., Natarajan, V., Zhen, L., Medler, T. R., Richter, A. T., Cho, C., Hubbard, W. C., Berdyshev, E. V, \& Tuder, R. M. (2005). Ceramide upregulation causes pulmonary cell apoptosis and emphysema-like disease in mice. Nature Medicine, 11(5), 491-498. https://doi.org/10.1038/nm1238

200. Pettus, B. J., Chalfant, C. E., \& Hannun, Y. A. (2002). Ceramide in apoptosis: An overview and current perspectives. In Biochimica et Biophysica Acta - Molecular and Cell Biology of Lipids (Vol. 1585, Issues 2-3, pp. 114-125). Biochim Biophys Acta. https://doi.org/10.1016/S1388-1981(02)00331-1

201. Pew, B. K., Harris, R. A., Sbrana, E., Guaman, M. C., Shope, C., Chen, R., Meloche, S., \& Aagaard, K. (2016). Structural and transcriptomic response to antenatal corticosteroids in an ERK3-null mouse model of respiratory distress. American Journal of Obstetrics and Gynecology, 215(3), 384.e1-384.e89. https://doi.org/10.1016/j.ajog.2016.04.043

202. Pham, N., Cheglakov, I., Koch, C. A., De Hoog, C. L., Moran, M. F., \& Rotin, D. (2000). The guanine nucleotide exchange factor CNrasGEF activates Ras in response to cAMP and cGMP. Current Biology, 10(9), 555-558. https://doi.org/10.1016/S0960-9822(00)00473-5

203. Phan, V. T., Wu, X., Cheng, J. H., Sheng, R. X., Chung, A. S., Zhuang, G., Tran, C., Song, Q., Kowanetz, M., Sambrone, A., Tan, M., Gloria Meng, Y., Jackson, E. L., Peale, F. V., Junttila, M. R., \& Ferrara, N. (2013). Oncogenic RAS pathway activation promotes resistance to anti-VEGF therapy through G-CSF-induced neutrophil recruitment. Proceedings of the National Academy of Sciences of the United States of America, 110(15), 6079-6084. https://doi.org/10.1073/pnas.1303302110

204. Pinilla-Macua, I., Watkins, S. C., \& Sorkin, A. (2016). Endocytosis separates EGF receptors 
from endogenous fluorescently labeled HRas and diminishes receptor signaling to MAP kinases in endosomes. Proceedings of the National Academy of Sciences of the United States of America, 113(8), 2122-2127. https://doi.org/10.1073/pnas.1520301113

205. Platta, H. W., \& Stenmark, H. (2011). Endocytosis and signaling. In C. Lamaze \& I. Prior (Eds.), Current Opinion in Cell Biology (Vol. 23, Issue 4, pp. 393-403). Springer International Publishing. https://doi.org/10.1016/j.ceb.2011.03.008

206. Plopper, C., \& Fanucchi, M. (2014). Development of Airway Epithelium. In The Lung: Development, Aging and the Environment: Second Edition (pp. 11-32). Academic Press. https://doi.org/10.1016/B978-0-12-799941-8.00002-X

207. Plowman, S. J., Williamson, D. J., O’Sullivan, M. J., Doig, J., Ritchie, A.-M., Harrison, D. J., Melton, D. W., Arends, M. J., Hooper, M. L., \& Patek, C. E. (2003). While K-ras is essential for mouse development, expression of the K-ras 4A splice variant is dispensable. Molecular and Cellular Biology, 23(24), 9245-9250. https://doi.org/10.1128/MCB.23.24.92459250.2003

208. PM, del M., SP, D. L., FG, S., JM, V., D, T., K, W., D, W., \& S, B. (2006). Differential role of FGF9 on epithelium and mesenchyme in mouse embryonic lung. Developmental Biology, 293(1), 77-89. https://doi.org/10.1016/J.YDBIO.2006.01.020

209. Potenza, N., Vecchione, C., Notte, A., De Rienzo, A., Rosica, A., Bauer, L., Affuso, A., De Felice, M., Russo, T., Poulet, R., Cifelli, G., De Vita, G., Lembo, G., \& Di Lauro, R. (2005). Replacement of K-Ras with H-Ras supports normal embryonic development despite inducing cardiovascular pathology in adult mice. EMBO Reports, 6(5), 432-437. https://doi.org/10.1038/SJ.EMBOR.7400397

210. Potey, P. M., Rossi, A. G., Lucas, C. D., \& Dorward, D. A. (2019). Neutrophils in the initiation and resolution of acute pulmonary inflammation: understanding biological function and therapeutic potential. In Journal of Pathology (Vol. 247, Issue 5, pp. 672-685). John Wiley \& Sons, Ltd. https://doi.org/10.1002/path.5221

211. Prescott, H. C., \& Rice, T. W. (2020). Corticosteroids in COVID-19 ARDS: Evidence and Hope During the Pandemic. JAMA, 324(13), 1292-1295. https://doi.org/10.1001/JAMA.2020.16747

212. Prior, I. A., \& Hancock, J. F. (2012). Ras trafficking, localization and compartmentalized signalling. Seminars in Cell and Developmental Biology, 23(2), 145-153. https://doi.org/10.1016/j.semcdb.2011.09.002

213. Prior, I. A., Hood, F. E., \& Hartley, J. L. (2020). The Frequency of Ras Mutations in Cancer. Cancer Research, 80(14), 2969-2974. https://doi.org/10.1158/0008-5472.CAN-19-3682

214. Qian, X., Esteban, L., Vass, W. C., Upadhyaya, C., Papageorge, A. G., Yienger, K., Ward, J. 
Bibliography

M., Lowy, D. R., \& Santos, E. (2000). The Sos1 and Sos2 Ras-specific exchange factors: Differences in placental expression and signaling properties. EMBO Journal, 19(4), 642-654. https://doi.org/10.1093/emboj/19.4.642

215. Quinlan, M. P., \& Settleman, J. (2009). Isoform-specific ras functions in development and cancer. Http://Dx.Doi.Org/10.2217/14796694.5.1.105, 5(1), 105-116. https://doi.org/10.2217/14796694.5.1.105

216. R Development Core Team, \& Development Core Team, R. (2011). R: A Language and Environment for Statistical Computing. $R$ Foundation for Statistical Computing Vienna Austria.

217. Rajalingam, K., Schreck, R., Rapp, U. R., \& Albert, Š. (2007). Ras oncogenes and their downstream targets. In Biochimica et Biophysica Acta - Molecular Cell Research (Vol. 1773, Issue 8, pp. 1177-1195). Elsevier. https://doi.org/10.1016/j.bbamcr.2007.01.012

218. Rämet, M., Haataja, R., Marttila, R., Floros, J., \& Hallman, M. (2000). Association between the Surfactant Protein A (SP-A) Gene Locus and Respiratory-Distress Syndrome in the Finnish Population. The American Journal of Human Genetics, 66(5), 1569-1579. https://doi.org/10.1086/302906

219. Rao, X., Huang, X., Zhou, Z., \& Lin, X. (2013). An improvement of the $2^{\wedge}$ (-delta delta CT) method for quantitative real-time polymerase chain reaction data analysis. Biostatistics, Bioinformatics and Biomathematics.

220. Ratola, A., Silva, H. M., Guedes, A., Mota, C., Braga, A. C., Oliveira, D., Alegria, A., Carvalho, C., Álvares, S., \& Proença, E. (2015). A Novel Noonan Syndrome RAF1 Mutation: Lethal Course in a Preterm Infant. Pediatric Reports, 7(2), 35-38. https://doi.org/10.4081/PR.2015.5955

221. Rawlins, E. L., \& Hogan, B. L. M. (2006). Epithelial stem cells of the lung: Privileged few or opportunities for many? In Development. https://doi.org/10.1242/dev.02407

222. Rey-Santano, C., Mielgo, V., Gomez-Solaetxe, M. A., Ricci, F., Bianco, F., Salomone, F., \& Loureiro, B. (2020). Dose-Response Study on Surfactant Nebulization Therapy during Nasal Continuous Positive Airway Pressure Ventilation in Spontaneously Breathing SurfactantDeficient Newborn Piglets*. Pediatric Critical Care Medicine. https://doi.org/10.1097/PCC.0000000000002313

223. Ricci, E., Roselletti, E., Gentili, M., Sabbatini, S., Perito, S., Riccardi, C., Migliorati, G., Monari, C., \& Ronchetti, S. (2021). Glucocorticoid-Induced Leucine Zipper-Mediated TLR2 Downregulation Accounts for Reduced Neutrophil Activity Following Acute DEX Treatment. Cells, 10(9), 2228. https://doi.org/10.3390/cells10092228

224. Ridsdale, R., \& Post, M. (2004). Surfactant lipid synthesis and lamellar body formation in 
glycogen-laden type II cells. Https://Doi.Org/10.1152/Ajplung.00146.2004, 287(4 31-4), 743751. https://doi.org/10.1152/AJPLUNG.00146.2004

225. Riller, Q., \& Rieux-Laucat, F. (2021). RASopathies: From germline mutations to somatic and multigenic diseases. Biomedical Journal, 44(4), 422-432. https://doi.org/https://doi.org/10.1016/j.bj.2021.06.004

226. Ringvall, M., \& Kjelln, L. (2010). Mice deficient in heparan sulfate n-deacetylase/nsulfotransferase 1. In Progress in Molecular Biology and Translational Science (Vol. 93, Issue C, pp. 35-58). Prog Mol Biol Transl Sci. https://doi.org/10.1016/S1877-1173(10)93003-2

227. Robbins, M. E., Cho, H. Y., Hansen, J. M., Luchsinger, J. R., Locy, M. L., Velten, M., Kleeberger, S. R., Rogers, L. K., \& Tipple, T. E. (2021). Glutathione reductase deficiency alters lung development and hyperoxic responses in neonatal mice. Redox Biology, 38. https://doi.org/10.1016/j.redox.2020.101797

228. Roberts, D., Brown, J., Medley, N., \& Dalziel, S. R. (2017). Antenatal corticosteroids for accelerating fetal lung maturation for women at risk of preterm birth. In Cochrane Database of Systematic Reviews (pp. 1465-1858). https://doi.org/10.1002/14651858.CD004454.pub3

229. Roberts, P. J., \& Der, C. J. (2007). Targeting the Raf-MEK-ERK mitogen-activated protein kinase cascade for the treatment of cancer. In Oncogene (Vol. 26, Issue 22, pp. 3291-3310). Nature Publishing Group. https://doi.org/10.1038/sj.onc.1210422

230. Rock, J. R., \& Hogan, B. L. M. (2011). Epithelial progenitor cells in lung development, maintenance, repair, and disease. Annual Review of Cell and Developmental Biology. https://doi.org/10.1146/annurev-cellbio-100109-104040

231. Rock, J. R., Randell, S. H., \& Hogan, B. L. M. (2010). Airway basal stem cells: A perspective on their roles in epithelial homeostasis and remodeling. In DMM Disease Models and Mechanisms. https://doi.org/10.1242/dmm.006031

232. Rodriguez-Viciana, P., Warne, P. H., Dhand, R., Vanhaesebroeck, B., Gout, I., Fry, M. J., Waterfield, M. D., \& Downward, J. (1994). Phosphatidylinositol-3-OH kinase direct target of Ras. Nature, 370(6490), 527-532. https://doi.org/10.1038/370527a0

233. Rogge, R. D., Karlovich, C. A., \& Banerjee, U. (1991). Genetic dissection of a neurodevelopmental pathway: Son of sevenless functions downstream of the sevenless and EGF receptor tyrosine kinases. Cell, 64(1), 39-48. https://doi.org/10.1016/00928674(91)90207-F

234. Rojas, José M., Oliva, J. L., \& Santos, E. (2011). Mammalian son of sevenless guanine nucleotide exchange factors: Old concepts and new perspectives. In Genes and Cancer (Vol. 2, Issue 3, pp. 298-305). Impact Journals, LLC. https://doi.org/10.1177/1947601911408078

235. Rojas, Jose M., \& Santos, E. (2006). Ras-Gefs and Ras Gaps. In RAS Family GTPases (pp. 
Bibliography

15-43). Springer, Dordrecht. https://doi.org/10.1007/1-4020-4708-8_2

236. Ronchetti, S., Ricci, E., Migliorati, G., Gentili, M., \& Riccardi, C. (2018). How glucocorticoids affect the neutrophil life. In International Journal of Molecular Sciences (Vol. 19, Issue 12). Multidisciplinary Digital Publishing Institute (MDPI). https://doi.org/10.3390/ijms19124090

237. Ross, D., \& Siegel, D. (2017). Functions of NQO1 in cellular protection and CoQ10 metabolism and its potential role as a redox sensitive molecular switch. In Frontiers in Physiology (Vol. 8, Issue AUG). Frontiers Media S.A. https://doi.org/10.3389/fphys.2017.00595

238. Roy, M. G., Rahmani, M., Hernandez, J. R., Alexander, S. N., Ehre, C., Ho, S. B., \& Evans, C. M. (2011). Mucin Production during Prenatal and Postnatal Murine Lung Development. American Journal of Respiratory Cell and Molecular Biology, 44(6), 755-760. https://doi.org/10.1165/rcmb.2010-0020oc

239. Sadowska, A. M., Manuel-Y-Keenoy, B., Vertongen, T., Schippers, G., RadomskaLesniewska, D., Heytens, E., \& De Backer, W. A. (2006). Effect of N-acetylcysteine on neutrophil activation markers in healthy volunteers: In vivo and in vitro study. Pharmacological Research, 53(3), 216-225. https://doi.org/10.1016/j.phrs.2005.11.003

240. Sah, S. K., Agrahari, G., \& Kim, T. Y. (2020). Insights into superoxide dismutase 3 in regulating biological and functional properties of mesenchymal stem cells. In Cell and Bioscience (Vol. 10, Issue 1, pp. 1-12). BioMed Central. https://doi.org/10.1186/s13578-02000386-3

241. Salerno, T., Peca, D., Menchini, L., Schiavino, A., Petreschi, F., Occasi, F., Cogo, P., Danhaive, O., \& Cutrera, R. (2014). Respiratory insufficiency in a newborn with congenital hypothyroidism due to a new mutation of TTF-1/NKX2.1 gene. Pediatric Pulmonology, 49(3). https://doi.org/10.1002/ppul.22788

242. Santos, E., \& Nebreda, A. R. (1989). Structural and functional properties of ras proteins. The FASEB Journal, 3(10), 2151-2163. https://doi.org/10.1096/fasebj.3.10.2666231

243. Sarbassov, D. D., Guertin, D. A., Ali, S. M., \& Sabatini, D. M. (2005). Phosphorylation and regulation of AKT/PKB by the rictor-mTOR complex. Science, 307(5712), 1098-1101. https://doi.org/10.1126/science.1106148

244. Schittny, J. C. (2017). Development of the lung. In Cell and Tissue Research. https://doi.org/10.1007/s00441-016-2545-0

245. Schmick, M., Vartak, N., Papke, B., Kovacevic, M., Truxius, D. C., Rossmannek, L., \& Bastiaens, P. I. H. (2014). KRas Localizes to the Plasma Membrane by Spatial Cycles of Solubilization, Trapping and Vesicular Transport. Cell, 157(2), 459-471. 
https://doi.org/10.1016/J.CELL.2014.02.051

246. Schreiber, M. D., Gin-Mestan, K., Marks, J. D., Huo, D., Lee, G., \& Srisuparp, P. (2003). Inhaled Nitric Oxide in Premature Infants with the Respiratory Distress Syndrome. New England Journal of Medicine. https://doi.org/10.1056/nejmoa031154

247. Shaw, A. T., Meissner, A., Dowdle, J. A., Crowley, D., Magendantz, M., Ouyang, C., Parisi, T., Rajagopal, J., Blank, L. J., Bronson, R. T., Stone, J. R., Tuveson, D. A., Jaenisch, R., \& Jacks, T. (2008). Sprouty-2 regulates oncogenic K-ras in lung development and tumorigenesis (Genes and Development (2007) 21, (694-707)). Genes and Development, 22(2), 277. https://doi.org/10.1101/gad.1526207.stream

248. Shen, W., Kuang, P., Wang, B., Zeng, Q., Chen, C., \& Lin, X. (2020). Genetic Polymorphisms of LPCAT1, CHPT1 and PCYT1B and Risk of Neonatal Respiratory Distress Syndrome among a Chinese Han Population. Pediatrics and Neonatology, 61(3), 318-324. https://doi.org/10.1016/j.pedneo.2019.12.012

249. Shi, L., Shan, G., Zhang, X., Zhang, B., Zhang, A., \& Liu, Z. (2020). Comparison of therapeutic effects of budesonide and ambroxol combined with bovine pulmonary surfactant on neonatal respiratory distress syndrome. International Journal of Clinical and Experimental Medicine, 13(8), 5754-5762. http://www.ijcem.com/files/ijcem0113632.pdf\%0Ahttp://ovidsp.ovid.com/ovidweb.cgi?T=J $\mathrm{S} \& \mathrm{PAGE}=$ reference $\& \mathrm{D}=\mathrm{emexc} \& \mathrm{NEWS}=\mathrm{N} \& \mathrm{AN}=2004989359$

250. Shiraishi, K., Shichino, S., Ueha, S., Nakajima, T., Hashimoto, S., Yamazaki, S., \& Matsushima, K. (2019). Mesenchymal-Epithelial Interactome Analysis Reveals Essential Factors Required for Fibroblast-Free Alveolosphere Formation. IScience, 11, 318-333. https://doi.org/10.1016/j.isci.2018.12.022

251. Simanshu, D. K., Nissley, D. V., \& McCormick, F. (2017). RAS Proteins and Their Regulators in Human Disease. In Cell (Vol. 170, pp. 17-33). https://doi.org/10.1016/j.cell.2017.06.009

252. Simon, M. A., Bowtell, D. D. L., Dodson, G. S., Laverty, T. R., \& Rubin, G. M. (1991). Ras1 and a putative guanine nucleotide exchange factor perform crucial steps in signaling by the sevenless protein tyrosine kinase. Cell, 67(4), 701-716. https://doi.org/10.1016/00928674(91)90065-7

253. Sparkman, L., Chandru, H., \& Boggaram, V. (2006). Ceramide decreases surfactant protein B gene expression via downregulation of TTF-1 DNA binding activity. American Journal of Physiology - Lung Cellular and Molecular Physiology, 290(2). https://doi.org/10.1152/ajplung.00275.2005

254. Sperlich, B., Kapoor, S., Waldmann, H., Winter, R., \& Weise, K. (2016). Regulation of KRas4B Membrane Binding by Calmodulin. Biophysical Journal, 111(1), 113-122. https://doi.org/10.1016/j.bpj.2016.05.042 
Bibliography

255. Stacey, D., Bilbao, A., Maroteaux, M., Jia, T., Easton, A. C., Longueville, S., Nymberg, C., Banaschewski, T., Barker, G. J., Büchel, C., Carvalho, F., Conrod, P. J., Desrivières, S., FauthBühler, M., Fernandez-Medarde, A., Flor, H., Gallinat, J., Garavan, H., Bokde, A. L. W., ... Consortium, the I. (2012). RASGRF2 regulates alcohol-induced reinforcement by influencing mesolimbic dopamine neuron activity and dopamine release. Proceedings of the National Academy of Sciences, 109(51), 21128-21133. https://doi.org/10.1073/PNAS.1211844110

256. Stone, J. C. (2011). Regulation and function of the rasGRP family of ras activators in blood cells. Genes and Cancer, 2, 320-334. https://doi.org/10.1177/1947601911408082

257. Sugimoto, T., Stewart, S., Han, M., \& Guan, K. L. (1998). The kinase suppressor of Ras (KSR) modulates growth factor and Ras signaling by uncoupling Elk-1 phosphorylation from MAP $\begin{array}{llll}\text { kinase } & \text { activation. } & \text { EMBO }\end{array}$ https://doi.org/10.1093/emboj/17.6.1717

258. Sun, Y., Chen, C., Zhang, X., Weng, X., Sheng, A., Zhu, Y., Chen, S., Zheng, X., \& Lu, C. (2019). High Neutrophil-to-Lymphocyte Ratio Is an Early Predictor of Bronchopulmonary Dysplasia. Frontiers in Pediatrics, 7, 464. https://doi.org/10.3389/fped.2019.00464

259. Suter, P. M., Domenighetti, G., Schaller, M. D., Laverriere, M. C., Ritz, R., \& Perret, C. (1994). N-acetylcysteine enhances recovery from acute lung injury in man: A randomized, double-blind, placebo-controlled clinical study. Chest, 105(1), 190-194. https://doi.org/10.1378/chest.105.1.190

260. Suwanjang, W., Wu, K. L. H., Prachayasittikul, S., Chetsawang, B., \& Charngkaew, K. (2019). Mitochondrial Dynamics Impairment in Dexamethasone-Treated Neuronal Cells. Neurochemical Research 2019 44:7, 44(7), 1567-1581. https://doi.org/10.1007/S11064-019$02779-4$

261. Sweet, D. G., Carnielli, V., Greisen, G., Hallman, M., Ozek, E., Plavka, R., Saugstad, O. D., Simeoni, U., Speer, C. P., Vento, M., \& Halliday, H. L. (2013). European Consensus Guidelines on the Management of Neonatal Respiratory Distress Syndrome in Preterm Infants - 2013 Update. Neonatology, 103(4), 353-368. https://doi.org/10.1159/000349928

262. Tabas-Madrid, D., Nogales-Cadenas, R., \& Pascual-Montano, A. (2012). GeneCodis3: A nonredundant and modular enrichment analysis tool for functional genomics. Nucleic Acids Research. https://doi.org/10.1093/nar/gks402

263. Tajan, M., Paccoud, R., Branka, S., Edouard, T., \& Yart, A. (2018). The RASopathy Family: Consequences of Germline Activation of the RAS/MAPK Pathway. Endocrine Reviews, 39(5), 676-700. https://doi.org/10.1210/er.2017-00232

264. Tang, N., Marshall, W. F., McMahon, M., Metzger, R. J., \& Martin, G. R. (2011). Control of mitotic spindle angle by the RAS-regulated ERK1/2 pathway determines lung tube shape. 
Science, 333(6040), 342-345. https://doi.org/10.1126/science.1204831

265. Tauskela, J. S. (2007). MitoQ - A mitochondria-targeted antioxidant. IDrugs.

266. Terrell, E. M., \& Morrison, D. K. (2019). Ras-mediated activation of the Raf family kinases. Cold Spring Harbor Perspectives in Medicine, 9(1). https://doi.org/10.1101/cshperspect.a033746

267. Thai, P., Loukoianov, A., Wachi, S., \& Wu, R. (2008). Regulation of Airway Mucin Gene $\begin{array}{lllll}\text { Expression. Annual Review of Physiology, } & \text { 70, } 405 .\end{array}$ https://doi.org/10.1146/ANNUREV.PHYSIOL.70.113006.100441

268. Tohgo, A., Pierce, K. L., Choy, E. W., Lefkowitz, R. J., \& Luttrell, L. M. (2002). $\beta$-arrestin scaffolding of the ERK cascade enhances cytosolic ERK activity but inhibits ERK-mediated transcription following angiotensin AT1a receptor stimulation. Journal of Biological Chemistry, 277(11), 9429-9436. https://doi.org/10.1074/jbc.M106457200

269. Toker, A., \& Newton, A. C. (2000). AKT/protein kinase B is regulated by autophosphorylation at the hypothetical PDK-2 site. Journal of Biological Chemistry, 275(12), 8271-8274. https://doi.org/10.1074/jbc.275.12.8271

270. Torii, S., Kusakabe, M., Yamamoto, T., Maekawa, M., \& Nishida, E. (2004). Sef is a spatial regulator for Ras/MAP kinase signaling. Developmental Cell, 7(1), 33-44. https://doi.org/10.1016/j.devcel.2004.05.019

271. Tripathi, O., Schreibmayer, W., \& Tritthart, H. A. (1993). Fendiline inhibits L-type calcium channels in guinea-pig ventricular myocytes: a whole-cell patch-clamp study. British Journal of Pharmacology, 108(4), 865-869. https://doi.org/10.1111/j.1476-5381.1993.tb13479.x

272. Tsitoura, M.-E. I., Stavrou, E. F., Maraziotis, I. A., Sarafidis, K., Athanassiadou, A., \& Dimitriou, G. (2016). Surfactant Protein A and B Gene Polymorphisms and Risk of Respiratory Distress Syndrome in Late-Preterm Neonates. PLOS ONE, 11(11), e0166516. https://doi.org/10.1371/JOURNAL.PONE.0166516

273. Tsutsumi, K., Fujioka, Y., Tsuda, M., Kawaguchi, H., \& Ohba, Y. (2009). Visualization of Ras-PI3K interaction in the endosome using BiFC. Cellular Signalling, 21(11), 1672-1679. https://doi.org/10.1016/j.cellsig.2009.07.004

274. Turjanski, A. G., Vaqué, J. P., \& Gutkind, J. S. (2007). MAP kinases and the control of nuclear events. In Oncogene (Vol. 26, Issue 22, pp. 3240-3253). Nature Publishing Group. https://doi.org/10.1038/sj.onc.1210415

275. Tusher, V. G., Tibshirani, R., \& Chu, G. (2001). Significance analysis of microarrays applied to the ionizing radiation response. Proceedings of the National Academy of Sciences of the United States of America. https://doi.org/10.1073/pnas.091062498

276. Tyutyunnykova, A., Telegeev, G., \& Dubrovska, A. (2017). The controversial role of 
Bibliography

phospholipase C epsilon (PLC $\varepsilon$ ) in cancer development and progression. In Journal of Cancer (Vol. 8, Issue 5, pp. 716-729). Ivyspring International Publisher. https://doi.org/10.7150/jca.17779

277. Umanoff, H., Edelmann, W., Pellicer, A., \& Kucherlapati, R. (1995). The murine N-ras gene is not essential for growth and development. Proceedings of the National Academy of Sciences of the United States of America, 92, 1709-1713. https://doi.org/10.1073/pnas.92.5.1709

278. Ustiyan, V., Zhang, Y., Perl, A. K. T., Whitsett, J. A., Kalin, T. V., \& Kalinichenko, V. V. (2016). $\beta$-catenin and Kras/Foxm1 signaling pathway are critical to restrict Sox9 in basal cells during pulmonary branching morphogenesis. Developmental Dynamics, 245(5), 590-604. https://doi.org/10.1002/dvdy.24393

279. van der Hoeven, D., Cho, K. -j., Ma, X., Chigurupati, S., Parton, R. G., \& Hancock, J. F. (2013). Fendiline Inhibits K-Ras Plasma Membrane Localization and Blocks K-Ras Signal Transmission. Molecular and Cellular Biology, 33(2), 237-251. https://doi.org/10.1128/mcb.00884-12

280. van Mastrigt, E., Zweekhorst, S., Bol, B., Tibboel, J., Van Rosmalen, J., Samsom, J. N., Kroon, A. A., De Jongste, J. C., Reiss, I. K. M., Post, M., \& Pijnenburg, M. W. (2018). Ceramides in tracheal aspirates of preterm infants: Marker for bronchopulmonary dysplasia. PLoS ONE, 13(1). https://doi.org/10.1371/journal.pone.0185969

281. Vetter, I. R., \& Wittinghofer, A. (2001). The guanine nucleotide-binding switch in three dimensions. In Science (Vol. 294, Issue 5545, pp. 1299-1304). American Association for the Advancement of Science. https://doi.org/10.1126/science.1062023

282. Volckaert, T., Campbell, A., Dill, E., Li, C., Minoo, P., \& De Langhe, S. (2013). Localized Fgf10 expression is not required for lung branching morphogenesis but prevents differentiation of epithelial progenitors. Development (Cambridge). https://doi.org/10.1242/dev.096560

283. Volckaert, T., \& De Langhe, S. P. (2015). Wnt and FGF mediated epithelial-mesenchymal crosstalk during lung development. In Developmental Dynamics (Vol. 244, Issue 3, pp. 342 366). NIH Public Access. https://doi.org/10.1002/dvdy.24234

284. Wambach, J. A., Yang, P., Wegner, D. J., An, P., Hackett, B. P., Cole, F. S., \& Hamvas, A. (2010). Surfactant protein-C promoter variants associated with neonatal respiratory distress syndrome reduce transcription. Pediatric Research. https://doi.org/10.1203/PDR.0b013e3181eb5d68

285. Wan, H., Dingle, S., Xu, Y., Besnard, V., Kaestner, K. H., Ang, S. L., Wert, S., Stahlman, M. T., \& Whitsett, J. A. (2005). Compensatory roles of Foxal and Foxa2 during lung morphogenesis. Journal of Biological Chemistry, 280(14), 13809-13816. 
https://doi.org/10.1074/jbc.M414122200

286. Wan, H., Xu, Y., Ikegami, M., Stahlman, M. T., Kaestner, K. H., Ang, S. L., \& Whitsett, J. A. (2004). Foxa2 is required for transition to air breathing at birth. Proceedings of the National Academy of Sciences of the United States of America, 101(40), 14449-14454. https://doi.org/10.1073/pnas.0404424101

287. Wang, D. Z. M., Hammond, V. E., Abud, H. E., Bertoncello, I., McAvoy, J. W., \& Bowtell, D. D. L. (1997). Mutation in Sos1 dominantly enhances a weak allele of the EGFR, demonstrating a requirement for sos1 in EGFR signaling and development. Genes and Development, 11(3), 309-320. https://doi.org/10.1101/gad.11.3.309

288. Wang, J., Ito, T., Udaka, N., Okudela, K., Yazawa, T., \& Kitamura, H. (2005). PI3K-AKT pathway mediates growth and survival signals during development of fetal mouse lung. Tissue and Cell. https://doi.org/10.1016/j.tice.2004.09.002

289. Wang, P., van der Hoeven, D., Ye, N., Chen, H., Liu, Z., Ma, X., Montufar-Solis, D., Rehl, K. M., Cho, K. J., Thapa, S., Chen, W., van der Hoeven, R., Frost, J. A., Hancock, J. F., \& Zhou, J. (2021). Scaffold repurposing of fendiline: Identification of potent KRAS plasma membrane localization inhibitors. European Journal of Medicinal Chemistry, 217, 113381. https://doi.org/10.1016/j.ejmech.2021.113381

290. Wang, W., Chen, J. X., Liao, R., Deng, Q., Zhou, J. J., Huang, S., \& Sun, P. (2002). Sequential Activation of the MEK-Extracellular Signal-Regulated Kinase and MKK3/6-p38 MitogenActivated Protein Kinase Pathways Mediates Oncogenic ras -Induced Premature Senescence. Molecular and $\quad$ Cellular 3iology, 22(10), 3389-3403. https://doi.org/10.1128/mcb.22.10.3389-3403.2002

291. Wang, X., Zhang, Y., Mei, H., An, C., Liu, C., Zhang, Y., Zhang, Y., \& Xin, C. (2020). Study on the Relationship Between Respiratory Distress Syndrome and SP-A1 (rs1059057) Gene Polymorphism in Mongolian Very Premature Infants. Frontiers in Pediatrics, 8, 81. https://doi.org/10.3389/fped.2020.00081

292. Warburton, D., Schwarz, M., Tefft, D., Flores-Delgado, G., Anderson, K. D., \& Cardoso, W. V. (2000). The molecular basis of lung morphogenesis. In Mechanisms of Development. https://doi.org/10.1016/S0925-4773(99)00325-1

293. Weaver, M., Dunn, N. R., \& Hogan, B. L. M. (2000). Bmp4 and Fgf10 play opposing roles during lung bud morphogenesis. Development.

294. Welberg, L. (2009). Glucocorticoids in mitochondria: getting it just right. Nature Reviews Neuroscience 2009 10:4, 10(4), 247-247. https://doi.org/10.1038/nrn2622

295. Wennerberg, K., Rossman, K. L., \& Der, C. J. (2005). The Ras superfamily at a glance. Journal of Cell Science, 118(5), 843-846. https://doi.org/10.1242/JCS.01660 
Bibliography

296. Weyandt, J. D., Carney, J. M., Pavlisko, E. N., Xu, M., \& Counter, C. M. (2016). IsoformSpecific Effects of Wild-Type Ras Genes on Carcinogen-Induced Lung Tumorigenesis in Mice. PLOS ONE, 11(12), 1-11. https://doi.org/10.1371/journal.pone.0167205

297. Whitsett, J. A., Kalin, T. V, Xu, Y., \& Kalinichenko, V. V. (2019). Building and regenerating the lung cell by cell. Physiological Reviews, 99(1), 513-554. https://doi.org/10.1152/physrev.00001.2018

298. Whitsett, J. A., Wert, S. E., \& Trapnell, B. C. (2004). Genetic disorders influencing lung formation and function at birth. In Human Molecular Genetics (Vol. 13, Issue REV. ISS. 2). Hum Mol Genet. https://doi.org/10.1093/hmg/ddh252

299. Wolfman, J. C., Planchon, S. M., Liao, J., \& Wolfman, A. (2006). Structural and functional consequences of c-N-Ras constitutively associated with intact mitochondria. Biochimica et Biophysica Acta, 1763(10), 1108-1124. https://doi.org/10.1016/j.bbamcr.2006.07.015

300. Wong, J. C., Perez-Mancera, P. A., Huang, T. Q., Kim, J., Grego-Bessa, J., Alzamora, M. del pilar, Kogan, S. C., Sharir, A., Keefe, S. H., Morales, C. E., Schanze, D., Castel, P., Hirose, K., Huang, G. N., Zenker, M., Sheppard, D., Klein, O. D., Tuveson, D. A., Braun, B. S., \& Shannon, K. (2020). KrasP34R and KrasT58I mutations induce distinct RASopathy phenotypes in mice. JCI Insight, 5(21). https://doi.org/10.1172/JCI.INSIGHT.140495

301. Wortzel, I., \& Seger, R. (2011). The ERK cascade: Distinct functions within various subcellular organelles. In Genes and Cancer (Vol. 2, Issue 3, pp. 195-209). Genes Cancer. https://doi.org/10.1177/1947601911407328

302. Xiang, J., \& Wang, P. (2019). Efficacy of pulmonary surfactant combined with high-dose ambroxol hydrochloride in the treatment of neonatal respiratory distress syndrome. Experimental and Therapeutic Medicine. https://doi.org/10.3892/etm.2019.7615

303. Yan, J., Roy, S., Apolloni, A., Lane, A., \& Hancock, J. F. (1998). Ras Isoforms Vary in Their Ability to Activate Raf-1 and Phosphoinositide 3-Kinase*. http://www.jbc.org

304. Yang, J., \& Chen, J. (2014). Developmental programs of lung epithelial progenitors: A balanced progenitor model. Wiley Interdisciplinary Reviews: Developmental Biology. https://doi.org/10.1002/wdev.141

305. Yang, S. C., Tsai, Y. F., Pan, Y. L., \& Hwang, T. L. (2020). Understanding the role of neutrophils in acute respiratory distress syndrome. In Biomedical Journal (Vol. 44, Issue 4). Biomed J. https://doi.org/10.1016/j.bj.2020.09.001

306. Yates, L. L., Schnatwinkel, C., Murdoch, J. N., Bogani, D., Formstone, C. J., Townsend, S., Greenfield, A., Niswander, L. A., \& Dean, C. H. (2010). The PCP genes Celsr1 and Vangl2 are required for normal lung branching morphogenesis. Human Molecular Genetics. https://doi.org/10.1093/hmg/ddq104 
307. Ye, W., Zhang, T., Shu, Y., Fang, C., Xie, L., Peng, K., \& Liu, C. (2020). The influence factors of neonatal respiratory distress syndrome in Southern China: a case-control study. Journal of Maternal-Fetal and Neonatal Medicine. https://doi.org/10.1080/14767058.2018.1526918

308. Yoshimura, S. I., Banno, Y., Nakashima, S., Hayashi, K., Yamakawa, H., Sawada, M., Sakai, N., \& Nozawa, Y. (1999). Inhibition of neutral sphingomyelinase activation and ceramide formation by glutathione in hypoxic PC 12 cell death. Journal of Neurochemistry, 73(2), 675683. https://doi.org/10.1046/j.1471-4159.1999.0730675.x

309. Young, S. L., Fram, E. K., Spain, C. L., \& Larson, E. W. (1991). Development of type II pneumocytes in rat lung. Https://Doi.Org/10.1152/Ajplung.1991.260.2.L113, 260(2 4-1). https://doi.org/10.1152/AJPLUNG.1991.260.2.L113

310. Yu, H., He, K., Li, L., Sun, L., Tang, F., Li, R., Ning, W., \& Jin, Y. (2013). Deletion of STK40 protein in mice causes respiratory failure and death at birth. Journal of Biological Chemistry, 288(8), 5342-5352. https://doi.org/10.1074/jbc.M112.409433

311. Yuan, T., Volckaert, T., Chanda, D., Thannickal, V. J., \& De Langhe, S. P. (2018). Fgf10 Signaling in Lung Development, Homeostasis, Disease, and Repair After Injury. Frontiers in Genetics. https://doi.org/10.3389/fgene.2018.00418

312. Yzaguirre, A. D., Padmanabhan, A., de Groh, E. D., Engleka, K. A., Li, J., Speck, N. A., \& Epstein, J. A. (2015). Loss of neurofibromin Ras-GAP activity enhances the formation of cardiac blood islands in murine embryos. ELife, 4, e07780. https://doi.org/10.7554/eLife.07780

313. Zafarullah, M., Li, W. Q., Sylvester, J., \& Ahmad, M. (2003). Molecular mechanisms of Nacetylcysteine actions. In Cellular and Molecular Life Sciences. https://doi.org/10.1007/s000180300001

314. Zarubin, T., \& Han, J. (2005). Activation and signaling of the p38 MAP kinase pathway. In Cell Research (Vol. 15, Issue 1, pp. 11-18). Nature Publishing Group. https://doi.org/10.1038/sj.cr.7290257

315. Zemans, R. L., Briones, N., Campbell, M., McClendon, J., Young, S. K., Suzuki, T., Yang, I. V., Langhe, S. De, Reynolds, S. D., Mason, R. J., Kahn, M., Henson, P. M., Colgan, S. P., \& Downey, G. P. (2011). Neutrophil transmigration triggers repair of the lung epithelium via $\beta$ catenin signaling. Proceedings of the National Academy of Sciences, 108(38), 15990-15995. https://doi.org/10.1073/PNAS.1110144108

316. Zepp, J. A., Zacharias, W. J., Frank, D. B., Cavanaugh, C. A., Zhou, S., Morley, M. P., \& Morrisey, E. E. (2017). Distinct Mesenchymal Lineages and Niches Promote Epithelial SelfRenewal and Myofibrogenesis in the Lung. Cell. https://doi.org/10.1016/j.cell.2017.07.034

317. Zhang, Y., Ding, S., Li, C., Wang, Y., Chen, Z., \& Wang, Z. (2017). Effects of N- 
Bibliography

acetylcysteine treatment in acute respiratory distress syndrome: A meta-analysis. Experimental and Therapeutic Medicine, 2863. https://doi.org/10.3892/ETM.2017.4891

318. Zhang, Y. Y., Vik, T. A., Ryder, J. W., Srour, E. F., Jacks, T., Shannon, K., \& Clapp, D. W. (1998). Nf1 regulates hematopoietic progenitor cell growth and ras signaling in response to multiple cytokines. The Journal of Experimental Medicine, 187(11), 1893-1902. https://doi.org/10.1084/jem.187.11.1893

319. Zhao, R. Z., Jiang, S., Zhang, L., \& Yu, Z. Bin. (2019). Mitochondrial electron transport chain, ROS generation and uncoupling (Review). In International Journal of Molecular Medicine (Vol. 44, Issue 1, pp. 3-15). Spandidos Publications. https://doi.org/10.3892/ijmm.2019.4188 


\section{ANNEX}





\title{
Concomitant deletion of HRAS and NRAS leads to pulmonary immaturity, respiratory failure and neonatal death in mice
}

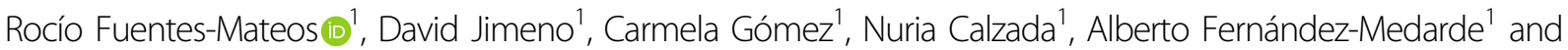 \\ Eugenio Santos (iD)
}

\begin{abstract}
We reported previously that adult $\left(\mathrm{HAS}^{-1-}\right.$; $\mathrm{NRAS}^{-1-}$ ) double knockout (DKO) mice showed no obvious external phenotype although lower-than-expected numbers of weaned DKO animals were consistently tallied after crossing NRAS-KO and HRAS-KO mice kept on mixed genetic backgrounds. Using mouse strains kept on pure C57BI/6 background, here we performed an extensive analysis of the offspring from crosses between HRAS-KO and NRAS-KO mice and uncovered the occurrence of very high rates of perinatal mortality of the resulting DKO littermates due to respiratory failure during the first postnatal 24-48 $\mathrm{h}$. The lungs of newborn DKO mice showed normal organ structure and branching but displayed marked defects of maturation including much-reduced alveolar space with thick separating septa and significant alterations of differentiation of alveolar (AT1, AT2 pneumocytes) and bronchiolar (ciliated, Clara cells) cell lineages. We also observed the retention of significantly increased numbers of undifferentiated progenitor precursor cells in distal lung epithelia and the presence of substantial accumulations of periodic acid-Schiffpositive (PAS+) material and ceramide in the lung airways of newborn DKO mice. Interestingly, antenatal dexamethasone treatment partially mitigated the defective lung maturation phenotypes and extended the lifespan of the DKO animals up to 6 days, but was not sufficient to abrogate lethality in these mice. RNA microarray hybridization analyses of the lungs of dexamethasone-treated and untreated mice uncovered transcriptional changes pointing to functional and metabolic alterations that may be mechanistically relevant for the defective lung phenotypes observed in DKO mice. Our data suggest that delayed alveolar differentiation, altered sphingolipid metabolism and ceramide accumulation are primary contributors to the respiratory stress and neonatal lethality shown by DKO mice and uncover specific, critical roles of HRAS and NRAS for correct lung differentiation that are essential for neonatal survival and cannot be substituted by the remaining KRAS function in this organ.
\end{abstract}

\section{Introduction}

RAS GTPases play critical roles in control of cellular proliferation, differentiation or death ${ }^{1-3}$ acting as biochemical switches shifting between inactive (RAS-GDP) and active (RAS-GTP) conformations in a cycle modulated

\footnotetext{
Correspondence: Alberto Fernández-Medarde (afm@usal.es) or

Eugenio Santos (esantos@usal.es)

${ }^{1}$ Centro de Investigación del Cáncer-Instituto de Biología Molecular y Celular del Cáncer (CSIC- Universidad de Salamanca) and CIBERONC, 37007 Salamanca, Spain
}

Edited by S. Tait by negative (GAPs, GTPase activating proteins) and positive (RAS-GEFs, guanine nucleotide exchange factors) regulators ${ }^{4-7}$. Activating point mutations trigger different tumor types (somatic mutations) or inherited developmental syndromes (germline mutations) ${ }^{2,5,8}$. Although the canonical RAS genes are ubiquitous in mammals, they exhibit different expression levels depending on cell type, tissue or developmental stage under study ${ }^{2,9}$.

Prior reports support the functional specificity of different Ras isoforms under various physiological and pathological contexts by demonstrating preferential association of

\section{(c) The Author(s) 2019}

(c) (i) Open Access This article is licensed under a Creative Commons Attribution 4.0 International License, which permits use, sharing, adaptation, distribution and reproduction in any medium or format, as long as you give appropriate credit to the original author(s) and the source, provide a link to the Creative Commons license, and indicate if changes were made. The images or other third party material in this article are included in the article's Creative Commons license, unless indicated otherwise in a credit line to the material. If material is not included in the article's Creative Commons license and your intended use is not permitted by statutory regulation or exceeds the permitted use, you will need to obtain permission directly from the copyright holder. To view a copy of this license, visit http://creativecommons.org/licenses/by/4.0/. 
specific RAS isoforms with different tumor types, intracellular processing pathways, subcellular locations or functional interactions with regulators and effectors $^{2,5,8,10,11}$. Analysis of genetically modified mouse strains also supports the functional specificity of the RAS isoforms. Among RAS family members, only KRAS is essential for mouse development and viability whereas HRAS and NRAS are dispensable ${ }^{12-18}$. Transcriptomic analyses have identified specific transcriptional programs controlled by each RAS isoform ${ }^{2}$ and suggested preferential involvement of HRAS with cell growth and proliferation, NRAS with immunomodulatory and apoptotic responses ${ }^{19,20}$, and KRAS with control of cell cycle progression ${ }^{21,22}$. Our earlier studies showed that HRAS/NRAS-DKO mice (expressing only KRAS) were viable and presented no obvious phenotypes, but significantly lower-than-expected numbers of adult DKO animals were obtained when breeding NRAS$\mathrm{KO}\left(\mathrm{HRAS}^{+/-}\right.$; $\left.\mathrm{NRAS}^{-1-}\right)$ and HRAS-KO $\left(\mathrm{HRAS}^{-1-}\right.$; NRAS $^{+/-}$) mice kept on mixed genetic background ${ }^{12}$.

To get mechanistic clues for these observations and to ascertain possible differential roles of RAS isoforms in control of tissue/organ development during embryonic or adult stages, here we carried out an extensive breeding program between mice kept on pure $\mathrm{C} 57 \mathrm{Bl} / 6$ background to generate single- or double-KO offspring for HRAS and NRAS that were then studied at different embryonic or adult stages by means of immunochemical or transcriptomic analyses. Most DKO offspring died immediately after birth while showing significant respiratory distress and marked signs of pulmonary immaturity and defective differentiation of specialized lung cell types. The lungs of these DKO mice showed also significant transcriptional alterations of components of sphingolipid metabolic pathways that correlated with abnormal accumulations of ceramide, a common feature of various lung diseases in humans ${ }^{23,24}$. These findings indicate that HRAS and NRAS play specific functions during lung maturation that are critical for neonatal survival and cannot be provided by the remaining KRAS isoform in this organ.

\section{Results}

\section{Simultaneous loss of HRAS and NRAS leads to neonatal} death in mice

We reported previously that significantly less-thanexpected adult HRAS/NRAS-DKO mice resulted from crosses between single HRAS-KO and NRAS-KO mice kept on mixed $(129 / \mathrm{Bl} / 6)$ genetic background ${ }^{12}$. To identify possible causes for that observation, here we crossed heterozygous HRAS-KO and NRAS-KO strains kept on pure $\mathrm{C} 57 \mathrm{Bl} / 6$ background and counted the numbers of Control, HRAS-KO, NRAS-KO and DKO offspring mice at different developmental stages including E18.5 embryos, P0 neonates or P21 weaned pups (Fig. 1a).
The number of alive DKO E18.5 embryos (not shown) and newborn P0 pups followed expected mendelian rates $(\sim 25 \%)$ and these frequencies were not sexually biased, as similar percentages were measured independently of the sex of the parental breeding partners (Fig. 1a). In contrast, the percentage of surviving DKO mice pups counted after weaning (P21) was 5-6 fold lower than around birth time (Fig. 1a), and we observed that concomitant HRAS and NRAS loss caused significant neonatal lethality within the first 1-2 postnatal days. The DKO neonates exhibited also significant reduction of body weight (15-20\%) and size in comparison to their Control or single HRAS-KO and NRAS-KO littermates (Fig. 1b, c). Notice that single NRAS-KO animals exhibit also a slight reduction of body weight as compared to the Control group (Fig. 1b).

\section{HRAS/NRAS-DKO mice exhibit impaired lung maturation}

In contrast to the other genotypes, most DKO neonates were cyanotic and showed severe respiratory distress, displaying noticeable breathing efforts (Fig. 1c; Supplementary movie 1). Given the recognized connection between impaired respiratory activity and neonatal mortality $^{25-28}$, we examined overall lung morphology and structure in the newborn pups. No morphological or branching differences were found between the lungs of DKO, HRAS-KO, NRAS-KO, and Control mice. However, the lungs of most PO DKO neonates showed extensive atelectasis and occasional hemorrhages probably related to their early postnatal death (Fig. 2a, arrows). Interestingly, the lungs of the small number of DKO mice that survived to adulthood displayed much smaller patches of atelectasis affecting only limited areas of the structure of otherwise normal organs (Supplementary Fig. S1).

Hematoxylin-Eosin-staining (H\&E) revealed that, in contrast to all other genotypes, the lungs of DKO neonates showed also very significant reduction of the alveolar saccular space with notably thicker separating septa (Fig. 2a). The reduced saccular space was already visible at earlier embryonal stages (E18.5) not only in DKO but also in HRAS-KO animals (Fig. 2b). Notice that this defect had disappeared at birth time in HRAS-KO but not in DKO lungs (Fig. 2a, b). PAS-staining of lung sections revealed also significant polysaccharide accumulations in alveolar areas of the lungs of E18.5 DKO mice that were not seen in the three other genotypes (Fig. 2d) and a similar tendency for PAS + accumulations, though not statistically significant, was also seen in the PO DKO lungs (Fig. 2c).

\section{Defective/delayed differentiation of alveolar cell lineages in HRAS/NRAS-DKO mice}

The differentiation of specialized cell types in distal alveolar epithelia, including gas-exchanging AT1 squamous pneumocytes and surfactant-producing AT2 

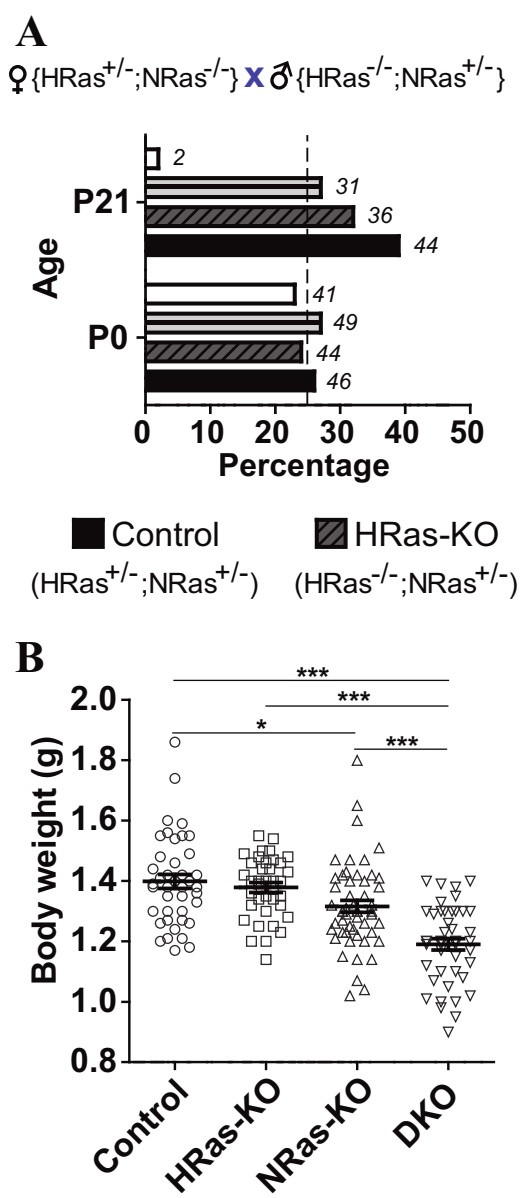

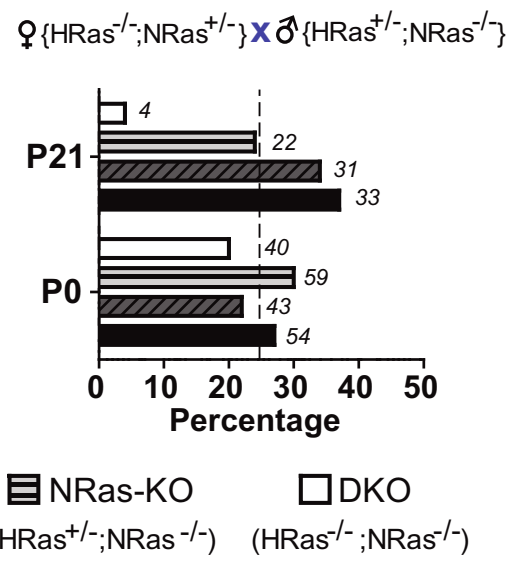

C

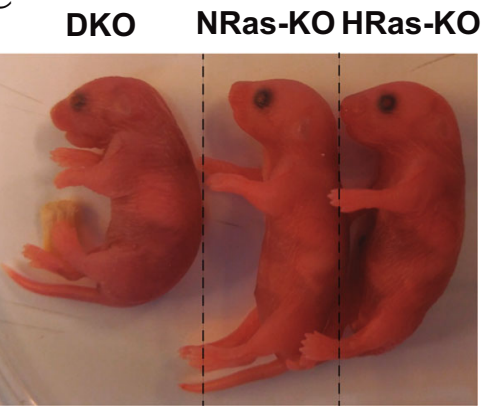

Fig. 1 Analysis of the offspring from crosses between HRAS-KO and/or NRAS-KO mice. a Bar graphs depicting percentage (\%) and absolute numbers (in italics) of individuals of the indicated genotypes (Control; HRAS-KO; NRAS-KO; DKO) counted at time of birth (PO) or at weaning time (P21) in the litters resulting from parental crosses between heterozygous HRAS-KO and NRAS-KO mice of the indicated sex. $\mathbf{b}$ Body weight distribution of living, newborn PO mice of the indicated genotypes at time of birth. Data represented as the mean \pm s.e.m. for each genotype. Control, $n=41$; HRAS-KO, $n=39$; NRAS-KO, $n=52$; DKO, $n=45$. ${ }^{*} p<0.05$, ${ }^{* *} p<0.001$. c Representative picture of a cyanotic DKO pup (left) next to healthy HRAS-KO and NRAS-KO littermates, immediately after birth (P0). See also respiratory distress in Supplementary video 1

cuboidal pneumocytes, was evaluated using specific markers $^{29,30}$ (Fig. 3).

Consistent with the notion of pulmonary immaturity ${ }^{31}$, the lungs of newborn DKO (and also HRAS-KO) mice exhibited significantly elevated numbers of Surfactant protein C-positive (SftpC + ) AT2 cells as compared to normal Controls and NRAS-KO, and these SftpC + pneumocytes were frequently misplaced in inner parenchymal accumulations instead of being exclusively distributed throughout the luminal surface of the alveolar sacs (Fig. 3a).

Enrichment in PAS + intracellular content is also a major feature of immature AT2 cells since cytoplasmic glycogen granules are building blocks for surfactant phospholipids $^{32,33}$. Consistent with the SftpC immunoassays, strong increase of PAS + immunostaining was observed in the lungs of P0 and E18.5 DKO mice as compared to the other genotypes (Fig. 2c, d).
Simultaneous immunoassays against Ricinus communis agglutinin-I (RCA-I) (AT1 lineage) and SftpC (AT2 lineage), two markers co-localizing only in the bi-potent alveolar progenitor cells known to differentiate and disappear from normal mouse embryonic lungs before E18.3 $29,34,35$, we observed that the lungs of E18.5 and P0 DKO embryos (also the E18.5 HRAS-KO lungs) retained abnormally high numbers of bi-potent alveolar progenitors (originating both AT1 and AT2 lineages) in comparison to normal Controls (Fig. 3b).

The retention of undifferentiated progenitors in alveoli of our KO strains was also monitored with immunoassays of Sex-determining region Y-box 9 (Sox9), a well-established marker of alveolar distal-tip progenitors ${ }^{36,37}$. We detected strong nuclear Sox9 staining in lung distal-tip structures of E18.5 HRAS-KO and DKO mice as compared to Controls. 

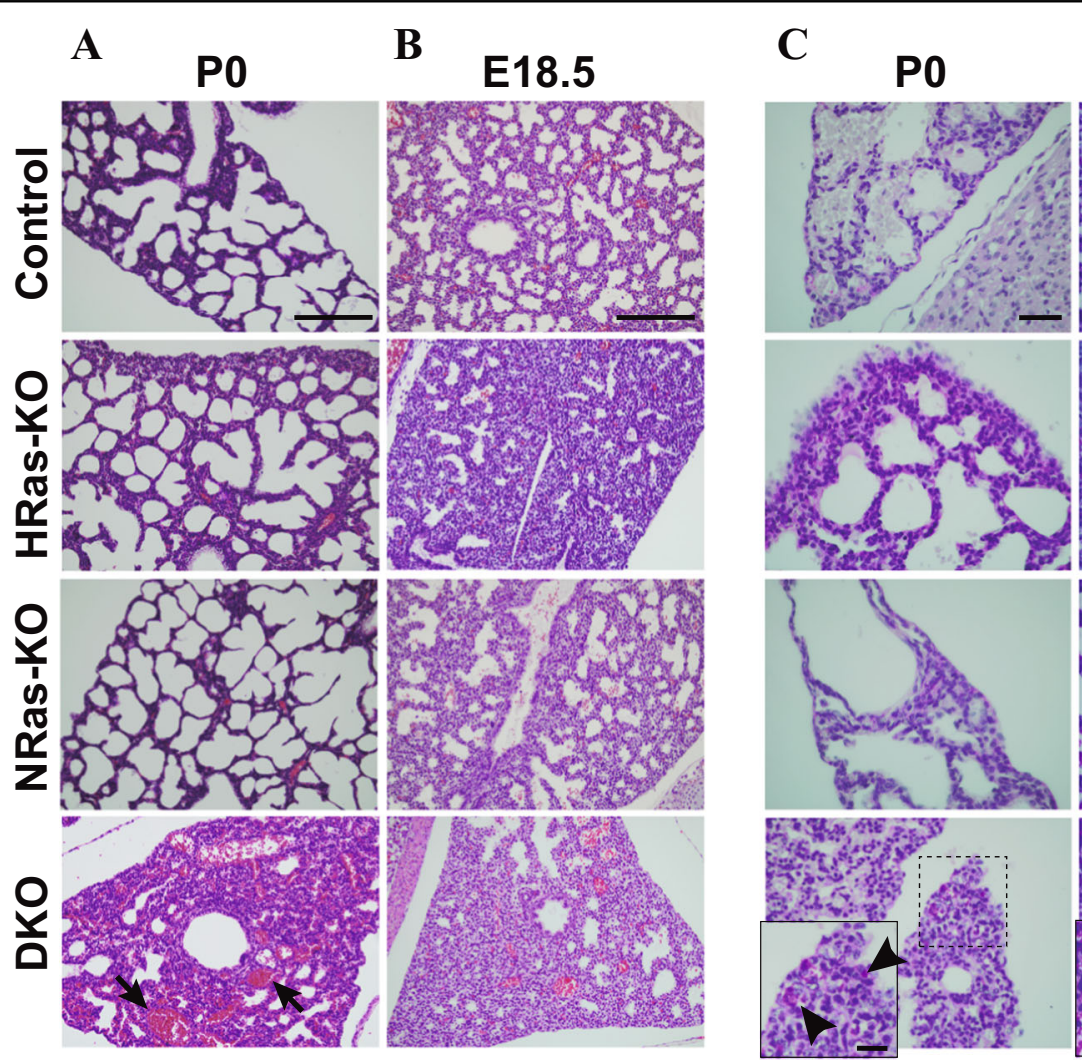

D $\quad$ E18.5
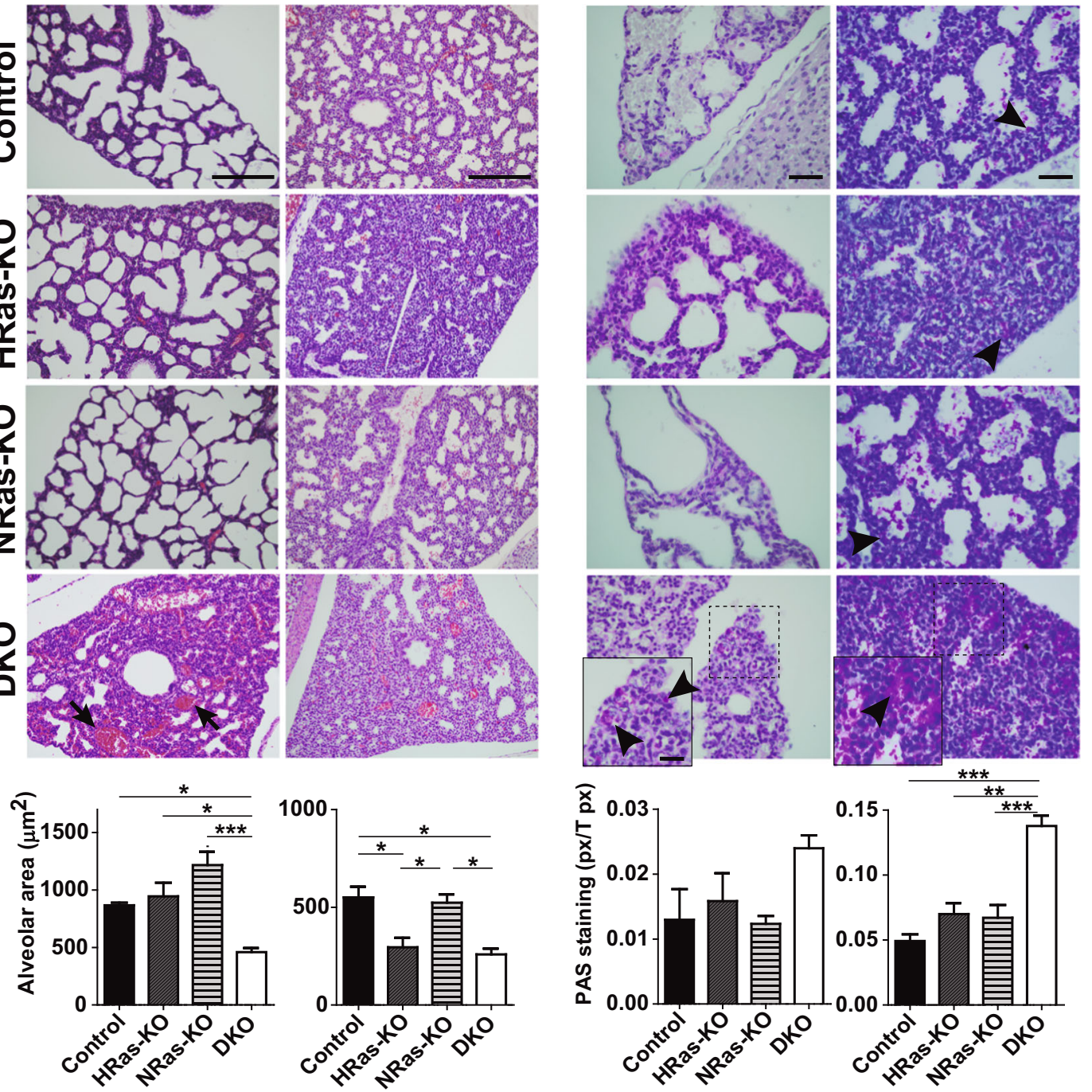

Fig. 2 Histological analysis of the lungs of newborn pups (PO) and late embryos (E18.5) of HRAS-KO and/or NRAS-KO mice. a, b Representative images of Hematoxylin-Eosin (H\&E)-stained sections of lungs from P0 newborn pups (panel a) or from E18.5 embryos (panel b) of the indicated genotypes. Arrows indicate hemorrhagic regions. Scale bars: $100 \mu \mathrm{m}$. The bar graphs at bottom of the panels quantify the average area $\left(\mu \mathrm{m}^{2}\right)$ of the individual alveolar sacs in the lungs of, respectively, PO (panel a) and E18.5 individuals (panel b) of the indicated genotypes. Data are expressed as the mean \pm s.e.m. $n=3$ individuals for each genotype. ${ }^{*} p<0.05,{ }^{* * *} p<0.001$. $\mathbf{c}$, d Representative images of PAS-stained lung sections from P0 newborn mice (panel c) or from E18.5 embryos (panel d) of the indicated genotypes. Scale bars: $20 \mu \mathrm{m}$ and $10 \mu \mathrm{m}$ in magnified, boxed areas. Black arrowheads point to cytoplasmic and extracellular accumulations of PAS-positive label in alveolar areas of the indicated genotypes. The bar graphs in these panels quantify the relative levels of PAS-staining (ratio of PAS+ pixels relative to total number of pixels) in the lungs of P0 (panel c) and E18.5 (panel d) individuals of the indicated genotypes. Data are expressed as the mean \pm s.e.m. $n=3$ individuals for all genotypes in E18.5 lungs; $n=4$ for DKO and $n=3$ for rest of genotypes of PO lungs. ${ }^{* *} p<0.01,{ }^{* * *} p<0.001$

These Sox9+ cells were detected not only in peripheral zones (where distal-tip structures are usually located) but also in inner parenchymal areas of the lungs of HRAS-KO and DKO mice (Fig. 3c). Altogether, these observations point to delayed differentiation of the alveolar cell lineages in DKO mice.
Alterations of bronchiolar cell lineages in HRAS/NRAS-DKO mice

PAS-staining of lung bronchioles from newborn P0 mice showed that the typical columnar morphology of the PAS + Clara cells (located in the luminal layer of the bronchioles of normal Control mice) was significantly 


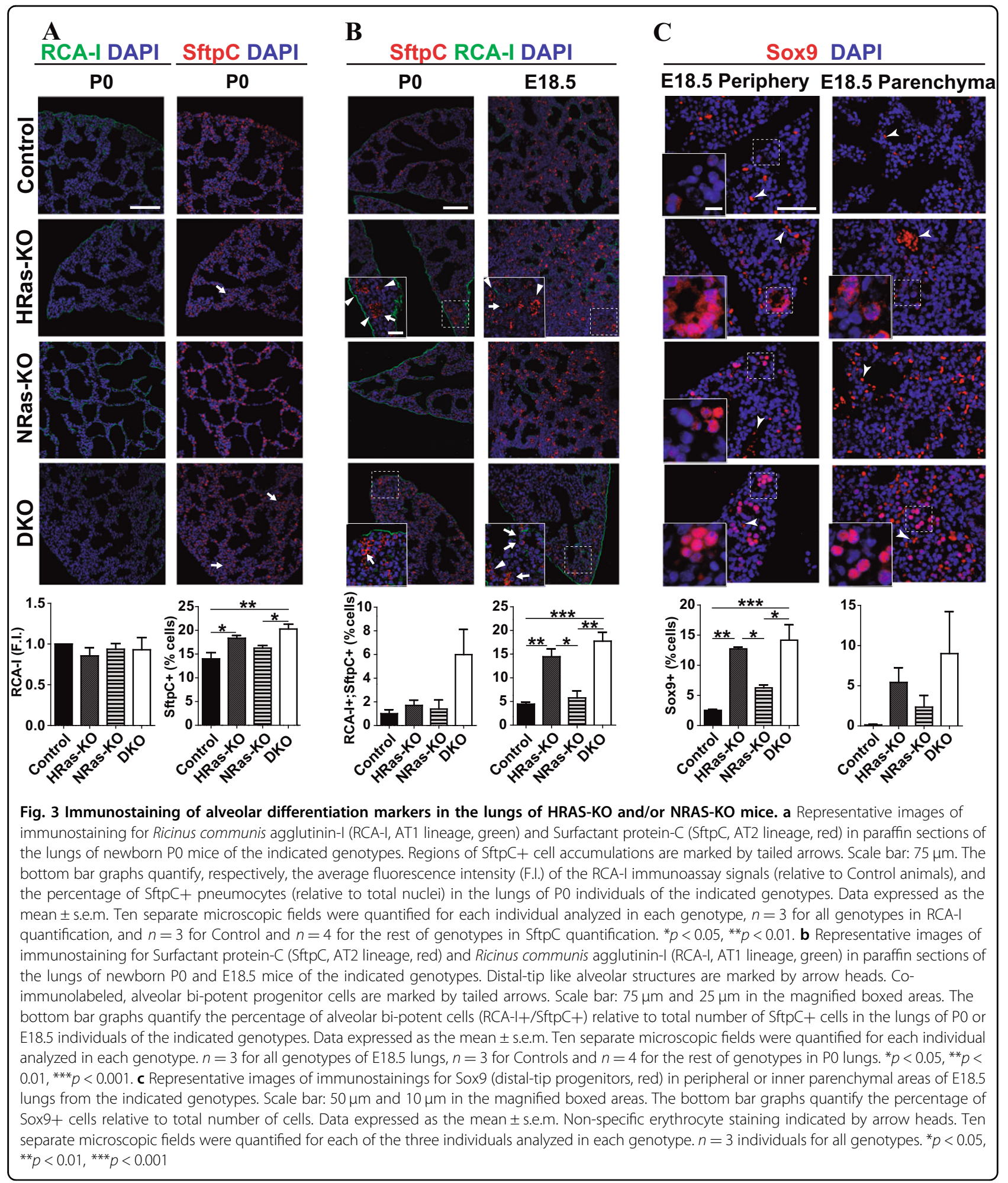

altered in DKO and single KO littermates (Fig. 4a). These alterations included overall reduction of glycosaminoglycan (PAS) labeling, as well as noticeable morphological flattening linked to shortening of their cytoplasmic, apical vesicular area (Fig. 4a, c).
Immunostaining Clara cells with antibodies to Secretoglobulin $(\mathrm{Scgb})$ and their derived ciliated cells with anti$\beta$-Tubulin ( $\beta$-Tub) confirmed the columnar-to-cuboidal morphological change occurring in Clara cells of DKO and single $\mathrm{KO}$ animals (Fig. 4b, c). Regarding ciliated cells, 


\section{A}

\section{PAS}
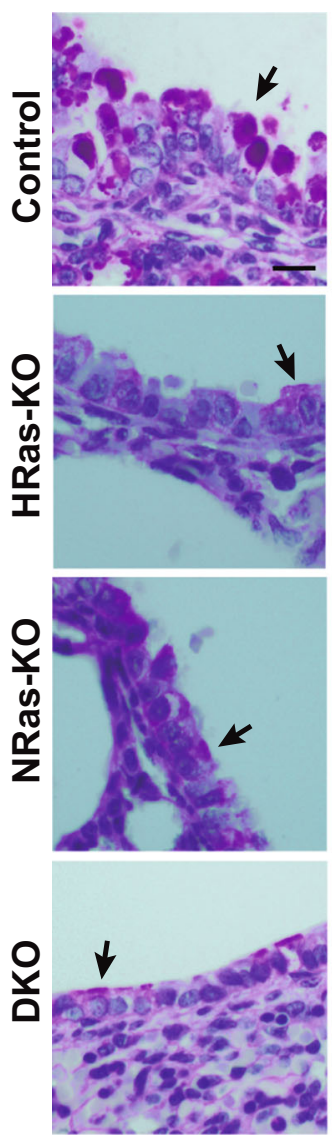

B

Scgb $\beta$-Tub DAPI
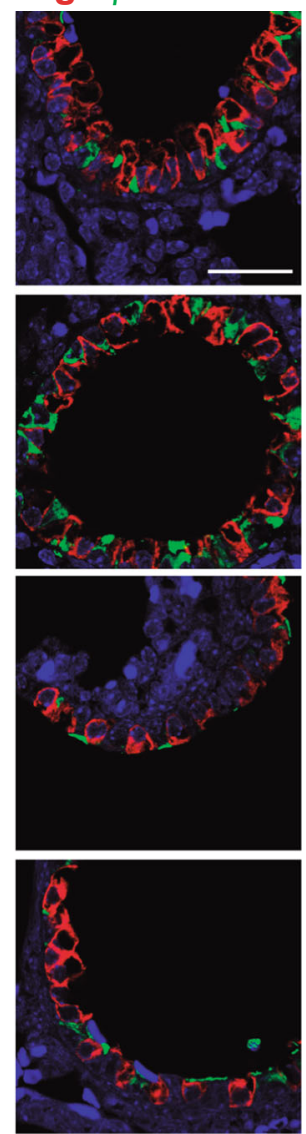

C

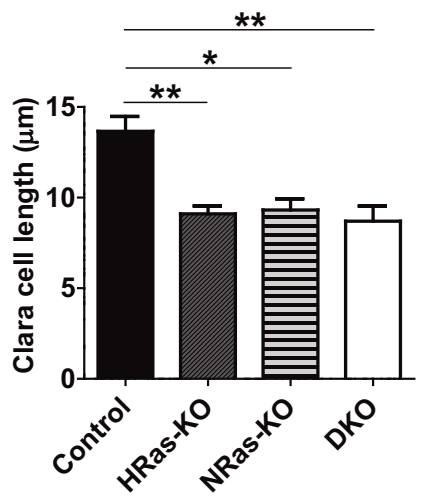

D

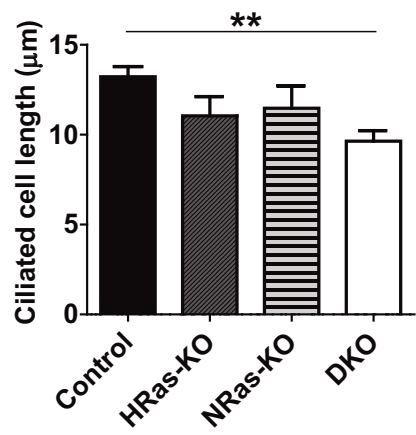

Fig. 4 Immunostaining of bronchiolar differentiation markers in the lungs of HRAS-KO and/or NRAS-KO mice. a Representative images of PAS-stained lung sections from PO mice of the indicated genotypes. Tailed arrows point to PAS+ accumulations located in the apical cytoplasmic region of bronchiolar Clara cells. Scale bar: $10 \mu \mathrm{m}$. b Representative images of immunostaining for Secretoglobulin (Scgb, Clara cells, red) and $\beta$-Tubulin ( $\beta$-Tub, Ciliated cells, green), counterstained with DAPI (blue), in paraffin sections of bronchiolar regions of lungs from P0 mice of the indicated genotypes. Scale bar: $25 \mu \mathrm{m}$. c, d Cell length measurements (from basal to apical membrane) of Clara (panel c) and Ciliated (panel d) cells stained as in panel (b). Data expressed as the mean \pm s.e.m. Ten separate microscopy fields were quantified for each individual analyzed in each genotype. $n=4$ individuals for DKO and $n=3$ for rest of genotypes. ${ }^{*} p<0.05$, ${ }^{* *} p<0.01$

we only detected statistically significant shortening of this cell type in DKO mice as compared to normal Controls (Fig. 4d).

Increased rates of proliferation, apoptosis, and infiltrating neutrophils in the lungs of HRAS/NRAS-DKO mice

Proliferation and cellular death are also well-balanced processes during normal embryonal and postnatal lung development ${ }^{29,38-40}$. We observed that the number of Bromodeoxyuridine (BrdU+), proliferating cells in lungs of E18.5 embryos was significantly higher in DKO and HRAS-KO mice than in Controls and NRAS-KO littermates. Our immunoassays showed also that a vast majority of these BrdU+ cells at E18.5 corresponded to SftpC + AT2 cells (Fig. 5a).
Cell death quantitation in lung sections of newborn P0 mice by means of Cleaved-caspase-3 (CC3) immunoassays yielded overall low rates in absolute terms, but we statistically verified significantly higher levels of apoptosis in the lungs of DKO mice than in all other genotypes (Fig. 5b).

Immunoassays for different immune cell types detected significantly higher levels of infiltrating neutrophils in DKO lungs as compared to the other genotypes (Supplementary Fig. S2).

\section{Specific transcriptomic alterations in the lungs of newborn HRAS/NRAS-DKO mice}

To search for mechanistic clues to the phenotypic defects of lung maturation exhibited by DKO mice, we compared the transcriptional profiles of lung tissues from 

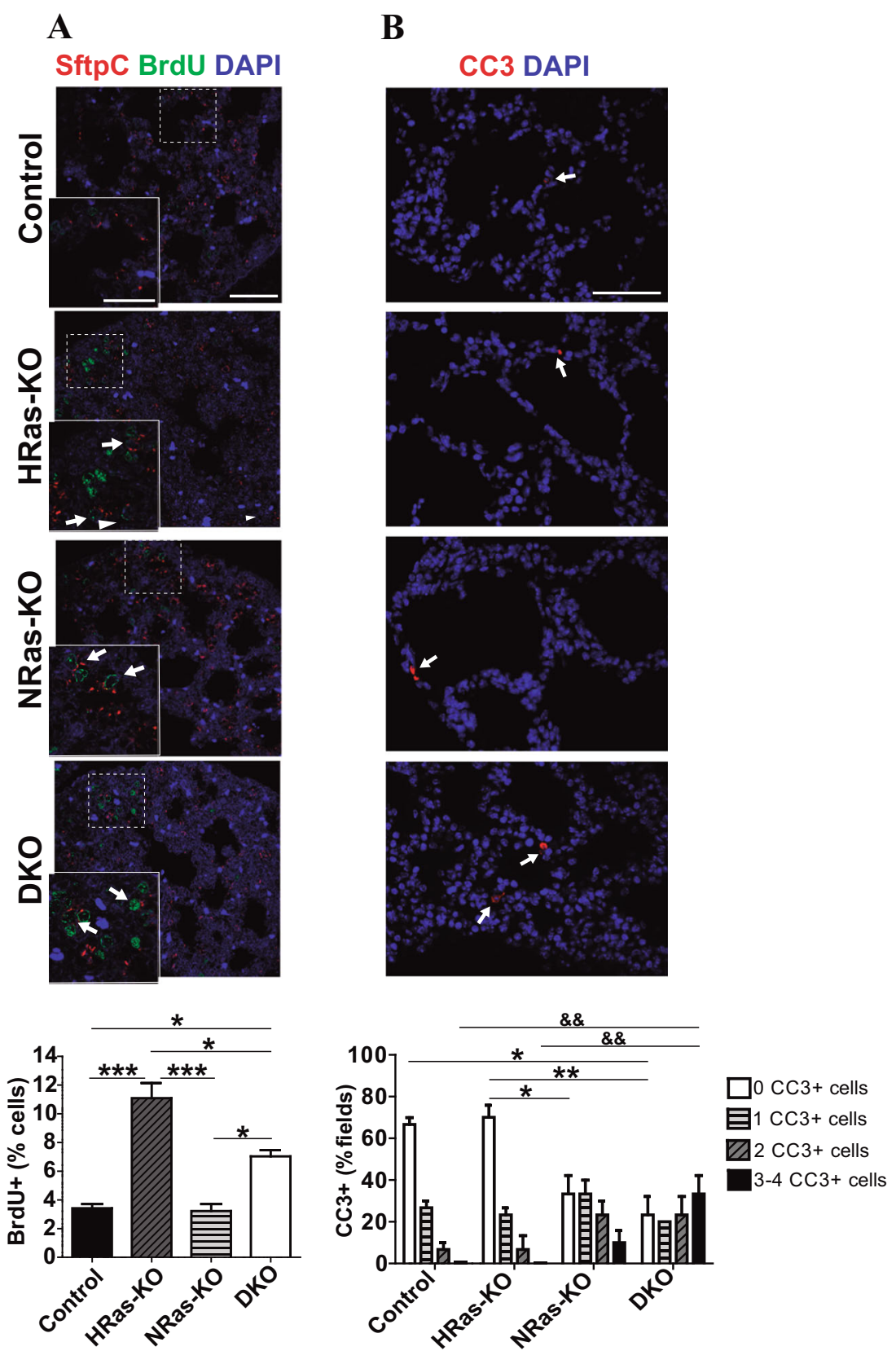

Fig. 5 Analysis of proliferative and apoptotic rates in the lungs of HRAS-KO and/or NRAS-KO mice. a Representative images of immunostaining for Bromodeoxyridine (BrdU, green) and SftpC (red), counterstained with DAPI (blue), in paraffin sections of E18.5 lungs of the indicated genotypes. Tailed arrows point to cells presenting double staining with BrdU and SftpC. Scale bar: $50 \mu \mathrm{m}$ and $25 \mu \mathrm{m}$ for magnified box areas. Bar graphs quantifying percentage of BrdU+ cells relative to total number of cells. Data expressed as mean \pm s.e.m. Ten separate microscopy fields were quantified for each individual analyzed. $n=3$ individuals for all genotypes. ${ }^{*} p<0.05,{ }^{* *} p<0.001$. b Representative images of immunostaining for Cleaved caspase-3 (CC3, red) and counterstaining with DAPI (blue) in paraffin sections of P0 lungs of the indicated genotypes. Scale bar: $50 \mu \mathrm{m}$. For quantitation of apoptotic (CC3+) cells, the bar plots represent the percentage (\%) of microscopy fields containing the specified number of apoptotic cells $(0,1,2$, or 3-4 per individual field) as indicated. Ten separate microscopy fields were quantified for each individual analyzed. $n=3$ animals for all genotypes. For analysis of statistical significance, the * and \& characters in the bar plot correspond, respectively, to comparisons between frequencies of samples of each genotype containing 0 apoptotic cells per microscopy field (clear bars) and frequencies of samples of each genotype containing 3-4 apoptotic cells per microscopic field (solid bars). ${ }^{*} p<0.05$, ${ }^{* *} p<0.01,{ }^{\& \&} p<0.01$ 


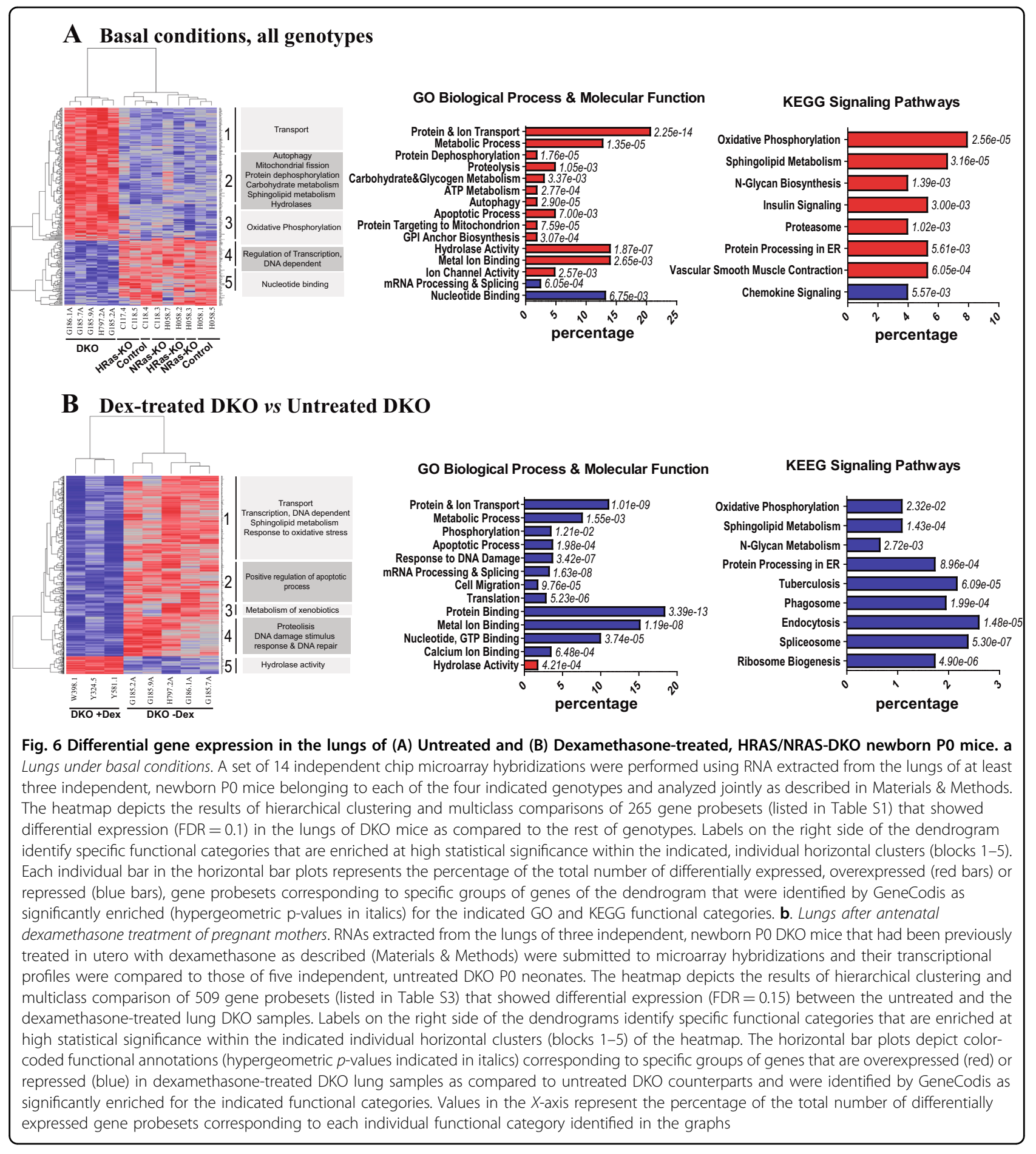

P0 neonate littermates of the relevant RAS genotypes (Fig. 6a). Multiclass comparisons of microarray expression data profiles generated with high stringency $(F D R=0.1)$ produced a dendrogram that clearly discriminated all our independent DKO samples from a separate group encompassing the rest of genotypes (Fig. 6a heatmap), suggesting the existence of a distinct pattern of transcriptional alterations specifically linked to HRAS and NRAS disappearance in the lungs of DKO mice. Most differentially expressed probesets in neonate DKO lungs were overexpressed ( $75 \%)$ whereas only $25 \%$ were repressed, suggesting that transcriptional repression is the 
predominant consequence of HRAS- and NRAS-driven signals in mouse lung tissues at this early developmental stage (Fig. 6a heatmap; Supplementary Table S1).

Functional annotation of the probesets in the dendrogram identified significant enrichment in components of various biological processes, molecular functions and signaling pathways that may be mechanistically significant for generation of the phenotypic alterations observed in newborn DKO lungs (Fig. 6a GO\&KEGG; Supplementary Table S2). The group of genes upregulated in DKO samples showed highly statistically significant enrichment in distinct GO (Gene Ontology) functional categories including "Transport and Metabolic Processes" or "Hydrolase activity". 34 overexpressed genes in DKO lungs were related with various functional subcategories of Transport, including "Protein transport", "Vesiclemediated transport", "Intracellular protein transport", "Ion transport", "Proton transport", or "Transmembrane transport". A separate group of 21 upregulated genes was specifically concerned with various "Protein Metabolic Processes" (Proteolysis, Ubiquitination and Dephosphorylation), as well as "Carbohydrate and Glycogen Metabolism” (Fig. 6a GO; Supplementary Table S2). Consistent with the GO annotations, the list of genes upregulated in DKO lungs was significantly enriched with components of different KEGG signaling pathways including "Oxidative phosphorylation", "N-glycan biosynthesis" and, particularly, "Sphingolipid metabolism", that are functionally significant for the developmental processes of lungs in mice (Fig. 6a KEGG; Supplementary Table S2).

\section{Metabolic/physiological alterations and increased ceramide levels in the lungs of HRAS/NRAS-DKO mice}

A mechanistic link was readily apparent between some transcriptional alterations (Supplementary Table S2) and the phenotypic defects observed in DKO lungs. The severe respiratory distress exhibited by P0 mice (Fig. 1c) was paralleled by transcriptional upregulation of significant numbers of loci involved in "Oxidative phosphorylation" and "ATP metabolism" or "Protein targeting to mitochondria" (Supplementary Table S2). The increased apoptotic rate detected in P0 lungs (Fig. 5b) was accompanied by overexpression of various loci coding for regulatory components of "Apoptotic and Autophagic processes" (Supplementary Table S2). The significant increase of PAS staining detected in P0 lungs (Fig. 2c, d) or the morphological flattening of secretory Clara cells (Fig. 4a) correlated with transcriptional upregulation of various loci involved in regulation of "Carbohydrate and glycogen metabolism" as well as "Intracellular vesiclemediated transport" (Supplementary Table S2).

The significant upregulation of genes related to "Sphingolipid metabolism" or "GPI anchor biosynthesis"
(Supplementary Table S2) is also likely to be relevant for the defective DKO lung phenotypes in view of the critical roles that ceramides and surfactants play in multiple physiological and pathological lung processes ${ }^{23,24,41-45}$. Of note, our transcriptomic analyses uncovered significant overexpression in DKO lungs of an important number of loci involved in sphingolipid metabolic pathways controlling the levels of cellular ceramide (Sptlc1, Serine palmitoyltransferase, long chain base subunit 1; Cers5, Ceramide synthase 5; Degs1, Delta(4)-desaturase, sphingolipid 1; Sgpp1, Sphingosine-1-phosphate phosphatase 1; Acer3, alkaline ceramidase 3) (Supplementary Table S2, Supplementary Fig S3). Consistent with the transcriptomic alterations, our parallel immunohistochemical studies of the lungs of PO mice detected significantly elevated levels of ceramide in lung alveoli of newborn DKO mice as compared to all three other genotypes (Fig. 7a).

\section{Partial rescue of defective DKO lung phenotypes by antenatal treatment with glucocorticoids}

To test the possibility of counteracting the defective developmental phenotypes observed in the lungs of newborn DKO mice by means of antenatal administration of glucocorticoids ${ }^{44-46}$, we gave subcutaneous dexamethasone injections to pregnant female mice on days E17.5 and E18.5 of gestation (see Materials and Methods for details), and the lungs of the pups in the resulting litters were subsequently examined at later developmental stages (E18.5, P0, P5) (Fig. 8a).

Antenatal dexamethasone treatment extended the lifespan of newborn DKO mice in comparison to untreated animals of the same genotype. Whereas most untreated DKO pups were routinely dead within the first two postnatal days, dexamethasone treatment caused a delay of 4-5 days in the timing of death for pups of the DKO genotype (Fig. 8c). As with untreated animals, the size of dexamethasone-treated DKO pups was significantly smaller than their similarly treated Control littermates (Fig. 8b).

Histological analysis showed that the glucocorticoid treatment was capable of resolving the alveolar differentiation defects observed in DKO mice. Comparison of H\&Estained, dexamethasone-treated and untreated E18.5 lung samples showed that a single antenatal dexamethasone injection (at E17.5) was enough to correct the altered saccular architecture and reduced alveolar area observed in the lungs of DKO mice. Indeed, the dexamethasone treatment produced a complete reversion to normal values of the reduced alveolar space typically seen in untreated newborn DKO animals (Fig. 8d). Consistently, the practical absence of RCA-I+/SftpC + cells (bi-potent progenitors) (Fig. 8e), and the significant reduction of Sox9+ cells (alveolar distaltip progenitors) found in the parenchymal and peripheral lung regions (Fig. 8f) of the dexamethasone-treated, E18.5 


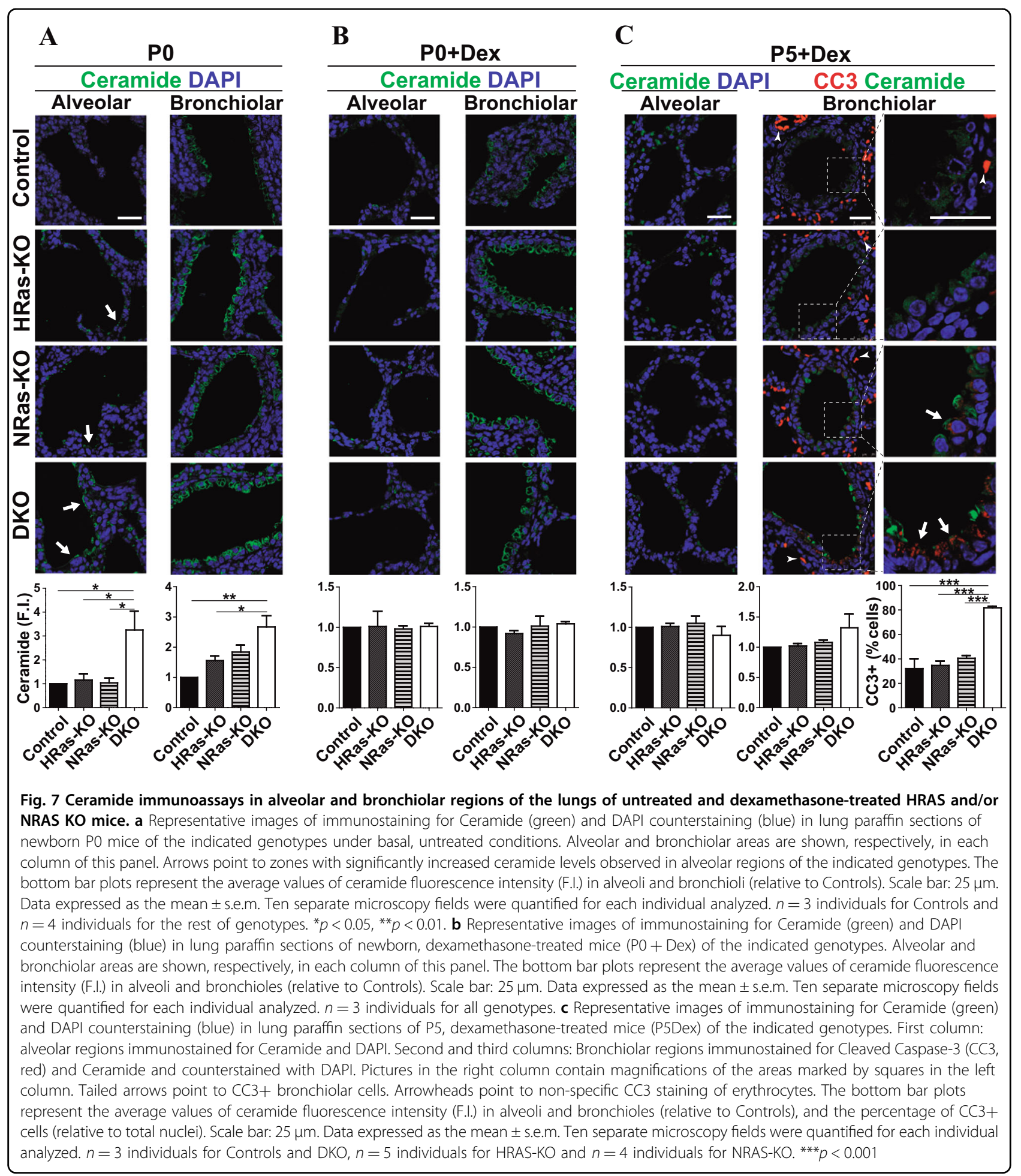

embryos of all four relevant genotypes confirmed that a single dexamethasone pretreatment was able to rescue the defective differentiation of pneumocytic lineages observed in the lungs of untreated DKO mice. Dexamethasone treatment also reverted the increase of infiltrating neutrophils specifically observed in PO DKO lungs (Supplementary Fig. S2).

In contrast, the administration of glucocorticoids could not fully recover other developmental defects in the lungs of DKO mice. We observed that 


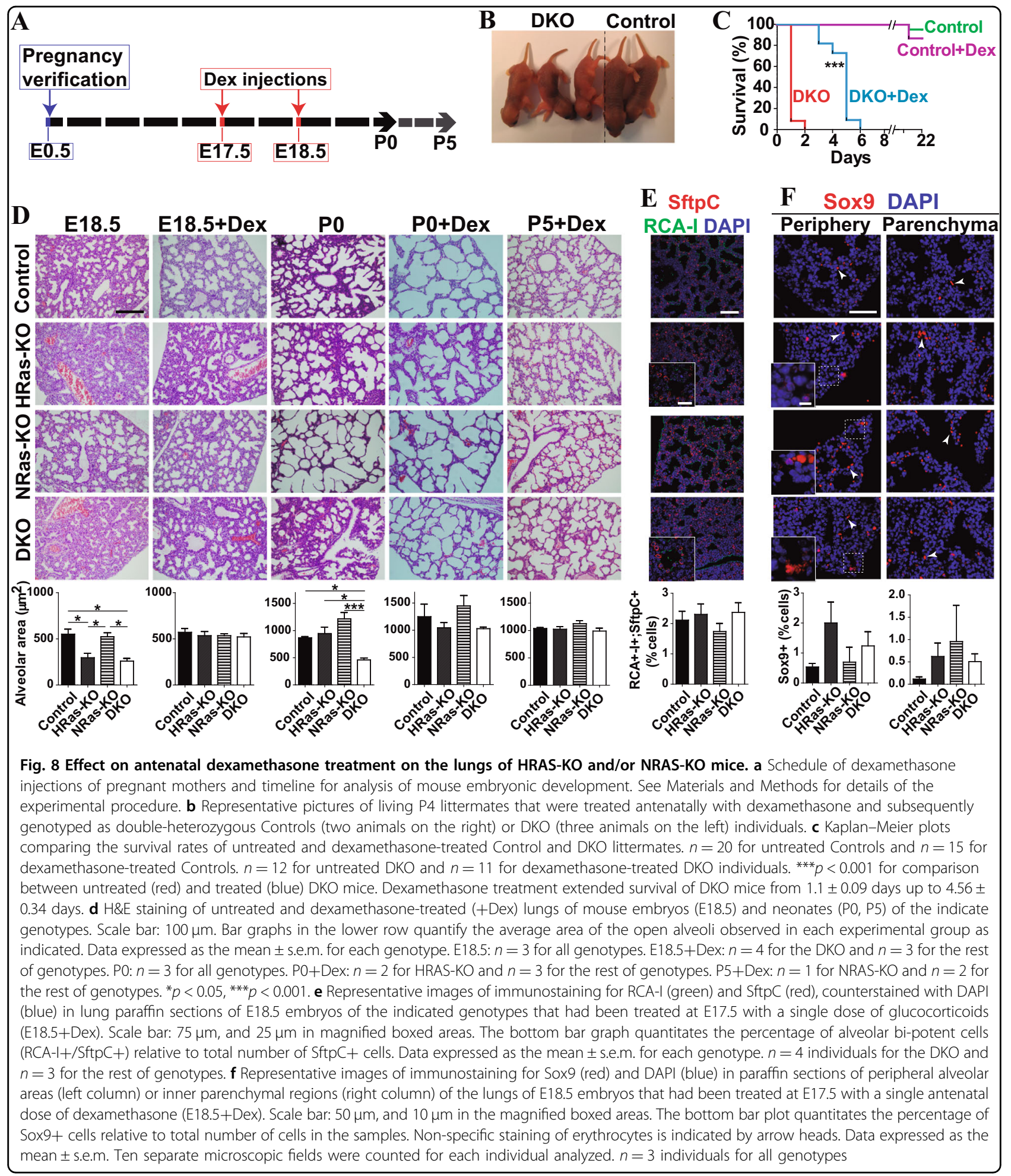

dexamethasone treatment reduced ceramide immunolabeling in the alveoli and bronchioles of P0 and P5 DKO mice to levels that were almost similar to those measured in the rest of genotypes (Fig. 7b, c). However, at P5 (nearing their death time), the lungs of dexamethasone-treated DKO mice showed substantially higher levels of bronchiolar cell apoptosis in comparison to all other genotypes (Fig. 7c). 


\section{Transcriptomic changes induced by antenatal dexamethasone treatment in the lungs of newborn PO DKO mice}

Figure $6 \mathrm{~b}$ shows a comparison between the transcriptional profiles of lungs from dexamethasone-treated (antenatal injections at E17.5 and E18.5) and untreated, newborn P0 DKO mice. Unsupervised hierarchical clustering of the normalized expression data profiles generated under high stringency produced a dendrogram that clearly discriminated the group of vertical branches corresponding to dexamethasone-treated newborn DKO lungs from the untreated DKO lung samples (Fig. 6b heatmap). More than $90 \%$ of differentially expressed genes in this heatmap corresponded to genes downregulated after treatment of the DKO animals with dexamethasone, whereas less than $10 \%$ were upregulated as a result of this treatment (Supplementary Table S3, Fig. 6b heatmap).

Functional annotation of the genes differentially expressed in dexamethasone-treated DKO samples identified a series of GO functional categories and KEGG signaling pathways (Fig. 6b GO\&KEGG; Supplementary Table S4) that, for the most part, mirrored, in exactly opposite direction (downregulation), the transcriptional behavior of similar functional categories that were previously found upregulated in the untreated DKO lungs (Fig. 6a GO\&KEGG). Notice for example the significant downregulation observed in functional categories such as "Protein Transport" and "Metabolic", "Phosphorylation" or "Apoptotic" cellular processes (Fig. 6b GO, Supplementary Table S4). It was also striking the significant downregulation of components of various signaling pathways that were otherwise upregulated in DKO lungs under basal conditions (Fig. 6a) and are known to be significant for lung functionality, such as "Oxidative phosphorylation", "N-glycan metabolism" and particularly, "Sphingolipid metabolism" (Fig. 6b KEGG; Supplementary Table S4). Indeed, the dexamethasone treatment of pregnant mothers caused in the lungs of the resulting DKO offspring a clear downregulation of several components of sphingolipid metabolic pathways (Supplementary Fig S3) that were previously found specifically upregulated in DKO lungs under basal conditions (Fig. 6a, Supplementary Table S2). Specifically, compared to untreated DKO lungs, the glucocorticoid treatment caused downregulation of loci such Alkaline ceramidase 2 (Acer2), Alkaline ceramidase 3 (Acer3), Delta(4)-desaturase, sphingolipid 1 (Degs1), Neuraminidase 3 (Neu3) and Sphingosine kinase 1 (Sphk1) (Fig. 6b, Supplementary Table S4).

\section{Discussion}

This report confirms and extend our initial observations on the viability of HRAS/NRAS-DKO mice kept on mixed genetic background ${ }^{12}$ by demonstrating that a majority of
DKO mice kept on pure C57Bl/6 background are unable to reach adulthood and die during their first postnatal days due to respiratory failure. Thus, despite the recognized functional dominance of KRAS regarding adult viability and lung tumorigenesis ${ }^{1,2,47,48}$, the HRAS and NRAS family members (undergoing markedly different intracellular processing than $\mathrm{KRAS}^{10}$ ) also exert critical functions regarding fetal lung development and survival of adult mice.

The hard-breathing, cyanotic newborn DKO mice showed unaltered lung morphology and internal branching but also exhibited significant defects of internal maturation/differentiation. We observed significant defects of alveolar development including markedly reduced saccular space and thicker septa, as well as abnormal accumulation of PAS + material in the alveolar cells. Consistent with defective/delayed differentiation of the alveolar (AT1, AT2) cell lineages, we also detected much-elevated levels of alveolar bi-potent progenitors ${ }^{29}$ and distal-tip progenitors ${ }^{36,37}$ in the lungs of DKO newborns as compared to Control littermates. In addition, the abnormally flattened morphology of Clara and ciliated cells, together with their altered PAS-staining patterns, suggest a secretory deficiency at the bronchiolar level which may also contribute to their respiratory stress of newborn DKO mice since correct mucin production is critical for normal lung function ${ }^{49}$. The small, but reproducible, increase of apoptotic rates in the lungs of DKO mice may have also contributed to the respiratory distress and postnatal death observed in these animals. Consistent with our observations in mice, human lung pathologies such as bronchopulmonary dysplasia (BPD) involve impaired alveolarization, dysregulated vascularization and high apoptosis in the alveolar epithelium ${ }^{40}$. It is unclear whether respiratory disorders seen in Costellosyndrome patients carrying HRAS germline mutations ${ }^{50}$ might be mechanistically related to phenotypes of the DKO mice. Anyhow, no major changes of RASdownstream-effector-activity were detected (not shown) in our DKO lung samples. Our detection of increased levels of proliferating BrdU+ cells in the lungs of DKO and HRAS-KO mice, and of apoptotic CC3+ cells in the lungs of DKO and NRAS-KO mice, is also consistent with our prior transcriptomic analyses of RAS-KO MEFs indicating a preferential link of HRAS with control of cellular proliferation and of NRAS with control of cell death $^{19,20,22}$. The elevated levels of infiltrating neutrophils detected in the lungs of newborn DKO mice may also contribute to their defective respiratory phenotype since neutrophil presence/activation is a well-established hallmark in acute-respiratory-distress-syndromes (ARDS) ${ }^{51}$.

Transcriptional analysis yielded additional mechanistic clues regarding the defective lung phenotypes of DKO mice. In DKO lungs under basal conditions, we uncovered the overexpression of several distinct groups of genes coding for 
components of various cellular processes required for correct lung function including "Transport", "Oxidative phosphorylation", "Carbohydrate/glycogen metabolism" or "Sphingolipid metabolism". The overexpression of components of signaling pathways regulating sphingolipid metabolism and ceramide production may be particularly relevant since our immunoassays also confirmed the significant accumulation of ceramides in the lungs of the DKO mice. In the context of mouse RAS genotypes studied here, it is also worth mentioning that sphingomyelin metabolism has been reported as a critical regulator of KRAS function and plasma membrane localization ${ }^{52,53}$. Given the recognized relation of ceramides with stress signals, tissue injury and apoptosis ${ }^{23,24,41,42,54}$, as well as with decreased surfactant production and various lung pathologies including ARDS (acute-respiratory-distress syndrome) and $\mathrm{BPD}^{23,24,41-45}$, we postulate that ceramide accumulation is a major factor for the respiratory stress and neonatal death of the DKO mice.

Treatment with glucocorticoids alleviates lung pathologies and improves pneumocytic differentiation in immature fetal lungs via upregulation of TTF-1, an essential transcription factor for correct lung morphogenesis and differentiation whose activity is also inhibited by ceramides ${ }^{44-46}$. Antenatal dexamethasone treatment significantly reverted the defects of differentiation of alveolar cell lineages and extended for 5-6 more days the lifespan of newborn DKO mice. Our transcriptional analyses showed also that this treatment reversed many transcriptional alterations observed in lung of untreated newborn DKOs, including several related to ceramide/ sphingosine metabolism. However, it was apparent that the antenatal glucocorticoid injections produced only partial rescue of the defective DKO lung phenotypes since the dexamethasone-treated DKO mice still died around P5/P6 due to likely respiratory failure while showing abnormally high apoptotic levels in their bronchiolar cells.

Our observations in HRAS/NRAS-DKO mice indicate that, despite the predominant role commonly attributed to KRAS regarding cell cycle progression, adult viability and lung tumorigenesis ${ }^{21,22,47,48,55,56}$, the HRAS and NRAS isoforms play crucial, specific functions during early lung maturation that are critical for neonatal survival and cannot be substituted by the action of the remaining KRAS isoform in this organ.

\section{materials and methods}

\section{Animal care, genotyping, and handling}

Laboratory mice were managed and handled according to EU and Spanish guidelines for the use and care of animals in research. All NRAS and HRAS knockout strains $^{12,57}$ to be used here were maintained on pure C57Bl/ 6 background and kept on a $12 \mathrm{~h}$ light/dark cycle. Single heterozygous $H R A S^{+/-}$or $N R A S^{+/-}$mice, as well

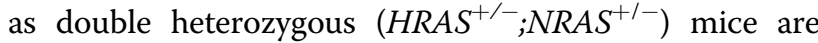
phenotypically indistinguishable from wild-type animals. Thus, we routinely set out parental crosses between mouse strains that were homozygous null-mutant for one of the HRAS or NRAS genes and heterozygous for the other one ( $\% / \mathrm{O}^{\prime} \mathrm{HRAS}^{+/-}$;NRAS ${ }^{-/-} \times \mathrm{O}^{\prime} / Q \mathrm{HRAS}^{-/-}$; $N R A S^{+/-}$) in order to more quickly and efficiently generate comparable sets of littermates of 4 relevant genotypes of interest for our studies $\left(\mathrm{HRAS}^{+/-} ; \mathrm{NRAS}^{+/-}\right.$, designated hereafter as Control; $\mathrm{HRAS}^{-/}$;NRAS ${ }^{+/-}$ designated as HRAS-KO; $\mathrm{HRAS}^{+/-}$;NRAS ${ }^{-/-}$designated NRAS-KO; and $H_{R A S^{-1}}$;NRAS ${ }^{-1-}$ designated as DKO).

Genotyping was done by PCR analysis of genomic DNA isolated from mouse tails using specific primers for the wild-type (WT) or the null-mutant alleles of HRAS or $N R A S$, as appropriate. Primers used were as follows. HRAS WT allele: (Forward 5'-AGCTCCCTGGCC CCTTGTGG- $3^{\prime}$ and reverse 5'-ACCTGCCAATGA GAAGCACACTTAGCC-3') generating a specific 434 bp fragment. HRAS null-mutant allele: (Forward 5'-AGCT CCCTGGCCCCTTGTGG-3' and reverse 5'-CTACCGGT GGATGTGGAATGTGTGCGA-3') generating a specific 336 bp fragment. NRAS WT allele: (Forward 5'-CCAG GATTCTTACCGAAAGCAAGTGGTG-3' and reverse $5^{\prime}$ GATGGCAAATACACAGAGGAACCCTTCG-3') generating a specific 185 bp fragment. NRAS null-mutant allele: (Forward 5'-CCAGGATTCTTACCGAAAGCAAG TGGTG- $3^{\prime}$ and reverse $5^{\prime}$-CATATGCGGTGTGAAA TACCGCACAGATGC-3') generating a specific 315 bp fragment.

For dexamethasone treatment of pregnant females, the beginning of gestation (E0.5) was timed via the detection of vaginal plugs, and pregnancy was later confirmed by weighting the females from day 10 post coitum (pc). Dexamethasone (SIGMA, D2915) or saline control $(\mathrm{NaCl}$ $0.9 \%)$ was injected subcutaneously $(0.4 \mathrm{mg} / \mathrm{kg})$ to pregnant females on days E17.5 and E18.5 of embryonic development, and survival of the newborn pups was monitored daily. Thus, embryos collected at E18.5 received only one dose of dexamethasone at E17.5 ${ }^{58,59}$.

\section{Histology and immunohistochemistry}

Mouse lung tissues were fixed in 4\% paraformaldehyde overnight at $4{ }^{\circ} \mathrm{C}$ for 3 days before dehydration and paraffin embedding. Three-micrometer sections were used for tissue staining with Hematoxylin-Eosin (H\&E) and five-micrometer sections for periodic acid-Schiff (PAS) according to standard procedures.

Immunohistochemical procedures were performed as previously described using deparaffinized and rehydrated, three-micrometer-thick sections ${ }^{60}$. Antigen retrieval was routinely performed to facilitate antibody binding to antigen using citrate buffer $0.01 \mathrm{M} \mathrm{pH} 6.0$ and heating in a microwave oven $(3 \times 3 \mathrm{~min}$ each, $250 \mathrm{~W})$. 
Detection of neutrophils in lung sections was carried out using an avidin-biotin-peroxidase procedure ${ }^{61}$. Sections were rinsed in PBS $(3 \times 10 \mathrm{~min})$ and endogenous peroxidase activity was blocked with $0.03 \%$ hydrogen peroxide for $15 \mathrm{~min}$. Sections were sequentially incubated in (1) primary antibody Neutrophil elastase (NE) (1:400, Abcam, ab68672) in PBS, 0.1\% Tween20, 2\% BSA and 2\% goat serum, overnight at $4{ }^{\circ} \mathrm{C}$; (2) 1:250 biotinylated goat anti rabbit IgG (Vector); and (3) 1:250 Vectastain Elite $\mathrm{ABC}$ reagent (Vector) in $\mathrm{PBS}$ for $1 \mathrm{~h}$ at room temperature. The sections were rinsed in PBS $(3 \times 10 \mathrm{~min})$ between each step. The reaction product was visualized by incubating the sections in $0.05 \% 3,3^{\prime}$-diaminobenzidine and 0.0033\% hydrogen peroxide in PBS until the desired staining intensity was reached.

For immunofluorescence, sections were washed with PBS and blocked in PBS containing 0.2\% Tween20 (Sigma Aldrich, 9005-64-5), 5\% Bovine serum albumin (BSA) (Sigma Aldrich, 9048-46-8) and 2\% goat serum (Sigma, G9023). Primary antibodies were diluted in PBS, $0.1 \%$ Tween $20,2 \%$ BSA and $2 \%$ goat serum, and incubated with the sections overnight at $4{ }^{\circ} \mathrm{C}$. Primary antibodies (dilution and origin) used in this study included: $\beta$-Tubulin ( $\beta$-Tub) (1:500, Sigma, T5293), Ceramide (1:100, Enzo, ALX-804-196), Cleaved-caspase-3 (CC3) (1:400, Cell Signaling, 9661), Ricinus communis agglutinin-I (RCA-I) (1:1000, Atom, FL-1081), Uteroglobin (Scgb) (1:1000, Abcam, ab40873), prosurfactant protein-C (SftpC) (1:500, Merck Millipore, AB3786), Sex-determining region Y-box 9 (Sox9) (1:500, Cell Signaling, 82630S). After 3 PBS washes, sections were incubated with secondary antibodies (from Jackson ImmunoResearch; diluted 1:500) including, as appropriate, goat anti-mouse Alexa 488 or Cy3, goat antirabbit Alexa 488 or $\mathrm{Cy} 3$, and counterstained with nuclear DAPI (Sigma) for $1 \mathrm{~h}$ at room temperature (RT), washed with PBS and mounted with ProLong Diamond antifading reagent (P36970, Life Technologies).

Images were acquired using a Leica TCS SP5 confocal microscope with the pinhole set to 1 Airy units and $40 \times$ $1.25 \mathrm{NA}$ or $63 \times 1.40 \mathrm{NA}$ immersion oil objectives. The proper laser lines, $405 \mathrm{~nm}, 488 \mathrm{~nm}$ and 561 were employed to excite Hoechst 33342, Alexa 488/FITC and Cy3, respectively. Images were acquired sequentially, starting first with Hoechst 33342 and following with the Cy3 and Alexa 488 staining. Images were imported to ImageJ software (NIH, Bethesda, MD, USA) using the LOCI Bioformats plug-in and Adobe Photoshop CS6 version 13.0 for minor adjustments of brightness and contrast.

\section{BrdU incorporation}

Cellular proliferation was measured by nuclear incorporation of BrdU in the lungs of E18.5 embryos. BrdU ( $0.1 \mathrm{mg} / \mathrm{g}$ body mass) (B5002, Sigma Aldrich) was injected intraperitoneally into pregnant female mice at E18.5 and $2 \mathrm{~h}$ later the animals were anesthetized with isoflurane prior to euthanasia by cervical dislocation and removal of the embryos. Each embryo was weighted and processed for paraffin sections. Three-micrometer sections were deparaffinized and rehydrated, submitted to antigen retrieval treatment as described above, treated with $2 \mathrm{~N} \mathrm{HCl}$ for $45 \mathrm{~min}$ at $37^{\circ} \mathrm{C}$, neutralized with borate buffer $(0.1 \mathrm{M} \mathrm{pH}$ 8.5 three times for $10 \mathrm{~min}$ each), washed with PBS and blocked with PBS, $0.2 \%$ Tween $20,5 \%$ BSA and $2 \%$ goat serum. After PBS washing, sections were immunostained overnight at $4{ }^{\circ} \mathrm{C}$ with a primary antibody for BrdU (1:2000, Accurate Chemical, OBT0030CX, diluted in PBS, 0.1\% Tween 20, 2\% BSA and 2\% goat serum). Sections were washed with PBS and incubated with the secondary antibody (1:500 diluted, goat anti-rat Alexa 488 from Jackson ImmunoResearch) and counterstained with nuclear marker DAPI (Sigma) for $1 \mathrm{~h}$ at RT. Preparations were then washed with PBS and mounted with ProLong Diamond anti-fading reagent (P36970, Life Technologies).

\section{Image analysis and quantifications}

For BrdU+, SftpC+, SfptC+/RCA-I+ and NE + cell density analyses, images from E18.5 or P0 lung sections of the four genotypes were obtained as described above. Equivalent lung sections from the four genotypes (Control, HRAS-KO, NRAS-KO and HRAS/NRAS-DKO) were selected for the analysis. BrdU-positive nuclei, SftpC + , $\mathrm{SftpC}+/ \mathrm{RCA}-\mathrm{I}+$ or $\mathrm{NE}+$ cells, and total nuclei, were counted using the command "Cell Counter" of the ImageJ software and were relativized to the total number of nuclei depending on the region. Cell density data are represented as percentage of $\mathrm{BrdU}+, \mathrm{SftpC}+$, SftpC+/RCA-I+or NE+cells.

For Ceramide and RCA-I quantification, digital images taken from equivalent alveolar or bronchiolar lung sections of control and $\mathrm{KO}$ animals were treated to balance the signal-to-noise ratio in such a way that the positive element (Ceramide or RCA-I) was clearly distinguishable from the background. The surface analyzed was then delimited using the original image as reference, and both the average fluorescent intensity and total number of nuclei were measured in the chosen area using the ImageJ software (NIH).

For PAS+ quantification, digital images were taken from equivalent lung sections of Control and $\mathrm{KO}$ animals. They were manually transformed into binary images in which only PAS positive staining elements appeared as black pixels. Then, the surface analyzed was delimited, using the original image as reference, and average PAS staining was measured as the black/white pixel ratio in the chosen area.

For alveolar area quantification, images taken from equivalent lung sections of Control and $\mathrm{KO}$ animals were manually transformed into binary images with the ImageJ software (NIH) where the alveolar spaces were recognized as positive element (Black). The alveolar surface analyzed 
was then delimited using the original image as reference, and the area $\left(\mu \mathrm{m}^{2}\right)$ of each alveolus was calculated using the Image J software $(\mathrm{NIH})$.

For measurements of Ciliate and Clara cell length, equivalent images of bronchiolar areas of Control and $\mathrm{KO}$ animals were obtained and the length of each cell type was manually measured using the ImageJ software (NIH).

\section{Microarray hybridizations}

Lungs were dissected from P0 neonate mice and RNA was extracted using Trizol following the manufacturer's instructions. After the extraction, the RNA was further purified using RNAse Mini Kit columns (QIAGEN, 74104). RNA quantification and quality was checked by RNA capillary electrophoresis columns (Agilent Technologies, RNA 6000 Nanochips).

Chip microarray hybridizations and data generated with Affymetrix GeneChip Mouse Gene 2.0 ST Array (26,515 genes) were used in this study. All microarray hybridization data were deposited and are available at the NCBI Gene Expression Omnibus (GEO) database (https://www.ncbi. nlm.nih.gov/geo/query/acc.cgi?acc=GSE130415). The RNAs were pre-amplified prior to microarray hybridization using the Gene Chip Expression 3'-Amplification TwoCycle cDNA Synthesis kit (Affymetrix, Santa Clara, CA, USA; \#900432), the Gene Chip Sample Cleanup Module (Affymetrix \#900371) and the MEGAscript T7 High Yield Transcription Kit (Ambion, Austin, TX, USA; \#1334), according to Affymetrix instruction manual \#701025 rev. 5. The pre-amplified RNAs were then submitted to the Gene Chip microarray hybridization protocol (Affymetrix Expression Analysis Technical Manual, (http://www. affymetrix.com/.\%20And\%20www.\%20affymetrix.com/

support/technical/manual/expression_manual.affx) as previously described ${ }^{19}$. Using Bioconductor ${ }^{62}$ and $R^{63}$ as computational tools, the robust microarray analysis (RMA) algorithm $^{64}$ was applied for background correction and normalization of fluorescent hybridization signals. The significance analysis of microarrays (SAM) algorithm ${ }^{65}$ was used to identify probe sets displaying significant differential expression when comparing the $\mathrm{KO}$ samples to their respective controls. This method uses permutations to provide robust statistical inference of the most significant genes and provides $\mathrm{P}$ values adjusted to multiple testing using false discovery rate (FDR) ${ }^{66}$. The GeneCodis (Gene Annotation Co-occurrence Discovery) software package (http://genecodis.cnb.csic.es/) was used for functional annotation analysis of differentially expressed gene sets in order to identify specific gene subsets sharing co-occurrent functional annotations linking them, with high statistical significance, to particular Gene Ontology (GO) Biological Process or Molecular Function categories and KEGG Signaling Pathways ${ }^{67}$.

\section{Statistical analysis}

Experiments were performed using at least three independent biological replicates in all cases, with actual experimental $n=$ values being specified in each figure legend. Animals were selected randomly. Data are expressed as mean \pm standard error of the mean (s.e.m.). Normal distribution of the data was tested using the IBM SPSS Statistics 23 package (SPSS, Chicago, IL, USA) and KolmogorovSmirnov test. One-way ANOVA followed by the Bonferroni post-test was used for the comparison of parametric values. Survival analysis was performed by the Kaplan-Meier method and between-group differences in survival were tested using the Log-rank (Mantel-Cox) test (GraphPad Prism 5.03 Software, Inc.). Differences between groups were considered statistically significant when $p<0.05$.

\section{Acknowledgements}

Work supported by grants from ISCIII-MCIU (FIS-PI16/02137; CIBERONC-CB16/ 12/003652), JCyL (SA043U16-UIC 076) and Foundations Ramón Areces (Madrid) and Solórzano (USAL). R.F.M. was supported by a predoctoral fellowship from Consejería de Educación, JCyL. CG was supported by a postdoctoral fellowship from AECC. Research co-financed by FEDER funds.

\section{Conflict of interest}

The authors declare that they have no conflict of interest.

\section{Publisher's note}

Springer Nature remains neutral with regard to jurisdictional claims in published maps and institutional affiliations.

Supplementary Information accompanies this paper at (https://doi.org/ 10.1038/s41419-019-2075-2).

Received: 15 July 2019 Revised: 7 October 2019 Accepted: 21 October 2019 Published online: 04 November 2019

\section{References}

1. Simanshu, D. K., Nissley, D. V. \& McCormick, F. RAS proteins and their regulators in human disease. Cell 170, 17-33 (2017).

2. Castellano, E. \& Santos, E. Functional specificity of ras isoforms: so similar but so different. Genes Cancer 2, 216-231 (2011).

3. Karnoub, A. E. \& Weinberg, R. A. Ras oncogenes: split personalities. Nat. Rev. Mol. Cell Biol. 9, 517-531 (2008).

4. Buday, L. \& Downward, J. Many faces of Ras activation. Biochim Biophys. Acta 1786, 178-187 (2008).

5. Fernandez-Medarde, A. \& Santos, E. Ras in cancer and developmental diseases. Genes Cancer 2, 344-358 (2011).

6. Stone, J. C. Regulation and function of the RasGRP family of Ras activators in blood cells. Genes Cancer 2, 320-334 (2011).

7. Hennig, A., Markwart, R., Esparza-Franco, M. A., Ladds, G. \& Rubio, I. Ras activation revisited: role of GEF and GAP systems. Biol. Chem. 396, 831-848 (2015).

8. Li, S., Balmain, A. \& Counter, C. M. A model for RAS mutation patterns in cancers: finding the sweet spot. Nat. Rev. Cancer 18, 767-777 (2018).

9. Omerovic, J., Hammond, D. E., Clague, M. J. \& Prior, I. A. Ras isoform abundance and signalling in human cancer cell lines. Oncogene 27, 2754-2762 (2008).

10. Johnson, C. W. et al. The small GTPases K-Ras, N-Ras, and H-Ras have distinct biochemical properties determined by allosteric effects. J. Biol. Chem. 292, 12981-12993 (2017).

11. Newlaczyl, A. U., Coulson, J. M. \& Prior, I. A. Quantification of spatiotemporal patterns of Ras isoform expression during development. Sci. Rep. 7, 41297 (2017). 
12. Esteban, L. M. et al. Targeted genomic disruption of $\mathrm{H}$-ras and $\mathrm{N}$-ras, individually or in combination, reveals the dispensability of both loci for mouse growth and development. Mol. Cell Biol. 21, 1444-1452 (2001).

13. Johnson, L. et al. K-ras is an essential gene in the mouse with partial functional overlap with N-ras. Genes Dev. 11, 2468-2481 (1997).

14. Nakamura, K. et al. Partial functional overlap of the three ras genes in mouse embryonic development. Oncogene 27, 2961-2968 (2008).

15. Potenza, N. et al. Replacement of K-Ras with H-Ras supports normal embryonic development despite inducing cardiovascular pathology in adult mice. EMBO Rep. 6, 432-437 (2005).

16. Iborra, S. et al. N-ras couples antigen receptor signaling to Eomesodermin and to functional CD8+ T cell memory but not to effector differentiation. J. Exp. Med 210, 1463-1479 (2013).

17. Iborra, S. et al. H-ras and $\mathrm{N}$-ras are dispensable for T-cell development and activation but critical for protective Th1 immunity. Blood 117, 5102-5111 (2011).

18. Chen, $X$. et al. Endogenous expression of Hras(G12V) induces developmental defects and neoplasms with copy number imbalances of the oncogene. Proc. Natl Acad. Sci. USA 106, 7979-7984 (2009).

19. Castellano, E., De Las Rivas, J., Guerrero, C. \& Santos, E. Transcriptional networks of knockout cell lines identify functional specificities of $\mathrm{H}$-Ras and $\mathrm{N}$-Ras: significant involvement of N-Ras in biotic and defense responses. Oncogene 26, 917-933 (2007).

20. Castellano, E., Guerrero, C., Nunez, A., De Las Rivas, J. \& Santos, E. Serumdependent transcriptional networks identify distinct functional roles for H-Ras and N-Ras during initial stages of the cell cycle. Genome Biol. 10, R123 (2009).

21. Drosten, M. et al. Genetic analysis of Ras signalling pathways in cell proliferation, migration and survival. EMBO J. 29, 1091-1104 (2010).

22. Azrak, S. S., Ginel-Picardo, A., Drosten, M., Barbacid, M. \& Santos, E. Reversible, interrelated mRNA and miRNA expression patterns in the transcriptome of Rasless fibroblasts: functional and mechanistic implications. BMC Genomics 14, 731 (2013).

23. van Mastrigt, E. et al. Ceramides in tracheal aspirates of preterm infants: Marker for bronchopulmonary dysplasia. PLOS ONE 13, e0185969 (2018).

24. Petrache, I. \& Berdyshev, E. V. Ceramide Signaling and metabolism in pathophysiological states of the lung. Annu Rev. Physiol. 78, 463-480 (2016).

25. Mohamed, B. A. et al. Respiratory distress and early neonatal lethality in Hspa4l/ Hspa4 double-mutant mice. Am. J. Respir. Cell Mol. Biol. 50, 817-824 (2014).

26. Leinwand, M. J. et al. Nitrofen inhibition of pulmonary growth and development occurs in the early embryonic mouse. J. Pediatr. Surg. 37, 1263-1268 (2002).

27. Suen, H. C., Catlin, E. A., Ryan, D. P., Wain, J. C. \& Donahoe, P. K. Biochemical immaturity of lungs in congenital diaphragmatic hernia. J. Pediatr. Surg. $\mathbf{2 8}$ 471-475 (1993). discussion 476-477.

28. Bhatt, A. J. et al. Disrupted pulmonary vasculature and decreased vascular endothelial growth factor, Flt-1, and TIE-2 in human infants dying with bronchopulmonary dysplasia. Am. J. Respir. Crit. Care Med 164, 1971-1980 (2001).

29. Desai, T. J., Brownfield, D. G. \& Krasnow, M. A. Alveolar progenitor and stem cells in lung development, renewal and cancer. Nature 507, 190-194 (2014).

30. Treutlein, B. et al. Reconstructing lineage hierarchies of the distal lung epithelium using single-cell RNA-seq. Nature 509, 371-375 (2014).

31. Ringvall, M. \& Kjellen, L. Mice deficient in heparan sulfate N-deacetylase/Nsulfotransferase 1. Prog. Mol. Biol. Transl. Sci. 93, 35-58 (2010).

32. Meyerholz, D. K., DeGraaff, J. A., Gallup, J. M., Olivier, A. K. \& Ackermann, M. R. Depletion of alveolar glycogen corresponds with immunohistochemical development of CD208 antigen expression in perinatal lamb lung. J. Histochem Cytochem 54, 1247-1253 (2006).

33. Young, S. L., Fram, E. K., Spain, C. L. \& Larson, E. W. Development of type ॥ pneumocytes in rat lung. Am. J. Physiol. 260, L113-L122 (1991).

34. Alanis, D. M., Chang, D. R., Akiyama, H., Krasnow, M. A. \& Chen, J. Two nested developmental waves demarcate a compartment boundary in the mouse lung. Nat. Commun. 5, 3923 (2014).

35. Rawlins, E. L., Clark, C. P., Xue, Y. \& Hogan, B. L. The $1 d 2+$ distal tip lung epithelium contains individual multipotent embryonic progenitor cells. Development 136, 3741-3745 (2009).

36. Perl, A. K., Kist, R., Shan, Z., Scherer, G. \& Whitsett, J. A. Normal lung development and function after Sox9 inactivation in the respiratory epithelium. Genesis 41, 23-32 (2005).

37. Chang, D. R. et al. Lung epithelial branching program antagonizes alveolar differentiation. Proc. Natl Acad. Sci. USA 110, 18042-18051 (2013).

38. Morrisey, E. E. \& Hogan, B. L. Preparing for the first breath: genetic and cellular mechanisms in lung development. Dev. Cell 18, 8-23 (2010).
39. Cardoso, W. V. \& Kotton, D. N. Specification and patterning of the respiratory system. StemBook, ed. The Stem Cell Research Community (2008). https://doi. org/10.3824/stembook.1.10.1.

40. Del Riccio, V., van Tuyl, M. \& Post, M. Apoptosis in lung development and neonatal lung injury. Pediatr. Res 55, 183-189 (2004).

41. Barnes, P. J. Ceramide lances the lungs. Nat. Med 10, 130-131 (2004).

42. Pettus, B. J., Chalfant, C. E. \& Hannun, Y. A. Ceramide in apoptosis: an overview and current perspectives. Biochim. Biophys. Acta 1585, 114-125 (2002).

43. Goggel, R. et al. PAF-mediated pulmonary edema: a new role for acid sphingomyelinase and ceramide. Nat. Med. 10, 155-160 (2004).

44. Boggaram, V. Thyroid transcription factor-1 (TTF-1/Nkx2.1/TITF1) gene regulation in the lung. Clin. Sci. (Lond.) 116, 27-35 (2009).

45. Sparkman, L., Chandru, H. \& Boggaram, V. Ceramide decreases surfactant protein B gene expression via downregulation of TTF-1 DNA binding activity. Am. J. Physiol. Lung Cell Mol. Physiol. 290, L351-L358 (2006).

46. Peca, D. et al. Altered surfactant homeostasis and recurrent respiratory failure secondary to TTF-1 nuclear targeting defect. Respir. Res 12, 115 (2011).

47. Drosten, M., Guerra, C. \& Barbacid, M. Genetically engineered mouse models of K-Ras-Driven lung and pancreatic tumors: validation of therapeutic targets. Cold Spring Harb. Perspect. Med. 8, a031542 (2018).

48. Gazdar, A. F., Hirsch, F. R. \& Minna, J. D. From mice to men and back: an assessment of preclinical model systems for the study of lung cancers. J. Thorac. Oncol. 11, 287-299 (2016).

49. Roy, M. G. et al. Mucin production during prenatal and postnatal murine lung development. Am. J. Respir. Cell Mol. Biol. 44, 755-760 (2011).

50. Gomez-Ospina, N. et al. Respiratory system involvement in Costello syndrome. Am. J. Med Genet A 170, 1849-1857 (2016).

51. Castillo, R. L., Carrasco Loza, R. \& Romero-Dapueto, C. Pathophysiological approaches of acute respiratory distress syndrome: novel bases for study of lung injury. Open Respir. Med J. 9, 83-91 (2015).

52. van der Hoeven, D. et al. Sphingomyelin Metabolism Is a Regulator of K-Ras Function. Mol Cell Biol 38, e00373-e00417 (2018).

53. Tan, L., Cho, K. J., Neupane, P., Capon, R. J. \& Hancock, J. F. An oxanthroquinone derivative that disrupts RAS plasma membrane localization inhibits cancer cell growth. J. Biol. Chem. 293, 13696-13706 (2018).

54. Petrache, I. et al. Ceramide upregulation causes pulmonary cell apoptosis and emphysema-like disease in mice. Nat. Med. 11, 491-498 (2005).

55. Hirsch, F. R. et al. Lung cancer: current therapies and new targeted treatments. Lancet 389, 299-311 (2017).

56. Gazdar, A. F. et al. The comparative pathology of genetically engineered mouse models for neuroendocrine carcinomas of the lung. J. Thorac. Oncol. 10, 553-564 (2015).

57. Umanoff, H., Edelmann, W., Pellicer, A. \& Kucherlapati, R. The murine N-ras gene is not essential for growth and development. Proc. Natl Acad. Sci. USA 92, 1709-1713 (1995)

58. Cuevas Guaman, M. et al. Administration of antenatal glucocorticoids and postnatal surfactant ameliorates respiratory distress syndrome-associated neonatal lethality in Erk3(-/-) mouse pups. Pediatr. Res. 76, 24-32 (2014).

59. Klinger, $\mathrm{S}$. et al. Loss of Erk3 function in mice leads to intrauterine growth restriction, pulmonary immaturity, and neonatal lethality. Proc. Natl Acad. Sci. USA 106, 16710-16715 (2009).

60. Jimeno, D. et al. RASGRF2 controls nuclear migration in postnatal retinal cone photoreceptors. J. Cell Sci. 129, 729-742 (2016).

61. Hsu, S. M., Raine, L. \& Fanger, H. Use of avidin-biotin-peroxidase complex (ABC) in immunoperoxidase techniques: a comparison between $A B C$ and unlabeled antibody (PAP) procedures. J. Histochem. Cytochem. 29, 577-580 (1981).

62. Gentleman, R. C. et al. Bioconductor: open software development for computational biology and bioinformatics. Genome Biol. 5, R80 (2004).

63. Dalgaard, P. R Development Core Team (2010): R: A language and environment for statistical computing v. 2010 (2010) Computer programme. http:// www.R-project.org.

64. Irizarry, R. A. et al. Exploration, normalization, and summaries of high density oligonucleotide array probe level data. Biostatistics 4, 249-264 (2003).

65. Tusher, V. G., Tibshirani, R. \& Chu, G. Significance analysis of microarrays applied to the ionizing radiation response. Proc. Natl Acad. Sci. USA 98, 5116-5121 (2001).

66. Benjamini, Y., Drai, D., Elmer, G., Kafkafi, N. \& Golani, I. Controlling the false discovery rate in behavior genetics research. Behav. Brain Res 125, 279-284 (2001).

67. Tabas-Madrid, D., Nogales-Cadenas, R. \& Pascual-Montano, A. GeneCodis3: a non-redundant and modular enrichment analysis tool for functional genomics. Nucleic Acids Res 40, W478-W483 (2012). 


\section{Concomitant deletion of HRAS and NRAS leads to pulmonary immaturity, respiratory failure and neonatal death in mice}

Rocío Fuentes-Mateos, David Jimeno, Carmela Gómez, Nuria Calzada, Alberto Fernández-Medarde and Eugenio Santos

\section{Supplementary Figure S1. Lungs of surviving, adult HRAS/NRAS-DKO mice show partial atelectasis.}

A. Representative images of H\&E staining of lung sections from one year-old mice of the indicated genotypes (Control and DKO). Scale bar: $500 \mu \mathrm{m}$ for the pictures in the first column and $50 \mu \mathrm{m}$ for the magnifications in the second and third columns. $n=4$ individuals for DKO and $n=3$ for Control.

B. Representative images of immunostaining for SftpC (red) and RCA-I (green), counterstained with DAPI, in alveolar areas of lung paraffin sections from adult mice of the indicated genotype. Scale bars: $75 \mu \mathrm{m}$, and $25 \mu \mathrm{m}$ on the higher magnification of boxed areas. $\mathrm{n}=4$ individuals for DKO and $\mathrm{n}=3$ for Control.

C. Representative images of immunostaining for Scgb (red) and $\beta$-Tubulin (green) counterstained with DAPI, in bronchiolar areas of lung paraffin sections from adult mice of the indicated genotypes. Scale bar: $25 \mu \mathrm{m} . \mathrm{n}=4$ individuals for DKO and $\mathrm{n}=3$ for Control.

Supplementary Figure S2. Increased neutrophil infiltration in the lungs of HRAS/NRAS-DKO mice.

Representative images of immunostaining for neutrophil elastase (NE), counterstained with hematoxilin (blue) in lung paraffin sections of untreated (P0) and dexamethasone-treated (P0+Dex) neonates. Arrows point to NE+ cells. Scale bar: $25 \mu \mathrm{m}$. The bottom bar graph quantitates percentage of $\mathrm{NE}+$ cells relative to total cells. Data expressed as the mean \pm s.e.m. for each genotype. $n=4$ individuals for Controls and NRAS-KO and $\mathrm{n}=3$ for HRAS-KO and DKO. ***p<0.001.

Supplementary Figure S3. Components of sphingolipid metabolic pathways that are differentially expressed in the lungs of HRAS/NRAS-DKO mice. 
The enzymatic components of sphingolipid metabolism pathways that showed differential expression in our transcriptomic analyses of P0 lungs extracts are marked in red (genes overexpressed in untreated DKO lungs) or blue (repressed in DKO upon dexamethasone treatment. Bi-colored genes showed opposite pattern of differential expression under these conditions (Untreated vs Dex-treated). Ceramide is indicated in green. Enzymatic reactions driving either de novo synthesis (yellow arrow) or sphingolipid transformation events (green arrow) lead to ceramide accumulation in the DKO lungs, a trend that was partially corrected after antenatal dexamethasone treatment. SGPL1- Sphingosine-1Phosphate Lyase 1, SGPP1- Sphinganine phosphate phosphatase 1, SPHK- Sphinganine kinase, SPTLC1- Serine Palmitoyltransferase Long Chain Base Subunit 1, KDSR- 3-Ketodihydrosphingosine Reductase, CERS- Ceramide Synthase, ACER1,2,3- Alkaline Ceramidase 1 to 3, DEGS- Delta 4Desaturase, Sphingolipid 1, SCPT- Sphingosine Choline Phosphotransferase, SGMS1-2Sphingomyelin Synthase 1 and 2, SMPD1-5- Sphingomyelin Phosphodiesterase 1 to 5, CERKCeramide kinase, UGT8A- UDP Glycosyltransferase 8a, GALC- Galactosylceramidase, NEU3Neuraminidase 3.

\section{Supplementary Video 1. Respiratory distress of HRAS-KO and NRAS-KO animals.}

Breathing difficulties and cyanotic appearance of a newborn DKO mouse next to two normalbreathing NRAS-KO littermates. (.mov).

\section{SUPPLEMENTARY TABLES.}

Supplementary Table S1. Differential gene expression in the lungs of Controls, HRAS-KO, NRASKO and HRAS/NRAS-DKO mice.

Supplementary Table S2. Functional annotation of differentially expressed genes (overexpressed and downregulated) in the lungs of HRAS/NRAS-DKO mice.

Supplementary Table S3. Differential gene expression in the lungs of HRAS/NRAS-DKO mice treated antenatally with dexamethasone.

Supplementary Table S4. Functional annotation of differentially expressed genes (downregulated and overexpressed) in the lungs of HRAS/NRAS-DKO mice treated antenatally with dexamethasone. 
Table S1. Differential gene expression in the lungs of Control, HRAS-KO, NRAS-KO and HRAS/NRAS-DKO mice.

List of 265 differentially expressed gene probesets ( $F D R=0.10)$ identified by means of SAM contrasts in multiclass comparisons (Fig. 6A heatmap) between the transcriptional profiles of lungs isolated from newborn (PO) mouse littermates of the four relevant genotypes (Control, single HRAS-KO, NRAS-KO and DKO) that were generated by RNA microarray hybridization assays using GeneChip(R) Mouse Gene 2.0 ST Arrays. The differentially expressed loci are identified by Affymetrix Probeset ID, Genename symbol or Description and listed according to their degree of overexpression or repression in the lung tissue analyzed. $d$-value is a parameter measuring the statistical distance separating the calculated expression value of each gene probeset from the null hypothesis (no-change). $q$-value is the estimated FDR at the largest $p$-value for which the probe set would be statistically significant. $R$-fold is a measure of the fold change of a probeset in the collection of microarrays provided by the SAM algorithm. Entries in red denote overexpression. Entries in green indicate transcriptional repression. The data list is organized here from maximal to minimal R-fold values.

\begin{tabular}{|c|c|c|c|c|c|c|}
\hline $\begin{array}{l}\text { probese } \\
\text { t ID }\end{array}$ & d.value & p.value & q.value & R.fold & Genename & Description \\
\hline 17379873 & 5,03201259 & 0,001545359 & 0,097524576 & 3,516549163 & $\begin{array}{l}\text { 1500012F01Ri } \\
\mathrm{k}\end{array}$ & RIKEN cDNA $1500012 F 01$ gene \\
\hline 17250744 & 4,962865131 & 0,001655063 & 0,098013534 & 2,744922707 & Snord65 & small nucleolar RNA, C/D box 65 \\
\hline 17257591 & 6,556047075 & 0,000371871 & 0,073866639 & 2,599529728 & Snord104 & small nucleolar RNA, C/D box 104 \\
\hline 17215370 & 4,778245344 & 0,001986766 & 0,100695956 & 2,393749726 & Atg16l1 & autophagy related 16 -like 1 (S. cerevisiae) \\
\hline 17231844 & 4,919809738 & 0,001731942 & 0,099252055 & 2,280357661 & Perp & PERP, TP53 apoptosis effector \\
\hline 17222564 & 8,037727227 & 0,000124821 & 0,054095556 & 2,239693089 & Snord89 & small nucleolar RNA, C/D box 89 \\
\hline 17483385 & 11,74553218 & $2,2891 \mathrm{E}-05$ & 0,027251476 & 2,158247887 & Phkg2 & phosphorylase kinase, gamma 2 (testis) \\
\hline 17487796 & 11,30283159 & $2,67782 \mathrm{E}-05$ & 0,027251476 & 2,123408157 & Rabac1 & Rab acceptor 1 (prenylated) \\
\hline 17527977 & 7,635218467 & 0,000161101 & 0,058717847 & 2,0855348 & Glce & glucuronyl C5-epimerase \\
\hline 17467359 & 6,75995963 & 0,000310108 & 0,070811709 & 2,01808281 & Tacstd2 & tumor-associated calcium signal transducer 2 \\
\hline 17480880 & 5,563628316 & 0,000907002 & 0,084873601 & 2,015857269 & Pde2a & phosphodiesterase $2 \mathrm{~A}$, cGMP-stimulated \\
\hline 17269911 & 5,280733333 & 0,001205449 & 0,094186645 & 1,89151228 & Vat1 & vesicle amine transport protein 1 homolog ( $T$ californica) \\
\hline 17384619 & 4,828541946 & 0,001886132 & 0,100511427 & 1,882488174 & Snord90 & small nucleolar RNA, C/D box 90 \\
\hline 17359008 & 5,506129216 & 0,000958831 & 0,086244922 & 1,849381554 & March5 & membrane-associated ring finger $(\mathrm{C} 3 \mathrm{HC} 4) 5$ \\
\hline 17280552 & 6,068434304 & 0,000585664 & 0,080976625 & 1,849277813 & Bcap29 & B cell receptor associated protein 29 \\
\hline 17497769 & 6,2041935 & 0,00052131 & 0,080717083 & 1,848026495 & Rnh1 & ribonuclease/angiogenin inhibitor 1 \\
\hline 17442588 & 4,864905186 & 0,0018153 & 0,10045378 & 1,842105439 & Atp6v0a2 & ATPase, $\mathrm{H}+$ transporting, lysosomal V0 subunit A2 \\
\hline 17214825 & 6,646675023 & 0,000341637 & 0,070811709 & 1,810437366 & Mff & mitochondrial fission factor \\
\hline 17435816 & 5,771473144 & 0,000751516 & 0,081210084 & 1,79977293 & Slc35f6 & solute carrier family 35, member F6 \\
\hline 17364932 & 4,83150626 & 0,001877926 & 0,100511427 & 1,791864034 & Got1 & glutamic-oxaloacetic transaminase 1 , soluble \\
\hline 17286962 & 4,933873263 & 0,0017043 & 0,099007076 & 1,790972248 & Mylip & myosin regulatory light chain interacting protein \\
\hline 17224587 & 6,983135268 & 0,000263031 & 0,070811709 & 1,78686799 & Dnpep & aspartyl aminopeptidase \\
\hline 17305856 & 5,937134599 & 0,000654337 & 0,080976625 & 1,731804056 & Exoc5 & exocyst complex component 5 \\
\hline 17222332 & 8,21300871 & 0,000111 & 0,05291631 & 1,7286873 & Mgat4a & mannoside acetylglucosaminyltransferase 4 , isoenzyme $A$ \\
\hline 17240357 & 4,830965134 & 0,001879222 & 0,100511427 & 1,727541565 & Gtf3c6 & general transcription factor IIIC, polypeptide 6 , alpha \\
\hline 17241032 & 5,077689276 & 0,001470639 & 0,096536895 & 1,70044515 & Ddit4 & DNA-damage-inducible transcript 4 \\
\hline 17247176 & 7,708465148 & 0,000155486 & 0,058518959 & 1,68375335 & Ramp3 & receptor (calcitonin) activity modifying protein 3 \\
\hline 17334948 & 6,805317546 & 0,000301038 & 0,070811709 & 1,673843547 & Atp6v0e & ATPase, $\mathrm{H}+$ transporting, lysosomal V0 subunit $\mathrm{E}$ \\
\hline 17497421 & 4,84072141 & 0,001862809 & 0,100511427 & 1,672648335 & Bnip3 & $\mathrm{BCL} 2 /$ adenovirus $\mathrm{E} 1 \mathrm{~B}$ interacting protein 3 \\
\hline 17365960 & 6,001079009 & 0,000618057 & 0,080976625 & 1,670981714 & Gfra1 & glial cell line derived neurotrophic factor family receptor alpha 1 \\
\hline 17478864 & 6,650632643 & 0,000339046 & 0,070811709 & 1,652730338 & Mtmr10 & myotubularin related protein 10 \\
\hline 17501260 & 5,06138785 & 0,001494826 & 0,096786837 & 1,649433545 & Fbxo8 & F-box protein 8 \\
\hline 17376274 & 6,131592116 & 0,00055068 & 0,080776169 & 1,647494514 & Nop56 & NOP56 ribonucleoprotein \\
\hline 17493556 & 4,963863341 & 0,001652039 & 0,098013534 & 1,646643515 & Acer3 & alkaline ceramidase 3 \\
\hline 17353241 & 5,61757118 & 0,000865971 & 0,084494765 & 1,642984978 & Slc25a46 & solute carrier family 25, member 46 \\
\hline 17318587 & 5,039766411 & 0,001530242 & 0,097524576 & 1,64248183 & Slc39a4 & solute carrier family 39 (zinc transporter), member 4 \\
\hline 17430609 & 6,362010068 & 0,000442703 & 0,07674456 & 1,629496894 & Serinc2 & serine incorporator 2 \\
\hline 17458734 & 5,495270584 & 0,000970492 & 0,087015147 & 1,620642067 & Plekha8 & $\begin{array}{l}\text { pleckstrin homology domain containing, family A (phosphoinositide } \\
\text { binding specific) member } 8\end{array}$ \\
\hline 17448960 & 6,019633868 & 0,000607691 & 0,080976625 & 1,613181097 & Clock & circadian locomotor output cycles kaput \\
\hline 17502954 & 6,704605592 & 0,000323929 & 0,070811709 & 1,61077619 & Dnajb1 & DnaJ (Hsp40) homolog, subfamily B, member 1 \\
\hline 17312905 & 7,282825613 & 0,000201268 & 0,062295714 & 1,601363183 & Eif3l & eukaryotic translation initiation factor 3 , subunit $\mathrm{L}$ \\
\hline 17278110 & 7,941885432 & 0,000134323 & 0,055000696 & 1,591745889 & Ubr7 & ubiquitin protein ligase E3 component $n$-recognin 7 (putative) \\
\hline 17289824 & 7,396792412 & 0,000187879 & 0,06106808 & 1,591270284 & Mier3 & mesoderm induction early response 1 , family member 3 \\
\hline 17333854 & 6,148157149 & 0,000543769 & 0,080717083 & 1,580789994 & Ppp2r1a & protein phosphatase 2 , regulatory subunit $A$, alpha \\
\hline 17231690 & 7,901798417 & 0,000135618 & 0,055000696 & 1,572277731 & Sf3b5 & splicing factor $3 b$, subunit 5 \\
\hline 17372119 & 6,385378996 & 0,000431474 & 0,07674456 & 1,570025464 & Mtx2 & metaxin 2 \\
\hline 17506137 & 5,27303679 & 0,001212359 & 0,094232839 & 1,559865339 & Wfdc1 & WAP four-disulfide core domain 1 \\
\hline 17413649 & 7,526646028 & 0,000171898 & 0,058717847 & 1,553338702 & Dcaf10 & DDB1 and CUL4 associated factor 10 \\
\hline 17302834 & 4,984539309 & 0,001616191 & 0,097826161 & 1,548654612 & Ubac2 & ubiquitin associated domain containing 2 \\
\hline 17252170 & 4,779530604 & 0,001982879 & 0,100695956 & 1,548560195 & Rnf167 & ring finger protein 167 \\
\hline 17435963 & 5,215850772 & 0,001279305 & 0,095993501 & 1,54745357 & Atraid & all-trans retinoic acid induced differentiation factor \\
\hline 17518585 & 5,577867724 & 0,000896636 & 0,084873601 & 1,546362321 & Spg21 & spastic paraplegia 21 homolog (human) \\
\hline 17402595 & 5,821946496 & 0,000718691 & 0,080976625 & 1,54547311 & Casp6 & caspase 6 \\
\hline 17336190 & 5,864503061 & 0,000696664 & 0,080976625 & 1,544772387 & Ndufa7 & NADH dehydrogenase (ubiquinone) 1 alpha subcomplex, 7 (B14.5a) \\
\hline 17395165 & 5,162998009 & 0,001360935 & 0,096476139 & 1,532801862 & Atp5e & $\begin{array}{l}\text { ATP synthase, } \mathrm{H}+\text { transporting, mitochondrial F1 complex, epsilon } \\
\text { subunit }\end{array}$ \\
\hline 17267430 & 7,853455205 & 0,000140369 & 0,055000696 & 1,521456865 & Gdpd1 & glycerophosphodiester phosphodiesterase domain containing 1 \\
\hline 17354282 & 6,936819632 & 0,000272533 & 0,070811709 & 1,518011338 & Cdo1 & cysteine dioxygenase 1 , cytosolic \\
\hline
\end{tabular}




\begin{tabular}{|c|c|c|c|c|c|c|}
\hline $\begin{array}{l}\text { probese } \\
\text { t ID }\end{array}$ & d.value & p.value & q.value & R.fold & Genename & Description \\
\hline 17233799 & 5,019197211 & 0,001562203 & 0,097524576 & 1,502612955 & Slc25a16 & $\begin{array}{l}\text { solute carrier family } 25 \text { (mitochondrial carrier, Graves disease } \\
\text { autoantigen), member } 16\end{array}$ \\
\hline 17321630 & 5,32109075 & 0,001157939 & 0,093087581 & 1,501758444 & Cers5 & ceramide synthase 5 \\
\hline 17440086 & 9,696637687 & $5,09649 \mathrm{E}-05$ & 0,031690702 & 1,50145908 & Rpap2 & RNA polymerase II associated protein 2 \\
\hline 17365384 & 7,587789429 & 0,000167148 & 0,058717847 & 1,499137335 & Actr1a & ARP1 actin-related protein $1 \mathrm{~A}$, centractin alpha \\
\hline 17239077 & 5,351524074 & 0,001130297 & 0,092109471 & 1,496818803 & Ginm1 & glycoprotein integral membrane 1 \\
\hline 17249700 & 5,525046255 & 0,000944146 & 0,085732855 & 1,495218821 & Oser1 & oxidative stress responsive serine rich 1 \\
\hline 17420996 & 7,895125885 & 0,000137778 & 0,055000696 & 1,488515612 & Rsg1 & REM2 and RAB-like small GTPase 1 \\
\hline 17447868 & 5,432223498 & 0,001034846 & 0,089000756 & 1,488208034 & Tapt1 & transmembrane anterior posterior transformation 1 \\
\hline 17394036 & 5,194815608 & 0,001305651 & 0,096069042 & 1,484415414 & Fitm2 & fat storage-inducing transmembrane protein 2 \\
\hline 17408135 & 4,791260711 & 0,001959556 & 0,100695956 & 1,483132038 & Gpr89 & G protein-coupled receptor 89 \\
\hline 17215968 & 6,795409484 & 0,00030363 & 0,070811709 & 1,481727438 & Ppp1r7 & protein phosphatase 1, regulatory (inhibitor) subunit 7 \\
\hline 17289037 & 6,395152226 & 0,000425427 & 0,07674456 & 1,47960716 & Ssbp2 & single-stranded DNA binding protein 2 \\
\hline 17222440 & 5,313464363 & 0,001167441 & 0,093276169 & 1,476617017 & Rev1 & REV1 homolog (S. cerevisiae) \\
\hline 17283923 & 6,600197218 & 0,00035805 & 0,072634525 & 1,472674146 & Slc25a29 & $\begin{array}{l}\text { solute carrier family } 25 \text { (mitochondrial carrier, palmitoylcarnitine } \\
\text { transporter), member } 29\end{array}$ \\
\hline 17482719 & 5,577253933 & 0,0008975 & 0,084873601 & 1,472282515 & Ubfd1 & ubiquitin family domain containing 1 \\
\hline 17216070 & 5,186843841 & 0,001317313 & 0,096121702 & 1,47221896 & Atg $4 b$ & autophagy related $4 \mathrm{~B}$, cysteine peptidase \\
\hline 17283445 & 5,921825897 & 0,000664271 & 0,080976625 & 1,468357734 & Lgmn & legumain \\
\hline 17350901 & 5,619933533 & 0,000862948 & 0,084494765 & 1,444393469 & $\begin{array}{l}\text { A730017C20Ri } \\
k\end{array}$ & RIKEN cDNA A730017C20 gene \\
\hline 17348957 & 9,093759575 & $6,69454 \mathrm{E}-05$ & 0,03682443 & 1,436272436 & Zfp35 & zinc finger protein 35 \\
\hline 17274184 & 10,55197265 & $3,45525 \mathrm{E}-05$ & 0,027453339 & 1,435293551 & Socs2 & suppressor of cytokine signaling 2 \\
\hline 17315669 & 6,245747322 & 0,000494964 & 0,080648038 & 1,435171048 & AW549877 & expressed sequence AW549877 \\
\hline 17350301 & 5,300771057 & 0,001182126 & 0,093815338 & 1,434075816 & Ythdc2 & YTH domain containing 2 \\
\hline 17317203 & 8,038419602 & 0,000124389 & 0,054095556 & 1,432409572 & Derl1 & Der1-like domain family, member 1 \\
\hline 17503596 & 4,965590085 & 0,001646425 & 0,097907395 & 1,430954978 & Papd5 & PAP associated domain containing 5 \\
\hline 17396315 & 5,76550591 & 0,000756699 & 0,081369219 & 1,428922762 & Tbl1xr1 & transducin (beta)-like $1 \mathrm{X}$-linked receptor 1 \\
\hline 17453617 & 6,302930844 & 0,000469482 & 0,078218073 & 1,427572676 & Tmem120a & transmembrane protein $120 \mathrm{~A}$ \\
\hline 17538356 & 4,901737045 & 0,001759584 & 0,09985745 & 1,426280856 & Alg13 & asparagine-linked glycosylation 13 \\
\hline 17253972 & 6,087929082 & 0,000574867 & 0,080976625 & 1,422131135 & Psmd11 & proteasome (prosome, macropain) 26S subunit, non-ATPase, 11 \\
\hline 17411732 & 6,860398405 & 0,0002924 & 0,070811709 & 1,416251912 & $\begin{array}{l}\text { 2610301B20Ri } \\
\mathrm{k}\end{array}$ & RIKEN cDNA 2610301B20 gene \\
\hline 17401768 & 6,304657478 & 0,000468618 & 0,078218073 & 1,415017373 & Clcc1 & chloride channel CLIC-like 1 \\
\hline 17407631 & 5,225371945 & 0,001270235 & 0,095993501 & 1,414158221 & Psmb4 & proteasome (prosome, macropain) subunit, beta type 4 \\
\hline 17339549 & 5,050749029 & 0,001511238 & 0,096786837 & 1,412732315 & Ypel5 & yippee-like 5 (Drosophila) \\
\hline 17341412 & 5,013261207 & 0,001574296 & 0,097524576 & 1,412591472 & Zfp944 & zinc finger protein 944 \\
\hline 17407886 & 5,602844028 & 0,000878928 & 0,084494765 & 1,411260989 & Rprd2 & regulation of nuclear pre-mRNA domain containing 2 \\
\hline 17510836 & 5,097737665 & 0,001445589 & 0,096476139 & 1,410134977 & Usp38 & ubiquitin specific peptidase 38 \\
\hline 17222106 & 5,617646147 & 0,000865539 & 0,084494765 & 1,40863918 & Uggt1 & UDP-glucose glycoprotein glucosyltransferase 1 \\
\hline 17304012 & 5,213521253 & 0,00128276 & 0,095993501 & 1,407416302 & Kcnma1 & $\begin{array}{l}\text { potassium large conductance calcium-activated channel, subfamily } \\
\mathrm{M} \text {, alpha member } 1\end{array}$ \\
\hline 17309065 & 5,727172289 & 0,000785205 & 0,082571972 & 1,396818292 & Mzt1 & mitotic spindle organizing protein 1 \\
\hline 17357700 & 5,090808131 & 0,001454227 & 0,096510269 & 1,391853147 & Ms4a4d & membrane-spanning 4-domains, subfamily $A$, member 4D \\
\hline 17501652 & 6,251705812 & 0,000490213 & 0,080585015 & 1,389690189 & Atp6v1b2 & ATPase, $\mathrm{H}+$ transporting, lysosomal V1 subunit B2 \\
\hline 17527421 & 6,383866807 & 0,000432338 & 0,07674456 & 1,388934252 & Tspan3 & tetraspanin 3 \\
\hline 17281354 & 4,874727219 & 0,001805366 & 0,10045378 & 1,385382471 & $\operatorname{Sec} 23 a$ & SEC23A (S. cerevisiae) \\
\hline 17539797 & 4,844711598 & 0,001855899 & 0,100511427 & 1,382420703 & Clcn5 & chloride channel 5 \\
\hline 17456381 & 5,680159009 & 0,000816734 & 0,083980931 & 1,379463376 & Ptprz1 & protein tyrosine phosphatase, receptor type $Z$, polypeptide 1 \\
\hline 17474136 & 4,77276715 & 0,001995836 & 0,100695956 & 1,372224394 & Ap2s1 & adaptor-related protein complex 2 , sigma 1 subunit \\
\hline 17303765 & 6,412026439 & 0,000420676 & 0,076642027 & 1,371971557 & Mrps16 & mitochondrial ribosomal protein S16 \\
\hline 17450098 & 5,126089454 & 0,001406285 & 0,096476139 & 1,370257017 & $\operatorname{Lin} 54$ & lin-54 homolog (C. elegans) \\
\hline 17211498 & 5,973677627 & 0,000635765 & 0,080976625 & 1,369483826 & Lmbrd1 & LMBR1 domain containing 1 \\
\hline 17526929 & 5,514431591 & 0,00095192 & 0,085893442 & 1,366224111 & $\begin{array}{l}1110032 \mathrm{A03Ri} \\
\mathrm{k}\end{array}$ & RIKEN cDNA 1110032A03 gene \\
\hline 17230153 & 6,165339661 & 0,000535995 & 0,080717083 & 1,363376887 & Fh1 & fumarate hydratase 1 \\
\hline 17277307 & 7,325651301 & 0,000196085 & 0,062295714 & 1,356044836 & Fcf1 & $\begin{array}{l}\text { FCF1 small subunit (SSU) processome component homolog (S. } \\
\text { cerevisiae) }\end{array}$ \\
\hline 17327069 & 6,186021727 & 0,000527789 & 0,080717083 & 1,355891044 & Ifnar1 & interferon (alpha and beta) receptor 1 \\
\hline 17254537 & 5,238643359 & 0,001247344 & 0,095652437 & 1,354748371 & Ppm1d & protein phosphatase 1D magnesium-dependent, delta isoform \\
\hline 17275718 & 6,052613541 & 0,000593438 & 0,080976625 & 1,347394723 & Mia2 & melanoma inhibitory activity 2 \\
\hline 17275785 & 5,855418479 & 0,000700551 & 0,080976625 & 1,347178164 & Fam179b & family with sequence similarity 179 , member B \\
\hline 17356182 & 5,775078829 & 0,000749356 & 0,081210084 & 1,346406898 & Ppp1ca & protein phosphatase 1 , catalytic subunit, alpha isoform \\
\hline 17391598 & 8,43427456 & $9,80426 \mathrm{E}-05$ & 0,048351009 & 1,344438136 & Snrpb & small nuclear ribonucleoprotein $\mathrm{B}$ \\
\hline 17436315 & 5,302860093 & 0,001180398 & 0,093815338 & 1,341241645 & Ppp1cb & protein phosphatase 1 , catalytic subunit, beta isoform \\
\hline 17491454 & 9,999205914 & $4,31906 \mathrm{E}-05$ & 0,031165779 & 1,33472512 & Nipa1 & $\begin{array}{l}\text { non imprinted in Prader-Willi/Angelman syndrome } 1 \text { homolog } \\
\text { (human) }\end{array}$ \\
\hline 17514247 & 6,661548942 & 0,000336023 & 0,070811709 & 1,329776715 & Tomm20 & translocase of outer mitochondrial membrane 20 homolog (yeast) \\
\hline 17403706 & 4,845278794 & 0,001854603 & 0,100511427 & 1,327749357 & Pigk & phosphatidylinositol glycan anchor biosynthesis, class $\mathrm{K}$ \\
\hline 17381184 & 4,798271406 & 0,001947463 & 0,100695956 & 1,325643318 & Polr3k & polymerase (RNA) III (DNA directed) polypeptide K \\
\hline 17411174 & 5,996663554 & 0,000618921 & 0,080976625 & 1,324076434 & Gipc2 & GIPC PDZ domain containing family, member 2 \\
\hline 17357425 & 9,753677147 & $4,79415 \mathrm{E}-05$ & 0,031165779 & 1,323221633 & Rab3il1 & RAB3A interacting protein (rabin3)-like 1 \\
\hline 17266581 & 4,857703157 & 0,001829121 & 0,100511427 & 1,32102589 & AU040972 & expressed sequence AU040972 \\
\hline 17419222 & 5,196554583 & 0,001301764 & 0,096069042 & 1,320756051 & Snrnp40 & small nuclear ribonucleoprotein 40 (U5) \\
\hline 17469775 & 6,364796035 & 0,000441408 & 0,07674456 & 1,31207617 & Emc3 & ER membrane protein complex subunit 3 \\
\hline 17462905 & 6,715465438 & 0,000322202 & 0,070811709 & 1,310459171 & Lpcat3 & lysophosphatidylcholine acyltransferase 3 \\
\hline 17281971 & 6,088035985 & 0,000574435 & 0,080976625 & 1,310311018 & Sgpp1 & sphingosine-1-phosphate phosphatase 1 \\
\hline 17424298 & 7,621291807 & 0,000162828 & 0,058717847 & 1,309983872 & Dctn3 & dynactin 3 \\
\hline 17523531 & 5,619674613 & 0,00086338 & 0,084494765 & 1,307198157 & Tmem42 & transmembrane protein 42 \\
\hline 17538109 & 5,403415416 & 0,001072422 & 0,090018052 & 1,306728387 & Tbc1d8b & TBC1 domain family, member 8B \\
\hline 17448863 & 5,064861306 & 0,001488779 & 0,096786837 & 1,303627173 & Scfd2 & Sec1 family domain containing 2 \\
\hline
\end{tabular}




\begin{tabular}{|c|c|c|c|c|c|c|}
\hline $\begin{array}{l}\text { probese } \\
\text { t ID }\end{array}$ & d.value & p.value & q.value & R.fold & Genename & Description \\
\hline 17306417 & 5,989552484 & 0,000626263 & 0,080976625 & 1,303250769 & Psmb5 & proteasome (prosome, macropain) subunit, beta type 5 \\
\hline 17230595 & 8,127783839 & 0,000118774 & 0,053926201 & 1,302775315 & Degs1 & degenerative spermatocyte homolog 1 (Drosophila) \\
\hline 17363779 & 5,233653758 & 0,001256846 & 0,095867058 & 1,302734136 & Ak3 & adenylate kinase 3 \\
\hline 17358617 & 4,954762629 & 0,001670611 & 0,098323624 & 1,302392046 & Uhrf2 & ubiquitin-like, containing PHD and RING finger domains 2 \\
\hline 17263877 & 4,786433494 & 0,00197165 & 0,100695956 & 1,301542327 & Prpsap2 & phosphoribosyl pyrophosphate synthetase-associated protein 2 \\
\hline 17464901 & 5,000026846 & 0,001593732 & 0,097826161 & 1,299857411 & Tmem168 & transmembrane protein 168 \\
\hline 17331828 & 7,525310298 & 0,00017233 & 0,058717847 & 1,299808115 & Cldn8 & claudin 8 \\
\hline 17440342 & 5,254826846 & 0,001233523 & 0,095102509 & 1,296142958 & Gm15446 & predicted gene 15446 \\
\hline 17428545 & 5,011168431 & 0,001576888 & 0,097524576 & 1,294827033 & Uqcrh & ubiquinol-cytochrome $\mathrm{c}$ reductase hinge protein \\
\hline 17386018 & 5,960355411 & 0,00063922 & 0,080976625 & 1,29076151 & Scn1a & sodium channel, voltage-gated, type I, alpha \\
\hline 17223138 & 4,93193203 & 0,001708619 & 0,099007076 & 1,289370561 & Pgap1 & post-GPI attachment to proteins 1 \\
\hline 17473219 & 9,505388999 & $5,4852 \mathrm{E}-05$ & 0,032686631 & 1,285812037 & Tsen34 & tRNA splicing endonuclease 34 homolog (S. cerevisiae) \\
\hline 17479628 & 4,791233924 & 0,001959988 & 0,100695956 & 1,272475718 & Alpk3 & alpha-kinase 3 \\
\hline 17444236 & 5,202617847 & 0,001295717 & 0,096069042 & 1,265939947 & Wipi2 & WD repeat domain, phosphoinositide interacting 2 \\
\hline 17404570 & 4,847148857 & 0,00184942 & 0,100511427 & 1,264786627 & Slc7a14 & $\begin{array}{l}\text { solute carrier family } 7 \text { (cationic amino acid transporter, } y+\text { system), } \\
\text { member } 14\end{array}$ \\
\hline 17364111 & 5,932228566 & 0,000657361 & 0,080976625 & 1,259197164 & Ch25h & cholesterol 25-hydroxylase \\
\hline 17229917 & 4,784711642 & 0,001975105 & 0,100695956 & 1,257935743 & Kcnj9 & potassium inwardly-rectifying channel, subfamily J, member 9 \\
\hline 17249888 & 5,652174648 & 0,00084308 & 0,084494765 & 1,256469624 & Mfap3 & microfibrillar-associated protein 3 \\
\hline 17300802 & 4,972048018 & 0,001637787 & 0,097826161 & 1,255135033 & Parp4 & poly (ADP-ribose) polymerase family, member 4 \\
\hline 17324932 & 5,571447501 & 0,000901387 & 0,084873601 & 1,251749353 & $\operatorname{Lrch} 3$ & $\begin{array}{l}\text { leucine-rich repeats and calponin homology }(\mathrm{CH}) \text { domain containing } \\
3\end{array}$ \\
\hline 17526265 & 5,835844021 & 0,000713508 & 0,080976625 & 1,251214202 & Trappc4 & trafficking protein particle complex 4 \\
\hline 17506512 & 5,659977771 & 0,000833578 & 0,084251677 & 1,251059546 & Cpne7 & copine VII \\
\hline 17460809 & 5,050113525 & 0,001512534 & 0,096786837 & 1,246052481 & Zxdc & ZXD family zinc finger $C$ \\
\hline 17343755 & 5,412560456 & 0,001061192 & 0,090018052 & 1,245935857 & $\mathrm{H} 2-\mathrm{Pb}$ & histocompatibility $2, \mathrm{P}$ region beta locus \\
\hline 17236102 & 4,812581737 & 0,001912047 & 0,100695956 & 1,240289903 & Btbd11 & BTB (POZ) domain containing 11 \\
\hline 17431516 & 6,689116524 & 0,000327385 & 0,070811709 & 1,228516901 & Pithd1 & $\begin{array}{l}\text { PITH (C-terminal proteasome-interacting domain of thioredoxin- } \\
\text { like) domain containing } 1\end{array}$ \\
\hline 17505260 & 5,61553232 & 0,000869426 & 0,084494765 & 1,224853356 & Nfat5 & nuclear factor of activated T cells 5 \\
\hline 17399396 & 6,715152031 & 0,000322634 & 0,070811709 & 1,217994932 & Dpm3 & dolichyl-phosphate mannosyltransferase polypeptide 3 \\
\hline 17546082 & 6,236092211 & 0,000501443 & 0,080648038 & 1,213307197 & Gm8817 & predicted gene 8817 \\
\hline 17500523 & 4,720073144 & 0,00211893 & 0,103782082 & 1,209649043 & Saraf & store-operated calcium entry-associated regulatory factor \\
\hline 17501234 & 5,129748451 & 0,00140283 & 0,096476139 & 1,208439667 & Glra3 & glycine receptor, alpha 3 subunit \\
\hline 17292654 & 4,985275004 & 0,001614464 & 0,097826161 & 1,208372101 & Sptlc1 & serine palmitoyltransferase, long chain base subunit 1 \\
\hline 17288501 & 5,319069835 & 0,001159235 & 0,093087581 & 1,206700182 & Clptm1l & CLPTM1-like \\
\hline 17425836 & 5,263353037 & 0,001224885 & 0,094948376 & 1,204688654 & Ptbp3 & polypyrimidine tract binding protein 3 \\
\hline 17311512 & 5,124465665 & 0,001408877 & 0,096476139 & 1,204573672 & Mal2 & mal, T cell differentiation protein 2 \\
\hline 17363851 & 5,137227157 & 0,001395056 & 0,096476139 & 1,196000691 & 9930021J03Rik & RIKEN cDNA 9930021103 gene \\
\hline 17494041 & 5,082145173 & 0,001465456 & 0,096536895 & 1,195518248 & Nup98 & nucleoporin 98 \\
\hline 17345029 & 5,013781884 & 0,001572569 & 0,097524576 & 1,190905972 & Gpr111 & G protein-coupled receptor 111 \\
\hline 17385303 & 4,849992153 & 0,001839918 & 0,100511427 & 1,190084555 & Stam2 & $\begin{array}{l}\text { signal transducing adaptor molecule (SH3 domain and ITAM motif) } \\
2\end{array}$ \\
\hline 17451823 & 5,902037852 & 0,000676364 & 0,080976625 & 1,182245636 & Srrm4 & serine/arginine repetitive matrix 4 \\
\hline 17438134 & 6,700459327 & 0,000325657 & 0,070811709 & 1,18106764 & Dcun1d4 & $\begin{array}{l}\text { DCN1, defective in cullin neddylation } 1 \text {, domain containing } 4 \text { (S. } \\
\text { cerevisiae) }\end{array}$ \\
\hline 17373120 & 4,933794237 & 0,001704732 & 0,099007076 & 1,180753741 & Celf1 & CUGBP, Elav-like family member 1 \\
\hline 17248975 & 6,183987662 & 0,000528653 & 0,080717083 & 1,1718909 & Olfr1386 & olfactory receptor 1386 \\
\hline 17240235 & 4,966994613 & 0,001643833 & 0,097907395 & 1,165643118 & Rfpl4b & ret finger protein-like $4 \mathrm{~B}$ \\
\hline 17480127 & 4,985361603 & 0,001613168 & 0,097826161 & 1,155466251 & Ccdc89 & coiled-coil domain containing 89 \\
\hline 17326938 & 5,36541344 & 0,001119068 & 0,091980518 & 1,153007742 & $\begin{array}{l}\text { LOC10263719 } \\
2\end{array}$ & keratin-associated protein 20 -2-like \\
\hline 17325770 & 4,789429718 & 0,001964307 & 0,100695956 & 1,141566245 & Gm608 & predicted gene 608 \\
\hline 17370350 & 4,900200845 & 0,001761744 & 0,09985745 & 1,100156666 & Olfr350 & olfactory receptor 350 \\
\hline 17357591 & $-6,244605593$ & 0,000497124 & 0,080648038 & 0,915780274 & $\begin{array}{l}\text { A430093F15Ri } \\
\mathrm{k}\end{array}$ & RIKEN cDNA A430093F15 gene \\
\hline 17350996 & $-7,065093548$ & 0,000240571 & 0,068811793 & 0,883285713 & Arsi & arylsulfatase i \\
\hline 17228057 & $-6,085778253$ & 0,000577026 & 0,080976625 & 0,865764147 & $\begin{array}{l}1700025 G 04 R i \\
k\end{array}$ & RIKEN cDNA $1700025 G 04$ gene \\
\hline 17341712 & $-5,010288042$ & 0,001578615 & 0,097524576 & 0,847553417 & Amdhd2 & amidohydrolase domain containing 2 \\
\hline 17476643 & $-7,159416819$ & 0,000223727 & 0,06597292 & 0,843392385 & $\begin{array}{l}\text { LOC10263716 } \\
3\end{array}$ & major allergen I polypeptide chain 1-like \\
\hline 17319134 & $-5,216820319$ & 0,001278009 & 0,095993501 & 0,838908108 & Sox10 & SRY (sex determining region Y)-box 10 \\
\hline 17368633 & $-5,405514407$ & 0,001070694 & 0,090018052 & 0,833814357 & F730016J06Rik & RIKEN cDNA F730016J06 gene \\
\hline 17322923 & $-6,514866398$ & 0,00038526 & 0,074618174 & 0,833335258 & Gm4262 & predicted gene 4262 \\
\hline 17213645 & $-6,647909491$ & 0,000340774 & 0,070811709 & 0,826618402 & Fastkd2 & FAST kinase domains 2 \\
\hline 17477774 & $-4,977900356$ & 0,001628285 & 0,097826161 & 0,826412828 & $\mathrm{Hrc}$ & histidine rich calcium binding protein \\
\hline 17396498 & $-5,11780908$ & 0,001419242 & 0,096476139 & 0,819481846 & Rpl22|1 & ribosomal protein L22 like 1 \\
\hline 17407905 & $-6,61293257$ & 0,000353731 & 0,072270914 & 0,819389164 & Prpf3 & PRP3 pre-mRNA processing factor 3 homolog (yeast) \\
\hline 17548894 & $-5,098484111$ & 0,001444725 & 0,096476139 & 0,810831415 & Tmlhe & trimethyllysine hydroxylase, epsilon \\
\hline 17273910 & $-5,750294189$ & 0,000768792 & 0,081600237 & 0,80768276 & Hs1bp3 & HCLS1 binding protein 3 \\
\hline 17481632 & $-5,227060346$ & 0,001265484 & 0,095993501 & 0,80657294 & Olfr510 & olfactory receptor 510 \\
\hline 17334419 & $-7,49192625$ & 0,000176649 & 0,058753337 & 0,80627617 & Msrb1 & methionine sulfoxide reductase $\mathrm{B} 1$ \\
\hline 17495207 & $-7,256807715$ & 0,000204723 & 0,062295714 & 0,800682844 & $\begin{array}{l}2310014 F 06 R i \\
k\end{array}$ & RIKEN cDNA $2310014 F 06$ gene \\
\hline 17259475 & $-5,294892089$ & 0,001187309 & 0,093815338 & 0,792338987 & Lrrc45 & leucine rich repeat containing 45 \\
\hline 17473477 & $-5,772061854$ & 0,000751084 & 0,081210084 & 0,783784091 & U2af2 & U2 small nuclear ribonucleoprotein auxiliary factor (U2AF) 2 \\
\hline 17217887 & $-4,974343153$ & 0,001633899 & 0,097826161 & 0,775300932 & 4930596121Rik & RIKEN cDNA 4930596121 gene \\
\hline 17296422 & $-6,549200782$ & 0,000375326 & 0,074038814 & 0,773952395 & Pelo & pelota homolog (Drosophila) \\
\hline 17506369 & $-6,738000916$ & 0,000316155 & 0,070811709 & 0,768006566 & Zc3h18 & zinc finger $\mathrm{CCCH}$-type containing 18 \\
\hline 17262178 & $-5,034364167$ & 0,001540176 & 0,097524576 & 0,765810185 & Trim41 & tripartite motif-containing 41 \\
\hline
\end{tabular}




\begin{tabular}{|c|c|c|c|c|c|c|}
\hline $\begin{array}{c}\text { probese } \\
\text { t ID }\end{array}$ & d.value & p.value & q.value & R.fold & Genename & Description \\
\hline 17234711 & $-5,669316077$ & 0,000825804 & 0,083980931 & 0,765137462 & Gm10142 & predicted gene 10142 \\
\hline 17244506 & $-5,644908828$ & 0,000846103 & 0,084494765 & 0,762041229 & Mrpl42 & mitochondrial ribosomal protein $\mathrm{L} 42$ \\
\hline 17452194 & $-6,387698454$ & 0,000429314 & 0,07674456 & 0,753836045 & Erp29 & endoplasmic reticulum protein 29 \\
\hline 17296128 & $-5,707717265$ & 0,000796866 & 0,082884125 & 0,747343206 & Gapt & Grb2-binding adaptor, transmembrane \\
\hline 17383848 & $-10,74790876$ & $3,15291 \mathrm{E}-05$ & 0,027251476 & 0,741433813 & Swi5 & SWI5 recombination repair homolog (yeast) \\
\hline 17498059 & $-5,431919004$ & 0,001036142 & 0,089000756 & 0,740031916 & Krtap5-2 & keratin associated protein $5-2$ \\
\hline 17236077 & $-6,221477007$ & 0,000510513 & 0,080717083 & 0,739548197 & Ric8b & resistance to inhibitors of cholinesterase 8 homolog B (C. elegans) \\
\hline 17470175 & $-6,131860148$ & 0,000550248 & 0,080776169 & 0,73954527 & Zfp248 & zinc finger protein 248 \\
\hline 17287247 & $-5,15516952$ & 0,001370005 & 0,096476139 & 0,737989697 & 2310081J21Rik & RIKEN cDNA $2310081 \mathrm{~J} 21$ gene \\
\hline 17528430 & $-5,112088093$ & 0,001424857 & 0,096476139 & 0,730768773 & Tpm1 & tropomyosin 1, alpha \\
\hline 17380268 & $-6,235980138$ & 0,000501874 & 0,080648038 & 0,729705006 & Vapb & vesicle-associated membrane protein, associated protein $B$ and $C$ \\
\hline 17440745 & $-5,565698702$ & 0,000905706 & 0,084873601 & 0,729066335 & Gm15736 & predicted gene 15736 \\
\hline 17329842 & $-5,088061448$ & 0,001458546 & 0,096536895 & 0,727438123 & Mir1946a & microRNA 1946a \\
\hline 17365243 & $-5,835863343$ & 0,000713076 & 0,080976625 & 0,725561679 & Npm3 & nucleoplasmin 3 \\
\hline 17467466 & $-5,099053614$ & 0,001442997 & 0,096476139 & 0,721848494 & Igkv4-55 & immunoglobulin kappa variable 4-55 \\
\hline 17408813 & $-5,366790579$ & 0,001118204 & 0,091980518 & 0,716906834 & Cttnbp2nl & CTTNBP2 N-terminal like \\
\hline 17219086 & $-6,190495876$ & 0,000526061 & 0,080717083 & 0,715544776 & Tmco1 & transmembrane and coiled-coil domains 1 \\
\hline 17244341 & $-5,770092046$ & 0,00075238 & 0,081210084 & 0,715519882 & Elk3 & ELK3, member of ETS oncogene family \\
\hline 17322254 & $-5,167897919$ & 0,001352297 & 0,096476139 & 0,714678626 & Npff & neuropeptide FF-amide peptide precursor \\
\hline 17258615 & $-4,983174887$ & 0,00162051 & 0,097826161 & 0,70839807 & Gm11744 & predicted gene 11744 \\
\hline 17237084 & $-5,445528947$ & 0,001019298 & 0,088888554 & 0,702515826 & Ccdc59 & coiled-coil domain containing 59 \\
\hline 17517105 & $-5,751221169$ & 0,000767928 & 0,081600237 & 0,701555281 & $\| 18$ & interleukin 18 \\
\hline 17486874 & $-5,381847693$ & 0,001098336 & 0,091326244 & 0,697668412 & Ccdc9 & coiled-coil domain containing 9 \\
\hline 17243659 & $-5,317624063$ & 0,001161826 & 0,093087581 & 0,69711834 & Gm6713 & predicted gene 6713 \\
\hline 17254591 & $-5,111272772$ & 0,001425721 & 0,096476139 & 0,692868074 & Tbx2 & T-box 2 \\
\hline 17536742 & $-6,697325279$ & 0,000326089 & 0,070811709 & 0,683861625 & Nono & non-POU-domain-containing, octamer binding protein \\
\hline 17520856 & $-6,67633391$ & 0,000332999 & 0,070811709 & 0,678196667 & Amotl2 & angiomotin-like 2 \\
\hline 17408074 & $-5,134275193$ & 0,001398079 & 0,096476139 & 0,677787116 & Polr3gl & polymerase (RNA) III (DNA directed) polypeptide G like \\
\hline 17250236 & $-5,743764089$ & 0,000771816 & 0,081600237 & 0,677784883 & $\begin{array}{l}\text { 4933439C10Ri } \\
\text { k }\end{array}$ & RIKEN cDNA 4933439C10 gene \\
\hline 17214910 & $-6,063503921$ & 0,000587392 & 0,080976625 & 0,67622792 & Gm2427 & predicted gene 2427 \\
\hline 17527495 & $-5,358153832$ & 0,001125978 & 0,092090263 & 0,667924383 & Commd4 & COMM domain containing 4 \\
\hline 17520624 & $-5,146475762$ & 0,001383394 & 0,096476139 & 0,66694127 & Rbp1 & retinol binding protein 1 , cellular \\
\hline 17453454 & $-5,276100621$ & 0,001208472 & 0,094186645 & 0,66646612 & Eln & elastin \\
\hline 17277521 & $-5,995106041$ & 0,000620649 & 0,080976625 & 0,664029815 & Vash1 & vasohibin 1 \\
\hline 17349304 & $-5,337752682$ & 0,001143254 & 0,092637519 & 0,66378557 & Tslp & thymic stromal lymphopoietin \\
\hline 17550042 & $-6,198037932$ & 0,00052347 & 0,080717083 & 0,65963552 & Mir1943 & microRNA 1943 \\
\hline 17319205 & $-6,198037932$ & 0,00052347 & 0,080717083 & 0,65963552 & Mir1943 & microRNA 1943 \\
\hline 17217619 & $-5,423112655$ & 0,001046508 & 0,089354471 & 0,656651815 & Phlda3 & pleckstrin homology-like domain, family A, member 3 \\
\hline 17357597 & $-5,672506897$ & 0,000822349 & 0,083980931 & 0,649934897 & Slc15a3 & solute carrier family 15, member 3 \\
\hline 17213189 & $-5,400101218$ & 0,001076309 & 0,090018052 & 0,643829403 & Gm20257 & caspase 8 pseudogene \\
\hline 17401117 & $-6,584363992$ & 0,000361073 & 0,072732014 & 0,639396195 & Nras & neuroblastoma ras oncogene \\
\hline 17270715 & $-5,194373362$ & 0,001306515 & 0,096069042 & 0,628000474 & Gm11651 & predicted gene 11651 \\
\hline 17366918 & $-5,180441052$ & 0,001331133 & 0,096476139 & 0,622552549 & Mir466d & microRNA 466d \\
\hline 17252013 & $-7,018203775$ & 0,000252665 & 0,070165936 & 0,612573124 & Arrb2 & arrestin, beta 2 \\
\hline 17254171 & $-5,225798416$ & 0,001269371 & 0,095993501 & 0,60580987 & Slfn1 & schlafen 1 \\
\hline 17333709 & $-5,132370803$ & 0,001399375 & 0,096476139 & 0,59901349 & Spaca6 & sperm acrosome associated 6 \\
\hline 17431174 & $-5,614726916$ & 0,000869858 & 0,084494765 & 0,593866736 & Cd52 & CD52 antigen \\
\hline 17548432 & $-5,448719809$ & 0,001014547 & 0,088744806 & 0,59141636 & Gm10091 & predicted gene 10091 \\
\hline 17403237 & $-5,057195389$ & 0,001501304 & 0,096786837 & 0,586959168 & Gbp3 & guanylate binding protein 3 \\
\hline 17357947 & $-7,042141299$ & 0,000248346 & 0,06964266 & 0,575545809 & Olfr1502 & olfactory receptor 1502 \\
\hline 17466768 & $-6,042861491$ & 0,000599053 & 0,080976625 & 0,549191127 & Hnrnpa2b1 & heterogeneous nuclear ribonucleoprotein A2/B1 \\
\hline 17448924 & $-5,210166839$ & 0,001285351 & 0,095993501 & 0,540414412 & $\mathrm{Kdr}$ & kinase insert domain protein receptor \\
\hline 17491323 & $-5,526778781$ & 0,000941123 & 0,085732855 & 0,51999887 & Mrgpra2a & MAS-related GPR, member A2A \\
\hline 17458362 & $-5,457848769$ & 0,001007636 & 0,088448594 & 0,500004863 & Gimap4 & GTPase, IMAP family member 4 \\
\hline 17238367 & $-6,458444801$ & 0,000404696 & 0,074681937 & 0,49335415 & Stat2 & signal transducer and activator of transcription 2 \\
\hline 17325324 & $-6,872414721$ & 0,000289809 & 0,070811709 & 0,484851567 & Stfa2l1 & stefin A2 like 1 \\
\hline 17329298 & $-6,225493105$ & 0,000507921 & 0,080717083 & 0,479676207 & Etv5 & ets variant 5 \\
\hline 17327557 & $-7,775252466$ & 0,000149871 & 0,057157851 & 0,46721545 & $\mathrm{M} \times 2$ & MX dynamin-like GTPase 2 \\
\hline 17451930 & $-5,984203798$ & 0,000630582 & 0,080976625 & 0,460512146 & AW549542 & expressed sequence AW549542 \\
\hline 17547909 & $-6,147338094$ & 0,000544633 & 0,080717083 & 0,446588513 & Gm19551 & predicted gene, 19551 \\
\hline 17366932 & $-4,992296959$ & 0,001604098 & 0,097826161 & 0,419490919 & Mir466h & microRNA 466h \\
\hline 17350925 & $-5,171913899$ & 0,001345818 & 0,096476139 & 0,374099363 & ligp1 & interferon inducible GTPase 1 \\
\hline 17254176 & $-5,35773351$ & 0,001126842 & 0,092090263 & 0,357441501 & SIfn4 & schlafen 4 \\
\hline 17510345 & $-5,345714464$ & 0,00113548 & 0,092268955 & 0,274123389 & Bst2 & bone marrow stromal cell antigen 2 \\
\hline
\end{tabular}


Table S2. Functional annotation of differentially expressed genes (overexpressed and downregulated) in the lungs of HRAS/NRAS-DKO mice

The GeneCodis (Gene Annotation Co-occurrence Discovery) functional annotation tool (http://genecodis.cnb.csic.es/ ) was used to identify statistically significant functional associations linking particular gene subsets contained within the list of differentially expressed gene probesets identified in HRAS/NRAS-DKO lungs [FDR = 0.1; heatmap Fig 6A; Table S1 including 165 overexpressed (red) and 76 repressed (blue) genes] to specific cellular functionalities, including particular GO Biological Processes (BP) or Molecular Functions (MF), KEGG Signaling Pathways, and Transcriptional Factors that may account for regulation of expression of the corresponding groups of loci listed in each case, as indicated. Red: overexpressed genes. Blue: repressed genes.

The columns labelled "Functional Category", "KEGG Pathway" and "Transcription Factor" identify the specific functional GO (BP or MF), KEGG or TF terms recognized in each case for the corresponding groups of loci listed under the column labelled "Genes". The column labelled "Number of Genes" indicates the specific number of genes annotated to the indicated functionality, out of the total number (in parenthesis) of genes recognized by GeneCodis in the lists of differentially expressed, overexpressed (red) or repressed (blue) genes identified in HRas/NRas-DKO lungs. The column labelled "Hypergeometric pValue" refers to the statistical significance assignated by Genecodis to each of the functional associations identified.

\section{GO Biological Process (BP) Enrichment Analysis of 165 Genes Overexpressed in HRAS/NRAS-DKO Lungs}

\begin{tabular}{|c|c|c|c|c|}
\hline Items & Functional categorys & $\begin{array}{l}\text { Number } \\
\text { of genes }\end{array}$ & $\begin{array}{l}\text { Hypergeom. } \\
\text { pValue }\end{array}$ & Genes \\
\hline $\begin{array}{r}\text { GO:0006810 } \\
\text { GO:0015031 } \\
\text { GO:0016192 } \\
\text { GO:0006886 } \\
\text { GO:0006811 } \\
\text { GO:0015991 } \\
\text { GO:0015992 } \\
\text { GO } 0055085 \\
\text { GO } 0034220\end{array}$ & $\begin{array}{l}\text { transport (BP) } \\
\text { protein transport (BP) } \\
\text { vesicle-mediated transport (BP) } \\
\text { intracellular protein transport (BP) } \\
\text { ion transport (BP) } \\
\text { ATP hydrolysis coupled proton transport (BP) } \\
\text { proton transport (BP) } \\
\text { transmembrane transport (BP) } \\
\text { ion transmembrane transport (BP) }\end{array}$ & 34 & $2.24556 \mathrm{e}-14$ & $\begin{array}{l}\text { Slc25a16, Pgap1, Atp5e, Scfd2, Derl1, } \\
\text { Atp6v1b2, Clcn5, Ap2s1, Exoc5, Kcnma1, } \\
\text { Gpr89, Kcnj9, Atp6v0a2, Stam2, Plekha8, } \\
\text { Atg4b, Slc25a29, Ic39a4, Mtx2, Uqcrh, } \\
\text { Ndufa7, Slc25a46, Trappc4, Tomm20, Scn1a, } \\
\text { Atp6v0e, Nipa1, Bcap29, Lmbrd1, Sec23a, } \\
\text { Slc7a14, Glra3, Atg16l1, Ramp3 }\end{array}$ \\
\hline $\begin{array}{l}\text { GO:0008152 } \\
\text { G0-000658 } \\
\text { G0-0016567 }\end{array}$ & $\begin{array}{l}\text { metabolic process (BP) } \\
\text { proteolysis } \\
\text { protein ubiquitination }\end{array}$ & 21 & $1.35098 \mathrm{e}-05$ & $\begin{array}{l}\text { Mylip, March5, Rpap2, Pde2a, Lgmn, Ubr7, } \\
\text { Atg4b, Pigk, Casp6, Glce, Ppp1ca, Fh1, Sptlc1, } \\
\text { Ifnar1, Atp6v0e, Rnf167, Uhrf2, Sgpp1, } \\
\text { Ptprz1, Uggt1, Ythdc2 }\end{array}$ \\
\hline GO:0046034 & ATP metabolic process (BP) & 3 & $2.77057 \mathrm{e}-04$ & Atp5e,Atp6v1b2,Ak3 \\
\hline GO:0006508 & proteolysis (BP) & 8 & $3.36783 e-03$ & $\begin{array}{l}\text { Psmb4,Dnpep,Psmb5,Lgmn,Usp38,Atg4b, } \\
\text { Pigk,Casp6 }\end{array}$ \\
\hline GO:0006511 & ubiquitin-dependent protein catabolic process (BP) & 3 & $1.59383 \mathrm{e}-02$ & Usp38, Fbxo8, Uhrf2 \\
\hline $\begin{array}{r}\text { GO:0005975 } \\
\text { GO } 005977 \\
\end{array}$ & $\begin{array}{l}\text { carbohydrate metabolic process (BP) } \\
\text { glycogen metabolic process (BP) }\end{array}$ & 5 & $1.04802 \mathrm{e}-03$ & Mgat4a, Ppp1ca, Phkg2, Alg13, Ppp1cb \\
\hline $\begin{array}{l}\text { GO:0006470 } \\
\text { GO:0007049 }\end{array}$ & protein dephosphorylation (BP), cell cycle (BP) & 3 & $1.76194 \mathrm{e}-05$ & Ppm1d, Ppp1ca, Ppp1cb \\
\hline GO:0006626 & protein targeting to mitochondrion (BP) & 3 & $7.58745 \mathrm{e}-05$ & Pde2a, Mtx2, Tomm20 \\
\hline GO:0042981 & regulation of apoptotic process (BP) & 3 & $3.19694 \mathrm{e}-02$ & Ppp2r1a, Bnip3, Casp6 \\
\hline $\begin{array}{l}\text { GO:0006915 } \\
\text { GO:0006917 }\end{array}$ & apoptotic process (BP) & 7 & $6.9995 e-03$ & $\begin{array}{l}\text { Bnip3, Clptm1l, Casp6, Perp, Bcap29, Sgpp1, } \\
\text { Ddit4 }\end{array}$ \\
\hline $\begin{array}{l}\text { GO:0006914 } \\
\text { GO:0000045 }\end{array}$ & autophagy (BP), autophagic vacuole assembly (BP) & 3 & $2.89647 e-05$ & Wipi2, Atg4b, Atg16l1 \\
\hline GO:0006506 & GPI anchor biosynthetic process (BP) & 3 & $3.06854 \mathrm{e}-04$ & Pgap1, Dpm3, Pigk \\
\hline
\end{tabular}

GO Molecular Function (MF) Enrichment Analysis of 165 Genes Overexpressed in HRAS/NRAS-DKO Lungs

\begin{tabular}{|c|c|c|c|c|}
\hline Items & Functional category & $\begin{array}{l}\text { Number } \\
\text { of genes }\end{array}$ & $\begin{array}{c}\text { Hypergeom. } \\
\text { pValue }\end{array}$ & Genes \\
\hline GO:0016787 & hydrolase activity (MF) & $23 / 165$ & $1.86758 \mathrm{e}-07$ & $\begin{array}{l}\text { Pgap1, Atp5e, Psmb4, Dnpep, Atp6v1b2, } \\
\text { Psmb5, Rpap2, Tsen34, Pde2a, Lgmn, Usp38, }\end{array}$ \\
\hline
\end{tabular}




\begin{tabular}{|l|l|l|l|l|}
\hline & & & & $\begin{array}{l}\text { Atg4b, Pigk, Acer3, Ppm1d, Casp6, Ppp1ca, } \\
\text { Atp6v0e, Sgpp1, Gdpd1, Ptprz1, Ythdc2, } \\
\text { Ppp1cb }\end{array}$ \\
\hline G0:0046872 & metal ion binding (MF) & $23 / 165$ & $2.65291 \mathrm{e}-03$ & $\begin{array}{l}\text { Dnpep, Mylip, Zxdc, March5, Mgat4a, Rpap2, } \\
\text { Kcnma1, Pde2a, Ubr7, Ch25h, Zfp35, Cdo1, } \\
\text { Zfp944, Rev1, Ppm1d, Ppp1ca, Polr3k, } \\
\text { Rnf167, Uhrf2, Gdpd1, Papd5, Ppp1cb, } \\
\text { Gm15446 }\end{array}$ \\
\hline GO:0005216 & ion channel activity (MF) & & & \\
\hline
\end{tabular}

\section{KEGG Pathways Enrichment analysis of 165 Genes Overexpressed in HRAS/NRAS-DKO Lungs}

\begin{tabular}{|c|c|c|c|c|}
\hline Items & KEGG pathways & $\begin{array}{l}\text { Number } \\
\text { of genes }\end{array}$ & $\begin{array}{l}\text { Hypergeom. } \\
\text { pValue }\end{array}$ & Genes \\
\hline Kegg:00190 & Oxidative phosphorylation & 7 & $2.55981 \mathrm{e}-05$ & $\begin{array}{l}\text { Atp6v0a2, Uqcrh, Atp6v0e, Ndufa7, } \\
\text { Atp6v1b2, Atp5e, Ak3 }\end{array}$ \\
\hline Kegg:00600 & Sphingolipid metabolism & 5 & $3.16363 e-05$ & Degs1, Sgpp1, Acer3, Sptlc1, Cers5 \\
\hline Kegg:00510 & N-Glycan biosynthesis & 3 & $1.38934 \mathrm{e}-03$ & Alg13, Mgat4a, Dpm3 \\
\hline Kegg:04910 & Insulin signaling pathway & 4 & $3.00635 \mathrm{e}-03$ & Ppp1cb, Ppp1ca, Phkg2, Socs2 \\
\hline Kegg:04270 & Vascular smooth muscle contraction & 4 & $1.68019 \mathrm{e}-03$ & Ppp1cb, Kcnma1, Ppp1ca, Ramp3 \\
\hline Kegg:03050 & Proteasome & 3 & $1.02209 \mathrm{e}-03$ & Psmb5, Psmd11, Psmb4 \\
\hline Kegg:04141 & Protein processing in endoplasmic reticulum & 4 & $5.61344 \mathrm{e}-03$ & Sec23a, Derl1, Dnajb1, Uggt1 \\
\hline
\end{tabular}

Functi

onal

annot

ation

\section{to "TRANSCRIPTION FACTORS" of 165 genes overexpressed in the lungs of HRAS/NRAS-DKO mice}

\begin{tabular}{|l|l|l|l|}
\hline Transcription Factor & $\begin{array}{c}\text { Number of } \\
\text { genes }\end{array}$ & \multicolumn{1}{|c|}{$\begin{array}{c}\text { Hypergeom } \\
\text { pValue }\end{array}$} & \\
\hline V\$ELK1_02 & $10 / 165$ & $1.88805 e-03$ & Ap2s1, Scfd2, Dnajb1, Uqcrh, Trappc4, Mtx2, Spg21, Tomm20, Snrpb, Fbxo8 \\
\hline V\$ERR1_Q2 & $9 / 165$ & $8.11141 \mathrm{e}-03$ & Uhrf2, Ppp1cb, Scfd2, Bnip3, Uqcrh, Actr1a, Mtx2, Got1, Socs2 \\
\hline V\$AP1_C & $9 / 165$ & $1.06567 \mathrm{e}-02$ & Clcn5, Bnip3, Vat1, Btbd11, Atp6v1b2, Gfra1, Perp, Psmd11, Gpr111 \\
\hline V\$LEF1_Q2 & $18(165)$ & $2.25861 \mathrm{e}-03$ & $\begin{array}{l}\text { Cdo1, Uhrf2, Clcn5, Papd5, Ppp1cb, Tacstd2, Nup98, Bnip3, Dctn3, Pgap1, Vat1, Actr1a, } \\
\text { Gfra1, Psmb5, Psmd11, Ddit4, Socs2, Psmb4 }\end{array}$ \\
\hline V\$NFY_Q6_01 & $9(165)$ & $1.07351 \mathrm{e}-02$ & Slc39a4, Nup98, Dnajb1, Actr1a, Ppp2r1a, Ppp1r7, Glra3, Got1, Ppm1d \\
\hline V\$MYB_Q6 & 4 & $6.37742 \mathrm{e}-03$ & Slc39a4, Ssbp2, Cldn8, Socs2 \\
\hline V\$CEBPA_01 & 4 & $7.79868 \mathrm{e}-03$ & Clock, Cldn8, Ppp1cb, Nfat5 \\
\hline V\$HSF1_01 & 5 & $1.61305 e-02$ & Dnajb1, Gipc2, Glra3, Socs2, Ppm1d \\
\hline V\$MYOGENIN_Q6 & 4 & $7.49923 e-03$ & Bnip3, Btbd11, Gfra1, Ddit4 \\
\hline V\$YY1_Q6 & 5 & $1.14333 e-02$ & Sec23a, Kcnma1, Uqcrh, Prpsap2, Psmb5 \\
\hline V\$FOXJ2_01 & 5 & $3.79737 e-04$ & Cldn8, Ppp1cb, Gfra1, Ch25h, Ppm1d \\
\hline V\$HFH1_01 & 4 & $8.26242 e-03$ & Cldn8, Ppp1cb, Tacstd2, Ppm1d \\
\hline
\end{tabular}

\section{Go Biological Process (BP) Enrichment Analysis of 76 Genes Downregulated in HRAS/NRAS-DKO} Lungs

\begin{tabular}{|c|l|l|l|l|}
\hline \multicolumn{1}{|c|}{ Items } & \multicolumn{1}{|c|}{ Functional category } & $\begin{array}{l}\text { Number } \\
\text { of genes }\end{array}$ & $\begin{array}{c}\text { Hypergeom. } \\
\text { pValue }\end{array}$ & Genes \\
\hline GO:0007204 & elevation of cytosolic calcium ion concentration (BP) & 3 & $1.24038 \mathrm{e}-03$ & $\mathrm{Kdr}, \mathrm{Cd} 52, \mathrm{Npff}$ \\
\hline $\begin{array}{l}\text { GO:0006397, } \\
\text { GO:0008380 }\end{array}$ & mRNA processing (BP),RNA splicing (BP) & 4 & $6.05231 \mathrm{e}-04$ & Hnrnpa2b1, Nono, U2af2, Prpf3 \\
\hline
\end{tabular}


Go Molecular Function (MF) Enrichment Analysis of 76 Genes Downregulated in HRAS/NRAS-DKO Lungs

\begin{tabular}{|l|l|l|l|l|}
\hline Items & Functional category & $\begin{array}{l}\text { Number } \\
\text { of genes }\end{array}$ & $\begin{array}{l}\text { Hypergeom. } \\
\text { pValue }\end{array}$ & \begin{tabular}{l} 
Genes \\
\hline GO:0000166
\end{tabular} \\
\hline
\end{tabular}

KEGG Pathways Enrichment analysis of 76 Genes Downregulated in HRAS/NRAS-DKO Lungs

\begin{tabular}{|c|l|l|l|l|}
\hline Items & \multicolumn{1}{|c|}{ KEGG pathways } & $\begin{array}{c}\text { Number } \\
\text { of genes }\end{array}$ & $\begin{array}{c}\text { Hypergeom. } \\
\text { pValue }\end{array}$ & \multicolumn{1}{|c|}{ Genes } \\
\hline Kegg:04062 & Chemokine signaling pathway & 3 & $5.5719 \mathrm{e}-03$ & Arrb2, Stat2, Nras \\
\hline Kegg:04060 & Cytokine-cytokine receptor interaction & 3 & $1.33254 \mathrm{e}-02$ & Tslp, Il18, Kdr \\
\hline
\end{tabular}

Functional annotation to "TRANSCRIPTION FACTORS" of 76 genes repressed in the lungs of HRAS/NRAS-DKO mice

\begin{tabular}{|l|l|l|l|}
\hline Transcription Factor & $\begin{array}{c}\text { Number of } \\
\text { genes } /\end{array}$ & $\begin{array}{c}\text { Hypergeom. } \\
\text { pValue }\end{array}$ & \multicolumn{1}{c|}{ Genes } \\
\hline V\$SP1_Q6 & $12 / 76$ & 0.000779745 & Elk3, Arrb2, Commd4, Eln, Npm3, Vapb, Etv5, Tbx2, Prpf3, Tmlhe, Trim41, Nras \\
\hline V\$GABP_B & $7(76)$ & 0.000117672 & Elk3, Arrb2, Etv5, Trim41, U2af2, Sox10, Nras \\
\hline V\$AML1_01 & 5 & $2.88692 \mathrm{e}-05$ & Slc15a3, Stat2, Tpm1, Mrpl42, Nras \\
\hline V\$AML1_Q6 & 5 & $2.88692 \mathrm{e}-05$ & Slc15a3, Stat2, Tpm1, Mrpl42, Nras \\
\hline V\$\$ETS_Q4 & 4 & 0.000425533 & Elk3, Arrb2, Etv5, Trim41 \\
\hline
\end{tabular}


Table S3. Differential gene expression in the lungs of HRAS/NRAS-DKO mice treated antenatally with dexamethasone.

List of 509 differentially expressed gene probesets identified by means of SAM contrasts (FDR=0.15) in multiclass comparison (Fig. 6B heatmap) between the transcriptional profiles of lungs from newborn (PO) DKO mice that had been treated in utero with dexamethasone (injections at E17.5 and E18.5) and the transcriptional profiles of untreated (P0) DKO littermates that were generated by RNA microarray hybridization assays using GeneChip(R) Mouse Gene 2.0 ST Arrays. The differentially expressed loci are identified by Affymetrix Probeset ID, Genename Symbol or Description and listed according to their degree of overexpression or repression in dexamethasone-treated lung tissue of DKO mice. $d$-value is a parameter measuring the statistical distance separating the calculated expression value of each gene probeset from the null hypothesis (no-change). $q$-value is the estimated FDR at the largest $p$-value for which the probe set would be statistically significant. $R$-fold is a measure of the fold change of a probeset in the collection of microarrays provided by the SAM algorithm. Entries in red denote overexpression. Entries in green denote transcriptional repression. The data list organized here from maximal to minimal d-values. Components of ceramide/sphingosine metabolism are printed in bold.

\begin{tabular}{|c|c|c|c|c|c|c|}
\hline $\begin{array}{c}\text { probeset } \\
\text { ID }\end{array}$ & d.value & p.value & q.value & R.fold & Genename & Description \\
\hline 17357648 & 5,800980166 & $3,88715 \mathrm{E}-05$ & 0,096074093 & 1,920712178 & Ms4a4c & membrane-spanning 4-domains, subfamily $\mathrm{A}$, member $4 \mathrm{C}$ \\
\hline 17259311 & 5,450425722 & $5,83073 \mathrm{E}-05$ & 0,096074093 & 1,568655314 & 0610009L18Rik & RIKEN cDNA 0610009L18 gene \\
\hline 17400622 & 5,443224101 & $5,91711 \mathrm{E}-05$ & 0,096074093 & 1,694496031 & Lix1l & Lix1-like \\
\hline 17509721 & 5,308217228 & $7,12644 \mathrm{E}-05$ & 0,096074093 & 1,495688964 & Tufm & Tu translation elongation factor, mitochondrial \\
\hline 17443683 & 5,126100134 & $8,59492 \mathrm{E}-05$ & 0,096074093 & 1,620692856 & Ppp1r35 & protein phosphatase 1 , regulatory subunit 35 \\
\hline 17240303 & 4,936497607 & 0,000108408 & 0,096074093 & 1,887549863 & G630090E17Rik & RIKEN cDNA G630090E17 gene \\
\hline 17458362 & 4,765692033 & 0,000141233 & 0,104761644 & 2,246207919 & Gimap4 & GTPase, IMAP family member 4 \\
\hline 17276182 & 4,755402964 & 0,000144688 & 0,104761644 & 1,552627184 & Jkamp & JNK1/MAPK8-associated membrane protein \\
\hline 17357910 & 4,526359256 & 0,000206451 & 0,116707116 & 2,052924326 & Olfr1463 & olfactory receptor 1463 \\
\hline 17411492 & 4,518059895 & 0,000209474 & 0,116707116 & 1,873515792 & Gm11783 & predicted gene 11783 \\
\hline 17402165 & 4,478487392 & 0,000228478 & 0,117965976 & 2,111983968 & A530020G20Rik & RIKEN cDNA A530020G20 gene \\
\hline 17376538 & 4,376356888 & 0,000262599 & 0,124070176 & 2,076853064 & 4930425F17Rik & RIKEN cDNA 4930425F17 gene \\
\hline 17244341 & 4,286378482 & 0,000302766 & 0,12603958 & 1,575044061 & Elk3 & ELK3, member of ETS oncogene family \\
\hline 17394829 & 4,152856254 & 0,000367984 & 0,127996194 & 1,513665594 & Atp9a & ATPase, class II, type $9 \mathrm{~A}$ \\
\hline 17433310 & 4,031688191 & 0,000440976 & 0,132184757 & 1,30850635 & Park7 & Parkinson disease (autosomal recessive, early onset) 7 \\
\hline 17466294 & 3,975815699 & 0,000480711 & 0,132184757 & 1,397874208 & Try5 & trypsin 5 \\
\hline 17255215 & 3,922649481 & 0,000522174 & 0,132737203 & 2,013953925 & Ankrd40 & ankyrin repeat domain 40 \\
\hline 17284702 & 3,862915192 & 0,000574003 & 0,132737203 & 1,533029579 & Gm10421 & predicted gene 10421 \\
\hline 17530915 & 3,852809951 & 0,000580913 & 0,132737203 & 2,122660087 & Gm9917 & predicted gene 9917 \\
\hline 17393225 & 3,833965601 & 0,000595166 & 0,132737203 & 1,71662334 & Pigu & phosphatidylinositol glycan anchor biosynthesis, class $\mathrm{U}$ \\
\hline 17234024 & 3,796165761 & 0,000629719 & 0,132737203 & 3,556466551 & Rtkn2 & rhotekin 2 \\
\hline 17234711 & 3,781923723 & 0,000647859 & 0,132737203 & 1,309959767 & Gm10142 & predicted gene 10142 \\
\hline 17511677 & 3,764405546 & 0,000661248 & 0,133061743 & 2,112141857 & Ces1c & carboxylesterase $1 \mathrm{C}$ \\
\hline 17386177 & 3,736929088 & 0,000693641 & 0,134289173 & 1,488797143 & Abcb11 & ATP-binding cassette, sub-family B (MDR/TAP), member 11 \\
\hline 17548940 & 3,728646209 & 0,000700551 & 0,134587111 & 1,414644871 & Soat1 & sterol O-acyltransferase 1 \\
\hline 17484682 & 3,72186879 & 0,000709621 & 0,1347626 & 1,680331399 & Athl1 & ATH1, acid trehalase-like 1 (yeast) \\
\hline 17442332 & 3,708490555 & 0,000725602 & 0,136174199 & 1,367416124 & Kntc1 & kinetochore associated 1 \\
\hline 17222825 & 3,688469985 & 0,000751948 & 0,136401701 & 2,799199546 & Nabp1 & nucleic acid binding protein 1 \\
\hline 17384205 & 3,676864141 & 0,000765769 & 0,136733781 & 1,52783397 & Mvb12b & multivesicular body subunit $12 \mathrm{~B}$ \\
\hline 17380567 & 3,655483338 & 0,000792547 & 0,137836277 & 2,303066348 & Gm14322 & predicted gene 14322 \\
\hline 17331848 & 3,587883431 & 0,000886702 & 0,139806133 & 1,297769639 & 2310061N02Rik & RIKEN cDNA 2310061N02 gene \\
\hline 17457609 & 3,576813513 & 0,00090873 & 0,139806133 & 1,452905967 & Mgam & maltase-glucoamylase \\
\hline 17516960 & 3,564595157 & 0,000929893 & 0,139806133 & 1,483860375 & Cadm1 & cell adhesion molecule 1 \\
\hline 17217946 & 3,527653586 & 0,000981722 & 0,139806133 & 1,32796367 & $\mathrm{~F} 13 \mathrm{~b}$ & coagulation factor XIII, beta subunit \\
\hline 17404923 & 3,511912934 & 0,001011523 & 0,139806133 & 1,338392838 & Gm5148 & predicted gene 5148 \\
\hline 17380555 & 3,511749727 & 0,001011955 & 0,139806133 & 2,070276047 & Gm14403 & predicted gene 14403 \\
\hline 17211375 & 3,483268058 & 0,00106292 & 0,139806133 & 1,342887651 & Paqr8 & progestin and adipoQ receptor family member VIII \\
\hline 17399314 & 3,456364806 & 0,001115613 & 0,139806133 & 1,565545328 & Fam189b & family with sequence similarity 189 , member B \\
\hline 17474906 & 3,452573562 & 0,001125546 & 0,139806133 & 1,307430512 & Zfp114 & zinc finger protein 114 \\
\hline 17265030 & 3,441950465 & 0,001144982 & 0,139806133 & 1,451421797 & Acap1 & ArfGAP with coiled-coil, ankyrin repeat and PH domains 1 \\
\hline 17217590 & 3,433246226 & 0,001163986 & 0,139806133 & 1,685692046 & Arl8a & ADP-ribosylation factor-like $8 \mathrm{~A}$ \\
\hline 17344251 & 3,396121838 & 0,001243889 & 0,139806133 & 1,608600529 & Prrc2a & proline-rich coiled-coil 2A \\
\hline 17354653 & 3,383791231 & 0,00127585 & 0,139806133 & 1,667081798 & Tcof1 & Treacher Collins Franceschetti syndrome 1, homolog \\
\hline 17400375 & 3,369432449 & 0,001309538 & 0,139806133 & 1,422660284 & Ctss & cathepsin S \\
\hline 17400072 & 3,3679807 & 0,001313857 & 0,139806133 & 1,284600442 & Tdrkh & tudor and $\mathrm{KH}$ domain containing protein \\
\hline 17333731 & 3,361028777 & 0,001329838 & 0,139806133 & 1,711764232 & Fpr2 & formyl peptide receptor 2 \\
\hline 17267184 & 3,359590182 & 0,001335453 & 0,139806133 & 1,485165506 & Mir5110 & microRNA 5110 \\
\hline 17430521 & 3,350265516 & 0,001360503 & 0,139806133 & 1,329751953 & Tmem39b & transmembrane protein 39b \\
\hline 17350921 & 3,335498453 & 0,001404989 & 0,139806133 & 1,995201274 & F830016B08Rik & RIKEN cDNA F830016B08 gene \\
\hline 17486299 & 3,319700979 & 0,001440406 & 0,139806133 & 1,429051565 & Vmn2r45 & vomeronasal 2 , receptor 45 \\
\hline 17501096 & 3,316466258 & 0,001449476 & 0,139806133 & 1,369257681 & Cldn24 & claudin 24 \\
\hline 17416460 & $-2,497229696$ & 0,0072206 & 0,18966393 & 0,581152779 & Yipf1 & Yip1 domain family, member 1 \\
\hline 17349926 & $-2,497634313$ & 0,007209803 & 0,189599092 & 0,626549827 & Pcdhb17 & protocadherin beta 17 \\
\hline 17361805 & $-2,498518842$ & 0,007197709 & 0,189514594 & 0,552596624 & Neat1 & nuclear paraspeckle assembly transcript 1 (non-protein coding) \\
\hline 17245248 & $-2,499813117$ & 0,00717741 & 0,189281753 & 0,53043319 & Mdm2 & transformed mouse $3 T 3$ cell double minute 2 \\
\hline 17419025 & $-2,501624413$ & 0,007154951 & 0,189281753 & 0,74309541 & Tmem234 & transmembrane protein 234 \\
\hline 17484140 & $-2,501726179$ & 0,007153655 & 0,189281753 & 0,757259058 & Bccip & $\mathrm{BRCA} 2$ and $\mathrm{CDKN} 1 \mathrm{~A}$ interacting protein \\
\hline 17290603 & $-2,508650142$ & 0,007056476 & 0,188347263 & 0,559173144 & Actn2 & actinin alpha 2 \\
\hline 17514257 & $-2,509510044$ & 0,007042655 & 0,188130325 & 0,668267065 & Rbm34 & RNA binding motif protein 34 \\
\hline 17374612 & $-2,509875138$ & 0,00703704 & 0,188130325 & 0,66473691 & Phgr1 & proline/histidine/glycine-rich 1 \\
\hline 17514247 & $-2,510042233$ & 0,007034881 & 0,188130325 & 0,724393976 & Tomm20 & translocase of outer mitochondrial membrane 20 homolog (yeast) \\
\hline 17305243 & $-2,512314764$ & 0,007007239 & 0,187720276 & 0,632938723 & Sftpd & surfactant associated protein $\mathrm{D}$ \\
\hline 17444664 & $-2,512425227$ & 0,007006375 & 0,187720276 & 0,529707651 & Zfp655 & zinc finger protein 655 \\
\hline 17407643 & $-2,51351983$ & 0,006988667 & 0,187598072 & 0,783699822 & B230398E01Rik & RIKEN CDNA B230398E01 gene \\
\hline
\end{tabular}




\begin{tabular}{|c|c|c|c|c|c|c|}
\hline $\begin{array}{l}\text { probeset } \\
\text { ID }\end{array}$ & d.value & p.value & q.value & R.fold & Genename & Description \\
\hline 17239113 & $-2,5141921$ & 0,006980029 & 0,187518528 & 0,592007731 & Sash1 & SAM and SH3 domain containing 1 \\
\hline 17305856 & $-2,516407566$ & 0,00694634 & 0,186765324 & 0,63893514 & Exoc5 & exocyst complex component 5 \\
\hline 17454345 & $-2,519971846$ & 0,00689883 & 0,185801914 & 0,797963632 & Pdgfa & platelet derived growth factor, alpha \\
\hline 17533553 & $-2,520733979$ & 0,006886737 & 0,185767399 & 0,640840867 & Jade3 & jade family PHD finger 3 \\
\hline 17369821 & $-2,52119706$ & 0,00688069 & 0,185767399 & 0,671838754 & Ptges2 & prostaglandin E synthase 2 \\
\hline 17438189 & $-2,52214049$ & 0,006866437 & 0,185767399 & 0,579690564 & Rasl11b & RAS-like, family 11 , member B \\
\hline 17333946 & $-2,524315277$ & 0,006842251 & 0,18547585 & 0,748224926 & Zfp948 & zinc finger protein 948 \\
\hline 17329692 & $-2,524740888$ & 0,006831453 & 0,18547585 & 0,557148174 & Lsg1 & large subunit GTPase 1 homolog (S. cerevisiae) \\
\hline 17301926 & $-2,52905349$ & 0,006778329 & 0,184499854 & 0,345126777 & $\mathrm{Htr} 2 \mathrm{a}$ & 5-hydroxytryptamine (serotonin) receptor $2 \mathrm{~A}$ \\
\hline 17248592 & $-2,529282868$ & 0,006775737 & 0,184499854 & 0,599455873 & Slu7 & SLU7 splicing factor homolog (S. cerevisiae) \\
\hline 17502378 & $-2,530378383$ & 0,006755438 & 0,184499854 & 0,646333559 & Tpm4 & tropomyosin 4 \\
\hline 17539898 & $-2,531075478$ & 0,006745936 & 0,184499854 & 0,628054031 & Ccdc120 & coiled-coil domain containing 120 \\
\hline 17547602 & $-2,531672848$ & 0,00673773 & 0,184499854 & 0,463432067 & Gm10099 & predicted gene 10099 \\
\hline 17548865 & $-2,532814626$ & 0,006719158 & 0,184499854 & 0,730586649 & Gm8692 & predicted gene 8692 \\
\hline 17217666 & $-2,532845692$ & 0,006718726 & 0,184499854 & 0,721297613 & Tmem9 & transmembrane protein 9 \\
\hline 17252183 & $-2,533220175$ & 0,006711815 & 0,184499854 & 0,563148488 & Eno3 & enolase 3 , beta muscle \\
\hline 17488975 & $-2,539511227$ & 0,006619387 & 0,183666593 & 0,571852116 & Tbcb & tubulin folding cofactor B \\
\hline 17211700 & $-2,540550192$ & 0,006605998 & 0,183666593 & 0,745274987 & Imp4 & IMP4, U3 small nucleolar ribonucleoprotein, homolog (yeast) \\
\hline 17354764 & $-2,541071114$ & 0,006598656 & 0,183666593 & 0,673204769 & Arhgef37 & Rho guanine nucleotide exchange factor (GEF) 37 \\
\hline 17415219 & $-2,541925716$ & 0,006586563 & 0,183666593 & 0,764944787 & Acer2 & alkaline ceramidase 2 \\
\hline 17301576 & $-2,54257559$ & 0,006575333 & 0,183664448 & 0,51894453 & Gnrh1 & gonadotropin releasing hormone 1 \\
\hline 17263837 & $-2,546844978$ & 0,006529119 & 0,182803277 & 0,764126348 & Epn2 & epsin 2 \\
\hline 17388551 & $-2,546893617$ & 0,006527823 & 0,182803277 & 0,729518007 & Alkbh3 & alkB, alkylation repair homolog 3 (E. coli) \\
\hline 17401041 & $-2,54718384$ & 0,006524368 & 0,182803277 & 0,64039461 & Casq2 & calsequestrin 2 \\
\hline 17274415 & $-2,547631489$ & 0,006518753 & 0,182803277 & 0,782585177 & Asap2 & ArfGAP with $\mathrm{SH} 3$ domain, ankyrin repeat and $\mathrm{PH}$ domain 2 \\
\hline 17323192 & $-2,548586565$ & 0,006509683 & 0,182803277 & 0,665701162 & Snai2 & snail family zinc finger 2 \\
\hline 17497044 & $-2,548985289$ & 0,006503637 & 0,182803277 & 0,620950933 & Ikzf5 & IKAROS family zinc finger 5 \\
\hline 17310432 & $-2,553274449$ & 0,006436259 & 0,181777348 & 0,64087704 & Mtmr12 & myotubularin related protein 12 \\
\hline 17473219 & $-2,553384254$ & 0,006434964 & 0,181777348 & 0,807714072 & Tsen34 & tRNA splicing endonuclease 34 homolog (S. cerevisiae) \\
\hline 17497421 & $-2,554435716$ & 0,006423302 & 0,181722039 & 0,65820401 & Bnip3 & $\mathrm{BCL} 2$ /adenovirus $\mathrm{E} 1 \mathrm{~B}$ interacting protein 3 \\
\hline 17497687 & $-2,555936938$ & 0,006401707 & 0,181266279 & 0,642662774 & Bet1l & blocked early in transport 1 homolog (S. cerevisiae)-like \\
\hline 17360216 & $-2,556471106$ & 0,00639566 & 0,181250378 & 0,580484835 & Gsto1 & glutathione S-transferase omega 1 \\
\hline 17399914 & $-2,556684235$ & 0,006391773 & 0,181250378 & 0,728164336 & Lce3d & late cornified envelope 3D \\
\hline 17280247 & $-2,557542789$ & 0,00637968 & 0,181250378 & 0,808351755 & 6030426L16Rik & RIKEN cDNA 6030426L16 gene \\
\hline 17338562 & $-2,559290579$ & 0,006358516 & 0,181250378 & 0,591810537 & Rab5a & RAB5A, member RAS oncogene family \\
\hline 17455231 & $-2,564868215$ & 0,00628682 & 0,181250378 & 0,539023618 & B230303012Rik & RIKEN cDNA B230303012 gene \\
\hline 17223793 & $-2,566114705$ & 0,006272135 & 0,181250378 & 0,538160081 & Fzd5 & frizzled homolog 5 (Drosophila) \\
\hline 17511259 & $-2,56785717$ & 0,006251404 & 0,181250378 & 0,616698437 & Junb & jun B proto-oncogene \\
\hline 17498032 & $-2,568871151$ & 0,00623931 & 0,181250378 & 0,59953482 & Mob2 & MOB kinase activator 2 \\
\hline 17256691 & $-2,56991057$ & 0,006227649 & 0,181250378 & 0,704756948 & Aoc2 & amine oxidase, copper containing 2 (retina-specific) \\
\hline 17245539 & $-2,570650658$ & 0,006219011 & 0,181250378 & 0,683037052 & Srgap1 & SLIT-ROBO Rho GTPase activating protein 1 \\
\hline 17327450 & $-2,570690228$ & 0,006217715 & 0,181250378 & 0,658200203 & Kcnj15 & potassium inwardly-rectifying channel, subfamily J, member 15 \\
\hline 17334749 & $-2,570763036$ & 0,006217283 & 0,181250378 & 0,7656193 & Narfl & nuclear prelamin A recognition factor-like \\
\hline 17319554 & $-2,571781758$ & 0,006201303 & 0,181250378 & 0,651679162 & Pmm1 & phosphomannomutase 1 \\
\hline 17344990 & $-2,571962197$ & 0,006199575 & 0,181250378 & 0,53083904 & Cenpq & centromere protein $Q$ \\
\hline 17339574 & $-2,574694349$ & 0,006167614 & 0,181250378 & 0,541281906 & Ehd3 & EH-domain containing 3 \\
\hline 17326816 & $-2,578933327$ & 0,006107579 & 0,181229766 & 0,716719895 & Gabpa & GA repeat binding protein, alpha \\
\hline 17292800 & $-2,578941992$ & 0,006106715 & 0,181229766 & 0,672990221 & Uimc1 & ubiquitin interaction motif containing 1 \\
\hline 17359449 & $-2,580315302$ & 0,006080369 & 0,181171751 & 0,652432117 & Gm340 & predicted gene 340 \\
\hline 17519568 & $-2,584695794$ & 0,006023789 & 0,180135616 & 0,78242384 & Gm19569 & predicted gene, 19569 \\
\hline 17523281 & $-2,58517594$ & 0,006017311 & 0,180104871 & 0,751641644 & Trak1 & trafficking protein, kinesin binding 1 \\
\hline 17450727 & $-2,589900355$ & 0,005959435 & 0,179280713 & 0,555872881 & Tmed5 & $\begin{array}{l}\text { transmembrane emp } 24 \text { protein transport domain containing } 5 \\
\text { nuclear factor of activated T cells, cytoplasmic, calcineurin dependent }\end{array}$ \\
\hline 17496183 & $-2,591950462$ & 0,005929634 & 0,178776083 & 0,735562411 & Nfatc2ip & 2 interacting protein \\
\hline 17328451 & $-2,592978075$ & 0,005920996 & 0,178776083 & 0,635783393 & Mzt2 & mitotic spindle organizing protein 2 \\
\hline 17211405 & $-2,595194891$ & 0,005894218 & 0,178776083 & 0,729772749 & Gsta3 & glutathione S-transferase, alpha 3 \\
\hline 17376252 & $-2,60021396$ & 0,005841093 & 0,178220547 & 0,6842111 & Nop56 & NOP56 ribonucleoprotein \\
\hline 17526614 & $-2,604116768$ & 0,005794879 & 0,177630577 & 0,669812652 & Pafah1b2 & platelet-activating factor acetylhydrolase, isoform $1 \mathrm{~b}$, subunit 2 \\
\hline 17222001 & $-2,604622014$ & 0,005791424 & 0,177630577 & 0,668269592 & Prim2 & DNA primase, $\mathrm{p} 58$ subunit \\
\hline 17510563 & $-2,606851859$ & 0,005768965 & 0,177630577 & 0,737027415 & Slc35e1 & solute carrier family 35, member E1 \\
\hline 17379417 & $-2,61233766$ & 0,005703747 & 0,17675478 & 0,633331235 & Sys1 & $\begin{array}{l}\text { SYS1 Golgi-localized integral membrane protein homolog (S. } \\
\text { cerevisiae) }\end{array}$ \\
\hline 17366039 & $-2,613876477$ & 0,005682584 & 0,176641293 & 0,683774407 & Pdzd8 & PDZ domain containing 8 \\
\hline 17299924 & $-2,615495996$ & 0,00566574 & 0,176454732 & 0,632618372 & Rps19 & ribosomal protein $\mathrm{S} 19$ \\
\hline 17299847 & $-2,615495996$ & 0,00566574 & 0,176454732 & 0,632618372 & Rps19 & ribosomal protein S19 \\
\hline 17234042 & $-2,616348527$ & 0,005656238 & 0,176454732 & 0,692929988 & Rhobtb1 & Rho-related BTB domain containing 1 \\
\hline 17323759 & $-2,616492732$ & 0,005655374 & 0,176454732 & 0,716603763 & Ufd1l & $\begin{array}{l}\text { ubiquitin fusion degradation } 1 \text { like } \\
\text { FERM, RhoGEF (Arhgef) and pleckstrin domain protein } 1\end{array}$ \\
\hline 17302802 & $-2,618405952$ & 0,005631619 & 0,176389566 & 0,787319767 & Farp1 & (chondrocyte-derived) \\
\hline 17277084 & $-2,619507906$ & 0,005622117 & 0,176259024 & 0,728845203 & Psen1 & presenilin 1 \\
\hline 17478864 & $-2,620770381$ & 0,005610456 & 0,176060466 & 0,631549514 & Mtmr10 & myotubularin related protein 10 \\
\hline 17454416 & $-2,622212535$ & 0,00559793 & 0,175834397 & 0,366636307 & Zfand2a & $\begin{array}{l}\text { zinc finger, AN1-type domain 2A } \\
\text { solute carrier family } 25 \text { (mitochondrial carrier, phosphate carrier), }\end{array}$ \\
\hline 17244175 & $-2,624796715$ & 0,005565969 & 0,175330474 & 0,70873077 & Slc25a3 & member 3 \\
\hline 17463883 & $-2,625266183$ & 0,00556165 & 0,175330474 & 0,662442292 & Strap & serine/threonine kinase receptor associated protein \\
\hline 17522971 & $-2,625563305$ & 0,005555604 & 0,175330474 & 0,662778337 & Vill & villin-like \\
\hline 17337513 & $-2,625791271$ & 0,005553444 & 0,175330474 & 0,766430093 & Gabbr1 & gamma-aminobutyric acid (GABA) B receptor, 1 \\
\hline 17237851 & $-2,626493184$ & 0,005543942 & 0,175305073 & 0,785328727 & Avil & advillin \\
\hline 17442909 & $-2,627890648$ & 0,005526234 & 0,175281605 & 0,678336469 & Gbas & glioblastoma amplified sequence \\
\hline 17371661 & $-2,628673989$ & 0,005515868 & 0,175255894 & 0,79092066 & Gorasp2 & golgi reassembly stacking protein 2 \\
\hline 17448607 & $-2,630949662$ & 0,005492113 & 0,175255894 & 0,650875127 & Commd8 & COMM domain containing 8 \\
\hline
\end{tabular}




\begin{tabular}{|c|c|c|c|c|c|c|}
\hline $\begin{array}{l}\text { probeset } \\
\text { ID }\end{array}$ & d.value & p.value & q.value & R.fold & Genename & Description \\
\hline 17514745 & $-2,631887548$ & 0,005483907 & 0,175255894 & 0,617876931 & Cwc15 & CWC15 homolog (S. cerevisiae) \\
\hline 17515045 & $-2,633336813$ & 0,005465335 & 0,175255894 & 0,581788798 & Ppan & peter pan homolog (Drosophila) \\
\hline 17368947 & $-2,63336743$ & 0,005464903 & 0,175255894 & 0,743592136 & Odf2 & outer dense fiber of sperm tails 2 \\
\hline 17271931 & $-2,63411414$ & 0,005456697 & 0,175255894 & 0,703313195 & Sumo2 & SMT3 suppressor of mif two 3 homolog 2 (yeast) \\
\hline 17326801 & $-2,636456437$ & 0,005432079 & 0,175255894 & 0,617708271 & Jam2 & junction adhesion molecule 2 \\
\hline 17443133 & $-2,63719045$ & 0,0054256 & 0,175255894 & 0,731736973 & Wbscr27 & Williams Beuren syndrome chromosome region 27 (human) \\
\hline 17249140 & $-2,638850463$ & 0,005407028 & 0,175255894 & 0,748149099 & 3010026009Rik & RIKEN cDNA 3010026009 gene \\
\hline 17492239 & $-2,638872953$ & 0,005406164 & 0,175255894 & 0,696284176 & AU020206 & expressed sequence AU020206 \\
\hline 17412552 & $-2,641472762$ & 0,005374203 & 0,175255894 & 0,646276466 & Ube2j1 & ubiquitin-conjugating enzyme E2J 1 \\
\hline 17262768 & $-2,641813714$ & 0,005370316 & 0,175255894 & 0,648609204 & Slc22a21 & solute carrier family 22 (organic cation transporter), member 21 \\
\hline 17533234 & $-2,642946783$ & 0,005358223 & 0,175255894 & 0,661347211 & Sytl5 & $\begin{array}{l}\text { synaptotagmin-like } 5 \\
\text { polycystic kidney disease (polycystin) and REJ (sperm receptor for egg }\end{array}$ \\
\hline 17320100 & $-2,643270686$ & 0,005354767 & 0,175255894 & 0,773630324 & Pkdrej & jelly homolog, sea urchin) \\
\hline 17318587 & $-2,644250407$ & 0,005342674 & 0,175255894 & 0,576918048 & Slc39a4 & solute carrier family 39 (zinc transporter), member 4 \\
\hline 17452396 & $-2,644408803$ & 0,005340083 & 0,175255894 & 0,784052782 & Fam216a & family with sequence similarity 216, member $A$ \\
\hline 17370309 & $-2,647746974$ & 0,005308553 & 0,174901557 & 0,556445032 & Ptgs1 & prostaglandin-endoperoxide synthase 1 \\
\hline 17469556 & $-2,647751481$ & 0,005308122 & 0,174901557 & 0,684449735 & Crbn & cereblon \\
\hline 17245850 & $-2,648349665$ & 0,005300779 & 0,174901557 & 0,65999114 & Mars & methionine-tRNA synthetase \\
\hline 17288387 & $-2,648493938$ & 0,00529862 & 0,174901557 & 0,761178211 & Nsun2 & NOL1/NOP2/Sun domain family member 2 \\
\hline 17304860 & $-2,649685851$ & 0,005286526 & 0,174901557 & 0,663727425 & Timm23 & translocase of inner mitochondrial membrane 23 \\
\hline 17349745 & $-2,650716874$ & 0,005277024 & 0,174901557 & 0,75270613 & lk & IK cytokine \\
\hline 17333854 & $-2,651528861$ & 0,005264931 & 0,174901557 & 0,615362746 & Ppp2r1a & protein phosphatase 2 , regulatory subunit $A$, alpha \\
\hline 17253972 & $-2,6520956$ & 0,005258452 & 0,174901557 & 0,623804796 & Psmd11 & proteasome (prosome, macropain) 26S subunit, non-ATPase, 11 \\
\hline 17269368 & $-2,653108381$ & 0,0052442 & 0,174901557 & 0,566773446 & Krt19 & keratin 19 \\
\hline 17352120 & $-2,654372022$ & 0,005231674 & 0,174901557 & 0,66554991 & Pard6g & par-6 family cell polarity regulator gamma \\
\hline 17331438 & $-2,654766189$ & 0,005226491 & 0,174901557 & 0,769791633 & Chmp2b & charged multivesicular body protein $2 \mathrm{~B}$ \\
\hline 17353796 & $-2,655642458$ & 0,005218285 & 0,174901557 & 0,594846218 & Taf7 & $\begin{array}{l}\text { TAF7 RNA polymerase II, TATA box binding protein (TBP)-associated } \\
\text { factor }\end{array}$ \\
\hline 17533746 & $-2,65573428$ & 0,005216126 & 0,174901557 & 0,723096003 & Agtr2 & angiotensin II receptor, type 2 \\
\hline 17512833 & $-2,664127643$ & 0,005127585 & 0,174901557 & 0,73046813 & Ist1 & increased sodium tolerance 1 homolog (yeast) \\
\hline 17262783 & $-2,66644136$ & 0,005102534 & 0,174901557 & 0,726446306 & Slc22a4 & solute carrier family 22 (organic cation transporter), member 4 \\
\hline 17398043 & $-2,667184195$ & 0,005092169 & 0,174901557 & 0,692908997 & Vmn2r1 & vomeronasal 2 , receptor 1 \\
\hline 17531154 & $-2,667203229$ & 0,005091737 & 0,174901557 & 0,733496752 & Bsn & bassoon \\
\hline 17479303 & $-2,670901419$ & 0,005051138 & 0,174834218 & 0,65753289 & Aen & apoptosis enhancing nuclease \\
\hline 17338233 & $-2,671984142$ & 0,005038612 & 0,174834218 & 0,638633305 & Mrps10 & $\begin{array}{l}\text { mitochondrial ribosomal protein S10 } \\
\text { TATA box binding protein (Tbp)-associated factor, RNA polymerase I, }\end{array}$ \\
\hline 17514806 & $-2,672162946$ & 0,005036021 & 0,174834218 & 0,71156109 & Taf1d & D \\
\hline 17361032 & $-2,672922179$ & 0,005026087 & 0,174822971 & 0,792850817 & Ndufs8 & NADH dehydrogenase (ubiquinone) Fe-S protein 8 \\
\hline 17250374 & $-2,673237391$ & 0,005023064 & 0,174822971 & 0,73845289 & Drg2 & developmentally regulated GTP binding protein 2 \\
\hline 17507094 & $-2,674917121$ & 0,005005356 & 0,174653402 & 0,742652221 & Xab2 & XPA binding protein 2 \\
\hline 17383284 & $-2,675053376$ & 0,005003196 & 0,174653402 & 0,828941042 & Vav2 & vav 2 oncogene \\
\hline 17459614 & $-2,675948716$ & 0,004994126 & 0,174630374 & 0,757689235 & 0610030E20Rik & RIKEN CDNA 0610030E20 gene \\
\hline 17517914 & $-2,677056042$ & 0,004982465 & 0,174407164 & 0,765272604 & Nptn & neuroplastin \\
\hline 17529647 & $-2,679319512$ & 0,004958278 & 0,173744578 & 0,499188659 & Morf4l1 & mortality factor 4 like 1 \\
\hline 17509682 & $-2,682520629$ & 0,004924589 & 0,17311482 & 0,52785366 & ВС030870 & cDNA sequence $\mathrm{BC} 030870$ \\
\hline 17373825 & $-2,683266564$ & 0,004916383 & 0,1730104 & 0,353978165 & Cd59a & CD59a antigen \\
\hline 17276386 & $-2,684230462$ & 0,004906017 & 0,1730104 & 0,618383974 & Rhoj & ras homolog gene family, member J \\
\hline 17281908 & $-2,685225943$ & 0,004898675 & 0,172939763 & 0,748004605 & Tmem30b & transmembrane protein $30 \mathrm{~B}$ \\
\hline 17359803 & $-2,687621799$ & 0,00487492 & 0,172469663 & 0,651471734 & Sfxn3 & sideroflexin 3 \\
\hline 17335467 & $-2,68834531$ & 0,004867578 & 0,172394473 & 0,298237151 & Cdkn1a & cyclin-dependent kinase inhibitor $1 \mathrm{~A}(\mathrm{P} 21)$ \\
\hline 17227464 & $-2,68942266$ & 0,004858076 & 0,172242556 & 0,702641089 & 5730559C18Rik & RIKEN CDNA 5730559 C18 gene \\
\hline 17324762 & $-2,691829135$ & 0,004831298 & 0,171477128 & 0,724984675 & Rnf168 & ring finger protein 168 \\
\hline 17294368 & $-2,694329859$ & 0,004803224 & 0,171216326 & 0,541978321 & Pdcd6 & programmed cell death 6 \\
\hline 17386396 & $-2,694769255$ & 0,004799768 & 0,171216326 & 0,641300545 & Slc25a12 & solute carrier family 25 (mitochondrial carrier, Aralar), member 12 \\
\hline 17376378 & $-2,694987373$ & 0,004797609 & 0,171216326 & 0,741134925 & Itpa & inosine triphosphatase (nucleoside triphosphate pyrophosphatase) \\
\hline 17344794 & $-2,697204468$ & 0,004778605 & 0,171216326 & 0,463904845 & Znrd1 & zinc ribbon domain containing, 1 \\
\hline 17357640 & $-2,700784828$ & 0,00474578 & 0,171216326 & 0,5234096 & Ms4a4a & membrane-spanning 4-domains, subfamily $\mathrm{A}$, member $4 \mathrm{~A}$ \\
\hline 17506042 & $-2,707981576$ & 0,004678835 & 0,170836722 & 0,64113798 & Cdh13 & cadherin 13 \\
\hline 17245383 & $-2,713910773$ & 0,004623551 & 0,169756037 & 0,637763452 & Helb & helicase (DNA) B \\
\hline 17457343 & $-2,714704844$ & 0,004617936 & 0,169738486 & 0,560431806 & Atp6v0c & ATPase, $\mathrm{H}+$ transporting, lysosomal VO subunit $\mathrm{C}$ \\
\hline 17291833 & $-2,71495106$ & 0,004615345 & 0,169738486 & 0,651694114 & Eci2 & enoyl-Coenzyme A delta isomerase 2 \\
\hline 17414802 & $-2,716110854$ & 0,00460757 & 0,169738486 & 0,442857133 & Pappa & pregnancy-associated plasma protein A \\
\hline 17346175 & $-2,716445687$ & 0,004603683 & 0,169738486 & 0,701397021 & Mydgf & myeloid derived growth factor \\
\hline 17414416 & $-2,718043851$ & 0,004585975 & 0,169738486 & 0,760183774 & Dnajc25 & DnaJ (Hsp40) homolog, subfamily C, member 25 \\
\hline 17526917 & $-2,719138653$ & 0,004576905 & 0,169738486 & 0,765064316 & 2310030G06Rik & RIKEN CDNA $2310030 \mathrm{G} 06$ gene \\
\hline 17378628 & $-2,72211682$ & 0,004554014 & 0,169462457 & 0,663841499 & Aar2 & AAR2 splicing factor homolog (S. cerevisiae) \\
\hline 17527977 & $-2,725253598$ & 0,004521189 & 0,169193657 & 0,630720734 & Glce & glucuronyl C5-epimerase \\
\hline 17486110 & $-2,731947434$ & 0,004454676 & 0,168614124 & 0,550626816 & Peg3 & paternally expressed 3 \\
\hline 17434310 & $-2,733188407$ & 0,004443446 & 0,168545619 & 0,556552899 & Fam133b & family with sequence similarity 133 , member $B$ \\
\hline 17470187 & $-2,733429711$ & 0,004440423 & 0,168545619 & 0,68765518 & Zfp9 & zinc finger protein 9 \\
\hline 17290083 & $-2,734867464$ & 0,004426602 & 0,168516657 & 0,74520962 & $\mathrm{Emb}$ & embigin \\
\hline 17255387 & $-2,735038979$ & 0,004424874 & 0,168516657 & 0,69471919 & Slc35b1 & solute carrier family 35, member B1 \\
\hline 17240199 & $-2,735765254$ & 0,004419691 & 0,168516657 & 0,614379542 & Nt5dc1 & $\begin{array}{l}\text { 5'-nucleotidase domain containing } 1 \\
\text { solute carrier family } 7 \text { (cationic amino acid transporter, y+ system), }\end{array}$ \\
\hline 17306477 & $-2,738227733$ & 0,004393345 & 0,167830663 & 0,688707645 & Slc7a8 & $\begin{array}{l}\text { member } 8 \\
\text { gamma-aminobutyric acid (GABA) A receptor-associated protein-like }\end{array}$ \\
\hline 17505774 & $-2,740217957$ & 0,004376069 & 0,167830663 & 0,725892034 & Gabarapl2 & 2 \\
\hline 17309065 & $-2,745347205$ & 0,004332878 & 0,167619692 & 0,695111508 & Mzt1 & mitotic spindle organizing protein 1 \\
\hline 17324274 & $-2,745612561$ & 0,004330287 & 0,167619692 & 0,642755047 & Senp2 & SUMO/sentrin specific peptidase 2 \\
\hline 17397344 & $-2,747310177$ & 0,004314306 & 0,167522644 & 0,679516798 & Jade1 & jade family PHD finger 1 \\
\hline 17541404 & $-2,747977485$ & 0,004309124 & 0,167518244 & 0,695152799 & Elf4 & E74-like factor 4 (ets domain transcription factor) \\
\hline
\end{tabular}




\begin{tabular}{|c|c|c|c|c|c|c|}
\hline $\begin{array}{l}\text { probeset } \\
\text { ID }\end{array}$ & d.value & p.value & q.value & R.fold & Genename & Description \\
\hline 17514832 & $-2,755338322$ & 0,004245202 & 0,166668489 & 0,632339155 & Taf1d & $\begin{array}{l}\text { TATA box binding protein (Tbp)-associated factor, RNA polymerase I, } \\
\text { D }\end{array}$ \\
\hline 17339529 & $-2,755541526$ & 0,004243474 & 0,166668489 & 0,54056209 & Clip4 & CAP-GLY domain containing linker protein family, member 4 \\
\hline 17425077 & $-2,757718462$ & 0,004221879 & 0,166668489 & 0,69635286 & Tbc1d2 & TBC1 domain family, member 2 \\
\hline 17327331 & $-2,757834385$ & 0,004220151 & 0,166668489 & 0,621553797 & Chaf1b & chromatin assembly factor 1 , subunit B (p60) \\
\hline 17259794 & $-2,763469001$ & 0,00417005 & 0,166668489 & 0,53911254 & Rnf185 & ring finger protein 185 \\
\hline 17376728 & $-2,763519677$ & 0,004168754 & 0,166668489 & 0,608161941 & Plcb4 & phospholipase $C$, beta 4 \\
\hline 17468520 & $-2,764192819$ & 0,004160548 & 0,166668489 & 0,750245536 & Snrnp27 & small nuclear ribonucleoprotein 27 (U4/U6.U5) \\
\hline 17303849 & $-2,766514473$ & 0,004145863 & 0,166668489 & 0,593515645 & Usp54 & ubiquitin specific peptidase 54 \\
\hline 17337228 & $-2,767751845$ & 0,004136361 & 0,166668489 & 0,470759535 & ler3 & immediate early response 3 \\
\hline 17335383 & $-2,769413109$ & 0,004121245 & 0,166668489 & 0,569199605 & Mapk13 & mitogen-activated protein kinase 13 \\
\hline 17479183 & $-2,773217809$ & 0,004086692 & 0,166668489 & 0,739552422 & Fam174b & family with sequence similarity 174 , member $B$ \\
\hline 17308881 & $-2,775050125$ & 0,004072871 & 0,166668489 & 0,668594339 & Rgcc & regulator of cell cycle \\
\hline 17246520 & $-2,779696262$ & 0,004041342 & 0,165684795 & 0,587135566 & Eif4enif1 & $\begin{array}{l}\text { eukaryotic translation initiation factor } 4 \text { E nuclear import factor } 1 \\
\text { KDEL (Lys-Asp-Glu-Leu) endoplasmic reticulum protein retention }\end{array}$ \\
\hline 17313000 & $-2,78126475$ & 0,004027521 & 0,165684795 & 0,64154094 & Kdelr3 & receptor 3 \\
\hline 17523861 & $-2,781305583$ & 0,004027089 & 0,165684795 & 0,641725926 & C330006D17Rik & RIKEN cDNA C330006D17 gene \\
\hline 17465942 & $-2,781636575$ & 0,004024498 & 0,165684795 & 0,411732126 & Atp6v0a4 & ATPase, $\mathrm{H}+$ transporting, lysosomal V0 subunit $\mathrm{A} 4$ \\
\hline 17550426 & $-2,783900712$ & 0,004008085 & 0,165553763 & 0,516733223 & Neat1 & nuclear paraspeckle assembly transcript 1 (non-protein coding) \\
\hline 17390249 & $-2,785898521$ & 0,0039934 & 0,165225099 & 0,677831841 & Lrrc57 & leucine rich repeat containing 57 \\
\hline 17364545 & $-2,787841075$ & 0,003976124 & 0,165058909 & 0,444027674 & Tctn3 & tectonic family member 3 \\
\hline 17393357 & $-2,795813643$ & 0,003916089 & 0,163801393 & 0,616693759 & Eif6 & eukaryotic translation initiation factor 6 \\
\hline 17458112 & $-2,80083754$ & 0,003871171 & 0,162953918 & 0,639585708 & Zfp398 & zinc finger protein 398 \\
\hline 17315946 & $-2,803407163$ & 0,003851303 & 0,162739538 & 0,678459253 & Dnajc21 & DnaJ (Hsp40) homolog, subfamily C, member 21 \\
\hline 17453205 & $-2,807766855$ & 0,003815455 & 0,162262231 & 0,658980052 & Sbds & Shwachman-Bodian-Diamond syndrome homolog (human) \\
\hline 17420347 & $-2,810183289$ & 0,003797315 & 0,161864409 & 0,722690664 & Ece1 & endothelin converting enzyme 1 \\
\hline 17211305 & $-2,811517469$ & 0,003789973 & 0,161803246 & 0,369164268 & Pi15 & peptidase inhibitor 15 \\
\hline 17299869 & $-2,822636784$ & 0,003701864 & 0,159369283 & 0,581031863 & Trav9d-3 & T cell receptor alpha variable 9D-3 \\
\hline 17431332 & $-2,822778262$ & 0,003699705 & 0,159369283 & 0,522164933 & Man1c1 & mannosidase, alpha, class $1 \mathrm{C}$, member 1 \\
\hline 17333465 & $-2,827291114$ & 0,003672063 & 0,159029476 & 0,475035242 & Smoc2 & SPARC related modular calcium binding 2 \\
\hline 17363374 & $-2,829136878$ & 0,003658674 & 0,158657562 & 0,511484521 & Ostf1 & osteoclast stimulating factor 1 \\
\hline 17288898 & $-2,833041462$ & 0,003633191 & 0,158209654 & 0,664284373 & Lysmd3 & LysM, putative peptidoglycan-binding, domain containing 3 \\
\hline 17349181 & $-2,835298323$ & 0,003618938 & 0,158209654 & 0,662003169 & Wdr33 & WD repeat domain 33 \\
\hline 17446775 & $-2,836700914$ & 0,003609868 & 0,158202043 & 0,659102403 & Preb & prolactin regulatory element binding \\
\hline 17222527 & $-2,837987894$ & 0,003599071 & 0,158202043 & 0,428180789 & Tbc1d8 & TBC1 domain family, member 8 \\
\hline 17312301 & $-2,840091064$ & 0,003583954 & 0,158114857 & 0,743693985 & Zfp707 & zinc finger protein 707 \\
\hline 17532455 & $-2,841218778$ & 0,00357618 & 0,157982799 & 0,515684212 & 1110059G10Rik & RIKEN CDNA 1110059 G10 gene \\
\hline 17506697 & $-2,842981118$ & 0,003565814 & 0,157947197 & 0,415862105 & Rhou & ras homolog gene family, member $U$ \\
\hline 17277134 & $-2,844512396$ & 0,003554152 & 0,157871853 & 0,609387983 & Acot2 & acyl-CoA thioesterase 2 \\
\hline 17364813 & $-2,844907076$ & 0,003550265 & 0,157871853 & 0,492644027 & Avpi1 & arginine vasopressin-induced 1 \\
\hline 17402181 & $-2,845262259$ & 0,003548969 & 0,157871853 & 0,43002184 & F3 & coagulation factor III \\
\hline 17453242 & $-2,848236859$ & 0,003530829 & 0,157871853 & 0,702887869 & Auts2 & autism susceptibility candidate 2 \\
\hline 17459250 & $-2,850424486$ & 0,003516145 & 0,15780396 & 0,759080524 & Gng12 & guanine nucleotide binding protein (G protein), gamma 12 \\
\hline 17309420 & $-2,851785456$ & 0,003509666 & 0,15780396 & 0,674755276 & Abcc4 & ATP-binding cassette, sub-family C (CFTR/MRP), member 4 \\
\hline 17306333 & $-2,853344362$ & 0,003498005 & 0,15780396 & 0,634075011 & Dad1 & defender against cell death 1 \\
\hline 17530406 & $-2,85467586$ & 0,003489366 & 0,15780396 & 0,673026503 & Acpp & acid phosphatase, prostate \\
\hline 17396614 & $-2,855539144$ & 0,003485479 & 0,15780396 & 0,766754821 & Prkci & protein kinase $\mathrm{C}$, iota \\
\hline 17340232 & $-2,856393878$ & 0,00348116 & 0,15780396 & 0,604969375 & Cript & cysteine-rich PDZ-binding protein \\
\hline 17257639 & $-2,858179968$ & 0,003469067 & 0,15780396 & 0,400220306 & 1810010H24Rik & RIKEN cDNA $1810010 \mathrm{H} 24$ gene \\
\hline 17436791 & $-2,860802131$ & 0,003450063 & 0,15780396 & 0,588290921 & Mir3097 & microRNA 3097 \\
\hline 17498199 & $-2,861743951$ & 0,003444016 & 0,15780396 & 0,685832791 & R74862 & expressed sequence $R 74862$ \\
\hline 17523994 & $-2,863694605$ & 0,003434083 & 0,15780396 & 0,686936842 & Cep57 & centrosomal protein 57 \\
\hline 17334241 & $-2,865718644$ & 0,003414215 & 0,157568688 & 0,670905447 & Rnps1 & ribonucleic acid binding protein S1 \\
\hline 17480312 & $-2,86824249$ & 0,003395211 & 0,157390409 & 0,570905384 & Gab2 & growth factor receptor bound protein 2-associated protein 2 \\
\hline 17467197 & $-2,870464458$ & 0,003378799 & 0,157390409 & 0,723038751 & Vmn1r27 & vomeronasal 1 receptor 27 \\
\hline 17306906 & $-2,872336583$ & 0,003366273 & 0,157333808 & 0,709115308 & Ripk3 & receptor-interacting serine-threonine kinase 3 \\
\hline 17375083 & $-2,876896935$ & 0,003329129 & 0,156929906 & 0,581598741 & Snap23 & synaptosomal-associated protein 23 \\
\hline 17243171 & $-2,878067666$ & 0,003319628 & 0,156929906 & 0,735257937 & Sgta & small glutamine-rich tetratricopeptide repeat (TPR)-containing, alpha \\
\hline 17454794 & $-2,878523691$ & 0,003316604 & 0,156929906 & 0,593664009 & Rbak & RB-associated KRAB repressor \\
\hline 17271968 & $-2,879556918$ & 0,003310126 & 0,156929906 & 0,72579217 & Mif4gd & MIF4G domain containing \\
\hline 17221215 & $-2,881478644$ & 0,003295441 & 0,156929906 & 0,67872552 & & $\begin{array}{l}\text { COP9 (constitutive photomorphogenic) homolog, subunit } 5 \\
\text { (Arabidopsis thaliana) }\end{array}$ \\
\hline 17219643 & $\begin{array}{l}-2,0014 / 8044 \\
-2,884187716\end{array}$ & 0,003280324 & 0,156929906 & 0,786531027 & Aim2 & absent in melanoma 2 \\
\hline 17478998 & $-2,885880821$ & 0,00327039 & 0,156929906 & 0,606187619 & Vimp & VCP-interacting membrane protein \\
\hline 17286354 & $-2,887874145$ & 0,003255705 & 0,156929906 & 0,583372733 & Serpinb6b & serine (or cysteine) peptidase inhibitor, clade $B$, member $6 b$ \\
\hline 17478942 & $-2,889643763$ & 0,003246635 & 0,156929906 & 0,631472797 & $\operatorname{Tm} 2 \mathrm{~d} 3$ & TM2 domain containing 3 \\
\hline 17516699 & $-2,892183784$ & 0,003232814 & 0,156927765 & 0,489560627 & Mpzl2 & myelin protein zero-like 2 \\
\hline 17428054 & $-2,89472776$ & 0,003218562 & 0,156633315 & 0,649509314 & Gpx7 & glutathione peroxidase 7 \\
\hline 17276139 & $-2,89535071$ & 0,003212083 & 0,156548588 & 0,573291672 & Dact1 & dapper homolog 1 , antagonist of beta-catenin (xenopus) \\
\hline 17399519 & $-2,896452995$ & 0,003205173 & 0,156548588 & 0,637401685 & Ube2q1 & ubiquitin-conjugating enzyme $E 2 Q$ (putative) 1 \\
\hline 17325093 & $-2,898678324$ & 0,00319092 & 0,156548588 & 0,647477629 & Muc13 & mucin 13 , epithelial transmembrane \\
\hline 17305221 & $-2,901217807$ & 0,003173643 & 0,156548588 & 0,556694248 & Fam213a & family with sequence similarity 213 , member $A$ \\
\hline 17391137 & $-2,902579033$ & 0,003165437 & 0,156548588 & 0,627357297 & Usp50 & ubiquitin specific peptidase 50 \\
\hline 17252170 & $-2,902980154$ & 0,003160686 & 0,156548588 & 0,564436041 & Rnf167 & ring finger protein 167 \\
\hline 17338472 & $-2,904412407$ & 0,003150752 & 0,156548588 & 0,570003757 & Mocs1 & molybdenum cofactor synthesis 1 \\
\hline 17379337 & $-2,906410464$ & 0,003136931 & 0,156548588 & 0,679466548 & Pabpc1l & poly $(\mathrm{A})$ binding protein, cytoplasmic 1 -like \\
\hline 17278073 & $-2,907920194$ & 0,003126134 & 0,156548588 & 0,60756407 & Golga5 & golgi autoantigen, golgin subfamily a, 5 \\
\hline 17432790 & $-2,910318428$ & 0,003115336 & 0,156548588 & 0,740855034 & Miip & migration and invasion inhibitory protein \\
\hline 17476866 & $-2,911514985$ & 0,003109289 & 0,156548588 & 0,755984695 & Tshz3 & teashirt zinc finger family member 3 \\
\hline 17501748 & $-2,9169817$ & 0,00307992 & 0,156115062 & 0,731911879 & Lpar2 & lysophosphatidic acid receptor 2 \\
\hline 17511377 & $-2,917357949$ & 0,003076465 & 0,156115062 & 0,399082015 & Neto2 & neuropilin (NRP) and tolloid (TLL)-like 2 \\
\hline
\end{tabular}




\begin{tabular}{|c|c|c|c|c|c|c|}
\hline $\begin{array}{l}\text { probeset } \\
\text { ID }\end{array}$ & d.value & p.value & q.value & R.fold & Genename & Description \\
\hline 17212874 & $-2,922889375$ & 0,003047959 & 0,155667125 & 0,40034037 & Coq10b & coenzyme Q10 homolog B (S. cerevisiae) \\
\hline 17434322 & $-2,92346396$ & 0,003043208 & 0,155665076 & 0,582549458 & NA & NA \\
\hline 17262575 & $-2,923977023$ & 0,003039753 & 0,155665076 & 0,680468229 & Ube2b & ubiquitin-conjugating enzyme E2B \\
\hline 17339460 & $-2,924478915$ & 0,003035434 & 0,155665076 & 0,390934376 & Lpin2 & lipin 2 \\
\hline 17379300 & $-2,926741977$ & 0,003018589 & 0,155367822 & 0,716735444 & 0610039K10Rik & RIKEN cDNA 0610039K10 gene \\
\hline 17285867 & $-2,928953441$ & 0,003006496 & 0,154986785 & 0,277871374 & Hist1h2ab & histone cluster 1, H2ab \\
\hline 17278110 & $-2,931061221$ & 0,002994403 & 0,154986785 & 0,659746808 & Ubr7 & ubiquitin protein ligase E3 component $n$-recognin 7 (putative) \\
\hline 17548498 & $-2,934899053$ & 0,002966761 & 0,154986785 & 0,471774487 & Gm10931 & predicted gene 10931 \\
\hline 17548294 & $-2,934899053$ & 0,002966761 & 0,154986785 & 0,471774487 & Gm10931 & predicted gene 10931 \\
\hline 17520560 & $-2,934915756$ & 0,002965897 & 0,154986785 & 0,620678896 & Tfdp2 & transcription factor $\mathrm{Dp} 2$ \\
\hline 17418413 & $-2,936511481$ & 0,002960714 & 0,154986785 & 0,706558314 & Gnl2 & guanine nucleotide binding protein-like 2 (nucleolar) \\
\hline 17340177 & $-2,93854291$ & 0,002945165 & 0,154986785 & 0,627239193 & Prkce & protein kinase $\mathrm{C}$, epsilon \\
\hline 17300251 & $-2,940000283$ & 0,002937823 & 0,154986785 & 0,551183972 & Abhd4 & abhydrolase domain containing 4 \\
\hline 17452209 & $-2,940104699$ & 0,002936527 & 0,154986785 & 0,813882741 & Mapkapk5 & MAP kinase-activated protein kinase 5 \\
\hline 17213580 & $-2,944413182$ & 0,002916228 & 0,154676898 & 0,764208062 & Eef1b2 & eukaryotic translation elongation factor 1 beta 2 \\
\hline 17468705 & $-2,948954722$ & 0,002889881 & 0,154554536 & 0,649242507 & Rab7 & RAB7, member RAS oncogene family \\
\hline 17464638 & $-2,955205287$ & 0,002854465 & 0,154499461 & 0,660399108 & Pon2 & paraoxonase 2 \\
\hline 17465924 & $-2,961750978$ & 0,002821208 & 0,153604887 & 0,758205304 & Svopl & SV2 related protein homolog (rat)-like \\
\hline 17535344 & $-2,962965749$ & 0,002810843 & 0,153604887 & 0,74143475 & Hmgb3 & high mobility group box 3 \\
\hline 17316057 & $-2,963196376$ & 0,002809979 & 0,153604887 & 0,699046058 & Sub1 & SUB1 homolog (S. cerevisiae) \\
\hline 17522430 & $-2,963305896$ & 0,002808683 & 0,153604887 & 0,698891063 & Ccdc12 & coiled-coil domain containing 12 \\
\hline 17531265 & $-2,964906188$ & 0,002798317 & 0,153604887 & 0,661295314 & Atrip & ATR interacting protein \\
\hline 17532045 & $-2,966402709$ & 0,00278536 & 0,153604887 & 0,503012573 & Plcd1 & phospholipase C, delta 1 \\
\hline 17443275 & $-2,974483855$ & 0,002743033 & 0,153133373 & 0,598569656 & Rhbdd2 & rhomboid domain containing 2 \\
\hline 17331642 & $-2,975078079$ & 0,002739578 & 0,153133373 & 0,798131229 & Mrpl39 & mitochondrial ribosomal protein L39 \\
\hline 17443972 & $-2,976104793$ & 0,0027331 & 0,153133373 & 0,690352306 & Get4 & golgi to ER traffic protein 4 homolog (S. cerevisiae) \\
\hline 17234667 & $-2,979301423$ & 0,002721006 & 0,153133373 & 0,437514462 & Pttg1ip & $\begin{array}{l}\text { pituitary tumor-transforming } 1 \text { interacting protein } \\
\text { glucosaminyl ( } \mathrm{N} \text {-acetyl) transferase } 4 \text {, core } 2 \text { (beta-1,6-N- }\end{array}$ \\
\hline 17289324 & $-2,982846927$ & 0,002705026 & 0,152916443 & 0,703496759 & Gent4 & acetylglucosaminyltransferase) \\
\hline 17241409 & $-2,983090787$ & 0,002704162 & 0,152916443 & 0,653022292 & Srgn & serglycin \\
\hline 17302061 & $-2,986356803$ & 0,002689045 & 0,152916443 & 0,544488517 & Nufip1 & nuclear fragile $\mathrm{X}$ mental retardation protein interacting protein 1 \\
\hline 17380185 & $-2,989257438$ & 0,002678679 & 0,152874224 & 0,668366125 & Rae1 & RAE1 RNA export 1 homolog (S. pombe) \\
\hline 17462395 & $-2,991552339$ & 0,002662267 & 0,152728896 & 0,429969007 & Slc25a18 & solute carrier family 25 (mitochondrial carrier), member 18 \\
\hline 17292689 & $-2,992155273$ & 0,00265838 & 0,152728896 & 0,424572015 & Thoc3 & THO complex 3 \\
\hline 17303804 & $-2,992202705$ & 0,002657516 & 0,152728896 & 0,701389748 & Anxa7 & annexin A7 \\
\hline 17301697 & $-2,992249601$ & 0,002657084 & 0,152728896 & 0,469105204 & Tnfrsf10b & tumor necrosis factor receptor superfamily, member $10 \mathrm{~b}$ \\
\hline 17439769 & $-3,003098324$ & 0,002600073 & 0,152587611 & 0,677476493 & Nudt9 & nudix (nucleoside diphosphate linked moiety X)-type motif 9 \\
\hline 17507623 & $-3,009902282$ & 0,002563361 & 0,151256401 & 0,724972922 & 1700029H14Rik & RIKEN CDNA $1700029 \mathrm{H} 14$ gene \\
\hline 17306532 & $-3,010216875$ & 0,002560337 & 0,151256401 & 0,693651303 & Myh6 & myosin, heavy polypeptide 6 , cardiac muscle, alpha \\
\hline 17321467 & $-3,010538183$ & 0,002558178 & 0,151256401 & 0,578520813 & Tuba1b & tubulin, alpha $1 \mathrm{~B}$ \\
\hline 17451661 & $-3,010792679$ & 0,002556882 & 0,151256401 & 0,756188227 & Unc119b & unc-119 homolog B (C. elegans) \\
\hline 17312295 & $-3,012227814$ & 0,00254954 & 0,151256401 & 0,742327979 & Zfp623 & zinc finger protein 623 \\
\hline 17502049 & $-3,016290912$ & 0,002528376 & 0,151256401 & 0,579175341 & 2010320M18Rik & RIKEN cDNA $2010320 \mathrm{M} 18$ gene \\
\hline 17238580 & $-3,022007492$ & 0,002507213 & 0,151256401 & 0,715589074 & Sarnp & SAP domain containing ribonucleoprotein \\
\hline 17430930 & $-3,037504717$ & 0,002432061 & 0,150014459 & 0,460384221 & Stx12 & syntaxin 12 \\
\hline 17453347 & $-3,041525609$ & 0,002418672 & 0,149667614 & 0,652307123 & Gtf2ird1 & general transcription factor II I repeat domain-containing 1 \\
\hline 17469572 & $-3,044939539$ & 0,002406147 & 0,149171898 & 0,615780636 & Sumf1 & sulfatase modifying factor 1 \\
\hline 17279230 & $-3,04524379$ & 0,002403555 & 0,149171898 & 0,594642292 & Zfyve21 & zinc finger, FYVE domain containing 21 \\
\hline 17508968 & $-3,050021389$ & 0,002377209 & 0,149171898 & 0,701068037 & Frg1 & FSHD region gene 1 \\
\hline 17241032 & $-3,050089073$ & 0,002376777 & 0,149171898 & 0,484360198 & Ddit4 & DNA-damage-inducible transcript 4 \\
\hline 17458641 & $-3,051693865$ & 0,002368139 & 0,149171898 & 0,652911438 & Tax1bp1 & Tax1 (human T cell leukemia virus type I) binding protein 1 \\
\hline 17469775 & $-3,052307919$ & 0,002364684 & 0,149171898 & 0,672987356 & Emc3 & ER membrane protein complex subunit 3 \\
\hline 17377177 & $-3,053117132$ & 0,002361661 & 0,149171898 & 0,667126324 & $\operatorname{Rin} 2$ & Ras and Rab interactor 2 \\
\hline 17341080 & $-3,056224366$ & 0,002348272 & 0,149171898 & 0,521115657 & Thbs2 & thrombospondin 2 \\
\hline 17363407 & $-3,060562651$ & 0,002328404 & 0,148532208 & 0,600757088 & Anxa1 & annexin $\mathrm{A} 1$ \\
\hline 17321078 & $-3,062682627$ & 0,002317606 & 0,148129375 & 0,439200746 & Vdr & vitamin $\mathrm{D}$ receptor \\
\hline 17419636 & $-3,067492111$ & 0,002296011 & 0,147319016 & 0,574142474 & Gpn2 & GPN-loop GTPase 2 \\
\hline & & & & & & COP9 (constitutive photomorphogenic) homolog, subunit 3 \\
\hline 17263482 & $-3,068086507$ & 0,002293419 & 0,147319016 & 0,583091296 & Cops3 & (Arabidopsis thaliana) \\
\hline 17534142 & $-3,068739652$ & 0,002290396 & 0,147319016 & 0,607534488 & Nkap & NFKB activating protein \\
\hline 17432927 & $-3,07491865$ & 0,002264914 & 0,147319016 & 0,741739853 & $\mathrm{FbxO} 44$ & F-box protein 44 \\
\hline 17493875 & $-3,077615058$ & 0,002255412 & 0,147319016 & 0,411239006 & P2ry2 & purinergic receptor P2Y, G-protein coupled 2 \\
\hline 17493556 & $-3,07894926$ & 0,002248933 & 0,147319016 & 0,568002505 & Acer3 & alkaline ceramidase 3 \\
\hline 17319124 & $-3,080983727$ & 0,002241159 & 0,147319016 & 0,580846043 & 1700088E04Rik & RIKEN CDNA $1700088 E 04$ gene \\
\hline 17417791 & $-3,084599703$ & 0,002228202 & 0,147257217 & 0,684555165 & Ebna1bp2 & EBNA1 binding protein 2 \\
\hline 17222072 & $-3,086012436$ & 0,002220427 & 0,147151909 & 0,436229852 & Ccdc115 & coiled-coil domain containing 115 \\
\hline 17349634 & $-3,091004395$ & 0,002192354 & 0,14614286 & 0,724890267 & Cystm1 & cysteine-rich transmembrane module containing 1 \\
\hline 17550492 & $-3,091240502$ & 0,002190194 & 0,14614286 & 0,678462934 & Gm11974 & predicted gene 11974 \\
\hline 17444494 & $-3,093731945$ & 0,002177669 & 0,145961053 & 0,692864083 & Gm15708 & predicted gene 15708 \\
\hline 17229166 & $-3,095001162$ & 0,002169463 & 0,145706572 & 0,678132114 & Blzf1 & basic leucine zipper nuclear factor 1 \\
\hline 17321169 & $-3,10532825$ & 0,002126704 & 0,145228743 & 0,727657558 & Asb8 & ankyrin repeat and SOCS box-containing 8 \\
\hline 17504281 & $-3,108913693$ & 0,002112451 & 0,14482105 & 0,712022873 & Usb1 & U6 snRNA biogenesis 1 \\
\hline 17515758 & $-3,11068183$ & 0,002102949 & 0,14482105 & 0,692146553 & Nfrkb & nuclear factor related to kappa B binding protein \\
\hline 17240819 & $-3,111093357$ & 0,002099926 & 0,14482105 & 0,448957228 & Cep851 & centrosomal protein 85 -like \\
\hline 17434127 & $-3,113606737$ & 0,002087832 & 0,144753326 & 0,49649057 & Gm10590 & predicted gene 10590 \\
\hline 17424410 & $-3,113606737$ & 0,002087832 & 0,144753326 & 0,49649057 & Gm10590 & predicted gene 10590 \\
\hline 17412998 & $-3,113606737$ & 0,002087832 & 0,144753326 & 0,49649057 & Gm10590 & predicted gene 10590 \\
\hline 17266489 & $-3,12058551$ & 0,002056303 & 0,144230612 & 0,71972203 & Tmem97 & transmembrane protein 97 \\
\hline 17287361 & $-3,126396885$ & 0,002040323 & 0,144230612 & 0,550516669 & Gadd45g & growth arrest and DNA-damage-inducible 45 gamma \\
\hline 17377199 & $-3,133163771$ & 0,002018727 & 0,143764545 & 0,438551428 & $\mathrm{Naa} 20$ & $\mathrm{~N}$ (alpha)-acetyltransferase $20, \mathrm{NatB}$ catalytic subunit \\
\hline 17468195 & $-3,133350954$ & 0,002017 & 0,143764545 & 0,578877605 & Stambp & STAM binding protein \\
\hline
\end{tabular}




\begin{tabular}{|c|c|c|c|c|c|c|}
\hline $\begin{array}{l}\text { probeset } \\
\text { ID }\end{array}$ & d.value & p.value & q.value & R.fold & Genename & Description \\
\hline 17443310 & $-3,133687298$ & 0,002013976 & 0,143764545 & 0,690897214 & Mdh2 & malate dehydrogenase 2, NAD (mitochondrial) \\
\hline 17463441 & $-3,135628498$ & 0,002009657 & 0,143764545 & 0,612454214 & Tom1 & target of myb1 homolog (chicken) \\
\hline 17451431 & $-3,136261558$ & 0,002005338 & 0,143764545 & 0,663245348 & Tmem119 & transmembrane protein 119 \\
\hline 17407647 & $-3,137383353$ & 0,001999292 & 0,143764545 & 0,603920306 & A730011C13Rik & RIKEN cDNA A730011C13 gene \\
\hline 17318461 & $-3,137775359$ & 0,001998428 & 0,143764545 & 0,74149168 & Sharpin & SHANK-associated RH domain interacting protein \\
\hline 17277592 & $-3,140056379$ & 0,001991949 & 0,143764545 & 0,691264997 & Gstz1 & glutathione transferase zeta 1 (maleylacetoacetate isomerase) \\
\hline 17529076 & $-3,140753941$ & 0,001989358 & 0,143764545 & 0,54757579 & Slc17a5 & solute carrier family 17 (anion/sugar transporter), member 5 \\
\hline 17403025 & $-3,142604954$ & 0,001981584 & 0,143764545 & 0,706140824 & Lamtor3 & late endosomal/lysosomal adaptor, MAPK and MTOR activator 3 \\
\hline 17319806 & $-3,142624914$ & 0,001981152 & 0,143764545 & 0,521405946 & Arfgap3 & ADP-ribosylation factor GTPase activating protein 3 \\
\hline 17440757 & $-3,143930082$ & 0,001975537 & 0,143764545 & 0,565081764 & Iscu & IscU iron-sulfur cluster scaffold homolog (E. coli) \\
\hline 17399672 & $-3,145056898$ & 0,001967331 & 0,143764545 & 0,676977068 & Slc39a1 & solute carrier family 39 (zinc transporter), member 1 \\
\hline 17538356 & $-3,145381723$ & 0,001966467 & 0,143764545 & 0,639182508 & Alg13 & asparagine-linked glycosylation 13 \\
\hline 17535752 & $-3,147256115$ & 0,001957829 & 0,143764545 & 0,596749711 & Emd & emerin \\
\hline 17429234 & $-3,15125999$ & 0,00194444 & 0,143764545 & 0,718142259 & Tmem125 & transmembrane protein 125 \\
\hline 17380134 & $-3,151436008$ & 0,001942712 & 0,143764545 & 0,62916817 & Rtfdc1 & replication termination factor 2 domain containing 1 \\
\hline 17378784 & $-3,151646776$ & 0,001941416 & 0,143764545 & 0,576910766 & Rprd1b & regulation of nuclear pre-mRNA domain containing $1 \mathrm{~B}$ \\
\hline 17496887 & $-3,152888829$ & 0,001938393 & 0,143764545 & 0,535242817 & Rgs10 & regulator of G-protein signalling 10 \\
\hline 17542149 & $-3,15459343$ & 0,001930619 & 0,143764545 & 0,672379998 & Ids & iduronate 2 -sulfatase \\
\hline 17287726 & $-3,157364859$ & 0,001923276 & 0,143764545 & 0,705374963 & Caml & calcium modulating ligand \\
\hline 17482366 & $-3,15840746$ & 0,001918525 & 0,143764545 & 0,396400538 & Tmem159 & transmembrane protein 159 \\
\hline 17291570 & $-3,162333663$ & 0,001905568 & 0,143764545 & 0,673320278 & Uqcrfs1 & ubiquinol-cytochrome c reductase, Rieske iron-sulfur polypeptide 1 \\
\hline 17502860 & $-3,164106453$ & 0,001898226 & 0,143764545 & 0,697755613 & Rnf150 & ring finger protein 150 \\
\hline 17460918 & $-3,164145273$ & 0,001897794 & 0,143764545 & 0,507691572 & Tmem43 & transmembrane protein 43 \\
\hline 17470627 & $-3,176124458$ & 0,001861082 & 0,143764545 & 0,583904837 & Clec4e & C-type lectin domain family 4 , member e \\
\hline 17355113 & $-3,17935585$ & 0,001848988 & 0,143764545 & 0,591547038 & Ccbe1 & collagen and calcium binding EGF domains 1 \\
\hline 17541008 & $-3,180767614$ & 0,001842942 & 0,143764545 & 0,417776766 & Snora69 & small nucleolar RNA, H/ACA box 69 \\
\hline 17498245 & $-3,183987927$ & 0,001833872 & 0,143764545 & 0,713236797 & Cdkn1c & cyclin-dependent kinase inhibitor 1C (P57) \\
\hline 17495097 & $-3,188406806$ & 0,001823506 & 0,143764545 & 0,496090994 & Lyve1 & $\begin{array}{l}\text { lymphatic vessel endothelial hyaluronan receptor } 1 \\
\text { excision repaiross-complementing rodent repair deficiency, }\end{array}$ \\
\hline 17289699 & $-3,193602942$ & 0,001803206 & 0,143764545 & 0,715513419 & Ercc8 & complementation group 8 \\
\hline 17422817 & $-3,203150732$ & 0,00177686 & 0,143764545 & 0,594126066 & Ube2j2 & $\begin{array}{l}\text { ubiquitin-conjugating enzyme E2J } 2 \\
\text { TATA box binding protein (Tbp)-associated factor, RNA polymerase I, }\end{array}$ \\
\hline 17514826 & $-3,209674254$ & 0,001759152 & 0,143764545 & 0,495265611 & Taf1d & D \\
\hline 17447831 & $-3,209859744$ & 0,001758288 & 0,143764545 & 0,572845219 & Fgfbp1 & fibroblast growth factor binding protein 1 \\
\hline 17481960 & $-3,212437555$ & 0,001748786 & 0,143764545 & 0,684533511 & Arntl & aryl hydrocarbon receptor nuclear translocator-like \\
\hline 17545459 & $-3,216846927$ & 0,001737989 & 0,143764545 & 0,720029811 & Shroom2 & shroom family member 2 \\
\hline 17444674 & $-3,219806088$ & 0,001725463 & 0,143764545 & 0,71364088 & Zscan25 & zinc finger and SCAN domain containing 25 \\
\hline 17413158 & $-3,227961342$ & 0,001693934 & 0,143764545 & 0,559648697 & Gm10590 & predicted gene 10590 \\
\hline 17526206 & $-3,228581149$ & 0,001691775 & 0,143764545 & 0,709094726 & $\mathrm{C} 2 \mathrm{~cd} 2 \mathrm{I}$ & C2 calcium-dependent domain containing 2-like \\
\hline 17368834 & $-3,230843617$ & 0,001680113 & 0,143764545 & 0,607217719 & Med27 & mediator complex subunit 27 \\
\hline 17453703 & $-3,236061044$ & 0,001661109 & 0,143764545 & 0,74253504 & Prkrip1 & Prkr interacting protein 1 (IL11 inducible) \\
\hline 17505967 & $-3,238036806$ & 0,001654631 & 0,143764545 & 0,750756228 & Cmip & c-Maf inducing protein \\
\hline 17396492 & $-3,242207228$ & 0,001647288 & 0,143764545 & 0,617044583 & Eif5a2 & eukaryotic translation initiation factor $5 \mathrm{~A} 2$ \\
\hline 17284037 & $-3,248485737$ & 0,001630444 & 0,143764545 & 0,434752184 & Mpc1 & mitochondrial pyruvate carrier 1 \\
\hline 17285863 & $-3,266749083$ & 0,001582071 & 0,141291573 & 0,690778604 & Hist1h2bb & histone cluster $1, \mathrm{H} 2 \mathrm{bb}$ \\
\hline 17368079 & $-3,26948524$ & 0,001576024 & 0,141291573 & 0,696214241 & Fbxw5 & F-box and WD-40 domain protein 5 \\
\hline 17440086 & $-3,272009537$ & 0,001566954 & 0,141085467 & 0,65213089 & Rpap2 & RNA polymerase II associated protein 2 \\
\hline 17431302 & $-3,273111252$ & 0,001563931 & 0,141085467 & 0,622978707 & Mtfril & mitochondrial fission regulator 1-like \\
\hline 17411054 & $-3,280888731$ & 0,001546223 & 0,141085467 & 0,686706455 & Rpf1 & ribosome production factor 1 homolog (S. cerevisiae) \\
\hline 17264153 & $-3,284077773$ & 0,001534561 & 0,141085467 & 0,482531323 & 1700086D15Rik & RIKEN cDNA 1700086D15 gene \\
\hline 17374686 & $-3,285053878$ & 0,001531106 & 0,141085467 & 0,59096413 & Rpusd2 & RNA pseudouridylate synthase domain containing 2 \\
\hline sphk & $-3,289287203$ & 0,001520308 & 0,141085467 & 0,699798841 & Neu3 & neuraminidase 3 \\
\hline 17402296 & $-3,292596748$ & 0,001512534 & 0,14107046 & 0,724573078 & Dnttip2 & deoxynucleotidyltransferase, terminal, interacting protein 2 \\
\hline 17465608 & $-3,295216588$ & 0,001508647 & 0,14107046 & 0,508548194 & Lincpint & long intergenic non-protein coding RNA, Trp53 induced transcript \\
\hline 17272519 & $-3,300420245$ & 0,001494826 & 0,14107046 & 0,697920319 & Jmjd6 & jumonji domain containing 6 \\
\hline 17256565 & $-3,302589284$ & 0,001487483 & 0,14107046 & 0,637032736 & Tubg2 & tubulin, gamma 2 \\
\hline 17231574 & $-3,309663144$ & 0,001467616 & 0,140567638 & 0,684961856 & Ppil4 & peptidylprolyl isomerase (cyclophilin)-like 4 \\
\hline 17240357 & $-3,309953408$ & 0,001466752 & 0,140567638 & 0,630537095 & Gtf3c6 & general transcription factor IIIC, polypeptide 6 , alpha \\
\hline 17298775 & $-3,315862757$ & 0,001451203 & 0,139806133 & 0,716051526 & Anxa8 & annexin A8 \\
\hline 17455093 & $-3,318774053$ & 0,001443429 & 0,139806133 & 0,766056229 & Zkscan14 & zinc finger with KRAB and SCAN domains 14 \\
\hline 17308842 & $-3,320097347$ & 0,001439974 & 0,139806133 & 0,461844373 & Dgkh & diacylglycerol kinase, eta \\
\hline 17308233 & $-3,321573275$ & 0,00143695 & 0,139806133 & 0,491003722 & 9930012K11Rik & RIKEN cDNA 9930012K11 gene \\
\hline 17500068 & $-3,323521437$ & 0,001433927 & 0,139806133 & 0,476706938 & Sfrp1 & secreted frizzled-related protein 1 \\
\hline 17374594 & $-3,324472957$ & 0,001429608 & 0,139806133 & 0,466066542 & Pak6 & p21 protein $(\mathrm{Cdc} 42 / \mathrm{Rac})$-activated kinase 6 \\
\hline 17375327 & $-3,331346212$ & 0,001416219 & 0,139806133 & 0,594416505 & Casc4 & cancer susceptibility candidate 4 \\
\hline 17220974 & $-3,331383696$ & 0,001415787 & 0,139806133 & 0,559654837 & Plxna2 & plexin A2 \\
\hline 17241637 & $-3,335331226$ & 0,001406285 & 0,139806133 & 0,620285776 & Nrbf2 & nuclear receptor binding factor 2 \\
\hline 17234192 & $-3,338915233$ & 0,001395487 & 0,139806133 & 0,538660813 & Zwint & ZW10 interactor \\
\hline 17214825 & $-3,341423389$ & 0,001387281 & 0,139806133 & 0,660268405 & Mff & mitochondrial fission factor \\
\hline 17473269 & $-3,344812939$ & 0,001378643 & 0,139806133 & 0,760651781 & Leng8 & leukocyte receptor cluster (LRC) member 8 \\
\hline 17313016 & $-3,345156374$ & 0,001377779 & 0,139806133 & 0,62691808 & Tomm22 & translocase of outer mitochondrial membrane 22 homolog (yeast) \\
\hline 17258457 & $-3,345165902$ & 0,001377347 & 0,139806133 & 0,754312856 & Sap30bp & SAP30 binding protein \\
\hline 17332851 & $-3,346145344$ & 0,001373892 & 0,139806133 & 0,695521805 & Gtf2h5 & general transcription factor $\mathrm{IIH}$, polypeptide 5 \\
\hline 17320947 & $-3,347039758$ & 0,001370005 & 0,139806133 & 0,362732325 & Slc38a4 & solute carrier family 38 , member 4 \\
\hline 17366437 & $-3,347143691$ & 0,001369141 & 0,139806133 & 0,516745636 & Fam107b & family with sequence similarity 107 , member $B$ \\
\hline 17506917 & $-3,348136414$ & 0,001366982 & 0,139806133 & 0,326006331 & Kcnk1 & potassium channel, subfamily K, member 1 \\
\hline 17292157 & $-3,350273602$ & 0,001360071 & 0,139806133 & 0,620463742 & Dtnbp1 & dystrobrevin binding protein 1 \\
\hline 17220018 & $-3,357718819$ & 0,001340635 & 0,139806133 & 0,577888053 & Cnst & consortin, connexin sorting protein \\
\hline 17362320 & $-3,359977929$ & 0,001333293 & 0,139806133 & 0,667915644 & Al846148 & expressed sequence Al846148 \\
\hline 17512740 & $-3,367262041$ & 0,001315153 & 0,139806133 & 0,590327769 & Nob1 & NIN1/RPN12 binding protein 1 homolog (S. cerevisiae) \\
\hline 17487952 & $-3,374525545$ & 0,001294853 & 0,139806133 & 0,481109546 & Ceacam1 & carcinoembryonic antigen-related cell adhesion molecule 1 \\
\hline
\end{tabular}




\begin{tabular}{|c|c|c|c|c|c|c|}
\hline $\begin{array}{l}\text { probeset } \\
\text { ID }\end{array}$ & d.value & p.value & q.value & R.fold & Genename & Description \\
\hline 17503377 & $-3,374898042$ & 0,001293558 & 0,139806133 & 0,689560337 & Dhps & deoxyhypusine synthase \\
\hline 17386135 & $-3,377816634$ & 0,001286215 & 0,139806133 & 0,683866066 & Stk39 & serine/threonine kinase 39 \\
\hline 17242773 & $-3,38173854$ & 0,001279305 & 0,139806133 & 0,476948811 & Polr2e & polymerase (RNA) II (DNA directed) polypeptide E \\
\hline 17444828 & $-3,3927032$ & 0,001252095 & 0,139806133 & 0,56651793 & Polr1d & polymerase (RNA) I polypeptide D \\
\hline 17381283 & $-3,396356237$ & 0,001243025 & 0,139806133 & 0,557166499 & Prpf18 & PRP18 pre-mRNA processing factor 18 homolog (yeast) \\
\hline 17488001 & $-3,39924399$ & 0,001237842 & 0,139806133 & 0,740683411 & Ccdc97 & coiled-coil domain containing 97 \\
\hline 17512732 & $-3,40372337$ & 0,001229636 & 0,139806133 & 0,578431755 & Nqo1 & $\begin{array}{l}\mathrm{NAD}(\mathrm{P}) \mathrm{H} \text { dehydrogenase, quinone } 1 \\
\text { translational activator of mitochondrially encoded cytochrome } \mathrm{c}\end{array}$ \\
\hline 17270724 & $-3,408107144$ & 0,001224021 & 0,139806133 & 0,732808744 & Tacolos & oxidase I, opposite strand \\
\hline 17467711 & $-3,41031971$ & 0,001218838 & 0,139806133 & 0,593266468 & Usp39 & ubiquitin specific peptidase 39 \\
\hline 17253885 & $-3,432870782$ & 0,001164418 & 0,139806133 & 0,545319177 & Adap2 & ArfGAP with dual PH domains 2 \\
\hline 17311821 & $-3,449762905$ & 0,001132457 & 0,139806133 & 0,671602404 & Nsmce2 & non-SMC element 2 homolog (MMS21, S. cerevisiae) \\
\hline 17301440 & $-3,453867795$ & 0,001122091 & 0,139806133 & 0,489198594 & Ccdc25 & coiled-coil domain containing 25 \\
\hline 17515062 & $-3,47139927$ & 0,001084947 & 0,139806133 & 0,665794484 & Mrpl4 & mitochondrial ribosomal protein $\mathrm{L} 4$ \\
\hline 17506754 & $-3,473541247$ & 0,00108106 & 0,139806133 & 0,544532568 & Cog2 & component of oligomeric golgi complex 2 \\
\hline 17442588 & $-3,473818467$ & 0,001080196 & 0,139806133 & 0,524454143 & Atp6v0a2 & ATPase, $\mathrm{H}+$ transporting, lysosomal $\mathrm{V} 0$ subunit $\mathrm{A} 2$ \\
\hline 17258341 & $-3,476527556$ & 0,00107415 & 0,139806133 & 0,712731984 & Llgl2 & lethal giant larvae homolog 2 (Drosophila) \\
\hline 17236800 & $-3,479401442$ & 0,001068535 & 0,139806133 & 0,496946546 & Den & decorin \\
\hline 17289289 & $-3,497048136$ & 0,001042189 & 0,139806133 & 0,69446935 & Poc5 & POC5 centriolar protein homolog (Chlamydomonas) \\
\hline 17463702 & $-3,504309212$ & 0,001027072 & 0,139806133 & 0,734493683 & Loh12cr1 & loss of heterozygosity, 12 , chromosomal region 1 homolog (human) \\
\hline 17358007 & $-3,510314282$ & 0,001016706 & 0,139806133 & 0,621860904 & Rfk & riboflavin kinase \\
\hline 17482681 & $-3,514125786$ & 0,00100634 & 0,139806133 & 0,631720937 & Scnn1g & sodium channel, nonvoltage-gated 1 gamma \\
\hline 17401169 & $-3,520312453$ & 0,000995975 & 0,139806133 & 0,59957355 & Bcas2 & breast carcinoma amplified sequence 2 \\
\hline 17275718 & $-3,556639111$ & 0,000943714 & 0,139806133 & 0,627317635 & Mia2 & melanoma inhibitory activity 2 \\
\hline 17327909 & $-3,559964149$ & 0,000937667 & 0,139806133 & 0,510715901 & Ppl & periplakin \\
\hline 17468511 & $-3,565659476$ & 0,000928165 & 0,139806133 & 0,660609484 & Mxd1 & MAX dimerization protein 1 \\
\hline 17249461 & $-3,56980662$ & 0,000919527 & 0,139806133 & 0,627153803 & Fstl4 & follistatin-like 4 \\
\hline 17408099 & $-3,569884607$ & 0,000919095 & 0,139806133 & 0,73518066 & Polr3c & polymerase (RNA) III (DNA directed) polypeptide C \\
\hline 17411732 & $-3,57430591$ & 0,000913049 & 0,139806133 & 0,697540976 & 2610301B20Rik & RIKEN cDNA 2610301B20 gene \\
\hline 17340397 & $-3,577868578$ & 0,000905706 & 0,139806133 & 0,533152124 & Nanp & $\mathrm{N}$-acetylneuraminic acid phosphatase \\
\hline 17354378 & $-3,586120674$ & 0,000890158 & 0,139806133 & 0,677136421 & Ppic & $\begin{array}{l}\text { peptidylprolyl isomerase } \mathrm{C} \\
\text { 1-acylglycerol-3-phosphate O-acyltransferase } 2 \text { (lysophosphatidic acid }\end{array}$ \\
\hline 17383104 & $-3,58950033$ & 0,000884543 & 0,139806133 & 0,719562327 & Agpat2 & acyltransferase, beta) \\
\hline 17363605 & $-3,594098108$ & 0,000876769 & 0,139806133 & 0,519354267 & Pip5k1b & phosphatidylinositol-4-phosphate 5-kinase, type 1 beta \\
\hline 17548850 & $-3,594787251$ & 0,000875473 & 0,139806133 & 0,578479595 & Arpc1b & actin related protein $2 / 3$ complex, subunit $1 B$ \\
\hline 17389009 & $-3,598350423$ & 0,000872018 & 0,139806133 & 0,655885569 & Eif3m & eukaryotic translation initiation factor 3 , subunit $\mathrm{M}$ \\
\hline 17230595 & $-3,600908628$ & 0,000866835 & 0,139806133 & 0,672541857 & Degs1 & degenerative spermatocyte homolog 1 (Drosophila) \\
\hline 17518585 & $-3,6041549$ & 0,00086122 & 0,139806133 & 0,492572435 & Spg21 & spastic paraplegia 21 homolog (human) \\
\hline 17254537 & $-3,604548687$ & 0,000860356 & 0,139806133 & 0,688280503 & Ppm1d & protein phosphatase 1D magnesium-dependent, delta isoform \\
\hline 17422612 & $-3,606694512$ & 0,000858629 & 0,139806133 & 0,546327082 & Slc35e2 & solute carrier family 35, member E2 \\
\hline 17334685 & $-3,615074037$ & 0,000847831 & 0,139806133 & 0,745631637 & Tekt4 & tektin 4 \\
\hline 17461606 & $-3,64627147$ & 0,000806368 & 0,138060066 & 0,671870433 & Brpf1 & bromodomain and PHD finger containing, 1 \\
\hline 17550428 & $-3,647607329$ & 0,000803345 & 0,138060066 & 0,32956441 & Neat1 & nuclear paraspeckle assembly transcript 1 (non-protein coding) \\
\hline 17499922 & $-3,661889709$ & 0,000783909 & 0,137055318 & 0,57111072 & Mrps31 & mitochondrial ribosomal protein S31 \\
\hline 17324305 & $-3,69670445$ & 0,000738991 & 0,136174199 & 0,71908677 & Dnajb11 & DnaJ (Hsp40) homolog, subfamily B, member 11 \\
\hline 17516921 & $-3,726462048$ & 0,00070487 & 0,134634114 & 0,712324047 & Zpr1 & ZPR1 zinc finger \\
\hline 17419222 & $-3,733573785$ & 0,000694936 & 0,134289173 & 0,739502152 & Snrnp40 & small nuclear ribonucleoprotein 40 (U5) \\
\hline 17276732 & $-3,740144462$ & 0,000689322 & 0,134289173 & 0,573178085 & Eif2s1 & eukaryotic translation initiation factor 2 , subunit 1 alpha \\
\hline 17462373 & $-3,757901224$ & 0,000670318 & 0,133061743 & 0,441843787 & Cecr2 & $\begin{array}{l}\text { cat eye syndrome chromosome region, candidate } 2 \\
\text { solute carrier family } 25 \text { (mitochondrial carrier, adenine nucleotide }\end{array}$ \\
\hline 17509171 & $-3,771567859$ & 0,000654337 & 0,132737203 & 0,676551639 & Slc25a4 & translocator), member 4 \\
\hline 17286254 & $-3,797324471$ & 0,000628855 & 0,132737203 & 0,48272016 & Mboat1 & membrane bound O-acyltransferase domain containing 1 \\
\hline 17223313 & $-3,797704456$ & 0,000627991 & 0,132737203 & 0,672558507 & Tyw5 & tRNA-yW synthesizing protein 5 \\
\hline 17428885 & $-3,80137802$ & 0,000624968 & 0,132737203 & 0,706298653 & Dmap1 & $\begin{array}{l}\text { DNA methyltransferase } 1 \text {-associated protein } 1 \\
\text { carbohydrate ( } \mathrm{N} \text {-acetylgalactosamine } 4 \text {-sulfate } 6-\mathrm{O} \text { ) sulfotransferase }\end{array}$ \\
\hline 17497076 & $-3,806519272$ & 0,000619353 & 0,132737203 & 0,690577859 & Chst15 & 15 \\
\hline 17231859 & $-3,807188167$ & 0,000618921 & 0,132737203 & 0,567789061 & Ifngr1 & interferon gamma receptor 1 \\
\hline 17356099 & $-3,83422815$ & 0,000594734 & 0,132737203 & 0,618854199 & Cdk2ap2 & CDK2-associated protein 2 \\
\hline 17479548 & $-3,839799454$ & 0,000590847 & 0,132737203 & 0,495682389 & Vps33b & vacuolar protein sorting 33B (yeast) \\
\hline 17342042 & $-3,846845987$ & 0,000585232 & 0,132737203 & 0,578845895 & Nubp2 & nucleotide binding protein 2 \\
\hline 17266185 & $-3,87471105$ & 0,000564501 & 0,132737203 & 0,705566022 & Ccdc55 & coiled-coil domain containing 55 \\
\hline 17464503 & $-3,877044124$ & 0,000563637 & 0,132737203 & 0,727517162 & 2810474019Rik & RIKEN CDNA 2810474019 gene \\
\hline 17427155 & $-3,881256759$ & 0,000560614 & 0,132737203 & 0,515698326 & Cdkn2b & cyclin-dependent kinase inhibitor 2B ( $p 15$, inhibits CDK4) \\
\hline 17329454 & $-3,943569936$ & 0,000508353 & 0,132267702 & 0,671211642 & P3h2 & prolyl 3-hydroxylase 2 \\
\hline 17548440 & $-3,947796389$ & 0,000503602 & 0,132184757 & 0,685442738 & Imp3 & IMP3, U3 small nucleolar ribonucleoprotein, homolog (yeast) \\
\hline 17524958 & $-3,9559556$ & 0,000498419 & 0,132184757 & 0,610775772 & Zfp810 & $\begin{array}{l}\text { zinc finger protein } 810 \\
\text { pleckstrin homology domain containing, family D (with coiled-coil }\end{array}$ \\
\hline 17276864 & $-3,960284316$ & 0,000494964 & 0,132184757 & 0,710433769 & Plekhd1 & domains) member 1 \\
\hline 17503431 & $-3,962058249$ & 0,000493668 & 0,132184757 & 0,559190937 & Orc6 & origin recognition complex, subunit 6 \\
\hline 17211998 & $-3,971919205$ & 0,000483734 & 0,132184757 & 0,714505212 & Mrpl30 & mitochondrial ribosomal protein $\mathrm{L} 30$ \\
\hline 17519967 & $-3,973869516$ & 0,000482007 & 0,132184757 & 0,711876703 & Tpbg & trophoblast glycoprotein \\
\hline 17384619 & $-3,982798977$ & 0,000473801 & 0,132184757 & 0,444753712 & Snord90 & small nucleolar RNA, C/D box 90 \\
\hline 17353131 & $-4,000032064$ & 0,000465162 & 0,132184757 & 0,583929509 & Ino80c & INO80 complex subunit C \\
\hline 17258584 & $-4,012350585$ & 0,000454365 & 0,132184757 & 0,560032735 & Sphk1 & sphingosine kinase 1 \\
\hline 17481936 & $-4,034499719$ & 0,000439248 & 0,132184757 & 0,572627747 & Tead1 & TEA domain family member 1 \\
\hline 17353241 & $-4,039429942$ & 0,000435793 & 0,132184757 & 0,626893294 & Slc25a46 & solute carrier family 25, member 46 \\
\hline 17379128 & $-4,051019678$ & 0,000428882 & 0,132184757 & 0,619618662 & Srsf6 & serine/arginine-rich splicing factor 6 \\
\hline 17365960 & $-4,06333677$ & 0,000422836 & 0,132184757 & 0,569277891 & Gfra1 & glial cell line derived neurotrophic factor family receptor alpha 1 \\
\hline 17349774 & $-4,067505514$ & 0,000420244 & 0,132184757 & 0,746684615 & Hars2 & histidyl-tRNA synthetase 2 , mitochondrial (putative) \\
\hline 17239077 & $-4,117804708$ & 0,000387419 & 0,130631356 & 0,532540427 & Ginm1 & glycoprotein integral membrane 1 \\
\hline 17219516 & $-4,132601094$ & 0,000380941 & 0,129771079 & 0,428596058 & Gm17224 & predicted gene 17224 \\
\hline
\end{tabular}




\begin{tabular}{|c|c|c|c|c|c|c|}
\hline $\begin{array}{l}\text { probeset } \\
\text { ID }\end{array}$ & d.value & p.value & q.value & R.fold & Genename & Description \\
\hline 17312905 & $-4,142561209$ & 0,000375326 & 0,129190212 & 0,583237458 & Eif3l & eukaryotic translation initiation factor 3 , subunit $\mathrm{L}$ \\
\hline 17533413 & $-4,184770505$ & 0,000349412 & 0,127526119 & 0,550405163 & $\mathrm{Rpl} 3$ & ribosomal protein $\mathrm{L} 3$ \\
\hline 17275706 & $-4,189147847$ & 0,000345956 & 0,127526119 & 0,583912648 & Pnn & pinin \\
\hline 17358020 & $-4,205642406$ & 0,00033991 & 0,127526119 & 0,576169778 & Nmrk1 & $\begin{array}{l}\text { nicotinamide riboside kinase } 1 \\
\text { solute carrier family } 25 \text { (mitochondrial carrier, Graves disease }\end{array}$ \\
\hline 17233799 & $-4,207168462$ & 0,000339478 & 0,127526119 & 0,5489078 & Slc25a16 & autoantigen), member 16 \\
\hline 17475818 & $-4,231375384$ & 0,000329544 & 0,127526119 & 0,454714086 & AF357399 & snoRNA AF357399 \\
\hline 17469636 & $-4,232978892$ & 0,000328248 & 0,127526119 & 0,433734787 & Rad18 & RAD18 homolog (S. cerevisiae) \\
\hline 17245399 & $-4,28387533$ & 0,000304062 & 0,12603958 & 0,562405539 & Irak3 & interleukin-1 receptor-associated kinase 3 \\
\hline 17339549 & $-4,337129628$ & 0,000274692 & 0,124070176 & 0,583455003 & Ypel5 & yippee-like 5 (Drosophila) \\
\hline 17277370 & $-4,340198331$ & 0,000273396 & 0,124070176 & 0,560278083 & Eif2b2 & eukaryotic translation initiation factor $2 B$, subunit 2 beta \\
\hline 17402595 & $-4,369250202$ & 0,000264326 & 0,124070176 & 0,423236106 & Casp6 & caspase 6 \\
\hline 17451203 & $-4,369544715$ & 0,000263894 & 0,124070176 & 0,667582272 & Srrd & SRR1 domain containing \\
\hline 17522577 & $-4,412134904$ & 0,000250937 & 0,124070176 & 0,710931014 & Lrrfip2 & leucine rich repeat (in FLII) interacting protein 2 \\
\hline 17345038 & $-4,487677474$ & 0,000224591 & 0,117799612 & 0,549881852 & Cd2ap & CD2-associated protein \\
\hline 17515074 & $-4,51117016$ & 0,000211634 & 0,116707116 & 0,465606464 & Icam1 & intercellular adhesion molecule 1 \\
\hline 17516217 & $-4,518129424$ & 0,000209042 & 0,116707116 & 0,331736836 & Olfr920 & olfactory receptor 920 \\
\hline 17346749 & $-4,537261637$ & 0,000202996 & 0,116707116 & 0,601773665 & Rab31 & RAB31, member RAS oncogene family \\
\hline 17265646 & $-4,567629358$ & 0,000194358 & 0,116707116 & 0,603423401 & Tekt1 & tektin 1 \\
\hline 17286998 & $-4,573030215$ & 0,000192198 & 0,116707116 & 0,384288188 & $\mathrm{Rbm} 24$ & RNA binding motif protein 24 \\
\hline 17283445 & $-4,58655048$ & 0,000184424 & 0,116707116 & 0,661719589 & Lgmn & legumain \\
\hline 17224587 & $-4,592282037$ & 0,00018356 & 0,116707116 & 0,529509117 & Dnpep & aspartyl aminopeptidase \\
\hline 17288716 & $-4,857157317$ & 0,000122661 & 0,096505067 & 0,417057385 & Glrx & glutaredoxin \\
\hline 17263511 & $-4,878684126$ & 0,00011791 & 0,096074093 & 0,271489219 & Rasd1 & RAS, dexamethasone-induced 1 \\
\hline 17347267 & $-4,892003271$ & 0,000115751 & 0,096074093 & 0,569578354 & Fez2 & fasciculation and elongation protein zeta 2 (zygin II) \\
\hline 17364932 & $-4,893219997$ & 0,000114887 & 0,096074093 & 0,459150059 & Got1 & glutamic-oxaloacetic transaminase 1 , soluble \\
\hline 17508609 & $-4,942716978$ & 0,000106249 & 0,096074093 & 0,441015297 & Nrg1 & neuregulin 1 \\
\hline 17483385 & $-4,955833031$ & 0,000104089 & 0,096074093 & 0,445527753 & Phkg2 & phosphorylase kinase, gamma 2 (testis) \\
\hline 17486099 & $-4,959257549$ & 0,000103225 & 0,096074093 & 0,490802608 & Zim1 & zinc finger, imprinted 1 \\
\hline 17219139 & $-5,100936289$ & $8,98364 \mathrm{E}-05$ & 0,096074093 & 0,513086673 & Rgs5 & regulator of G-protein signaling 5 \\
\hline 17497769 & $-5,248765774$ & $7,51516 \mathrm{E}-05$ & 0,096074093 & 0,550423956 & Rnh1 & ribonuclease/angiogenin inhibitor 1 \\
\hline 17519649 & $-5,37953875$ & $6,60816 \mathrm{E}-05$ & 0,096074093 & 0,483671338 & Gsta4 & glutathione S-transferase, alpha 4 \\
\hline 17424298 & $-5,623221226$ & 4,83734E-05 & 0,096074093 & 0,609742159 & Dctn3 & dynactin 3 \\
\hline 17274184 & $-6,947647979$ & 1,33891E-05 & 0,088009903 & 0,33593012 & Socs2 & suppressor of cytokine signaling 2 \\
\hline
\end{tabular}




\section{Table S3. Differential gene expression in the lungs of HRAS/NRAS-DKO mice treated antenatally with}

dexamethasone.

List of 509 differentially expressed gene probesets identified by means of SAM contrasts (FDR=0.15) in multiclass comparison (Fig. 6B heatmap) between the transcriptional profiles of lungs from newborn (PO) DKO mice that had been treated in utero with dexamethasone (injections at E17.5 and E18.5) and the transcriptional profiles of untreated (P0) DKO littermates that were generated by RNA microarray hybridization assays using GeneChip(R) Mouse Gene 2.0 ST Arrays. The differentially expressed loci are identified by Affymetrix Probeset ID, Genename Symbol or Description and listed according to their degree of overexpression or repression in dexamethasone-treated lung tissue of DKO mice. $d$-value is a parameter measuring the statistical distance separating the calculated expression value of each gene probeset from the null hypothesis (no-change). $q$-value is the estimated FDR at the largest $p$-value for which the probe set would be statistically significant. $R$-fold is a measure of the fold change of a probeset in the collection of microarrays provided by the SAM algorithm. Entries in red denote overexpression. Entries in green denote transcriptional repression. The data list organized here from maximal to minimal d-values. Components of ceramide/sphingosine metabolism are printed in bold.

\begin{tabular}{|c|c|c|c|c|c|c|}
\hline $\begin{array}{c}\text { probeset } \\
\text { ID }\end{array}$ & d.value & p.value & q.value & R.fold & Genename & Description \\
\hline 17357648 & 5,800980166 & $3,88715 \mathrm{E}-05$ & 0,096074093 & 1,920712178 & Ms4a4c & membrane-spanning 4-domains, subfamily $\mathrm{A}$, member $4 \mathrm{C}$ \\
\hline 17259311 & 5,450425722 & $5,83073 \mathrm{E}-05$ & 0,096074093 & 1,568655314 & 0610009L18Rik & RIKEN cDNA 0610009L18 gene \\
\hline 17400622 & 5,443224101 & 5,91711E-05 & 0,096074093 & 1,694496031 & Lix1l & Lix1-like \\
\hline 17509721 & 5,308217228 & 7,12644E-05 & 0,096074093 & 1,495688964 & Tufm & Tu translation elongation factor, mitochondrial \\
\hline 17443683 & 5,126100134 & $8,59492 \mathrm{E}-05$ & 0,096074093 & 1,620692856 & Ppp1r35 & protein phosphatase 1 , regulatory subunit 35 \\
\hline 17240303 & 4,936497607 & 0,000108408 & 0,096074093 & 1,887549863 & G630090E17Rik & RIKEN cDNA G630090E17 gene \\
\hline 17458362 & 4,765692033 & 0,000141233 & 0,104761644 & 2,246207919 & Gimap4 & GTPase, IMAP family member 4 \\
\hline 17276182 & 4,755402964 & 0,000144688 & 0,104761644 & 1,552627184 & Jkamp & JNK1/MAPK8-associated membrane protein \\
\hline 17357910 & 4,526359256 & 0,000206451 & 0,116707116 & 2,052924326 & Olfr1463 & olfactory receptor 1463 \\
\hline 17411492 & 4,518059895 & 0,000209474 & 0,116707116 & 1,873515792 & Gm11783 & predicted gene 11783 \\
\hline 17402165 & 4,478487392 & 0,000228478 & 0,117965976 & 2,111983968 & A530020G20Rik & RIKEN cDNA A530020G20 gene \\
\hline 17376538 & 4,376356888 & 0,000262599 & 0,124070176 & 2,076853064 & 4930425F17Rik & RIKEN cDNA $4930425 F 17$ gene \\
\hline 17244341 & 4,286378482 & 0,000302766 & 0,12603958 & 1,575044061 & Elk3 & ELK3, member of ETS oncogene family \\
\hline 17394829 & 4,152856254 & 0,000367984 & 0,127996194 & 1,513665594 & Atp9a & ATPase, class II, type 9A \\
\hline 17433310 & 4,031688191 & 0,000440976 & 0,132184757 & 1,30850635 & Park7 & Parkinson disease (autosomal recessive, early onset) 7 \\
\hline 17466294 & 3,975815699 & 0,000480711 & 0,132184757 & 1,397874208 & Try5 & $\operatorname{tryp} \sin 5$ \\
\hline 17255215 & 3,922649481 & 0,000522174 & 0,132737203 & 2,013953925 & Ankrd40 & ankyrin repeat domain 40 \\
\hline 17284702 & 3,862915192 & 0,000574003 & 0,132737203 & 1,533029579 & Gm10421 & predicted gene 10421 \\
\hline 17530915 & 3,852809951 & 0,000580913 & 0,132737203 & 2,122660087 & Gm9917 & predicted gene 9917 \\
\hline 17393225 & 3,833965601 & 0,000595166 & 0,132737203 & 1,71662334 & Pigu & phosphatidylinositol glycan anchor biosynthesis, class $U$ \\
\hline 17234024 & 3,796165761 & 0,000629719 & 0,132737203 & 3,556466551 & Rtkn2 & rhotekin 2 \\
\hline 17234711 & 3,781923723 & 0,000647859 & 0,132737203 & 1,309959767 & Gm10142 & predicted gene 10142 \\
\hline 17511677 & 3,764405546 & 0,000661248 & 0,133061743 & 2,112141857 & Ces1c & carboxylesterase $1 \mathrm{C}$ \\
\hline 17386177 & 3,736929088 & 0,000693641 & 0,134289173 & 1,488797143 & Abcb11 & ATP-binding cassette, sub-family B (MDR/TAP), member 11 \\
\hline 17548940 & 3,728646209 & 0,000700551 & 0,134587111 & 1,414644871 & Soat1 & sterol O-acyltransferase 1 \\
\hline 17484682 & 3,72186879 & 0,000709621 & 0,1347626 & 1,680331399 & Athl1 & ATH1, acid trehalase-like 1 (yeast) \\
\hline 17442332 & 3,708490555 & 0,000725602 & 0,136174199 & 1,367416124 & Kntc1 & kinetochore associated 1 \\
\hline 17222825 & 3,688469985 & 0,000751948 & 0,136401701 & 2,799199546 & Nabp1 & nucleic acid binding protein 1 \\
\hline 17384205 & 3,676864141 & 0,000765769 & 0,136733781 & 1,52783397 & Mvb12b & multivesicular body subunit $12 \mathrm{~B}$ \\
\hline 17380567 & 3,655483338 & 0,000792547 & 0,137836277 & 2,303066348 & Gm14322 & predicted gene 14322 \\
\hline 17331848 & 3,587883431 & 0,000886702 & 0,139806133 & 1,297769639 & 2310061N02Rik & RIKEN cDNA 2310061N02 gene \\
\hline 17457609 & 3,576813513 & 0,00090873 & 0,139806133 & 1,452905967 & Mgam & maltase-glucoamylase \\
\hline 17516960 & 3,564595157 & 0,000929893 & 0,139806133 & 1,483860375 & Cadm1 & cell adhesion molecule 1 \\
\hline 17217946 & 3,527653586 & 0,000981722 & 0,139806133 & 1,32796367 & F13b & coagulation factor XIII, beta subunit \\
\hline 17404923 & 3,511912934 & 0,001011523 & 0,139806133 & 1,338392838 & Gm5148 & predicted gene 5148 \\
\hline 17380555 & 3,511749727 & 0,001011955 & 0,139806133 & 2,070276047 & Gm14403 & predicted gene 14403 \\
\hline 17211375 & 3,483268058 & 0,00106292 & 0,139806133 & 1,342887651 & Paqr8 & progestin and adipoQ receptor family member VIII \\
\hline 17399314 & 3,456364806 & 0,001115613 & 0,139806133 & 1,565545328 & Fam189b & family with sequence similarity 189 , member B \\
\hline 17474906 & 3,452573562 & 0,001125546 & 0,139806133 & 1,307430512 & Zfp114 & zinc finger protein 114 \\
\hline 17265030 & 3,441950465 & 0,001144982 & 0,139806133 & 1,451421797 & Acap1 & ArfGAP with coiled-coil, ankyrin repeat and PH domains 1 \\
\hline 17217590 & 3,433246226 & 0,001163986 & 0,139806133 & 1,685692046 & Arl8a & ADP-ribosylation factor-like $8 \mathrm{~A}$ \\
\hline 17344251 & 3,396121838 & 0,001243889 & 0,139806133 & 1,608600529 & Prrc2a & proline-rich coiled-coil $2 \mathrm{~A}$ \\
\hline 17354653 & 3,383791231 & 0,00127585 & 0,139806133 & 1,667081798 & Tcof1 & Treacher Collins Franceschetti syndrome 1, homolog \\
\hline 17400375 & 3,369432449 & 0,001309538 & 0,139806133 & 1,422660284 & Ctss & cathepsin S \\
\hline 17400072 & 3,3679807 & 0,001313857 & 0,139806133 & 1,284600442 & Tdrkh & tudor and $\mathrm{KH}$ domain containing protein \\
\hline 17333731 & 3,361028777 & 0,001329838 & 0,139806133 & 1,711764232 & Fpr2 & formyl peptide receptor 2 \\
\hline 17267184 & 3,359590182 & 0,001335453 & 0,139806133 & 1,485165506 & Mir5110 & microRNA 5110 \\
\hline 17430521 & 3,350265516 & 0,001360503 & 0,139806133 & 1,329751953 & Tmem39b & transmembrane protein $39 \mathrm{~b}$ \\
\hline 17350921 & 3,335498453 & 0,001404989 & 0,139806133 & 1,995201274 & F830016B08Rik & RIKEN cDNA F830016B08 gene \\
\hline 17486299 & 3,319700979 & 0,001440406 & 0,139806133 & 1,429051565 & Vmn2r45 & vomeronasal 2, receptor 45 \\
\hline 17501096 & 3,316466258 & 0,001449476 & 0,139806133 & 1,369257681 & Cldn24 & claudin 24 \\
\hline 17416460 & $-2,497229696$ & 0,0072206 & 0,18966393 & 0,581152779 & Yipf1 & Yip1 domain family, member 1 \\
\hline 17349926 & $-2,497634313$ & 0,007209803 & 0,189599092 & 0,626549827 & Pcdhb17 & protocadherin beta 17 \\
\hline 17361805 & $-2,498518842$ & 0,007197709 & 0,189514594 & 0,552596624 & Neat1 & nuclear paraspeckle assembly transcript 1 (non-protein coding) \\
\hline 17245248 & $-2,499813117$ & 0,00717741 & 0,189281753 & 0,53043319 & Mdm2 & transformed mouse $3 T 3$ cell double minute 2 \\
\hline 17419025 & $-2,501624413$ & 0,007154951 & 0,189281753 & 0,74309541 & Tmem234 & transmembrane protein 234 \\
\hline 17484140 & $-2,501726179$ & 0,007153655 & 0,189281753 & 0,757259058 & Bccip & BRCA2 and CDKN1A interacting protein \\
\hline 17290603 & $-2,508650142$ & 0,007056476 & 0,188347263 & 0,559173144 & Actn2 & actinin alpha 2 \\
\hline 17514257 & $-2,509510044$ & 0,007042655 & 0,188130325 & 0,668267065 & $\mathrm{Rbm} 34$ & RNA binding motif protein 34 \\
\hline 17374612 & $-2,509875138$ & 0,00703704 & 0,188130325 & 0,66473691 & Phgr1 & proline/histidine/glycine-rich 1 \\
\hline 17514247 & $-2,510042233$ & 0,007034881 & 0,188130325 & 0,724393976 & Tomm20 & translocase of outer mitochondrial membrane 20 homolog (yeast) \\
\hline
\end{tabular}




\begin{tabular}{|c|c|c|c|c|c|c|}
\hline $\begin{array}{l}\text { probeset } \\
\text { ID }\end{array}$ & d.value & p.value & q.value & R.fold & Genename & Description \\
\hline 17305243 & $-2,512314764$ & 0,007007239 & 0,187720276 & 0,632938723 & Sftpd & surfactant associated protein D \\
\hline 17444664 & $-2,512425227$ & 0,007006375 & 0,187720276 & 0,529707651 & Zfp655 & zinc finger protein 655 \\
\hline 17407643 & $-2,51351983$ & 0,006988667 & 0,187598072 & 0,783699822 & B230398E01Rik & RIKEN cDNA B230398E01 gene \\
\hline 17239113 & $-2,5141921$ & 0,006980029 & 0,187518528 & 0,592007731 & Sash1 & SAM and SH3 domain containing 1 \\
\hline 17305856 & $-2,516407566$ & 0,00694634 & 0,186765324 & 0,63893514 & Exoc5 & exocyst complex component 5 \\
\hline 17454345 & $-2,519971846$ & 0,00689883 & 0,185801914 & 0,797963632 & Pdgfa & platelet derived growth factor, alpha \\
\hline 17533553 & $-2,520733979$ & 0,006886737 & 0,185767399 & 0,640840867 & Jade3 & jade family PHD finger 3 \\
\hline 17369821 & $-2,52119706$ & 0,00688069 & 0,185767399 & 0,671838754 & Ptges2 & prostaglandin E synthase 2 \\
\hline 17438189 & $-2,52214049$ & 0,006866437 & 0,185767399 & 0,579690564 & Rasl11b & RAS-like, family 11 , member B \\
\hline 17333946 & $-2,524315277$ & 0,006842251 & 0,18547585 & 0,748224926 & Zfp948 & zinc finger protein 948 \\
\hline 17329692 & $-2,524740888$ & 0,006831453 & 0,18547585 & 0,557148174 & Lsg1 & large subunit GTPase 1 homolog (S. cerevisiae) \\
\hline 17301926 & $-2,52905349$ & 0,006778329 & 0,184499854 & 0,345126777 & $\mathrm{Htr} 2 \mathrm{a}$ & 5-hydroxytryptamine (serotonin) receptor $2 \mathrm{~A}$ \\
\hline 17248592 & $-2,529282868$ & 0,006775737 & 0,184499854 & 0,599455873 & Slu7 & SLU7 splicing factor homolog (S. cerevisiae) \\
\hline 17502378 & $-2,530378383$ & 0,006755438 & 0,184499854 & 0,646333559 & Tpm4 & tropomyosin 4 \\
\hline 17539898 & $-2,531075478$ & 0,006745936 & 0,184499854 & 0,628054031 & Ccdc120 & coiled-coil domain containing 120 \\
\hline 17547602 & $-2,531672848$ & 0,00673773 & 0,184499854 & 0,463432067 & Gm10099 & predicted gene 10099 \\
\hline 17548865 & $-2,532814626$ & 0,006719158 & 0,184499854 & 0,730586649 & Gm8692 & predicted gene 8692 \\
\hline 17217666 & $-2,532845692$ & 0,006718726 & 0,184499854 & 0,721297613 & Tmem9 & transmembrane protein 9 \\
\hline 17252183 & $-2,533220175$ & 0,006711815 & 0,184499854 & 0,563148488 & Eno3 & enolase 3 , beta muscle \\
\hline 17488975 & $-2,539511227$ & 0,006619387 & 0,183666593 & 0,571852116 & Tbcb & tubulin folding cofactor B \\
\hline 17211700 & $-2,540550192$ & 0,006605998 & 0,183666593 & 0,745274987 & Imp4 & IMP4, U3 small nucleolar ribonucleoprotein, homolog (yeast) \\
\hline 17354764 & $-2,541071114$ & 0,006598656 & 0,183666593 & 0,673204769 & Arhgef37 & Rho guanine nucleotide exchange factor (GEF) 37 \\
\hline 17415219 & $-2,541925716$ & 0,006586563 & 0,183666593 & 0,764944787 & Acer2 & alkaline ceramidase 2 \\
\hline 17301576 & $-2,54257559$ & 0,006575333 & 0,183664448 & 0,51894453 & Gnrh1 & gonadotropin releasing hormone 1 \\
\hline 17263837 & $-2,546844978$ & 0,006529119 & 0,182803277 & 0,764126348 & Epn2 & epsin 2 \\
\hline 17388551 & $-2,546893617$ & 0,006527823 & 0,182803277 & 0,729518007 & Alkbh3 & alkB, alkylation repair homolog 3 (E. coli) \\
\hline 17401041 & $-2,54718384$ & 0,006524368 & 0,182803277 & 0,64039461 & Casq2 & calsequestrin 2 \\
\hline 17274415 & $-2,547631489$ & 0,006518753 & 0,182803277 & 0,782585177 & Asap2 & ArfGAP with SH3 domain, ankyrin repeat and PH domain 2 \\
\hline 17323192 & $-2,548586565$ & 0,006509683 & 0,182803277 & 0,665701162 & Snai2 & snail family zinc finger 2 \\
\hline 17497044 & $-2,548985289$ & 0,006503637 & 0,182803277 & 0,620950933 & lkzf5 & IKAROS family zinc finger 5 \\
\hline 17310432 & $-2,553274449$ & 0,006436259 & 0,181777348 & 0,64087704 & Mtmr12 & myotubularin related protein 12 \\
\hline 17473219 & $-2,553384254$ & 0,006434964 & 0,181777348 & 0,807714072 & Tsen34 & tRNA splicing endonuclease 34 homolog (S. cerevisiae) \\
\hline 17497421 & $-2,554435716$ & 0,006423302 & 0,181722039 & 0,65820401 & Bnip3 & $\mathrm{BCL} 2$ /adenovirus E1B interacting protein 3 \\
\hline 17497687 & $-2,555936938$ & 0,006401707 & 0,181266279 & 0,642662774 & Bet1l & blocked early in transport 1 homolog (S. cerevisiae)-like \\
\hline 17360216 & $-2,556471106$ & 0,00639566 & 0,181250378 & 0,580484835 & Gsto1 & glutathione S-transferase omega 1 \\
\hline 17399914 & $-2,556684235$ & 0,006391773 & 0,181250378 & 0,728164336 & Lce3d & late cornified envelope 3D \\
\hline 17280247 & $-2,557542789$ & 0,00637968 & 0,181250378 & 0,808351755 & 6030426L16Rik & RIKEN cDNA 6030426L16 gene \\
\hline 17338562 & $-2,559290579$ & 0,006358516 & 0,181250378 & 0,591810537 & Rab5a & RAB5A, member RAS oncogene family \\
\hline 17455231 & $-2,564868215$ & 0,00628682 & 0,181250378 & 0,539023618 & B230303012Rik & RIKEN cDNA B230303012 gene \\
\hline 17223793 & $-2,566114705$ & 0,006272135 & 0,181250378 & 0,538160081 & Fzd5 & frizzled homolog 5 (Drosophila) \\
\hline 17511259 & $-2,56785717$ & 0,006251404 & 0,181250378 & 0,616698437 & Junb & jun B proto-oncogene \\
\hline 17498032 & $-2,568871151$ & 0,00623931 & 0,181250378 & 0,59953482 & Mob2 & MOB kinase activator 2 \\
\hline 17256691 & $-2,56991057$ & 0,006227649 & 0,181250378 & 0,704756948 & Aoc2 & amine oxidase, copper containing 2 (retina-specific) \\
\hline 17245539 & $-2,570650658$ & 0,006219011 & 0,181250378 & 0,683037052 & Srgap1 & SLIT-ROBO Rho GTPase activating protein 1 \\
\hline 17327450 & $-2,570690228$ & 0,006217715 & 0,181250378 & 0,658200203 & Kcnj15 & potassium inwardly-rectifying channel, subfamily J, member 15 \\
\hline 17334749 & $-2,570763036$ & 0,006217283 & 0,181250378 & 0,7656193 & Narfl & nuclear prelamin A recognition factor-like \\
\hline 17319554 & $-2,571781758$ & 0,006201303 & 0,181250378 & 0,651679162 & Pmm1 & phosphomannomutase 1 \\
\hline 17344990 & $-2,571962197$ & 0,006199575 & 0,181250378 & 0,53083904 & Cenpq & centromere protein $\mathrm{Q}$ \\
\hline 17339574 & $-2,574694349$ & 0,006167614 & 0,181250378 & 0,541281906 & Ehd3 & EH-domain containing 3 \\
\hline 17326816 & $-2,578933327$ & 0,006107579 & 0,181229766 & 0,716719895 & Gabpa & GA repeat binding protein, alpha \\
\hline 17292800 & $-2,578941992$ & 0,006106715 & 0,181229766 & 0,672990221 & Uimc1 & ubiquitin interaction motif containing 1 \\
\hline 17359449 & $-2,580315302$ & 0,006080369 & 0,181171751 & 0,652432117 & Gm340 & predicted gene 340 \\
\hline 17519568 & $-2,584695794$ & 0,006023789 & 0,180135616 & 0,78242384 & Gm19569 & predicted gene, 19569 \\
\hline 17523281 & $-2,58517594$ & 0,006017311 & 0,180104871 & 0,751641644 & Trak1 & trafficking protein, kinesin binding 1 \\
\hline 17450727 & $-2,589900355$ & 0,005959435 & 0,179280713 & 0,555872881 & Tmed5 & $\begin{array}{l}\text { transmembrane emp } 24 \text { protein transport domain containing } 5 \\
\text { nuclear factor of activated T cells, cytoplasmic, calcineurin dependent }\end{array}$ \\
\hline 17496183 & $-2,591950462$ & 0,005929634 & 0,178776083 & 0,735562411 & Nfatc2ip & 2 interacting protein \\
\hline 17328451 & $-2,592978075$ & 0,005920996 & 0,178776083 & 0,635783393 & Mzt2 & mitotic spindle organizing protein 2 \\
\hline 17211405 & $-2,595194891$ & 0,005894218 & 0,178776083 & 0,729772749 & Gsta3 & glutathione S-transferase, alpha 3 \\
\hline 17376252 & $-2,60021396$ & 0,005841093 & 0,178220547 & 0,6842111 & Nop56 & NOP56 ribonucleoprotein \\
\hline 17526614 & $-2,604116768$ & 0,005794879 & 0,177630577 & 0,669812652 & Pafah1b2 & platelet-activating factor acetylhydrolase, isoform $1 b$, subunit 2 \\
\hline 17222001 & $-2,604622014$ & 0,005791424 & 0,177630577 & 0,668269592 & Prim2 & DNA primase, $\mathrm{p} 58$ subunit \\
\hline 17510563 & $-2,606851859$ & 0,005768965 & 0,177630577 & 0,737027415 & Slc35e1 & solute carrier family 35 , member E1 \\
\hline 17379417 & $-2,61233766$ & 0,005703747 & 0,17675478 & 0,633331235 & Sys1 & $\begin{array}{l}\text { SYS1 Golgi-localized integral membrane protein homolog (S. } \\
\text { cerevisiae) }\end{array}$ \\
\hline 17366039 & $-2,613876477$ & 0,005682584 & 0,176641293 & 0,683774407 & Pdzd8 & PDZ domain containing 8 \\
\hline 17299924 & $-2,615495996$ & 0,00566574 & 0,176454732 & 0,632618372 & Rps19 & ribosomal protein $\mathrm{S} 19$ \\
\hline 17299847 & $-2,615495996$ & 0,00566574 & 0,176454732 & 0,632618372 & Rps19 & ribosomal protein S19 \\
\hline 17234042 & $-2,616348527$ & 0,005656238 & 0,176454732 & 0,692929988 & Rhobtb1 & Rho-related BTB domain containing 1 \\
\hline 17323759 & $-2,616492732$ & 0,005655374 & 0,176454732 & 0,716603763 & Ufd1l & ubiquitin fusion degradation 1 like \\
\hline 17302802 & $-2,618405952$ & 0,005631619 & 0,176389566 & 0,787319767 & Farp1 & $\begin{array}{l}\text { FERM, RhoGEF (Arhgef) and pleckstrin domain protein } 1 \\
\text { (chondrocyte-derived) }\end{array}$ \\
\hline 17277084 & $-2,619507906$ & 0,005622117 & 0,176259024 & 0,728845203 & Psen1 & presenilin 1 \\
\hline 17478864 & $-2,620770381$ & 0,005610456 & 0,176060466 & 0,631549514 & Mtmr10 & myotubularin related protein 10 \\
\hline 17454416 & $-2,622212535$ & 0,00559793 & 0,175834397 & 0,366636307 & Zfand2a & zinc finger, AN1-type domain $2 \mathrm{~A}$ \\
\hline 17244175 & $-2,624796715$ & 0,005565969 & 0,175330474 & 0,70873077 & Slc25a3 & $\begin{array}{l}\text { solute carrier family } 25 \text { (mitochondrial carrier, phosphate carrier), } \\
\text { member } 3\end{array}$ \\
\hline 17463883 & $-2,625266183$ & 0,00556165 & 0,175330474 & 0,662442292 & Strap & serine/threonine kinase receptor associated protein \\
\hline 17522971 & $-2,625563305$ & 0,005555604 & 0,175330474 & 0,662778337 & Vill & villin-like \\
\hline 17337513 & $-2,625791271$ & 0,005553444 & 0,175330474 & 0,766430093 & Gabbr1 & gamma-aminobutyric acid (GABA) B receptor, 1 \\
\hline 17237851 & $-2,626493184$ & 0,005543942 & 0,175305073 & 0,785328727 & Avil & advillin \\
\hline
\end{tabular}




\begin{tabular}{|c|c|c|c|c|c|c|}
\hline $\begin{array}{l}\text { probeset } \\
\text { ID }\end{array}$ & d.value & p.value & q.value & R.fold & Genename & Description \\
\hline 17442909 & $-2,627890648$ & 0,005526234 & 0,175281605 & 0,678336469 & Gbas & glioblastoma amplified sequence \\
\hline 17371661 & $-2,628673989$ & 0,005515868 & 0,175255894 & 0,79092066 & Gorasp2 & golgi reassembly stacking protein 2 \\
\hline 17448607 & $-2,630949662$ & 0,005492113 & 0,175255894 & 0,650875127 & Commd8 & COMM domain containing 8 \\
\hline 17514745 & $-2,631887548$ & 0,005483907 & 0,175255894 & 0,617876931 & Cwc15 & CWC15 homolog (S. cerevisiae) \\
\hline 17515045 & $-2,633336813$ & 0,005465335 & 0,175255894 & 0,581788798 & Ppan & peter pan homolog (Drosophila) \\
\hline 17368947 & $-2,63336743$ & 0,005464903 & 0,175255894 & 0,743592136 & Odf2 & outer dense fiber of sperm tails 2 \\
\hline 17271931 & $-2,63411414$ & 0,005456697 & 0,175255894 & 0,703313195 & Sumo2 & SMT3 suppressor of mif two 3 homolog 2 (yeast) \\
\hline 17326801 & $-2,636456437$ & 0,005432079 & 0,175255894 & 0,617708271 & Jam2 & junction adhesion molecule 2 \\
\hline 17443133 & $-2,63719045$ & 0,0054256 & 0,175255894 & 0,731736973 & Wbscr27 & Williams Beuren syndrome chromosome region 27 (human) \\
\hline 17249140 & $-2,638850463$ & 0,005407028 & 0,175255894 & 0,748149099 & 3010026009Rik & RIKEN cDNA 3010026009 gene \\
\hline 17492239 & $-2,638872953$ & 0,005406164 & 0,175255894 & 0,696284176 & AU020206 & expressed sequence AU020206 \\
\hline 17412552 & $-2,641472762$ & 0,005374203 & 0,175255894 & 0,646276466 & Ube2j1 & ubiquitin-conjugating enzyme E2J 1 \\
\hline 17262768 & $-2,641813714$ & 0,005370316 & 0,175255894 & 0,648609204 & Slc22a21 & solute carrier family 22 (organic cation transporter), member 21 \\
\hline 17533234 & $-2,642946783$ & 0,005358223 & 0,175255894 & 0,661347211 & Syt15 & $\begin{array}{l}\text { synaptotagmin-like } 5 \\
\text { polycystic kidney disease (polycystin) and REJ (sperm receptor for egg }\end{array}$ \\
\hline 17320100 & $-2,643270686$ & 0,005354767 & 0,175255894 & 0,773630324 & Pkdrej & jelly homolog, sea urchin) \\
\hline 17318587 & $-2,644250407$ & 0,005342674 & 0,175255894 & 0,576918048 & SIc39a4 & solute carrier family 39 (zinc transporter), member 4 \\
\hline 17452396 & $-2,644408803$ & 0,005340083 & 0,175255894 & 0,784052782 & Fam216a & family with sequence similarity 216 , member $A$ \\
\hline 17370309 & $-2,647746974$ & 0,005308553 & 0,174901557 & 0,556445032 & Ptgs1 & prostaglandin-endoperoxide synthase 1 \\
\hline 17469556 & $-2,647751481$ & 0,005308122 & 0,174901557 & 0,684449735 & Crbn & cereblon \\
\hline 17245850 & $-2,648349665$ & 0,005300779 & 0,174901557 & 0,65999114 & Mars & methionine-tRNA synthetase \\
\hline 17288387 & $-2,648493938$ & 0,00529862 & 0,174901557 & 0,761178211 & Nsun2 & NOL1/NOP2/Sun domain family member 2 \\
\hline 17304860 & $-2,649685851$ & 0,005286526 & 0,174901557 & 0,663727425 & Timm23 & translocase of inner mitochondrial membrane 23 \\
\hline 17349745 & $-2,650716874$ & 0,005277024 & 0,174901557 & 0,75270613 & lk & IK cytokine \\
\hline 17333854 & $-2,651528861$ & 0,005264931 & 0,174901557 & 0,615362746 & Ppp2r1a & protein phosphatase 2 , regulatory subunit $A$, alpha \\
\hline 17253972 & $-2,6520956$ & 0,005258452 & 0,174901557 & 0,623804796 & Psmd11 & proteasome (prosome, macropain) 26S subunit, non-ATPase, 11 \\
\hline 17269368 & $-2,653108381$ & 0,0052442 & 0,174901557 & 0,566773446 & Krt19 & keratin 19 \\
\hline 17352120 & $-2,654372022$ & 0,005231674 & 0,174901557 & 0,66554991 & Pard6g & par-6 family cell polarity regulator gamma \\
\hline 17331438 & $-2,654766189$ & 0,005226491 & 0,174901557 & 0,769791633 & Chmp2b & $\begin{array}{l}\text { charged multivesicular body protein } 2 \mathrm{~B} \\
\text { TAF7 RNA polymerase II, TATA box binding protein (TBP)-associated }\end{array}$ \\
\hline 17353796 & $-2,655642458$ & 0,005218285 & 0,174901557 & 0,594846218 & Taf7 & factor \\
\hline 17533746 & $-2,65573428$ & 0,005216126 & 0,174901557 & 0,723096003 & Agtr2 & angiotensin II receptor, type 2 \\
\hline 17512833 & $-2,664127643$ & 0,005127585 & 0,174901557 & 0,73046813 & Ist1 & increased sodium tolerance 1 homolog (yeast) \\
\hline 17262783 & $-2,66644136$ & 0,005102534 & 0,174901557 & 0,726446306 & Slc22a4 & solute carrier family 22 (organic cation transporter), member 4 \\
\hline 17398043 & $-2,667184195$ & 0,005092169 & 0,174901557 & 0,692908997 & Vmn2r1 & vomeronasal 2 , receptor 1 \\
\hline 17531154 & $-2,667203229$ & 0,005091737 & 0,174901557 & 0,733496752 & Bsn & bassoon \\
\hline 17479303 & $-2,670901419$ & 0,005051138 & 0,174834218 & 0,65753289 & Aen & apoptosis enhancing nuclease \\
\hline 17338233 & $-2,671984142$ & 0,005038612 & 0,174834218 & 0,638633305 & Mrps10 & $\begin{array}{l}\text { mitochondrial ribosomal protein } \mathrm{S} 10 \\
\text { TATA box binding protein (Tbp)-associated factor, RNA polymerase I, }\end{array}$ \\
\hline 17514806 & $-2,672162946$ & 0,005036021 & 0,174834218 & 0,71156109 & Taf1d & D \\
\hline 17361032 & $-2,672922179$ & 0,005026087 & 0,174822971 & 0,792850817 & Ndufs8 & NADH dehydrogenase (ubiquinone) Fe-S protein 8 \\
\hline 17250374 & $-2,673237391$ & 0,005023064 & 0,174822971 & 0,73845289 & Drg2 & developmentally regulated GTP binding protein 2 \\
\hline 17507094 & $-2,674917121$ & 0,005005356 & 0,174653402 & 0,742652221 & Xab2 & XPA binding protein 2 \\
\hline 17383284 & $-2,675053376$ & 0,005003196 & 0,174653402 & 0,828941042 & Vav2 & vav 2 oncogene \\
\hline 17459614 & $-2,675948716$ & 0,004994126 & 0,174630374 & 0,757689235 & 0610030E20Rik & RIKEN cDNA 0610030 E20 gene \\
\hline 17517914 & $-2,677056042$ & 0,004982465 & 0,174407164 & 0,765272604 & Nptn & neuroplastin \\
\hline 17529647 & $-2,679319512$ & 0,004958278 & 0,173744578 & 0,499188659 & Morf4l1 & mortality factor 4 like 1 \\
\hline 17509682 & $-2,682520629$ & 0,004924589 & 0,17311482 & 0,52785366 & BC030870 & cDNA sequence $B C 030870$ \\
\hline 17373825 & $-2,683266564$ & 0,004916383 & 0,1730104 & 0,353978165 & Cd59a & CD59a antigen \\
\hline 17276386 & $-2,684230462$ & 0,004906017 & 0,1730104 & 0,618383974 & Rhoj & ras homolog gene family, member J \\
\hline 17281908 & $-2,685225943$ & 0,004898675 & 0,172939763 & 0,748004605 & Tmem30b & transmembrane protein $30 \mathrm{~B}$ \\
\hline 17359803 & $-2,687621799$ & 0,00487492 & 0,172469663 & 0,651471734 & Sfxn3 & sideroflexin 3 \\
\hline 17335467 & $-2,68834531$ & 0,004867578 & 0,172394473 & 0,298237151 & Cdkn1a & cyclin-dependent kinase inhibitor $1 \mathrm{~A}(\mathrm{P} 21)$ \\
\hline 17227464 & $-2,68942266$ & 0,004858076 & 0,172242556 & 0,702641089 & 5730559C18Rik & RIKEN cDNA 5730559C18 gene \\
\hline 17324762 & $-2,691829135$ & 0,004831298 & 0,171477128 & 0,724984675 & Rnf168 & ring finger protein 168 \\
\hline 17294368 & $-2,694329859$ & 0,004803224 & 0,171216326 & 0,541978321 & Pdcd6 & programmed cell death 6 \\
\hline 17386396 & $-2,694769255$ & 0,004799768 & 0,171216326 & 0,641300545 & Slc25a12 & solute carrier family 25 (mitochondrial carrier, Aralar), member 12 \\
\hline 17376378 & $-2,694987373$ & 0,004797609 & 0,171216326 & 0,741134925 & Itpa & inosine triphosphatase (nucleoside triphosphate pyrophosphatase) \\
\hline 17344794 & $-2,697204468$ & 0,004778605 & 0,171216326 & 0,463904845 & Znrd1 & zinc ribbon domain containing, 1 \\
\hline 17357640 & $-2,700784828$ & 0,00474578 & 0,171216326 & 0,5234096 & $\mathrm{Ms} 4 \mathrm{a} 4 \mathrm{a}$ & membrane-spanning 4-domains, subfamily $\mathrm{A}$, member $4 \mathrm{~A}$ \\
\hline 17506042 & $-2,707981576$ & 0,004678835 & 0,170836722 & 0,64113798 & Cdh13 & cadherin 13 \\
\hline 17245383 & $-2,713910773$ & 0,004623551 & 0,169756037 & 0,637763452 & Helb & helicase (DNA) B \\
\hline 17457343 & $-2,714704844$ & 0,004617936 & 0,169738486 & 0,560431806 & Atp6v0c & ATPase, $\mathrm{H}+$ transporting, lysosomal Vo subunit $\mathrm{C}$ \\
\hline 17291833 & $-2,71495106$ & 0,004615345 & 0,169738486 & 0,651694114 & Eci2 & enoyl-Coenzyme A delta isomerase 2 \\
\hline 17414802 & $-2,716110854$ & 0,00460757 & 0,169738486 & 0,442857133 & Pappa & pregnancy-associated plasma protein $A$ \\
\hline 17346175 & $-2,716445687$ & 0,004603683 & 0,169738486 & 0,701397021 & Mydgf & myeloid derived growth factor \\
\hline 17414416 & $-2,718043851$ & 0,004585975 & 0,169738486 & 0,760183774 & Dnajc25 & DnaJ (Hsp40) homolog, subfamily C, member 25 \\
\hline 17526917 & $-2,719138653$ & 0,004576905 & 0,169738486 & 0,765064316 & 2310030G06Rik & RIKEN CDNA 2310030 G06 gene \\
\hline 17378628 & $-2,72211682$ & 0,004554014 & 0,169462457 & 0,663841499 & Aar2 & AAR2 splicing factor homolog (S. cerevisiae) \\
\hline 17527977 & $-2,725253598$ & 0,004521189 & 0,169193657 & 0,630720734 & Glce & glucuronyl C5-epimerase \\
\hline 17486110 & $-2,731947434$ & 0,004454676 & 0,168614124 & 0,550626816 & Peg3 & paternally expressed 3 \\
\hline 17434310 & $-2,733188407$ & 0,004443446 & 0,168545619 & 0,556552899 & Fam133b & family with sequence similarity 133 , member $B$ \\
\hline 17470187 & $-2,733429711$ & 0,004440423 & 0,168545619 & 0,68765518 & Zfp9 & zinc finger protein 9 \\
\hline 17290083 & $-2,734867464$ & 0,004426602 & 0,168516657 & 0,74520962 & Emb & embigin \\
\hline 17255387 & $-2,735038979$ & 0,004424874 & 0,168516657 & 0,69471919 & Slc35b1 & solute carrier family 35 , member B1 \\
\hline 17240199 & $-2,735765254$ & 0,004419691 & 0,168516657 & 0,614379542 & $\mathrm{Nt5dc1}$ & $\begin{array}{l}\text { 5'-nucleotidase domain containing } 1 \\
\text { solute carrier family } 7 \text { (cationic amino acid transporter, } y+\text { system), }\end{array}$ \\
\hline 17306477 & $-2,738227733$ & 0,004393345 & 0,167830663 & 0,688707645 & Slc7a8 & $\begin{array}{l}\text { member } 8 \\
\text { gamma-aminobutyric acid (GABA) A receptor-associated protein-like }\end{array}$ \\
\hline 17505774 & $-2,740217957$ & 0,004376069 & 0,167830663 & 0,725892034 & Gabarapl2 & 2 \\
\hline 17309065 & $-2,745347205$ & 0,004332878 & 0,167619692 & 0,695111508 & Mzt1 & mitotic spindle organizing protein 1 \\
\hline
\end{tabular}




\begin{tabular}{|c|c|c|c|c|c|c|}
\hline $\begin{array}{l}\text { probeset } \\
\text { ID }\end{array}$ & d.value & p.value & q.value & R.fold & Genename & Description \\
\hline 17324274 & $-2,745612561$ & 0,004330287 & 0,167619692 & 0,642755047 & Senp2 & SUMO/sentrin specific peptidase 2 \\
\hline 17397344 & $-2,747310177$ & 0,004314306 & 0,167522644 & 0,679516798 & Jade1 & jade family PHD finger 1 \\
\hline 17541404 & $-2,747977485$ & 0,004309124 & 0,167518244 & 0,695152799 & Elf4 & $\begin{array}{l}\text { E74-like factor } 4 \text { (ets domain transcription factor) } \\
\text { TATA box binding protein (Tbp)-associated factor, RNA polymerase I, }\end{array}$ \\
\hline 17514832 & $-2,755338322$ & 0,004245202 & 0,166668489 & 0,632339155 & Taf1d & D \\
\hline 17339529 & $-2,755541526$ & 0,004243474 & 0,166668489 & 0,54056209 & Clip4 & CAP-GLY domain containing linker protein family, member 4 \\
\hline 17425077 & $-2,757718462$ & 0,004221879 & 0,166668489 & 0,69635286 & Tbc1d 2 & TBC1 domain family, member 2 \\
\hline 17327331 & $-2,757834385$ & 0,004220151 & 0,166668489 & 0,621553797 & Chaf1b & chromatin assembly factor 1 , subunit B (p60) \\
\hline 17259794 & $-2,763469001$ & 0,00417005 & 0,166668489 & 0,53911254 & Rnf185 & ring finger protein 185 \\
\hline 17376728 & $-2,763519677$ & 0,004168754 & 0,166668489 & 0,608161941 & Plcb4 & phospholipase $C$, beta 4 \\
\hline 17468520 & $-2,764192819$ & 0,004160548 & 0,166668489 & 0,750245536 & Snrnp27 & small nuclear ribonucleoprotein 27 (U4/U6.U5) \\
\hline 17303849 & $-2,766514473$ & 0,004145863 & 0,166668489 & 0,593515645 & Usp54 & ubiquitin specific peptidase 54 \\
\hline 17337228 & $-2,767751845$ & 0,004136361 & 0,166668489 & 0,470759535 & ler3 & immediate early response 3 \\
\hline 17335383 & $-2,769413109$ & 0,004121245 & 0,166668489 & 0,569199605 & Mapk13 & mitogen-activated protein kinase 13 \\
\hline 17479183 & $-2,773217809$ & 0,004086692 & 0,166668489 & 0,739552422 & Fam174b & family with sequence similarity 174 , member $B$ \\
\hline 17308881 & $-2,775050125$ & 0,004072871 & 0,166668489 & 0,668594339 & Rgcc & regulator of cell cycle \\
\hline 17246520 & $-2,779696262$ & 0,004041342 & 0,165684795 & 0,587135566 & Eif4enif1 & $\begin{array}{l}\text { eukaryotic translation initiation factor } 4 \text { E nuclear import factor } 1 \\
\text { KDEL (Lys-Asp-Glu-Leu) endoplasmic reticulum protein retention }\end{array}$ \\
\hline 17313000 & $-2,78126475$ & 0,004027521 & 0,165684795 & 0,64154094 & Kdelr3 & receptor 3 \\
\hline 17523861 & $-2,781305583$ & 0,004027089 & 0,165684795 & 0,641725926 & C330006D17Rik & RIKEN cDNA C330006D17 gene \\
\hline 17465942 & $-2,781636575$ & 0,004024498 & 0,165684795 & 0,411732126 & Atp6v0a4 & ATPase, $\mathrm{H}+$ transporting, lysosomal V0 subunit A4 \\
\hline 17550426 & $-2,783900712$ & 0,004008085 & 0,165553763 & 0,516733223 & Neat1 & nuclear paraspeckle assembly transcript 1 (non-protein coding) \\
\hline 17390249 & $-2,785898521$ & 0,0039934 & 0,165225099 & 0,677831841 & Lrrc57 & leucine rich repeat containing 57 \\
\hline 17364545 & $-2,787841075$ & 0,003976124 & 0,165058909 & 0,444027674 & Tctn3 & tectonic family member 3 \\
\hline 17393357 & $-2,795813643$ & 0,003916089 & 0,163801393 & 0,616693759 & Eif6 & eukaryotic translation initiation factor 6 \\
\hline 17458112 & $-2,80083754$ & 0,003871171 & 0,162953918 & 0,639585708 & Zfp398 & zinc finger protein 398 \\
\hline 17315946 & $-2,803407163$ & 0,003851303 & 0,162739538 & 0,678459253 & Dnajc21 & DnaJ (Hsp40) homolog, subfamily C, member 21 \\
\hline 17453205 & $-2,807766855$ & 0,003815455 & 0,162262231 & 0,658980052 & Sbds & Shwachman-Bodian-Diamond syndrome homolog (human) \\
\hline 17420347 & $-2,810183289$ & 0,003797315 & 0,161864409 & 0,722690664 & Ece1 & endothelin converting enzyme 1 \\
\hline 17211305 & $-2,811517469$ & 0,003789973 & 0,161803246 & 0,369164268 & Pi15 & peptidase inhibitor 15 \\
\hline 17299869 & $-2,822636784$ & 0,003701864 & 0,159369283 & 0,581031863 & Trav9d-3 & T cell receptor alpha variable 9D-3 \\
\hline 17431332 & $-2,822778262$ & 0,003699705 & 0,159369283 & 0,522164933 & Man1c1 & mannosidase, alpha, class $1 \mathrm{C}$, member 1 \\
\hline 17333465 & $-2,827291114$ & 0,003672063 & 0,159029476 & 0,475035242 & Smoc2 & SPARC related modular calcium binding 2 \\
\hline 17363374 & $-2,829136878$ & 0,003658674 & 0,158657562 & 0,511484521 & Ostf1 & osteoclast stimulating factor 1 \\
\hline 17288898 & $-2,833041462$ & 0,003633191 & 0,158209654 & 0,664284373 & Lysmd3 & LysM, putative peptidoglycan-binding, domain containing 3 \\
\hline 17349181 & $-2,835298323$ & 0,003618938 & 0,158209654 & 0,662003169 & Wdr33 & WD repeat domain 33 \\
\hline 17446775 & $-2,836700914$ & 0,003609868 & 0,158202043 & 0,659102403 & Preb & prolactin regulatory element binding \\
\hline 17222527 & $-2,837987894$ & 0,003599071 & 0,158202043 & 0,428180789 & Tbc1d8 & TBC1 domain family, member 8 \\
\hline 17312301 & $-2,840091064$ & 0,003583954 & 0,158114857 & 0,743693985 & Zfp 707 & zinc finger protein 707 \\
\hline 17532455 & $-2,841218778$ & 0,00357618 & 0,157982799 & 0,515684212 & 1110059G10Rik & RIKEN cDNA $1110059 \mathrm{G} 10$ gene \\
\hline 17506697 & $-2,842981118$ & 0,003565814 & 0,157947197 & 0,415862105 & Rhou & ras homolog gene family, member $U$ \\
\hline 17277134 & $-2,844512396$ & 0,003554152 & 0,157871853 & 0,609387983 & Acot2 & acyl-CoA thioesterase 2 \\
\hline 17364813 & $-2,844907076$ & 0,003550265 & 0,157871853 & 0,492644027 & Avpi1 & arginine vasopressin-induced 1 \\
\hline 17402181 & $-2,845262259$ & 0,003548969 & 0,157871853 & 0,43002184 & F3 & coagulation factor III \\
\hline 17453242 & $-2,848236859$ & 0,003530829 & 0,157871853 & 0,702887869 & Auts2 & autism susceptibility candidate 2 \\
\hline 17459250 & $-2,850424486$ & 0,003516145 & 0,15780396 & 0,759080524 & Gng12 & guanine nucleotide binding protein (G protein), gamma 12 \\
\hline 17309420 & $-2,851785456$ & 0,003509666 & 0,15780396 & 0,674755276 & Abcc4 & ATP-binding cassette, sub-family C (CFTR/MRP), member 4 \\
\hline 17306333 & $-2,853344362$ & 0,003498005 & 0,15780396 & 0,634075011 & Dad1 & defender against cell death 1 \\
\hline 17530406 & $-2,85467586$ & 0,003489366 & 0,15780396 & 0,673026503 & Acpp & acid phosphatase, prostate \\
\hline 17396614 & $-2,855539144$ & 0,003485479 & 0,15780396 & 0,766754821 & Prkci & protein kinase $\mathrm{C}$, iota \\
\hline 17340232 & $-2,856393878$ & 0,00348116 & 0,15780396 & 0,604969375 & Cript & cysteine-rich PDZ-binding protein \\
\hline 17257639 & $-2,858179968$ & 0,003469067 & 0,15780396 & 0,400220306 & 1810010H24Rik & RIKEN cDNA $1810010 \mathrm{H} 24$ gene \\
\hline 17436791 & $-2,860802131$ & 0,003450063 & 0,15780396 & 0,588290921 & Mir3097 & microRNA 3097 \\
\hline 17498199 & $-2,861743951$ & 0,003444016 & 0,15780396 & 0,685832791 & R74862 & expressed sequence $R 74862$ \\
\hline 17523994 & $-2,863694605$ & 0,003434083 & 0,15780396 & 0,686936842 & Cep57 & centrosomal protein 57 \\
\hline 17334241 & $-2,865718644$ & 0,003414215 & 0,157568688 & 0,670905447 & Rnps1 & ribonucleic acid binding protein $\mathrm{S} 1$ \\
\hline 17480312 & $-2,86824249$ & 0,003395211 & 0,157390409 & 0,570905384 & Gab2 & growth factor receptor bound protein 2-associated protein 2 \\
\hline 17467197 & $-2,870464458$ & 0,003378799 & 0,157390409 & 0,723038751 & Vmn1r27 & vomeronasal 1 receptor 27 \\
\hline 17306906 & $-2,872336583$ & 0,003366273 & 0,157333808 & 0,709115308 & Ripk3 & receptor-interacting serine-threonine kinase 3 \\
\hline 17375083 & $-2,876896935$ & 0,003329129 & 0,156929906 & 0,581598741 & Snap23 & synaptosomal-associated protein 23 \\
\hline 17243171 & $-2,878067666$ & 0,003319628 & 0,156929906 & 0,735257937 & Sgta & small glutamine-rich tetratricopeptide repeat (TPR)-containing, alpha \\
\hline 17454794 & $-2,878523691$ & 0,003316604 & 0,156929906 & 0,593664009 & Rbak & RB-associated KRAB repressor \\
\hline 17271968 & $-2,879556918$ & 0,003310126 & 0,156929906 & 0,72579217 & Mif4gd & $\begin{array}{l}\text { MIF4G domain containing } \\
\text { COP9 (constitutive photomorphogenic) homolog, subunit } 5\end{array}$ \\
\hline 17221215 & $-2,881478644$ & 0,003295441 & 0,156929906 & 0,67872552 & Cops5 & (Arabidopsis thaliana) \\
\hline 17219643 & $-2,884187716$ & 0,003280324 & 0,156929906 & 0,786531027 & Aim2 & absent in melanoma 2 \\
\hline 17478998 & $-2,885880821$ & 0,00327039 & 0,156929906 & 0,606187619 & Vimp & VCP-interacting membrane protein \\
\hline 17286354 & $-2,887874145$ & 0,003255705 & 0,156929906 & 0,583372733 & Serpinb6b & serine (or cysteine) peptidase inhibitor, clade $B$, member $6 \mathrm{~b}$ \\
\hline 17478942 & $-2,889643763$ & 0,003246635 & 0,156929906 & 0,631472797 & $\operatorname{Tm} 2 \mathrm{~d} 3$ & TM2 domain containing 3 \\
\hline 17516699 & $-2,892183784$ & 0,003232814 & 0,156927765 & 0,489560627 & Mpzl2 & myelin protein zero-like 2 \\
\hline 17428054 & $-2,89472776$ & 0,003218562 & 0,156633315 & 0,649509314 & Gpx7 & glutathione peroxidase 7 \\
\hline 17276139 & $-2,89535071$ & 0,003212083 & 0,156548588 & 0,573291672 & Dact1 & dapper homolog 1 , antagonist of beta-catenin (xenopus) \\
\hline 17399519 & $-2,896452995$ & 0,003205173 & 0,156548588 & 0,637401685 & Ube2q1 & ubiquitin-conjugating enzyme E2Q (putative) 1 \\
\hline 17325093 & $-2,898678324$ & 0,00319092 & 0,156548588 & 0,647477629 & Muc13 & mucin 13 , epithelial transmembrane \\
\hline 17305221 & $-2,901217807$ & 0,003173643 & 0,156548588 & 0,556694248 & Fam213a & family with sequence similarity 213 , member $A$ \\
\hline 17391137 & $-2,902579033$ & 0,003165437 & 0,156548588 & 0,627357297 & Usp50 & ubiquitin specific peptidase 50 \\
\hline 17252170 & $-2,902980154$ & 0,003160686 & 0,156548588 & 0,564436041 & Rnf167 & ring finger protein 167 \\
\hline 17338472 & $-2,904412407$ & 0,003150752 & 0,156548588 & 0,570003757 & Mocs1 & molybdenum cofactor synthesis 1 \\
\hline 17379337 & $-2,906410464$ & 0,003136931 & 0,156548588 & 0,679466548 & Pabpc1l & poly(A) binding protein, cytoplasmic 1-like \\
\hline 17278073 & $-2,907920194$ & 0,003126134 & 0,156548588 & 0,60756407 & Golga5 & golgi autoantigen, golgin subfamily a, 5 \\
\hline 17432790 & $-2,910318428$ & 0,003115336 & 0,156548588 & 0,740855034 & Miip & migration and invasion inhibitory protein \\
\hline
\end{tabular}




\begin{tabular}{|c|c|c|c|c|c|c|}
\hline $\begin{array}{l}\text { probeset } \\
\text { ID }\end{array}$ & d.value & p.value & q.value & R.fold & Genename & Description \\
\hline 17476866 & $-2,911514985$ & 0,003109289 & 0,156548588 & 0,755984695 & Tshz3 & teashirt zinc finger family member 3 \\
\hline 17501748 & $-2,9169817$ & 0,00307992 & 0,156115062 & 0,731911879 & Lpar2 & lysophosphatidic acid receptor 2 \\
\hline 17511377 & $-2,917357949$ & 0,003076465 & 0,156115062 & 0,399082015 & Neto2 & neuropilin (NRP) and tolloid (TLL)-like 2 \\
\hline 17212874 & $-2,922889375$ & 0,003047959 & 0,155667125 & 0,40034037 & Coq10b & coenzyme Q10 homolog B (S. cerevisiae) \\
\hline 17434322 & $-2,92346396$ & 0,003043208 & 0,155665076 & 0,582549458 & NA & NA \\
\hline 17262575 & $-2,923977023$ & 0,003039753 & 0,155665076 & 0,680468229 & Ube $2 b$ & ubiquitin-conjugating enzyme E2B \\
\hline 17339460 & $-2,924478915$ & 0,003035434 & 0,155665076 & 0,390934376 & Lpin2 & lipin 2 \\
\hline 17379300 & $-2,926741977$ & 0,003018589 & 0,155367822 & 0,716735444 & 0610039K10Rik & RIKEN cDNA 0610039K10 gene \\
\hline 17285867 & $-2,928953441$ & 0,003006496 & 0,154986785 & 0,277871374 & Hist1h2ab & histone cluster 1, H2ab \\
\hline 17278110 & $-2,931061221$ & 0,002994403 & 0,154986785 & 0,659746808 & Ubr7 & ubiquitin protein ligase E3 component $n$-recognin 7 (putative) \\
\hline 17548498 & $-2,934899053$ & 0,002966761 & 0,154986785 & 0,471774487 & Gm10931 & predicted gene 10931 \\
\hline 17548294 & $-2,934899053$ & 0,002966761 & 0,154986785 & 0,471774487 & Gm10931 & predicted gene 10931 \\
\hline 17520560 & $-2,934915756$ & 0,002965897 & 0,154986785 & 0,620678896 & Tfdp2 & transcription factor $\mathrm{Dp} 2$ \\
\hline 17418413 & $-2,936511481$ & 0,002960714 & 0,154986785 & 0,706558314 & Gnl2 & guanine nucleotide binding protein-like 2 (nucleolar) \\
\hline 17340177 & $-2,93854291$ & 0,002945165 & 0,154986785 & 0,627239193 & Prkce & protein kinase $\mathrm{C}$, epsilon \\
\hline 17300251 & $-2,940000283$ & 0,002937823 & 0,154986785 & 0,551183972 & Abhd4 & abhydrolase domain containing 4 \\
\hline 17452209 & $-2,940104699$ & 0,002936527 & 0,154986785 & 0,813882741 & Mapkapk5 & MAP kinase-activated protein kinase 5 \\
\hline 17213580 & $-2,944413182$ & 0,002916228 & 0,154676898 & 0,764208062 & Eef1b2 & eukaryotic translation elongation factor 1 beta 2 \\
\hline 17468705 & $-2,948954722$ & 0,002889881 & 0,154554536 & 0,649242507 & Rab7 & RAB7, member RAS oncogene family \\
\hline 17464638 & $-2,955205287$ & 0,002854465 & 0,154499461 & 0,660399108 & Pon2 & paraoxonase 2 \\
\hline 17465924 & $-2,961750978$ & 0,002821208 & 0,153604887 & 0,758205304 & Svopl & SV2 related protein homolog (rat)-like \\
\hline 17535344 & $-2,962965749$ & 0,002810843 & 0,153604887 & 0,74143475 & Hmgb3 & high mobility group box 3 \\
\hline 17316057 & $-2,963196376$ & 0,002809979 & 0,153604887 & 0,699046058 & Sub1 & SUB1 homolog (S. cerevisiae) \\
\hline 17522430 & $-2,963305896$ & 0,002808683 & 0,153604887 & 0,698891063 & Ccdc12 & coiled-coil domain containing 12 \\
\hline 17531265 & $-2,964906188$ & 0,002798317 & 0,153604887 & 0,661295314 & Atrip & ATR interacting protein \\
\hline 17532045 & $-2,966402709$ & 0,00278536 & 0,153604887 & 0,503012573 & Plcd1 & phospholipase C, delta 1 \\
\hline 17443275 & $-2,974483855$ & 0,002743033 & 0,153133373 & 0,598569656 & Rhbdd2 & rhomboid domain containing 2 \\
\hline 17331642 & $-2,975078079$ & 0,002739578 & 0,153133373 & 0,798131229 & Mrpl39 & mitochondrial ribosomal protein L39 \\
\hline 17443972 & $-2,976104793$ & 0,0027331 & 0,153133373 & 0,690352306 & Get4 & golgi to ER traffic protein 4 homolog (S. cerevisiae) \\
\hline 17234667 & $-2,979301423$ & 0,002721006 & 0,153133373 & 0,437514462 & Pttg1ip & $\begin{array}{l}\text { pituitary tumor-transforming } 1 \text { interacting protein } \\
\text { glucosaminyl ( } \mathrm{N} \text {-acetyl) transferase } 4 \text {, core } 2 \text { (beta-1,6-N- }\end{array}$ \\
\hline 17289324 & $-2,982846927$ & 0,002705026 & 0,152916443 & 0,703496759 & Gent4 & acetylglucosaminyltransferase) \\
\hline 17241409 & $-2,983090787$ & 0,002704162 & 0,152916443 & 0,653022292 & Srgn & serglycin \\
\hline 17302061 & $-2,986356803$ & 0,002689045 & 0,152916443 & 0,544488517 & Nufip1 & nuclear fragile $\mathrm{X}$ mental retardation protein interacting protein 1 \\
\hline 17380185 & $-2,989257438$ & 0,002678679 & 0,152874224 & 0,668366125 & Rae1 & RAE1 RNA export 1 homolog (S. pombe) \\
\hline 17462395 & $-2,991552339$ & 0,002662267 & 0,152728896 & 0,429969007 & Slc25a18 & solute carrier family 25 (mitochondrial carrier), member 18 \\
\hline 17292689 & $-2,992155273$ & 0,00265838 & 0,152728896 & 0,424572015 & Thoc3 & THO complex 3 \\
\hline 17303804 & $-2,992202705$ & 0,002657516 & 0,152728896 & 0,701389748 & Anxa7 & annexin $A 7$ \\
\hline 17301697 & $-2,992249601$ & 0,002657084 & 0,152728896 & 0,469105204 & Tnfrsf10b & tumor necrosis factor receptor superfamily, member 10b \\
\hline 17439769 & $-3,003098324$ & 0,002600073 & 0,152587611 & 0,677476493 & Nudt9 & nudix (nucleoside diphosphate linked moiety X)-type motif 9 \\
\hline 17507623 & $-3,009902282$ & 0,002563361 & 0,151256401 & 0,724972922 & 1700029H14Rik & RIKEN cDNA $1700029 \mathrm{H} 14$ gene \\
\hline 17306532 & $-3,010216875$ & 0,002560337 & 0,151256401 & 0,693651303 & Myh6 & myosin, heavy polypeptide 6 , cardiac muscle, alpha \\
\hline 17321467 & $-3,010538183$ & 0,002558178 & 0,151256401 & 0,578520813 & Tuba1b & tubulin, alpha $1 \mathrm{~B}$ \\
\hline 17451661 & $-3,010792679$ & 0,002556882 & 0,151256401 & 0,756188227 & Unc119b & unc-119 homolog B (C. elegans) \\
\hline 17312295 & $-3,012227814$ & 0,00254954 & 0,151256401 & 0,742327979 & Zfp623 & zinc finger protein 623 \\
\hline 17502049 & $-3,016290912$ & 0,002528376 & 0,151256401 & 0,579175341 & 2010320M18Rik & RIKEN cDNA $2010320 \mathrm{M} 18$ gene \\
\hline 17238580 & $-3,022007492$ & 0,002507213 & 0,151256401 & 0,715589074 & Sarnp & SAP domain containing ribonucleoprotein \\
\hline 17430930 & $-3,037504717$ & 0,002432061 & 0,150014459 & 0,460384221 & Stx12 & syntaxin 12 \\
\hline 17453347 & $-3,041525609$ & 0,002418672 & 0,149667614 & 0,652307123 & Gtf2ird1 & general transcription factor II I repeat domain-containing 1 \\
\hline 17469572 & $-3,044939539$ & 0,002406147 & 0,149171898 & 0,615780636 & Sumf1 & sulfatase modifying factor 1 \\
\hline 17279230 & $-3,04524379$ & 0,002403555 & 0,149171898 & 0,594642292 & Zfyve21 & zinc finger, FYVE domain containing 21 \\
\hline 17508968 & $-3,050021389$ & 0,002377209 & 0,149171898 & 0,701068037 & Frg1 & FSHD region gene 1 \\
\hline 17241032 & $-3,050089073$ & 0,002376777 & 0,149171898 & 0,484360198 & Ddit4 & DNA-damage-inducible transcript 4 \\
\hline 17458641 & $-3,051693865$ & 0,002368139 & 0,149171898 & 0,652911438 & Tax1bp1 & Tax1 (human T cell leukemia virus type I) binding protein 1 \\
\hline 17469775 & $-3,052307919$ & 0,002364684 & 0,149171898 & 0,672987356 & Emc3 & ER membrane protein complex subunit 3 \\
\hline 17377177 & $-3,053117132$ & 0,002361661 & 0,149171898 & 0,667126324 & $\operatorname{Rin} 2$ & Ras and Rab interactor 2 \\
\hline 17341080 & $-3,056224366$ & 0,002348272 & 0,149171898 & 0,521115657 & Thbs2 & thrombospondin 2 \\
\hline 17363407 & $-3,060562651$ & 0,002328404 & 0,148532208 & 0,600757088 & Anxa1 & annexin $\mathrm{A} 1$ \\
\hline 17321078 & $-3,062682627$ & 0,002317606 & 0,148129375 & 0,439200746 & Vdr & vitamin $\mathrm{D}$ receptor \\
\hline 17419636 & $-3,067492111$ & 0,002296011 & 0,147319016 & 0,574142474 & Gpn2 & GPN-loop GTPase 2 \\
\hline & & & & & & COP9 (constitutive photomorphogenic) homolog, subunit 3 \\
\hline 17263482 & $-3,068086507$ & 0,002293419 & 0,147319016 & 0,583091296 & Cops3 & (Arabidopsis thaliana) \\
\hline 17534142 & $-3,068739652$ & 0,002290396 & 0,147319016 & 0,607534488 & Nkap & NFKB activating protein \\
\hline 17432927 & $-3,07491865$ & 0,002264914 & 0,147319016 & 0,741739853 & $\mathrm{FbxO} 44$ & F-box protein 44 \\
\hline 17493875 & $-3,077615058$ & 0,002255412 & 0,147319016 & 0,411239006 & P2ry2 & purinergic receptor P2Y, G-protein coupled 2 \\
\hline 17493556 & $-3,07894926$ & 0,002248933 & 0,147319016 & 0,568002505 & Acer3 & alkaline ceramidase 3 \\
\hline 17319124 & $-3,080983727$ & 0,002241159 & 0,147319016 & 0,580846043 & 1700088E04Rik & RIKEN cDNA $1700088 E 04$ gene \\
\hline 17417791 & $-3,084599703$ & 0,002228202 & 0,147257217 & 0,684555165 & Ebna1bp2 & EBNA1 binding protein 2 \\
\hline 17222072 & $-3,086012436$ & 0,002220427 & 0,147151909 & 0,436229852 & Ccdc115 & coiled-coil domain containing 115 \\
\hline 17349634 & $-3,091004395$ & 0,002192354 & 0,14614286 & 0,724890267 & Cystm1 & cysteine-rich transmembrane module containing 1 \\
\hline 17550492 & $-3,091240502$ & 0,002190194 & 0,14614286 & 0,678462934 & Gm11974 & predicted gene 11974 \\
\hline 17444494 & $-3,093731945$ & 0,002177669 & 0,145961053 & 0,692864083 & Gm15708 & predicted gene 15708 \\
\hline 17229166 & $-3,095001162$ & 0,002169463 & 0,145706572 & 0,678132114 & Blzf1 & basic leucine zipper nuclear factor 1 \\
\hline 17321169 & $-3,10532825$ & 0,002126704 & 0,145228743 & 0,727657558 & Asb8 & ankyrin repeat and SOCS box-containing 8 \\
\hline 17504281 & $-3,108913693$ & 0,002112451 & 0,14482105 & 0,712022873 & Usb1 & U6 snRNA biogenesis 1 \\
\hline 17515758 & $-3,11068183$ & 0,002102949 & 0,14482105 & 0,692146553 & Nfrkb & nuclear factor related to kappa B binding protein \\
\hline 17240819 & $-3,111093357$ & 0,002099926 & 0,14482105 & 0,448957228 & Cep851 & centrosomal protein 85 -like \\
\hline 17434127 & $-3,113606737$ & 0,002087832 & 0,144753326 & 0,49649057 & Gm10590 & predicted gene 10590 \\
\hline 17424410 & $-3,113606737$ & 0,002087832 & 0,144753326 & 0,49649057 & Gm10590 & predicted gene 10590 \\
\hline 17412998 & $-3,113606737$ & 0,002087832 & 0,144753326 & 0,49649057 & Gm10590 & predicted gene 10590 \\
\hline 17266489 & $-3,12058551$ & 0,002056303 & 0,144230612 & 0,71972203 & Tmem97 & transmembrane protein 97 \\
\hline
\end{tabular}




\begin{tabular}{|c|c|c|c|c|c|c|}
\hline $\begin{array}{l}\text { probeset } \\
\text { ID }\end{array}$ & d.value & p.value & q.value & R.fold & Genename & Description \\
\hline 17287361 & $-3,126396885$ & 0,002040323 & 0,144230612 & 0,550516669 & Gadd45g & growth arrest and DNA-damage-inducible 45 gamma \\
\hline 17377199 & $-3,133163771$ & 0,002018727 & 0,143764545 & 0,438551428 & Naa20 & N(alpha)-acetyltransferase 20, NatB catalytic subunit \\
\hline 17468195 & $-3,133350954$ & 0,002017 & 0,143764545 & 0,578877605 & Stambp & STAM binding protein \\
\hline 17443310 & $-3,133687298$ & 0,002013976 & 0,143764545 & 0,690897214 & Mdh2 & malate dehydrogenase 2 , NAD (mitochondrial) \\
\hline 17463441 & $-3,135628498$ & 0,002009657 & 0,143764545 & 0,612454214 & Tom1 & target of myb1 homolog (chicken) \\
\hline 17451431 & $-3,136261558$ & 0,002005338 & 0,143764545 & 0,663245348 & Tmem119 & transmembrane protein 119 \\
\hline 17407647 & $-3,137383353$ & 0,001999292 & 0,143764545 & 0,603920306 & A730011C13Rik & RIKEN cDNA A730011C13 gene \\
\hline 17318461 & $-3,137775359$ & 0,001998428 & 0,143764545 & 0,74149168 & Sharpin & SHANK-associated RH domain interacting protein \\
\hline 17277592 & $-3,140056379$ & 0,001991949 & 0,143764545 & 0,691264997 & Gstz1 & glutathione transferase zeta 1 (maleylacetoacetate isomerase) \\
\hline 17529076 & $-3,140753941$ & 0,001989358 & 0,143764545 & 0,54757579 & Slc17a5 & solute carrier family 17 (anion/sugar transporter), member 5 \\
\hline 17403025 & $-3,142604954$ & 0,001981584 & 0,143764545 & 0,706140824 & Lamtor3 & late endosomal/lysosomal adaptor, MAPK and MTOR activator 3 \\
\hline 17319806 & $-3,142624914$ & 0,001981152 & 0,143764545 & 0,521405946 & Arfgap3 & ADP-ribosylation factor GTPase activating protein 3 \\
\hline 17440757 & $-3,143930082$ & 0,001975537 & 0,143764545 & 0,565081764 & Iscu & IscU iron-sulfur cluster scaffold homolog (E. coli) \\
\hline 17399672 & $-3,145056898$ & 0,001967331 & 0,143764545 & 0,676977068 & Slc39a1 & solute carrier family 39 (zinc transporter), member 1 \\
\hline 17538356 & $-3,145381723$ & 0,001966467 & 0,143764545 & 0,639182508 & Alg13 & asparagine-linked glycosylation 13 \\
\hline 17535752 & $-3,147256115$ & 0,001957829 & 0,143764545 & 0,596749711 & Emd & emerin \\
\hline 17429234 & $-3,15125999$ & 0,00194444 & 0,143764545 & 0,718142259 & Tmem125 & transmembrane protein 125 \\
\hline 17380134 & $-3,151436008$ & 0,001942712 & 0,143764545 & 0,62916817 & Rtfdc1 & replication termination factor 2 domain containing 1 \\
\hline 17378784 & $-3,151646776$ & 0,001941416 & 0,143764545 & 0,576910766 & Rprd1b & regulation of nuclear pre-mRNA domain containing $1 \mathrm{~B}$ \\
\hline 17496887 & $-3,152888829$ & 0,001938393 & 0,143764545 & 0,535242817 & Rgs10 & regulator of G-protein signalling 10 \\
\hline 17542149 & $-3,15459343$ & 0,001930619 & 0,143764545 & 0,672379998 & Ids & iduronate 2-sulfatase \\
\hline 17287726 & $-3,157364859$ & 0,001923276 & 0,143764545 & 0,705374963 & Caml & calcium modulating ligand \\
\hline 17482366 & $-3,15840746$ & 0,001918525 & 0,143764545 & 0,396400538 & Tmem159 & transmembrane protein 159 \\
\hline 17291570 & $-3,162333663$ & 0,001905568 & 0,143764545 & 0,673320278 & Uqcrfs1 & ubiquinol-cytochrome c reductase, Rieske iron-sulfur polypeptide 1 \\
\hline 17502860 & $-3,164106453$ & 0,001898226 & 0,143764545 & 0,697755613 & Rnf150 & ring finger protein 150 \\
\hline 17460918 & $-3,164145273$ & 0,001897794 & 0,143764545 & 0,507691572 & Tmem43 & transmembrane protein 43 \\
\hline 17470627 & $-3,176124458$ & 0,001861082 & 0,143764545 & 0,583904837 & Clec4e & C-type lectin domain family 4 , member e \\
\hline 17355113 & $-3,17935585$ & 0,001848988 & 0,143764545 & 0,591547038 & Ccbe1 & collagen and calcium binding EGF domains 1 \\
\hline 17541008 & $-3,180767614$ & 0,001842942 & 0,143764545 & 0,417776766 & Snora69 & small nucleolar RNA, H/ACA box 69 \\
\hline 17498245 & $-3,183987927$ & 0,001833872 & 0,143764545 & 0,713236797 & Cdkn1c & cyclin-dependent kinase inhibitor $1 \mathrm{C}$ (P57) \\
\hline 17495097 & $-3,188406806$ & 0,001823506 & 0,143764545 & 0,496090994 & Lyve1 & $\begin{array}{l}\text { lymphatic vessel endothelial hyaluronan receptor } 1 \\
\text { excision repaiross-complementing rodent repair deficiency, }\end{array}$ \\
\hline 17289699 & $-3,193602942$ & 0,001803206 & 0,143764545 & 0,715513419 & Ercc8 & complementation group 8 \\
\hline 17422817 & $-3,203150732$ & 0,00177686 & 0,143764545 & 0,594126066 & Ube2j2 & $\begin{array}{l}\text { ubiquitin-conjugating enzyme E2J } 2 \\
\text { TATA box binding protein (Tbp)-associated factor, RNA polymerase I, }\end{array}$ \\
\hline 17514826 & $-3,209674254$ & 0,001759152 & 0,143764545 & 0,495265611 & Taf1d & D \\
\hline 17447831 & $-3,209859744$ & 0,001758288 & 0,143764545 & 0,572845219 & Fgfbp1 & fibroblast growth factor binding protein 1 \\
\hline 17481960 & $-3,212437555$ & 0,001748786 & 0,143764545 & 0,684533511 & Arntl & aryl hydrocarbon receptor nuclear translocator-like \\
\hline 17545459 & $-3,216846927$ & 0,001737989 & 0,143764545 & 0,720029811 & Shroom2 & shroom family member 2 \\
\hline 17444674 & $-3,219806088$ & 0,001725463 & 0,143764545 & 0,71364088 & Zscan25 & zinc finger and SCAN domain containing 25 \\
\hline 17413158 & $-3,227961342$ & 0,001693934 & 0,143764545 & 0,559648697 & Gm10590 & predicted gene 10590 \\
\hline 17526206 & $-3,228581149$ & 0,001691775 & 0,143764545 & 0,709094726 & $\mathrm{C} 2 \mathrm{~cd} 2 \mathrm{l}$ & C2 calcium-dependent domain containing 2-like \\
\hline 17368834 & $-3,230843617$ & 0,001680113 & 0,143764545 & 0,607217719 & Med27 & mediator complex subunit 27 \\
\hline 17453703 & $-3,236061044$ & 0,001661109 & 0,143764545 & 0,74253504 & Prkrip1 & Prkr interacting protein 1 (IL11 inducible) \\
\hline 17505967 & $-3,238036806$ & 0,001654631 & 0,143764545 & 0,750756228 & Cmip & c-Maf inducing protein \\
\hline 17396492 & $-3,242207228$ & 0,001647288 & 0,143764545 & 0,617044583 & Eif5a2 & eukaryotic translation initiation factor $5 \mathrm{~A} 2$ \\
\hline 17284037 & $-3,248485737$ & 0,001630444 & 0,143764545 & 0,434752184 & Mpc1 & mitochondrial pyruvate carrier 1 \\
\hline 17285863 & $-3,266749083$ & 0,001582071 & 0,141291573 & 0,690778604 & Hist1h2bb & histone cluster $1, \mathrm{H} 2 \mathrm{bb}$ \\
\hline 17368079 & $-3,26948524$ & 0,001576024 & 0,141291573 & 0,696214241 & Fbxw5 & F-box and WD-40 domain protein 5 \\
\hline 17440086 & $-3,272009537$ & 0,001566954 & 0,141085467 & 0,65213089 & Rpap2 & RNA polymerase II associated protein 2 \\
\hline 17431302 & $-3,273111252$ & 0,001563931 & 0,141085467 & 0,622978707 & Mtfr1l & mitochondrial fission regulator 1-like \\
\hline 17411054 & $-3,280888731$ & 0,001546223 & 0,141085467 & 0,686706455 & Rpf1 & ribosome production factor 1 homolog (S. cerevisiae) \\
\hline 17264153 & $-3,284077773$ & 0,001534561 & 0,141085467 & 0,482531323 & 1700086D15Rik & RIKEN cDNA 1700086D15 gene \\
\hline 17374686 & $-3,285053878$ & 0,001531106 & 0,141085467 & 0,59096413 & Rpusd2 & RNA pseudouridylate synthase domain containing 2 \\
\hline sphk & $-3,289287203$ & 0,001520308 & 0,141085467 & 0,699798841 & Neu3 & neuraminidase 3 \\
\hline 17402296 & $-3,292596748$ & 0,001512534 & 0,14107046 & 0,724573078 & Dnttip2 & deoxynucleotidyltransferase, terminal, interacting protein 2 \\
\hline 17465608 & $-3,295216588$ & 0,001508647 & 0,14107046 & 0,508548194 & Lincpint & long intergenic non-protein coding RNA, Trp53 induced transcript \\
\hline 17272519 & $-3,300420245$ & 0,001494826 & 0,14107046 & 0,697920319 & Jmjd6 & jumonji domain containing 6 \\
\hline 17256565 & $-3,302589284$ & 0,001487483 & 0,14107046 & 0,637032736 & Tubg2 & tubulin, gamma 2 \\
\hline 17231574 & $-3,309663144$ & 0,001467616 & 0,140567638 & 0,684961856 & Ppil4 & peptidylprolyl isomerase (cyclophilin)-like 4 \\
\hline 17240357 & $-3,309953408$ & 0,001466752 & 0,140567638 & 0,630537095 & Gtf3c6 & general transcription factor IIIC, polypeptide 6 , alpha \\
\hline 17298775 & $-3,315862757$ & 0,001451203 & 0,139806133 & 0,716051526 & Anxa8 & annexin A8 \\
\hline 17455093 & $-3,318774053$ & 0,001443429 & 0,139806133 & 0,766056229 & Zkscan14 & zinc finger with KRAB and SCAN domains 14 \\
\hline 17308842 & $-3,320097347$ & 0,001439974 & 0,139806133 & 0,461844373 & Dgkh & diacylglycerol kinase, eta \\
\hline 17308233 & $-3,321573275$ & 0,00143695 & 0,139806133 & 0,491003722 & 9930012K11Rik & RIKEN CDNA 9930012K11 gene \\
\hline 17500068 & $-3,323521437$ & 0,001433927 & 0,139806133 & 0,476706938 & Sfrp1 & secreted frizzled-related protein 1 \\
\hline 17374594 & $-3,324472957$ & 0,001429608 & 0,139806133 & 0,466066542 & Pak6 & p21 protein (Cdc42/Rac)-activated kinase 6 \\
\hline 17375327 & $-3,331346212$ & 0,001416219 & 0,139806133 & 0,594416505 & Casc4 & cancer susceptibility candidate 4 \\
\hline 17220974 & $-3,331383696$ & 0,001415787 & 0,139806133 & 0,559654837 & Plxna2 & plexin A2 \\
\hline 17241637 & $-3,335331226$ & 0,001406285 & 0,139806133 & 0,620285776 & Nrbf2 & nuclear receptor binding factor 2 \\
\hline 17234192 & $-3,338915233$ & 0,001395487 & 0,139806133 & 0,538660813 & Zwint & ZW10 interactor \\
\hline 17214825 & $-3,341423389$ & 0,001387281 & 0,139806133 & 0,660268405 & Mff & mitochondrial fission factor \\
\hline 17473269 & $-3,344812939$ & 0,001378643 & 0,139806133 & 0,760651781 & Leng8 & leukocyte receptor cluster (LRC) member 8 \\
\hline 17313016 & $-3,345156374$ & 0,001377779 & 0,139806133 & 0,62691808 & Tomm22 & translocase of outer mitochondrial membrane 22 homolog (yeast) \\
\hline 17258457 & $-3,345165902$ & 0,001377347 & 0,139806133 & 0,754312856 & Sap30bp & SAP30 binding protein \\
\hline 17332851 & $-3,346145344$ & 0,001373892 & 0,139806133 & 0,695521805 & Gtf2h5 & general transcription factor $\mathrm{IH}$, polypeptide 5 \\
\hline 17320947 & $-3,347039758$ & 0,001370005 & 0,139806133 & 0,362732325 & Slc38a4 & solute carrier family 38 , member 4 \\
\hline 17366437 & $-3,347143691$ & 0,001369141 & 0,139806133 & 0,516745636 & Fam107b & family with sequence similarity 107 , member $B$ \\
\hline 17506917 & $-3,348136414$ & 0,001366982 & 0,139806133 & 0,326006331 & Kcnk1 & potassium channel, subfamily K, member 1 \\
\hline 17292157 & $-3,350273602$ & 0,001360071 & 0,139806133 & 0,620463742 & Dtnbp1 & dystrobrevin binding protein 1 \\
\hline 17220018 & $-3,357718819$ & 0,001340635 & 0,139806133 & 0,577888053 & Cnst & consortin, connexin sorting protein \\
\hline
\end{tabular}




\begin{tabular}{|c|c|c|c|c|c|c|}
\hline $\begin{array}{l}\text { probeset } \\
\text { ID }\end{array}$ & d.value & p.value & q.value & R.fold & Genename & Description \\
\hline 17362320 & $-3,359977929$ & 0,001333293 & 0,139806133 & 0,667915644 & Al846148 & expressed sequence Al846148 \\
\hline 17512740 & $-3,367262041$ & 0,001315153 & 0,139806133 & 0,590327769 & Nob1 & NIN1/RPN12 binding protein 1 homolog (S. cerevisiae) \\
\hline 17487952 & $-3,374525545$ & 0,001294853 & 0,139806133 & 0,481109546 & Ceacam1 & carcinoembryonic antigen-related cell adhesion molecule 1 \\
\hline 17503377 & $-3,374898042$ & 0,001293558 & 0,139806133 & 0,689560337 & Dhps & deoxyhypusine synthase \\
\hline 17386135 & $-3,377816634$ & 0,001286215 & 0,139806133 & 0,683866066 & Stk39 & serine/threonine kinase 39 \\
\hline 17242773 & $-3,38173854$ & 0,001279305 & 0,139806133 & 0,476948811 & Polr2e & polymerase (RNA) II (DNA directed) polypeptide E \\
\hline 17444828 & $-3,3927032$ & 0,001252095 & 0,139806133 & 0,56651793 & Polr1d & polymerase (RNA) I polypeptide $D$ \\
\hline 17381283 & $-3,396356237$ & 0,001243025 & 0,139806133 & 0,557166499 & Prpf18 & PRP18 pre-mRNA processing factor 18 homolog (yeast) \\
\hline 17488001 & $-3,39924399$ & 0,001237842 & 0,139806133 & 0,740683411 & Ccdc97 & coiled-coil domain containing 97 \\
\hline 17512732 & $-3,40372337$ & 0,001229636 & 0,139806133 & 0,578431755 & Nqo1 & $\begin{array}{l}\mathrm{NAD}(\mathrm{P}) \mathrm{H} \text { dehydrogenase, quinone } 1 \\
\text { translational activator of mitochondrially encoded cytochrome } \mathrm{c}\end{array}$ \\
\hline 17270724 & $-3,408107144$ & 0,001224021 & 0,139806133 & 0,732808744 & Taco1os & oxidase I, opposite strand \\
\hline 17467711 & $-3,41031971$ & 0,001218838 & 0,139806133 & 0,593266468 & Usp39 & ubiquitin specific peptidase 39 \\
\hline 17253885 & $-3,432870782$ & 0,001164418 & 0,139806133 & 0,545319177 & Adap2 & ArfGAP with dual PH domains 2 \\
\hline 17311821 & $-3,449762905$ & 0,001132457 & 0,139806133 & 0,671602404 & Nsmce2 & non-SMC element 2 homolog (MMS21, S. cerevisiae) \\
\hline 17301440 & $-3,453867795$ & 0,001122091 & 0,139806133 & 0,489198594 & Ccdc25 & coiled-coil domain containing 25 \\
\hline 17515062 & $-3,47139927$ & 0,001084947 & 0,139806133 & 0,665794484 & Mrpl4 & mitochondrial ribosomal protein $\mathrm{L} 4$ \\
\hline 17506754 & $-3,473541247$ & 0,00108106 & 0,139806133 & 0,544532568 & $\operatorname{Cog} 2$ & component of oligomeric golgi complex 2 \\
\hline 17442588 & $-3,473818467$ & 0,001080196 & 0,139806133 & 0,524454143 & Atp6v0a2 & ATPase, $\mathrm{H}+$ transporting, lysosomal V0 subunit A2 \\
\hline 17258341 & $-3,476527556$ & 0,00107415 & 0,139806133 & 0,712731984 & $\operatorname{Llg} 12$ & lethal giant larvae homolog 2 (Drosophila) \\
\hline 17236800 & $-3,479401442$ & 0,001068535 & 0,139806133 & 0,496946546 & Den & decorin \\
\hline 17289289 & $-3,497048136$ & 0,001042189 & 0,139806133 & 0,69446935 & Poc5 & POC5 centriolar protein homolog (Chlamydomonas) \\
\hline 17463702 & $-3,504309212$ & 0,001027072 & 0,139806133 & 0,734493683 & Loh12cr1 & loss of heterozygosity, 12 , chromosomal region 1 homolog (human) \\
\hline 17358007 & $-3,510314282$ & 0,001016706 & 0,139806133 & 0,621860904 & Rfk & riboflavin kinase \\
\hline 17482681 & $-3,514125786$ & 0,00100634 & 0,139806133 & 0,631720937 & Scnn1g & sodium channel, nonvoltage-gated 1 gamma \\
\hline 17401169 & $-3,520312453$ & 0,000995975 & 0,139806133 & 0,59957355 & Bcas2 & breast carcinoma amplified sequence 2 \\
\hline 17275718 & $-3,556639111$ & 0,000943714 & 0,139806133 & 0,627317635 & Mia2 & melanoma inhibitory activity 2 \\
\hline 17327909 & $-3,559964149$ & 0,000937667 & 0,139806133 & 0,510715901 & Ppl & periplakin \\
\hline 17468511 & $-3,565659476$ & 0,000928165 & 0,139806133 & 0,660609484 & Mxd1 & MAX dimerization protein 1 \\
\hline 17249461 & $-3,56980662$ & 0,000919527 & 0,139806133 & 0,627153803 & Fstl4 & follistatin-like 4 \\
\hline 17408099 & $-3,569884607$ & 0,000919095 & 0,139806133 & 0,73518066 & Polr3c & polymerase (RNA) III (DNA directed) polypeptide C \\
\hline 17411732 & $-3,57430591$ & 0,000913049 & 0,139806133 & 0,697540976 & 2610301B20Rik & RIKEN cDNA 2610301B20 gene \\
\hline 17340397 & $-3,577868578$ & 0,000905706 & 0,139806133 & 0,533152124 & Nanp & $\mathrm{N}$-acetylneuraminic acid phosphatase \\
\hline 17354378 & $-3,586120674$ & 0,000890158 & 0,139806133 & 0,677136421 & Ppic & $\begin{array}{l}\text { peptidylprolyl isomerase } C \\
\text { 1-acylglycerol-3-phosphate O-acyltransferase } 2 \text { (lysophosphatidic acid }\end{array}$ \\
\hline 17383104 & $-3,58950033$ & 0,000884543 & 0,139806133 & 0,719562327 & Agpat2 & acyltransferase, beta) \\
\hline 17363605 & $-3,594098108$ & 0,000876769 & 0,139806133 & 0,519354267 & Pip5k1b & phosphatidylinositol-4-phosphate 5-kinase, type 1 beta \\
\hline 17548850 & $-3,594787251$ & 0,000875473 & 0,139806133 & 0,578479595 & Arpc1b & actin related protein $2 / 3$ complex, subunit $1 \mathrm{~B}$ \\
\hline 17389009 & $-3,598350423$ & 0,000872018 & 0,139806133 & 0,655885569 & Eif3m & eukaryotic translation initiation factor 3 , subunit $\mathrm{M}$ \\
\hline 17230595 & $-3,600908628$ & 0,000866835 & 0,139806133 & 0,672541857 & Degs1 & degenerative spermatocyte homolog 1 (Drosophila) \\
\hline 17518585 & $-3,6041549$ & 0,00086122 & 0,139806133 & 0,492572435 & Spg21 & spastic paraplegia 21 homolog (human) \\
\hline 17254537 & $-3,604548687$ & 0,000860356 & 0,139806133 & 0,688280503 & Ppm1d & protein phosphatase 1D magnesium-dependent, delta isoform \\
\hline 17422612 & $-3,606694512$ & 0,000858629 & 0,139806133 & 0,546327082 & Slc35e2 & solute carrier family 35, member E2 \\
\hline 17334685 & $-3,615074037$ & 0,000847831 & 0,139806133 & 0,745631637 & Tekt4 & tektin 4 \\
\hline 17461606 & $-3,64627147$ & 0,000806368 & 0,138060066 & 0,671870433 & Brpf1 & bromodomain and PHD finger containing, 1 \\
\hline 17550428 & $-3,647607329$ & 0,000803345 & 0,138060066 & 0,32956441 & Neat1 & nuclear paraspeckle assembly transcript 1 (non-protein coding) \\
\hline 17499922 & $-3,661889709$ & 0,000783909 & 0,137055318 & 0,57111072 & Mrps31 & mitochondrial ribosomal protein S31 \\
\hline 17324305 & $-3,69670445$ & 0,000738991 & 0,136174199 & 0,71908677 & Dnajb11 & DnaJ (Hsp40) homolog, subfamily B, member 11 \\
\hline 17516921 & $-3,726462048$ & 0,00070487 & 0,134634114 & 0,712324047 & Zpr1 & ZPR1 zinc finger \\
\hline 17419222 & $-3,733573785$ & 0,000694936 & 0,134289173 & 0,739502152 & Snrnp40 & small nuclear ribonucleoprotein 40 (U5) \\
\hline 17276732 & $-3,740144462$ & 0,000689322 & 0,134289173 & 0,573178085 & Eif2s1 & eukaryotic translation initiation factor 2 , subunit 1 alpha \\
\hline 17462373 & $-3,757901224$ & 0,000670318 & 0,133061743 & 0,441843787 & Cecr2 & $\begin{array}{l}\text { cat eye syndrome chromosome region, candidate } 2 \\
\text { solute carrier family } 25 \text { (mitochondrial carrier, adenine nucleotide }\end{array}$ \\
\hline 17509171 & $-3,771567859$ & 0,000654337 & 0,132737203 & 0,676551639 & Slc25a4 & translocator), member 4 \\
\hline 17286254 & $-3,797324471$ & 0,000628855 & 0,132737203 & 0,48272016 & Mboat1 & membrane bound O-acyltransferase domain containing 1 \\
\hline 17223313 & $-3,797704456$ & 0,000627991 & 0,132737203 & 0,672558507 & Tyw5 & tRNA-yW synthesizing protein 5 \\
\hline 17428885 & $-3,80137802$ & 0,000624968 & 0,132737203 & 0,706298653 & Dmap1 & $\begin{array}{l}\text { DNA methyltransferase } 1 \text {-associated protein } 1 \\
\text { carbohydrate ( } \mathrm{N} \text {-acetylgalactosamine } 4 \text {-sulfate } 6 \text {-O) sulfotransferase }\end{array}$ \\
\hline 17497076 & $-3,806519272$ & 0,000619353 & 0,132737203 & 0,690577859 & Chst15 & 15 \\
\hline 17231859 & $-3,807188167$ & 0,000618921 & 0,132737203 & 0,567789061 & Ifngr1 & interferon gamma receptor 1 \\
\hline 17356099 & $-3,83422815$ & 0,000594734 & 0,132737203 & 0,618854199 & Cdk2ap2 & CDK2-associated protein 2 \\
\hline 17479548 & $-3,839799454$ & 0,000590847 & 0,132737203 & 0,495682389 & Vps33b & vacuolar protein sorting 33B (yeast) \\
\hline 17342042 & $-3,846845987$ & 0,000585232 & 0,132737203 & 0,578845895 & Nubp2 & nucleotide binding protein 2 \\
\hline 17266185 & $-3,87471105$ & 0,000564501 & 0,132737203 & 0,705566022 & Ccdc55 & coiled-coil domain containing 55 \\
\hline 17464503 & $-3,877044124$ & 0,000563637 & 0,132737203 & 0,727517162 & 2810474019Rik & RIKEN CDNA 2810474019 gene \\
\hline 17427155 & $-3,881256759$ & 0,000560614 & 0,132737203 & 0,515698326 & Cdkn2b & cyclin-dependent kinase inhibitor 2B ( $p 15$, inhibits CDK4) \\
\hline 17329454 & $-3,943569936$ & 0,000508353 & 0,132267702 & 0,671211642 & P3h2 & prolyl 3-hydroxylase 2 \\
\hline 17548440 & $-3,947796389$ & 0,000503602 & 0,132184757 & 0,685442738 & Imp3 & IMP3, U3 small nucleolar ribonucleoprotein, homolog (yeast) \\
\hline 17524958 & $-3,9559556$ & 0,000498419 & 0,132184757 & 0,610775772 & Zfp810 & $\begin{array}{l}\text { zinc finger protein } 810 \\
\text { pleckstrin homology domain containing, family D (with coiled-coil }\end{array}$ \\
\hline 17276864 & $-3,960284316$ & 0,000494964 & 0,132184757 & 0,710433769 & Plekhd1 & domains) member 1 \\
\hline 17503431 & $-3,962058249$ & 0,000493668 & 0,132184757 & 0,559190937 & Orc6 & origin recognition complex, subunit 6 \\
\hline 17211998 & $-3,971919205$ & 0,000483734 & 0,132184757 & 0,714505212 & Mrpl30 & mitochondrial ribosomal protein $\mathrm{L} 30$ \\
\hline 17519967 & $-3,973869516$ & 0,000482007 & 0,132184757 & 0,711876703 & Tpbg & trophoblast glycoprotein \\
\hline 17384619 & $-3,982798977$ & 0,000473801 & 0,132184757 & 0,444753712 & Snord90 & small nucleolar RNA, C/D box 90 \\
\hline 17353131 & $-4,000032064$ & 0,000465162 & 0,132184757 & 0,583929509 & Ino80c & INO80 complex subunit C \\
\hline 17258584 & $-4,012350585$ & 0,000454365 & 0,132184757 & 0,560032735 & Sphk1 & sphingosine kinase 1 \\
\hline 17481936 & $-4,034499719$ & 0,000439248 & 0,132184757 & 0,572627747 & Tead1 & TEA domain family member 1 \\
\hline 17353241 & $-4,039429942$ & 0,000435793 & 0,132184757 & 0,626893294 & Slc25a46 & solute carrier family 25 , member 46 \\
\hline 17379128 & $-4,051019678$ & 0,000428882 & 0,132184757 & 0,619618662 & Srsf6 & serine/arginine-rich splicing factor 6 \\
\hline 17365960 & $-4,06333677$ & 0,000422836 & 0,132184757 & 0,569277891 & Gfra1 & glial cell line derived neurotrophic factor family receptor alpha 1 \\
\hline
\end{tabular}




\begin{tabular}{|c|c|c|c|c|c|c|}
\hline $\begin{array}{c}\text { probeset } \\
\text { ID }\end{array}$ & d.value & p.value & q.value & R.fold & Genename & Description \\
\hline 17349774 & $-4,067505514$ & 0,000420244 & 0,132184757 & 0,746684615 & Hars2 & histidyl-tRNA synthetase 2 , mitochondrial (putative) \\
\hline 17239077 & $-4,117804708$ & 0,000387419 & 0,130631356 & 0,532540427 & Ginm1 & glycoprotein integral membrane 1 \\
\hline 17219516 & $-4,132601094$ & 0,000380941 & 0,129771079 & 0,428596058 & Gm17224 & predicted gene 17224 \\
\hline 17312905 & $-4,142561209$ & 0,000375326 & 0,129190212 & 0,583237458 & Eif3l & eukaryotic translation initiation factor 3 , subunit $\mathrm{L}$ \\
\hline 17533413 & $-4,184770505$ & 0,000349412 & 0,127526119 & 0,550405163 & $\mathrm{Rpl3}$ & ribosomal protein $\mathrm{L} 3$ \\
\hline 17275706 & $-4,189147847$ & 0,000345956 & 0,127526119 & 0,583912648 & Pnn & pinin \\
\hline 17358020 & $-4,205642406$ & 0,00033991 & 0,127526119 & 0,576169778 & Nmrk1 & $\begin{array}{l}\text { nicotinamide riboside kinase } 1 \\
\text { solute carrier family } 25 \text { (mitochondrial carrier, Graves disease }\end{array}$ \\
\hline 17233799 & $-4,207168462$ & 0,000339478 & 0,127526119 & 0,5489078 & Slc25a16 & autoantigen), member 16 \\
\hline 17475818 & $-4,231375384$ & 0,000329544 & 0,127526119 & 0,454714086 & AF357399 & SnoRNA AF357399 \\
\hline 17469636 & $-4,232978892$ & 0,000328248 & 0,127526119 & 0,433734787 & Rad18 & RAD18 homolog (S. cerevisiae) \\
\hline 17245399 & $-4,28387533$ & 0,000304062 & 0,12603958 & 0,562405539 & Irak3 & interleukin-1 receptor-associated kinase 3 \\
\hline 17339549 & $-4,337129628$ & 0,000274692 & 0,124070176 & 0,583455003 & Ypel5 & yippee-like 5 (Drosophila) \\
\hline 17277370 & $-4,340198331$ & 0,000273396 & 0,124070176 & 0,560278083 & Eif2b2 & eukaryotic translation initiation factor $2 B$, subunit 2 beta \\
\hline 17402595 & $-4,369250202$ & 0,000264326 & 0,124070176 & 0,423236106 & Casp6 & caspase 6 \\
\hline 17451203 & $-4,369544715$ & 0,000263894 & 0,124070176 & 0,667582272 & Srrd & SRR1 domain containing \\
\hline 17522577 & $-4,412134904$ & 0,000250937 & 0,124070176 & 0,710931014 & Lrrfip2 & leucine rich repeat (in FLII) interacting protein 2 \\
\hline 17345038 & $-4,487677474$ & 0,000224591 & 0,117799612 & 0,549881852 & Cd2ap & $\mathrm{CD} 2$-associated protein \\
\hline 17515074 & $-4,51117016$ & 0,000211634 & 0,116707116 & 0,465606464 & Icam1 & intercellular adhesion molecule 1 \\
\hline 17516217 & $-4,518129424$ & 0,000209042 & 0,116707116 & 0,331736836 & Olfr920 & olfactory receptor 920 \\
\hline 17346749 & $-4,537261637$ & 0,000202996 & 0,116707116 & 0,601773665 & Rab31 & RAB31, member RAS oncogene family \\
\hline 17265646 & $-4,567629358$ & 0,000194358 & 0,116707116 & 0,603423401 & Tekt1 & tektin 1 \\
\hline 17286998 & $-4,573030215$ & 0,000192198 & 0,116707116 & 0,384288188 & $\mathrm{Rbm} 24$ & RNA binding motif protein 24 \\
\hline 17283445 & $-4,58655048$ & 0,000184424 & 0,116707116 & 0,661719589 & Lgmn & legumain \\
\hline 17224587 & $-4,592282037$ & 0,00018356 & 0,116707116 & 0,529509117 & Dnpep & aspartyl aminopeptidase \\
\hline 17288716 & $-4,857157317$ & 0,000122661 & 0,096505067 & 0,417057385 & Glrx & glutaredoxin \\
\hline 17263511 & $-4,878684126$ & 0,00011791 & 0,096074093 & 0,271489219 & Rasd1 & RAS, dexamethasone-induced 1 \\
\hline 17347267 & $-4,892003271$ & 0,000115751 & 0,096074093 & 0,569578354 & Fez2 & fasciculation and elongation protein zeta 2 (zygin II) \\
\hline 17364932 & $-4,893219997$ & 0,000114887 & 0,096074093 & 0,459150059 & Got1 & glutamic-oxaloacetic transaminase 1 , soluble \\
\hline 17508609 & $-4,942716978$ & 0,000106249 & 0,096074093 & 0,441015297 & Nrg1 & neuregulin 1 \\
\hline 17483385 & $-4,955833031$ & 0,000104089 & 0,096074093 & 0,445527753 & Phkg2 & phosphorylase kinase, gamma 2 (testis) \\
\hline 17486099 & $-4,959257549$ & 0,000103225 & 0,096074093 & 0,490802608 & Zim1 & zinc finger, imprinted 1 \\
\hline 17219139 & $-5,100936289$ & $8,98364 \mathrm{E}-05$ & 0,096074093 & 0,513086673 & Rgs5 & regulator of G-protein signaling 5 \\
\hline 17497769 & $-5,248765774$ & 7,51516E-05 & 0,096074093 & 0,550423956 & Rnh1 & ribonuclease/angiogenin inhibitor 1 \\
\hline 17519649 & $-5,37953875$ & $6,60816 \mathrm{E}-05$ & 0,096074093 & 0,483671338 & Gsta4 & glutathione S-transferase, alpha 4 \\
\hline 17424298 & $-5,623221226$ & 4,83734E-05 & 0,096074093 & 0,609742159 & Dctn3 & dynactin 3 \\
\hline 17274184 & $-6,947647979$ & 1,33891E-05 & 0,088009903 & 0,33593012 & Socs 2 & suppressor of cytokine signaling 2 \\
\hline
\end{tabular}


Supplementary Figure S1. Lungs of surviving, adult HRAS/NRAS-DKO mice show partial atelectasis.

A
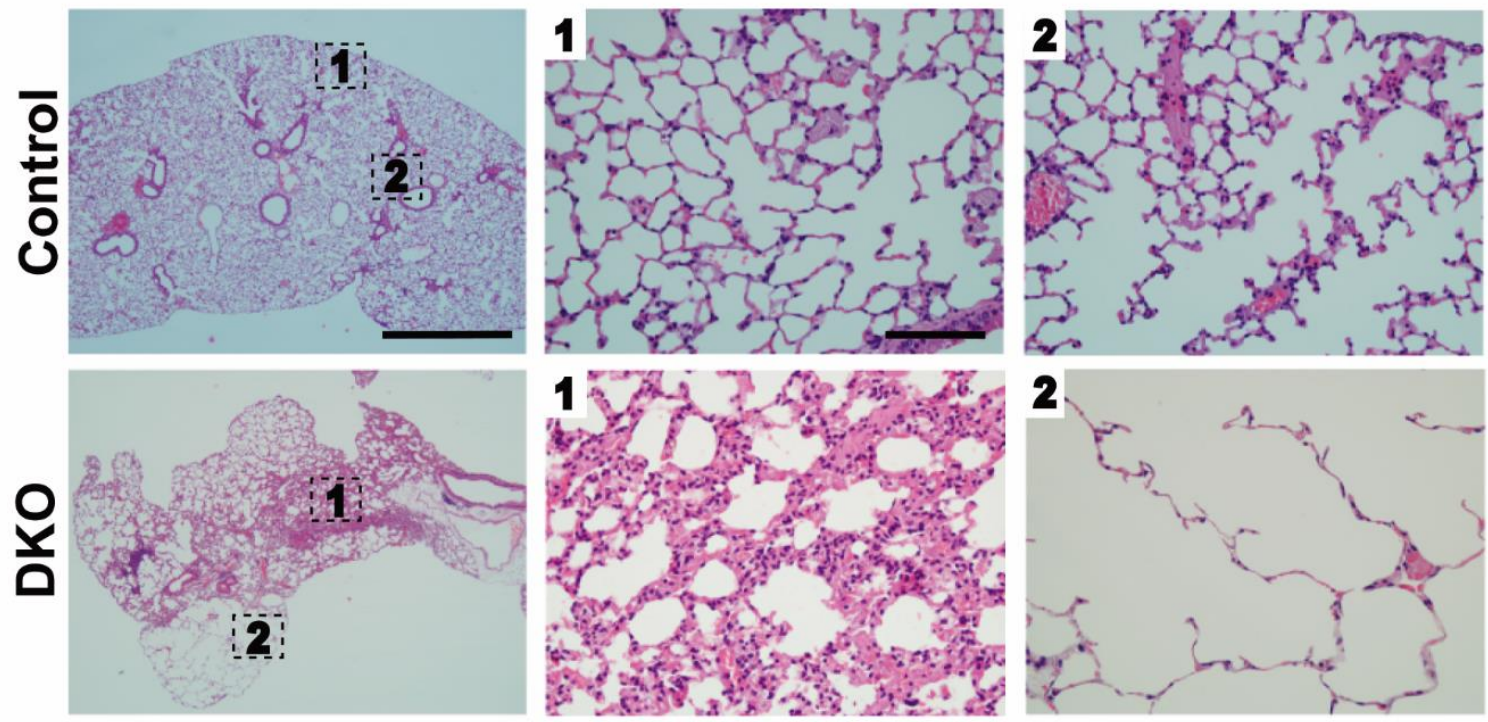

B

C

SftpC RCA-I DAPI

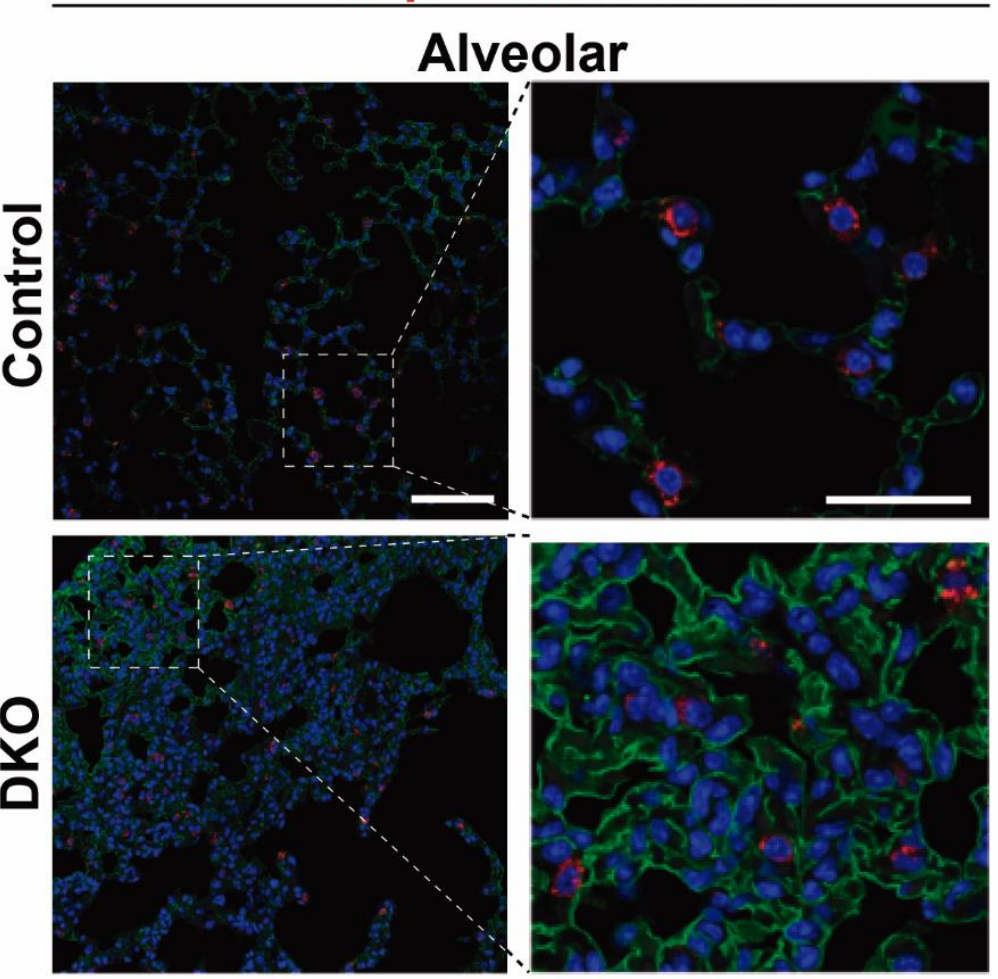

Scgb $\beta$-Tub DAPI
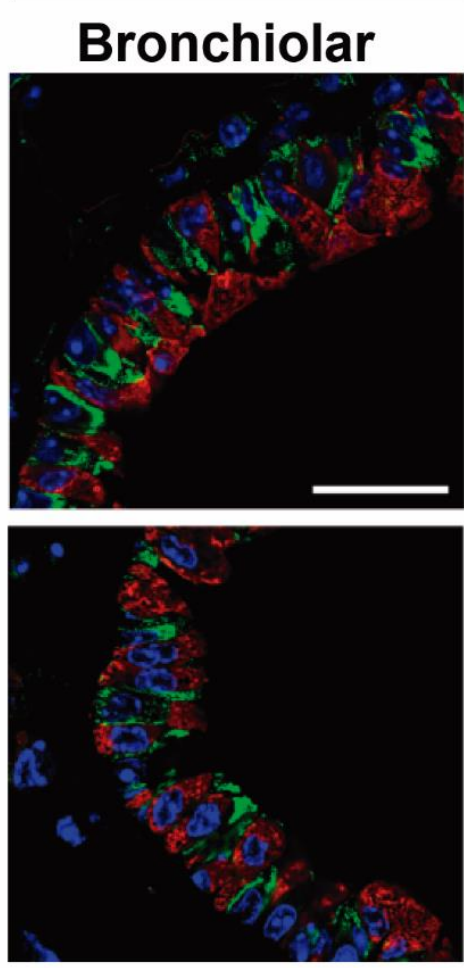
Supplementary Figure S2. Increased neutrophil infiltration in the lungs of HRAS/NRAS-DKO mice.

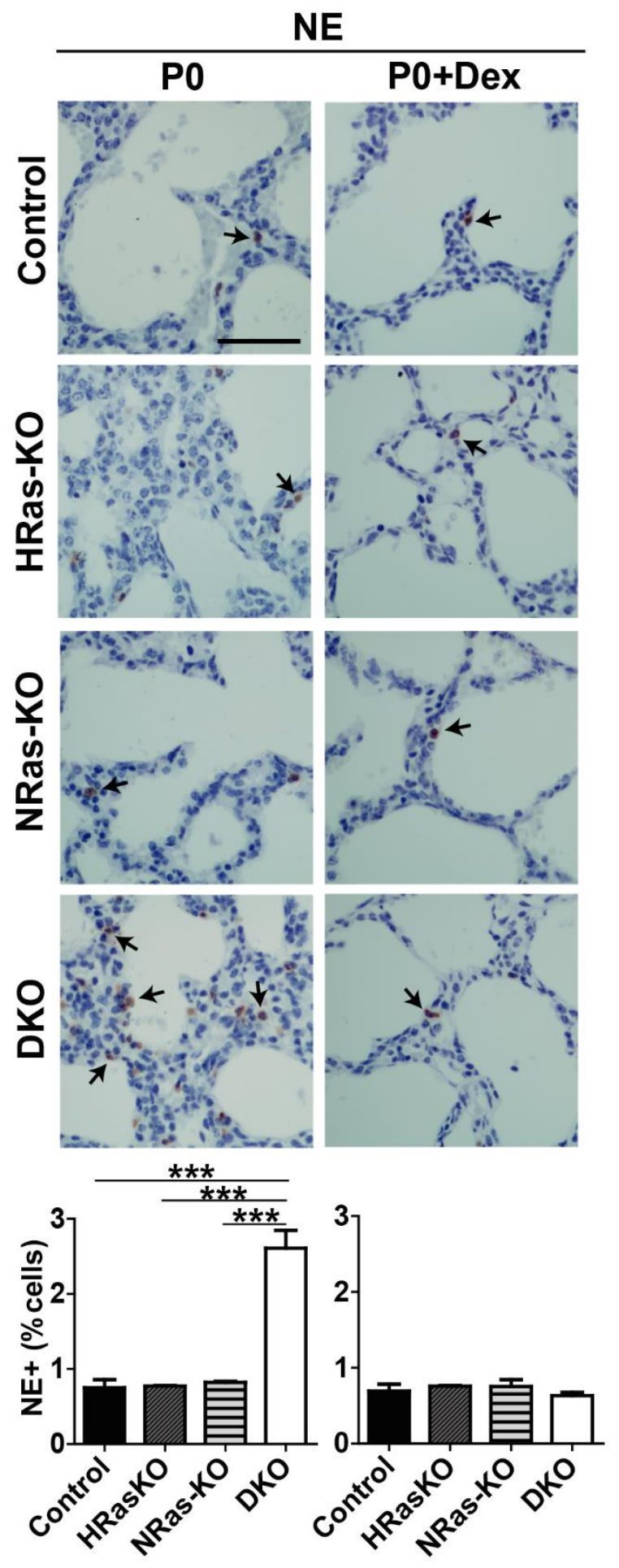


Supplementary Figure S3. Components of sphingolipid metabolic pathways that are differentially expressed in the lungs of HRAS/NRAS-DKO mice.

\section{Sphingolipid metabolism in HRAS/NRAS-DKO lungs}

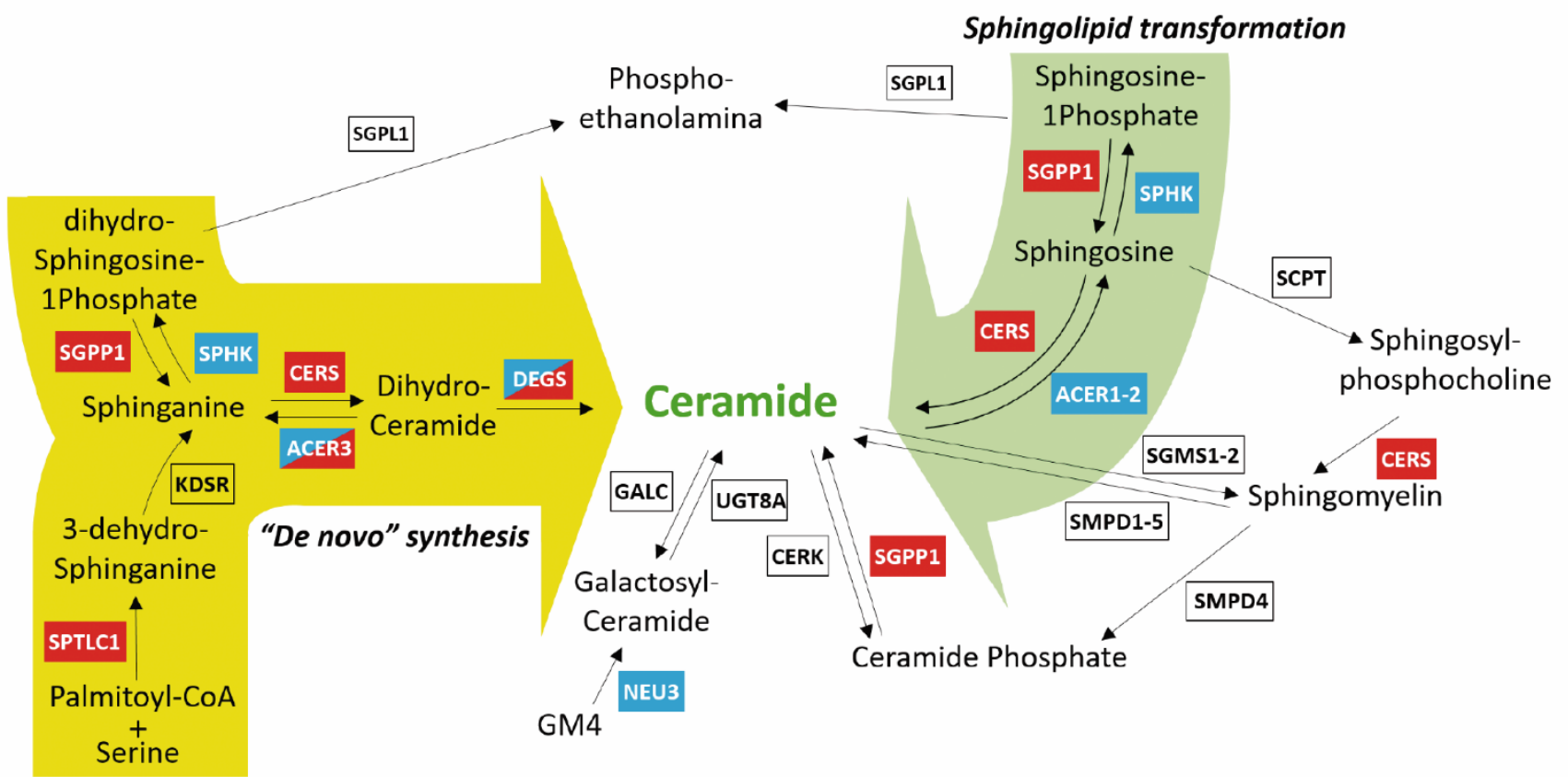

